U.S. Department of the Interior

U.S. Geological Survey

\title{
Water-Quality Data (July 1994 through September 1996) and Statistical Summaries of Data for Surface Water in the Sand Coulee Coal Area, Montana
}

By Philip L. Karper

Open-File Report 98-94

In cooperation with the MONTANA DEPARTMENT OF ENVIRONMENTAL QUALITY 
U.S. Department of the Interior

BRUCE BABBITT, Secretary

\section{U.S. Geological Survey}

Thomas J. Casadevall, Acting Director

Helena, Montana

May 1998

For additional information write to:

District Chief

U.S. Geological Survey

Federal Building, Drawer 10076

Helena, MT 59626-0076

Copies of this report may be purchased from:

U.S. Geological Survey

Branch of Information Services

Box 25286

Denver, CO 80225-0286 


\section{CONTENTS}

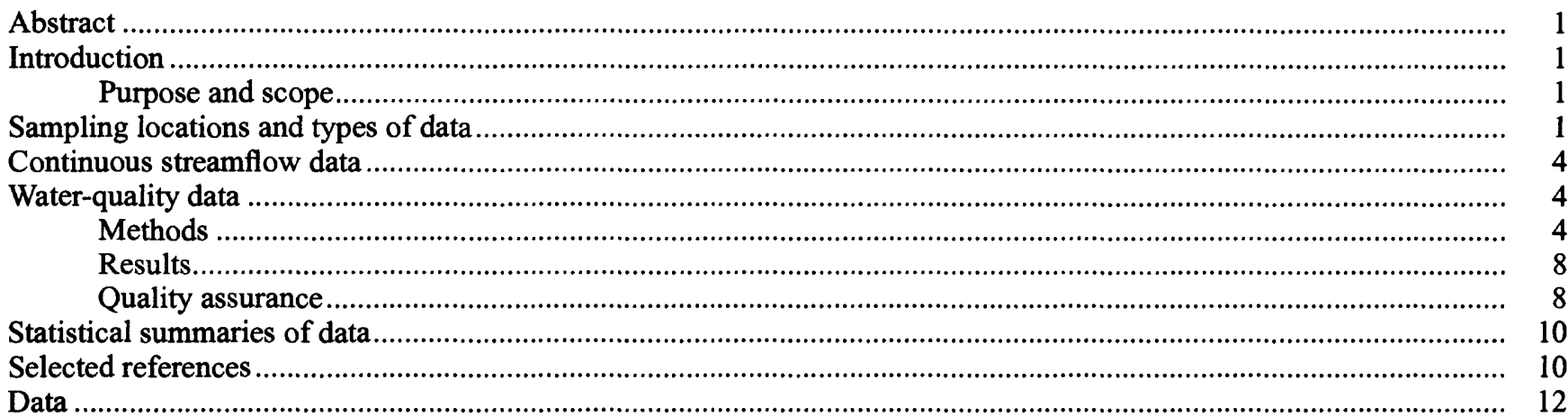

\section{ILLUSTRATIONS}

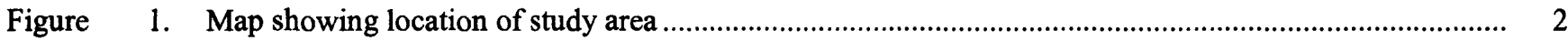

2. Map showing location of sampling sites in the Sand Coulee Creek basin, Montana ................................ 3

3. Map showing location of sampling sites in the Belt Creek basin, Montana ................................................ 4

4.-8. Hydrographs showing daily mean discharge for:

4. Sand Coulee Creek above Cottonwood Creek at Centerville, Montana ........................................... 6

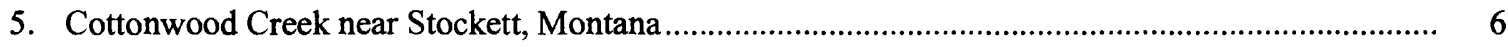

6. Number Five Coulee below Giffen Spring near Stockett, Montana .................................................... 7

7. Sand Coulee at Sand Coulee, Montana .................................................................................... 7

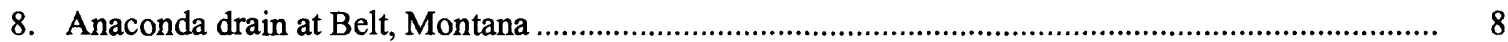

\section{TABLES}

Table 1. Type and period of data collection at sampling sites in the Sand Coulee Coal Area, Montana .................... 5

2. Properties and dissolved constituents analyzed in water samples from sites in the Sand Coulee Coal Area, Montana

3. Water-quality data for sites in the Sand Coulee Coal Area, Montana, July 1994 through September 1996.
4. Chemical analyses of field replicates for water samples from sites in the the Sand Coulee Coal Area, Montana, July 1994 through September 1996.

5. Chemical analyses of field blanks for water samples

6. Statistical summary of water-quality data for sites in the Sand Coulee Coal Area, Montana, July 1994 


\section{CONVERSION FACTORS AND ABBREVIATED WATER-QUALITY UNITS}

\begin{tabular}{rll}
\hline Multiply & By & To obtain \\
\hline cubic foot per second (ft $\left.\mathrm{ft}^{3} / \mathrm{s}\right)$ & 0.028317 & cubic meter per second \\
gallon $(\mathrm{gal})$ & 3.785 & liter $(\mathrm{L})$ \\
mile $(\mathrm{mi})$ & 1.609 & kilometer \\
acre-foot (acre-ft) & 1,233 & cubic meter $\left(\mathrm{m}^{3}\right)$ \\
ton per day (ton/d) & 907.2 & kilogram per day \\
\hline
\end{tabular}

Temperature can be converted from degrees Celsius $\left({ }^{\circ} \mathrm{C}\right)$ to degrees Fahrenheit $\left({ }^{\circ} \mathrm{F}\right)$ by the equation:

${ }^{\circ} \mathrm{F}=9 / 5\left({ }^{\circ} \mathrm{C}\right)+32$

Abbreviated water-quality units used in this report:

$\mu \mathrm{g} / \mathrm{L} \quad$ micrograms per liter

$\mu \mathrm{m} \quad$ micrometer

$\mu \mathrm{S} / \mathrm{cm}$ microsiemens per centimeter at 25 degrees Celsius

$\mathrm{mg} / \mathrm{L}$ milligrams per liter

Water-year definition:

A water year is the 12-month period from October 1 through September 30. It is designated by the calendar year in which it ends.

Chemical concentration in water is reported in milligrams per liter $(\mathrm{mg} / \mathrm{L})$ or micrograms per liter $(\mu \mathrm{g} / \mathrm{L})$. Milligrams per liter is a unit expressing the solute mass (milligram) per unit volume (liter) of water and is about the same as parts per million unless concentrations are more than 7,000 milligrams per liter (Hem, 1989, p. 55). One thousand micrograms per liter is equivalent to 1 milligram per liter. 


\title{
WATER-QUALITY DATA (JULY 1994 THROUGH SEPTEMBER 1996) AND STATISTICAL SUMMARIES OF DATA FOR SURFACE WATER IN THE SAND COULEE COAL AREA, MONTANA
}

\author{
By Philip L. Karper
}

\section{Abstract}

Water was sampled from sites in abandoned coalmine areas in the drainages of Sand Coulee Creek and Belt Creek, southeast of Great Falls, to obtain baseline data for the evaluation of the effects of ongoing and future remedial activities. Water-quality and streamflow data were collected periodically at 27 sites during July 1994 through September 1996. Water-quality data include laboratory concentrations of major ions and trace elements, and field measurements of $\mathrm{pH}$ and specific conductance. Quality-assurance data and statistical summaries are reported for the analytical results.

\section{INTRODUCTION}

The Sand Coulee Coal Area of the Great Falls Coal Field lies southeast of Great Falls in Cascade County, Montana (fig. 1). Sand Coulee Creek and Belt Creek are two major drainages in this area. The study area lies within the Sand Coulee Coal Area in drainages of the heavily mined zones in the vicinity of Belt, Sand Coulee, Tracy, Centerville, and Stockett, Montana. Coal beds in the upper part of the Morrison Formation (Jurassic age) were first mined in 1876 (Fisher, 1909). Coal was extensively mined from about 1889 to 1930 and most mines were abandoned by 1950 . Acidic water containing high concentrations of metals has discharged from much of the mined area since abandonment of the underground coal mines.

The Mine Waste Cleanup Bureau of the Montana Department of Environmental Quality (formerly the Abandoned Mines Reclamation Bureau of the Montana Department of State Lands) has been active in reclamation of the study area since 1979. Considerable progress has been made in reclamation of mine dumps and documentation of sources of acid-mine drainage. Some progress also has been made on treatment of mine drainage. However, acid mine drainage into local streams continues to be a major water-quality problem in the area.
The Montana Department of Environmental Quality initiated a sampling program to develop a longterm database that can be used to detect trends over time in order to evaluate the effectiveness of remediation projects. As part of that program, water-quality data were collected by the U.S. Geological Survey (USGS) in cooperation with the Montana Department of Environmental Quality at selected sites.

\section{Purpose and Scope}

The purpose of this report is to present waterquality data for 27 sites in the abandoned coal-mine areas in the drainages of Sand Coulee Creek and Belt Creek collected from July 1994 through September 1996. Quality-assurance data and statistical summaries for the data also are presented.

\section{SAMPLING LOCATIONS AND TYPES OF DATA}

Most of the sampling stations in the basins of Sand Coulee Creek (fig. 2) and Belt Creek (fig. 3) are outflows from mine adits where ground water discharges to the surface. At several sites the discharging ground water mixes with surface water only during periods of precipitation runoff. Several sites are located on streams, only one of which flows perennially. Others flow intermittently. Nine sites were sampled upstream and downstream of constructed wetlands which were developed as a remediation effort. Outflow was absent at several of the wetlands because of small inflows and evaporation of the ponded water. A list of stations and type and period of data collection are given in table 1 .

Quality assurance of data was maintained through the use of documented procedures designed to provide environmentally representative data. Acceptable performance of the procedures was verified with qualitycontrol samples that were collected systematically to provide a measure of the accuracy, precision, and bias of the environmental data and to identify problems associated with sampling, processing, or analysis. 

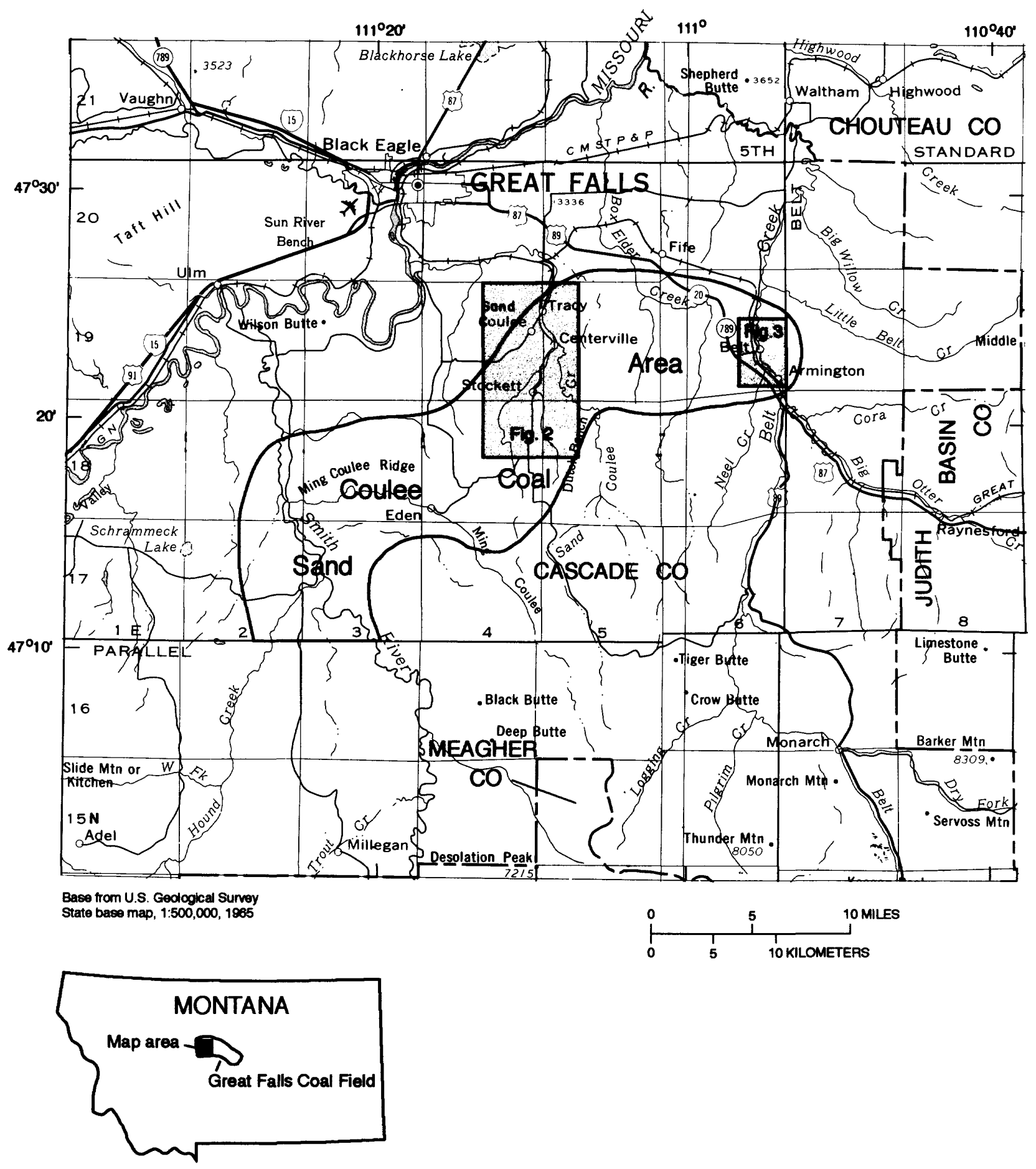

Figure 1. Location of study area. 


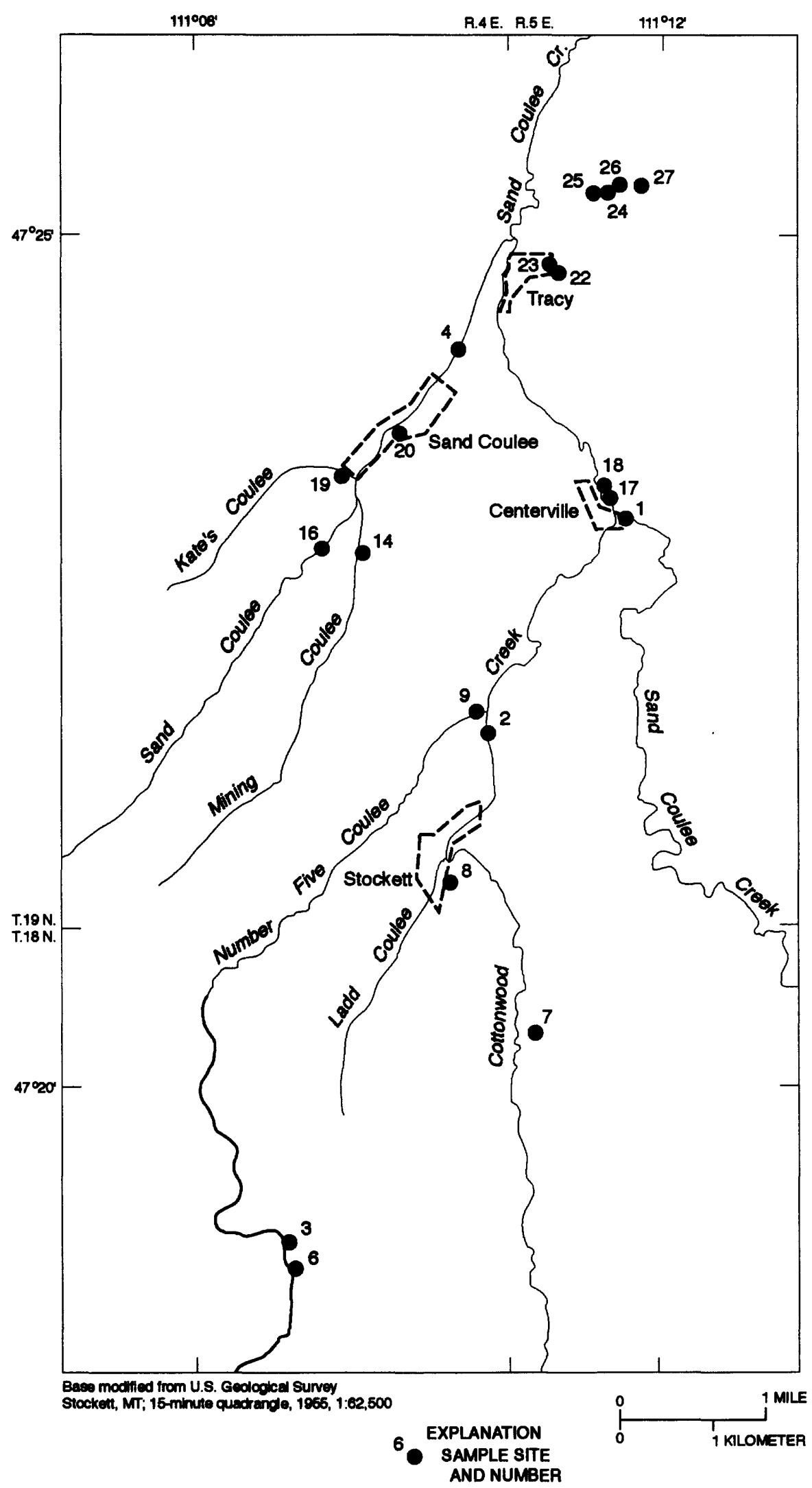

Figure 2. Location of sampling sites in the Sand Coulee Creek basin, Montana. 


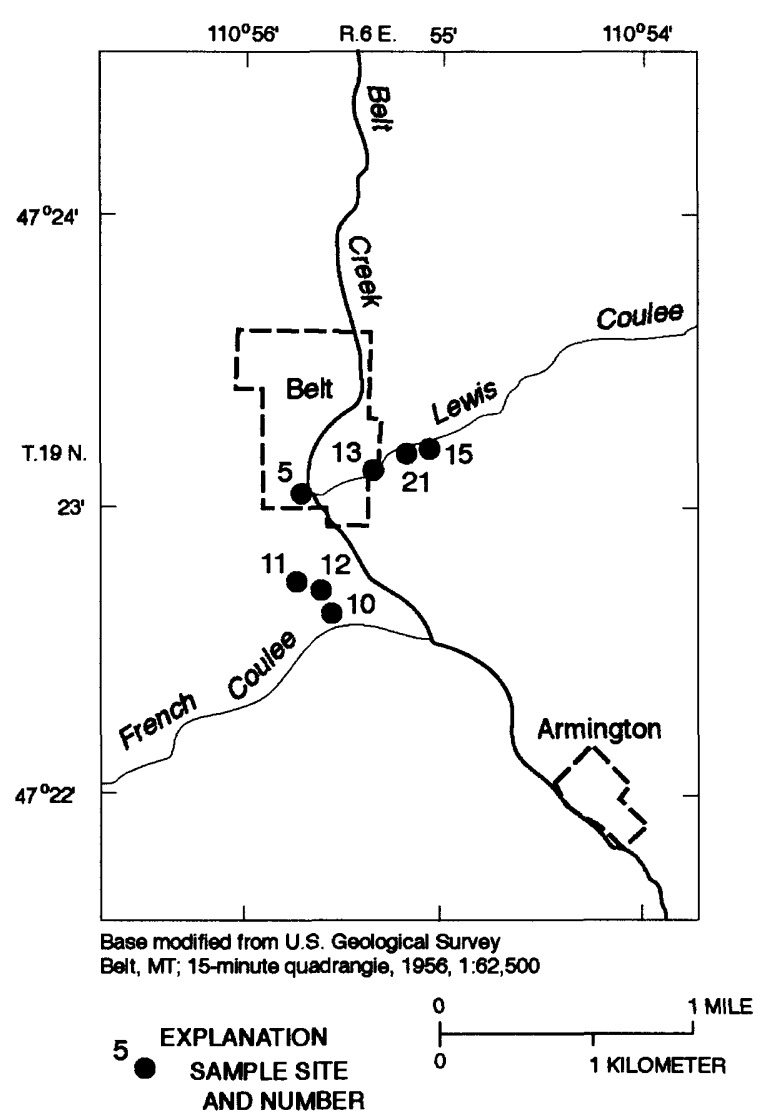

Figure 3. Location of sampling sites in the Belt Creek basin, Montana.

\section{CONTINUOUS STREAMFLOW DATA}

Continuous streamflow records are available for five of the sampling sites for this project. Stream gages were located on four of the major drainages in the Sand Coulee Creek basin (sites 1-4) and on Anaconda drain in the Belt Creek basin (site 5). Flow was intermittent for the 2-year period that the stream gages were operated on Sand Coulee Creek, Cottonwood Creek, and Sand Coulee. Water flowed perennially at the gages on Number Five Coulee below Giffen Spring, near Stockett and Anaconda drain at Belt.

Discharge measurements were made periodically to develop stage-discharge relation ratings. These ratings were applied to the continuous stage records to compute daily mean discharges for each of the gaging stations according to methods described by Rantz and others (1982). Hydrographs for each of the five continuous streamflow-gaging stations are presented in figures 4-8.

\section{WATER-QUALITY DATA}

Water-quality data consist of measurements of physical properties and concentrations of chemical constituents analyzed in water samples. Samples were collected 25 times (approximately once-monthly) during the July 1994 through September 1996 period, except where sites were dry during the times of sampling visits. Site 7, Cottonwood Mine No. 6 drain to Cottonwood Creek near Stockett, was added in February 1995. Flow through the French Coulee wetland ponds was shut off and diverted in March 1996, after which samples were collected from French Coulee wetlands inflow No. 2 at Belt (site 12).

\section{Methods}

Because of the small flows and shallow depths at the sites, grab samples were collected using 2-liter acid-rinsed polyethylene bottles. Onsite measurements of specific conductance, water temperature, and $\mathrm{pH}$ were made during collection of periodic water-quality samples. Onsite sample filtration for dissolved 
Table 1. Type and period of data collection at sampling sites in the Sand Coulee Coal Area, Montana

[Station number: Eight-digit numbers represent standard USGS numbering system for streamflow-gaging stations in downstream order. Fifteen-digit numbers represent approximate latitude and longitude of the site, plus a sequence number. Symbol: --, no data]

\begin{tabular}{|c|c|c|c|c|}
\hline $\begin{array}{c}\text { Site no. } \\
\text { (fig. } 2,3 \text { ) }\end{array}$ & Station number & Station name & $\begin{array}{l}\text { Continuous } \\
\text { record } \\
\text { streamflow }\end{array}$ & $\begin{array}{l}\text { Periodlc } \\
\text { water } \\
\text { quality }\end{array}$ \\
\hline 1 & 06078230 & Sand Coulee Creek above Cottonwood Creek, at Centerville & $10 / 94-09 / 96$ & $07 / 94-09 / 96$ \\
\hline 2 & 06078250 & Cottonwood Creek near Stockett & $10 / 94-09 / 96$ & 07/94-09/96 \\
\hline 3 & 06078260 & Number Five Coulee below Giffen Spring, near Stockett & $10 / 94-09 / 96$ & 07/94-09/96 \\
\hline 4 & 06078270 & Sand Coulee at Sand Coulee & $10 / 94-09 / 96$ & 07/94-09/96 \\
\hline 5 & 06090590 & Anaconda drain at Belt & $10 / 94-09 / 96$ & 07/94-09/96 \\
\hline 6 & 471851111111101 & Giffen Spring near Stockett & -- & 07/94-09/96 \\
\hline 7 & 472016111085701 & Cottonwood Mine No. 6 drain to Cottonwood Creek near Stockett & - & $02 / 95-09 / 96$ \\
\hline 8 & 472114111095001 & Cottonwood Mine No. 2 drain to Ladd Coulee at Stockett & -- & 07/94-09/96 \\
\hline 9 & 472212111093301 & Number Five Coulee near Stockett & -- & $07 / 94-09 / 96$ \\
\hline 10 & 472233110552601 & French Coulee wetlands outflow at Belt & -- & 07/94-04/96 \\
\hline 11 & 472235110553201 & French Coulee wetlands inflow at Belt & -- & 07/94-02/96 \\
\hline 12 & 472235110553202 & French Coulee wetlands inflow No. 2 at Belt & -- & 04/96-09/96 \\
\hline 13 & 472305110551701 & Lewis Coulee above Castner Park, at Belt & -- & $07 / 94-09 / 96$ \\
\hline 14 & 472306111103601 & Mine drain to Mining Coulee near Sand Coulee & -- & 07/94-09/96 \\
\hline 15 & 472310110550801 & Lewis Coulee above mine adit, at Belt & - & 07/94-09/96 \\
\hline 16 & 472313111104901 & Mine drain to Sand Coulee near Sand Coulee & -- & 07/94-09/96 \\
\hline 17 & 472330111082801 & Centerville wetlands inflow at Centerville & -- & 07/94-09/96 \\
\hline 18 & 472331111083001 & Centerville wetlands outflow at Centerville & -- & 07/94-09/96 \\
\hline 19 & 472334111104401 & Mount Oregon Mine drain to Kate's Coulee at Sand Coulee & -- & 07/94-09/96 \\
\hline 20 & 472346111102401 & Nelson Mine drain to Sand Coulee at Sand Coulee & -- & 07/94-09/96 \\
\hline 21 & 472309110551201 & Lewis Coulee below mine adit, at Belt & -- & 07/94-09/96 \\
\hline 22 & 472446111085101 & Pipe spring at Tracy & -- & 07/94-09/96 \\
\hline 23 & 472447111085301 & Stock tank spring at Tracy & -- & 07/94-09/96 \\
\hline 24 & 472513111082501 & Johnson Badwater Mine small wetlands inflow near Tracy & -- & 07/94-09/96 \\
\hline 25 & 472513111082901 & Johnson Badwater Mine large wetlands inflow near Tracy & -- & 07/94-09/96 \\
\hline 26 & 472514111082301 & Johnson Badwater Mine small wetlands outflow near Tracy & -- & 07/94-09/96 \\
\hline 27 & 472517111081001 & Johnson Goodwater Mine small wetlands inflow near Tracy & -- & 07/94-09/96 \\
\hline
\end{tabular}

constituents was performed using a peristaltic pump, silicone tubing, and $0.45-\mu \mathrm{m}$ pore-size cartridge filters. Cross contamination of the filtered samples was prevented by rinsing the silicone tubing with 5-percent hydrochloric acid solution and deionized water and using a new cartridge filter for each sample. Nitric acid preservative was added onsite to the filtered samples. Vinyl gloves were worn during all sample-handling procedures. All field meters were calibrated onsite with standards and results were documented on standard USGS water-quality field forms. Instantaneous streamflow at the time of water sampling was determined at all stations, except for several instances when the discharge could not be determined because reservoir outlets were not accessible. Flow values were determined either by direct measurement or from stage-discharge rating tables (Rantz and others, 1982).

Water samples were analyzed for the dissolved constituents listed in table 2 by the USGS National Water Quality Laboratory (NWQL) in Arvada, Colo. Analytical methods are described by Fishman and Friedman (1989) and Fishman (1993). 


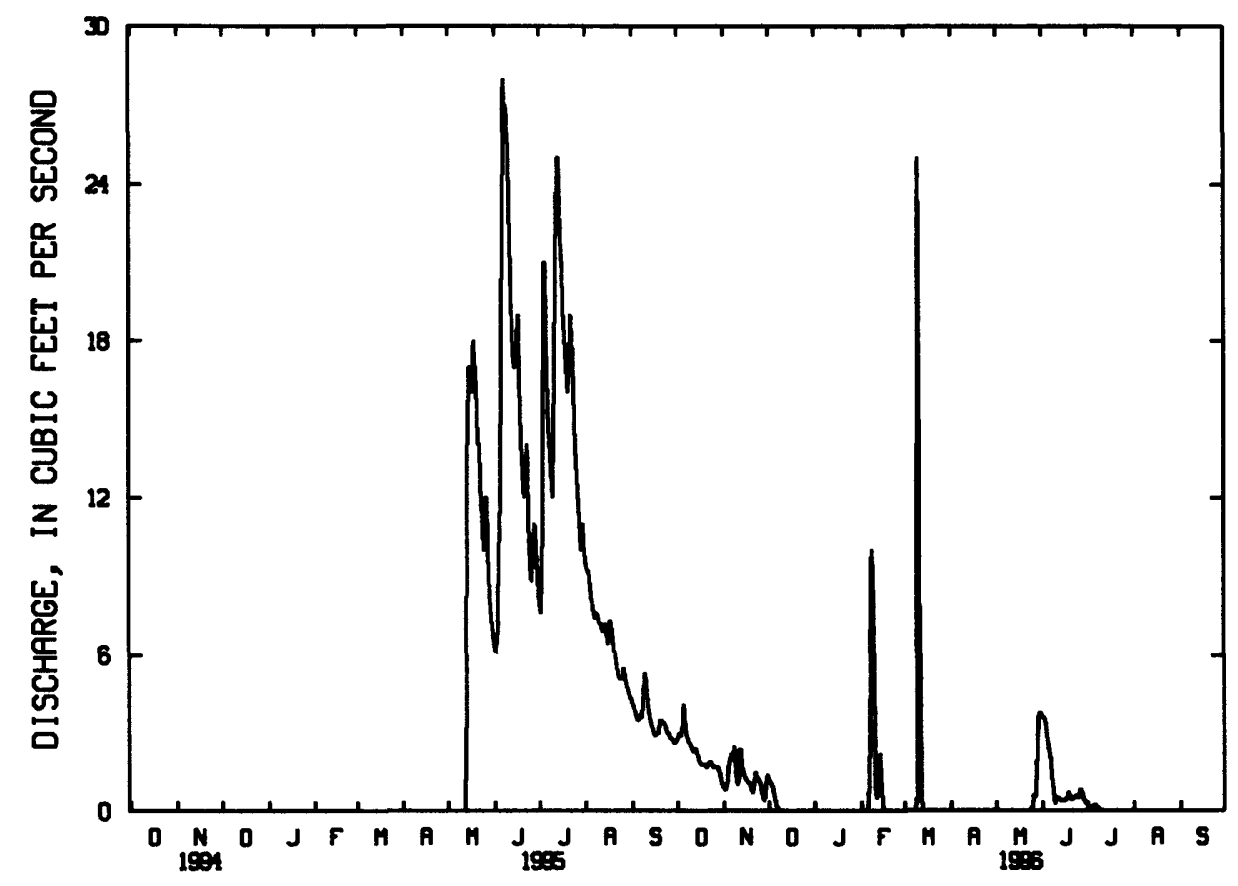

Figure 4. Daily mean discharge for Sand Coulee Creek above Cottonwood Creek, at Centerville, Montana (site 1), water years 1995-96. No flow occurred during several periods between October 1994 and September 1996.

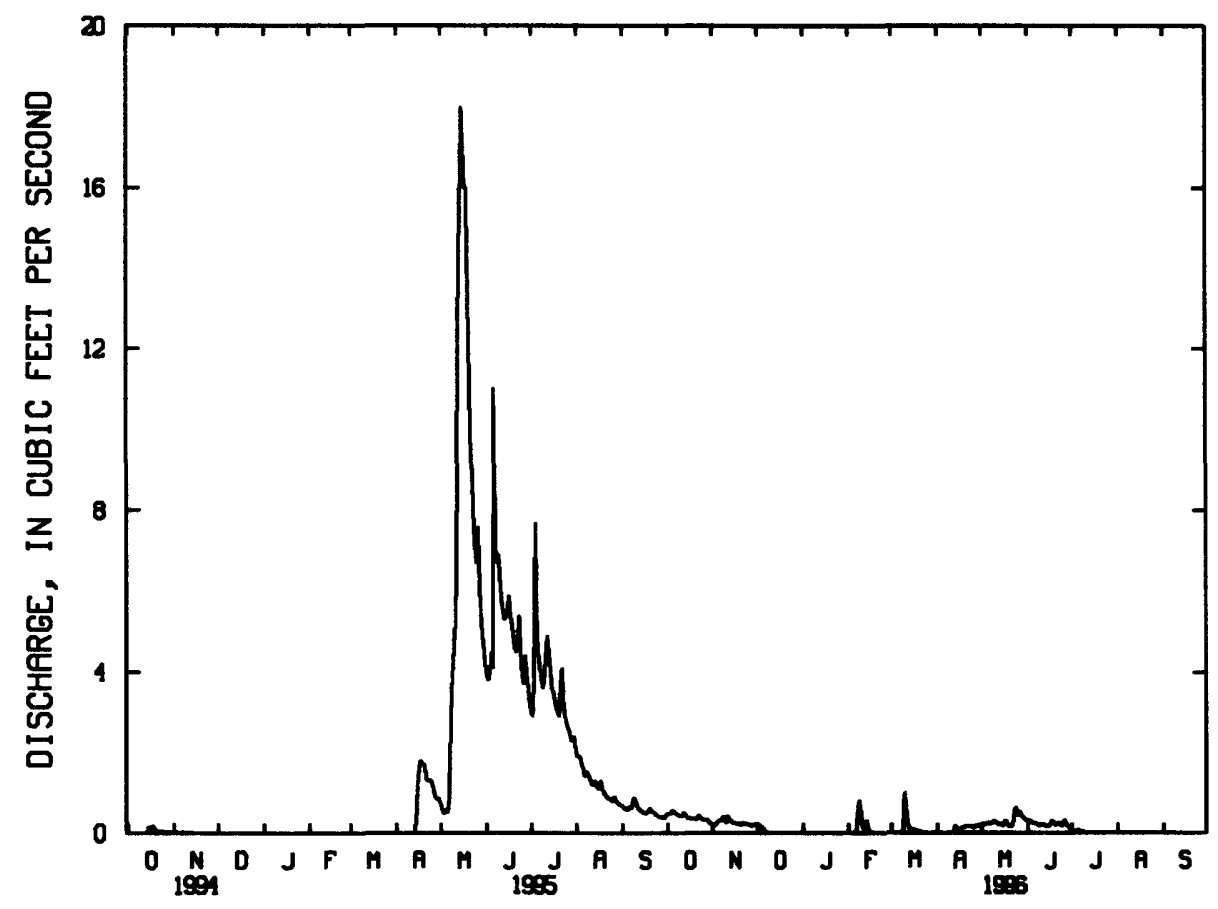

Figure 5. Daily mean discharge for Cottonwood Creek near Stockett, Montana (site 2), water years 1995-96. No flow occurred during several periods between October 1994 and September 1996. 


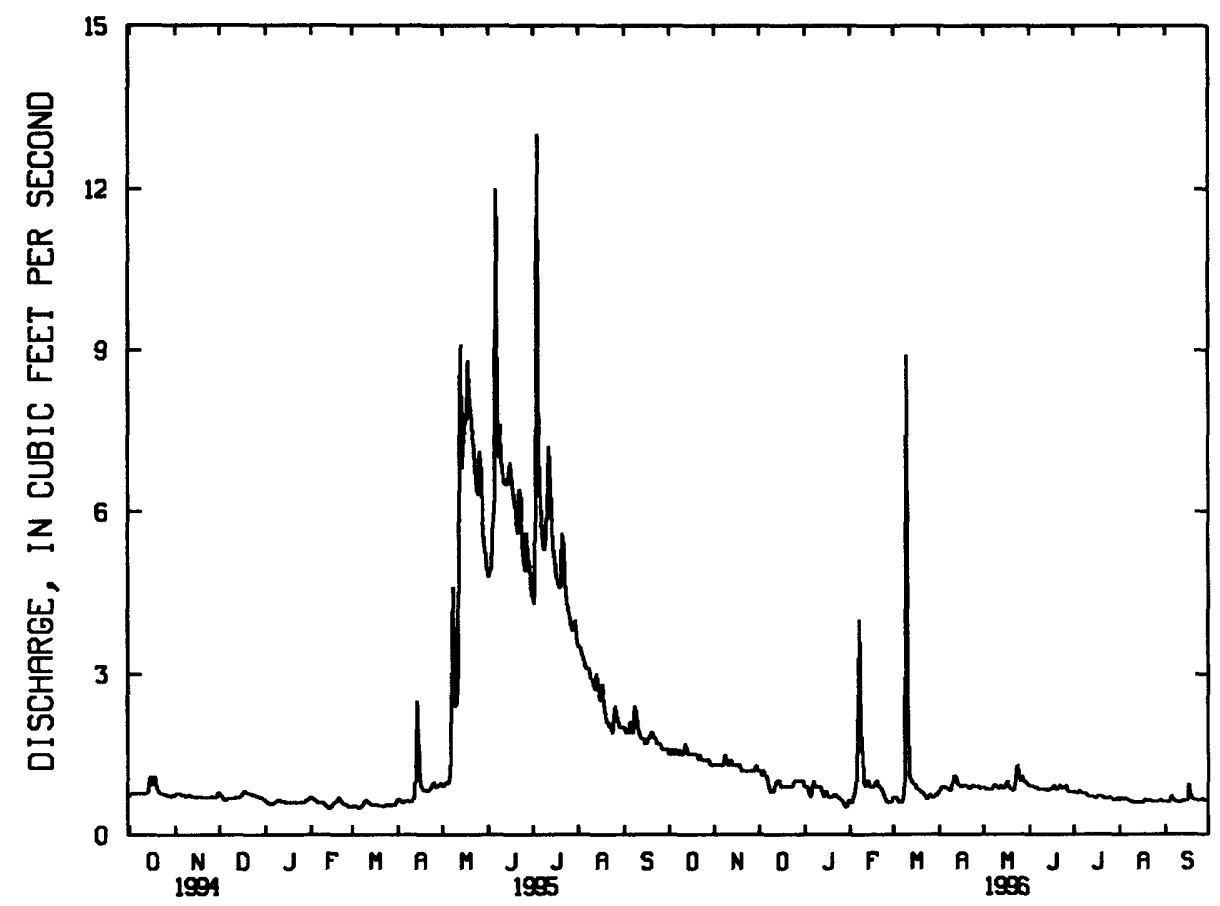

Figure 6. Daily mean discharge for Number Five Coulee below Giffen Spring, near Stockett, Montana (site 3), water years 1995-96.

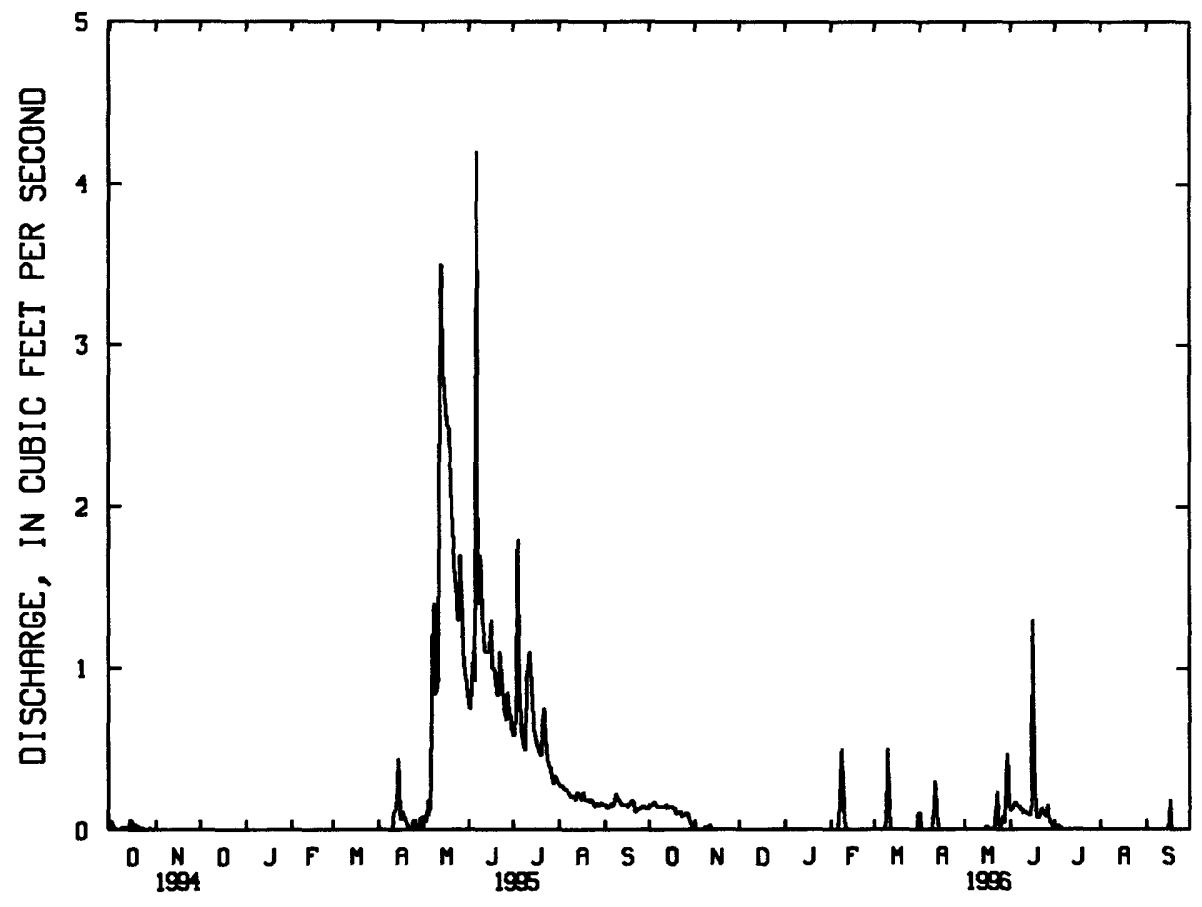

Figure 7. Daily mean discharge for Sand Coulee at Sand Coulee, Montana (site 4), water years 1995-96. No flow occurred during several periods between October 1994 and September 1996. 


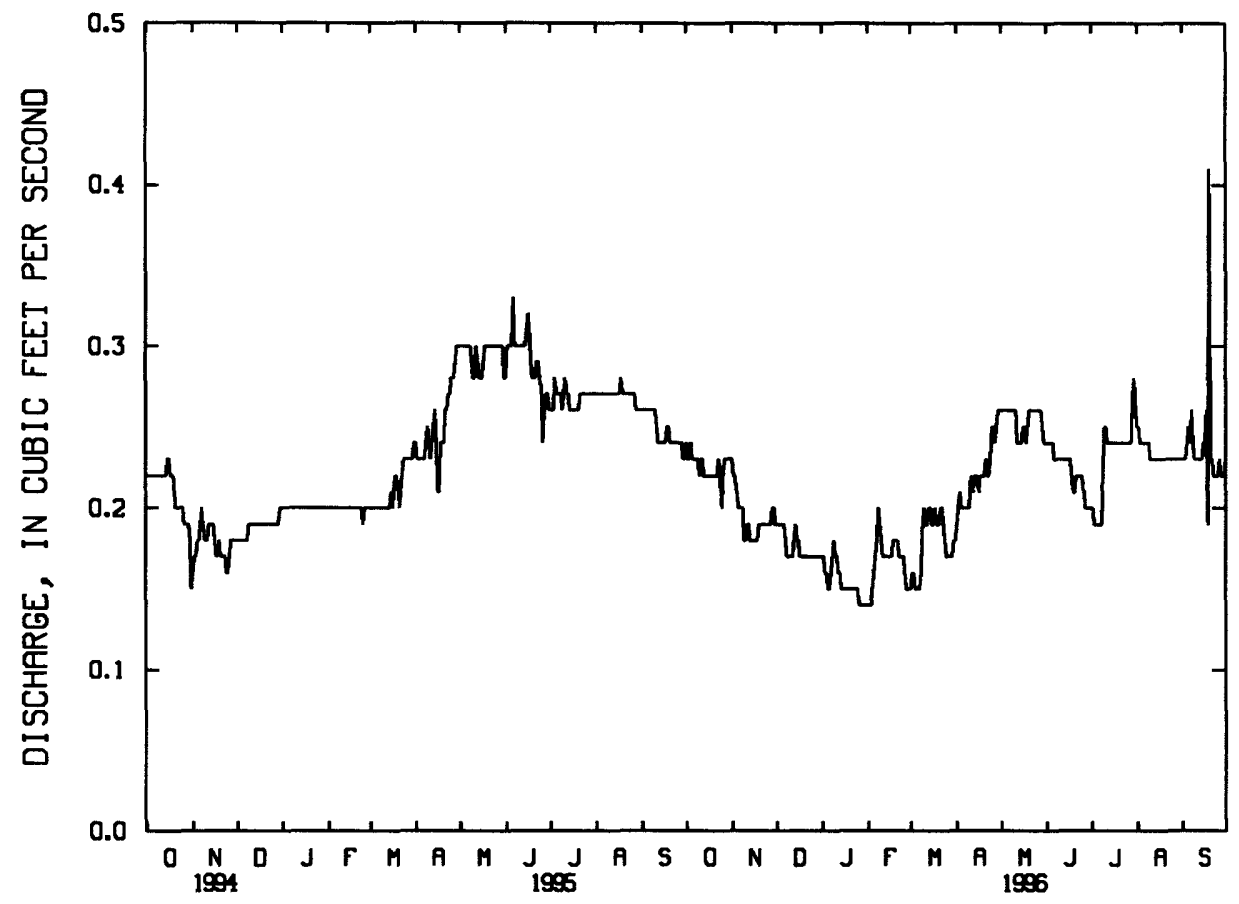

Figure 8. Daily mean discharge for Anaconda drain at Belt, Montana (site 5), water years 1995-96.

\section{Results}

Water-quality data for samples collected periodically during July 1994 through September 1996 are presented in table 3 (at the back of the report). The types of data include instantaneous streamflow, onsite measurements of water-quality properties, and analytical results for dissolved chemical constituents. Selenium analyses were discontinued in July 1995.

The field-determined $\mathrm{pH}$ for many samples was less than 4.5 units, below which alkalinity is negligible or absent. Because of the instability of acid-mine drain water, commonly having high concentrations of ferrous iron which can oxidize over time in the sample bottles, the $\mathrm{pH}$ measured weeks later by the NWQL would decrease and often be less than 4.5. Alkalinity for those samples was not reported by the NWQL. A value for alkalinity as well as uncensored (above detection limit) concentrations for common ions is required in the computer algorithm to calculate a value for dissolved-solids concentration. A value of $0.1 \mathrm{mg} / \mathrm{L}$ was substituted for alkalinity, chloride, and potassium for each sample which did not have a reported or uncensored value to perform the dissolved-solids concentration calculation. Alkalinity values for these samples are stored in the database as an estimated value of less than one $(e<1)$. The dissolved-solids concentrations for these samples are also qualified with an "e" (estimated).

\section{Quality Assurance}

Quality-assurance procedures used for the collection and field processing of water-quality samples are described by Horowitz and others (1994), Ward and Harr (1990), Edwards and Glysson (1988), Knapton and Nimick (1991), and Knapton (1985). Standard procedures used by the NWQL for internal sample handling and quality assurance are described by Friedman and Erdmann (1982), Jones (1987), and Pritt and Raese (1992).

The quality of analytical results reported for water-quality samples was evaluated by quality-control samples that were submitted from the field and analyzed concurrently in the laboratory with routine samples. These quality-control samples consisted of field replicates and blanks, which provide quantitative information on the precision and bias of the overall field and laboratory process. Each type of quality-control sample was submitted at a proportion equivalent to about 5 percent of the total number of water-quality samples. Therefore, the total number of quality-control samples submitted from the field represented about 10 percent of the total number of water-quality samples. 
Table 2. Properties and dissolved constituents analyzed in water samples from sites in the Sand Coulee Coal Area, Montana

[Analytical methods are listed in parentheses to the right of property or constituent]

\begin{tabular}{|c|c|c|}
\hline \multirow{2}{*}{$\begin{array}{l}\text { Physical or chemical property } \\
\text { (analytical method) }\end{array}$} & \multicolumn{2}{|c|}{ Dissolved constituent $^{1}$} \\
\hline & $\begin{array}{c}\text { Major ions } \\
\text { (analytical method) }\end{array}$ & $\begin{array}{c}\text { Trace elements } \\
\text { (analytical method) }\end{array}$ \\
\hline $\begin{array}{l}\text { Streamflow } \\
\text { Specific conductance (meter) } \\
\text { pH (meter) } \\
\text { Temperature } \\
\text { Acidity }\left({ }^{l} \text { ET) }\right. \\
\text { Alkalinity ( }{ }^{\mathrm{ET}} \text { ) } \\
\text { Dissolved solids (calculated) } \\
\text { Hardness (calculated) }\end{array}$ & $\begin{array}{l}\text { Calcium }\left({ }^{2} \mathrm{ICP}\right) \\
\text { Magnesium }\left({ }^{2} \mathrm{ICP}\right) \\
\text { Sodium }\left({ }^{2} \mathrm{ICP}\right) \\
\text { Potassium }\left({ }^{2} \mathrm{ICP}\right) \\
\text { Sulfate }\left({ }^{3} \mathrm{IC}\right) \\
\text { Chloride }\left({ }^{3} \mathrm{IC}\right) \\
\text { Fluoride }(\mathrm{Col} \text { orimetry) } \\
\text { Silica }\left({ }^{2} \mathrm{ICP}\right)\end{array}$ & $\begin{array}{l}\text { Aluminum }\left({ }^{4} \mathrm{DCP}\right) \\
\text { Arsenic }\left({ }^{5} \mathrm{AA}\right) \\
\text { Barium }\left({ }^{2} \mathrm{ICP}\right) \\
\text { Beryllium }\left({ }^{2} \mathrm{ICP}\right) \\
\text { Boron }\left({ }^{4} \mathrm{DCP}\right) \\
\text { Cadmium }\left({ }^{2} \mathrm{ICP} /{ }^{6} \mathrm{GFAA}\right) \\
\text { Chromium }\left({ }^{2} \mathrm{ICP}\right) \\
\text { Cobalt }\left({ }^{2} \mathrm{ICP} / 6 \mathrm{GFAA}\right) \\
\text { Copper }\left({ }^{2} \mathrm{ICP}\right) \\
\text { Iron }\left({ }^{2} \mathrm{ICP}\right) \\
\text { Lead }\left({ }^{2} \mathrm{ICP} /{ }^{6} \mathrm{GFAA}\right) \\
\text { Lithium }\left({ }^{2} \mathrm{ICP}\right) \\
\text { Manganese }\left({ }^{2} \mathrm{ICP}\right) \\
\text { Molybdenum }\left({ }^{2} \mathrm{ICP} /{ }^{6} \mathrm{GFAA}\right) \\
\text { Nickel }\left({ }^{2} \mathrm{ICP}\right) \\
\text { Selenium }\left({ }^{5} \mathrm{AA}\right) \\
\text { Silver }\left({ }^{2} \mathrm{ICP}\right) \\
\text { Strontium }\left({ }^{2} \mathrm{ICP}\right) \\
\text { Vanadium }\left({ }^{2} \mathrm{ICP}\right) \\
\text { Zinc }\left({ }^{2} \mathrm{ICP}\right)\end{array}$ \\
\hline
\end{tabular}

All laboratory analytical methods are referenced from Fishman and Friedman, (1989).

${ }^{1}$ Electrometric titration.

${ }^{2}$ Inductively-coupled argon radiofrequency plasma atomic emission spectrometry.

${ }^{3}$ Ion-exchange chromatography.

${ }^{4}$ Direct-current argon radiofrequency plasma atomic emission spectrometry.

${ }^{5}$ Atomic absorption spectrometry, hydride generation.

${ }^{6}$ Atomic absorption spectrometry, graphite furnace. Analyses for the concentrations of cadmium, cobalt, lead, and molybdenum in samples for all sites collected from July to October 1994 were made by the ICP method. For samples from sites where high iron and aluminum concentrations caused matrix interference, the GFAA method was used for these constituents from November 1994 to September 1996.

In addition to quality-control samples submitted from the field, internal quality-assurance practices at the NWQL are performed systematically to provide quality control of analytical procedures (Pritt and Raese, 1992). These internal practices include analyses of quality-control samples such as calibration standards, standard reference water samples, replicate samples, deionized-water blanks, or spiked samples at a proportion equivalent to at least 10 percent of the sample load. The NWQL participates in a blindsample program in which standard reference water samples prepared by the USGS Branch of Technical Development and Quality Systems are routinely inserted into the sample line for each analytical method at a frequency proportional to the sample load. The laboratory also participates in external evaluation studies twice-yearly with the U.S. Environmental Protection Agency, the Canadian Center for Inland Water, and the Branch of Technical Development and Quality Systems to assess analytical performance.
Replicate samples are two or more samples considered to be essentially identical in composition. Analyses of replicate samples indicate the precision (reproducibility) of results. Precision is affected by numerous sources of variability within the field and laboratory environments, including sample collection, sample processing, and sample analysis. To provide data on precision for samples exposed to all sources of variability combined, replicate samples were obtained in the field by splitting a composite water sample. Analyses of these field replicates indicate the reproducibility of environmental data that are affected by both field and laboratory processes (sample splitting, filtration, preservation, transportation, and laboratory handling). Water-quality data for field replicates are listed in table 4 (at the back of the report).

Blank samples of deionized water were routinely analyzed to identify the presence and magnitude of contamination that potentially could bias analytical 
results. The particular type of blank sample routinely tested was a "field" blank. Field blanks are aliquots of deionized water that are certified as trace-element free and are processed through the sampling equipment used to collect water samples. These blanks are then subjected to the same processing and handling as stream samples. Blank samples are analyzed for the same constituents as those of water samples to identify whether any detectable concentrations were introduced from non-environmental sources. Water-quality data for field blanks are listed in table 5 (at the back of the report).

Most analytical results for blanks were below minimum reporting levels. Of the few detectable concentrations, occurrences were random and values generally were low, indicating no systematic contamination persistent in the process that would bias the results, with two possible exceptions. Occurrences of detectable concentrations of sulfate and silica were common enough and of sufficient magnitude to indicate possible low-level bias at sites where these constituents occur in low concentrations. Although not confirmed, a possible cause for detectable sulfate is carry-over contamination from samples having very high sulfate concentrations. The source of silica is uncertain, but may be partly attributed to borosilicate glass of the ampules containing the nitric acid preservative.

Most of the samples were representative of an acid-mine drainage matrix that contained very high concentrations of dissolved aluminum, iron, and sulfate. High concentrations of these constituents cause analytical interference that limits the ability to accurately quantify concentrations of other, less concentrated, constituents. To compensate for matrix interference, most samples were diluted prior to analysis to decrease the concentration of the interfering constituents and thus allow other constituents to be quantified. Dilution of samples, however, decreases the accuracy of analytical results, especially for lessconcentrated constituents. As concentrations of some of the less-concentrated constituents in the diluted sample aliquots recede to near the detection limit of the analysis, readings are apt to be less accurate and precise. These less-reliable readings are multiplied by the dilution factor, yielding potentially unreliable results for those constituents. Dilution of some sample aliquots also caused the concentration of some constituents to recede below the detection limit, yielding results reported as "less than minimum reporting level." Because the detection limit of the analysis increases proportionally with the dilution factor, the resulting censoring of the value may be at a consider- ably higher level than for an undiluted sample. For example, a two-fold dilution results in a doubling of the minimum reporting level. Greater dilutions result in a correspondingly greater increase in the minimum reporting level. This loss of resolution for low-level concentrations (most notably for chloride, fluoride, and several trace elements) may impair the ability to track changes over time or to assess concentrations relative to water-quality standards.

Lack of analytical accuracy and precision from matrix interference was suspected as the cause of large changes in some trace-metal concentrations in samples for several sites collected during the first few sampling trips. A decision was made to switch from the ICP (inductively-coupled argon radiofrequency plasma atomic emission spectrometry) method of analysis to the graphite furnace (atomic absorption spectrometry) method for cadmium, cobalt, lead, and molybdenum analyses for samples from sites where high iron and aluminum concentrations were present. More consistent values for these constituents appear to have resulted from this change. As a result, some of the values determined by the ICP method from July to October 1994 were considered unreliable.

Where matrix interference resulted in unreliable analytical results, questionable data were deleted if reruns and verification checks could not resolve the uncertainty. For three sites, chloride values were deleted entirely. More expensive custom sample preparation and analysis may be required to provide reliable quantification of low-level concentrations for selected constituents when matrix interference is extensive.

\section{STATISTICAL SUMMARIES OF DATA}

Statistical summaries of water-quality data are provided in table 6 for the period of record since 1994 . Statistical summaries were computed for stations with five or more samples collected during the period of record. For one station, Site 15, Lewis Coulee above mine adit, at Belt, less than five samples were collected and no statistical summaries are computed. The summaries include the period of record; number of samples; and maximum, minimum, mean, and median concentrations.

\section{SELECTED REFERENCES}

Edwards, T.K., and Glysson, G.D., eds., 1988, Field methods for measurement of fluvial sediment: U.S. Geological Survey Open-File Report 86-531, 118 p. 
Fisher, Cassius A., 1909, Geology of the Great Falls Coal Field, Montana: U.S. Geological Survey Bulletin 356, $85 \mathrm{p}$.

Fishman, M.J., 1993, Methods of analysis by the U.S. Geological Survey National Water Quality Laboratory--Determination of inorganic and organic constituents in water and fluvial sediments: U.S. Geological Survey Open-File Report 93-125, 217 p.

Fishman, M.J., and Friedman, L.C., 1989, Methods for determination of inorganic substances in water and fluvial sediments: U.S. Geological Survey Techniques of Water-Resources Investigations, book 5, chap. Al, $709 \mathrm{p}$.

Friedman, L.C., and Erdman, D.E., 1982, Quality assurance practices for the chemical and biological analyses of water and fluvial sediments: U.S. Geological Survey Techniques of Water-Resources Investigations, book 5 , chap. A6, $181 \mathrm{p}$.

Helsel, D.R., and Cohn, T.A., 1988, Estimation of descriptive statistics for multiply censored water quality data: Water Resources Research, v. 24, no. 12, p. 1997-2004.

Hem, J.D., 1989, Study and interpretation of the chemical characteristics of natural water ( $3 \mathrm{~d}$ ed.): U.S. Geological Survey Water-Supply Paper 2254, 263 p.

Horowitz, A.J., Demas, C.R., Fitzgerald, K.K., Miller, T.L., and Rickert, D.A., 1994, U.S. Geological Survey protocol for the collection and processing of surfacewater samples for the subsequent determination of inorganic constituents in filtered water: U.S.

Geological Survey Open-File Report 94-539, 57 p.

Jones, B.E., 1987, Quality control manual of the U.S.

Geological Survey's National Water Quality

Laboratory: U.S. Geological Survey Open-File Report $87-457,17 \mathrm{p}$.

Knapton, J.R., 1985, Field guidelines for collection, treatment, and analysis of water samples, Montana District: U.S. Geological Survey Open-File Report $85-409,86 \mathrm{p}$.

Knapton, J.R., and Nimick, D.A., 1991, Quality assurance for water-quality activities of the U.S. Geological Survey in Montana: U.S. Geological Survey Open-File Report 91-216, 41 p.

Pritt, J.W., and Raese, J.W., eds., 1992, Quality assurance/ quality control manual--National Water Quality Laboratory: U.S. Geological Survey Open-File Report 92-495, $33 \mathrm{p}$.

Rantz, S.E., and others, 1982, Computation of discharge: U.S. Geological Survey Water-Supply Paper 2175, 2 v., $631 \mathrm{p}$.

Ward, J.R., and Harr, C.A., eds., 1990, Methods for collection and processing of surface-water and bedmaterial samples for physical and chemical analyses: U.S. Geological Survey Open-File Report 90-140, 71 p. 


\section{DATA}


Table 3. Water-quality data for sites in the Sand Coulee Coal Area, Montana, July 1994 through September 1996

[Site number shown in figure 2 or 3 . Abbreviations: $\mathrm{ft}^{3} / \mathrm{s}$, cubic feet per second; ${ }^{\circ} \mathrm{C}$, degrees Celsius; e, estimated; lab, laboratory; $\mu \mathrm{g} / \mathrm{L}$, micrograms per liter; $\mu \mathrm{S} / \mathrm{cm}$, microsiemens per centimeter at $25^{\circ} \mathrm{C} ; \mathrm{mg} / \mathrm{L}$, milligrams per liter; ton/acre-ft, tons per acre-foot, ton/d, tons per day. Symbols: $<$, less than minimum reporting level; --, no data]

SITE 1, 06078230--SAND COULEE CREEK ABOVE COTTONWOOD CREEK, AT CENTERVILLE, MT

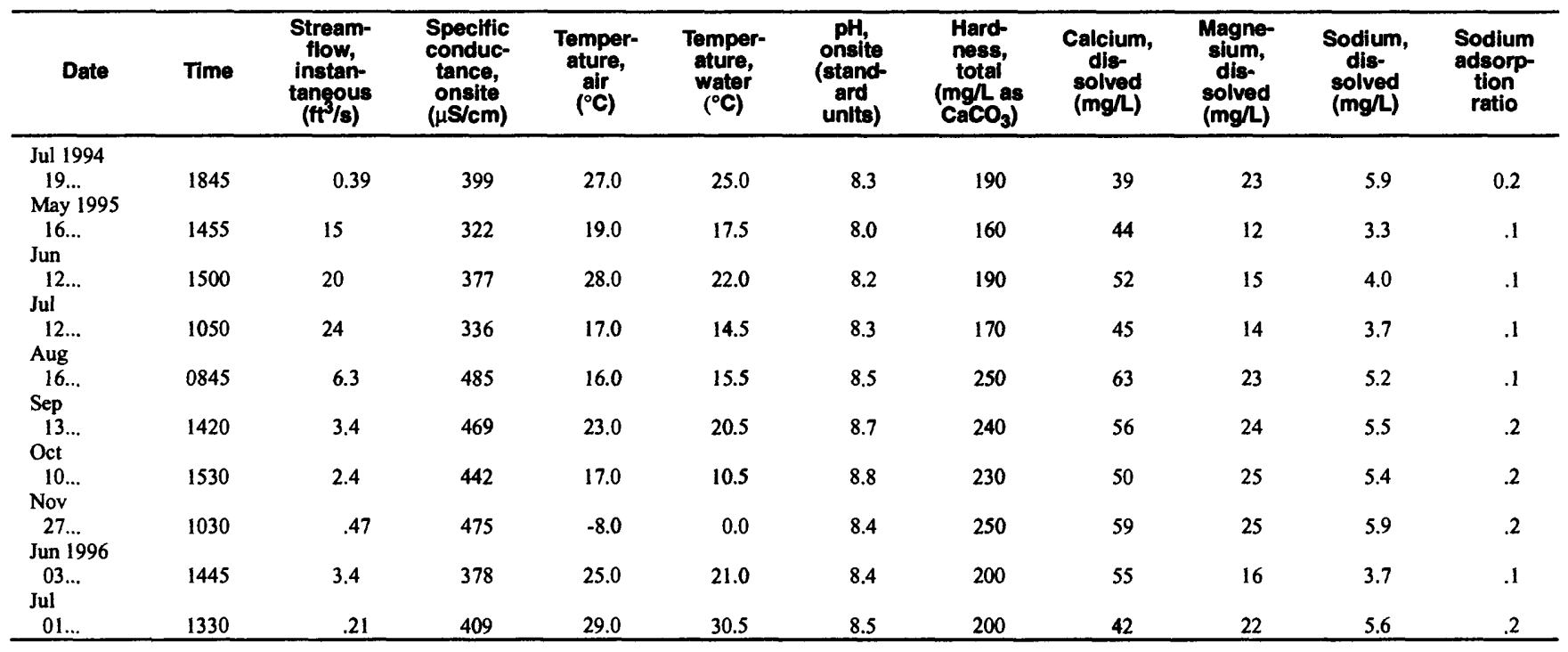

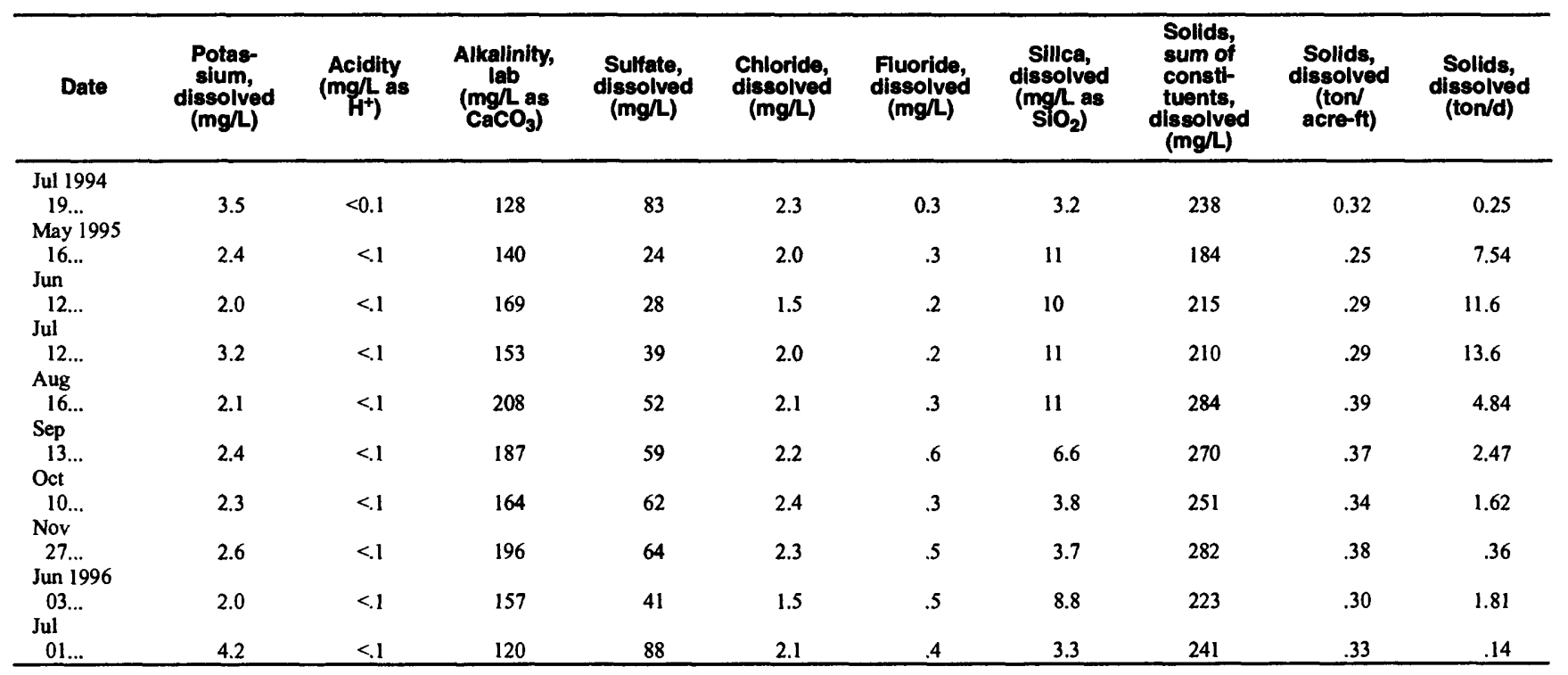


Table 3. Water-quality data for sites in the Sand Coulee Coal Area, Montana, July 1994 through September 1996 (Continued) SITE 1, 06078230--SAND COULEE CREEK ABOVE COTTONWOOD CREEK, AT CENTERVILLE, MT--Continued

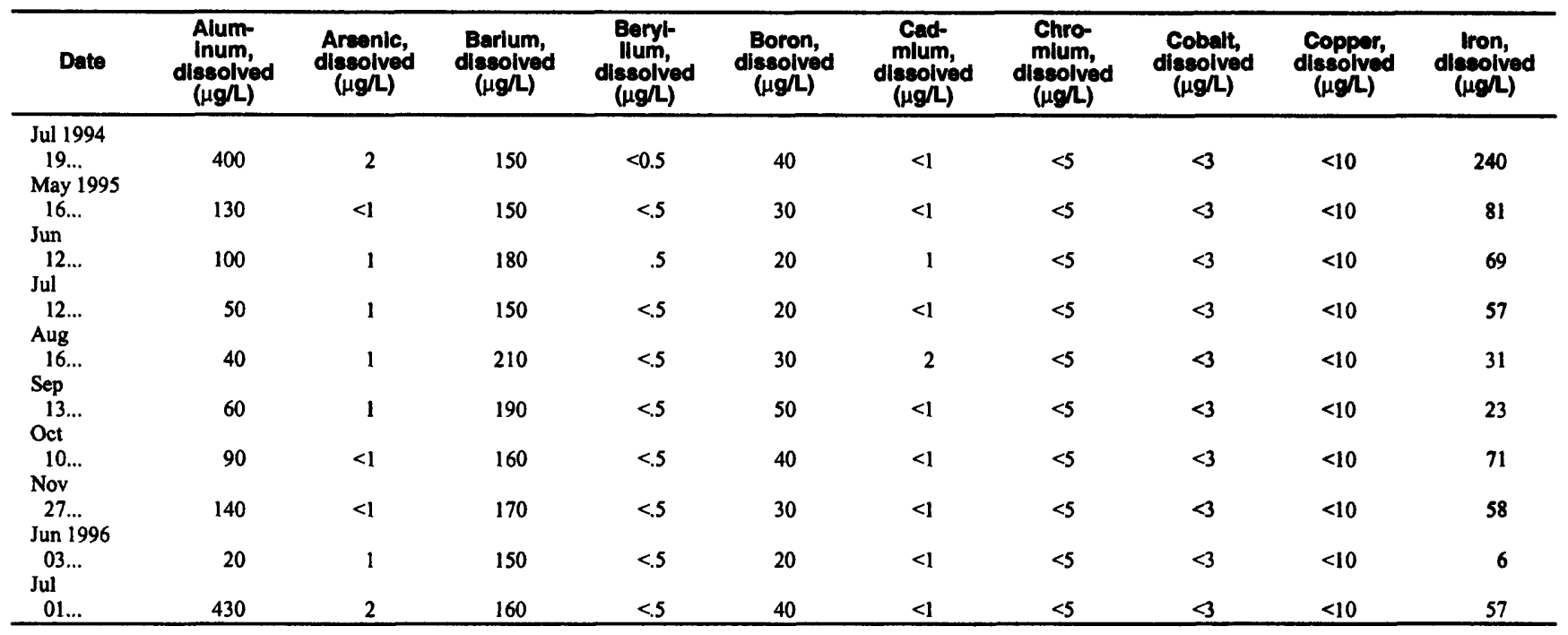

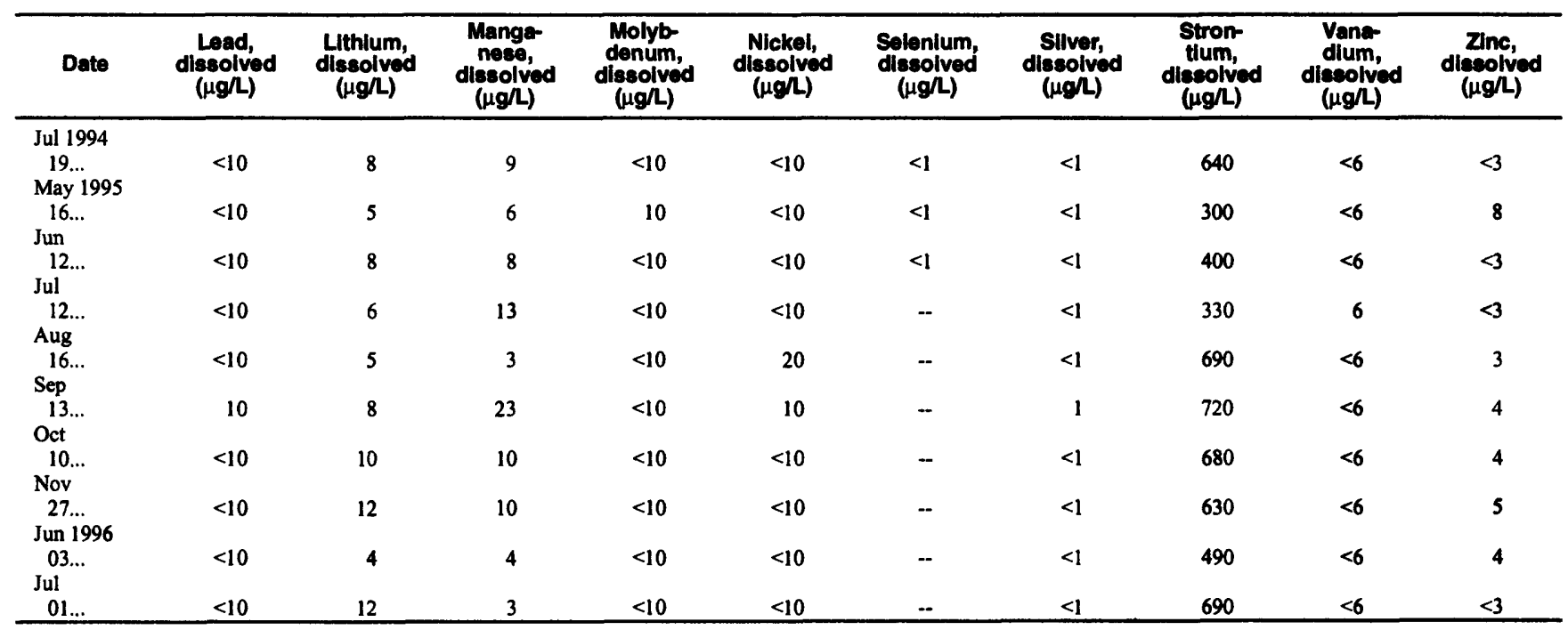


Table 3. Water-quality data for sites in the Sand Coulee Coal Area, Montana, July 1994 through September 1996-Continued SITE 2, $06078250--C O T T O N W O O D$ CREEK NEAR STOCKETT, MT

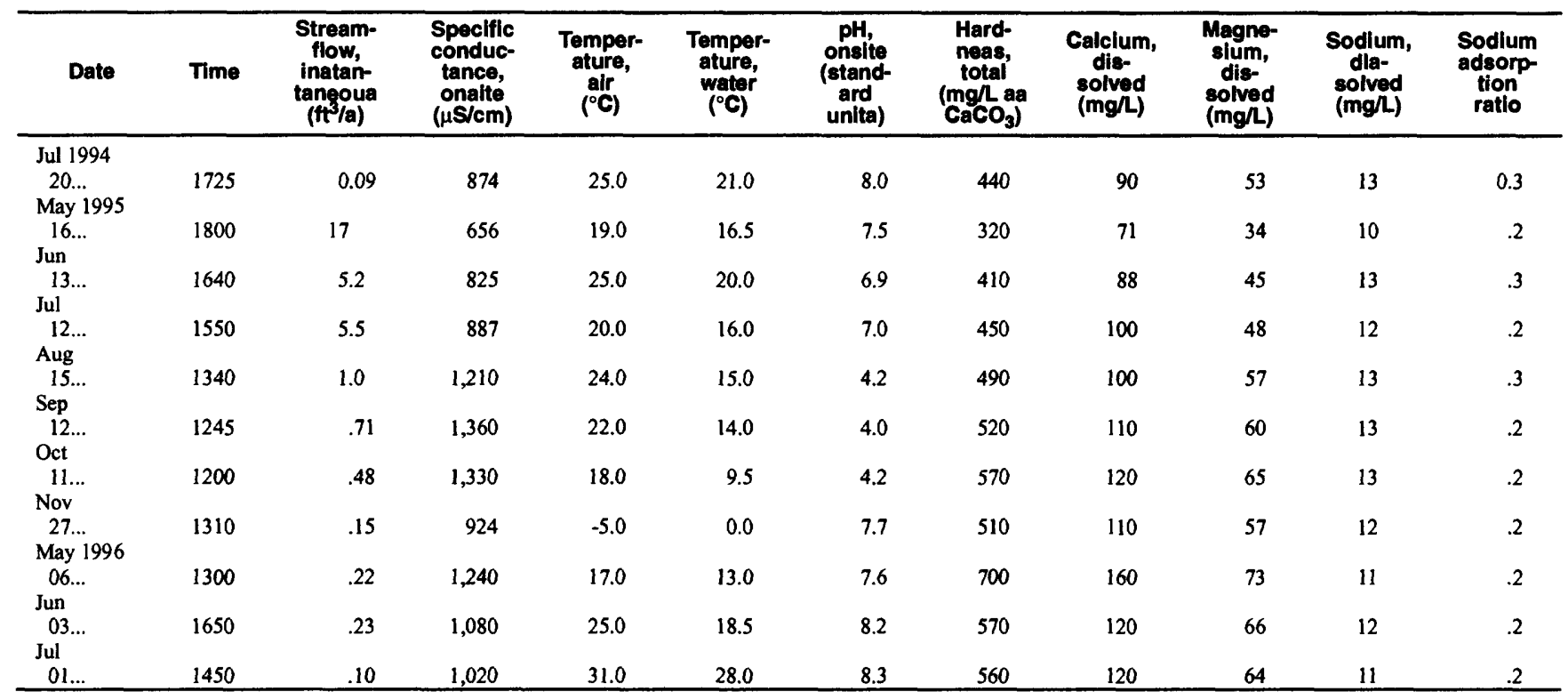

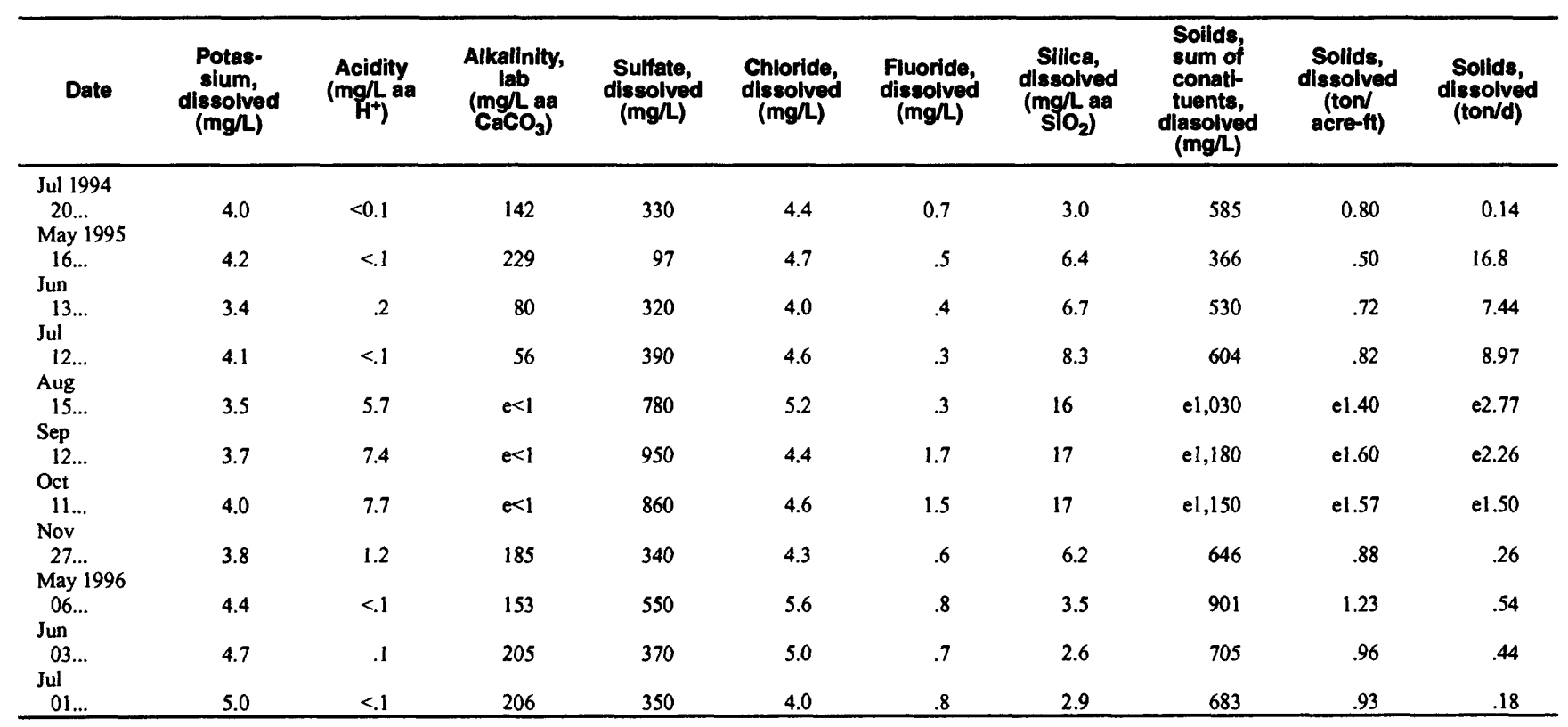


Table 3. Water-quality data for sites in the Sand Coulee Coal Area, Montana, July 1994 through September 1996 (Continued)

SITE 2, 06078250--COTTONWOOD CREEK NEAR STOCKETT, MT--Continued

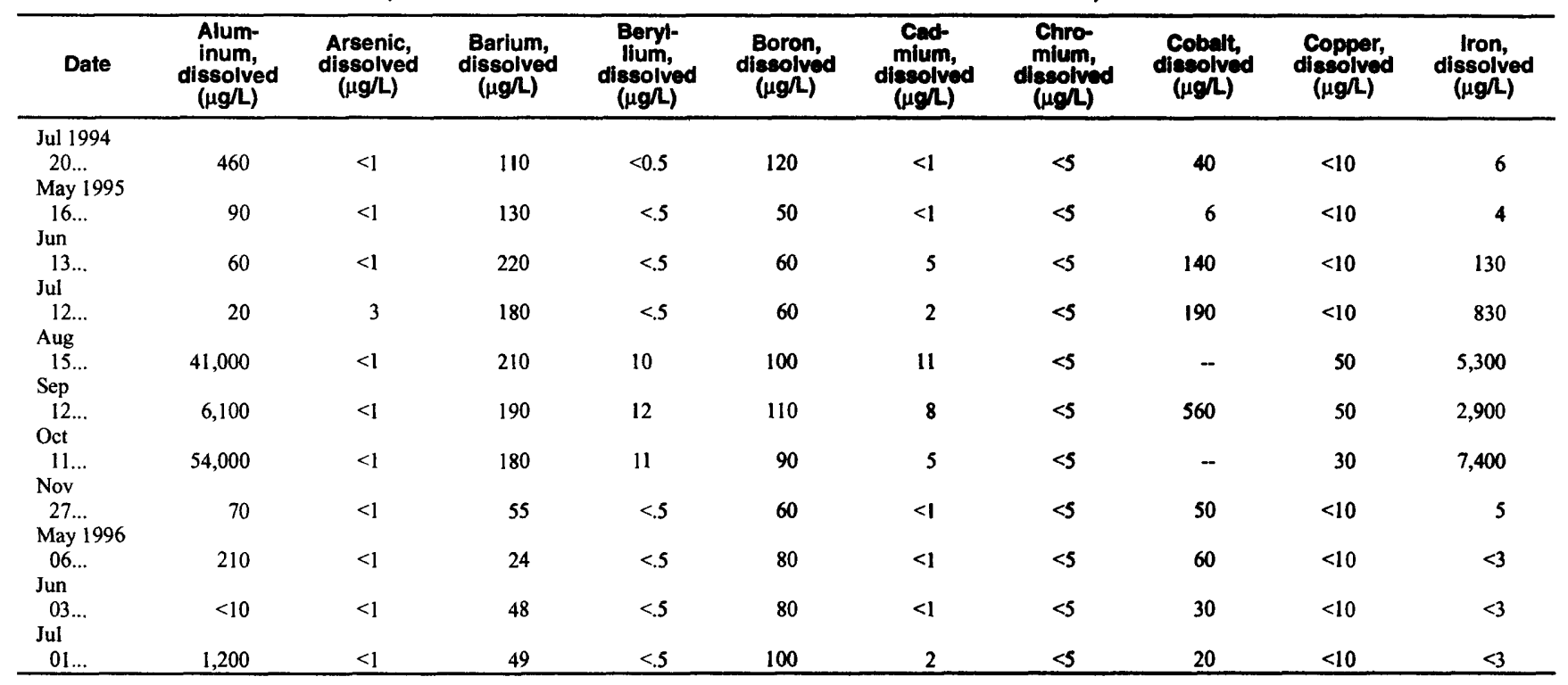

\begin{tabular}{|c|c|c|c|c|c|c|c|c|c|c|}
\hline Date & $\begin{array}{c}\text { Lead, } \\
\text { dissoived } \\
(\mu \mathrm{g} / \mathrm{L})\end{array}$ & $\begin{array}{c}\text { Lithium, } \\
\text { dissolved } \\
(\mu g / L)\end{array}$ & $\begin{array}{l}\text { Manga- } \\
\text { nese, } \\
\text { dissolved } \\
(\mu \mathrm{g} / \mathrm{L})\end{array}$ & $\begin{array}{c}\text { Molyb- } \\
\text { denum, } \\
\text { dissolved } \\
(\mu g /)\end{array}$ & $\begin{array}{c}\text { Nickel, } \\
\text { dissolved } \\
(\mu g h)\end{array}$ & $\begin{array}{c}\text { Selonium, } \\
\text { diseolved } \\
(\mu g / L)\end{array}$ & $\begin{array}{l}\text { Silwer, } \\
\text { dlasolved } \\
\text { (ugl) }\end{array}$ & $\begin{array}{c}\text { Stron- } \\
\text { tium, } \\
\text { diseolved } \\
(\mu \varphi /)\end{array}$ & $\begin{array}{c}\text { Vana- } \\
\text { dium, } \\
\text { dissolved } \\
(\mu g / 2)\end{array}$ & $\begin{array}{c}\text { Zinc, } \\
\text { dissolved } \\
(\mu g / L)\end{array}$ \\
\hline $\begin{array}{l}\text { Jul } 1994 \\
20 \ldots \\
\text { May } 1995\end{array}$ & $<10$ & 43 & 100 & $<10$ & 70 & $<1$ & $<1$ & 360 & $<6$ & 8 \\
\hline $\begin{array}{l}16 \ldots \\
\text { Jun }\end{array}$ & $<10$ & 16 & 25 & $<10$ & 20 & $<1$ & 1 & 300 & $<6$ & 12 \\
\hline $\begin{array}{l}13 \ldots \\
\text { Jul }\end{array}$ & $<10$ & 46 & 200 & $<10$ & 230 & $<1$ & $<1$ & 380 & $<6$ & 210 \\
\hline $\begin{array}{l}15 \ldots \\
\text { Sep }\end{array}$ & $<10$ & 96 & 380 & $<10$ & 810 & -- & $<1$ & 440 & $<6$ & 3,700 \\
\hline $\begin{array}{l}12 \ldots \\
\text { Oct }\end{array}$ & $<10$ & 110 & 430 & $<10$ & 1,000 & - & 1 & 450 & $<6$ & 4,400 \\
\hline $\begin{array}{l}11 \ldots \\
\text { Nov }\end{array}$ & $<10$ & 110 & 420 & $<10$ & 1,000 & - & $<1$ & 480 & $<6$ & 4,800 \\
\hline
\end{tabular}


Table 3. Water-quality data for sites in the Sand Coulee Coal Area, Montana, July 1994 through September 1996 (Continued)

SITE 3, 06078260--NUMBER FIVE COULEE BELOW GIFFEN SPRING, NEAR STOCKETT, MT

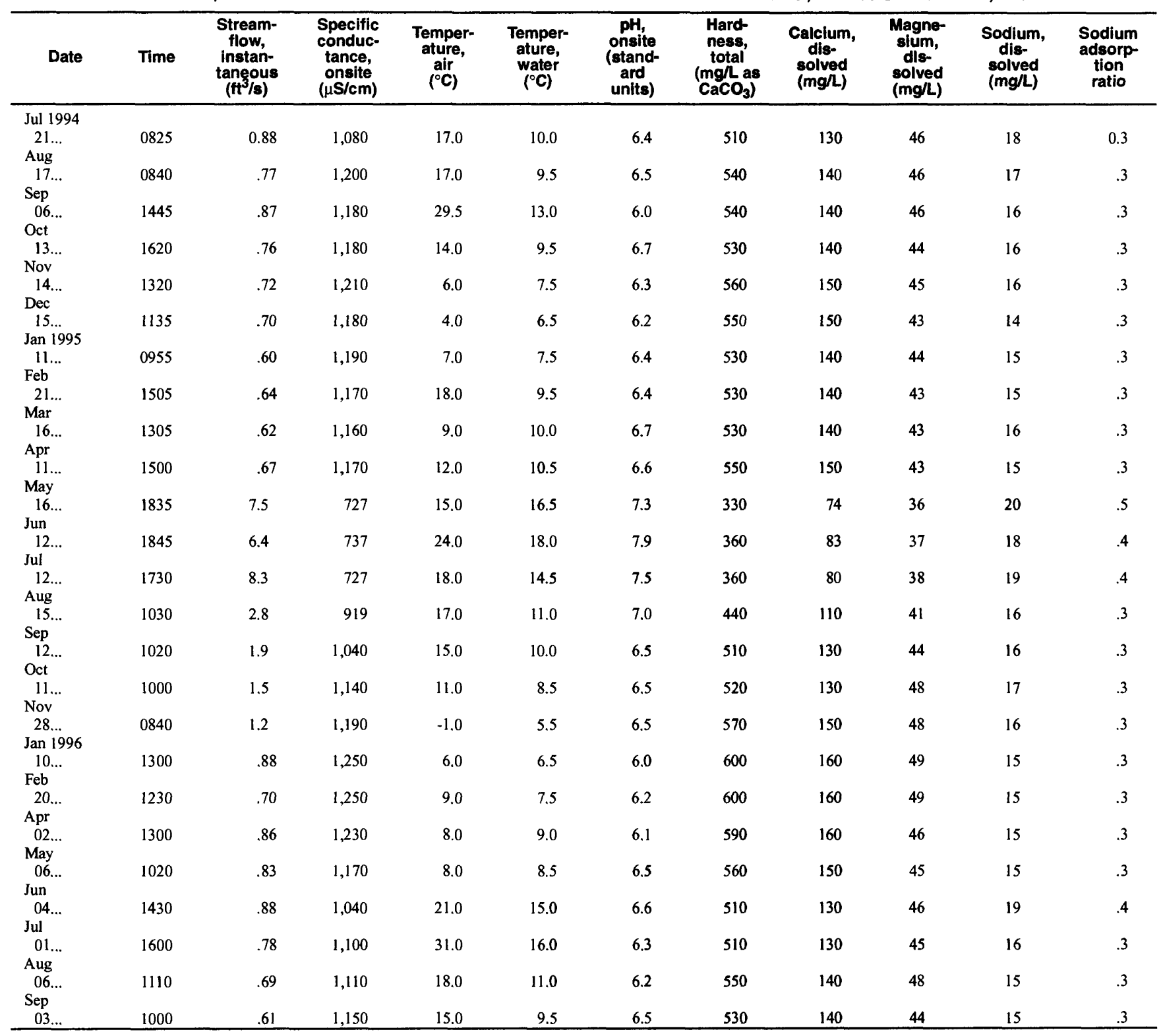


Table 3. Water-quality data for sites in the Sand Coulee Coal Area, Montana, July 1994 through September 1996 (Continued) SITE 3, 06078260--NUMBER FIVE COULEE BELOW GIFFEN SPRING, NEAR STOCKETT, MT--Continued

\begin{tabular}{|c|c|c|c|c|c|c|c|c|c|c|}
\hline Date & $\begin{array}{l}\text { Potas- } \\
\text { sium, } \\
\text { dissolved } \\
\text { (mg/l })\end{array}$ & $\begin{array}{c}\text { Acidity } \\
\left(\mathbf{m g}^{\prime} \mathbf{H}^{+}\right)\end{array}$ & $\begin{array}{c}\text { Alkalinity, } \\
\text { lab } \\
\left(\mathrm{mg}_{\mathrm{L}} \mathrm{as}\right. \\
\left.\mathrm{CaCO}_{3}\right)\end{array}$ & $\begin{array}{l}\text { Sulfate, } \\
\text { dissolved } \\
\text { (mg/l) }\end{array}$ & $\begin{array}{l}\text { Chloride, } \\
\text { dissolved } \\
\text { (mg/l) }\end{array}$ & $\begin{array}{l}\text { Fuorlde, } \\
\text { dissolved } \\
\text { (mg/L) }\end{array}$ & $\begin{array}{l}\text { Sllica, } \\
\text { dissolved } \\
\text { (mg/has } \\
\left.\text { Slo }_{2}\right)\end{array}$ & $\begin{array}{l}\text { Solids, } \\
\text { sum of } \\
\text { consti- } \\
\text { tuents, } \\
\text { dissolved } \\
\text { (mg/l) }\end{array}$ & $\begin{array}{l}\text { Solids, } \\
\text { dissolved } \\
\text { (ton/ } \\
\text { acre-ft) }\end{array}$ & $\begin{array}{l}\text { Solids, } \\
\text { dissolved } \\
\text { (ton/d) }\end{array}$ \\
\hline Jul 1994 & & & & & & & & & & \\
\hline $\begin{array}{l}21 \ldots \\
\text { Aug }\end{array}$ & 5.2 & 0.4 & 7.9 & 570 & 3.9 & 0.6 & 14 & 842 & 1.14 & 2.00 \\
\hline $17 \ldots$ & 5.5 & 3.2 & $e<1$ & 670 & 38 & 2 & 14 & e956 & el 30 & el 99 \\
\hline Sep & & & & (2) & & .2 & & & el. .50 & e1.99 \\
\hline $\begin{array}{l}06 \ldots . . \\
\text { Oct }\end{array}$ & 6.1 & 1.7 & $e<1$ & 720 & 3.4 & .3 & 15 & $\mathrm{e} 1,010$ & $e, 1.37$ & e2.37 \\
\hline $\begin{array}{l}13 . . \\
\text { Nov }\end{array}$ & 6.2 & 0.9 & $e<1$ & 650 & 3.8 & .5 & 14 & e928 & e 1.26 & el.91 \\
\hline $\begin{array}{l}14 \ldots \\
\text { Dec }\end{array}$ & 5.9 & 1.5 & $e<1$ & 670 & 3.4 & -- & 14 & e965 & el.31 & el. 88 \\
\hline $\begin{array}{c}15 \ldots \\
\text { Jan } 1995\end{array}$ & 5.9 & 1.5 & $\mathbf{e}<1$ & 630 & 3.3 & $<.5$ & 15 & e919 & el. 25 & el.74 \\
\hline $\begin{array}{l}11 \ldots \\
\text { Feb }\end{array}$ & 5.8 & 1.3 & $e<1$ & 610 & 3.4 & .7 & 14 & e885 & e 1.20 & el. 43 \\
\hline $\begin{array}{c}21 \ldots \\
\operatorname{Mar}\end{array}$ & 4.9 & .8 & $e<1$ & 680 & 4.5 & .2 & 14 & e950 & el. 29 & el. 64 \\
\hline $\begin{array}{l}16 \ldots \\
\text { Apr }\end{array}$ & 5.1 & 1.5 & $e<1$ & 590 & 3.8 & .3 & 14 & e860 & el.17 & e 1.44 \\
\hline $\begin{array}{l}11 \ldots \\
\text { May }\end{array}$ & 5.8 & .6 & 4.4 & 660 & 4.6 & .3 & 13 & 935 & 1.27 & 1.69 \\
\hline $\begin{array}{l}16 \ldots \\
\text { Jun }\end{array}$ & 5.3 & $<.1$ & 257 & 110 & 5.0 & .6 & 9.2 & 415 & .56 & 8.41 \\
\hline $\begin{array}{l}12 \ldots \\
\text { Jul }\end{array}$ & 4.1 & $<.1$ & 252 & 130 & 4.0 & .6 & 8.3 & 437 & .59 & 7.56 \\
\hline $\begin{array}{l}12 \ldots \\
\text { Aug }\end{array}$ & 4.4 & $<.1$ & 252 & 140 & 4.5 & .5 & 11 & 450 & .61 & 10.1 \\
\hline $\begin{array}{l}15 \ldots \\
\text { Sep }\end{array}$ & 4.1 & $<.1$ & 148 & 380 & 3.6 & .6 & 10 & 661 & .90 & 5.00 \\
\hline $\begin{array}{c}12 \ldots \\
\text { Oct }\end{array}$ & 5.7 & $<.1$ & 67 & 500 & 4.3 & .3 & 11 & 771 & 1.05 & 3.96 \\
\hline $\begin{array}{l}11 \ldots \\
\text { Nov }\end{array}$ & 5.7 & .5 & 27 & 560 & 4.7 & .4 & 13 & 833 & 1.13 & 3.33 \\
\hline $\begin{array}{c}28 \ldots \\
\text { Jan } 1996\end{array}$ & 5.3 & 1.1 & $e<1$ & 700 & 3.8 & 1.2 & 14 & e944 & el. .35 & $\mathrm{e} 3.22$ \\
\hline $\begin{array}{l}10 \ldots \\
\text { Feb }\end{array}$ & 7.0 & 2.1 & $e<1$ & 790 & 5.6 & 1.3 & 17 & el, 110 & el. 51 & $\mathrm{e} 2.65$ \\
\hline $\begin{array}{l}20 \ldots \\
\text { Apr }\end{array}$ & 5.9 & 1.6 & $e<1$ & 680 & 3.9 & .2 & 15 & e987 & el.34 & el. 1.87 \\
\hline $\begin{array}{l}02 \ldots \\
\text { May }\end{array}$ & 5.5 & 1.1 & $e<1$ & 700 & 4.5 & .8 & 15 & e 1,000 & e1.36 & e2.33 \\
\hline $\begin{array}{l}06 \ldots \\
\text { Jun }\end{array}$ & 6.1 & .3 & 2.3 & 680 & 5.6 & .8 & 13 & 958 & 1.30 & 2.15 \\
\hline $\begin{array}{c}04 \ldots \\
\text { Jul }\end{array}$ & 5.7 & $<.1$ & 5.6 & 570 & 7.3 & .9 & 11 & 844 & 1.15 & 2.01 \\
\hline $\begin{array}{l}01 \ldots . . . \\
\text { Aug }\end{array}$ & 5.9 & .6 & 5.6 & 680 & 4.3 & .8 & 13 & 934 & 1.27 & 1.97 \\
\hline $\begin{array}{l}06 . . \\
\text { Sep }\end{array}$ & 6.5 & .6 & 4.0 & 630 & 5.3 & .7 & 13 & 898 & 1.22 & 1.67 \\
\hline $03 \ldots$ & 5.7 & .5 & $e<1$ & 600 & 5.2 & .8 & 14 & e868 & el. 18 & el. 43 \\
\hline
\end{tabular}


Table 3. Water-quality data for sites in the Sand Coulee Coal Area, Montana, July 1994 through September 1996 (Continued)

SITE 3, 06078260--NUMBER FIVE COULEE BELOW GIFFEN SPRING, NEAR STOCKETT, MT--Continued

\begin{tabular}{|c|c|c|c|c|c|c|c|c|c|c|}
\hline Date & $\begin{array}{c}\text { Alum- } \\
\text { inum, } \\
\text { dissolved } \\
(\mu g / L)\end{array}$ & $\begin{array}{c}\text { Arsenic, } \\
\text { dissolved } \\
(\mu g / L)\end{array}$ & $\begin{array}{c}\text { Barium, } \\
\text { dissolved } \\
(\mu \mathrm{g} / \mathrm{L})\end{array}$ & $\begin{array}{c}\text { Beryl- } \\
\text { lium, } \\
\text { dissolved } \\
(\mu g / L)\end{array}$ & $\begin{array}{l}\text { Boron, } \\
\text { dissolved } \\
(\mu g / L)\end{array}$ & $\begin{array}{c}\text { Cad- } \\
\text { mium, } \\
\text { dissolved } \\
(\mu \mathrm{g} / \mathrm{L})\end{array}$ & $\begin{array}{c}\text { Chro- } \\
\text { mium, } \\
\text { diesolved } \\
(\mu \mathrm{g} / \mathrm{L})\end{array}$ & $\begin{array}{c}\text { Cobalt, } \\
\text { disesolved } \\
(\mu g / L)\end{array}$ & $\begin{array}{c}\text { Copper, } \\
\text { disaolved } \\
(\mu g / L)\end{array}$ & $\begin{array}{l}\text { Iron, } \\
\text { diesolved } \\
\text { ( } \mu g / L)\end{array}$ \\
\hline $\begin{array}{c}\text { Jul } 1994 \\
21 \ldots\end{array}$ & 60 & $<1$ & 48 & $<0.5$ & 80 & 6 & $<5$ & 330 & $<10$ & 46,000 \\
\hline Aug & & & & & & & & & & \\
\hline $\begin{array}{l}17 \ldots \\
\text { Sep }\end{array}$ & 190 & $<1$ & 40 & $<.5$ & 90 & 13 & $<5$ & 150 & $<10$ & 55,000 \\
\hline $\begin{array}{l}06 . . . \\
\text { Oct }\end{array}$ & 160 & $<1$ & 35 & 1 & 100 & 14 & $<5$ & 270 & $<10$ & 57,000 \\
\hline $\begin{array}{l}13 \ldots \\
\text { Nov }\end{array}$ & 170 & $<1$ & 32 & .5 & 90 & 5 & $<5$ & 260 & $<10$ & 50,000 \\
\hline $\begin{array}{l}14 \ldots \\
\text { Dec }\end{array}$ & 310 & $<1$ & 26 & 1 & 100 & 9 & $<5$ & 270 & $<10$ & 56,000 \\
\hline $\begin{array}{l}15 \ldots \\
\text { Jan } 1995\end{array}$ & 290 & $<1$ & 22 & 1 & 90 & 3 & $<5$ & 410 & $<10$ & 53,000 \\
\hline $\begin{array}{l}11 \ldots \\
\text { Feb }\end{array}$ & 220 & $<1$ & 22 & 1 & 90 & 9 & $<5$ & 300 & $<10$ & 48,000 \\
\hline $\begin{array}{l}21 \ldots \\
\mathrm{Mar}\end{array}$ & 150 & $<1$ & 22 & 1 & 80 & 2 & $<5$ & 170 & $<10$ & 45,000 \\
\hline $\begin{array}{l}16 \ldots \\
\text { Apr }\end{array}$ & 130 & $<1$ & 22 & 1 & 80 & 2 & $<5$ & 180 & $<10$ & 44,000 \\
\hline $\begin{array}{l}11 . . \\
\text { May }\end{array}$ & 60 & $<1$ & 22 & .7 & 80 & 2 & $<5$ & 150 & $<10$ & 38,000 \\
\hline $\begin{array}{c}16 \ldots \\
\text { Jun }\end{array}$ & 60 & $<1$ & 140 & $<.5$ & 50 & $<1$ & $<5$ & 10 & $<10$ & 430 \\
\hline $\begin{array}{l}12 \ldots \\
\text { Jul }\end{array}$ & 70 & $<1$ & 210 & $<.5$ & 50 & $<1$ & $<5$ & 20 & $<10$ & 340 \\
\hline $\begin{array}{l}12 \ldots \\
\text { Aug }\end{array}$ & 30 & $<1$ & 170 & $<.5$ & 60 & $<1$ & $<5$ & 20 & $<10$ & 390 \\
\hline $\begin{array}{l}15 \ldots \\
\text { Sep }\end{array}$ & 30 & $<1$ & 120 & $<.5$ & 60 & 1 & $<5$ & 100 & $<10$ & 5,600 \\
\hline $\begin{array}{l}12 \ldots \\
\text { Oct }\end{array}$ & 50 & $<2$ & 87 & $<.5$ & 80 & 3 & $<5$ & 140 & $<10$ & 17,000 \\
\hline $\begin{array}{l}11 \ldots \\
\text { Nov }\end{array}$ & 70 & $<1$ & 70 & $<.5$ & 80 & 3 & $<5$ & 190 & $<10$ & 35,000 \\
\hline $\begin{array}{l}28 \ldots \\
\operatorname{Jan} 1996\end{array}$ & 140 & $<1$ & 50 & $<.5$ & 80 & 4 & $<5$ & 230 & $<10$ & 51,000 \\
\hline $\begin{array}{l}10 \ldots \\
\text { Feb }\end{array}$ & 400 & $<2$ & 39 & .9 & 80 & 5 & $<5$ & 250 & $<10$ & 63,000 \\
\hline $\begin{array}{c}20 \ldots \\
\mathrm{Apr}\end{array}$ & 220 & $<1$ & 35 & .9 & 90 & 4 & $<5$ & 290 & $<10$ & 53,000 \\
\hline $\begin{array}{l}02 \ldots \\
\text { May }\end{array}$ & 100 & $<1$ & 34 & .6 & 90 & 3 & $<5$ & 220 & $<10$ & 50,000 \\
\hline $\begin{array}{c}06 \ldots \\
\text { Jun }\end{array}$ & 50 & $<1$ & 32 & 1 & 80 & 2 & $<5$ & 200 & $<10$ & 38,000 \\
\hline $\begin{array}{c}04 \ldots \\
\text { Jul }\end{array}$ & 30 & $<1$ & 46 & $<.5$ & 60 & 1 & $<5$ & 160 & $<10$ & 18,000 \\
\hline $\begin{array}{l}01 \ldots \\
\text { Aug }\end{array}$ & 30 & $<1$ & 35 & $<.5$ & 60 & 2 & $<5$ & 170 & $<10$ & 33,000 \\
\hline $\begin{array}{l}06 . . \\
\text { Sep }\end{array}$ & 20 & $<1$ & 28 & 1 & 70 & 2 & $<5$ & 160 & $<10$ & 34,000 \\
\hline $03 \ldots$ & 40 & $<1$ & 25 & .9 & 60 & 2 & $<5$ & 190 & $<10$ & 40,000 \\
\hline
\end{tabular}


Table 3. Water-quality data for sites in the Sand Coulee Coal Area, Montana, July 1994 through September 1996 (Continued)

SITE 3, 06078260--NUMBER FIVE COULEE BELOW GIFFEN SPRING, NEAR STOCKETT, MT--Continued

\begin{tabular}{|c|c|c|c|c|c|c|c|c|c|c|}
\hline Date & $\begin{array}{c}\text { Lead, } \\
\text { dissolved } \\
(\mu g / L)\end{array}$ & $\begin{array}{l}\text { Lithium, } \\
\text { dissolved } \\
(\mu g / L)\end{array}$ & $\begin{array}{l}\text { Mange- } \\
\text { nese, } \\
\text { dissolved } \\
(\mu \mathrm{g} / \mathrm{L})\end{array}$ & $\begin{array}{c}\text { Molyb- } \\
\text { denum, } \\
\text { dissolved } \\
(\mu g / L)\end{array}$ & $\begin{array}{l}\text { Nickel, } \\
\text { dissolved } \\
(\mu g / L)\end{array}$ & $\begin{array}{c}\text { Selenium, } \\
\text { dissolved } \\
(\mu \mathrm{g} / \mathrm{L})\end{array}$ & $\begin{array}{l}\text { Silver, } \\
\text { diseolved } \\
(\mu g / L)\end{array}$ & $\begin{array}{c}\text { Stron- } \\
\text { tlum, } \\
\text { dissolved } \\
(\mu g / L)\end{array}$ & $\begin{array}{l}\text { Vana- } \\
\text { dium, } \\
\text { diseolved } \\
(\mu g / L)\end{array}$ & $\begin{array}{c}\text { Zinc, } \\
\text { dissolved } \\
(\mu \mathrm{g} / L)\end{array}$ \\
\hline \multicolumn{11}{|l|}{ Jul 1994} \\
\hline $21 \ldots$ & $<10$ & 59 & 740 & $<10$ & 320 & $<1$ & $<1$ & 370 & $<6$ & 1,100 \\
\hline \multicolumn{11}{|l|}{ Aug } \\
\hline $17 \ldots$ & $<10$ & 66 & 850 & $<10$ & 340 & - & $<1$ & 390 & 12 & 1,300 \\
\hline \multicolumn{11}{|l|}{ Sep } \\
\hline \multicolumn{10}{|l|}{ Oct } & 1,400 \\
\hline $13 \ldots$ & $<10$ & 65 & 830 & $<10$ & 310 & $<1$ & $<1$ & 360 & 9 & 1,200 \\
\hline \multicolumn{11}{|l|}{ Nov } \\
\hline $14 \ldots$ & $<10$ & 68 & 930 & $<10$ & 340 & $<1$ & $<1$ & 380 & 9 & 1,400 \\
\hline \multicolumn{11}{|l|}{ Dec } \\
\hline \multicolumn{11}{|l|}{ Jan 1995} \\
\hline $11 \ldots$ & $<10$ & 68 & 870 & $<10$ & 320 & $<1$ & $<1$ & 390 & 8 & 1,200 \\
\hline \multicolumn{11}{|l|}{ Feb } \\
\hline $21 \ldots$ & $<1$ & 62 & 820 & $<1$ & 290 & $<1$ & $<1$ & 380 & $<6$ & 1,000 \\
\hline \multicolumn{11}{|l|}{ Mar } \\
\hline $16 \ldots$ & $<1$ & 68 & 780 & $<1$ & 280 & $<1$ & $<1$ & 380 & $<6$ & 1,000 \\
\hline \multicolumn{11}{|l|}{ Apr } \\
\hline $11 \ldots$ & $<1$ & 63 & 860 & $<1$ & 260 & $<1$ & $<1$ & 380 & $<6$ & 900 \\
\hline \multicolumn{11}{|l|}{ May } \\
\hline $16 \ldots$ & $<1$ & 26 & 130 & 1 & 30 & 1 & $<1$ & 290 & $<6$ & 30 \\
\hline \multicolumn{11}{|l|}{ Jun } \\
\hline $12 \ldots$ & $<1$ & 24 & 170 & $<1$ & 30 & $<1$ & $<1$ & 320 & $<6$ & 50 \\
\hline \multicolumn{11}{|l|}{ Jul } \\
\hline $12 \ldots$ & $<1$ & 11 & 180 & $<1$ & 30 & -- & $<1$ & 300 & $<6$ & 60 \\
\hline \multicolumn{11}{|l|}{ Aug } \\
\hline $15 \ldots$ & $<1$ & 36 & 490 & $<1$ & 150 & -- & $<1$ & 330 & $<6$ & 380 \\
\hline \multicolumn{11}{|l|}{ Sep } \\
\hline $12 \ldots$ & $<1$ & 47 & 740 & $<1$ & 250 & -. & 1 & 340 & $<6$ & 750 \\
\hline \multicolumn{11}{|l|}{ Oct } \\
\hline $11 \ldots$ & $<1$ & 54 & 890 & $<1$ & 310 & -- & $<1$ & 370 & $<6$ & 1,100 \\
\hline \multicolumn{11}{|l|}{ Nov } \\
\hline $28 \ldots$ & $<1$ & 61 & 990 & $<1$ & 380 & -- & $<1$ & 390 & $<6$ & 1,500 \\
\hline \multicolumn{11}{|l|}{ Jan 1996} \\
\hline $10 \ldots$ & $<1$ & 69 & 1,300 & $<1$ & 470 & - & $<1$ & 430 & $<6$ & 1,900 \\
\hline \multicolumn{11}{|l|}{ Feb } \\
\hline $20 \ldots$ & $<1$ & 62 & 1,300 & $<1$ & 430 & -- & 2 & 390 & $<6$ & 1,700 \\
\hline Apr & & & & & & & & & & \\
\hline $02 \ldots$ & $<1$ & 66 & 1,200 & $<1$ & 400 & -- & $<1$ & 400 & $<6$ & 1,500 \\
\hline May & & & & & & & & & & \\
\hline $06 .$. & $<1$ & 59 & 930 & $<1$ & 330 & - & $<1$ & 380 & $<6$ & 1,200 \\
\hline Jun & & & & & & & & & & \\
\hline $04 \ldots$ & $<1$ & 55 & 730 & $<1$ & 260 & - & $<1$ & 380 & $<6$ & 750 \\
\hline Jul & & & & & & & & & & \\
\hline $01 \ldots$ & $<1$ & 58 & 730 & 1 & 290 & -- & $<1$ & 360 & $<6$ & 1,000 \\
\hline Aug & & & & & & & & & & \\
\hline $06 .$. & $<1$ & 67 & 870 & $<1$ & 290 & -- & $<1$ & 360 & $<6$ & 1,100 \\
\hline Sep & & & & & & & & & & \\
\hline $03 \ldots$ & $<1$ & 67 & 830 & $<1$ & 320 & - & $<1$ & 370 & $<6$ & 1,100 \\
\hline
\end{tabular}


Table 3. Water-quality data for sites in the Sand Coulee Coal Area, Montana, July 1994 through September 1996 (Continued)

SITE 4, 06078270--SAND COULEE AT SAND COULEE, MT

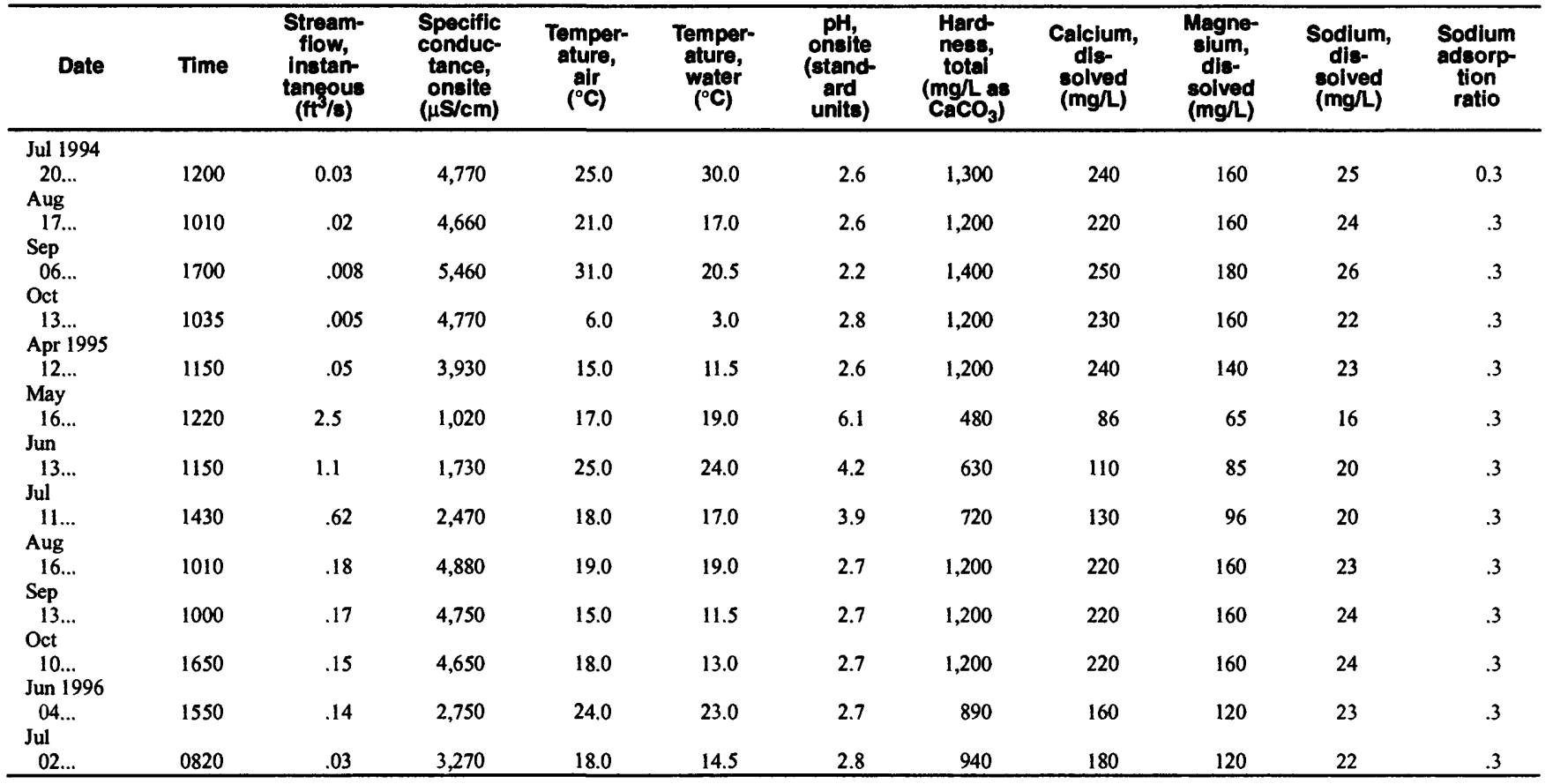

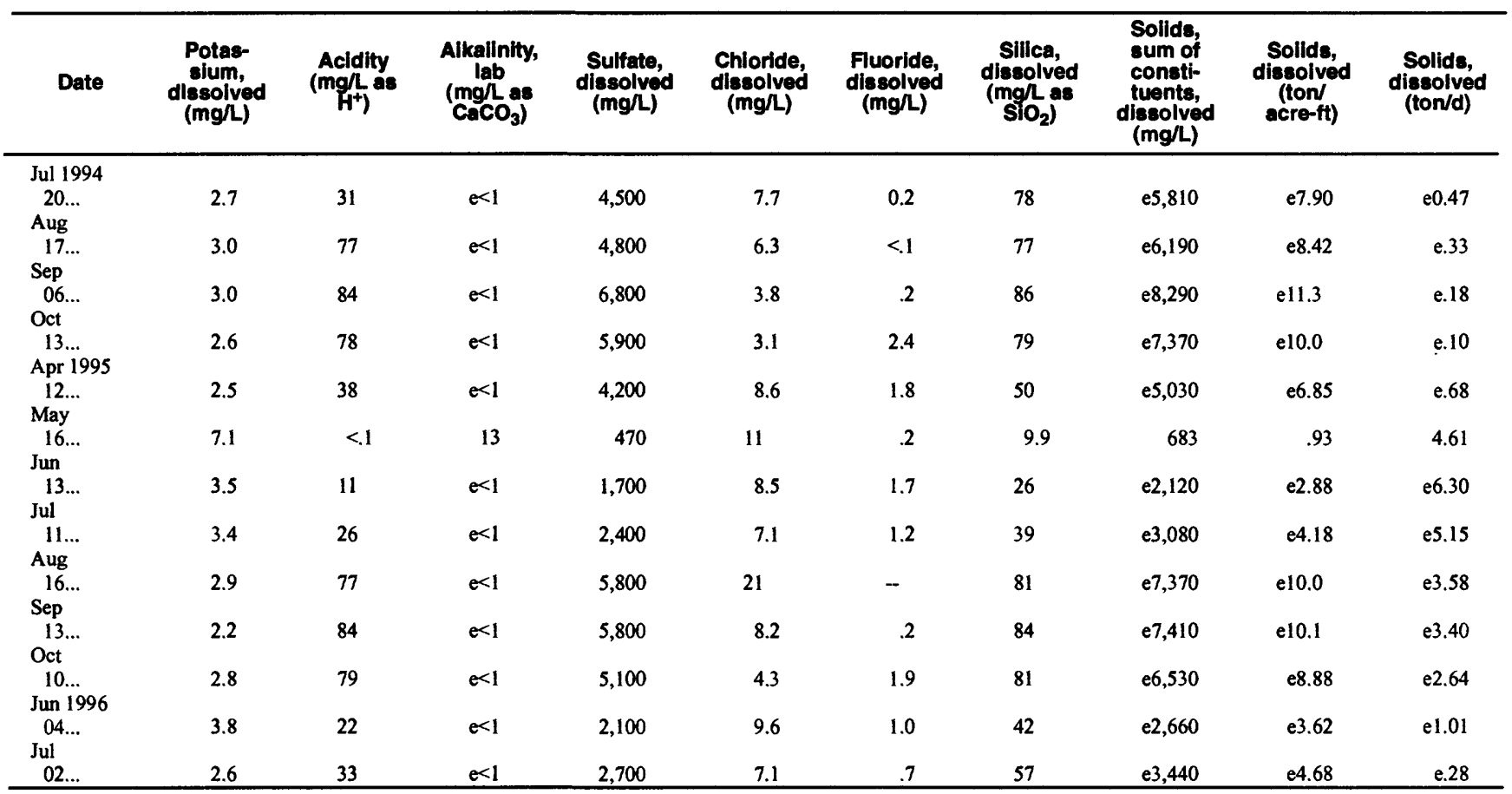


Table 3. Water-quality data for sites in the Sand Coulee Coal Area, Montana, July 1994 through September 1996 (Continued)

SITE 4, 06078270--SAND COULEE AT SAND COULEE, MT--Continued

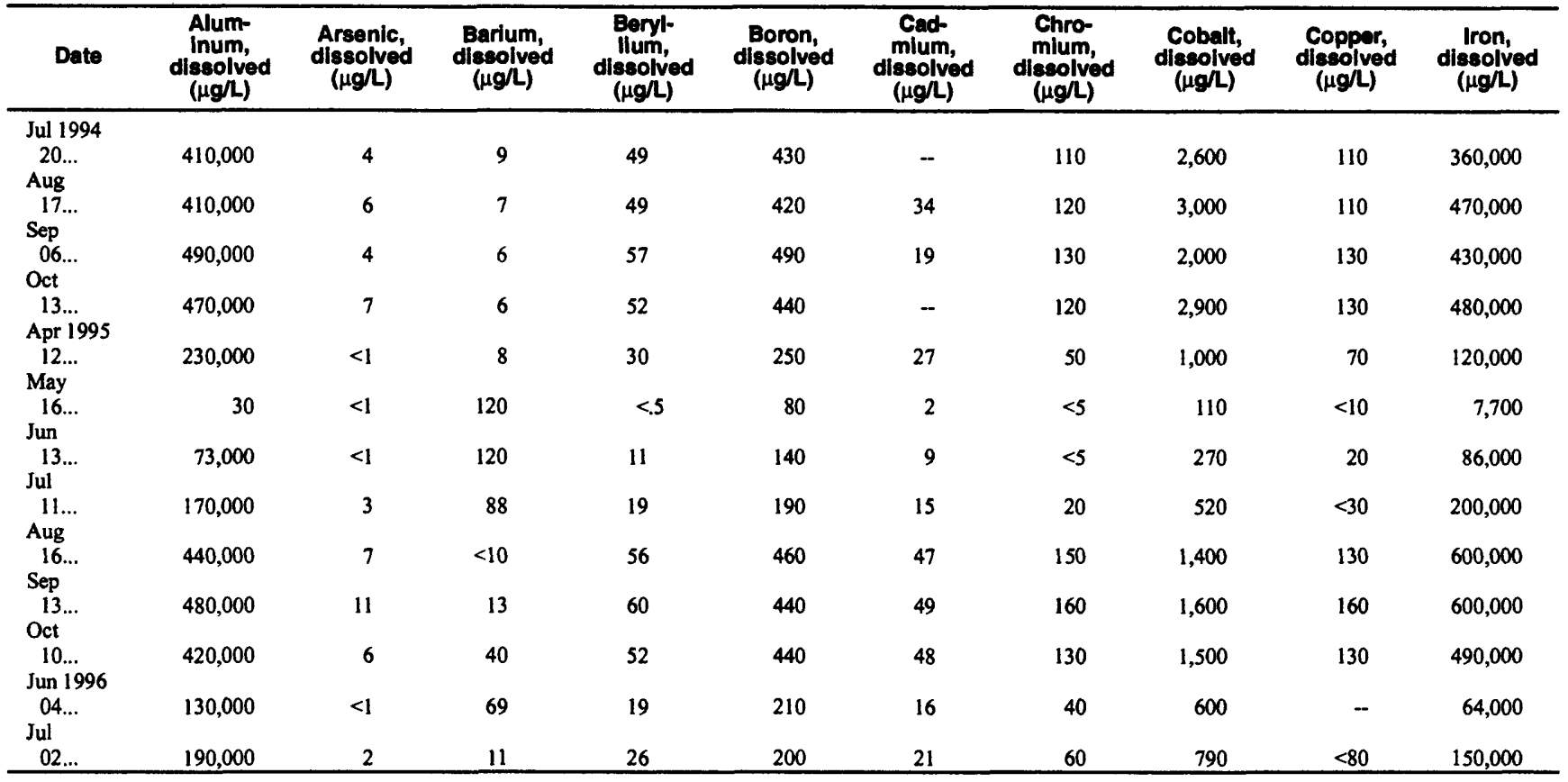

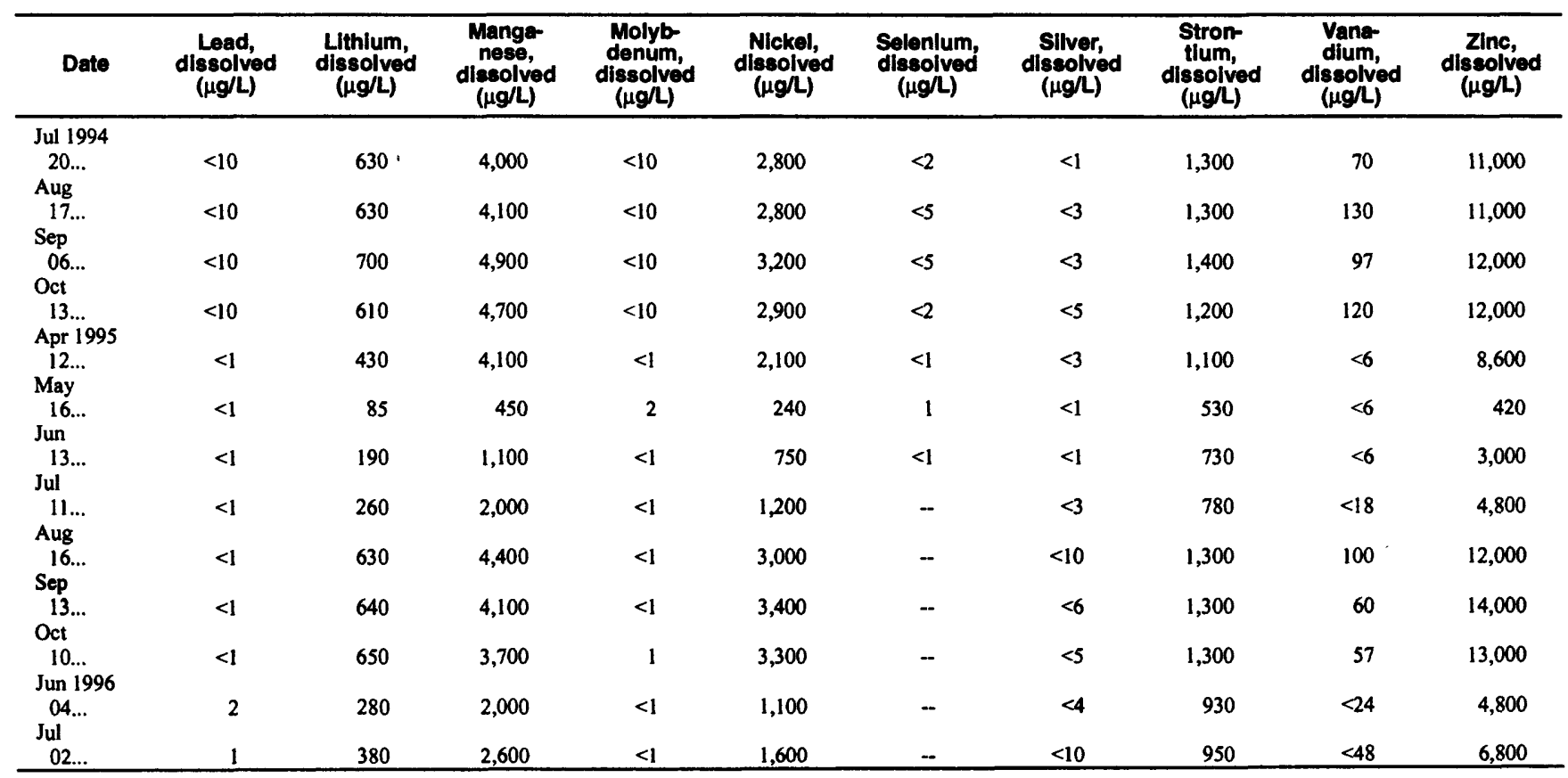


Table 3. Water-quality data for sites in the Sand Coulee Coal Area, Montana July 1994 through September 1996 (Continued) SITE 5, 06090590--ANACONDA DRAIN AT BELT, MT

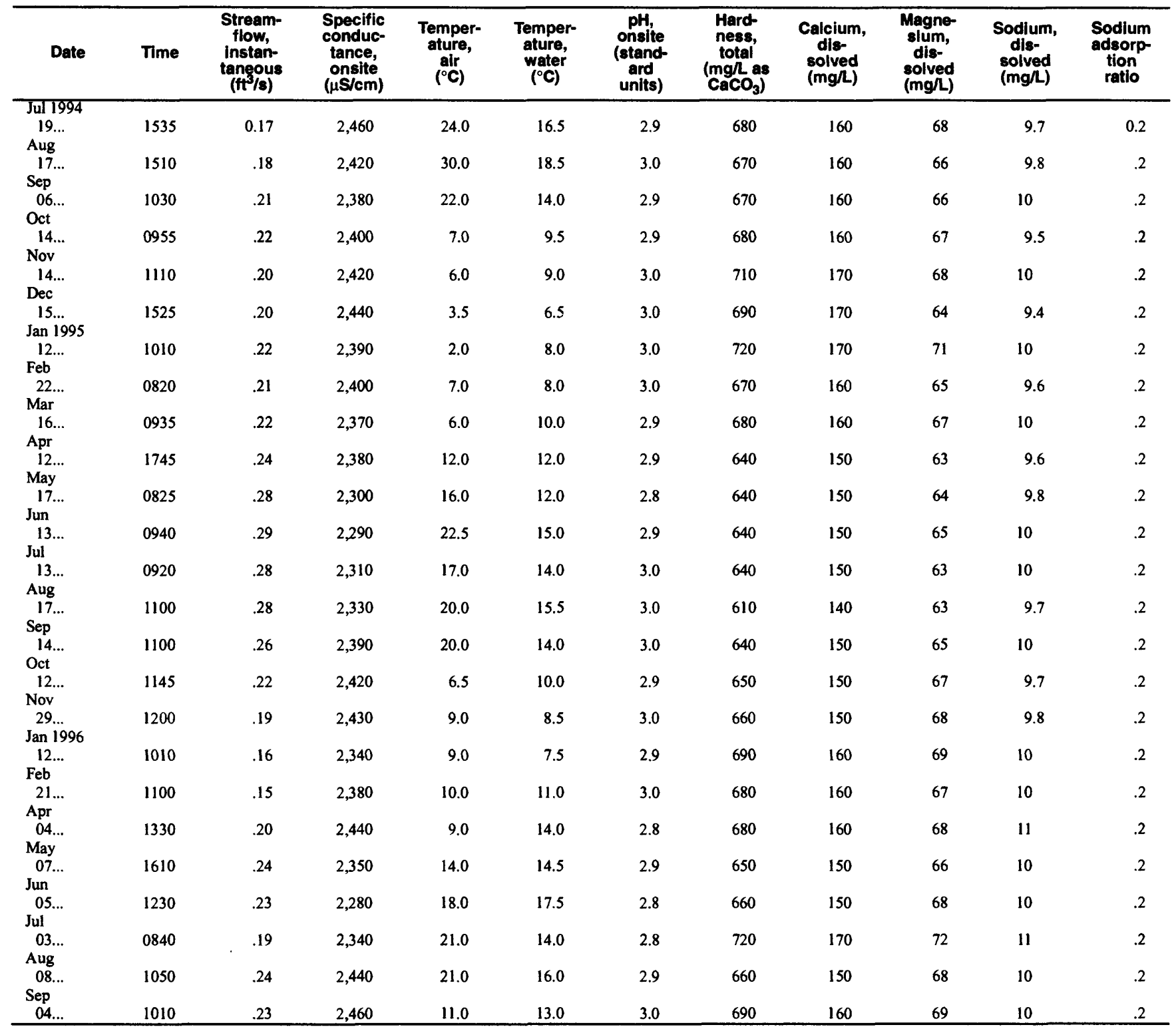


Table 3. Water-quality data for sites in the Sand Coulee Coal Area, Montana July 1994 through September 1996 (Continued) SITE 5, 06090590--ANACONDA DRAIN AT BELT, MT--Continued

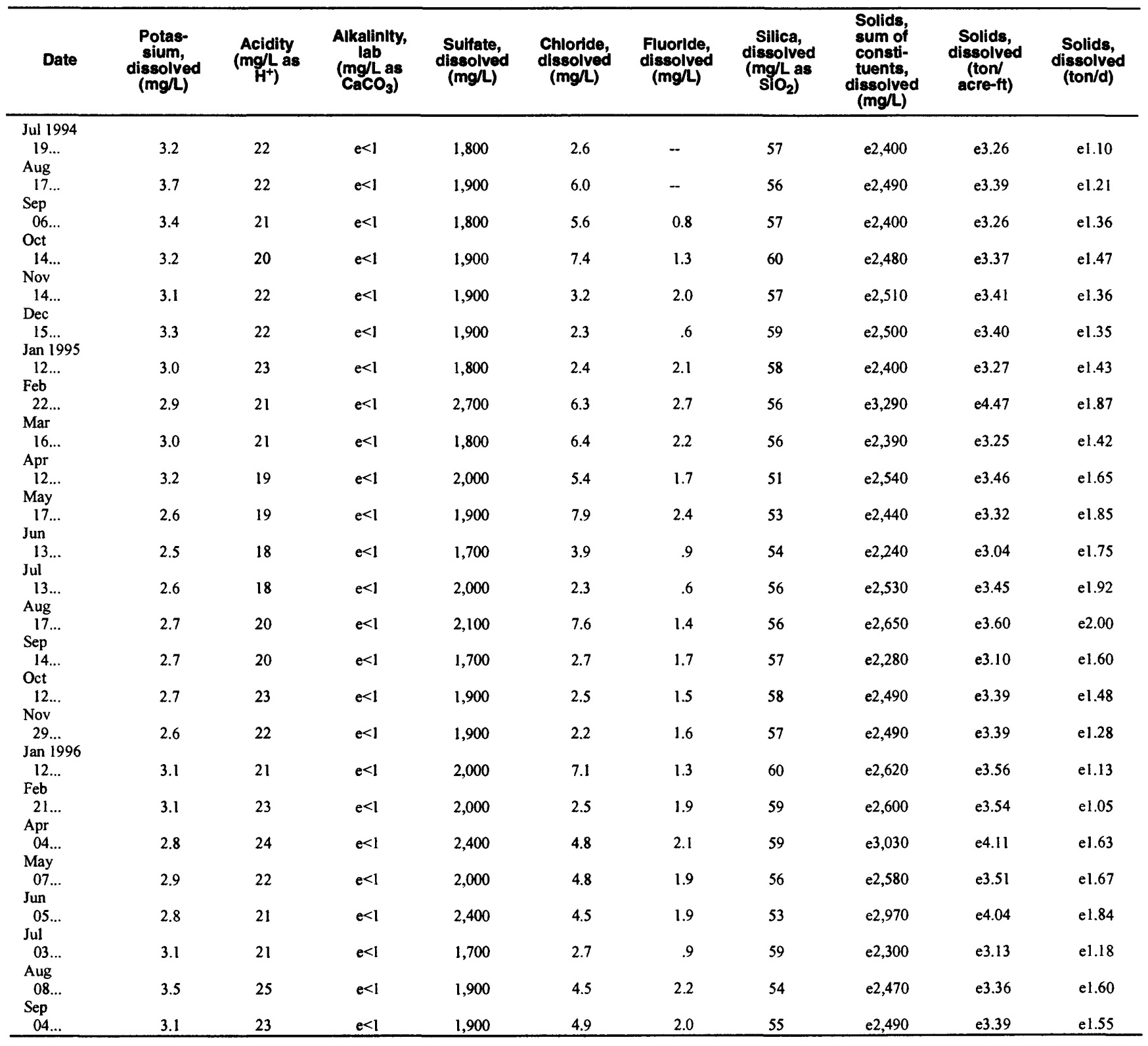


Table 3. Water-quality data for sites in the Sand Coulee Coal Area, Montana July 1994 through September 1996 (Continued) SITE 5, 06090590--ANACONDA DRAIN AT BELT, MT--Continued

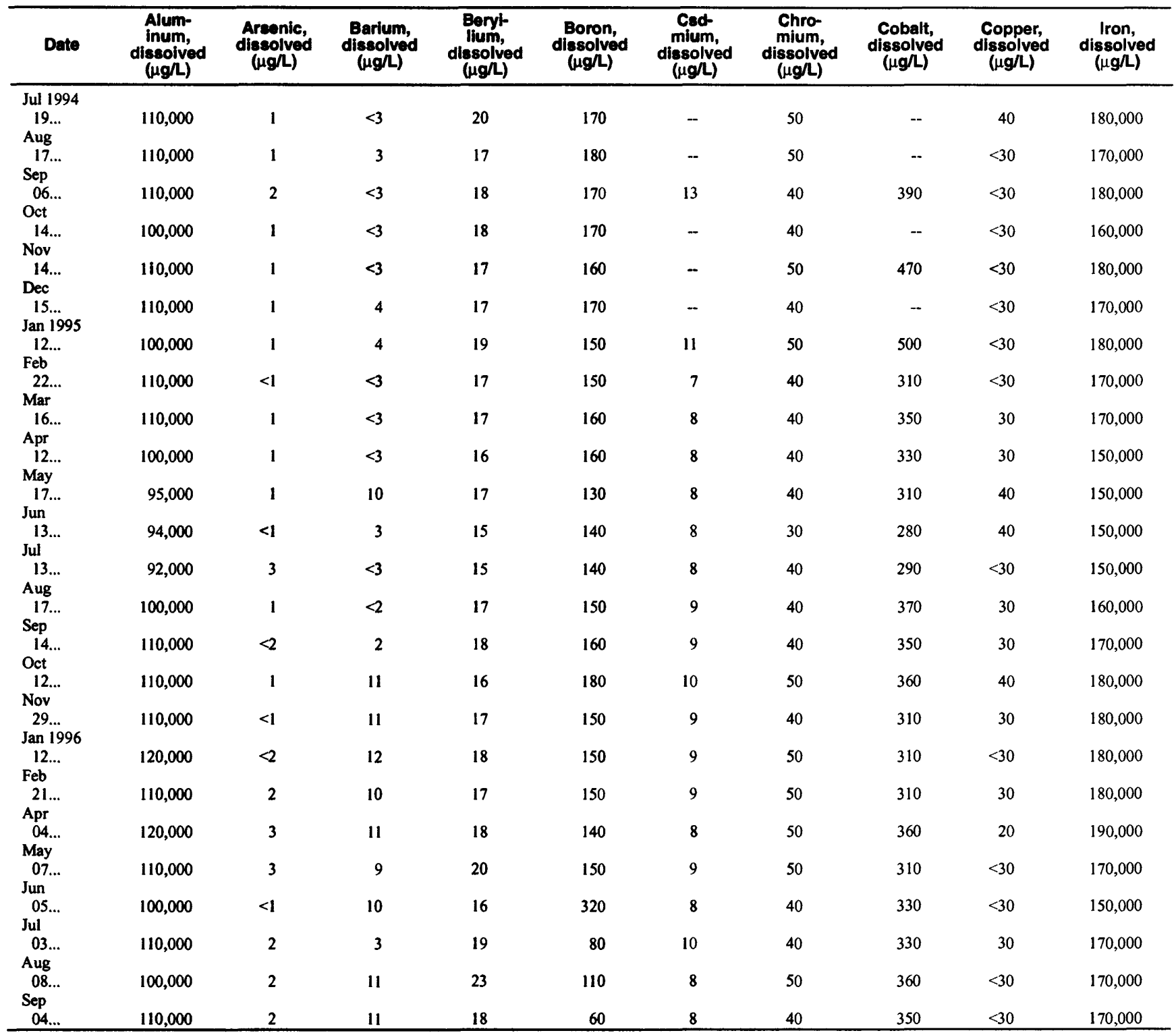


Table 3. Water-quality data for sites in the Sand Coulee Coal Area, Montana July 1994 through September 1996 (Continued) SITE 5, 06090590--ANACONDA DRAIN AT BELT, MT--Continued

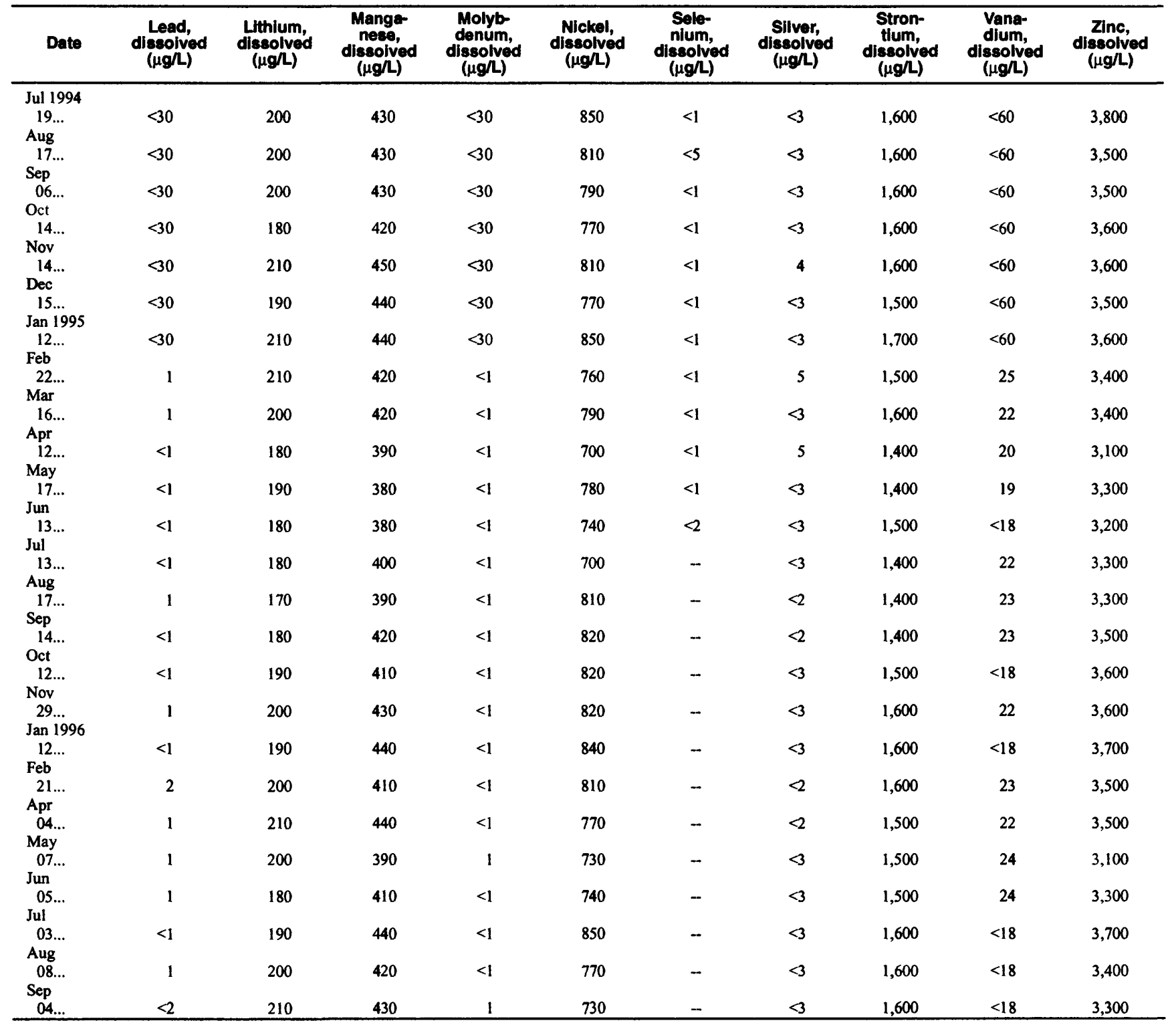


Table 3. Water-quality data for sites in the Sand Coulee Coal Area, Montana, July 1994 through September 1996 (Continued) SITE 6, 471851111111101--GIFFEN SPRING NEAR STOCKETT, MT

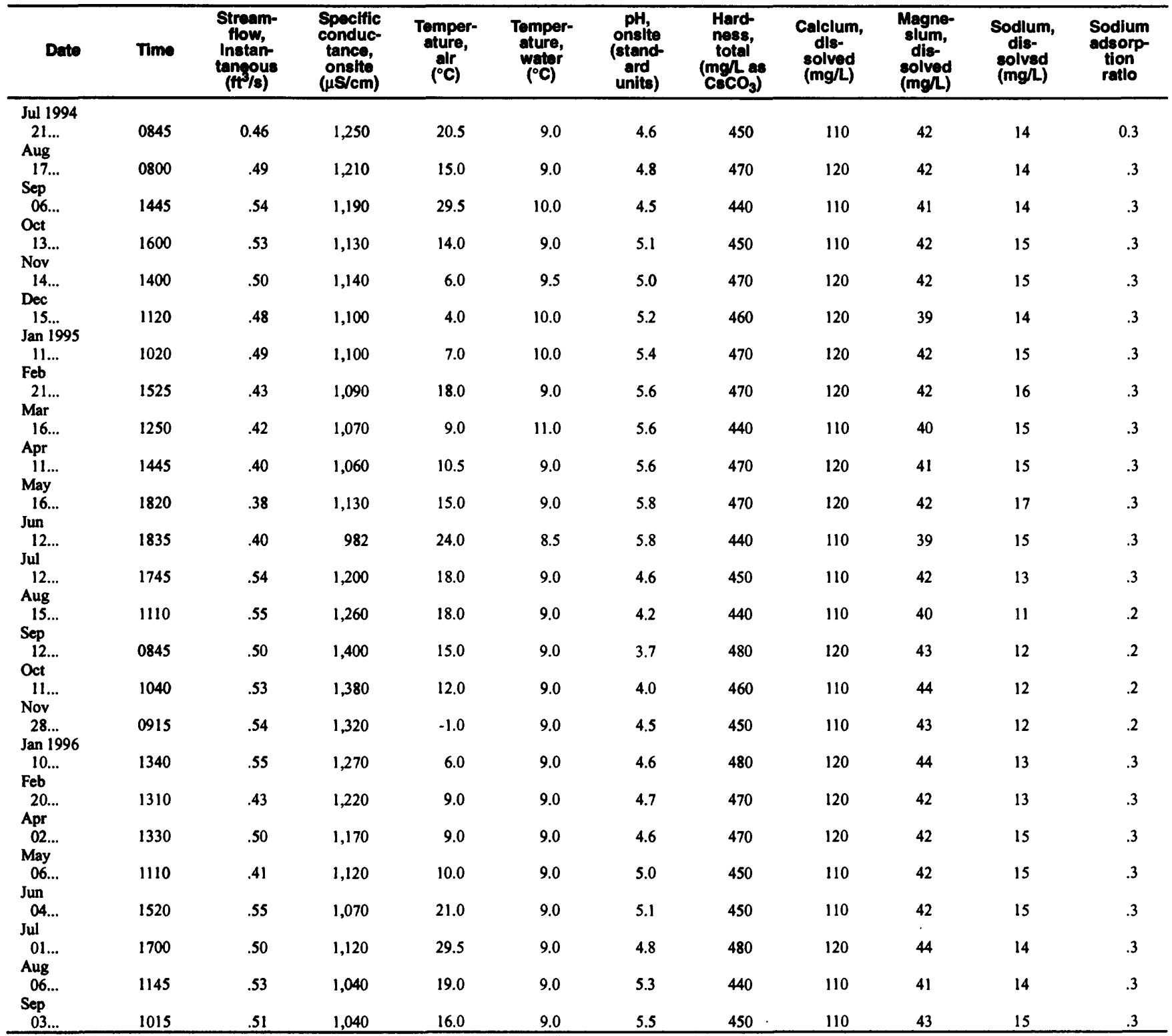


Table 3. Water-quality data for sites in the Sand Coulee Coal Area, Montana, July 1994 through September 1996 (Continued)

SITE 6, 471851111111101--GIFFEN SPRING NEAR STOCKETT, MT--Continued

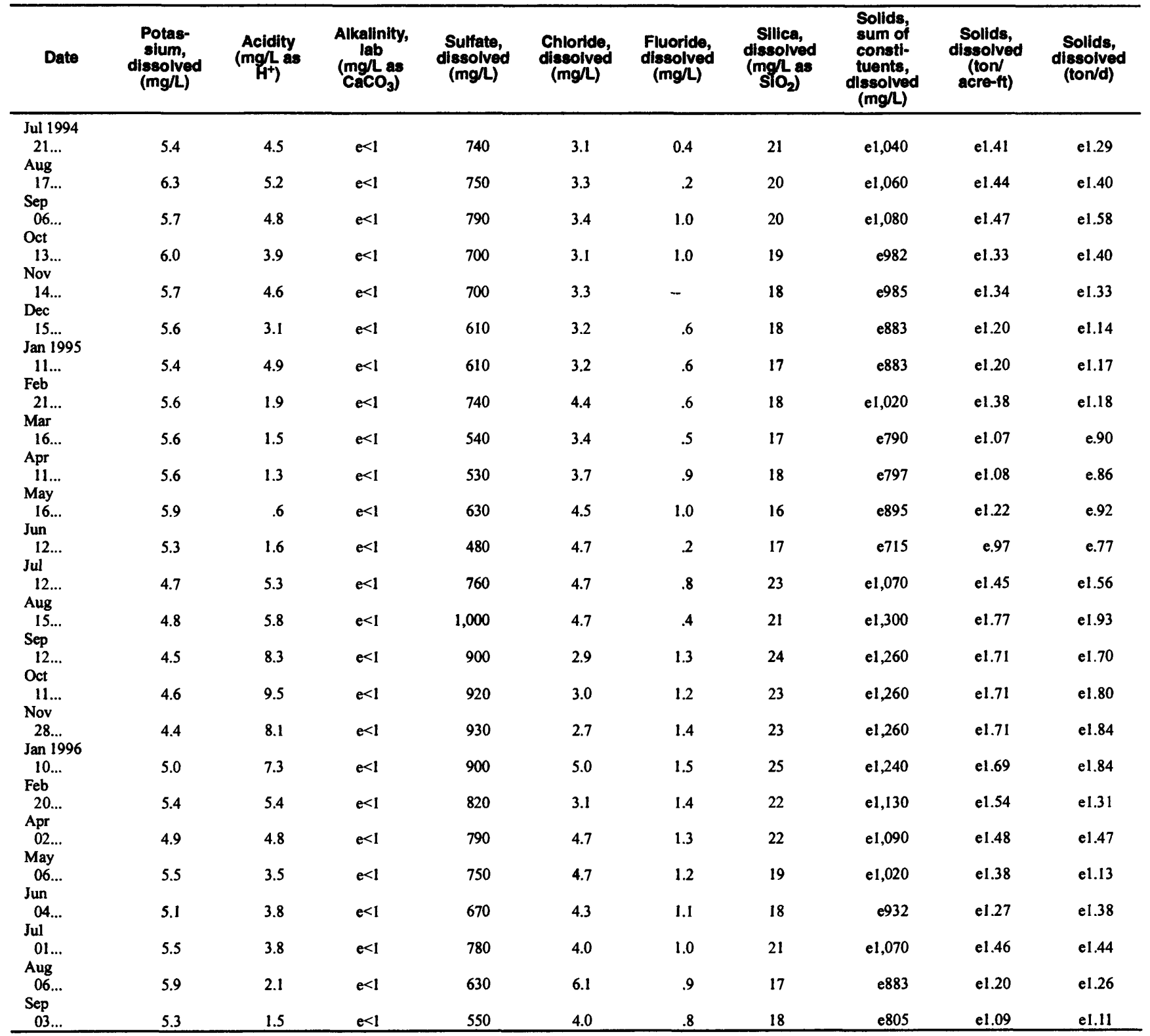


Table 3. Water-quality data for sites in the Sand Coulee Coal Area, Montana, July 1994 through September 1996 (Continued) SITE 6, 471851111111101--GIFFEN SPRING NEAR STOCKETT, MT--Continued

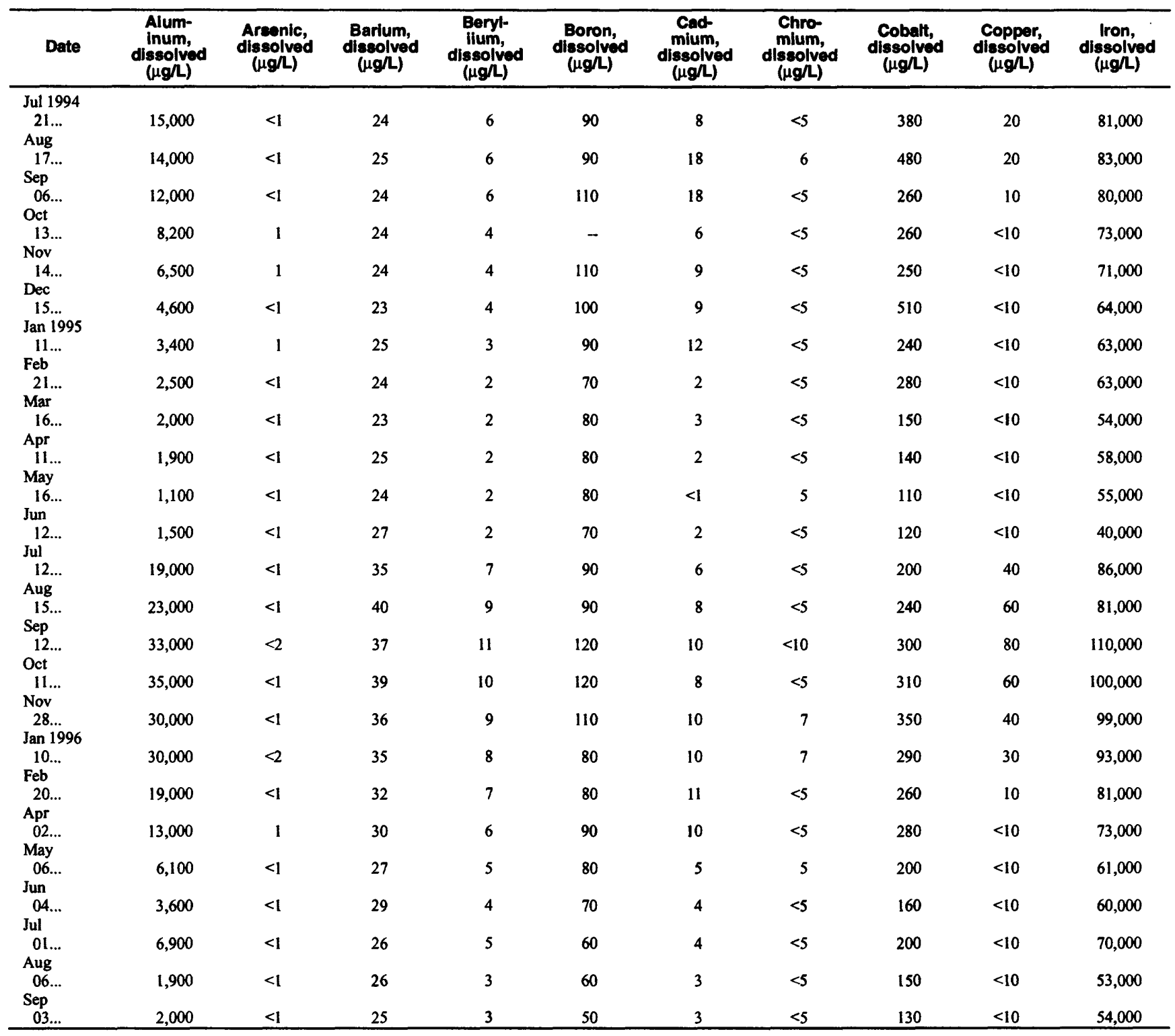


Table 3. Water-quality data for sites in the Sand Coulee Coal Area, Montana, July 1994 through September 1996 (Continued) SITE 6, 471851111111101--GIFFEN SPRING NEAR STOCKETT, MT--Continued

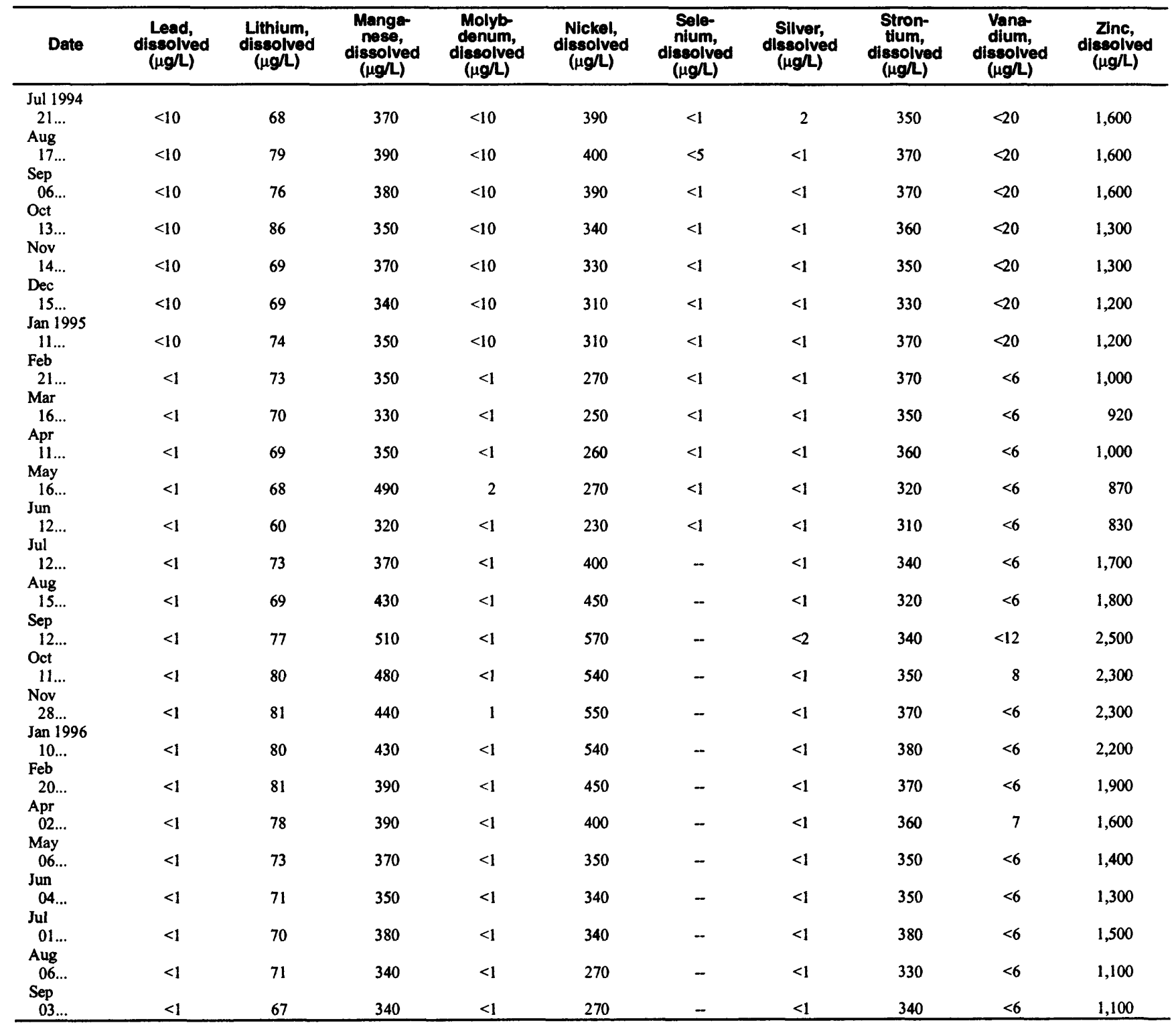


Table 3. Water-quality data for sites in the Sand Coulee Coal Area, Montana, July 1994 through September 1996 (Continued) SITE 7, 472016111085701--COTTONWOOD MINE NO. 6 DRAIN TO COTTONWOOD CREEK NEAR STOCKETT, MT

\begin{tabular}{|c|c|c|c|c|c|c|c|c|c|c|c|}
\hline Date & Time & $\begin{array}{c}\text { Stream- } \\
\text { flow, } \\
\text { instan- } \\
\text { taneous } \\
\left(\mathrm{ft}^{3} / \mathrm{s}\right)\end{array}$ & $\begin{array}{c}\text { Specific } \\
\text { conduc- } \\
\text { tance, } \\
\text { onsite } \\
(\mu S / \mathrm{cm})\end{array}$ & $\begin{array}{c}\text { Temper- } \\
\text { ature, } \\
\text { air } \\
\left({ }^{\circ} \mathrm{C}\right)\end{array}$ & $\begin{array}{c}\text { Temper- } \\
\text { ature, } \\
\text { water } \\
\left({ }^{\circ} \mathrm{C}\right)\end{array}$ & $\begin{array}{l}\text { pH, } \\
\text { onsite } \\
\text { (stand- } \\
\text { ard } \\
\text { units) }\end{array}$ & $\begin{array}{c}\text { Hard- } \\
\text { ness, } \\
\text { total } \\
\left(\mathrm{mg}^{2} \text { as }\right. \\
\left.\mathrm{CaCO}_{3}\right)\end{array}$ & $\begin{array}{c}\text { Calcium, } \\
\text { dis- } \\
\text { solved } \\
\text { (mg/L) }\end{array}$ & $\begin{array}{l}\text { Magne- } \\
\text { sium, } \\
\text { dis- } \\
\text { solved } \\
\text { (mgll) }\end{array}$ & $\begin{array}{c}\text { Sodium, } \\
\text { dis- } \\
\text { solved } \\
\text { (mg/L) }\end{array}$ & $\begin{array}{l}\text { Sodium } \\
\text { adsorp- } \\
\text { tion ratio }\end{array}$ \\
\hline $\begin{array}{l}\text { Feb } 1995 \\
21 \ldots \\
\text { Mar }\end{array}$ & 1720 & 0.02 & 6,020 & 10.5 & 9.0 & 2.6 & 1,300 & 330 & 120 & 13 & 0.2 \\
\hline $\begin{array}{l}16 \ldots \\
\text { Apr }\end{array}$ & 1215 & .02 & 5,940 & 11.0 & 9.5 & 2.6 & 1,400 & 350 & 130 & 14 & .2 \\
\hline $\begin{array}{l}11 \ldots \\
\text { May }\end{array}$ & 1400 & .02 & 5,960 & 8.0 & 9.5 & 2.6 & 1,300 & 340 & 120 & 13 & .2 \\
\hline $\begin{array}{l}17 \ldots \\
\text { Jun }\end{array}$ & 1345 & .05 & 5,620 & 21.0 & 11.5 & 2.5 & 1,400 & 340 & 130 & 13 & .2 \\
\hline $\begin{array}{c}12 \ldots \\
\text { Jul }\end{array}$ & 1750 & .15 & 5,610 & 26.0 & 10.0 & 2.7 & $\mathrm{I}, 400$ & 350 & 130 & 15 & .2 \\
\hline $\begin{array}{l}12 \ldots \\
\text { Aug }\end{array}$ & 1610 & .12 & 5,580 & 20.0 & 10.0 & 2.8 & 1,500 & 360 & 140 & 15 & .2 \\
\hline $\begin{array}{c}15 \ldots \\
\text { Sep }\end{array}$ & 1150 & .09 & 5,730 & 21.0 & 11.0 & 2.7 & 1,500 & 350 & 140 & 14 & .2 \\
\hline $\begin{array}{l}12 \ldots \\
\text { Oct }\end{array}$ & 1100 & .06 & 5,770 & 19.0 & 10.5 & 2.6 & 1,500 & 370 & 140 & 14 & .2 \\
\hline $\begin{array}{l}11 \ldots \\
\text { Nov }\end{array}$ & 1130 & .05 & 5,790 & 16.0 & 10.0 & 2.6 & 1,400 & 340 & 140 & 14 & .2 \\
\hline $\begin{array}{c}28 \ldots \\
\operatorname{Jan} 1996\end{array}$ & 1000 & .04 & 5,880 & -1.0 & 6.0 & 2.6 & 1,400 & 340 & 140 & 14 & .2 \\
\hline $\begin{array}{l}10 \ldots \\
\text { Feb }\end{array}$ & 1450 & .03 & 5,870 & 7.0 & 8.0 & 2.6 & 1,500 & 380 & 140 & 13 & .1 \\
\hline $\begin{array}{l}20 \ldots \\
\text { Apr }\end{array}$ & 1400 & .02 & 5,980 & 9.0 & 8.0 & 2.6 & 1,500 & 350 & 140 & 14 & .2 \\
\hline $\begin{array}{l}02 \ldots \\
\text { May }\end{array}$ & 1410 & .02 & 5,890 & 10.0 & 8.0 & 2.6 & 1,400 & 360 & 130 & 14 & .2 \\
\hline $\begin{array}{l}06 \ldots \\
\text { Jun }\end{array}$ & 1145 & .02 & 5,820 & 15.0 & 9.0 & 2.7 & 1,400 & 350 & 130 & 13 & .2 \\
\hline $\begin{array}{l}03 \ldots \\
\text { Jul }\end{array}$ & 1710 & .03 & 5,790 & 25.0 & 10.0 & 2.7 & 1,500 & 350 & 140 & 14 & .2 \\
\hline $\begin{array}{l}01 \ldots . . \\
\text { Aug }\end{array}$ & 1510 & .02 & 5,570 & 31.0 & 11.0 & 2.7 & 1,400 & 340 & 130 & 13 & .2 \\
\hline $\begin{array}{l}06 \ldots \\
\text { Sep }\end{array}$ & 1220 & .02 & 5,680 & 21.0 & 11.0 & 2.5 & 1,400 & 330 & 130 & 14 & .2 \\
\hline 03 & 1110 & .02 & 5,680 & 18.0 & 10.5 & 2.7 & 1,400 & 340 & 130 & 13 & .2 \\
\hline
\end{tabular}


Table 3. Water-quality data for sites in the Sand Coulee Coal Area, Montana, July 1994 through September 1996 (Continued)

SITE 7, 472016111085701--COTTONWOOD MINE NO. 6 DRAIN TO COTTONWOOD CREEK NEAR STOCKETT, MT--Continued

\begin{tabular}{|c|c|c|c|c|c|c|c|c|c|c|}
\hline Date & $\begin{array}{l}\text { Potas- } \\
\text { sium, } \\
\text { dissolved } \\
\text { (mg/L) }\end{array}$ & $\begin{array}{c}\text { Acldity } \\
\left(\mathbf{m g}^{\prime} / \mathrm{h} \text { as }\right. \\
\left.\mathrm{H}^{+}\right)\end{array}$ & $\begin{array}{c}\text { Alkalinity, } \\
\text { lab } \\
\left(\mathrm{mg}_{\mathrm{L}} \text { as }\right. \\
\left.\mathrm{CaCO}_{3}\right)\end{array}$ & $\begin{array}{l}\text { Sulfate, } \\
\text { dissolved } \\
\text { (mg/L) }\end{array}$ & $\begin{array}{l}\text { Chloride, } \\
\text { dissolved } \\
\text { (mg/L) }\end{array}$ & $\begin{array}{l}\text { Fluoride, } \\
\text { dissolved } \\
\text { (mg/l) }\end{array}$ & $\begin{array}{l}\text { Silica, } \\
\text { dissolved } \\
\text { (mgh as } \\
\left.\mathrm{SlO}_{2}\right)\end{array}$ & $\begin{array}{l}\text { Solids, } \\
\text { sum of } \\
\text { consti- } \\
\text { tuents, } \\
\text { dissolved } \\
\text { (mg/L) }\end{array}$ & $\begin{array}{l}\text { Solids, } \\
\text { diseolved } \\
\text { (ton/ } \\
\text { acre-ft) }\end{array}$ & $\begin{array}{l}\text { Sollds, } \\
\text { dissolved } \\
\text { (ton/d) }\end{array}$ \\
\hline $\begin{array}{c}\text { Feb } 1995 \\
21 \ldots \\
\text { Mar }\end{array}$ & 2.1 & 81 & $e<1$ & 7,200 & 14 & 3.7 & 93 & $\mathrm{e} 8,950$ & el2.2 & $\mathrm{e} 0.60$ \\
\hline $\begin{array}{l}16 \ldots \\
\text { Apr }\end{array}$ & 2.1 & 77 & $e<1$ & 5,900 & 15 & 2.8 & 100 & e7,750 & e 10.5 & e. 50 \\
\hline $\begin{array}{l}11 \ldots \\
\text { May }\end{array}$ & 2.3 & 81 & $e<1$ & 6,800 & 20 & 4.0 & 96 & e8,580 & ell.7 & e. 53 \\
\hline $\begin{array}{l}17 \ldots \\
\text { Jun }\end{array}$ & 1.7 & 77 & $e<1$ & 5,500 & 1.3 & 5.6 & 97 & e6,530 & e8.89 & e. 92 \\
\hline $\begin{array}{c}12 \ldots \\
\mathrm{Jul}\end{array}$ & 3.9 & 74 & $e<1$ & 5,600 & 3.8 & $<1$ & 83 & e6,600 & e8.98 & e2.67 \\
\hline $\begin{array}{l}12 \ldots \\
\text { Aug }\end{array}$ & 5.7 & 75 & $e<1$ & 5,800 & 3.3 & $<1$ & 83 & e7,650 & e 10.4 & e2.48 \\
\hline $\begin{array}{l}15 \ldots \\
\text { Sep }\end{array}$ & 3.7 & 83 & $e<1$ & 6,600 & 18 & - & 87 & e8,490 & ell.5 & e2.15 \\
\hline Oct & .5 & 84 & $e<1$ & 5,600 & 1.5 & -- & 87 & e7,560 & el0.3 & el. 22 \\
\hline $\begin{array}{l}11 \ldots \\
\text { Nov }\end{array}$ & 2.1 & 83 & $e<1$ & 5,800 & 1.3 & 1.6 & 86 & e7,640 & el0.4 & el. 03 \\
\hline $\begin{array}{l}28 \ldots \\
\text { Jan } 1996\end{array}$ & 2.1 & 83 & $e<1$ & 5,600 & 12 & 1.5 & 86 & e 7,470 & e 10.2 & e.73 \\
\hline $\begin{array}{l}10 \ldots \\
\text { Feb }\end{array}$ & 2.4 & 87 & $e<1$ & 6,200 & $<1$ & - & 99 & e8,170 & 11.1 & $e .66$ \\
\hline $\begin{array}{l}20 \ldots \\
\text { Apr }\end{array}$ & 2.6 & 86 & $e<1$ & 5,900 & $<1$ & 1.3 & 97 & e 7,830 & 11.7 & e.53 \\
\hline $\begin{array}{l}02 \ldots \\
\text { May }\end{array}$ & 2.7 & 84 & $e<1$ & 6,000 & 4.8 & 2.9 & 99 & e7,920 & el0.8 & e.43 \\
\hline $\begin{array}{l}06 \ldots . . \\
\text { Jun }\end{array}$ & .6 & 85 & $e<1$ & 6,500 & 2.0 & 2.8 & 98 & e8,350 & ell.4 & e. 56 \\
\hline Jul & 3.0 & 85 & $e<1$ & 7,200 & 3.8 & $<1$ & 98 & e9,140 & el2.4 & e.69 \\
\hline $\begin{array}{l}01 \ldots \\
\text { Aug }\end{array}$ & 3.6 & 82 & $e<1$ & 5,800 & 13 & $<1$ & 97 & e7,640 & el0.4 & e. 52 \\
\hline $\begin{array}{l}06 \ldots \\
\text { Sep }\end{array}$ & 4.0 & 86 & $e<1$ & 5,400 & 2.3 & 4.1 & 94 & e 7,210 & e9.80 & e.49 \\
\hline & 3.6 & 85 & $e<1$ & 5,400 & 3.4 & 3.9 & 92 & e 7,180 & e9.76 & e.39 \\
\hline
\end{tabular}


Table 3. Water-quality data for sites in the Sand Coulee Coal Area, Montana, July 1994 through September 1996 (Continued) SITE 7, 472016111085701--COTTONWOOD MINE NO. 6 DRAIN TO COTTONWOOD CREEK NEAR STOCKETT, MT--Continued

\begin{tabular}{|c|c|c|c|c|c|c|c|c|c|c|}
\hline Date & $\begin{array}{c}\text { Alum- } \\
\text { inum, } \\
\text { dissolved } \\
(\mu \mathrm{g} / \mathrm{L})\end{array}$ & $\begin{array}{l}\text { Arsenic, } \\
\text { dissolved } \\
(\mu \mathrm{g} / \mathrm{L})\end{array}$ & $\begin{array}{c}\text { Barium, } \\
\text { dissolved } \\
\left(\mu g^{\prime} / L\right)\end{array}$ & $\begin{array}{c}\text { Beryl- } \\
\text { lium, } \\
\text { dissolved } \\
(\mu \mathrm{g} / \mathrm{L})\end{array}$ & $\begin{array}{c}\text { Boron, } \\
\text { dissolved } \\
(\mu \mathrm{g} / \mathrm{L})\end{array}$ & $\begin{array}{c}\text { Cad- } \\
\text { mium, } \\
\text { dissolved } \\
(\mu \mathrm{g} / \mathrm{L})\end{array}$ & $\begin{array}{c}\text { Chro- } \\
\text { mium, } \\
\text { dissolved } \\
(\mu g / L)\end{array}$ & $\begin{array}{l}\text { Cobalt, } \\
\text { dissolved } \\
\left(\mu g^{\prime} L\right)\end{array}$ & $\begin{array}{l}\text { Copper, } \\
\text { dissolved } \\
\left(\mu g^{\prime} L\right)\end{array}$ & $\begin{array}{c}\text { Iron, } \\
\text { dissolved } \\
(\mu g / L)\end{array}$ \\
\hline $\begin{array}{c}\text { Feb } 1995 \\
21 \ldots\end{array}$ & 410,000 & $<10$ & -- & 120 & 480 & 82 & 40 & 6,000 & 80 & 690,000 \\
\hline $\begin{array}{l}16 \ldots \\
\text { Apr }\end{array}$ & 410,000 & $<5$ & -- & 120 & 500 & 79 & 40 & 6,000 & 80 & 750,000 \\
\hline $\begin{array}{l}11 \ldots \\
\text { May }\end{array}$ & 410,000 & $<10$ & - & 120 & 520 & 79 & 40 & 6,000 & 80 & 700,000 \\
\hline $\begin{array}{l}17 \ldots \\
\text { Jun }\end{array}$ & 380,000 & $<1$ & 100 & 94 & 470 & 73 & $<50$ & 5,700 & $<100$ & 660,000 \\
\hline $\begin{array}{c}12 \ldots \\
\text { Jul }\end{array}$ & 350,000 & $<1$ & -- & 110 & 490 & 110 & 50 & 3,900 & 210 & 740,000 \\
\hline $\begin{array}{l}12 \ldots \\
\text { Aug }\end{array}$ & 350,000 & $<5$ & -- & 100 & 480 & 80 & $<50$ & 5,100 & 140 & 830,000 \\
\hline $\begin{array}{l}15 \ldots \\
\text { Sep }\end{array}$ & 390,000 & $<5$ & -- & 110 & 570 & 100 & 60 & 6,600 & 180 & 810,000 \\
\hline oct & 430,000 & $<25$ & -- & 120 & 500 & 100 & $<50$ & 6,900 & 170 & 840,000 \\
\hline $\begin{array}{l}11 \ldots \\
\text { Nov }\end{array}$ & 380,000 & $<5$ & 110 & 110 & 520 & 81 & $<50$ & 6,000 & 130 & 800,000 \\
\hline $\begin{array}{c}28 \ldots \\
\operatorname{Jan} 1996\end{array}$ & 410,000 & $<1$ & 110 & 110 & 520 & 82 & 60 & 6,300 & 110 & 790,000 \\
\hline $\begin{array}{l}10 \ldots \\
\text { Feb }\end{array}$ & 450,000 & $<2$ & 130 & 120 & 530 & 72 & 60 & -- & $<100$ & 810,000 \\
\hline $\begin{array}{c}20 \ldots \\
\text { Apr }\end{array}$ & 440,000 & $<5$ & 120 & 120 & 490 & 79 & 60 & 9,700 & 140 & 810,000 \\
\hline $\begin{array}{l}02 \ldots \\
\text { May }\end{array}$ & 420,000 & $<1$ & 120 & 120 & 520 & 75 & $<50$ & 6,500 & 100 & 810,000 \\
\hline $\begin{array}{c}06 \ldots \\
\text { Jun }\end{array}$ & 400,000 & $<5$ & 120 & 130 & 540 & 98 & $<50$ & 6,600 & $<100$ & 780,000 \\
\hline $\begin{array}{l}03 \ldots \\
\text { Jul }\end{array}$ & 420,000 & $<10$ & 120 & 130 & - & 84 & $<80$ & 6,200 & 110 & 840,000 \\
\hline $\begin{array}{c}01 \ldots \\
\text { Aug }\end{array}$ & 390,000 & $<5$ & 120 & 110 & - & 83 & 80 & 6,300 & 110 & 770,000 \\
\hline $\begin{array}{c}06 \ldots \\
\text { Sep }\end{array}$ & 390,000 & $<5$ & 110 & 120 & - & 78 & $<50$ & 5,800 & $<100$ & 770,000 \\
\hline $03 \ldots$ & 380,000 & $<5$ & 120 & 120 & 510 & 77 & $<50$ & 6,200 & $<100$ & 740,000 \\
\hline
\end{tabular}


Table 3. Water-quality data for sites in the Sand Coulee Coal Area, Montana, July 1994 through September 1996 (Continued) SITE 7, 472016111085701--COTTONWOOD MINE NO. 6 DRAIN TO COTTONWOOD CREEK NEAR STOCKETT, MT--Continued

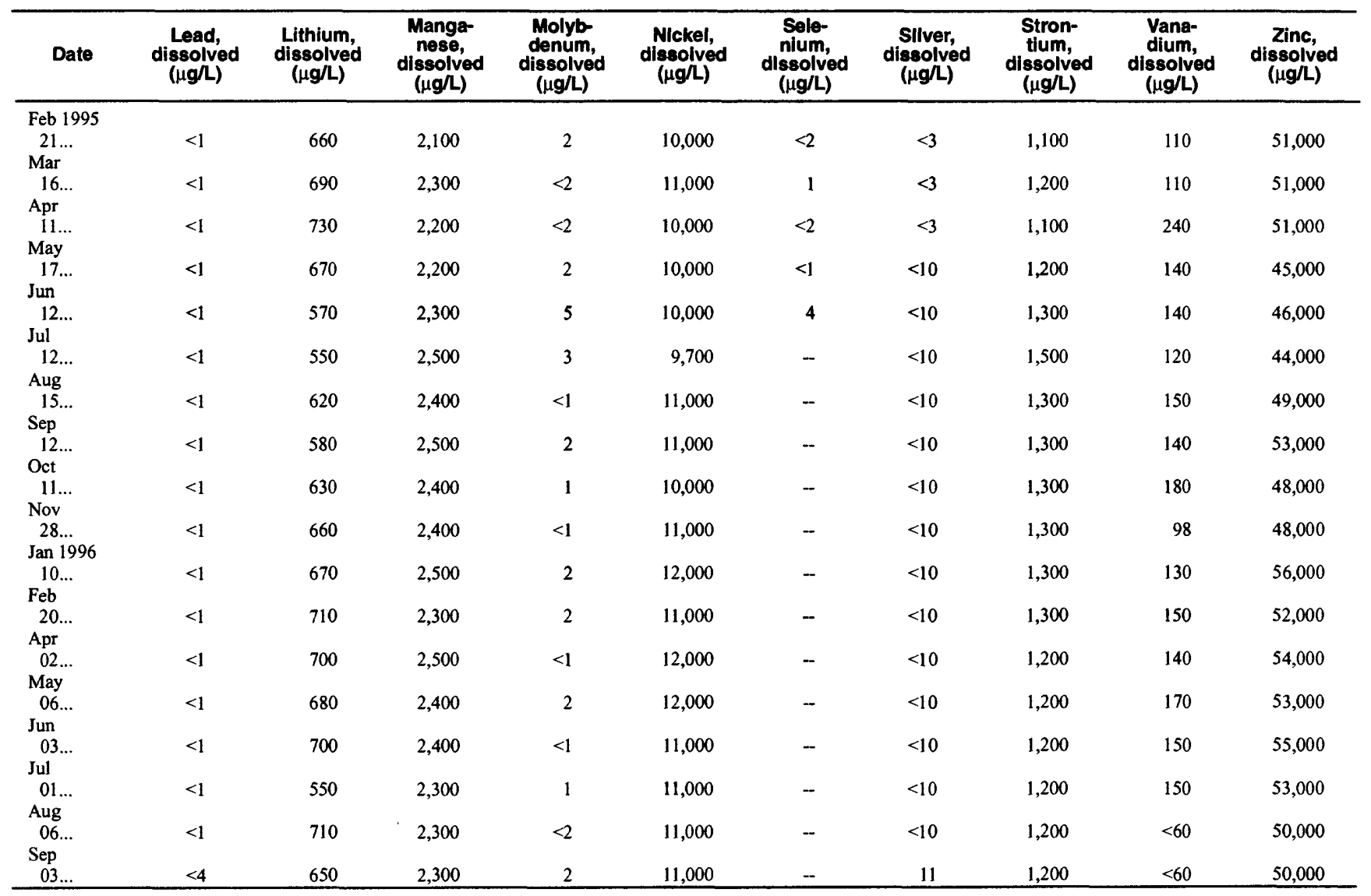


Table 3. Water-quality data for sites in the Sand Coulee Coal Area, Montana, July 1994 through September 1996 (Continued) SITE 8, 472114111095001--COTTONWOOD MINE NO. 2 DRAIN TO LADD COULEE AT STOCKETT, MT

\begin{tabular}{|c|c|c|c|c|c|c|c|c|c|c|c|}
\hline Date & Time & $\begin{array}{c}\text { Stream- } \\
\text { flow, } \\
\text { instan- } \\
\text { taneous } \\
\left(\mathrm{ft}^{3} / \mathrm{s}\right)\end{array}$ & $\begin{array}{c}\text { Specific } \\
\text { conduc- } \\
\text { tance, } \\
\text { onsite } \\
(\mu S / \mathrm{cm})\end{array}$ & $\begin{array}{c}\text { Temper- } \\
\text { ature, } \\
\text { air } \\
\left({ }^{\circ} \mathrm{C}\right)\end{array}$ & $\begin{array}{l}\text { Temper- } \\
\text { ature, } \\
\text { water } \\
\left({ }^{\circ} \mathrm{C}\right)\end{array}$ & $\begin{array}{c}\text { pH, } \\
\text { onsite } \\
\text { (stand- } \\
\text { ard } \\
\text { units) }\end{array}$ & $\begin{array}{c}\text { Hard- } \\
\text { ness, } \\
\text { total } \\
(\mathrm{mg} / \mathrm{L} \text { as } \\
\left.\mathrm{CaCO}_{3}\right)\end{array}$ & $\begin{array}{l}\text { Calclum, } \\
\text { dis- } \\
\text { solved } \\
\text { (mg/l) }\end{array}$ & $\begin{array}{l}\text { Magne- } \\
\text { slum, } \\
\text { dis- } \\
\text { solved } \\
\text { (mg/l) } \\
\end{array}$ & $\begin{array}{l}\text { Sodium, } \\
\text { dis- } \\
\text { solved } \\
\text { (mg/L) }\end{array}$ & $\begin{array}{l}\text { Sodium } \\
\text { adsorp- } \\
\text { tion ratio }\end{array}$ \\
\hline $\begin{array}{l}\text { Jul } 1994 \\
21 \ldots \\
\text { Aug }\end{array}$ & 0720 & 0.01 & 9,610 & 12.5 & 11.0 & 2.7 & 2,700 & 470 & 360 & 10 & 0.1 \\
\hline $\begin{array}{l}17 \ldots \\
\text { Sep }\end{array}$ & 0720 & .01 & 9,780 & 13.0 & 12.0 & 2.6 & 2,600 & 480 & 350 & 12 & .1 \\
\hline $\begin{array}{l}06 \ldots \\
\text { Oct }\end{array}$ & 1420 & .005 & 9,860 & 29.5 & 19.0 & 2.4 & 2,900 & 450 & 430 & 11 & .1 \\
\hline $\begin{array}{l}13 \ldots \\
\text { Nov }\end{array}$ & 1525 & .009 & 9,120 & 14.0 & 11.0 & 2.5 & 2,400 & 430 & 320 & 8.9 & .1 \\
\hline $\begin{array}{l}14 \ldots \\
\text { Dec }\end{array}$ & 1450 & .02 & 9,430 & 6.0 & 0.0 & 2.6 & 2,400 & 400 & 330 & 4.7 & $<.1$ \\
\hline $\begin{array}{c}15 \ldots \\
\text { Jan } 1995\end{array}$ & 1100 & -- & 10,100 & 3.5 & 0.0 & 2.6 & 2,700 & 450 & 370 & 9.8 & .1 \\
\hline $\begin{array}{l}11 \ldots \\
\text { Feb }\end{array}$ & 1105 & .01 & 8,650 & 8.0 & 1.0 & 2.8 & 2,200 & 360 & 310 & 7.3 & .1 \\
\hline $\begin{array}{l}21 \ldots \\
\text { Mar }\end{array}$ & 1710 & .007 & 7,770 & 12.0 & 3.0 & 2.6 & 2,100 & 390 & 270 & 6.9 & .1 \\
\hline $\begin{array}{l}16 \ldots \\
\text { Apr }\end{array}$ & 1230 & .006 & 8,680 & 9.0 & 4.5 & 2.5 & 2,300 & 410 & 320 & 8.8 & .1 \\
\hline $\begin{array}{l}11 \ldots \\
\text { May }\end{array}$ & 1430 & .05 & 6,470 & 10.5 & 10.5 & 2.6 & 1,700 & 370 & 200 & 5.9 & .1 \\
\hline $\begin{array}{l}17 \ldots \\
\text { Jun }\end{array}$ & 1315 & .02 & 7,270 & 21.0 & 22.0 & 2.3 & 2,300 & 460 & 270 & 9.0 & .1 \\
\hline $\begin{array}{c}12 \ldots \\
\text { Jul }\end{array}$ & 1815 & .07 & 8,390 & 26.0 & 25.5 & 2.4 & 2,000 & 460 & 210 & 9.5 & .1 \\
\hline $\begin{array}{l}12 \ldots \\
\text { Aug }\end{array}$ & 1630 & .10 & 8,170 & 21.0 & 25.5 & 2.4 & 1,800 & 390 & 200 & 9.1 & .1 \\
\hline $\begin{array}{l}15 \ldots \\
\text { Sep }\end{array}$ & 1130 & .06 & 8,530 & 21.0 & 20.5 & 2.4 & 1,800 & 390 & 210 & 9.2 & .1 \\
\hline $\begin{array}{l}12 \ldots \\
\text { Oct }\end{array}$ & 1040 & .04 & 8,530 & 18.0 & 15.0 & 2.4 & 2,000 & 430 & 230 & 9.8 & .1 \\
\hline $\begin{array}{l}11 \ldots \\
\text { Nov }\end{array}$ & 1100 & .03 & 8,800 & 13.0 & 11.5 & 2.4 & 2,000 & 390 & 240 & 8.9 & .1 \\
\hline $\begin{array}{c}28 \ldots \\
\text { Jan } 1996\end{array}$ & 0930 & .03 & 8,750 & -1.0 & .5 & 2.5 & 1,900 & 370 & 240 & 8.4 & .1 \\
\hline $\begin{array}{l}10 \ldots \\
\text { Feb }\end{array}$ & 1420 & .001 & 8,620 & 7.0 & 0.0 & 2.6 & 2,000 & 370 & 260 & 8.5 & .1 \\
\hline $\begin{array}{l}20 \ldots \\
\text { Apr }\end{array}$ & 1330 & .02 & 8,490 & 10.0 & 2.5 & 2.5 & 2,000 & 370 & 250 & 7.4 & .1 \\
\hline $\begin{array}{l}02 \ldots \\
\text { May }\end{array}$ & 1345 & .04 & 7,040 & 10.0 & 7.5 & 2.5 & 1,600 & 320 & 200 & 6.4 & .1 \\
\hline $\begin{array}{l}06 \ldots \\
\text { Jun }\end{array}$ & 1130 & .008 & 9,200 & 12.0 & 11.0 & 2.5 & 2,400 & 430 & 310 & 8.6 & .1 \\
\hline $\begin{array}{c}03 \ldots \\
\text { Jul }\end{array}$ & 1750 & .006 & 9,820 & 25.0 & 17.0 & 2.5 & 2,700 & 490 & 360 & 8.9 & .1 \\
\hline $\begin{array}{l}01 \ldots \\
\text { Aug }\end{array}$ & 1540 & .003 & 10,400 & 31.0 & 26.0 & 2.2 & 3,000 & 520 & 410 & 9.4 & .1 \\
\hline $\begin{array}{l}06 \ldots \\
\text { Sep }\end{array}$ & 1200 & .005 & 10,800 & 21.0 & 20.0 & 2.2 & 3,000 & 510 & 420 & 10 & .1 \\
\hline $03 \ldots$ & 1045 & .007 & 10,600 & 17.0 & 15.0 & 2.5 & 3,100 & 520 & 430 & 11 & .1 \\
\hline
\end{tabular}


Table 3. Water-quality data for sites in the Sand Coulee Coal Area, Montana, July 1994 through September 1996 (Continued)

SITE 8, 472114111095001 --COTTONWOOD MINE NO. 2 DRAIN TO LADD COULEE AT STOCKETT, MT--Continued

\begin{tabular}{|c|c|c|c|c|c|c|c|c|c|}
\hline Date & $\begin{array}{l}\text { Potas- } \\
\text { sium, } \\
\text { dissolved } \\
\text { (mg/L) }\end{array}$ & $\begin{array}{c}\text { Acidity } \\
\left(\mathrm{mgl}^{+} \text {as }\right. \\
\left.\mathrm{H}^{+}\right)\end{array}$ & $\begin{array}{c}\text { Alkalinity, } \\
\text { lab } \\
\left(\mathrm{mgl}_{\mathrm{as}}\right. \\
\left.\mathrm{CaCO}_{3}\right)\end{array}$ & $\begin{array}{l}\text { Sulfate, } \\
\text { dissolved } \\
\text { (mg/l) }\end{array}$ & $\begin{array}{l}\text { Fluoride, } \\
\text { dissolved } \\
\text { (mg/L) }\end{array}$ & $\begin{array}{l}\text { Sillica, } \\
\text { dissolved } \\
\text { (mg/h as } \\
\left.\mathrm{SIO}_{2}\right)\end{array}$ & $\begin{array}{l}\text { Sollds, } \\
\text { sum of } \\
\text { constl- } \\
\text { tuents, } \\
\text { dissolved } \\
\text { (mg/l) }\end{array}$ & $\begin{array}{l}\text { Solids, } \\
\text { dissolved } \\
\text { (ton/ } \\
\text { acre-t) }\end{array}$ & $\begin{array}{l}\text { Solids, } \\
\text { dissolved } \\
\text { (ton/d) }\end{array}$ \\
\hline $\begin{array}{l}\text { Jul } 1994 \\
21 \ldots \\
\text { Aug }\end{array}$ & 5.7 & 230 & $e<1$ & 14,000 & $<1$ & 60 & el8,000 & e24.5 & e0.54 \\
\hline $\begin{array}{l}\text { Aug } \\
17 \ldots \\
\text { Sep }\end{array}$ & 5.0 & 230 & $e<1$ & 13,000 & $<1$ & 41 & el7,000 & e23.1 & e.46 \\
\hline $\begin{array}{l}06 \ldots \\
\text { Oct }\end{array}$ & 4.4 & 240 & $e<1$ & 15,000 & $<1$ & 39 & el8,900 & e25.8 & e. 26 \\
\hline $\begin{array}{l}13 \ldots \\
\text { Nov }\end{array}$ & 4.8 & 210 & $e<1$ & 13,000 & $<1$ & 110 & el 6,600 & e22.6 & e. 40 \\
\hline $\begin{array}{c}14 \ldots \\
\text { Dec }\end{array}$ & 2.7 & 220 & $e<1$ & 14,000 & - & 66 & el 7,800 & e24.2 & e. 72 \\
\hline $\begin{array}{c}15 \ldots \\
\operatorname{Jan} 1995\end{array}$ & 1.2 & 240 & $e<1$ & 16,000 & $<1$ & 56 & e 20,700 & e28.2 & -- \\
\hline $\begin{array}{l}11 \ldots \\
\text { Feb }\end{array}$ & 1.9 & 190 & $e<1$ & 11,000 & 8.5 & 38 & el3,900 & e 18.9 & e. 45 \\
\hline $\begin{array}{l}21 \ldots \\
\text { Mar }\end{array}$ & .3 & 100 & $e<1$ & 11,000 & 3.2 & 73 & e12,800 & el 7.5 & $e .24$ \\
\hline $\begin{array}{l}16 . . \\
\text { Apr }\end{array}$ & - & 190 & $e<1$ & 15,000 & 6.6 & 92 & e18,200 & e24.8 & e.29 \\
\hline $\begin{array}{l}11 . . \\
\text { May }\end{array}$ & .8 & 120 & $e<1$ & 8,600 & 3.4 & 68 & e10,800 & el4.7 & el. 45 \\
\hline $\begin{array}{l}17 \ldots \\
\text { Jun }\end{array}$ & 1.7 & 130 & $e<1$ & 9,700 & 3.7 & 91 & e 12,200 & e16.6 & e. 63 \\
\hline$\underset{\text { Jul }}{12 \ldots}$ & 2.2 & 180 & $e<1$ & 15,000 & 2.1 & 130 & el8,100 & e24.6 & e3.61 \\
\hline $\begin{array}{l}12 \ldots \\
\text { Aug }\end{array}$ & 1.9 & 170 & $e<1$ & 12,000 & $<1$ & 130 & e15,000 & e20.4 & e3.97 \\
\hline $\begin{array}{l}15 \ldots \\
\text { Sep }\end{array}$ & 1.6 & 200 & $e<1$ & 14,000 & - & 140 & e17,400 & e23.6 & e2.72 \\
\hline $\begin{array}{c}12 \ldots \\
\text { Oct }\end{array}$ & 1.7 & 200 & $e<1$ & 12,000 & - & 140 & e15,600 & e 21.2 & el. 60 \\
\hline $\begin{array}{l}11 \ldots \\
\text { Nov }\end{array}$ & 1.9 & 210 & $e<1$ & 13,000 & 4.9 & 130 & el6,600 & e22.5 & el. .34 \\
\hline $\begin{array}{c}28 \ldots \\
\text { Jan } 1996\end{array}$ & 1.8 & 210 & $e<1$ & 14,000 & $<1$ & 120 & el7,600 & e23.9 & el. 1.28 \\
\hline $\begin{array}{l}10 \ldots \\
\text { Feb }\end{array}$ & 2.1 & 190 & $e<1$ & 11,000 & - & 100 & el4,500 & e19.8 & e. 04 \\
\hline $\begin{array}{l}20 \ldots \\
\text { Apr }\end{array}$ & .3 & 190 & $e<1$ & 10,000 & - & 90 & el3,200 & e 18.0 & e.82 \\
\hline $\begin{array}{l}02 \ldots \\
\text { May }\end{array}$ & .7 & 150 & $e<1$ & 10,000 & 5.0 & 79 & el2,500 & el7.0 & el.28 \\
\hline $\begin{array}{c}06 . . \\
\text { Jun }\end{array}$ & 1.2 & 210 & $e<1$ & 15,000 & 1.1 & 110 & el 18,600 & e 25.2 & e.40 \\
\hline $\begin{array}{c}03 \ldots \\
\text { Jul }\end{array}$ & .3 & 210 & $e<1$ & 15,000 & 2.0 & 120 & e 18,900 & e25.7 & e. 31 \\
\hline $\begin{array}{l}01 \ldots \\
\text { Aug }\end{array}$ & 1.1 & 250 & $e<1$ & 16,000 & $<1$ & 130 & e20,000 & e 27.2 & e. 16 \\
\hline $\begin{array}{l}06 . . \\
\text { Sep }\end{array}$ & 4.4 & 270 & $e<1$ & 16,000 & 6.2 & 130 & e20,600 & e28.0 & e.28 \\
\hline $03 \ldots$ & 4.2 & 260 & $e<1$ & 15,000 & 6.6 & 130 & e 19,600 & e26.6 & e.37 \\
\hline
\end{tabular}


Table 3. Water-quality data for sites in the Sand Coulee Coal Area, Montana, July 1994 through September 1996 (Continued)

SITE 8, 472114111095001--COTTONWOOD MINE NO. 2 DRAIN TO LADD COULEE AT STOCKETT, MT--Continued

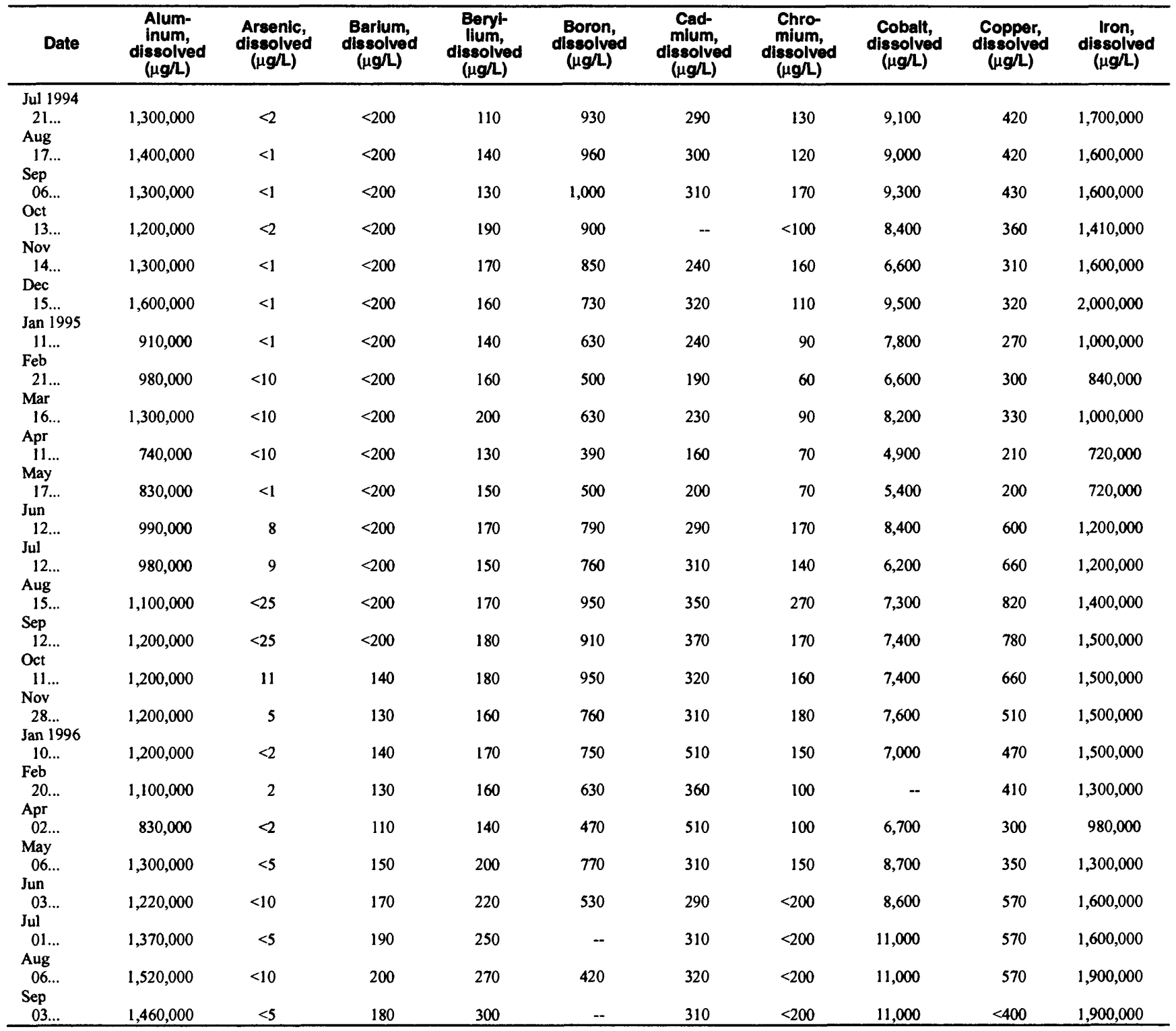


Table 3. Water-quality data for sites in the Sand Coulee Coal Area, Montana, July 1994 through September 1996 (Continued) SITE 8, 472114111095001--COTTONWOOD MINE NO. 2 DRAIN TO LADD COULEE AT STOCKETT, MT--Continued

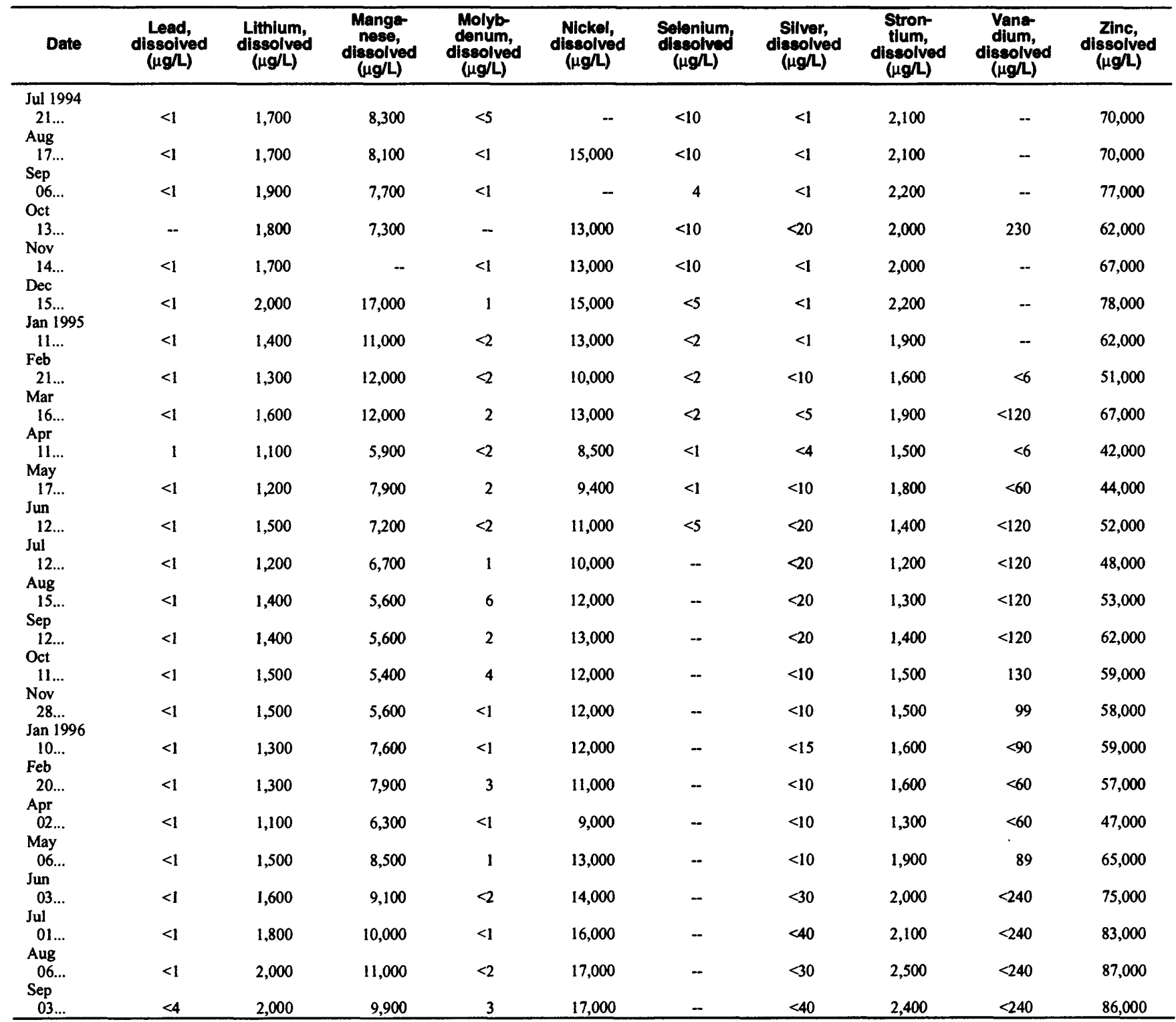


Table 3. Water-quality data for sites in the Sand Coulee Coal Area, Montana, July 1994 through September 1996 (Continued) SITE 9, 472212111093301--NUMBER FIVE COULEE NEAR STOCKETT, MT

\begin{tabular}{|c|c|c|c|c|c|c|c|c|c|c|c|}
\hline Date & Time & $\begin{array}{c}\text { Stream- } \\
\text { flow, } \\
\text { instan- } \\
\text { taneous } \\
\left(\mathrm{ft}^{3} / \mathrm{s}\right)\end{array}$ & $\begin{array}{c}\text { Specific } \\
\text { conduc- } \\
\text { tance, } \\
\text { onsite } \\
(\mu \mathrm{S} / \mathrm{cm})\end{array}$ & $\begin{array}{c}\text { Temper- } \\
\text { ature, } \\
\text { air } \\
\left({ }^{\circ} \mathbf{C}\right)\end{array}$ & $\begin{array}{l}\text { Temper- } \\
\text { ature, } \\
\text { water } \\
\left({ }^{\circ} \mathrm{C}\right)\end{array}$ & $\begin{array}{l}\text { pH, } \\
\text { onsite } \\
\text { (stand- } \\
\text { ard } \\
\text { units) }\end{array}$ & $\begin{array}{c}\text { Hard- } \\
\text { ness, } \\
\text { total } \\
(\mathrm{mg} / \mathrm{l} \text { as } \\
\left.\mathrm{CaCO}_{3}\right) \\
\end{array}$ & $\begin{array}{l}\text { Calcium, } \\
\text { dis- } \\
\text { solved } \\
\text { (mg/l) }\end{array}$ & $\begin{array}{l}\text { Magne- } \\
\text { sium, } \\
\text { dis- } \\
\text { solved } \\
\text { (mg/L) }\end{array}$ & $\begin{array}{c}\text { Sodium, } \\
\text { dis- } \\
\text { solved } \\
\text { (mg/L) }\end{array}$ & $\begin{array}{c}\text { Sodium } \\
\text { adsorp- } \\
\text { tion } \\
\text { ratio }\end{array}$ \\
\hline $\begin{array}{c}\text { Jul } 1994 \\
20 \ldots \\
\text { Jan } 1995\end{array}$ & 1745 & 0.02 & 1,010 & 27.0 & 29.0 & 8.4 & 490 & 120 & 45 & 19 & 0.4 \\
\hline $\begin{array}{l}11 \ldots \\
\text { Apr }\end{array}$ & 1155 & .12 & 964 & 4.0 & .5 & 6.8 & 500 & 140 & 37 & 14 & .3 \\
\hline $\begin{array}{l}11 \ldots \\
\text { May }\end{array}$ & 1800 & .40 & 845 & 5.0 & 11.0 & 7.6 & 390 & 98 & 35 & 24 & .5 \\
\hline $\begin{array}{l}17 \ldots \\
\text { Jun }\end{array}$ & 1405 & 9.5 & 645 & 21.0 & 17.0 & 8.2 & 300 & 62 & 34 & 25 & .6 \\
\hline $\begin{array}{c}13 \ldots \\
\text { Jul }\end{array}$ & 1600 & 5.2 & 660 & 25.0 & 22.5 & 8.4 & 310 & 61 & 38 & 22 & .5 \\
\hline $\begin{array}{l}12 \ldots \\
\text { Aug }\end{array}$ & 1510 & 7.1 & 698 & 20.0 & 16.5 & 8.2 & 340 & 72 & 38 & 23 & .5 \\
\hline $\begin{array}{l}15 \ldots \\
\text { Sep }\end{array}$ & 1430 & 1.5 & 899 & 25.0 & 19.0 & 7.8 & 430 & 99 & 44 & 21 & .4 \\
\hline $\begin{array}{l}12 \ldots \\
\text { Oct }\end{array}$ & 1315 & .80 & 1,000 & 24.0 & 18.0 & 6.9 & 470 & 110 & 47 & 20 & .4 \\
\hline $\begin{array}{l}11 \ldots \\
\text { Nov }\end{array}$ & 1250 & .81 & 1,080 & 20.0 & 13.0 & 6.5 & 530 & 130 & 50 & 20 & .4 \\
\hline $\begin{array}{c}27 \ldots \\
\text { Jan } 1996\end{array}$ & 1145 & .72 & 998 & -7.0 & 0.0 & 6.8 & 580 & 150 & 50 & 19 & .3 \\
\hline $\begin{array}{l}10 \ldots \\
\text { Feb }\end{array}$ & 1100 & .52 & 1,160 & 5.0 & 0.0 & 5.3 & 590 & 160 & 46 & 14 & .3 \\
\hline $\begin{array}{c}20 \ldots \\
\text { Apr }\end{array}$ & 1120 & .02 & 1,040 & 9.0 & 0.0 & 5.1 & 530 & 140 & 43 & 14 & .3 \\
\hline $\begin{array}{l}02 \ldots \\
\text { May }\end{array}$ & 1500 & .20 & 924 & 10.0 & 3.0 & 6.1 & 470 & 130 & 35 & 11 & .2 \\
\hline $06 \ldots$ & 1330 & .01 & 1,130 & 18.0 & 22.0 & 7.5 & 620 & 170 & 48 & 19 & .3 \\
\hline
\end{tabular}

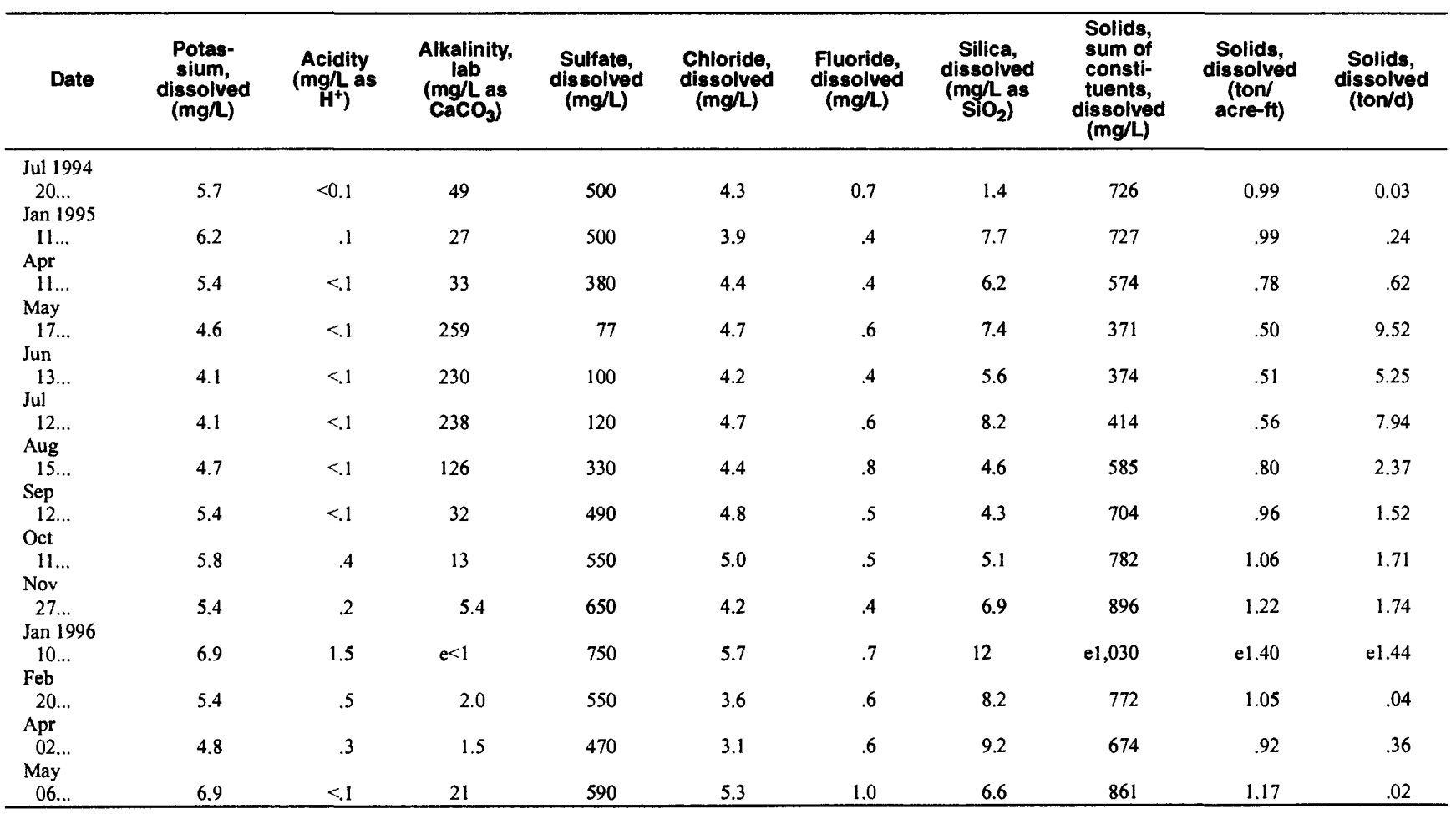


Table 3. Water-quality data for sites in the Sand Coulee Coal Area, Montana, July 1994 through September 1996 (Continued) SITE 9, 472212111093301--NUMBER FIVE COULEE NEAR STOCKETT, MT--Continued

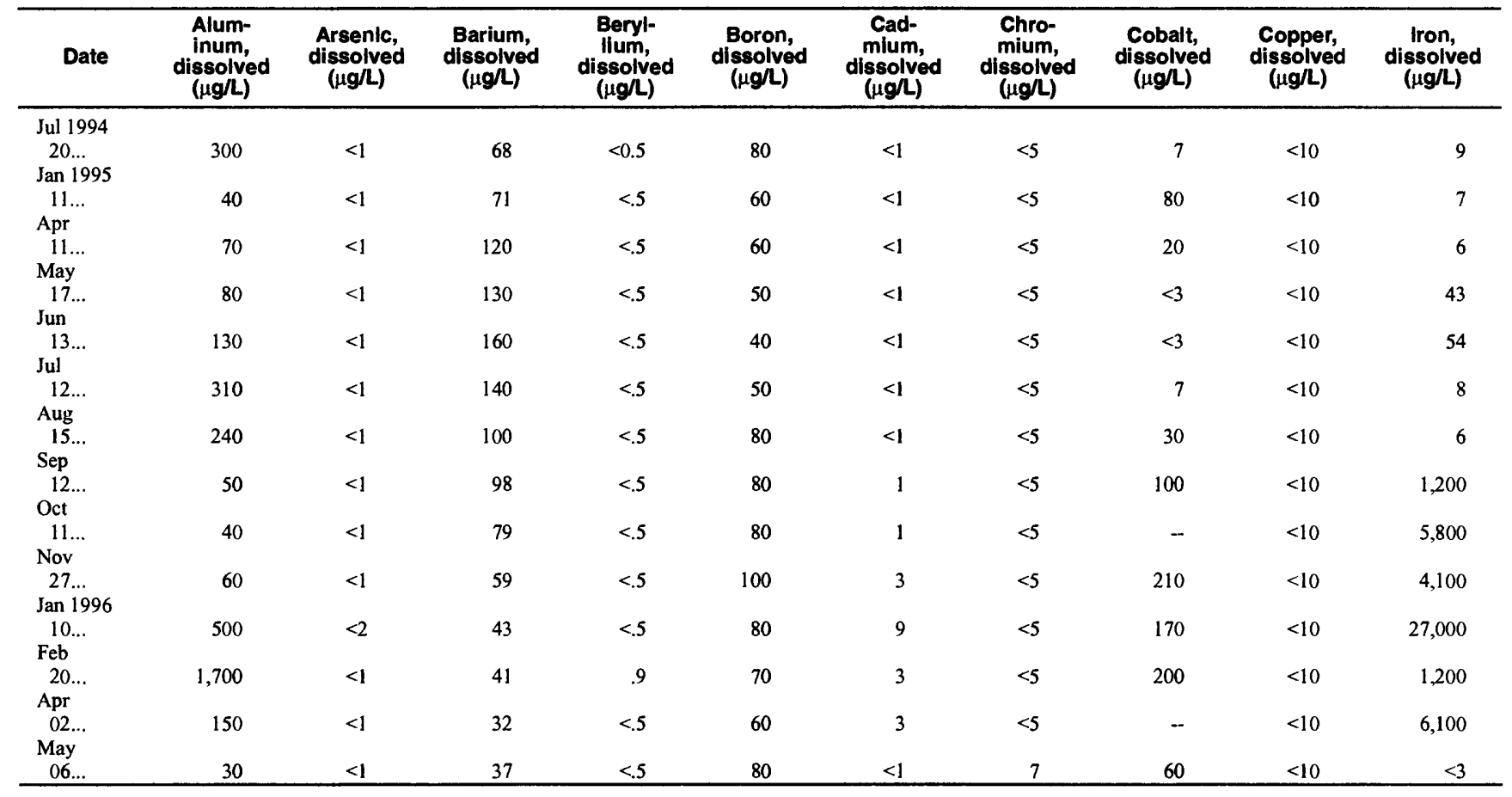

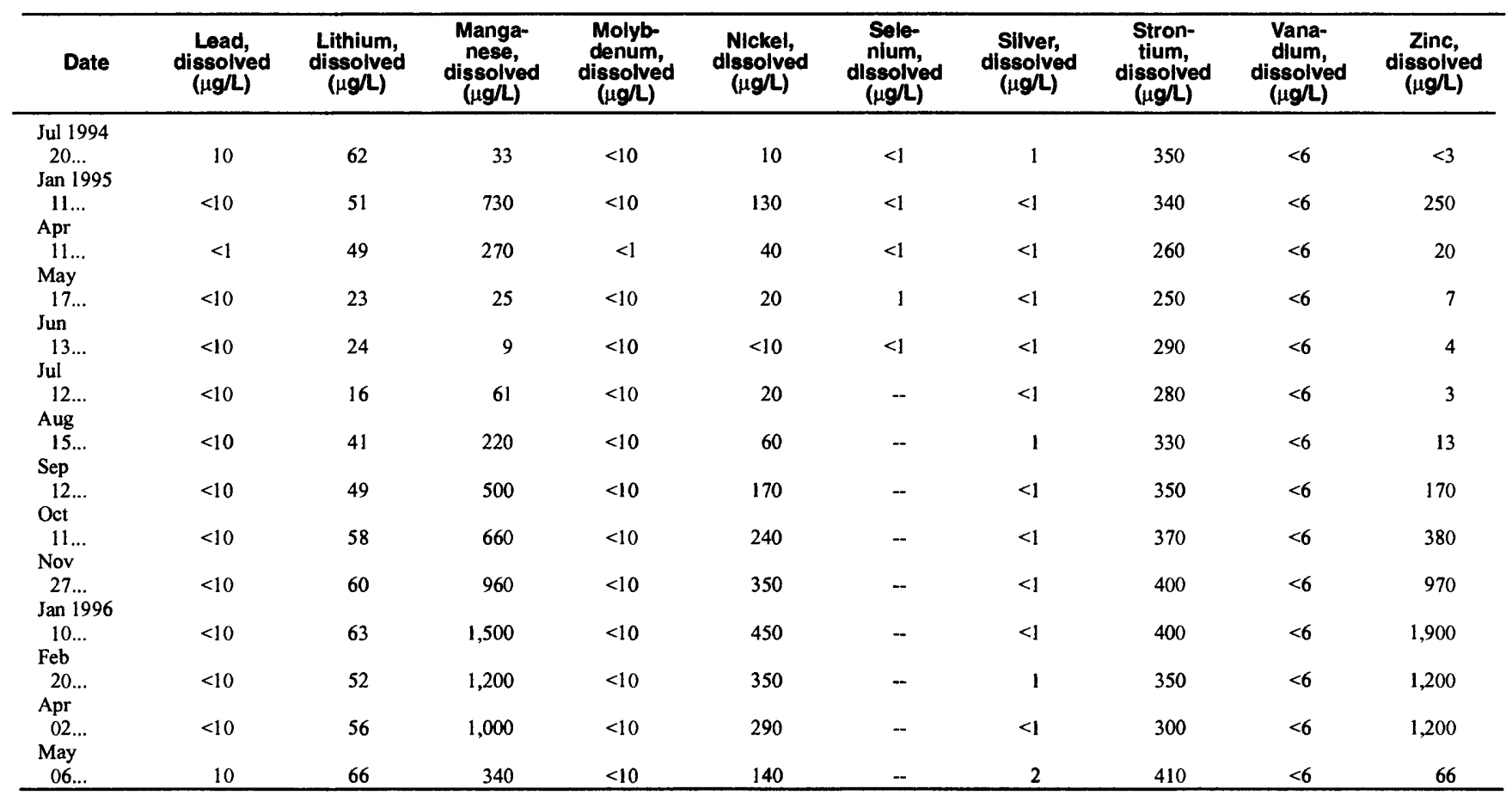


Table 3. Water-quality data for sites in the Sand Coulee Coal Area, Montana, July 1994 through September 1996 (Continued)

SITE 10, 472233110552601--FRENCH COULEE WETLANDS OUTFLOW AT BELT, MT

\begin{tabular}{|c|c|c|c|c|c|c|c|c|c|c|c|}
\hline Date & Time & $\begin{array}{c}\text { Stream- } \\
\text { flow, } \\
\text { instan- } \\
\text { taneous } \\
\left(\mathrm{ft}^{3} / \mathrm{s}\right)\end{array}$ & $\begin{array}{c}\text { Specific } \\
\text { conduc- } \\
\text { tance, } \\
\text { onsite } \\
(\mu \mathrm{S} / \mathrm{cm})\end{array}$ & $\begin{array}{l}\text { Temper- } \\
\text { ature, } \\
\text { air } \\
\left({ }^{\circ} \mathrm{C}\right)\end{array}$ & $\begin{array}{c}\text { Temper- } \\
\text { ature, } \\
\text { water } \\
\left({ }^{\circ} \mathbf{C}\right)\end{array}$ & $\begin{array}{l}\text { pH, } \\
\text { onsite } \\
\text { (stand- } \\
\text { ard } \\
\text { units) }\end{array}$ & $\begin{array}{c}\text { Hsrd- } \\
\text { ness, } \\
\text { total } \\
\left(\mathrm{mg}^{\prime} / \mathrm{as}\right. \\
\left.\mathrm{CaCO}_{3}\right)\end{array}$ & $\begin{array}{l}\text { Calcium, } \\
\text { dis- } \\
\text { solved } \\
\text { (mg/L) }\end{array}$ & $\begin{array}{l}\text { Magne- } \\
\text { sium, } \\
\text { dis- } \\
\text { solved } \\
\text { (mg/h) }\end{array}$ & $\begin{array}{l}\text { Sodium, } \\
\text { dis- } \\
\text { solved } \\
\text { (mg/L) }\end{array}$ & $\begin{array}{l}\text { Sodium } \\
\text { adsorp- } \\
\text { tion ratio }\end{array}$ \\
\hline $\begin{array}{l}\text { Jul } 1994 \\
19 \ldots \\
\text { Aug }\end{array}$ & 1145 & 0.02 & 5,780 & 28.0 & 17.0 & 2.8 & 2,000 & 490 & 190 & 38 & 0.4 \\
\hline $\begin{array}{l}17 \ldots \\
\text { Sep }\end{array}$ & 1630 & .01 & 6,090 & 31.0 & 20.0 & 2.8 & 2,000 & 470 & 210 & 44 & .6 \\
\hline $\begin{array}{l}\text { Sep } \\
06 \ldots \\
\text { Oct }\end{array}$ & 1010 & .02 & 6,180 & 22.0 & 10.0 & 2.8 & 2,000 & 450 & 210 & 37 & .4 \\
\hline $\begin{array}{l}14 \ldots \\
\text { Nov }\end{array}$ & 0940 & .03 & 5,610 & 7.0 & 5.5 & 3.0 & 1,700 & 410 & 170 & 26 & .3 \\
\hline $\begin{array}{l}14 \ldots \\
\text { Dec }\end{array}$ & 1040 & .02 & 5,450 & 5.5 & 1.0 & 3.2 & 1,600 & 390 & 150 & 23 & .3 \\
\hline $\begin{array}{c}15 \ldots \\
\text { Jan } 1995\end{array}$ & 1505 & .03 & 5,570 & 3.5 & .5 & 3.6 & 1,700 & 410 & 160 & 25 & .3 \\
\hline $\begin{array}{l}12 \ldots \\
\text { Feb }\end{array}$ & 0950 & .02 & 5,140 & 1.0 & .5 & 3.9 & 1,500 & 370 & 150 & 24 & .3 \\
\hline $\begin{array}{c}22 \ldots \\
\text { Mar }\end{array}$ & 0800 & .02 & 4,140 & 6.0 & 1.0 & 3.5 & 1,200 & 300 & 100 & 16 & .2 \\
\hline $\begin{array}{l}16 \ldots \\
\text { Apr }\end{array}$ & 0910 & .02 & 4,730 & 8.0 & 6.0 & 3.5 & 1,500 & 350 & 140 & 26 & .3 \\
\hline $\begin{array}{l}12 \ldots \\
\text { May }\end{array}$ & 1710 & .03 & 4,580 & 16.0 & 8.5 & 3.0 & 1,300 & 320 & 110 & 18 & .2 \\
\hline $\begin{array}{l}17 \ldots \\
\text { Jun }\end{array}$ & 0750 & .05 & 4,220 & 11.0 & 11.5 & 2.5 & 1,300 & 330 & 110 & 19 & .2 \\
\hline$\underset{\text { Jul }}{13 \ldots}$ & 0910 & .06 & 3,570 & 20.0 & 15.5 & 2.7 & 1,100 & 270 & 92 & 19 & .3 \\
\hline $\begin{array}{l}13 \ldots \\
\text { Aug }\end{array}$ & 0820 & .10 & 2,880 & 17.0 & 16.0 & 2.7 & 810 & 200 & 75 & 18 & .3 \\
\hline $\begin{array}{l}17 \ldots \\
\text { Sep }\end{array}$ & 1250 & .07 & 3,270 & 18.0 & 18.5 & 2.7 & 880 & 210 & 86 & 19 & .3 \\
\hline $\begin{array}{l}14 \ldots \\
\text { Oct }\end{array}$ & 0930 & .04 & 3,610 & 15.0 & 12.5 & 2.8 & 1,000 & 250 & 92 & 18 & .2 \\
\hline $\begin{array}{l}12 \ldots \\
\text { Nov }\end{array}$ & 1100 & .05 & 3,580 & 6.0 & 8.5 & 2.7 & 990 & 240 & 95 & 18 & .2 \\
\hline $\begin{array}{l}29 \ldots \\
\operatorname{Jan} 1996\end{array}$ & 0950 & .01 & 4,210 & 9.0 & 2.5 & 2.7 & 1,000 & 250 & 100 & 17 & .2 \\
\hline $\begin{array}{l}12 \ldots \\
\text { Feb }\end{array}$ & 0800 & .003 & 4,690 & 9.0 & 0.0 & 2.8 & 1,100 & 250 & 110 & 14 & .2 \\
\hline $\begin{array}{l}21 \ldots \\
\mathrm{Apr}\end{array}$ & 1200 & $<.001$ & 4,040 & 12.0 & 0.0 & 2.8 & 860 & 200 & 87 & 12 & .2 \\
\hline $04 \ldots$ & 1100 & .01 & 4,680 & 8.0 & 4.0 & 2.6 & 1,300 & 310 & 120 & 23 & .3 \\
\hline
\end{tabular}


Table 3. Water-quality data for sites in the Sand Coulee Coal Area, Montana, July 1994 through September 1996 (Continued) SITE 10, 472233110552601--FRENCH COULEE WETLANDS OUTFLOW AT BELT, MT--Continued

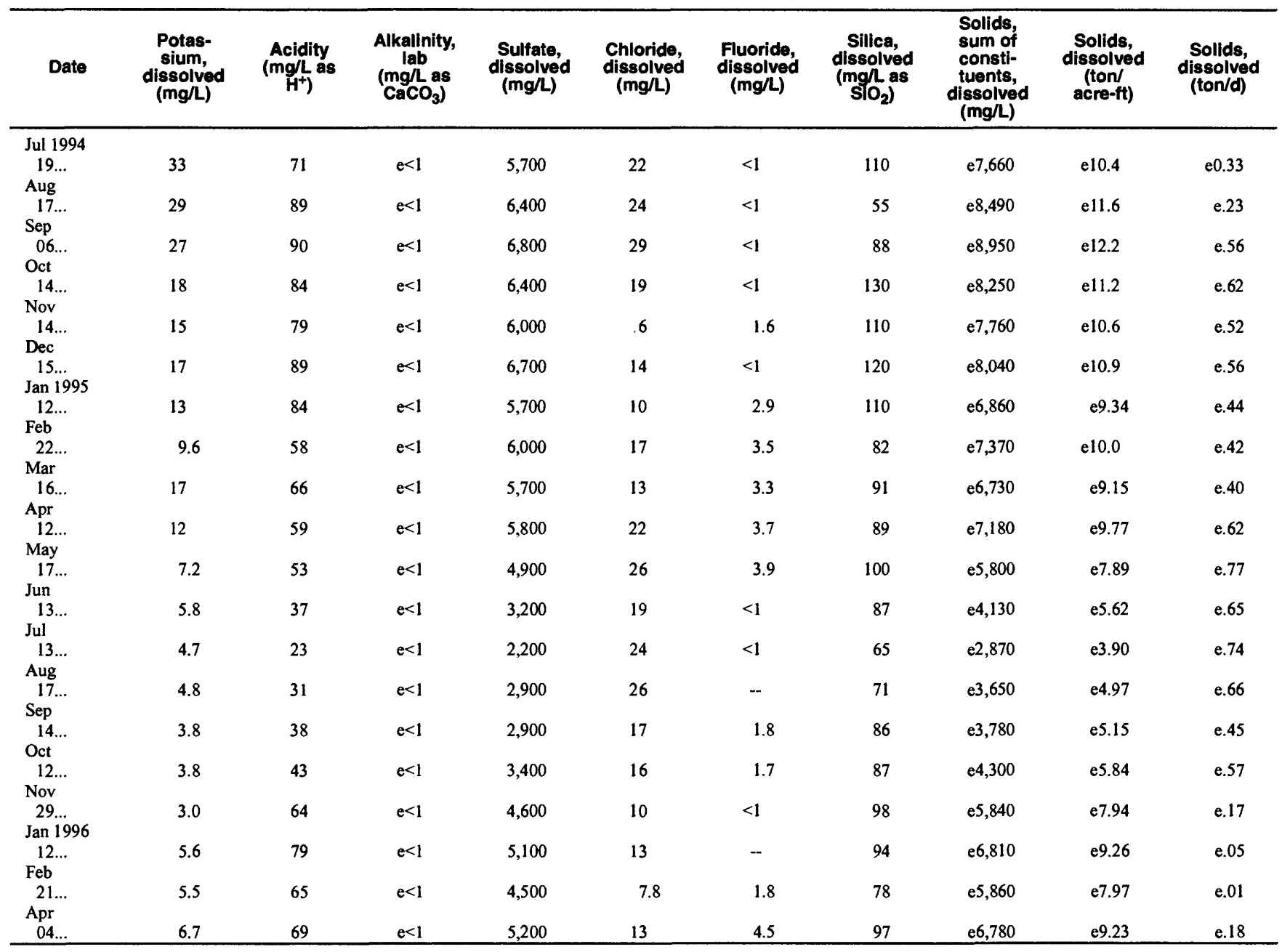


Table 3. Water-quality data for sites in the Sand Coulee Coal Area, Montana, July 1994 through September 1996 (Continued)

SITE 10, 472233110552601--FRENCH COULEE WETLANDS OUTFLOW AT BELT, MT--Continued

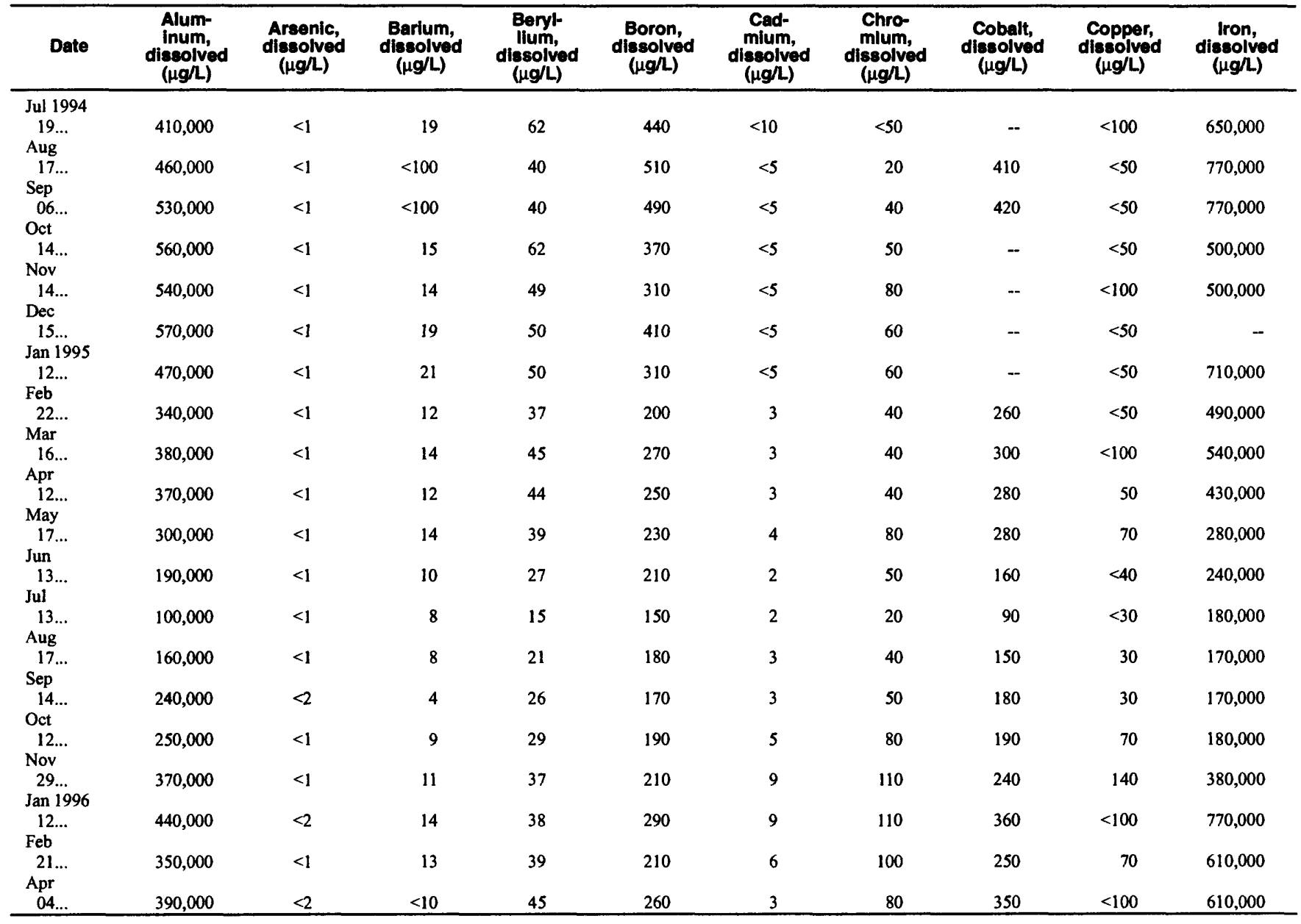


Table 3. Water-quality data for sites in the Sand Coulee Coal Area, Montana, July 1994 through September 1996 (Continued) SITE 10, 472233110552601 --FRENCH COULEE WETLANDS OUTFLOW AT BELT, MT--Continued

\begin{tabular}{|c|c|c|c|c|c|c|c|c|c|c|}
\hline Date & $\begin{array}{l}\text { Lead, } \\
\text { dissoived } \\
(\mu \mathrm{g} / \mathrm{L})\end{array}$ & $\begin{array}{l}\text { Lithium, } \\
\text { dissoived } \\
(\mu g / \text { l) }\end{array}$ & $\begin{array}{l}\text { Manga- } \\
\text { nese, } \\
\text { dissolved } \\
(\mu g / L)\end{array}$ & $\begin{array}{c}\text { Molyb- } \\
\text { denum, } \\
\text { dissoived } \\
(\mu g / L)\end{array}$ & $\begin{array}{c}\text { Nickel, } \\
\text { dissoived } \\
\text { ( } \mu g / L)\end{array}$ & $\begin{array}{c}\text { Sele- } \\
\text { nium, } \\
\text { diseoived } \\
(\mu \mathrm{g} / \mathrm{L})\end{array}$ & $\begin{array}{l}\text { Sliver, } \\
\text { dissoived } \\
(\mu g / L)\end{array}$ & $\begin{array}{l}\text { Stron- } \\
\text { tlum, } \\
\text { dissoived } \\
\left(\mu g^{\prime} L\right)\end{array}$ & $\begin{array}{c}\text { Vana- } \\
\text { dlum, } \\
\text { dissolved } \\
(\mu g / L)\end{array}$ & $\begin{array}{c}\text { Zinc, } \\
\text { dissoived } \\
(\mu g / L)\end{array}$ \\
\hline $\begin{array}{l}\text { Jul } 1994 \\
19 \ldots \\
\text { Aug }\end{array}$ & $<10$ & 660 & 5,600 & $<10$ & 750 & $<10$ & $<10$ & 2,900 & $<100$ & -- \\
\hline $\begin{array}{l}17 \ldots \\
\text { Sep }\end{array}$ & $<10$ & 740 & 6,400 & $<10$ & 760 & $<5$ & $<1$ & 2,900 & -- & -- \\
\hline $\begin{array}{l}06 \ldots \\
\text { Oct }\end{array}$ & $<10$ & 750 & 5,600 & $<10$ & 720 & $<5$ & $<1$ & 2,900 & - & - \\
\hline $\begin{array}{l}14 \ldots \\
\text { Nov }\end{array}$ & $<10$ & 630 & 4,500 & $<10$ & 820 & $<1$ & $<5$ & 2,600 & $<100$ & 3,100 \\
\hline $\begin{array}{c}14 \ldots \\
\text { Dec }\end{array}$ & $<10$ & 600 & 3,900 & $<10$ & 840 & $<1$ & 18 & 2,300 & $<100$ & 7,400 \\
\hline $\begin{array}{c}15 \ldots \\
\operatorname{Jan} 1995\end{array}$ & $<10$ & 590 & 3,900 & $<10$ & 1,100 & $<$ & $<5$ & 2,500 & $<100$ & 6,800 \\
\hline $\begin{array}{l}12 \ldots \\
\text { Feb }\end{array}$ & $<10$ & 560 & 3,300 & $<10$ & 1,000 & $<1$ & $<5$ & 2,600 & $<100$ & 4,600 \\
\hline $\begin{array}{l}22 \ldots . . \\
\text { Mar }\end{array}$ & $<1$ & 420 & 2,000 & $<1$ & 680 & $<1$ & 4 & 1,900 & $<30$ & 2,500 \\
\hline $\begin{array}{l}16 \ldots \\
\text { Apr }\end{array}$ & $<1$ & 470 & 3,800 & $<1$ & 690 & $<1$ & $<3$ & 2,200 & $<100$ & 2,400 \\
\hline $\begin{array}{c}12 \ldots \\
\text { May }\end{array}$ & $<1$ & 460 & 2,400 & $<1$ & 670 & $<1$ & $<3$ & 2,000 & $<30$ & 2,300 \\
\hline $\begin{array}{c}17 \ldots \\
\text { Jun }\end{array}$ & 2 & 430 & 1,700 & $<1$ & 680 & $<1$ & 3 & 1,900 & 12 & 1,900 \\
\hline $\begin{array}{l}13 \ldots \\
\text { Jul }\end{array}$ & 2 & 350 & 1,200 & $<1$ & 410 & $<5$ & 7 & 1,500 & $<24$ & 1,000 \\
\hline $\begin{array}{l}13 \ldots \\
\text { Aug }\end{array}$ & $<1$ & 200 & 790 & $<1$ & 230 & -- & $<3$ & 1,100 & $<18$ & 810 \\
\hline $\begin{array}{l}17 \ldots \\
\text { Sep }\end{array}$ & 1 & 260 & 870 & $<1$ & 270 & - & $<2$ & 1,200 & $<12$ & 1,100 \\
\hline $\begin{array}{l}14 \ldots \\
\text { Oct }\end{array}$ & $<1$ & 280 & 1,100 & $<1$ & 350 & - & $<2$ & 1,300 & $<12$ & 1,300 \\
\hline $\begin{array}{l}12 \ldots \\
\text { Nov }\end{array}$ & 1 & 310 & 1,000 & $<1$ & 410 & - & $<3$ & 1,400 & $<18$ & 1,900 \\
\hline $\begin{array}{c}29 . \ldots \\
\text { Jan } 1996\end{array}$ & 3 & 440 & 1,200 & $<1$ & 670 & - & $<5$ & 1,800 & $<30$ & 3,700 \\
\hline $\begin{array}{l}12 \ldots \\
\text { Feb }\end{array}$ & 4 & 450 & 1,600 & $<1$ & 890 & - & $<10$ & 1,600 & $<60$ & 5,100 \\
\hline $\begin{array}{l}21 \ldots \\
\text { Apr }\end{array}$ & 6 & 410 & 1,400 & $<1$ & 780 & - & $<5$ & 1,600 & 40 & 3,100 \\
\hline $04 \ldots$ & $<1$ & 510 & 3,100 & $<1$ & 700 & - & $<10$ & 2,000 & $<60$ & 2,300 \\
\hline
\end{tabular}


Table 3. Water-quality data for sites in the Sand Coulee Coal Area, Montana, July 1994 through September 1996 (Continued) SITE 11, 472235110553201 --FRENCH COULEE WETLANDS INFLOW AT BELT, MT

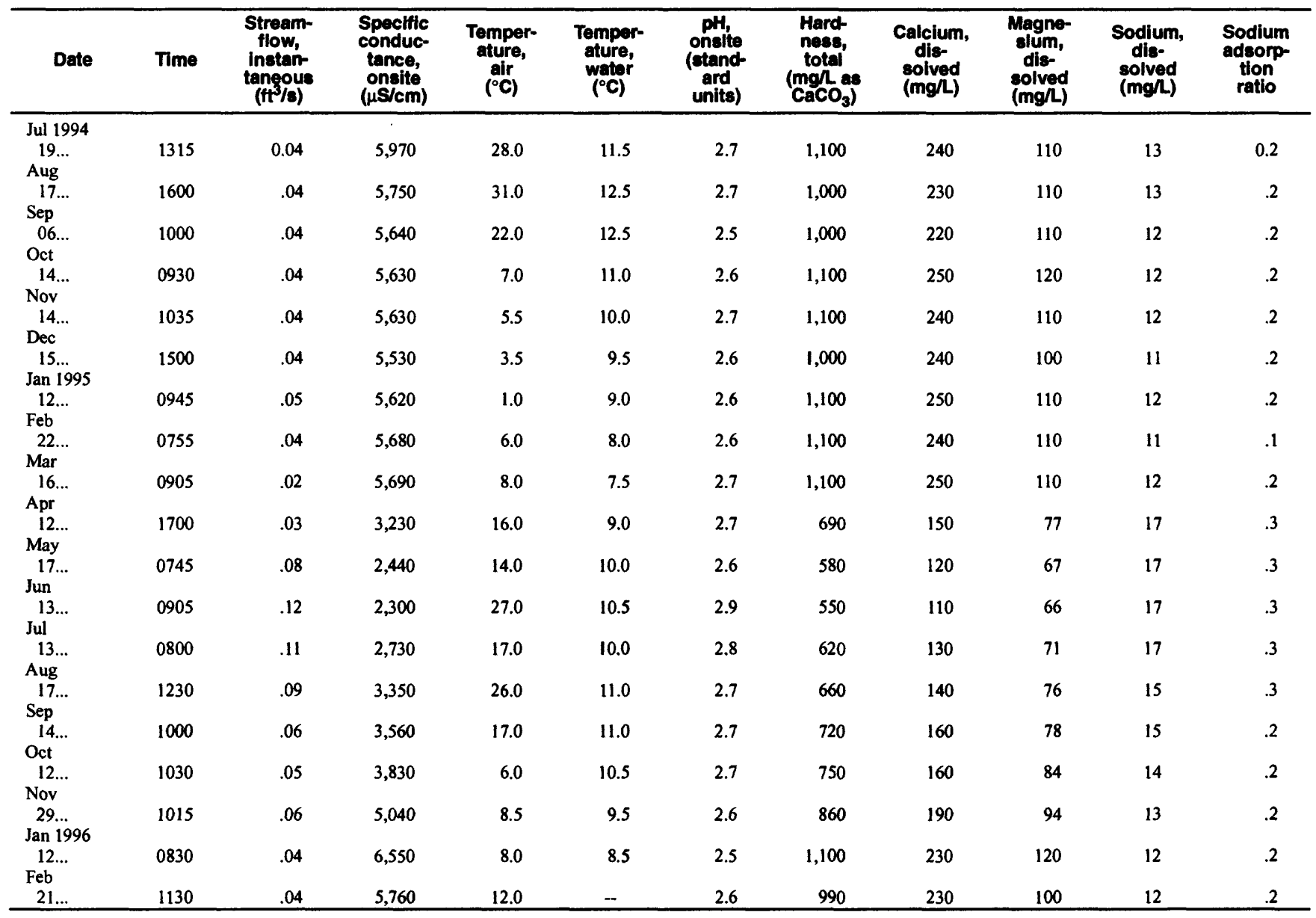


Table 3. Water-quality data for sites in the Sand Coulee Coal Area, Montana, July 1994 through September 1996 (Continued) SITE 11, 472235110553201--FRENCH COULEE WETLANDS INFLOW AT BELT, MT--Continued

\begin{tabular}{|c|c|c|c|c|c|c|c|c|c|c|}
\hline Date & $\begin{array}{l}\text { Potas- } \\
\text { sium, } \\
\text { diasolved } \\
\text { (mgll) }\end{array}$ & $\begin{array}{c}\text { Acidity } \\
\left(\mathrm{mg}^{\prime /} \text { as }\right.\end{array}$ & $\begin{array}{c}\text { Alkalinity, } \\
\text { lab } \\
\left(\mathrm{mg}^{\prime} \mathrm{Las}\right. \\
\left.\mathrm{CaCO}_{3}\right)\end{array}$ & $\begin{array}{c}\text { Sulfate, } \\
\text { dissolved } \\
\text { (mg/L) }\end{array}$ & $\begin{array}{l}\text { Chloride, } \\
\text { dissolved } \\
\text { (mg/l) }\end{array}$ & $\begin{array}{l}\text { Fluoride, } \\
\text { dlssolved } \\
(\mathrm{mg} / \mathrm{L})\end{array}$ & $\begin{array}{l}\text { Silica, } \\
\text { dlissolved } \\
\text { (mgl' as } \\
\left.\text { Sion }^{2}\right)\end{array}$ & $\begin{array}{l}\text { Solids, } \\
\text { sum of } \\
\text { consti- } \\
\text { tuenta, } \\
\text { dissolved } \\
\text { (mg/l) }\end{array}$ & $\begin{array}{l}\text { Solids, } \\
\text { diseolved } \\
\text { (ton' } \\
\text { acre-ft) }\end{array}$ & $\begin{array}{l}\text { Solids, } \\
\text { dissolved } \\
\text { (ton/d) }\end{array}$ \\
\hline $\begin{array}{l}\text { Jul } 1994 \\
19 \ldots \\
\text { Aug }\end{array}$ & 5.6 & 99 & $e<1$ & 6,000 & 7.9 & $<1$ & 100 & e7,990 & e10.9 & e0.91 \\
\hline $\begin{array}{l}17 . . \\
\text { Sep }\end{array}$ & 5.6 & 98 & $e<1$ & 6,000 & 8.3 & $<1$ & 100 & e 7,840 & el0.7 & e.74 \\
\hline $\begin{array}{l}06 \ldots \\
\text { Oct }\end{array}$ & 5.6 & 98 & $e<1$ & 6,100 & 4.5 & $<1$ & 99 & e7,950 & e10.8 & e.84 \\
\hline $\begin{array}{l}14 \ldots \\
\text { Nov }\end{array}$ & 5.1 & 94 & $e<1$ & 6,100 & 9.1 & $<1$ & 110 & e8,100 & ell. 0 & e.85 \\
\hline $\begin{array}{c}14 \ldots \\
\text { Dec }\end{array}$ & 5.2 & 91 & $e<1$ & 6,000 & 9.7 & - & 100 & e 7,810 & el0.6 & e.89 \\
\hline $\begin{array}{l}15 \ldots \\
\operatorname{Jan} 1995\end{array}$ & 5.9 & 93 & $e<1$ & 6,300 & 5.7 & $<1$ & 100 & e 7,260 & e 9.87 & e.76 \\
\hline $\begin{array}{l}12 \ldots \\
\text { Feb }\end{array}$ & 5.4 & 97 & $e<1$ & 5,900 & 4.9 & 1.4 & 110 & e 6,860 & e9.33 & e.93 \\
\hline $\begin{array}{c}22 \ldots \\
\text { Mar }\end{array}$ & 5.8 & 91 & $e<1$ & 7,100 & 20 & 3.6 & 100 & e8,980 & el2.2 & $e .90$ \\
\hline $\begin{array}{l}16 \ldots \\
\text { Apr }\end{array}$ & 5.8 & 92 & $e<1$ & 6,700 & 38 & 2.2 & 110 & e8,680 & ell.8 & e.59 \\
\hline $\begin{array}{c}12 \ldots \\
\text { May }\end{array}$ & 3.8 & 32 & $e<1$ & 2,800 & 24 & 1.7 & 64 & e3,620 & e4.93 & e.29 \\
\hline $\begin{array}{l}17 \ldots \\
\text { Jun }\end{array}$ & 3.5 & 23 & $e<1$ & 2,100 & 31 & 2.4 & 45 & e2,690 & e3.66 & e.54 \\
\hline $\begin{array}{c}13 \ldots \\
\text { Jul }\end{array}$ & 3.6 & 22 & $e<1$ & 2,000 & 23 & $<1$ & 41 & e2,530 & e3.45 & $e .82$ \\
\hline $\begin{array}{l}13 \ldots \\
\text { Aug }\end{array}$ & 3.4 & 27 & $e<1$ & 2,300 & 23 & $<1$ & 50 & $\mathrm{e} 2,960$ & e4.02 & e.88 \\
\hline $\begin{array}{l}17 \ldots \\
\text { Sep }\end{array}$ & 3.3 & 40 & $e<1$ & 3,100 & 22 & $<1$ & 64 & $e 3,980$ & es.41 & e.97 \\
\hline $\begin{array}{l}14 \ldots \\
\text { Oct }\end{array}$ & 3.1 & 46 & $e<1$ & 2,900 & 13 & 1.6 & 70 & e 3,940 & e5.35 & e.64 \\
\hline $\begin{array}{l}12 \ldots \\
\text { Nov }\end{array}$ & 2.8 & 54 & $e<1$ & 3,700 & 12 & 1.6 & 75 & e 4,830 & e6.56 & e.65 \\
\hline $\begin{array}{c}29 \ldots \\
\text { Jan } 1996\end{array}$ & 3.0 & 78 & $e<1$ & 5,000 & 8.2 & 1.1 & 90 & e6,560 & e8.92 & el.06 \\
\hline $\begin{array}{l}12 \ldots \\
\text { Feb }\end{array}$ & 5.7 & 120 & $e<1$ & 7,400 & 18 & - & 120 & e9,860 & el3.4 & el.06 \\
\hline $21 \ldots$ & 5.2 & 95 & $e<1$ & 6,500 & 7.3 & 1.1 & 110 & e8,470 & el1.5 & e.91 \\
\hline
\end{tabular}


Table 3. Water-quality data for sites in the Sand Coulee Coal Area, Montana, July 1994 through September 1996 (Continued)

SITE 11, 472235110553201--FRENCH COULEE WETLANDS INFLOW AT BELT, MT--Continued

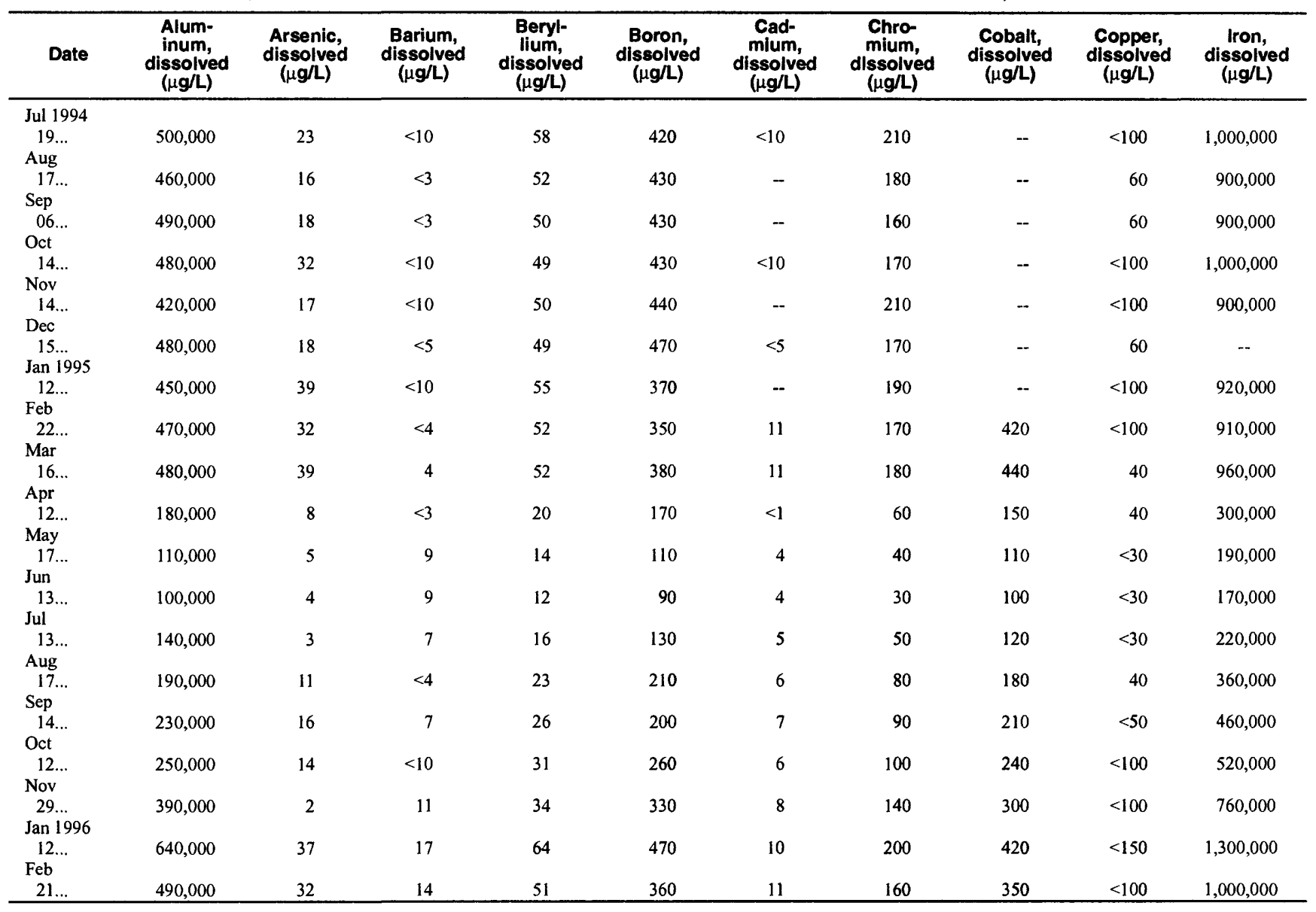


Table 3. Water-quality data for sites in the Sand Coulee Coal Area, Montana, July 1994 through September 1996 (Continued) SITE 11, 472235110553201--FRENCH COULEE WETLANDS INFLOW AT BELT, MT--Continued

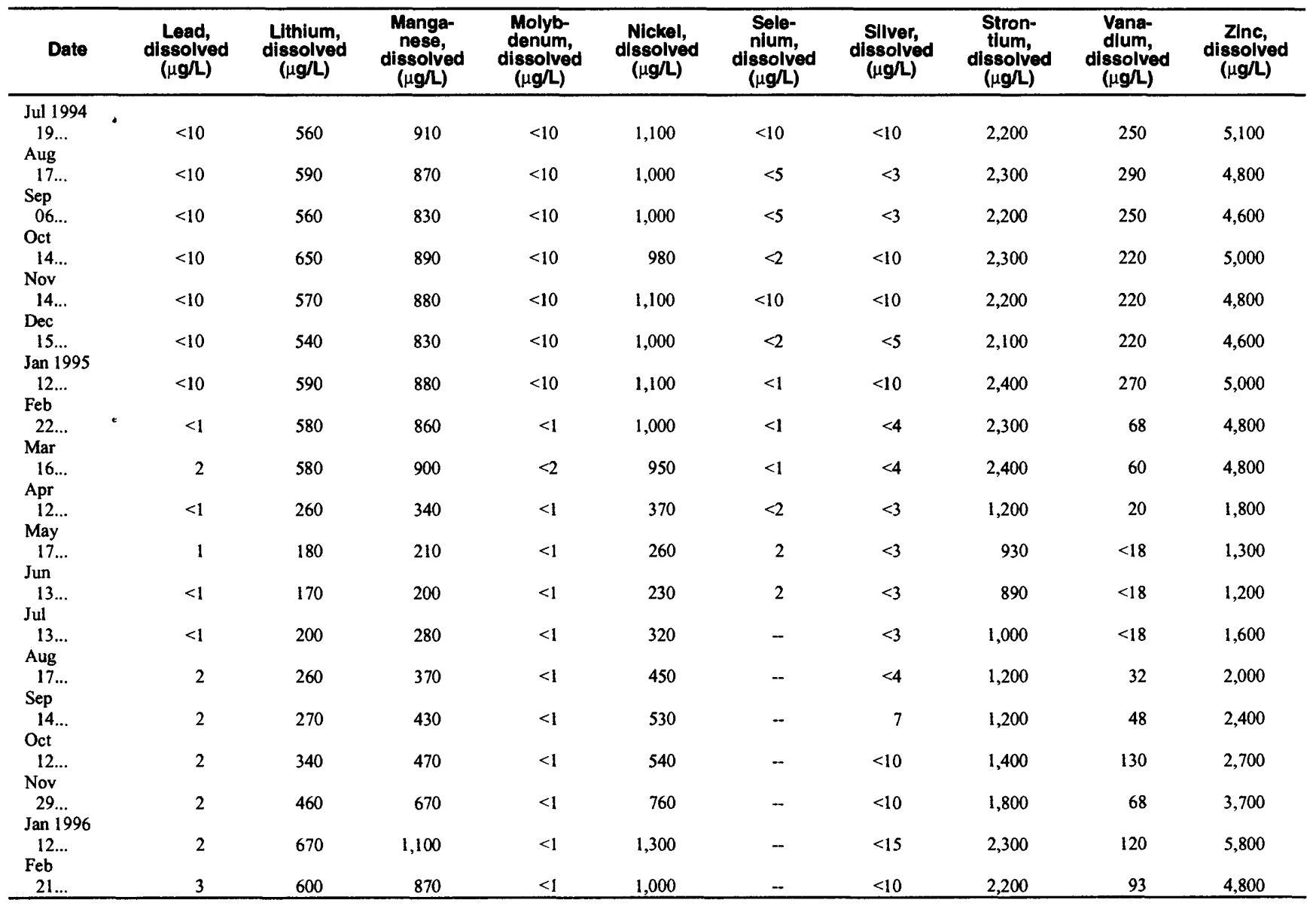


Table 3. Water-quality data for sites in the Sand Coulee Coal Area, Montana, July 1994 through September 1996 (Continued) SITE 12, 472235110553202--FRENCH COULEE WETLANDS INFLOW NO. 2 AT BELT, MT

\begin{tabular}{|c|c|c|c|c|c|c|c|c|c|c|c|}
\hline Date & Time & $\begin{array}{c}\text { Stream- } \\
\text { flow, } \\
\text { instan- } \\
\text { tangous } \\
\left(\mathrm{ft}^{3} / \mathrm{s}\right)\end{array}$ & $\begin{array}{c}\text { Specific } \\
\text { conduc- } \\
\text { tance, } \\
\text { onsite } \\
(\mu \mathrm{S} / \mathrm{cm})\end{array}$ & $\begin{array}{c}\text { Temper- } \\
\text { ature, } \\
\text { air } \\
\left.\text { ( }{ }^{\circ} \mathbf{C}\right)\end{array}$ & $\begin{array}{c}\text { Temper- } \\
\text { ature, } \\
\text { water } \\
\left({ }^{\circ} \mathbf{C}\right)\end{array}$ & $\begin{array}{c}\mathrm{pH}, \\
\text { onsite } \\
\text { (stand- } \\
\text { ard } \\
\text { units) }\end{array}$ & $\begin{array}{c}\text { Hard- } \\
\text { ness, } \\
\text { total } \\
\left(\mathrm{mg}^{\prime} \mathrm{L} \text { as }\right. \\
\left.\mathrm{CaCO}_{3}\right)\end{array}$ & $\begin{array}{l}\text { Calcium, } \\
\text { dis- } \\
\text { solved } \\
\text { (mg/L) }\end{array}$ & $\begin{array}{l}\text { Magne- } \\
\text { sium, } \\
\text { dis- } \\
\text { solved } \\
\text { (mg/L) }\end{array}$ & $\begin{array}{l}\text { Sodium, } \\
\text { dis- } \\
\text { solved } \\
\text { (mg/L) }\end{array}$ & $\begin{array}{l}\text { Sodium } \\
\text { adsorp- } \\
\text { tion ratio }\end{array}$ \\
\hline $\begin{array}{c}\text { Apr } 1996 \\
04 \ldots \\
\text { May }\end{array}$ & 1130 & 0.04 & 5,050 & 9.0 & 8.5 & 2.6 & 860 & 190 & 94 & 13 & 0.2 \\
\hline $\begin{array}{c}07 \ldots \\
\text { Jun }\end{array}$ & 1630 & .03 & 3,640 & 17.0 & 10.5 & 2.8 & 690 & 150 & 76 & 13 & .2 \\
\hline $\begin{array}{c}05 \ldots \\
\text { Jul }\end{array}$ & 1250 & .03 & 3,660 & 18.0 & 11.0 & 2.8 & 660 & 140 & 75 & 13 & .2 \\
\hline $\begin{array}{l}03 \ldots \\
\text { Aug }\end{array}$ & 0900 & .03 & 4,160 & 22.0 & 12.0 & 27 & 860 & 190 & 92 & 12 & .2 \\
\hline $\begin{array}{l}08 . . \\
\text { Sep }\end{array}$ & 1100 & .03 & 4,540 & 22.0 & 13.0 & 2.8 & 890 & 200 & 94 & 12 & .2 \\
\hline $04 \ldots$ & 1040 & .03 & 5,550 & 12.0 & 12.0 & 2.8 & 1,000 & 230 & 110 & 12 & .2 \\
\hline
\end{tabular}

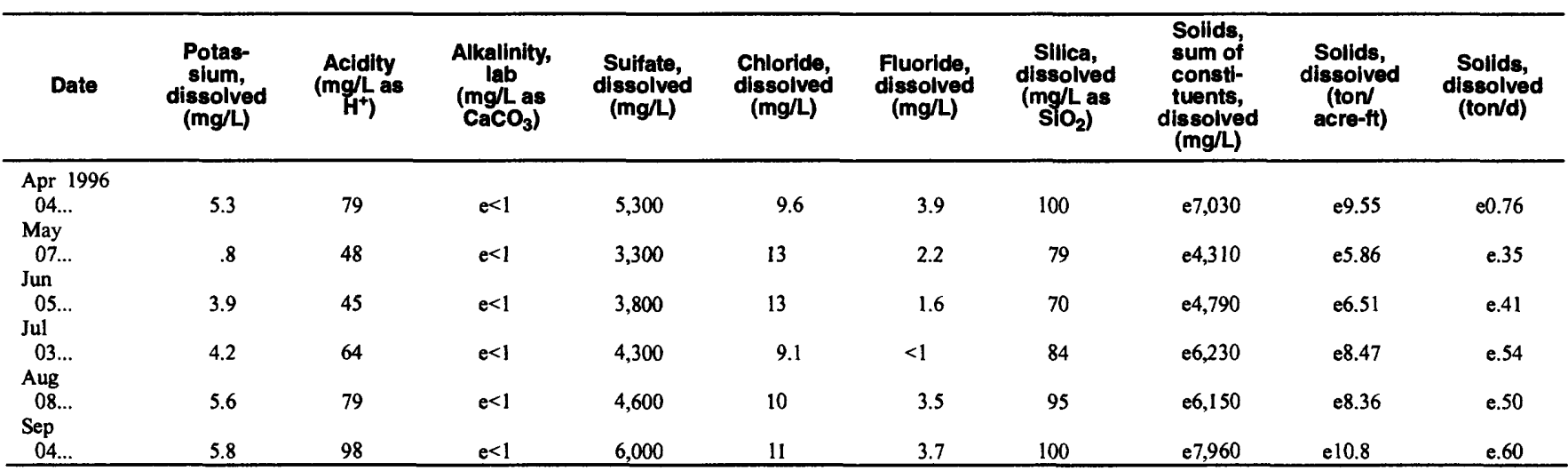

\begin{tabular}{|c|c|c|c|c|c|c|c|c|c|c|}
\hline Date & $\begin{array}{c}\text { Alum- } \\
\text { inum, } \\
\text { dissolved } \\
\text { ( } \mu \mathrm{g} / \mathrm{L})\end{array}$ & $\begin{array}{c}\text { Arsenic, } \\
\text { dissolved } \\
\left(\mu g^{\prime} L\right)\end{array}$ & $\begin{array}{l}\text { Barlum, } \\
\text { dissolved } \\
(\mu g / L)\end{array}$ & $\begin{array}{c}\text { Beryl- } \\
\text { lium, } \\
\text { dissolved } \\
(\mu \mathrm{g} / \mathrm{L})\end{array}$ & $\begin{array}{c}\text { Boron, } \\
\text { dissolved } \\
(\mu g / L)\end{array}$ & $\begin{array}{c}\text { Cad- } \\
\text { mium, } \\
\text { dissolved } \\
(\mu \mathrm{g} / \mathrm{L})\end{array}$ & $\begin{array}{c}\text { Chro- } \\
\text { mium, } \\
\text { dissolved } \\
(\mu \mathrm{g} / \mathrm{L})\end{array}$ & $\begin{array}{c}\text { Cobalt, } \\
\text { dissolved } \\
(\mu \mathrm{g} / \mathrm{L})\end{array}$ & $\begin{array}{c}\text { Copper, } \\
\text { dissolved } \\
(\mu \mathrm{g} / \mathrm{L})\end{array}$ & $\begin{array}{c}\text { Iron, } \\
\text { dissolved } \\
(\mu g / L)\end{array}$ \\
\hline $\begin{array}{l}\text { Apr } 1996 \\
04 \ldots \\
\text { May }\end{array}$ & 430,000 & $<2$ & 13 & 48 & 330 & 7 & 140 & 350 & $<100$ & 870,000 \\
\hline $\begin{array}{l}07 . . . \\
\text { Jun }\end{array}$ & 230,000 & 5 & 8 & 33 & 230 & 7 & 100 & 220 & $<50$ & 440,000 \\
\hline $\begin{array}{c}05 \ldots \\
\text { Jul }\end{array}$ & 210,000 & 5 & 11 & 26 & -- & 5 & 120 & 220 & $<100$ & 450,000 \\
\hline $\begin{array}{l}03 \ldots \\
\text { Aug }\end{array}$ & 310,000 & 14 & 11 & 38 & 240 & 8 & 100 & 320 & $<100$ & 640,000 \\
\hline $\begin{array}{l}08 . . . \\
\text { Sep }\end{array}$ & 360,000 & 22 & 13 & 46 & -- & 8 & 130 & 350 & $<100$ & 760,000 \\
\hline $04 \ldots$ & 480,000 & 8 & 19 & 56 & 390 & 10 & 190 & 480 & $<120$ & $1,000,000$ \\
\hline
\end{tabular}

\begin{tabular}{|c|c|c|c|c|c|c|c|c|c|}
\hline Date & $\begin{array}{l}\text { Lead, } \\
\text { dissoived } \\
\text { ( } \mu g / L)\end{array}$ & $\begin{array}{l}\text { Lithium, } \\
\text { dissolved } \\
(\mu g / L)\end{array}$ & $\begin{array}{l}\text { Mange } \\
\text { neese, } \\
\text { dissolved } \\
\text { ( } \mu \mathrm{g} / \mathrm{L})\end{array}$ & $\begin{array}{l}\text { Molyb- } \\
\text { denum, } \\
\text { dissolved } \\
\text { ( } \mu \mathrm{g} / \mathrm{L})\end{array}$ & $\begin{array}{c}\text { Nickel, } \\
\text { dissolved } \\
(\mu \mathrm{g} / \mathrm{L})\end{array}$ & $\begin{array}{c}\text { Silver, } \\
\text { dissolved } \\
(\mu g / L)\end{array}$ & $\begin{array}{c}\text { Stron- } \\
\text { tium, } \\
\text { dissolved } \\
(\mu g / L)\end{array}$ & $\begin{array}{c}\text { Vane- } \\
\text { dium, } \\
\text { dissolved } \\
(\mu g / L)\end{array}$ & $\begin{array}{c}\text { Znc, } \\
\text { dissolved } \\
(\mu \mathrm{g} / L)\end{array}$ \\
\hline $\begin{array}{c}\text { Apr } 1996 \\
04 \ldots\end{array}$ & 2 & 520 & 750 & $<1$ & 870 & $<10$ & 1,800 & 72 & 4,000 \\
\hline $\begin{array}{l}\text { May } \\
07 . . \\
\text { Jun }\end{array}$ & 2 & 340 & 450 & $<1$ & 560 & $<5.0$ & 1,400 & 69 & 2,500 \\
\hline $\begin{array}{l}05 . . . \\
\text { Jul }\end{array}$ & 2 & 320 & 430 & $<1$ & 490 & 29 & 1,200 & 87 & 2,300 \\
\hline $03 \ldots$ & 2 & 410 & 620 & $<1$ & 850 & $<10$ & 1,700 & 62 & 3,400 \\
\hline $08 . .$. & 1 & 510 & 720 & $<1$ & 830 & $<10$ & 1,900 & $<60$ & 4,000 \\
\hline $\begin{array}{c}\text { ep } \\
04 \ldots\end{array}$ & $<4$ & 650 & 930 & 2 & 1,100 & 28 & 2,300 & $<72$ & 5,100 \\
\hline
\end{tabular}


Table 3. Water-quality data for sites in the Sand Coulee Coal Area, Montana, July 1994 through September 1996 (Continued) SITE 13, 472305110551701 --LEWIS COULEE ABOVE CASTNER PARK, AT BELT, MT

\begin{tabular}{|c|c|c|c|c|c|c|c|c|c|c|c|}
\hline Date & Time & $\begin{array}{c}\text { Stream- } \\
\text { flow, } \\
\text { instan- } \\
\text { taneous } \\
\left(\mathrm{ft}^{3} / \mathrm{s}\right)\end{array}$ & $\begin{array}{c}\text { Specific } \\
\text { conduc- } \\
\text { tance, } \\
\text { onsite } \\
(\mu S / \mathrm{cm})\end{array}$ & $\begin{array}{c}\text { Temper- } \\
\text { ature, } \\
\text { alr } \\
\left.\text { ( }{ }^{\circ} \mathrm{C}\right)\end{array}$ & $\begin{array}{c}\text { Temper- } \\
\text { ature, } \\
\text { water } \\
\left.{ }^{\circ} \mathrm{C}\right)\end{array}$ & $\begin{array}{l}\text { pH, } \\
\text { onsite } \\
\text { (stand- } \\
\text { ard } \\
\text { units) }\end{array}$ & $\begin{array}{c}\text { Hard- } \\
\text { ness, } \\
\text { total } \\
\left(\mathrm{mghl}_{\mathrm{as}}\right. \\
\left.\mathrm{CaCO}_{3}\right)\end{array}$ & $\begin{array}{l}\text { Calcium, } \\
\text { dls- } \\
\text { solved } \\
\text { (mg/h) }\end{array}$ & $\begin{array}{l}\text { Magne- } \\
\text { slum, } \\
\text { dis- } \\
\text { solved } \\
\text { (mg/L) }\end{array}$ & $\begin{array}{l}\text { Sodium, } \\
\text { dis- } \\
\text { solved } \\
\text { (mg/l) }\end{array}$ & $\begin{array}{l}\text { Sodium } \\
\text { edsorp- } \\
\text { tion } \\
\text { ratio }\end{array}$ \\
\hline $\begin{array}{c}\text { Jul } 1994 \\
19 \ldots\end{array}$ & 1500 & 0.02 & 3,890 & 23.0 & 12.5 & 2.8 & 880 & 170 & 110 & 25 & 0.4 \\
\hline Oct.. & 1100 & .01 & 3,940 & 22.0 & 12.0 & 2.7 & 850 & 160 & 110 & 26 & .4 \\
\hline $\begin{array}{l}14 \ldots \\
\text { Nov }\end{array}$ & 0910 & .009 & 4,100 & 7.0 & 9.0 & 2.8 & 920 & 170 & 120 & 25 & .4 \\
\hline $\begin{array}{l}14 \ldots \\
\text { Dec }\end{array}$ & 1145 & .004 & 4,170 & 5.5 & 7.5 & 2.8 & 950 & 180 & 120 & 26 & .4 \\
\hline $\begin{array}{l}15 \ldots \\
\text { Jan } 1995\end{array}$ & 1545 & .002 & 4,130 & 2.5 & 6.5 & 2.8 & 900 & 180 & 110 & 25 & .4 \\
\hline $\begin{array}{l}12 \ldots \\
\text { Feb }\end{array}$ & 1020 & .008 & 4,250 & 3.0 & 7.0 & 2.8 & 950 & 180 & 120 & 26 & .4 \\
\hline $\begin{array}{l}22 \ldots \\
\text { Mar }\end{array}$ & 0900 & .008 & 4,360 & 7.0 & 7.5 & 2.8 & 950 & 180 & 120 & 25 & .4 \\
\hline $\begin{array}{c}16 \ldots \\
\text { Apr }\end{array}$ & 1000 & .006 & 4,340 & 9.0 & 7.5 & 2.7 & 970 & 190 & 120 & 26 & .4 \\
\hline $\begin{array}{l}12 \ldots \\
\text { May }\end{array}$ & 1810 & .009 & 4,660 & 10.0 & 9.0 & 2.6 & . 900 & 180 & 110 & 24 & .3 \\
\hline $\begin{array}{l}17 \ldots \\
\text { Jun }\end{array}$ & 0845 & .01 & 4,750 & 16.0 & 10.5 & 2.4 & 1,000 & 190 & 130 & 26 & .4 \\
\hline $13 \ldots$ & 1015 & .02 & 4,850 & 23.0 & 13.0 & 2.6 & 970 & 190 & 120 & 26 & .4 \\
\hline $\begin{array}{l}13 \ldots \\
\text { Aug }\end{array}$ & 0950 & .01 & 4,820 & 17.0 & 14.0 & 2.6 & 970 & 190 & 120 & 27 & .4 \\
\hline $\begin{array}{l}17 \ldots \\
\text { Sep }\end{array}$ & 1000 & .01 & 4,550 & 17.0 & 11.5 & 2.6 & 950 & 180 & 120 & 26 & .4 \\
\hline $\begin{array}{l}14 \ldots \\
\text { Oct }\end{array}$ & 1120 & .02 & 4,550 & 21.0 & 12.5 & 2.6 & 1,000 & 200 & 130 & 27 & .4 \\
\hline $\begin{array}{l}12 \ldots \\
\text { Nov }\end{array}$ & 1010 & .01 & 4,500 & 5.5 & 10.0 & 2.6 & 920 & 170 & 120 & 26 & .4 \\
\hline $\begin{array}{c}29 \ldots \\
\text { Jan } 1996\end{array}$ & 1120 & .01 & 4,500 & 9.0 & 8.0 & 2.6 & 920 & 170 & 120 & 26 & .4 \\
\hline $\begin{array}{l}12 \ldots \\
\text { Feb }\end{array}$ & 0945 & .01 & 4,240 & 9.0 & 7.0 & 2.6 & 920 & 170 & 120 & 26 & .4 \\
\hline $\begin{array}{l}21 \ldots \\
\text { Apr }\end{array}$ & 0925 & .01 & 4,190 & 8.0 & 6.5 & 2.7 & 950 & 180 & 120 & 26 & .4 \\
\hline $\begin{array}{c}04 \ldots \\
\text { May }\end{array}$ & 1200 & .02 & 4,090 & 9.0 & 7.5 & 2.7 & 920 & 170 & 120 & 27 & .4 \\
\hline $\begin{array}{c}07 . . . \\
\text { Jun }\end{array}$ & 1500 & .02 & 4,210 & 9.0 & 10.5 & 2.7 & 920 & 170 & 120 & 27 & .4 \\
\hline $\begin{array}{c}05 \ldots \\
\text { Jul }\end{array}$ & 1310 & .01 & 4,180 & 19.0 & 9.0 & 2.5 & 920 & 170 & 120 & 26 & .4 \\
\hline $\begin{array}{l}03 \ldots \\
\text { Aug }\end{array}$ & 0920 & .01 & 4,050 & 23.0 & 11.0 & 2.7 & 920 & 170 & 120 & 26 & .4 \\
\hline $\begin{array}{l}08 \ldots \\
\text { Sep }\end{array}$ & 1130 & .009 & 4,040 & 24.0 & 13.0 & 2.7 & 900 & 160 & 120 & 26 & .4 \\
\hline $04 \ldots$ & 0920 & .01 & 4,070 & 10.0 & 10.0 & 2.8 & 960 & 170 & 130 & 26 & .4 \\
\hline
\end{tabular}


Table 3. Water-quality data for sites in the Sand Coulee Coal Area, Montana, July 1994 through September 1996 (Continued) SITE 13, 472305110551701--LEWIS COULEE ABOVE CASTNER PARK, AT BELT, MT--Continued

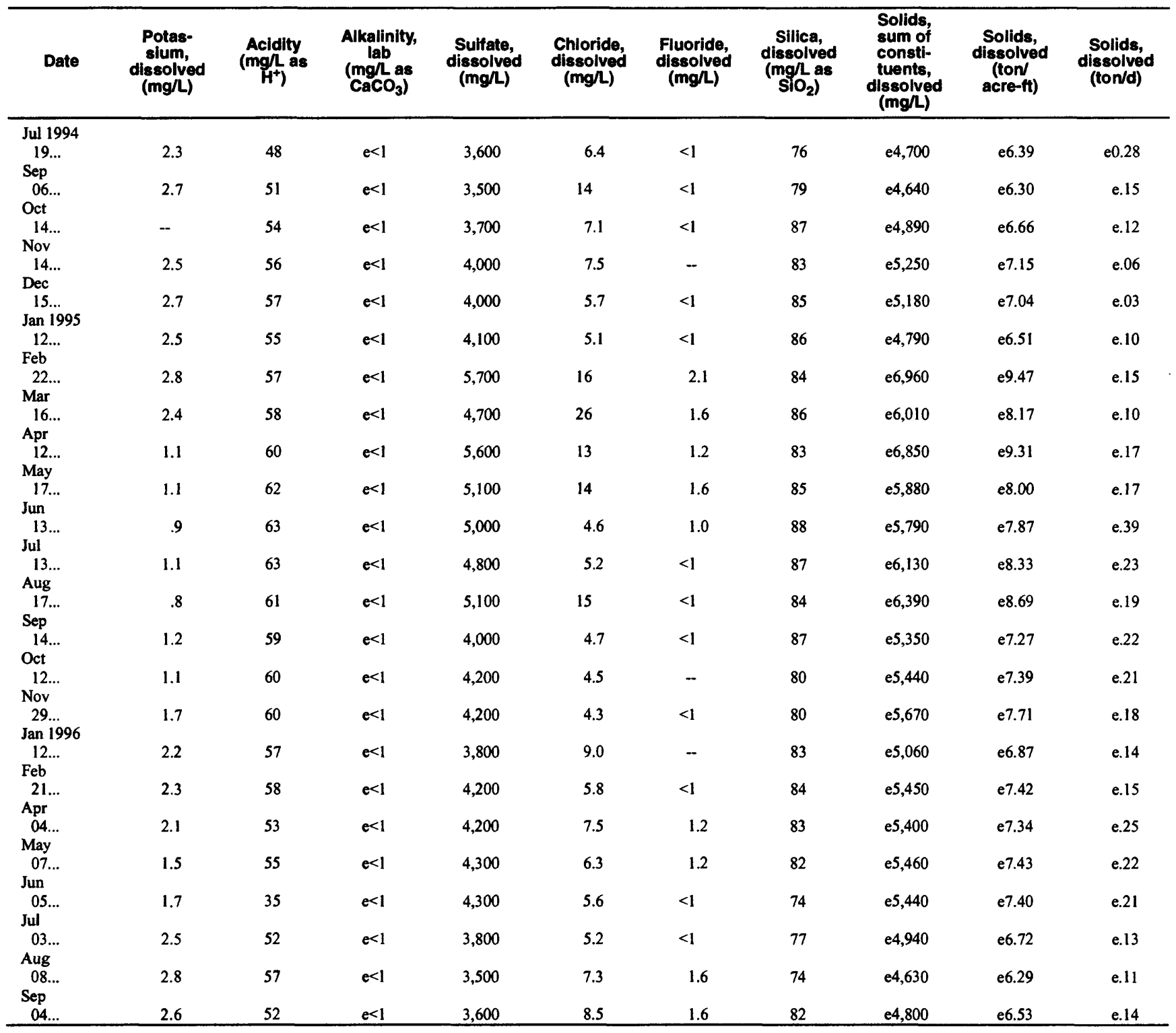


Table 3. Water-quality data for sites in the Sand Coulee Coal Area, Montana, July 1994 through September 1996 (Continued) SITE 13, 472305110551701--LEWIS COULEE ABOVE CASTNER PARK, AT BELT, MT--Continued

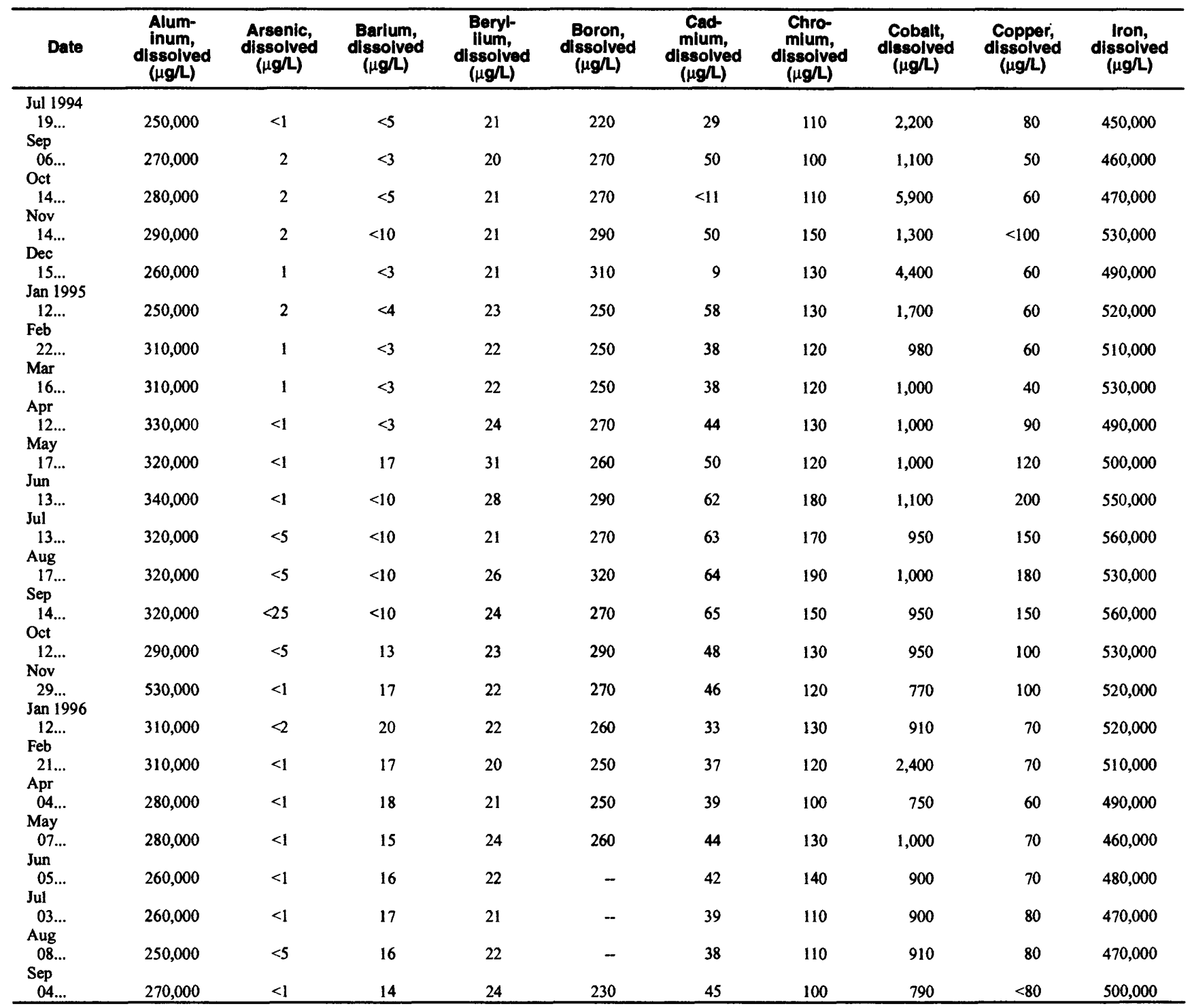


Table 3. Water-quality data for sites in the Sand Coulee Coal Area, Montana, July 1994 through September 1996 (Continued) SITE 13, 472305110551701--LEWIS COULEE ABOVE CASTNER PARK, AT BELT, MT--Continued

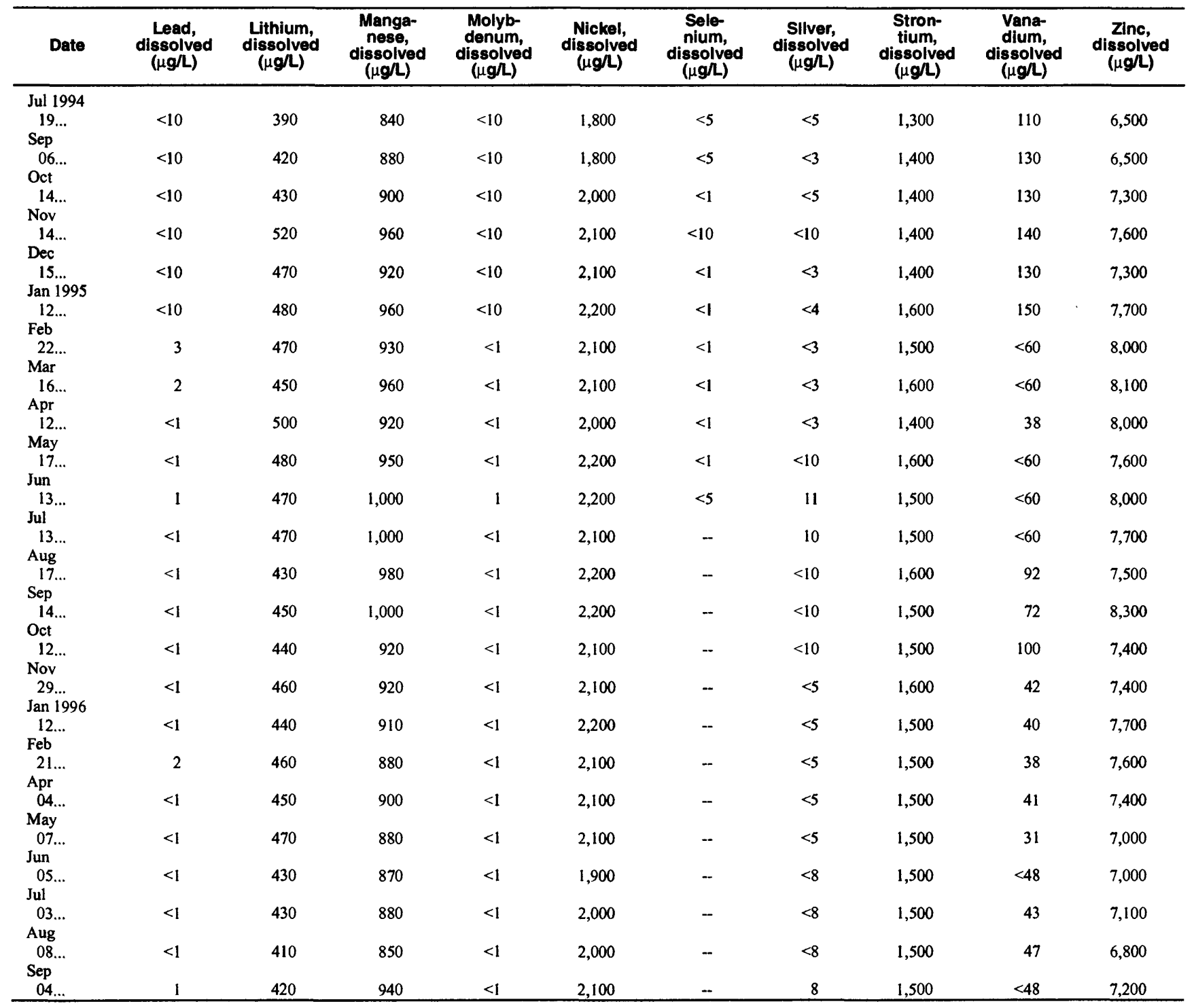


Table 3. Water-quality data for sites in the Sand Coulee Coal Area, Montana, July 1994 through September 1996 (Continued)

SITE 14, 472306111103601--MINE DRAIN TO MINING COULEE NEAR SAND COULEE, MT

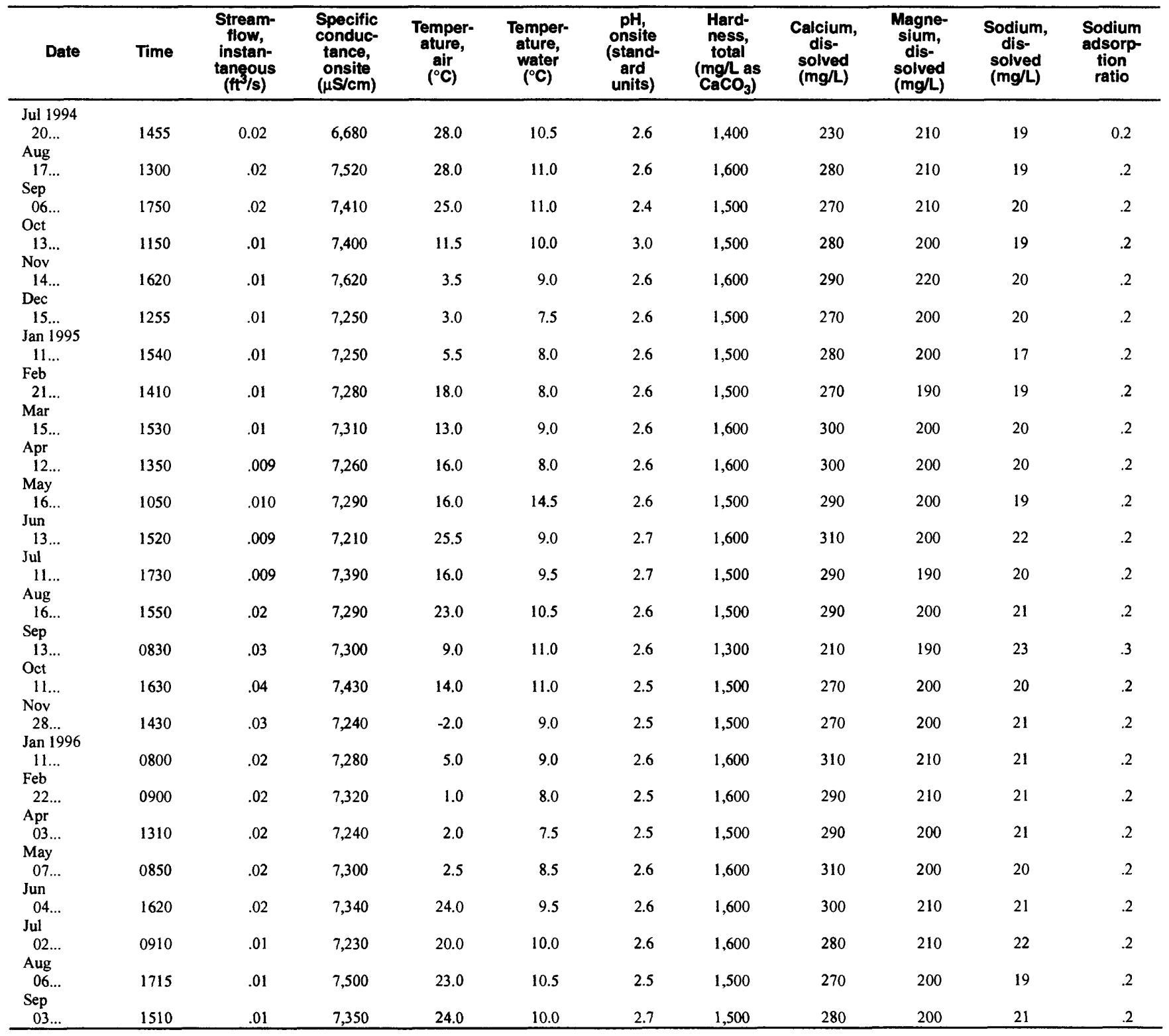


Table 3. Water-quality data for sites in the Sand Coulee Coal Area, Montana, July 1994 through September 1996 (Continued) SITE 14, 472306111103601--MINE DRAIN TO MINING COULEE NEAR SAND COULEE, MT--Continued

\begin{tabular}{|c|c|c|c|c|c|c|c|c|c|}
\hline Date & $\begin{array}{l}\text { Potas- } \\
\text { sium, } \\
\text { dissolved } \\
\text { (mg/L) }\end{array}$ & $\begin{array}{c}\text { Acidity } \\
\left(\mathrm{mg}_{\mathrm{H}} / \mathrm{L} \text { as }\right.\end{array}$ & $\begin{array}{c}\text { Alkalinity, } \\
\text { lab } \\
\left(\mathrm{mgg}_{\text {as }}\right. \\
\left.\mathrm{CaCO}_{3}\right)\end{array}$ & $\begin{array}{l}\text { Sulfate, } \\
\text { dissolved } \\
\text { (mg/l) }\end{array}$ & $\begin{array}{l}\text { Fluoride, } \\
\text { dissolved } \\
\text { (mg/L) }\end{array}$ & $\begin{array}{l}\text { Silica, } \\
\text { dissolved } \\
\text { (mg/l as } \\
\mathrm{siO}_{2} \text { ) }\end{array}$ & $\begin{array}{l}\text { Solids, } \\
\text { sum of } \\
\text { constl- } \\
\text { tuents, } \\
\text { dissolved } \\
\text { (mg/l) }\end{array}$ & $\begin{array}{l}\text { Solids, } \\
\text { dlssolved } \\
\text { (ton/ } \\
\text { acre-ft) }\end{array}$ & $\begin{array}{l}\text { Solids, } \\
\text { dissolved } \\
\text { (ton/d) }\end{array}$ \\
\hline $\begin{array}{l}\text { Jul } 1994 \\
20 \ldots . . \\
\text { Aug }\end{array}$ & 0.2 & 150 & $e<1$ & 9,200 & $<1$ & 71 & el1,800 & el6.0 & e 0.54 \\
\hline $\begin{array}{l}17 \ldots \\
\text { Sep }\end{array}$ & .2 & 160 & $e<1$ & 8,900 & $<1$ & 55 & e11,600 & el 5.8 & e. 57 \\
\hline $\begin{array}{l}06 \ldots . . \\
\text { Oct }\end{array}$ & .1 & 150 & $e<1$ & 9,800 & $<1$ & 72 & e 12,500 & el7.1 & e.61 \\
\hline $\begin{array}{l}13 \ldots \\
\text { Nov }\end{array}$ & .2 & 150 & $e<1$ & 9,800 & 1.4 & 130 & e12,400 & el6.8 & e. 37 \\
\hline $\begin{array}{l}14 \ldots \\
\text { Dec }\end{array}$ & $<.1$ & 150 & $e<1$ & 9,400 & - & 98 & $e 12,100$ & el 6.4 & e. 42 \\
\hline $\begin{array}{l}15 \ldots \\
\operatorname{Jan} 1995\end{array}$ & .2 & 150 & $e<1$ & 9,800 & - & 93 & el2,500 & el 17.0 & e.41 \\
\hline $\begin{array}{l}11 \ldots \\
\text { Feb }\end{array}$ & .4 & 150 & $e<1$ & 9,600 & 8.4 & 57 & el2,300 & el6.7 & e.32 \\
\hline $\begin{array}{l}21 \ldots \\
\text { Mar }\end{array}$ & .2 & 150 & $e<1$ & 11,000 & 2.6 & 120 & e 13,500 & el 8.3 & e.47 \\
\hline $\begin{array}{l}15 \ldots \\
\text { Apr }\end{array}$ & .3 & 150 & $e<1$ & 12,000 & $<1$ & 130 & el 14,600 & el9.8 & e.43 \\
\hline $\begin{array}{l}12 \ldots \\
\text { May }\end{array}$ & .2 & 150 & $e<1$ & 12,000 & 3.7 & 130 & el4,600 & el9.8 & e. 35 \\
\hline $\begin{array}{l}16 \ldots \\
\text { Jun }\end{array}$ & .3 & 150 & $e<1$ & 11,000 & 2.6 & 130 & el3,600 & e 18.5 & e.38 \\
\hline $\begin{array}{l}13 \ldots \\
\text { Jul }\end{array}$ & .3 & 150 & $e<1$ & 9,600 & $<1$ & 130 & el2,300 & el6.8 & e. 30 \\
\hline $\begin{array}{l}11 \ldots \\
\text { Aug }\end{array}$ & .2 & 150 & $e<1$ & 9,500 & $<1$ & 140 & el2,100 & 16.4 & e. 29 \\
\hline $\begin{array}{l}16 \ldots \\
\text { Sep }\end{array}$ & .4 & 150 & $e<1$ & 9,700 & 1.7 & 140 & el 12,300 & 16.7 & e. 73 \\
\hline $\begin{array}{l}13 \ldots \\
\text { Oct }\end{array}$ & .4 & 140 & $e<1$ & 9,800 & - & - & el2,300 & el6.7 & e.93 \\
\hline $\begin{array}{l}11 . . \\
\text { Nov }\end{array}$ & .2 & 150 & $e<1$ & 9,400 & 5.0 & 130 & ell,900 & el6.2 & el.16 \\
\hline $\begin{array}{l}28 \ldots \ldots \\
\operatorname{Jan} 1996\end{array}$ & .2 & 150 & $e<1$ & 8,900 & $<1$ & 130 & ell,500 & el5.6 & $e .93$ \\
\hline $\begin{array}{l}11 \ldots \\
\text { Feb }\end{array}$ & .1 & 160 & $e<1$ & 9,300 & -. & 140 & e 12,100 & el6.5 & e. 79 \\
\hline $\begin{array}{l}22 \ldots \\
\text { Apr }\end{array}$ & .2 & 150 & $e<1$ & 10,000 & -- & 140 & el2,800 & el 7.3 & e.79 \\
\hline $\begin{array}{l}03 \ldots \\
\text { May }\end{array}$ & $<.1$ & 150 & $e<1$ & 10,000 & 4.1 & 140 & el2,700 & el7.3 & e. 65 \\
\hline $\begin{array}{l}07 . . . \\
\text { Jun }\end{array}$ & .2 & 150 & $e<1$ & 10,000 & 1.8 & 140 & el2,600 & e) 7.1 & e.61 \\
\hline $\begin{array}{c}04 \ldots \\
\text { Jul }\end{array}$ & .1 & 150 & $e<1$ & 11,000 & $<1$ & 140 & el3,700 & el 8.7 & e. 59 \\
\hline $\begin{array}{l}02 \ldots \\
\text { Aug }\end{array}$ & .2 & 150 & $e<1$ & 9,200 & $<1$ & 130 & el1,800 & el6.0 & e. 45 \\
\hline $\begin{array}{l}06 . . \\
\text { Sep }\end{array}$ & .2 & 150 & $e<1$ & 8,900 & 3.8 & 120 & e 11,400 & e 15.5 & e.40 \\
\hline $03 \ldots$ & .2 & 150 & $\mathbf{e}<1$ & 8,700 & 3.7 & 130 & el1, 200 & el 15.3 & e. 36 \\
\hline
\end{tabular}


Table 3. Water-quality data for sites in the Sand Coulee Coal Area, Montana, July 1994 through September 1996 (Continued) SITE 14, 472306111103601--MINE DRAIN TO MINING COULEE NEAR SAND COULEE, MT--Continued

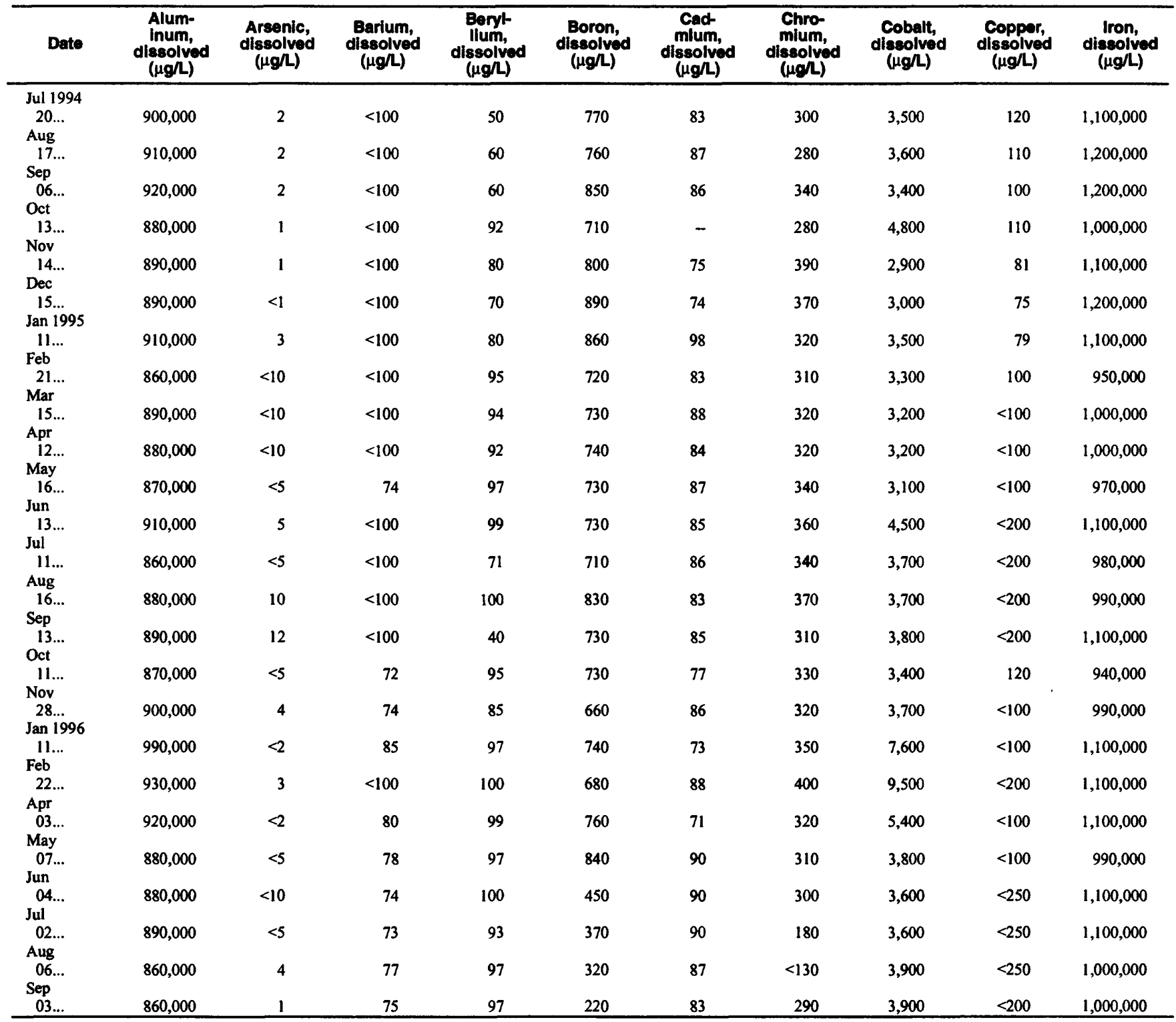


Table 3. Water-quality data for sites in the Sand Coulee Coal Area, Montana, July 1994 through September 1996 (Continued)

SITE 14, 472306111103601--MINE DRAIN TO MINING COULEE NEAR SAND COULEE, MT--Continued

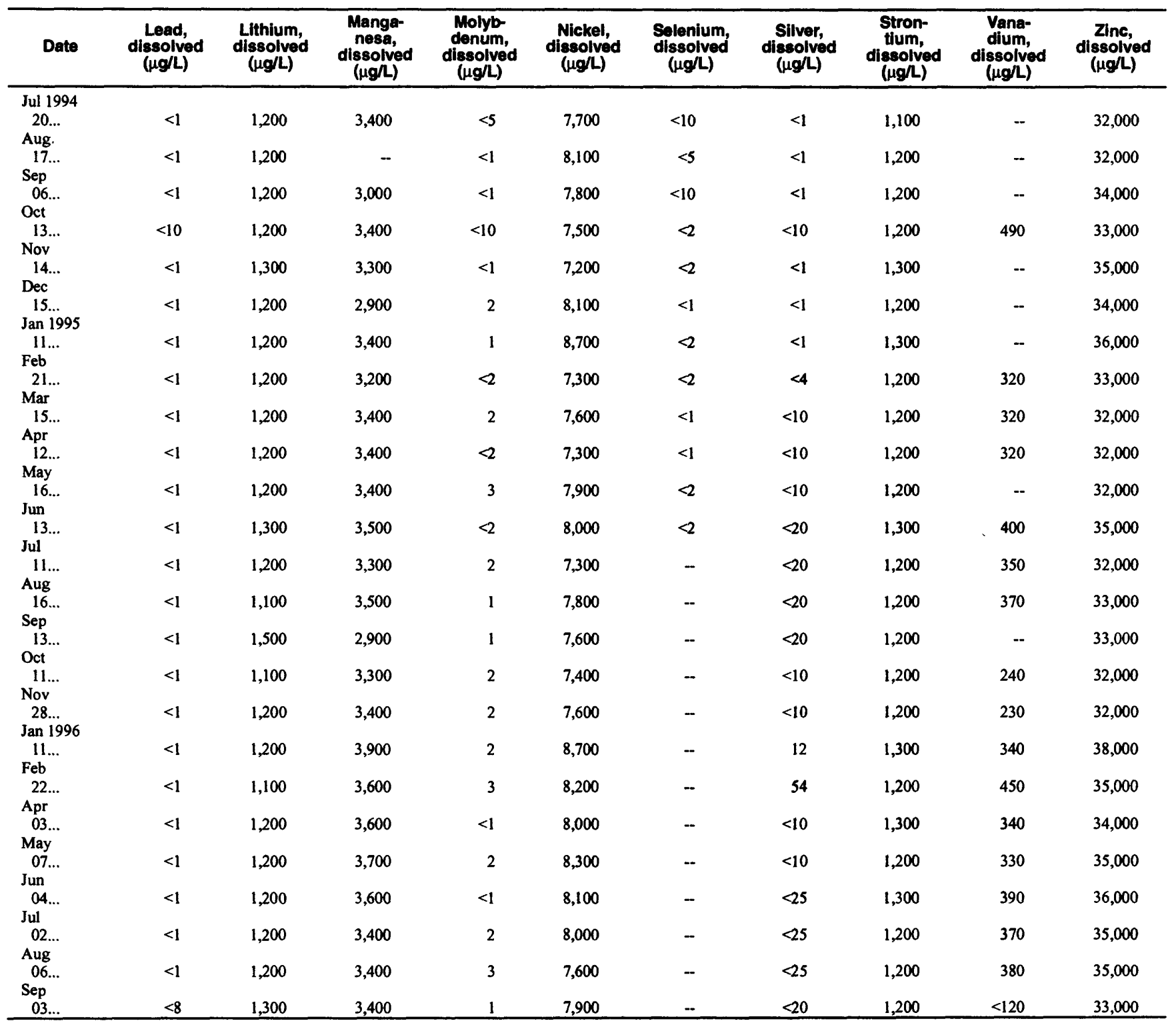


Table 3. Water-quality data for sites in the Sand Coulee Coal Area, Montana, July 1994 through September 1996 (Continued) SITE 15, 472310110550801--LEWIS COULEE ABOVE MINE ADIT, AT BELT, MT

\begin{tabular}{|c|c|c|c|c|c|c|c|c|c|c|c|}
\hline Date & Time & $\begin{array}{c}\text { Stream- } \\
\text { flow, } \\
\text { instan- } \\
\text { taneous } \\
\left(\mathrm{ft}^{\mathrm{j}} / \mathrm{s}\right)\end{array}$ & $\begin{array}{c}\text { Specific } \\
\text { conduc- } \\
\text { tance, } \\
\text { onsite } \\
\text { ( } \mu \text { S/cm) }\end{array}$ & $\begin{array}{c}\text { Temper- } \\
\text { ature, } \\
\text { air } \\
\left({ }^{\circ} \mathbf{C}\right)\end{array}$ & $\begin{array}{c}\text { Temper- } \\
\text { ature, } \\
\text { water } \\
\left({ }^{\circ} \mathrm{C}\right)\end{array}$ & $\begin{array}{c}\text { pH, } \\
\text { onsite } \\
\text { (stand- } \\
\text { ard } \\
\text { units) }\end{array}$ & $\begin{array}{c}\text { Hard- } \\
\text { ness, } \\
\text { total } \\
(\mathrm{mg} / \mathrm{Las} \\
\left.\mathrm{CaCO}_{3}\right)\end{array}$ & $\begin{array}{l}\text { Calcium, } \\
\text { dls- } \\
\text { solved } \\
\text { (mg/l) }\end{array}$ & $\begin{array}{l}\text { Magne- } \\
\text { slum, } \\
\text { dis- } \\
\text { solved } \\
\text { (mg/L) }\end{array}$ & $\begin{array}{c}\text { Sodlum, } \\
\text { dlis- } \\
\text { solved } \\
\text { (mg/h) }\end{array}$ & $\begin{array}{c}\text { Sodlum } \\
\text { adsorp- } \\
\text { tion } \\
\text { ratio }\end{array}$ \\
\hline \multicolumn{12}{|l|}{ Apr 1995} \\
\hline $\begin{array}{c}12 \ldots \\
\text { May }\end{array}$ & 1840 & 0.22 & 735 & 10.0 & 8.5 & 8.1 & 350 & 53 & 52 & 18 & 0.4 \\
\hline $\begin{array}{l}17 \ldots \\
\text { Jun }\end{array}$ & 0920 & .22 & 818 & 18.0 & 9.5 & 8.3 & 400 & 59 & 61 & 18 & .4 \\
\hline $\begin{array}{c}13 \ldots \\
\text { Jul }\end{array}$ & 1040 & .11 & 905 & 18.5 & 14.5 & 8.6 & 450 & 65 & 70 & 25 & .5 \\
\hline $13 \ldots$ & 1030 & .28 & 1300 & 17.0 & 14.0 & 8.6 & 600 & 100 & 85 & 62 & 1 \\
\hline
\end{tabular}

\begin{tabular}{|c|c|c|c|c|c|c|c|c|c|c|}
\hline Date & $\begin{array}{l}\text { Potas- } \\
\text { sium, } \\
\text { dissolved } \\
\text { (mg/li) }\end{array}$ & $\begin{array}{c}\text { Acidity } \\
(\mathbf{m g} / \mathrm{l} \text { as } \\
\left.\mathrm{H}^{+}\right)\end{array}$ & $\begin{array}{c}\text { Alkalinity, } \\
\text { lab } \\
\text { (mg/L as } \\
\text { CaCo })\end{array}$ & $\begin{array}{l}\text { Sulfate, } \\
\text { dlssolved } \\
\text { (mg/L) }\end{array}$ & $\begin{array}{l}\text { Chloride, } \\
\text { dlssolved } \\
\text { (mg/L) }\end{array}$ & $\begin{array}{c}\text { Fluoride, } \\
\text { diseolved } \\
\text { (mg/L) }\end{array}$ & $\begin{array}{l}\text { Sllica, } \\
\text { dissolved } \\
\text { (mg/l as } \\
\left.\text { Sio }_{2}\right)\end{array}$ & $\begin{array}{l}\text { Solids, } \\
\text { sum of } \\
\text { consti- } \\
\text { tuents, } \\
\text { dissolved } \\
\text { (mg/l) }\end{array}$ & $\begin{array}{l}\text { Solids, } \\
\text { dissolved } \\
\text { (ton/ } \\
\text { acre-ft) }\end{array}$ & $\begin{array}{l}\text { Solids, } \\
\text { dissolved } \\
\text { (ton/d) }\end{array}$ \\
\hline \multicolumn{11}{|l|}{ Apr 1995} \\
\hline $\begin{array}{c}12 \ldots \\
\text { May }\end{array}$ & 6.2 & $<0.1$ & 271 & 100 & 12 & 0.4 & 8.5 & 413 & 0.56 & 0.25 \\
\hline $\begin{array}{l}17 . . \\
\text { Jun }\end{array}$ & 4.3 & $<.1$ & 313 & 100 & 14 & .5 & 7.6 & 453 & .62 & .27 \\
\hline $\begin{array}{c}13 \ldots \\
\text { Jul }\end{array}$ & 4.9 & $<.1$ & 325 & 130 & 17 & .5 & 8.1 & 516 & .70 & .15 \\
\hline $13 \ldots$ & 7.9 & $<.1$ & 335 & 390 & 17 & .3 & 12 & 877 & 1.19 & .66 \\
\hline
\end{tabular}

\begin{tabular}{|c|c|c|c|c|c|c|c|c|c|c|}
\hline Date & $\begin{array}{l}\text { Alum- } \\
\text { Inum, } \\
\text { dissolved } \\
\text { ( } \mu \mathrm{g} / \mathrm{L})\end{array}$ & $\begin{array}{l}\text { Arsenic, } \\
\text { dissolved } \\
(\mu \mathrm{g} / \mathrm{L})\end{array}$ & $\begin{array}{l}\text { Barium, } \\
\text { dissolved } \\
\text { (ug/L) }\end{array}$ & $\begin{array}{l}\text { Beryl- } \\
\text { lium, } \\
\text { dissolved } \\
\text { ( } \mu g^{\prime} / \text { L) }\end{array}$ & $\begin{array}{l}\text { Boron, } \\
\text { dissolved } \\
\text { (ug/h) }\end{array}$ & $\begin{array}{c}\text { Cad- } \\
\text { mium, } \\
\text { dissolved } \\
(\mu \mathrm{g} / \mathrm{L})\end{array}$ & $\begin{array}{l}\text { Chro- } \\
\text { mium, } \\
\text { dissolved } \\
(\mu g / L)\end{array}$ & 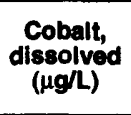 & $\begin{array}{l}\text { Copper, } \\
\text { dissolved } \\
(\mu \mathrm{g} / \mathrm{L})\end{array}$ & $\begin{array}{l}\text { Iron, } \\
\text { dissolved } \\
(\mu g / L)\end{array}$ \\
\hline Apr 1995 & & & & & & & & & & \\
\hline $\begin{array}{l}12 \ldots \\
\text { May }\end{array}$ & 20 & 1 & 93 & $<0.5$ & 40 & $<1$ & $<5$ & $<1$ & $<10$ & 18 \\
\hline $\begin{array}{l}17 \ldots \\
\text { Jun }\end{array}$ & 20 & $<1$ & 110 & $<.5$ & 50 & $<1$ & $<5$ & $<3$ & $<10$ & 5 \\
\hline $\begin{array}{l}13 \ldots \\
\text { Jul }\end{array}$ & $<10$ & $<1$ & 110 & $<.5$ & 50 & 2 & $<5$ & 3 & $<10$ & 130 \\
\hline $13 \ldots$ & 20 & 1 & 170 & $<.5$ & 110 & $<1$ & $<5$ & $<3$ & $<10$ & 48 \\
\hline
\end{tabular}

\begin{tabular}{|c|c|c|c|c|c|c|c|c|c|c|}
\hline Date & $\begin{array}{l}\text { Lead, } \\
\text { dissolved } \\
(\mu \mathrm{g} / \mathrm{L})\end{array}$ & $\begin{array}{l}\text { Lithium, } \\
\text { dlesolved } \\
(\mu \mathrm{g} / \mathrm{L})\end{array}$ & $\begin{array}{l}\text { Manga- } \\
\text { nese, } \\
\text { dissolved } \\
(\mu \mathrm{g} / \mathrm{L})\end{array}$ & $\begin{array}{l}\text { Molyb- } \\
\text { denum, } \\
\text { dissolved } \\
(\mu \mathrm{g} / \mathrm{L})\end{array}$ & $\begin{array}{l}\text { Nickel, } \\
\text { dissolved } \\
(\mu g / L)\end{array}$ & $\begin{array}{c}\text { Sele- } \\
\text { nlum, } \\
\text { dissolved } \\
(\mu \mathrm{g} / \mathrm{L})\end{array}$ & $\begin{array}{l}\text { Silver, } \\
\text { dlssolved } \\
\text { ( } \mu \mathrm{g} / \mathrm{L})\end{array}$ & $\begin{array}{l}\text { Stron- } \\
\text { tium, } \\
\text { dissolved } \\
\text { (ug/L) }\end{array}$ & $\begin{array}{l}\text { Vana- } \\
\text { dlum, } \\
\text { dissolved } \\
(\mu \mathrm{g} / \mathrm{L})\end{array}$ & $\begin{array}{l}\text { Zlnc, } \\
\text { dissolved } \\
(\mu \mathrm{g} / \mathrm{L})\end{array}$ \\
\hline $\begin{array}{c}\text { Jul } 1994 \\
12 \ldots \\
\text { May }\end{array}$ & $<1$ & 35 & 4 & 2 & $<10$ & 2 & 2 & 380 & $<6$ & $<3$ \\
\hline $\begin{array}{l}17 . . . \\
\text { Jun }\end{array}$ & 10 & 43 & 2 & $<10$ & $<10$ & 3 & $<1$ & 460 & $<6$ & 6 \\
\hline$\underset{\text { Jul }}{13 \ldots}$ & $<10$ & 54 & $<1$ & $<10$ & $<10$ & 2 & $<1$ & 540 & $<6$ & 10 \\
\hline $13 \ldots$ & $<10$ & 110 & 5 & $<10$ & $<10$ & - & $<1$ & 1,000 & $<6$ & $<3$ \\
\hline
\end{tabular}


Table 3. Water-quality data for sites in the Sand Coulee Coal Area, Montana, July 1994 through September 1996 (Continued)

SITE 16, $472313111104901--M I N E$ DRAIN TO SAND COULEE NEAR SAND COULEE, MT

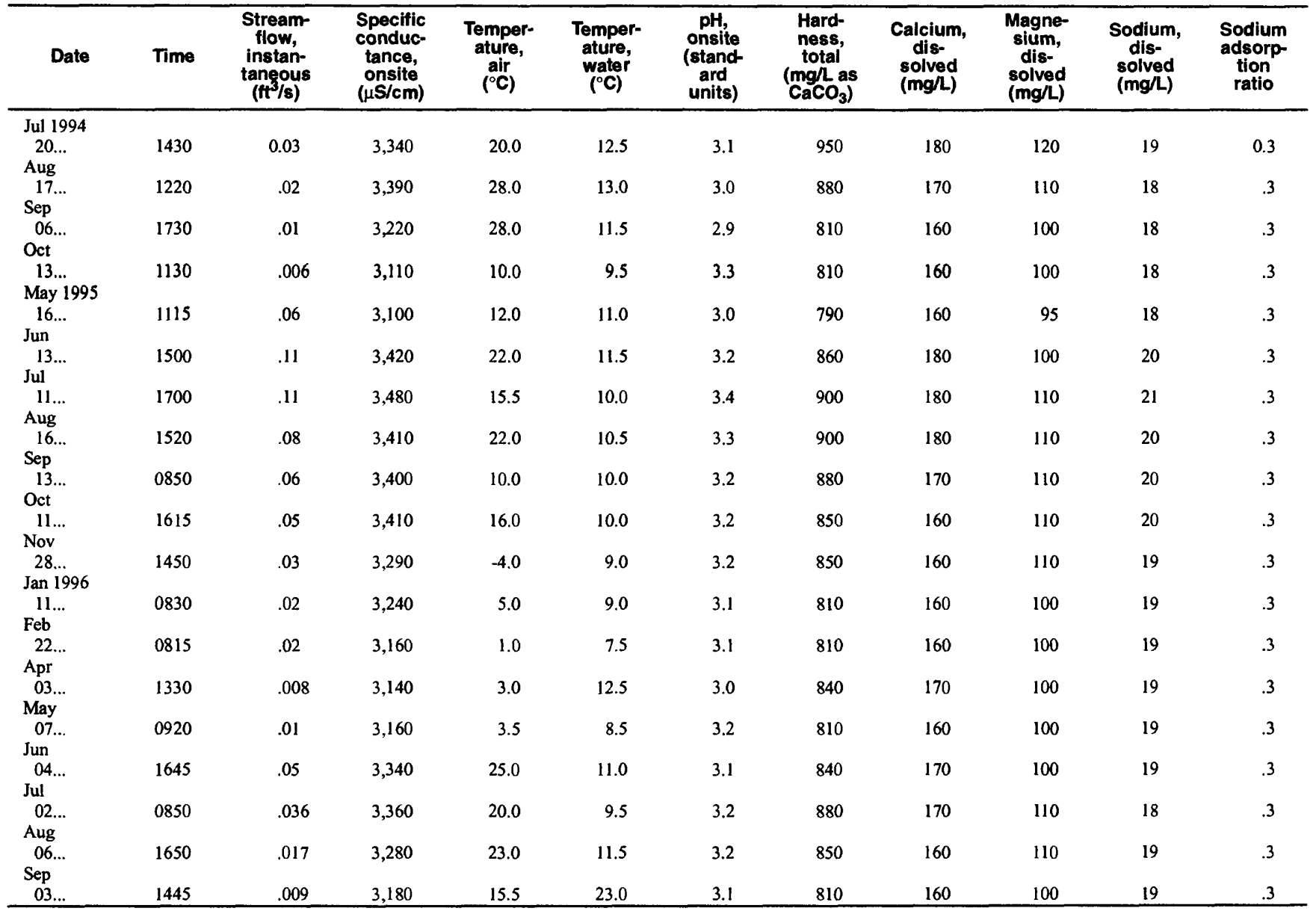


Table 3. Water-quality data for sites in the Sand Coulee Coal Area, Montana, July 1994 through September 1996 (Continued)

SITE 16, 472313111104901--MINE DRAIN TO SAND COULEE NEAR SAND COULEE, MT--Continued

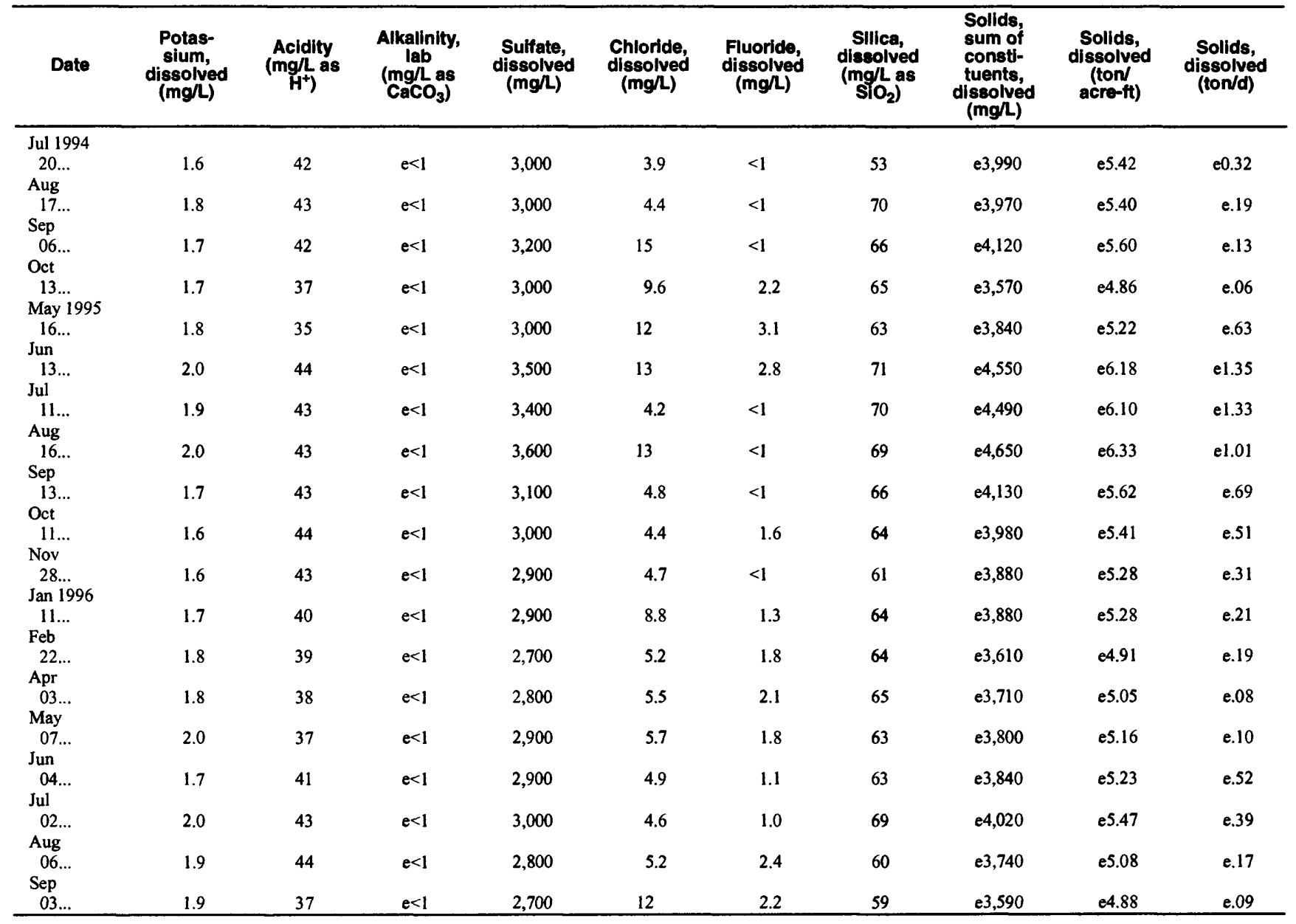


Table 3. Water-quality data for sites in the Sand Coulee Coal Area, Montana, July 1994 through September 1996 (Continued)

SITE 16, 472313111104901--MINE DRAIN TO SAND COULEE NEAR SAND COULEE, MT--Continued

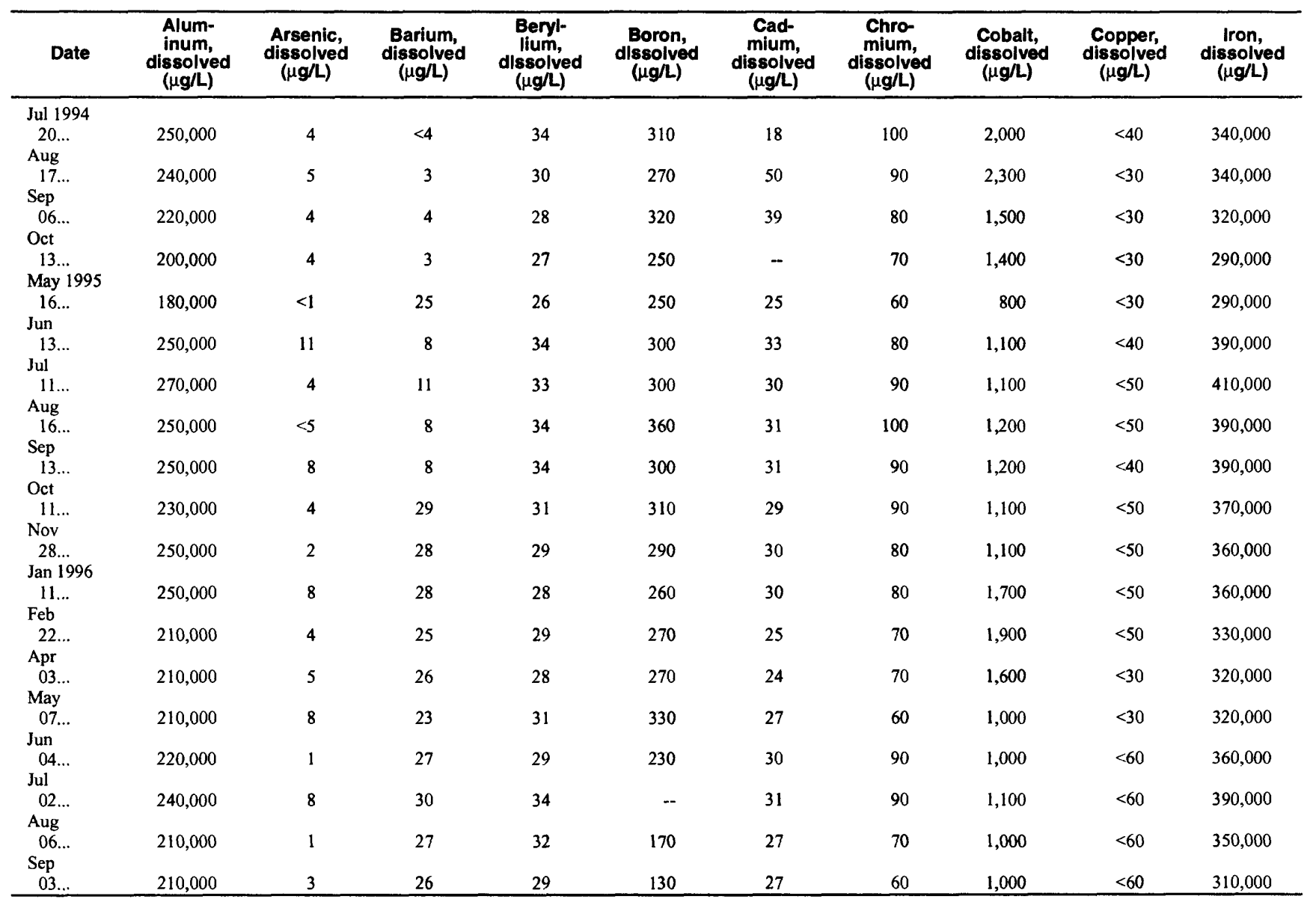


Table 3. Water-quality data for sites in the Sand Coulee Coal Area, Montana, July 1994 through September 1996 (Continued) SITE 16,472313111104901 --MINE DRAIN TO SAND COULEE NEAR SAND COULEE, MT--Continued

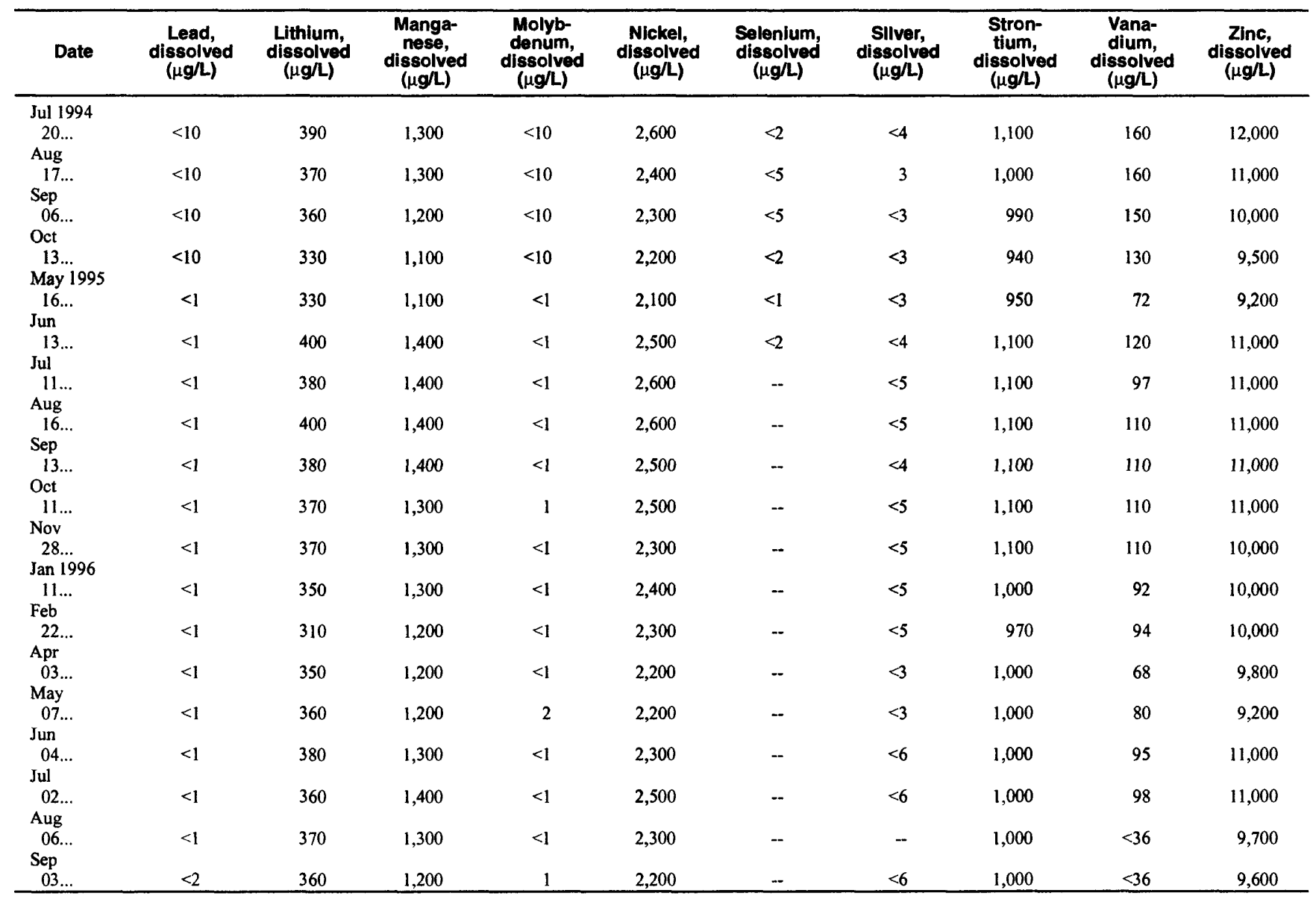


Table 3. Water-quality data for sites in the Sand Coulee Coal Area, Montana, July 1994 through September 1996 (Continued)

SITE 17, 472330111082801--CENTERVILLE WETLANDS INFLOW AT CENTERVILLE, MT

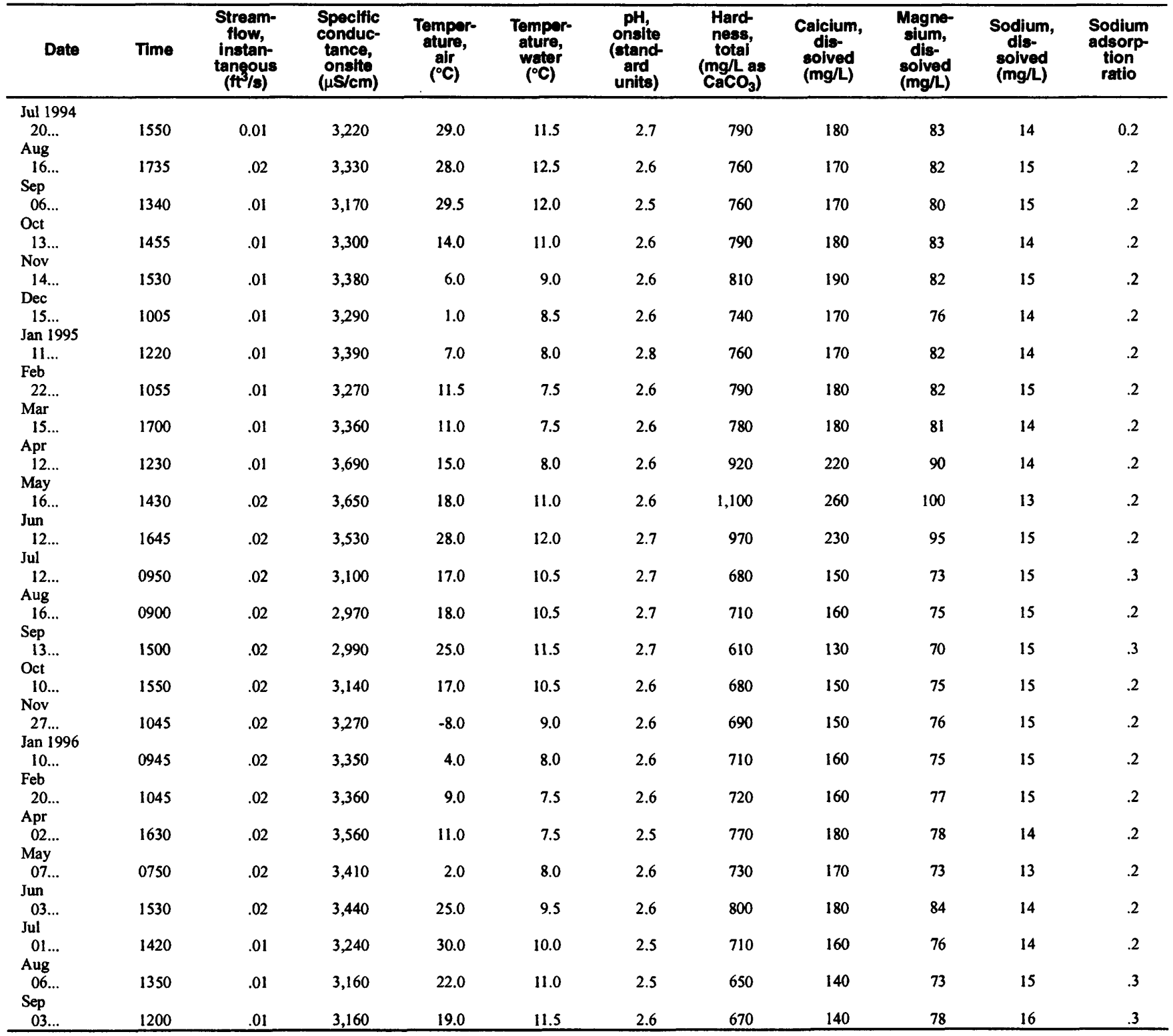


Table 3. Water-quality data for sites in the Sand Coulee Coal Area, July 1994 through September 1996 (Continued)

SITE 17,472330111082801 --CENTERVILLE WETLANDS INFLOW AT CENTERVILLE, MT--Continued

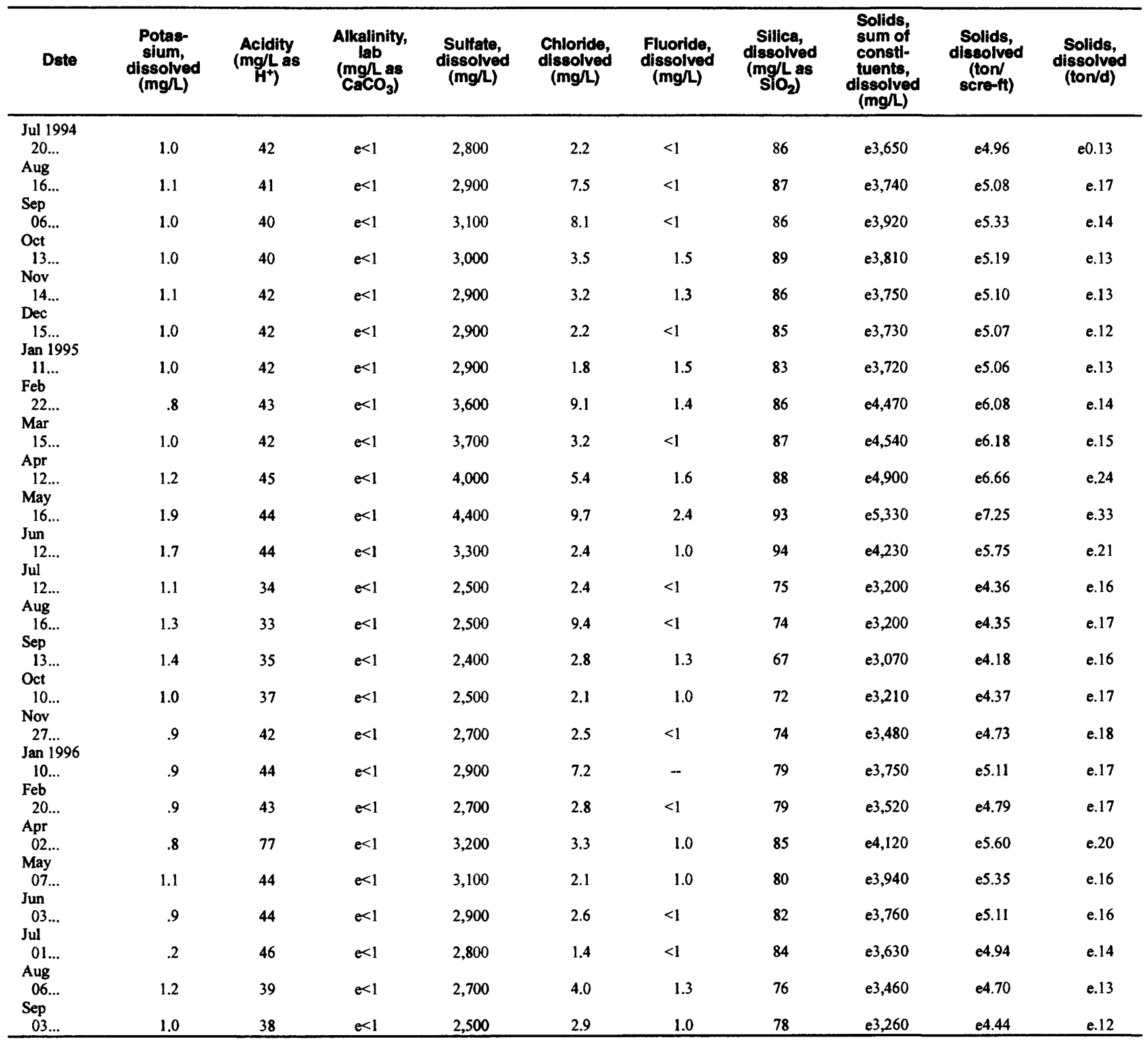


Table 3. Water-quality data for sites in the Sand Coulee Coal Area, Montana, July 1994 through September 1996 (Continued)

SITE 17, 472330111082801--CENTERVILLE WETLANDS INFLOW AT CENTERVILLE, MT--Continued

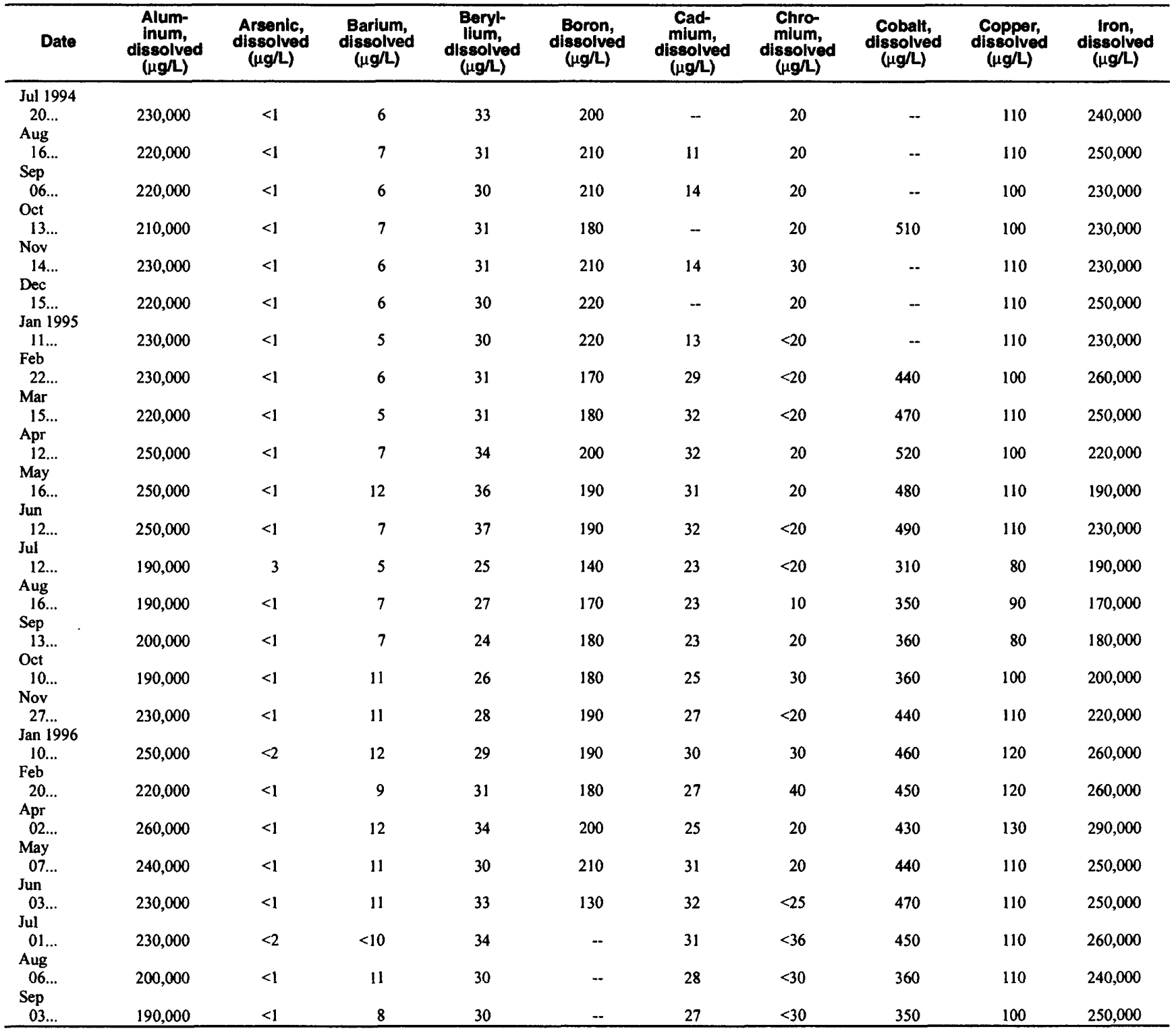


Table 3. Water-quality data for sites in the Sand Coulee Coal Area, Montana, July 1994 through September 1996 (Continued)

SITE 17, 472330111082801--CENTERVILLE WETLANDS INFLOW AT CENTERVILLE, MT--Continued

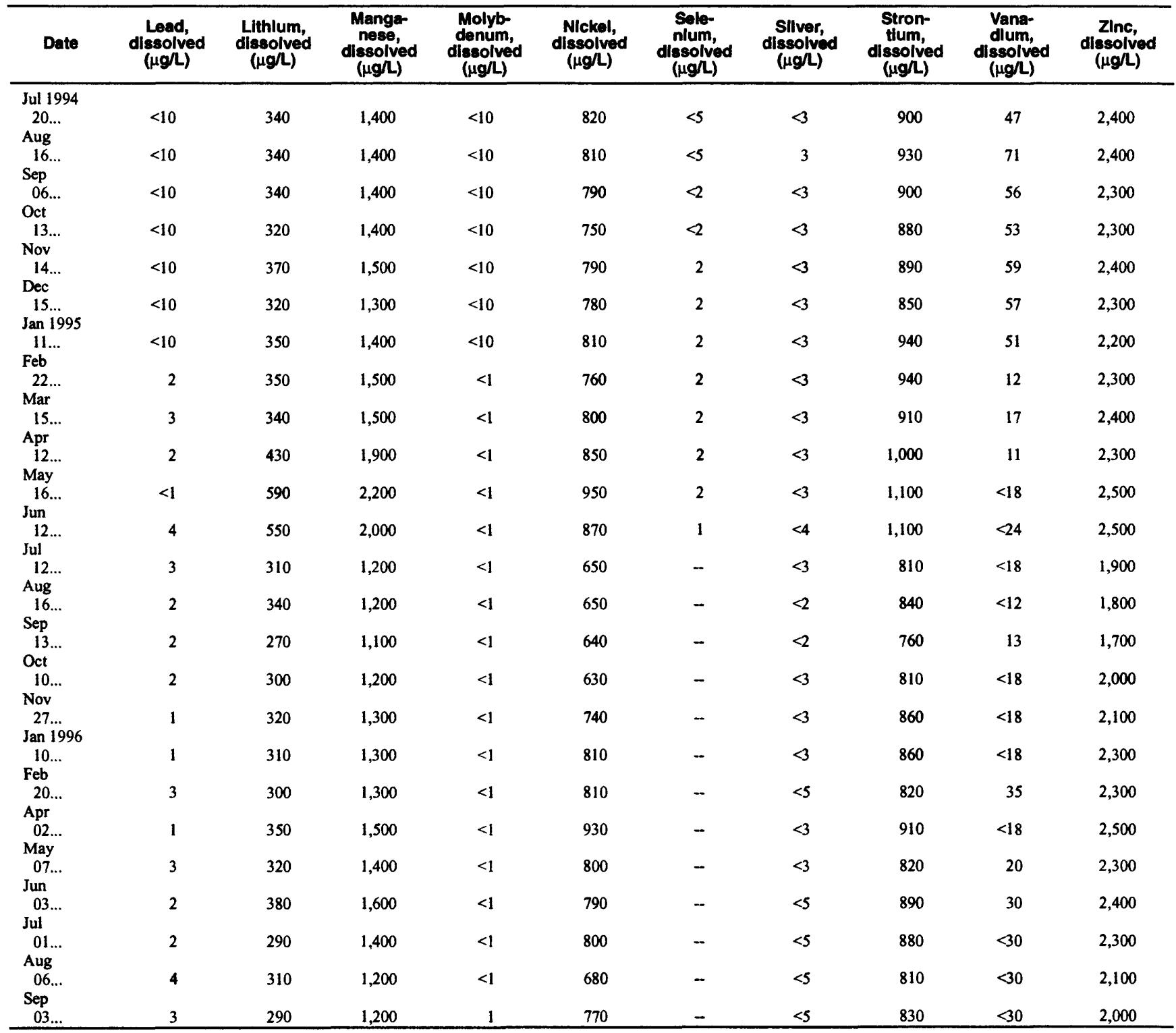


Table 3. Water-quality data for sites in the Sand Coulee Coal Area, Montana, July 1994 through September 1996 (Continued)

SITE 18,472331111083001 --CENTERVILLE WETLANDS OUTFLOW AT CENTERVILLE, MT

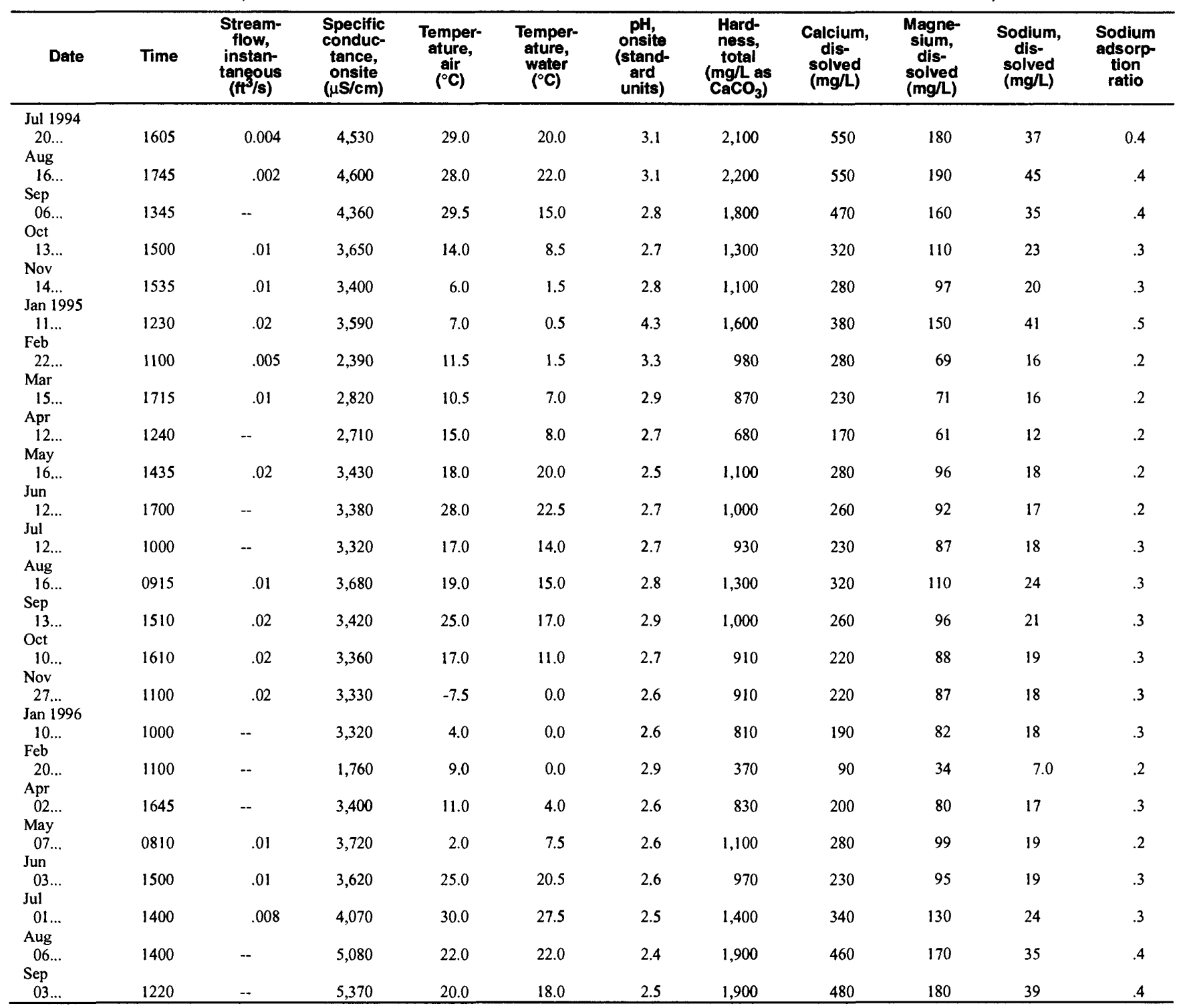


Table 3. Water-quality data for sites in the Sand Coulee Coal Area, Montana, July 1994 through September 1996 (Continued) SITE 18, 472331111083001--CENTERVILLE WETLANDS OUTFLOW AT CENTERVILLE, MT--Continued

\begin{tabular}{|c|c|c|c|c|c|c|c|c|c|c|}
\hline Date & $\begin{array}{l}\text { Potas- } \\
\text { sium, } \\
\text { dissolved } \\
\text { (mg/L) }\end{array}$ & $\begin{array}{c}\text { Acidity } \\
\left(\mathbf{m g}^{\prime} \mathbf{H}^{+} \text {as }\right.\end{array}$ & $\begin{array}{c}\text { Alkalinity, } \\
\text { lab } \\
(\mathrm{mg} / \mathrm{Las} \\
\left.\mathrm{CaCO}_{3}\right)\end{array}$ & $\begin{array}{l}\text { Sulfate, } \\
\text { dissolved } \\
\text { (mg/L) }\end{array}$ & $\begin{array}{l}\text { Chloride, } \\
\text { dissolved } \\
\text { (mg/L) }\end{array}$ & $\begin{array}{l}\text { Fluoride, } \\
\text { dissolved } \\
\text { (mg/L) }\end{array}$ & $\begin{array}{l}\text { Sillica, } \\
\text { dissolved } \\
\text { (mg/L as } \\
\left.\mathrm{SiO}_{2}\right)\end{array}$ & $\begin{array}{l}\text { Solids, } \\
\text { sum of } \\
\text { consti- } \\
\text { tuents, } \\
\text { dissolved } \\
\text { (mg/L) }\end{array}$ & $\begin{array}{l}\text { Solids, } \\
\text { dissolved } \\
\text { (ton/ } \\
\text { acre-ft) }\end{array}$ & $\begin{array}{l}\text { Solids, } \\
\text { dissolved } \\
\text { (ton/d) }\end{array}$ \\
\hline Jul 1994 & & & & & & & & & & \\
\hline $20 \ldots$ & 140 & 24 & $e<1$ & 3,600 & 40 & $<1$ & 65 & e4,840 & e6.59 & $\mathrm{e} 0.05$ \\
\hline Aug & & & & & & & & & & \\
\hline $\begin{array}{l}16 \ldots \\
\text { Sep }\end{array}$ & 120 & 28 & $e<1$ & 3,700 & 42 & $<1$ & 56 & e 4,960 & e6.74 & e. 03 \\
\hline $\begin{array}{l}06 \ldots \\
\text { Oct }\end{array}$ & 99 & 37 & $e<1$ & 3,900 & 36 & $<1$ & 48 & e5,120 & e6.96 & -- \\
\hline $\begin{array}{l}13 \ldots \\
\text { Nov }\end{array}$ & 45 & 32 & $e<1$ & 3,100 & 16 & 1.4 & 64 & $\mathrm{e} 4,000$ & e5.44 & e. 15 \\
\hline $\begin{array}{c}14 \ldots \\
\operatorname{Jan} 1995\end{array}$ & 36 & 32 & $e<1$ & 2,900 & 12 & 1.2 & 73 & $\mathrm{e} 3,760$ & e5.11 & e. 12 \\
\hline $\begin{array}{l}11 \ldots \\
\text { Feb }\end{array}$ & 140 & 23 & $e<1$ & 2,800 & 44 & 1.2 & 70 & e3,900 & e5.30 & e. 23 \\
\hline $\begin{array}{c}22 \ldots \\
\mathrm{Mar}\end{array}$ & 46 & 14 & $e<1$ & 2,600 & 17 & $<1$ & 38 & e3,220 & e4.38 & e. 05 \\
\hline $\begin{array}{c}15 \ldots \\
\text { Apr }\end{array}$ & 37 & 27 & $e<1$ & 3,300 & 26 & $<1$ & 51 & e3,950 & 5.37 & e.12 \\
\hline $\begin{array}{l}12 \ldots \\
\text { May }\end{array}$ & 13 & 24 & $e<1$ & 3,500 & 21 & 1.2 & 49 & e 4,070 & e5.54 &.- \\
\hline $\begin{array}{l}16 \ldots \\
\text { Jun }\end{array}$ & 36 & 30 & $e<1$ & 3,000 & 20 & 1.4 & 70 & e3,810 & e5.18 & e. 20 \\
\hline $\begin{array}{c}12 \ldots \\
\text { Jul }\end{array}$ & 22 & 32 & $e<1$ & 3,000 & 8.9 & $<1$ & 60 & e3,770 & e5.12 & -- \\
\hline $\begin{array}{l}12 \ldots \\
\text { Aug }\end{array}$ & 27 & 29 & $e<1$ & 2,600 & 11 & $<1$ & 46 & $e 3,330$ & e4.52 & -- \\
\hline $\begin{array}{l}16 \ldots \\
\text { Sep }\end{array}$ & 36 & 31 & $\mathrm{e}<1$ & 3,100 & 20 & $<1$ & 34 & $\mathrm{e} 3,960$ & e5.38 & e.13 \\
\hline Oct & 25 & 31 & $e<1$ & 2,800 & 10 & 1.3 & 42 & $e 3,560$ & e4.84 & e. 16 \\
\hline $\begin{array}{l}10 \ldots \\
\text { Nov }\end{array}$ & 17 & 32 & $e<1$ & 2,600 & 9.3 & 1.0 & 61 & $e 3,320$ & e4.52 & e. 14 \\
\hline $\begin{array}{c}27 \ldots \\
\text { Jan } 1996\end{array}$ & 13 & 35 & $\mathrm{e}<1$ & 2,800 & 6.2 & $<1$ & 65 & e3,580 & e4.87 & e.16 \\
\hline $\begin{array}{l}10 \ldots \\
\text { Feb }\end{array}$ & 21 & 39 & $e<1$ & 2,800 & 9.9 & -- & 72 & $\mathrm{e} 3,650$ & e4.96 & -- \\
\hline $\begin{array}{l}20 \ldots \\
\text { Apr }\end{array}$ & 4.2 & 16 & $e<1$ & 1,100 & 2.4 & $<1$ & 28 & el, 140 & el. 95 & -- \\
\hline $\begin{array}{l}02 \ldots \\
\text { May }\end{array}$ & 7.2 & 39 & $\mathrm{e}<1$ & 2,900 & 5.8 & 1.0 & 70 & e3,720 & e5.06 & - \\
\hline $\begin{array}{c}07 \ldots \\
\text { Jun }\end{array}$ & 13 & 42 & $\mathrm{e}<1$ & 3,200 & 8.9 & 1.1 & 75 & $\mathrm{e} 4,120$ & e5.61 & e.11 \\
\hline $\begin{array}{c}03 \ldots \\
\text { Jul }\end{array}$ & 9.9 & 38 & $e<1$ & 3,100 & 7.9 & $<1$ & 65 & e3,940 & e5.35 & e.11 \\
\hline $\begin{array}{l}01 \ldots \\
\text { Aug }\end{array}$ & 22 & 44 & $e<1$ & 3,500 & 12 & $<1$ & 63 & $\mathrm{e} 4,550$ & e6.19 & e.01 \\
\hline $\begin{array}{l}06 \ldots \\
\text { Sep }\end{array}$ & 34 & 55 & $e<1$ & 4,600 & 22 & 1.5 & 26 & e5,870 & e 7.99 & - \\
\hline $03 \ldots$ & 30 & 52 & $e<1$ & 4,600 & 25 & $<1$ & -- & e5,860 & e7.97 & -- \\
\hline
\end{tabular}


Table 3. Water-quality data for sites in the Sand Coulee Coal Area, Montana, July 1994 through September 1996 (Continued) SITE 18, 472331111083001--CENTERVILLE WETLANDS OUTFLOW AT CENTERVILLE, MT--Continued

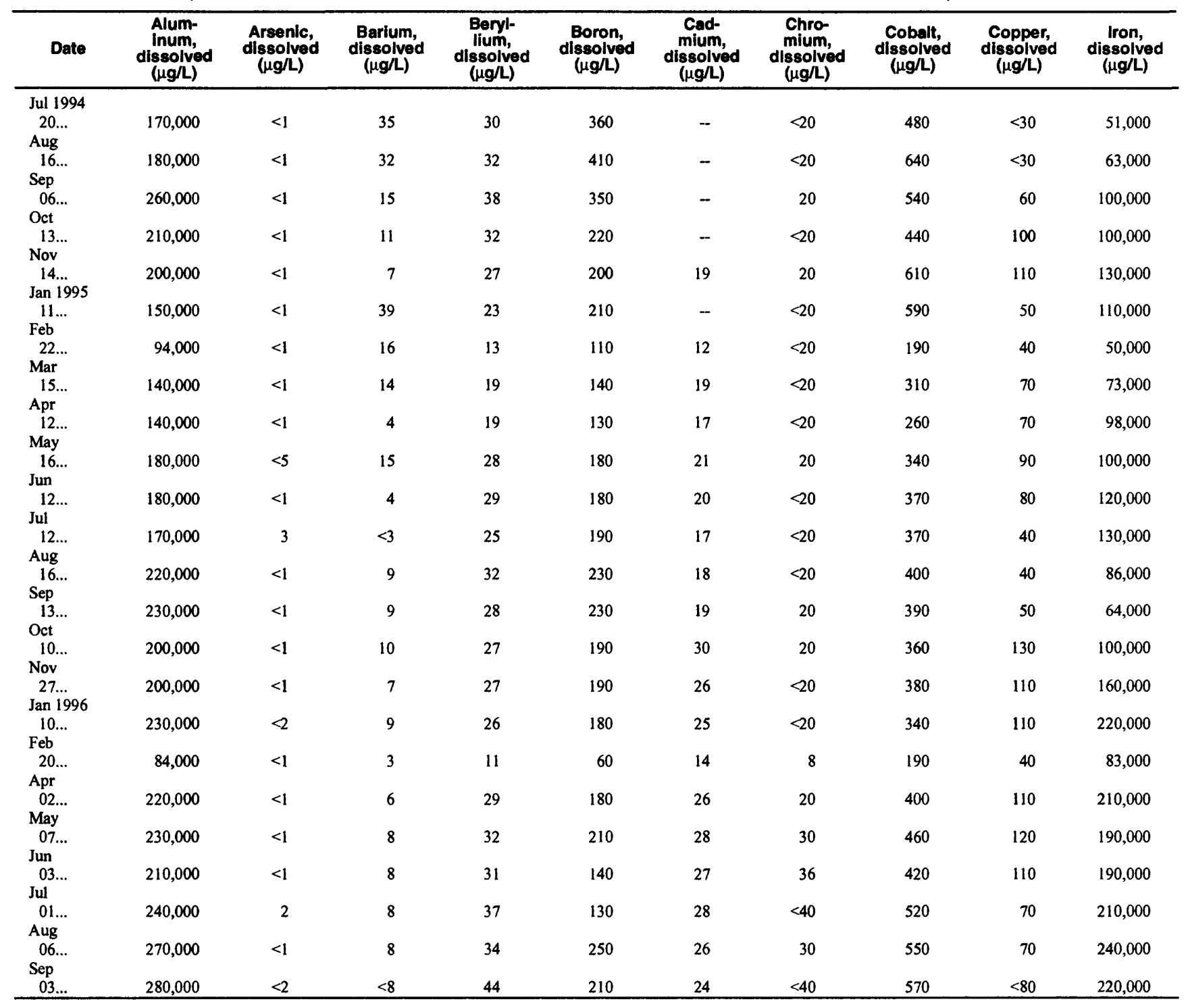


Table 3. Water-quality data for sites in the Sand Coulee Coal Area, Montana, July 1994 through September 1996 (Continued) SITE 18, 472331111083001--CENTERVILLE WETLANDS OUTFLOW AT CENTERVILLE, MT--Continued

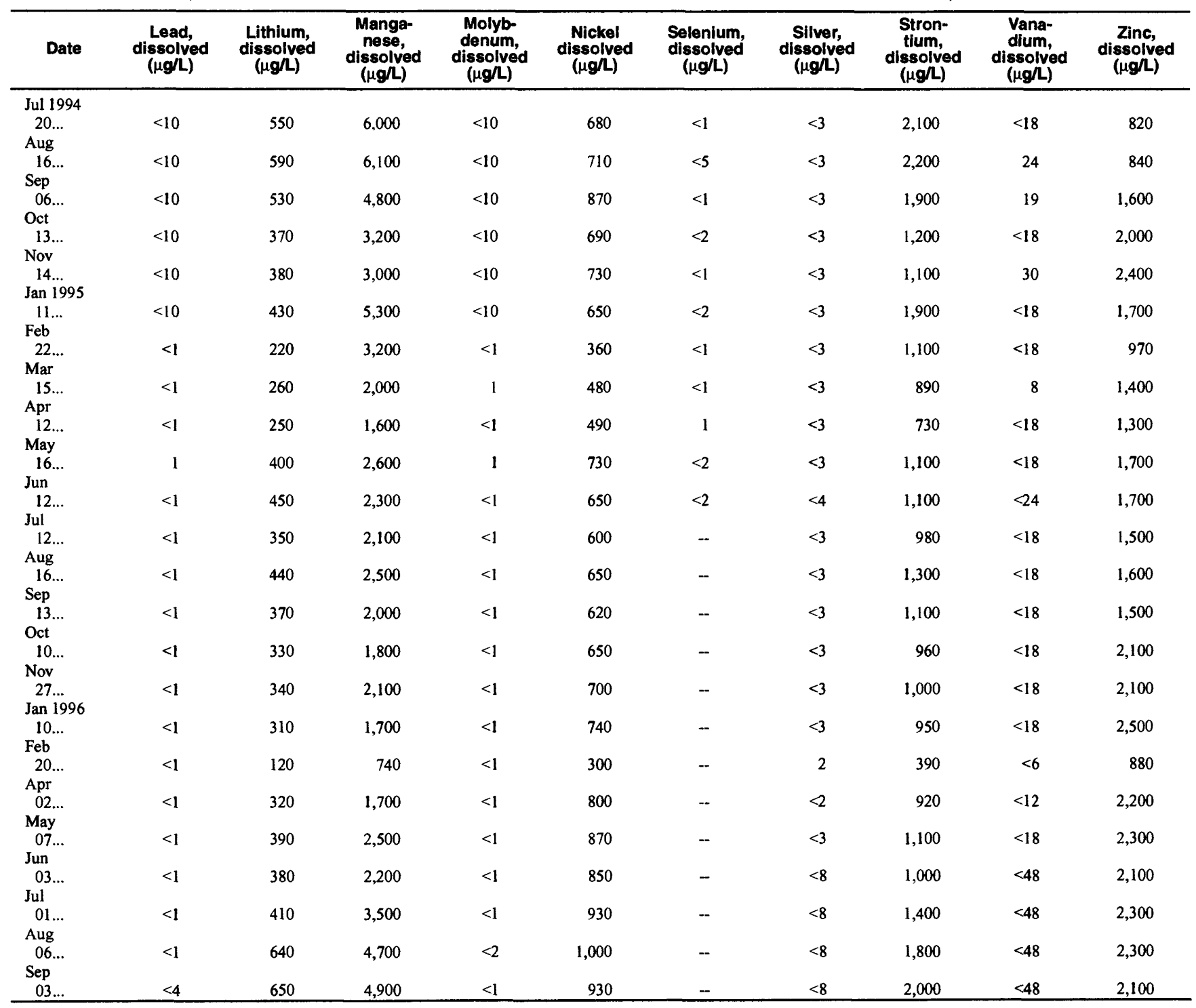


Table 3. Water-quality data for sites in the Sand Coulee Coal Area, Montana, July 1994 through September 1996 (Continued) SITE 19, 472334111104401--MOUNT OREGON MINE DRAIN TO KATE'S COULEE AT SAND COULEE, MT

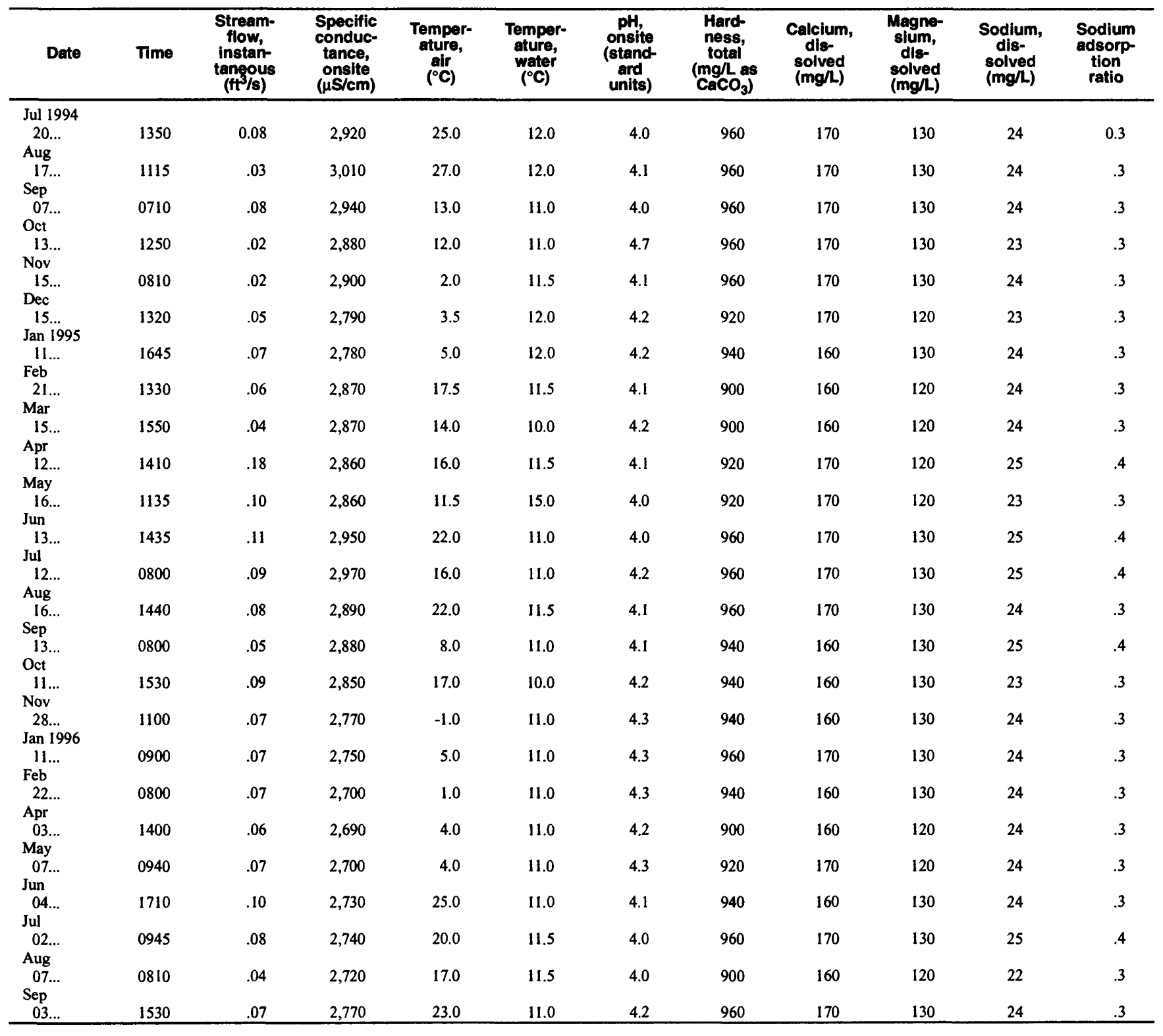


Table 3. Water-quality data for sites in the Sand Coulee Coal Area, Montana, July 1994 through September 1996 (Continued) SITE 19, 472334111104401 -MOUNT OREGON MINE DRAIN TO KATE'S COULEE AT SAND COULEE, MT--Continued

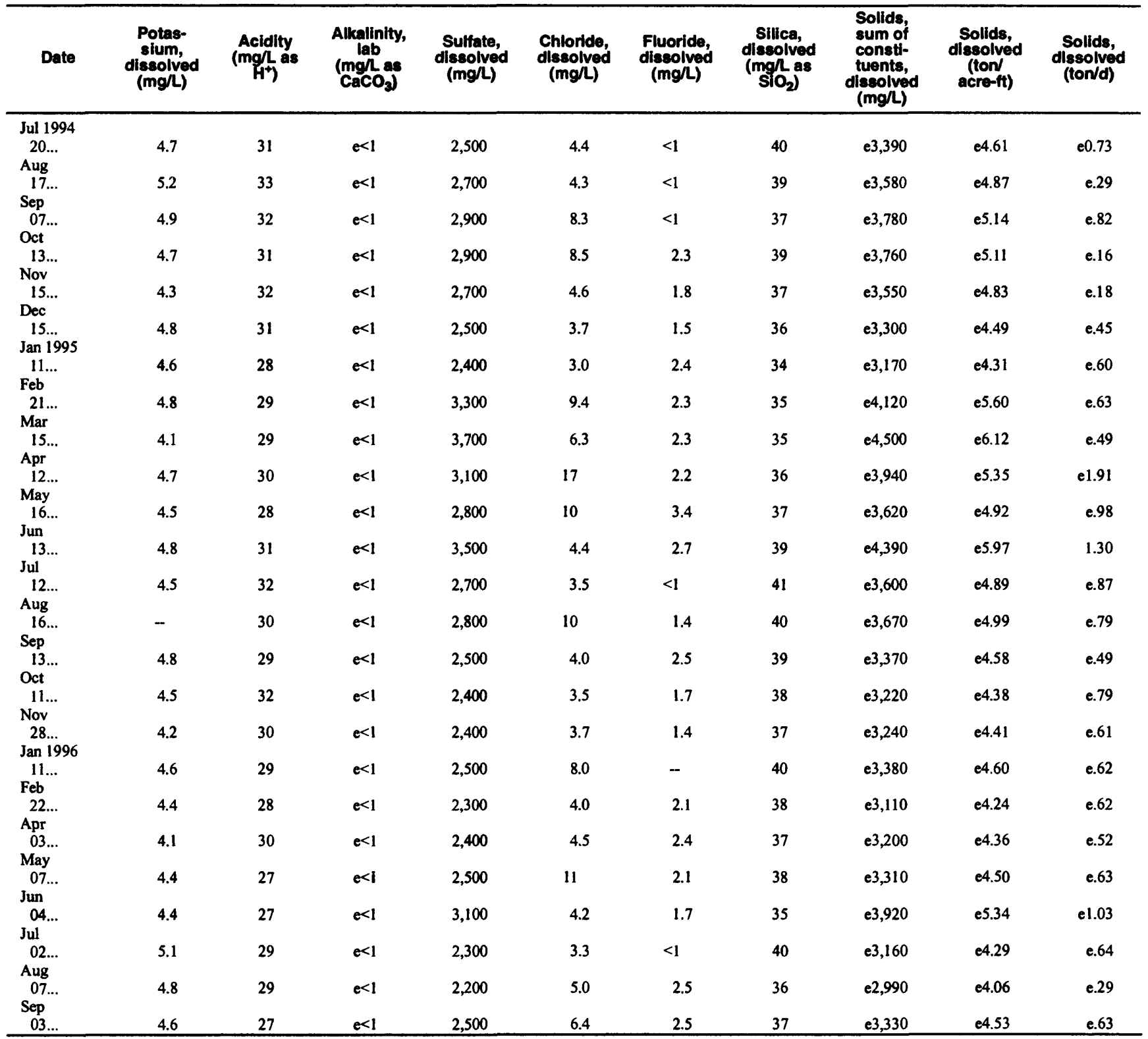


Table 3. Water-quality data for sites in the Sand Coulee Coal Area, Montana, July 1994 through September 1996 (Continued)

SITE 19, 472334111104401--MOUNT OREGON MINE DRAIN TO KATE'S COULEE AT SAND COULEE, MT--Continued

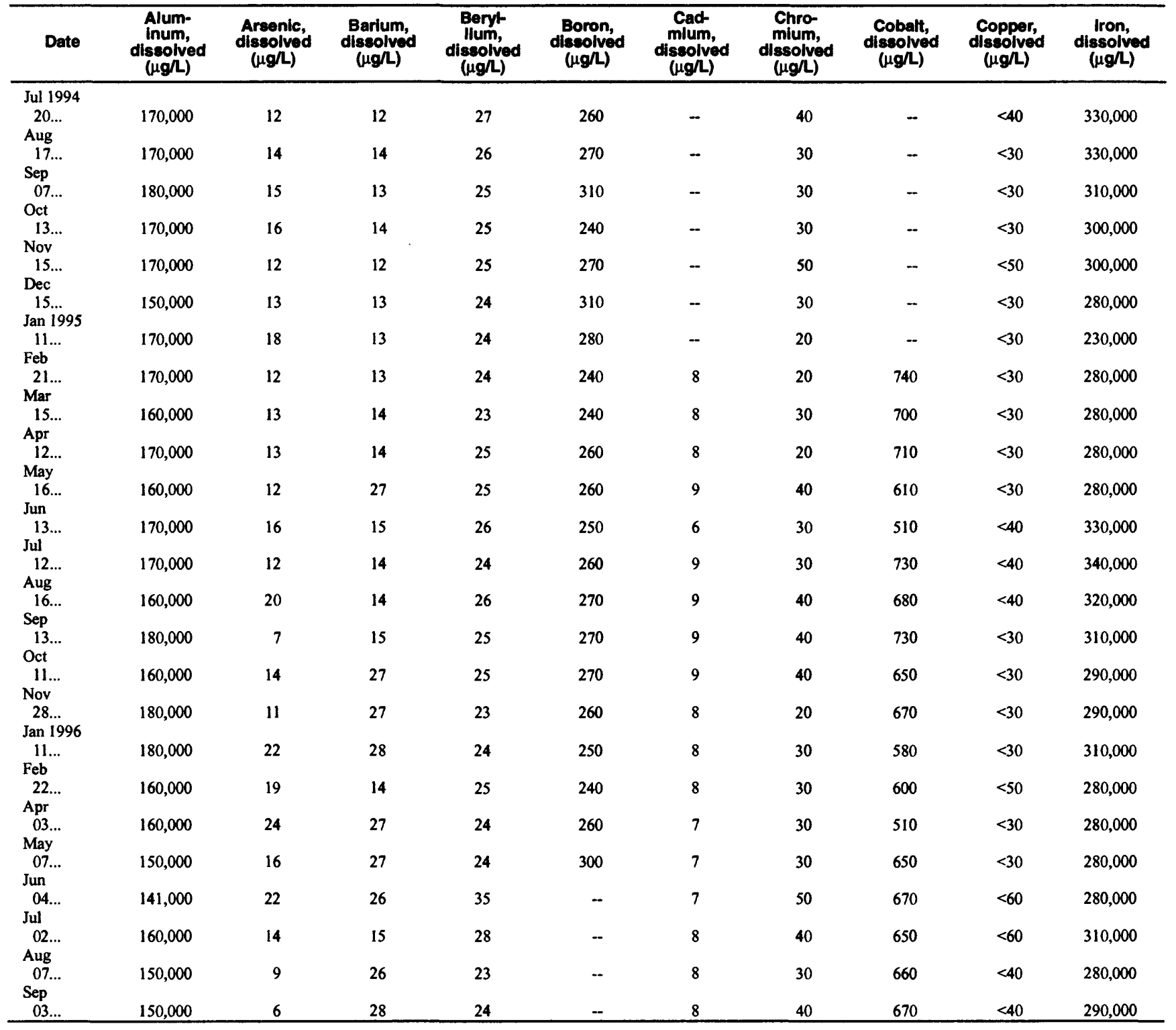


Table 3. Water-quality data for sites in the Sand Coulee Coal Area, Montana, July 1994 through September 1996 (Continued) SITE 19, 472334111104401--MOUNT OREGON MINE DRAIN TO KATE'S COULEE AT SAND COULEE, MT--Continued

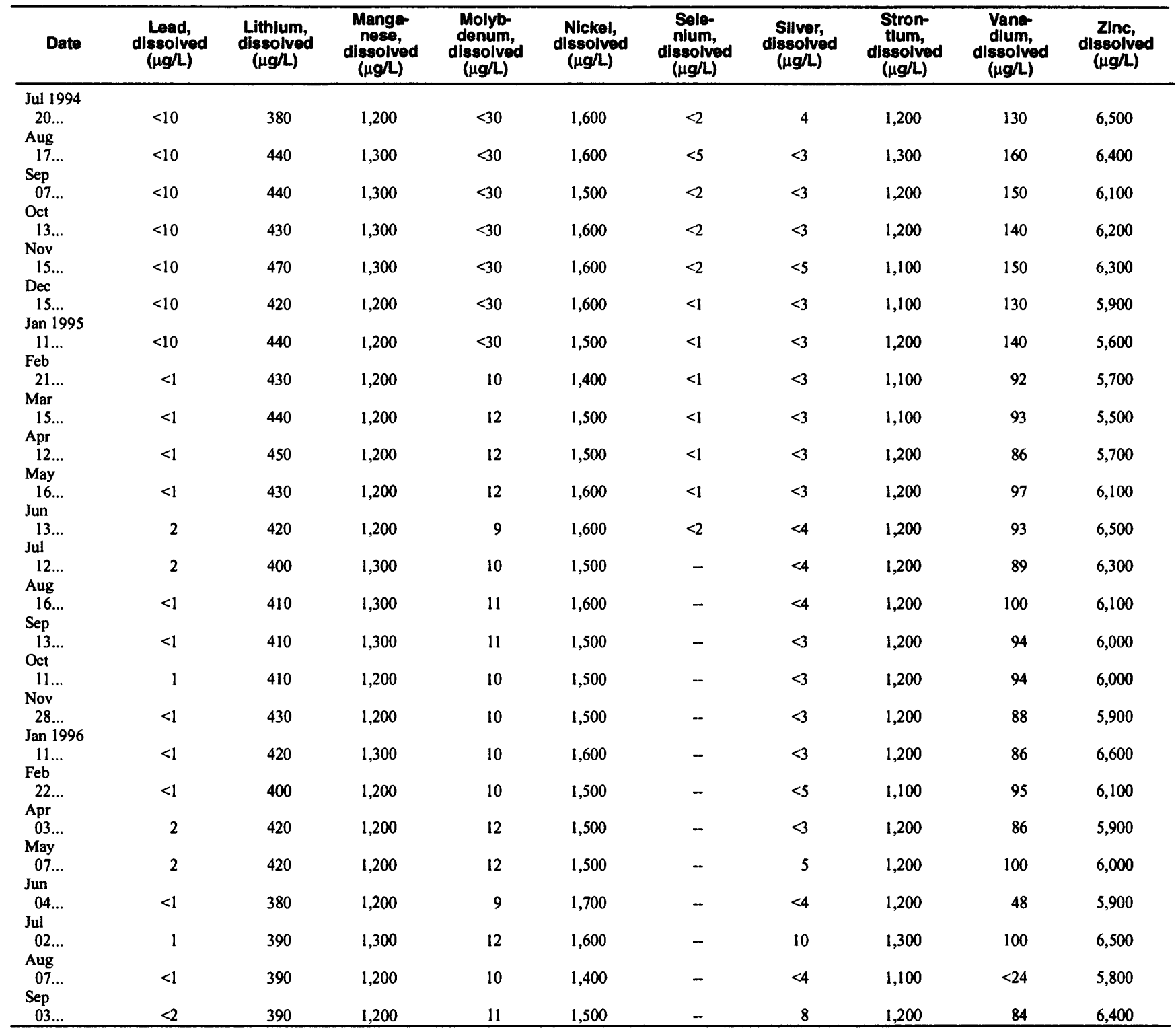


Table 3. Water-quality data for sites in the Sand Coulee Coal Area, Montana, July 1994 through September 1996 (Continued) SITE 20, 472346111102401 --NELSON MINE DRAIN TO SAND COULEE AT SAND COULEE, MT

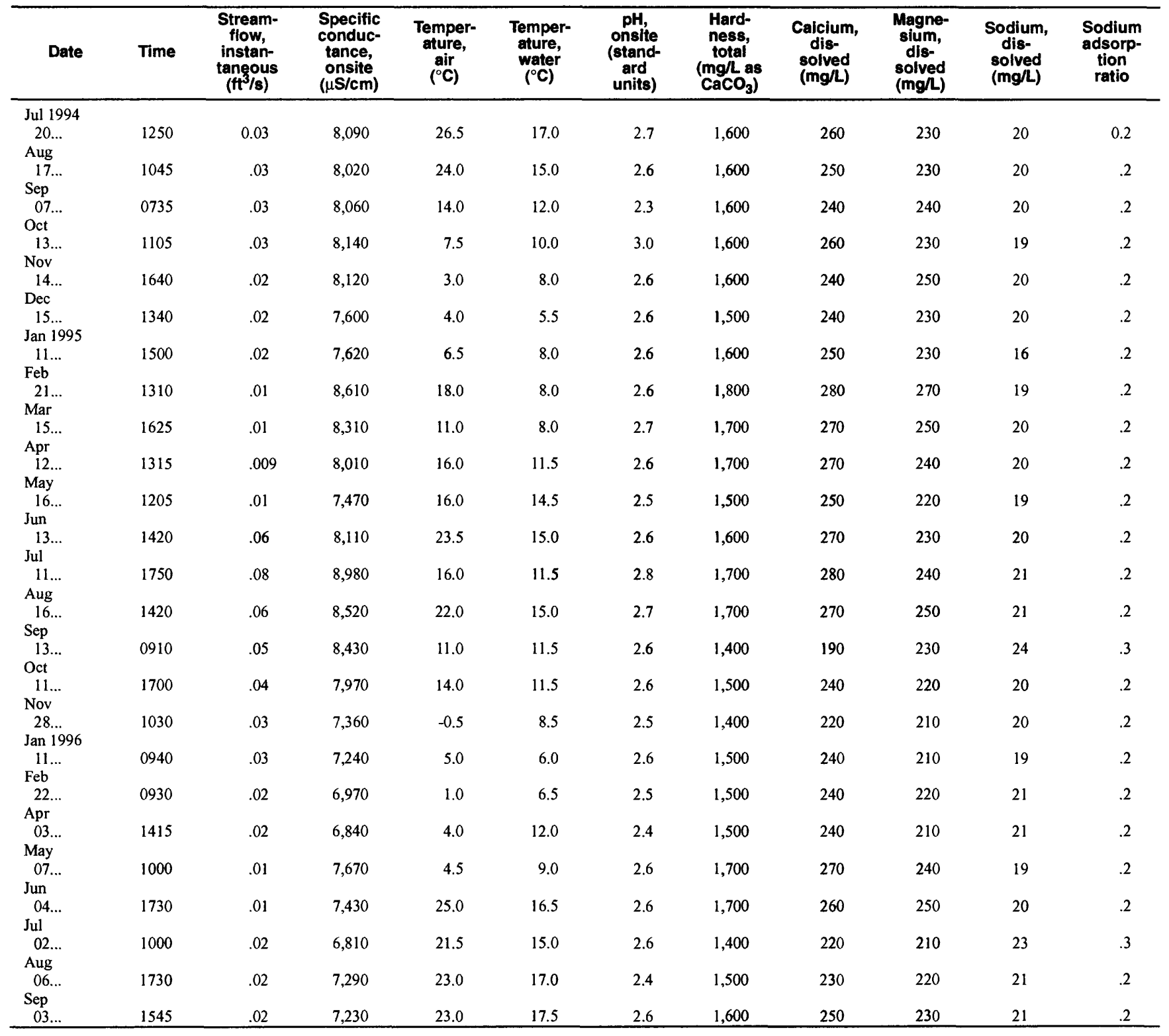


Table 3. Water-quality data for sites in the Sand Coulee Coal Area, Montana, July 1994 through September 1996 (Continued)

SITE 20, 472346111102401--NELSON MINE DRAIN TO SAND COULEE AT SAND COULEE, MT--Continued

\begin{tabular}{|c|c|c|c|c|c|c|c|c|c|}
\hline Date & $\begin{array}{l}\text { Potas- } \\
\text { sium, } \\
\text { dissolved } \\
\text { (mg/L) }\end{array}$ & $\begin{array}{c}\text { Acidity } \\
\left(\mathrm{mg}^{\prime} / \text { as }\right. \\
\left.\mathrm{H}^{+}\right)\end{array}$ & $\begin{array}{c}\text { Alkalinity, } \\
\text { lab } \\
\left(\mathrm{mgl}^{\prime} \mathrm{Ls}\right. \\
\left.\mathrm{CaCO}_{3}\right)\end{array}$ & $\begin{array}{c}\text { Sulfate, } \\
\text { dissolved } \\
(\mathrm{mg} / \mathrm{L})\end{array}$ & $\begin{array}{c}\text { Fluoride, } \\
\text { dissolved } \\
\text { (mg/L) }\end{array}$ & $\begin{array}{c}\text { Silica, } \\
\text { dissolved } \\
\text { (mg/L as } \\
\left.\mathrm{SiO}_{2}\right)\end{array}$ & $\begin{array}{l}\text { Solids, } \\
\text { sum of } \\
\text { consti- } \\
\text { tuents, } \\
\text { dissolved } \\
\text { (mg/h) }\end{array}$ & $\begin{array}{l}\text { Solids, } \\
\text { dissolved } \\
\text { (ton } \\
\text { acre-ft) }\end{array}$ & $\begin{array}{l}\text { Solids, } \\
\text { dissolved } \\
\text { (ton/d) }\end{array}$ \\
\hline $\begin{array}{c}\text { Jul } 1994 \\
20 \ldots\end{array}$ & 1.4 & 170 & $e<1$ & 10,000 & $<1$ & 87 & el3,200 & el7.9 & el. 14 \\
\hline $\begin{array}{l}\text { Aug } \\
17 \ldots \\
\text { Sep }\end{array}$ & 1.5 & 170 & $e<1$ & - & $<1$ & 84 & - & -- & -- \\
\hline $\begin{array}{l}\text { Sep } \\
07 . . . \\
\text { Oct }\end{array}$ & 2.0 & 170 & $e<1$ & 11,000 & $<1$ & 88 & el4,200 & el9.4 & el. 0 \\
\hline $\begin{array}{l}13 \ldots \\
\text { Nov }\end{array}$ & .6 & 170 & $e<1$ & 11,000 & 1.4 & 140 & el4,100 & e19.2 & el.07 \\
\hline $\begin{array}{l}14 \ldots \\
\text { Dec }\end{array}$ & .7 & 170 & $e<1$ & 10,000 & -- & 100 & el3,000 & el7.7 & e 8.1 \\
\hline $\begin{array}{c}15 \ldots \\
\operatorname{Jan} 1995\end{array}$ & .9 & 170 & $e<1$ & 10,000 & - & 75 & el3,000 & el17.7 & e.74 \\
\hline $\begin{array}{l}11 \ldots \\
\text { Feb }\end{array}$ & .9 & 170 & $e<1$ & 11,000 & 7.3 & 59 & el4,000 & el9.0 & e. 68 \\
\hline $\begin{array}{l}21 \ldots \\
\text { Mar }\end{array}$ & 1.1 & 190 & $\mathbf{e}<1$ & 13,000 & 4.5 & 140 & el6,500 & e22.4 & e.53 \\
\hline $\begin{array}{l}15 . . \\
\text { Apr }\end{array}$ & .7 & 180 & $e<1$ & 13,000 & 5.4 & 140 & el6,300 & e22. 2 & e. 43 \\
\hline $\begin{array}{c}12 \ldots \\
\text { May }\end{array}$ & .8 & 170 & $\mathbf{e}<1$ & 14,000 & 4.5 & 140 & el7,200 & e23.3 & e. 42 \\
\hline $\begin{array}{c}16 \ldots \\
\text { Jun }\end{array}$ & .9 & 160 & $\mathbf{e}<1$ & 12,000 & 5.7 & 140 & e 15,000 & e20.4 & e. 44 \\
\hline $\begin{array}{l}13 \ldots \\
\text { Jul }\end{array}$ & .6 & 180 & $e<1$ & 11,000 & $<1$ & 140 & el 4,300 & e 19.5 & $\mathrm{e} 2.12$ \\
\hline $\begin{array}{l}11 \ldots \\
\text { Aug }\end{array}$ & .6 & 200 & $e<1$ & 13,000 & $<1$ & 140 & el 6,700 & e22.7 & 3.60 \\
\hline $\begin{array}{l}16 . . \\
\text { Sep }\end{array}$ & .8 & 190 & $\mathbf{e}<1$ & 13,000 & -- & 140 & el 6,500 & e22.5 & 2.86 \\
\hline $\begin{array}{l}13 \ldots \\
\text { Oct }\end{array}$ & .4 & 180 & $e<1$ & 11,000 & -- & - & el 4,400 & el9.6 & el. 17 \\
\hline $\begin{array}{l}11 \ldots \\
\text { Nov }\end{array}$ & .6 & 170 & $\mathrm{e}<1$ & 10,000 & -- & 130 & el3,000 & el7.7 & el. 33 \\
\hline $\begin{array}{c}28 \ldots \\
\text { Jan } 1996\end{array}$ & .5 & 160 & $e<1$ & 9,600 & $<1$ & 130 & el2,400 & el 6.8 & el. .00 \\
\hline $\begin{array}{l}11 \ldots \\
\text { Feb }\end{array}$ & .3 & 160 & $\mathbf{e}<1$ & 9,100 & - & 140 & el1,900 & el 6.2 & e.87 \\
\hline $\begin{array}{c}22 \ldots \\
\mathrm{Apr}\end{array}$ & .4 & 150 & $\mathbf{e}<1$ & 11,000 & - & 140 & el3,700 & el 8.6 & e.78 \\
\hline $\begin{array}{c}03 \ldots \\
\text { May }\end{array}$ & .5 & 140 & $\mathbf{e}<1$ & 9,500 & 4.4 & 140 & el 2,200 & el 6.7 & e. 56 \\
\hline $\begin{array}{c}07 . . . \\
\text { Jun }\end{array}$ & .7 & 170 & $\mathbf{e}<1$ & 11,000 & 1.6 & 150 & el 4,000 & el9.1 & e. 53 \\
\hline $\begin{array}{l}04 \ldots \\
\text { Jul }\end{array}$ & .5 & 160 & $e<1$ & 11,000 & 2.4 & 140 & el 4,100 & el9.2 & e.42 \\
\hline $\begin{array}{r}02 \ldots \\
\text { Aug, }\end{array}$ & .5 & 150 & $\mathbf{e}<1$ & 9,000 & $<1$ & 140 & el1, 600 & e15.8 & e. 47 \\
\hline $\begin{array}{l}06 \ldots \\
\text { Sep, }\end{array}$ & .7 & 160 & $\mathbf{e}<1$ & 8,900 & 3.4 & 140 & e 11,700 & el5.9 & e. 57 \\
\hline $03 \ldots$ & .8 & 150 & $e<1$ & 8,900 & 3.5 & 150 & e 11,800 & el6.1 & e. 48 \\
\hline
\end{tabular}


Table 3. Water-quality data for sites in the Sand Coulee Coal Area, Montana, July 1994 through September 1996 (Continued)

SITE 20, 47234611102401--NELSON MINE DRAIN TO SAND COULEE AT SAND COULEE, MT--Continued

\begin{tabular}{|c|c|c|c|c|c|c|c|c|c|c|}
\hline Date & $\begin{array}{c}\text { Alum- } \\
\text { inum, } \\
\text { dissolved } \\
(\mu g / L)\end{array}$ & $\begin{array}{c}\text { Arsenic, } \\
\text { dissolved } \\
(\mu g / L)\end{array}$ & $\begin{array}{l}\text { Barlum, } \\
\text { dissolved } \\
(\mu g / L)\end{array}$ & $\begin{array}{l}\text { Beryl- } \\
\text { Ilum, } \\
\text { dissolved } \\
(\mu g / L)\end{array}$ & $\begin{array}{l}\text { Boron, } \\
\text { dissolved } \\
\left(\mu g^{\prime} L\right)\end{array}$ & $\begin{array}{c}\text { Cad- } \\
\text { mlum, } \\
\text { dissolved } \\
(\mu g / L)\end{array}$ & $\begin{array}{c}\text { Chro- } \\
\text { mium, } \\
\text { dissolved } \\
(\mu g / L)\end{array}$ & $\begin{array}{l}\text { Cobalt, } \\
\text { dissolved } \\
\left(\mu g^{\prime} L\right)\end{array}$ & $\begin{array}{l}\text { Copper, } \\
\text { dissolved } \\
(\mu g / L)\end{array}$ & $\begin{array}{l}\text { Iron, } \\
\text { dissolved } \\
(\mu g / L)\end{array}$ \\
\hline $\begin{array}{l}\text { Jul } 1994 \\
20 \ldots \\
\text { Aug }\end{array}$ & 910,000 & 75 & $<100$ & 60 & 750 & 100 & 310 & 2,200 & 310 & $1,600,000$ \\
\hline $\begin{array}{l}17 \ldots \\
\text { Sep }\end{array}$ & 910,000 & 19 & $<100$ & 70 & 800 & 110 & 290 & 2,000 & 300 & $1,600,000$ \\
\hline $\begin{array}{l}07 \ldots \\
\text { Oct }\end{array}$ & 910,000 & 67 & $<100$ & 60 & 850 & 110 & 350 & 2,100 & 310 & $1,700,000$ \\
\hline $\begin{array}{l}13 \ldots \\
\text { Nov }\end{array}$ & 930,000 & 38 & $<20$ & 110 & 730 & -- & 320 & 5,600 & 380 & $1,490,000$ \\
\hline $\begin{array}{l}14 \ldots \\
\text { Dec }\end{array}$ & 890,000 & 16 & $<100$ & 80 & 830 & 110 & 400 & 1,700 & 290 & $1,500,000$ \\
\hline $\begin{array}{c}15 \ldots \\
\text { Jan } 1995\end{array}$ & 860,000 & - & $<100$ & 70 & 900 & 100 & 370 & 1,900 & 290 & $1,500,000$ \\
\hline $\begin{array}{l}11 \ldots \\
\text { Feb }\end{array}$ & 920,000 & 63 & $<100$ & 80 & 830 & 110 & 320 & 2,200 & 300 & $1,400,000$ \\
\hline $\begin{array}{l}21 \ldots \\
\text { Mar }\end{array}$ & $1,000,000$ & 76 & $<10$ & 140 & 790 & 120 & 320 & 2,200 & 430 & $1,600,000$ \\
\hline $\begin{array}{l}15 \ldots \\
\text { Apr }\end{array}$ & 970,000 & 75 & $<10$ & 120 & 770 & 120 & 310 & 2,200 & 370 & $1,600,000$ \\
\hline $\begin{array}{l}12 \ldots \\
\text { May }\end{array}$ & 940,000 & 74 & $<10$ & 120 & 770 & 110 & 320 & 1,900 & 330 & $1,500,000$ \\
\hline $\begin{array}{l}16 \ldots \\
\text { Jun }\end{array}$ & 870,000 & 51 & 40 & 110 & 710 & 96 & 330 & 1,700 & 260 & $1,400,000$ \\
\hline$\underset{\text { Jul }}{13 \ldots}$ & $1,000,000$ & 45 & $<20$ & 110 & 810 & 110 & 330 & 2,700 & 310 & $1,600,000$ \\
\hline $\begin{array}{l}11 \ldots \\
\text { Aug }\end{array}$ & $1,000,000$ & 27 & $<20$ & 120 & 840 & 100 & 280 & 2,000 & 230 & $1,900,000$ \\
\hline $\begin{array}{l}16 \ldots \\
\text { Sep }\end{array}$ & 870,000 & 65 & $<25$ & 130 & 1,000 & 110 & 400 & 2,200 & 410 & $1,900,000$ \\
\hline $\begin{array}{l}13 \ldots \\
\text { Oct }\end{array}$ & 960,000 & 65 & $<100$ & 40 & 860 & 110 & 360 & 2,400 & 430 & $2,000,000$ \\
\hline $\begin{array}{l}11 . . \\
\text { Nov }\end{array}$ & 850,000 & 73 & 35 & 99 & 780 & 110 & 280 & 2,000 & 450 & $1,500,000$ \\
\hline $\begin{array}{l}28 \ldots \\
\text { Jan } 1996\end{array}$ & 830,000 & 60 & 37 & 83 & 620 & 100 & 300 & 2,100 & 390 & $1,300,000$ \\
\hline $\begin{array}{l}11 \ldots \\
\text { Feb }\end{array}$ & 880,000 & 50 & 38 & 91 & 660 & 97 & 330 & 3,900 & 390 & $1,300,000$ \\
\hline $\begin{array}{l}22 \ldots \\
\text { Apr }\end{array}$ & 740,000 & 48 & $<20$ & 98 & 630 & 84 & 390 & 5,200 & 400 & $1,300,000$ \\
\hline $\begin{array}{l}03 . . \\
\text { May }\end{array}$ & 790,000 & 29 & 37 & 95 & 660 & 87 & 290 & 4,900 & 380 & $1,300,000$ \\
\hline $\begin{array}{l}07 . . . \\
\text { Jun }\end{array}$ & 910,000 & 46 & 41 & 100 & 820 & 110 & 300 & 2,400 & 330 & $1,400,000$ \\
\hline $\begin{array}{c}04 \ldots \\
\text { Jul }\end{array}$ & 880,000 & 51 & 41 & 100 & - & 120 & 300 & 2,200 & 480 & $1,400,000$ \\
\hline $\begin{array}{l}02 \ldots \\
\text { Aug }\end{array}$ & 765,000 & 51 & 38 & 95 & - & 110 & 270 & 2,000 & 650 & $1,200,000$ \\
\hline $\begin{array}{l}06 . . \\
\text { Sep }\end{array}$ & 800,000 & 65 & 38 & 110 & - & 110 & 280 & 2,000 & 340 & $1,300,000$ \\
\hline $03 \ldots$ & 820,000 & 51 & 44 & 100 & $=$ & 110 & 380 & 1,900 & 460 & $1,400,000$ \\
\hline
\end{tabular}


Table 3. Water-quality data for sites in the Sand Coulee Coal Area, Montana, July 1994 through September 1996 (Continued) SITE 20, 472346111102401--NELSON MINE DRAIN TO SAND COULEE AT SAND COULEE, MT--Continued

\begin{tabular}{|c|c|c|c|c|c|c|c|c|c|c|}
\hline Date & $\begin{array}{l}\text { Lead, } \\
\text { dissolved } \\
(\mu \mathrm{g} / \mathrm{L})\end{array}$ & $\begin{array}{l}\text { Lithlum, } \\
\text { dissolved } \\
\left(\mu \mathrm{g}^{\prime} \mathrm{L}\right)\end{array}$ & $\begin{array}{c}\text { Manga- } \\
\text { nese, } \\
\text { dissolved } \\
(\mu g / L)\end{array}$ & $\begin{array}{c}\text { Molyb- } \\
\text { denum, } \\
\text { dissolved } \\
(\mu g / L)\end{array}$ & $\begin{array}{c}\text { Nickel, } \\
\text { dissolved } \\
(\mu \mathrm{g} / \mathrm{L})\end{array}$ & $\begin{array}{l}\text { Selenium, } \\
\text { dissolved } \\
(\mu g / L)\end{array}$ & $\begin{array}{l}\text { Silver, } \\
\text { dissolved } \\
(\mu \mathrm{g} / \mathrm{L})\end{array}$ & $\begin{array}{l}\text { Stron- } \\
\text { tlum, } \\
\text { dissolved } \\
(\mu \mathrm{g} / \mathrm{L})\end{array}$ & $\begin{array}{c}\text { Vana- } \\
\text { dium, } \\
\text { dissolved } \\
(\mu g / L)\end{array}$ & $\begin{array}{c}\text { Zinc, } \\
\text { dissolved } \\
(\mu g / L)\end{array}$ \\
\hline Jul 1994 & & & & & & & & & & \\
\hline $\begin{array}{l}20 \ldots \\
\text { Aug }\end{array}$ & 1.0 & 900 & 7,800 & $<5$ & 4,900 & $<25$ & $<1$ & 1,500 & -- & 16,000 \\
\hline $\begin{array}{l}17 \ldots \\
\text { Sep }\end{array}$ & $<1$ & 910 & 7,700 & $<1$ & 5,000 & $<10$ & $<1$ & 1,600 & -- & 18,000 \\
\hline $07 \ldots$ & $<1$ & 970 & 7,100 & 1 & 4,700 & $<10$ & $<1$ & 1,600 & -- & 19,000 \\
\hline $\begin{array}{l}13 \ldots \\
\text { Nov }\end{array}$ & $<10$ & 930 & 8,700 & $<10$ & 4,400 & $<5$ & $<20$ & 1,500 & 660 & 18,000 \\
\hline $\begin{array}{l}14 \ldots \\
\text { Dec }\end{array}$ & $<1$ & 930 & 8,000 & $<1$ & 4,600 & $<5$ & $<1$ & 1,600 & -- & 19,000 \\
\hline $\begin{array}{l}15 \ldots \\
\operatorname{Jan} 1995\end{array}$ & $<1$ & 1,000 & 7,100 & 4 & 4,300 & $<2$ & $<1$ & 1,500 & -- & 18,000 \\
\hline $\begin{array}{l}11 \ldots \\
\text { Feb }\end{array}$ & $<1$ & 940 & 8,500 & 3 & 5,100 & $<5$ & $<1$ & 1,600 & - & 19,000 \\
\hline $\begin{array}{l}21 \ldots \\
\text { Mar }\end{array}$ & $<1$ & 1,400 & 11,000 & 5 & 5,500 & $<2$ & $<10$ & 1,600 & 420 & 19,000 \\
\hline $15 \ldots$ & $<1$ & 1,100 & 9,900 & 6 & 5,200 & $<2$ & $<10$ & 1,600 & 360 & 19,000 \\
\hline $\begin{array}{l}12 \ldots \\
\text { May }\end{array}$ & $<1$ & 1,100 & 9,400 & $<2$ & 4,800 & $<2$ & $<10$ & 1,600 & 330 & 18,000 \\
\hline $\begin{array}{l}16 \ldots \\
\text { Jun }\end{array}$ & $<1$ & 1,000 & 8,200 & 5 & 4,700 & $<2$ & $<20$ & 1,600 & 410 & 17,000 \\
\hline Jul & $<1$ & 1,000 & 8,800 & $<4$ & 5,000 & $<5$ & $<20$ & 1,600 & 430 & 19,000 \\
\hline $\begin{array}{l}11 \ldots \\
\text { Aug }\end{array}$ & $<1$ & 1,100 & 12,000 & 3 & 5,000 & - & $<20$ & 1,700 & 520 & 19,000 \\
\hline $\begin{array}{l}16 \ldots \\
\text { Sep }\end{array}$ & $<1$ & 1,000 & 11,000 & 2 & 5,100 & - & $<25$ & 1,800 & 650 & 18,000 \\
\hline $\begin{array}{l}13 \ldots \\
\text { Oct }\end{array}$ & $<1$ & 1,400 & 8,200 & 4 & 4,900 & - & $<20$ & 1,800 & -- & 18,000 \\
\hline $\begin{array}{l}11 \ldots \\
\text { Nov }\end{array}$ & $<1$ & 950 & 8,200 & 4 & 4,400 & - & $<10$ & 1,600 & 420 & 16,000 \\
\hline $\begin{array}{c}28 \ldots \\
\operatorname{Jan} 1996\end{array}$ & $<1$ & 920 & 7,900 & 4 & 4,300 & - & $<10$ & 1,500 & 340 & 15,000 \\
\hline $\begin{array}{l}11 \ldots \\
\text { Feb }\end{array}$ & $<1$ & 900 & 8,000 & 1 & 4,500 & - & $<20$ & 1,500 & 320 & 16,000 \\
\hline $\begin{array}{l}22 \ldots \\
\mathrm{Apr}\end{array}$ & $<1$ & 880 & 8,000 & 4 & 4,700 & - & $<20$ & 1,400 & 380 & 17,000 \\
\hline $\begin{array}{c}03 \ldots \\
\text { May }\end{array}$ & $<1$ & 910 & 8,300 & 3 & 4,400 & - & $<12$ & 1,500 & 290 & 16,000 \\
\hline $\begin{array}{c}07 . . . \\
\text { Jun }\end{array}$ & $<1$ & 1,100 & 10,000 & 3 & 5,100 & - & $<25$ & 1,400 & 350 & 18,000 \\
\hline $\begin{array}{l}04 \ldots \\
\text { Jul }\end{array}$ & $<1$ & 990 & 9,600 & 4 & 5,000 & - & $<25$ & 1,500 & $<150$ & 18,000 \\
\hline $\begin{array}{l}02 \ldots \\
\text { Aug }\end{array}$ & $<1$ & 890 & 7,600 & $<1$ & 4,400 & - & $<25$ & 1,500 & $<150$ & 17,000 \\
\hline $\begin{array}{l}06 . . \\
\text { Sep }\end{array}$ & $<1$ & 970 & 7,500 & 4 & 4,300 & - & $<20$ & 1,500 & $<120$ & 16,000 \\
\hline $03 \ldots$ & $<4$ & 990 & 7,800 & 5 & 4,800 & - & - & 1,500 & 360 & 18,000 \\
\hline
\end{tabular}


Table 3. Water-quality data for sites in the Sand Coulee Coal Area, Montana, July 1994 through September 1996 (Continued)

SITE 21, 472309110551201--LEWIS COULEE BELOW MINE ADIT, AT BELT, MT

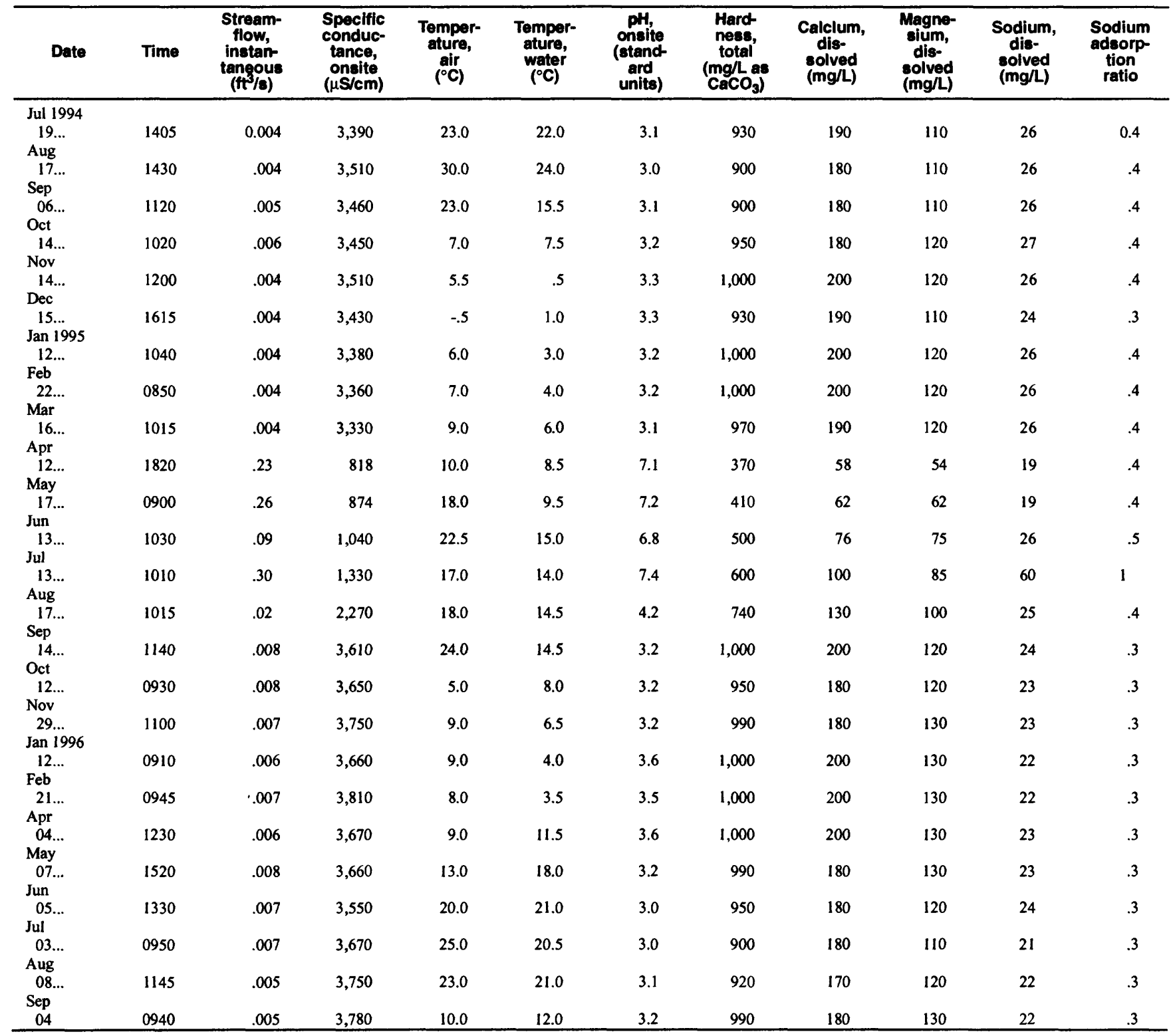


Table 3. Water-quality data for sites in the Sand Coulee Coal Area, Montana, July 1994 through September 1996 (Continued) SITE 21, 472309110551201--LEWIS COULEE BELOW MINE ADIT, AT BELT, MT--Continued

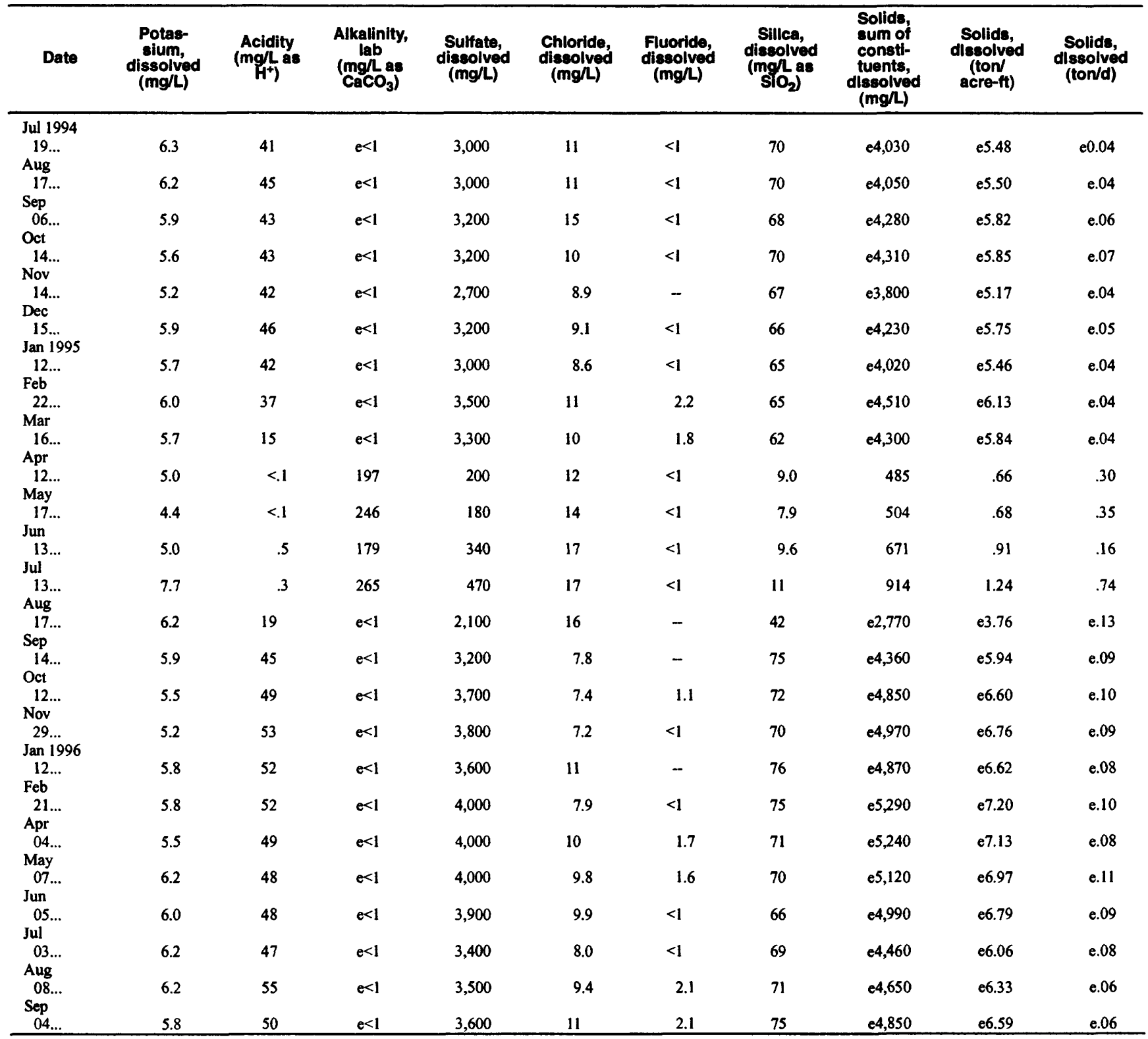


Table 3. Water-quality data for sites in the Sand Coulee Coal Area, Montana, July 1994 through September 1996 (Continued)

SITE 21, 472309110551201--LEWIS COULEE BELOW MINE ADIT, AT BELT, MT--Continued

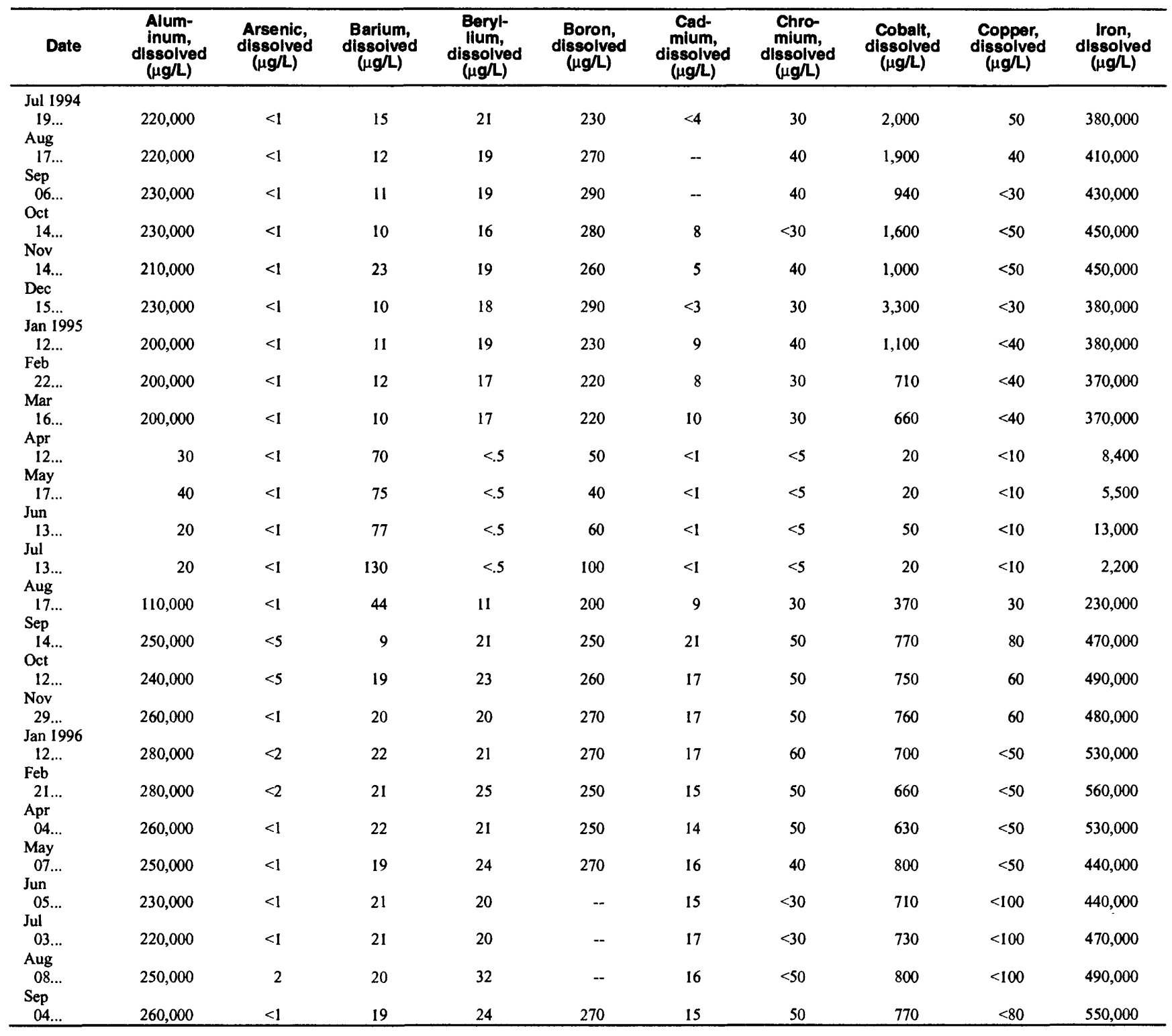


Table 3. Water-quality data for sites in the Sand Coulee Coal Area, Montana, July 1994 through September 1996 (Continued)

SITE 21, 472309110551201--LEWIS COULEE BELOW MINE ADIT AT BELT, MT--Continued

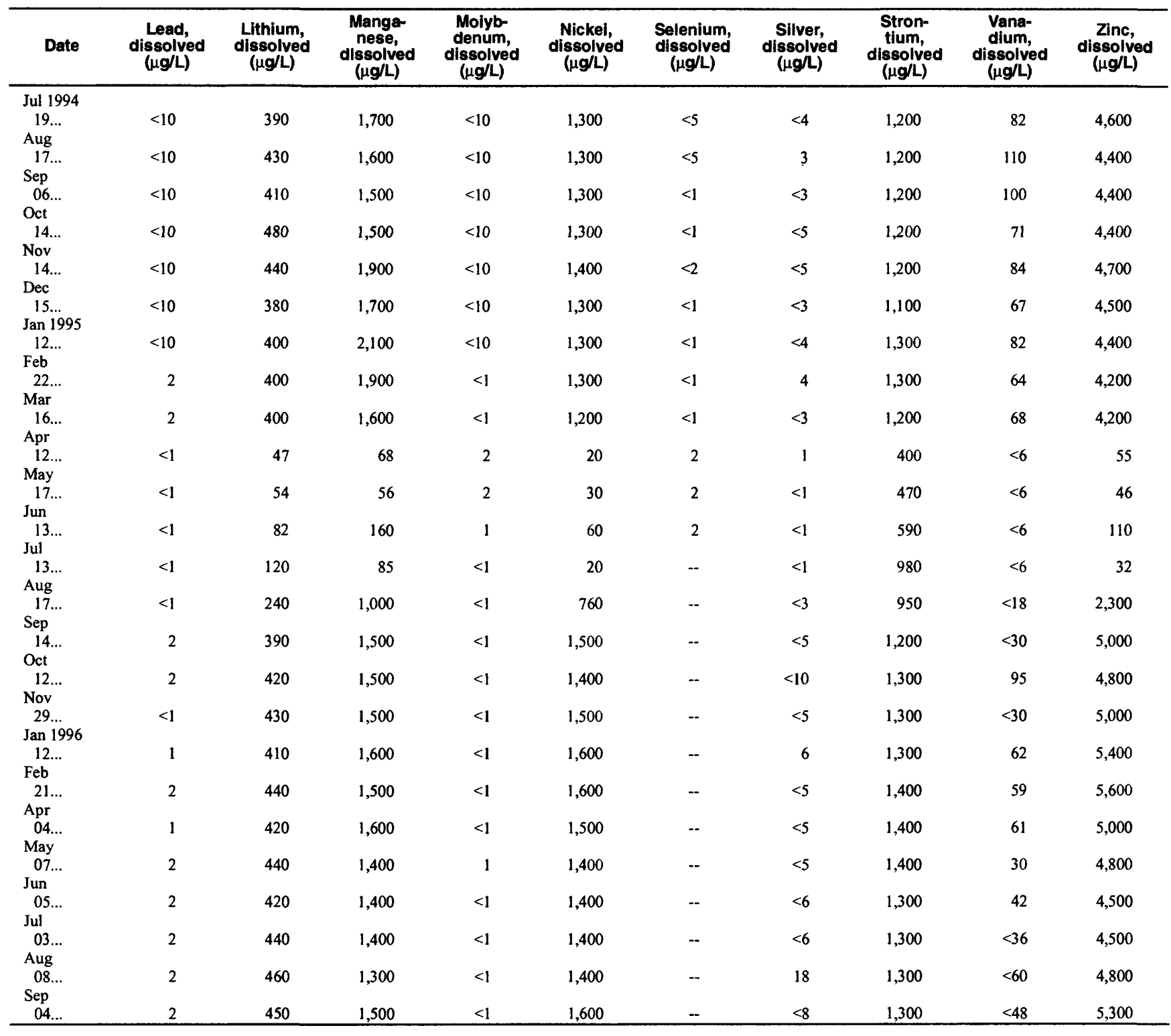


Table 3. Water-quality data for sites in the Sand Coulee Coal Area, Montana, July 1994 through September 1996 (Continued) SITE 22, 472446111085101--PIPE SPRING AT TRACY, MT

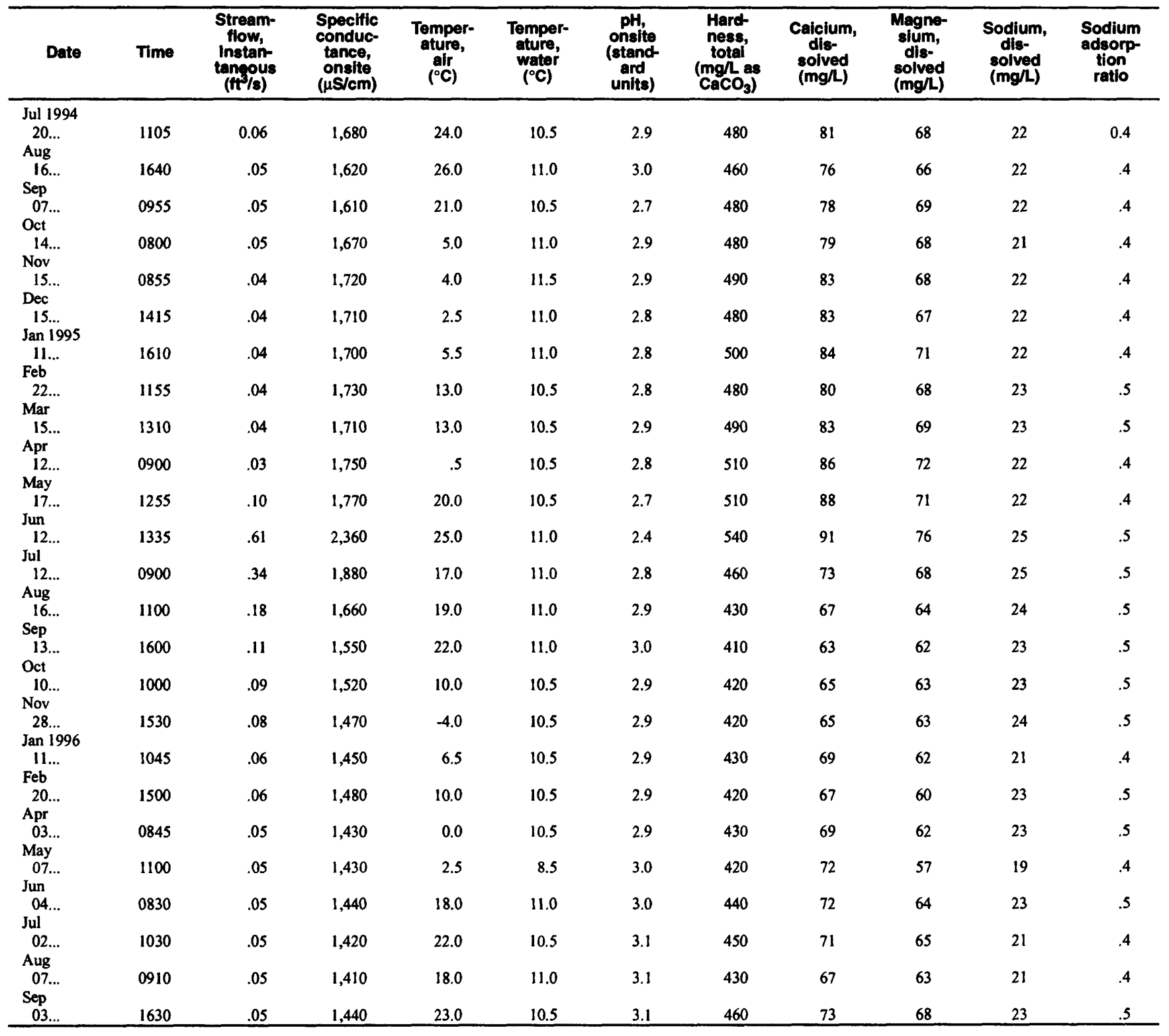


Table 3. Water-quality data for sites in the Sand Coulee Coal Area, Montana, July 1994 through September 1996 (Continued) SITE 22, 472446111085101--PIPE SPRING AT TRACY, MT--Continued

\begin{tabular}{|c|c|c|c|c|c|c|c|c|c|c|}
\hline Date & $\begin{array}{l}\text { Potas- } \\
\text { sium, } \\
\text { dissolved } \\
\text { (mg/L) }\end{array}$ & $\begin{array}{c}\text { Acidity } \\
\left(\mathbf{m g / h}_{\left.H^{+}\right)}^{\text {as }}\right.\end{array}$ & $\begin{array}{c}\text { Alkalinity, } \\
\text { lab } \\
\left(\mathrm{mg}^{2} \mathrm{as}\right. \\
\left.\mathrm{CaCO}_{3}\right)\end{array}$ & $\begin{array}{l}\text { Sulfate, } \\
\text { dissoived } \\
\text { (mg/L) }\end{array}$ & $\begin{array}{l}\text { Chloride, } \\
\text { dissolved } \\
\text { (mg/ll) }\end{array}$ & $\begin{array}{l}\text { Fluoride, } \\
\text { dissoived } \\
\text { (mg/L) }\end{array}$ & $\begin{array}{l}\text { Sillica, } \\
\text { dissoived } \\
\text { (mg/h as } \\
\text { sio })_{2}\end{array}$ & $\begin{array}{l}\text { Solids, } \\
\text { sum of } \\
\text { consti- } \\
\text { tuents, } \\
\text { dissoived } \\
\text { (mg/l) }\end{array}$ & $\begin{array}{c}\text { Solids, } \\
\text { dissolved } \\
\text { (ton/ } \\
\text { acre-ft) }\end{array}$ & $\begin{array}{l}\text { Solids, } \\
\text { dissoived } \\
\text { (ton/d) }\end{array}$ \\
\hline $\begin{array}{l}\text { Jul } 1994 \\
20 \ldots \ldots \\
\text { Aug }\end{array}$ & 2.6 & 6.4 & $e<1$ & 790 & 5.6 & 0.9 & 49 & el,, 070 & el. 45 & e0.17 \\
\hline $\begin{array}{l}16 \ldots \\
\text { Sep }\end{array}$ & 2.7 & 6.3 & $e<1$ & 810 & 5.6 & .8 & 47 & el,080 & el. .46 & e.16 \\
\hline $\begin{array}{l}07 \ldots \\
\text { Oct }\end{array}$ & 2.5 & 6.2 & $e<1$ & 890 & 5.4 & .6 & 47 & el,160 & el. 58 & e.16 \\
\hline $\begin{array}{l}14 \ldots \\
\text { Nov }\end{array}$ & 2.5 & 6.1 & $e<1$ & 910 & 5.6 & 2.0 & 50 & el,180 & el.61 & e. 15 \\
\hline $\begin{array}{c}15 \ldots \\
\text { Dec }\end{array}$ & 2.5 & - & $e<1$ & 830 & 5.6 & 2.0 & 49 & el,110 & el.51 & e. 13 \\
\hline $\begin{array}{c}15 \ldots \\
\text { Jan } 1995\end{array}$ & 2.5 & 6.5 & $e<1$ & 830 & 5.6 & .6 & 51 & el,110 & e1.51 & e. 12 \\
\hline $\begin{array}{l}11 \ldots \\
\text { Feb }\end{array}$ & 2.4 & 6.4 & $e<1$ & 860 & 5.7 & 2.1 & 51 & el,150 & el.56 & e. 12 \\
\hline $\begin{array}{c}22 \ldots \\
\text { Mar }\end{array}$ & 2.6 & 6.3 & $e<1$ & 970 & 5.9 & 1.6 & 50 & el,240 & el.69 & e. 12 \\
\hline $\begin{array}{l}15 \ldots \\
\text { Apr }\end{array}$ & 2.6 & 6.6 & $e<1$ & 950 & 8.0 & 1.7 & 51 & el,230 & el.68 & e.11 \\
\hline $\begin{array}{l}12 \ldots \\
\text { May }\end{array}$ & 2.6 & 6.3 & $e<1$ & 950 & 8.1 & 1.7 & 54 & e1,240 & el. 69 & e.11 \\
\hline $\begin{array}{l}17 \ldots \\
\text { Jun }\end{array}$ & 2.5 & 7.6 & $e<1$ & 1,000 & 7.2 & 1.6 & 54 & e 1,300 & el.77 & e.35 \\
\hline $\begin{array}{l}12 \ldots \\
\text { Jul }\end{array}$ & 1.7 & 13 & $e<1$ & 1,300 & 5.9 & 1.8 & 54 & el,670 & e2.27 & e2.75 \\
\hline $\begin{array}{l}12 \ldots \\
\text { Aug }\end{array}$ & 2.1 & 7.6 & $e<1$ & 1,000 & 6.6 & 2.0 & 42 & el, 270 & el.73 & el.17 \\
\hline $\begin{array}{l}16 \ldots \\
\text { Sep }\end{array}$ & 4.9 & 5.8 & $e<1$ & 910 & 7.9 & 1.2 & 39 & el,160 & el. 58 & e. 56 \\
\hline Oct & 2.2 & 5.3 & $e<1$ & 800 & 6.3 & 1.0 & 38 & el,030 & el.40 & e.31 \\
\hline $\begin{array}{l}10 \ldots \\
\text { Nov }\end{array}$ & 2.2 & 5.2 & $e<1$ & 700 & 6.3 & 1.2 & 39 & e933 & el.27 & e. 23 \\
\hline $\begin{array}{c}28 \ldots \\
\operatorname{Jan} 1996\end{array}$ & 2.2 & 4.4 & $e<1$ & 720 & 5.8 & .8 & 38 & e949 & el.29 & e.19 \\
\hline $\begin{array}{l}11 \ldots \\
\text { Feb }\end{array}$ & 2.4 & 4.5 & $e<1$ & 710 & 5.5 & .5 & 41 & e944 & el. 28 & e.17 \\
\hline $\begin{array}{l}20 \ldots \\
\text { Apr }\end{array}$ & 2.3 & 4.5 & $e<1$ & 640 & 6.2 & 2.5 & 41 & e875 & el.19 & e. 13 \\
\hline $\begin{array}{l}03 \ldots \\
\text { May }\end{array}$ & 2.3 & 4.4 & $e<1$ & 720 & 7.1 & .6 & 43 & e957 & el.30 & e. 14 \\
\hline $\begin{array}{l}07 . . . \\
\text { Jun }\end{array}$ & 2.6 & 4.4 & $e<1$ & 710 & 6.5 & .6 & 41 & e938 & el.28 & e. 13 \\
\hline $\begin{array}{l}04 \ldots \\
\text { Jui }\end{array}$ & 2.5 & 4.4 & $e<1$ & 670 & 5.8 & .7 & 44 & e911 & el.24 & e. 12 \\
\hline $\begin{array}{l}02 \ldots \\
\text { Aug }\end{array}$ & 2.7 & 4.2 & $e<1$ & 780 & 5.6 & .8 & 45 & el,020 & el.39 & e. 15 \\
\hline $\begin{array}{l}07 \ldots \\
\text { Sep }\end{array}$ & 2.8 & 4.5 & $e<1$ & 670 & 6.2 & .6 & 41 & e901 & el.23 & e. 13 \\
\hline $03 \ldots$ & 2.5 & 5.0 & $e<1$ & 650 & 6.0 & .9 & 44 & e898 & el.22 & e.12 \\
\hline
\end{tabular}


Table 3. Water-quality data for sites in the Sand Coulee Coal Area, Montana, July 1994 through September 1996 (Continued) SITE 22, 472446111085101--PIPE SPRING AT TRACY, MT--Continued

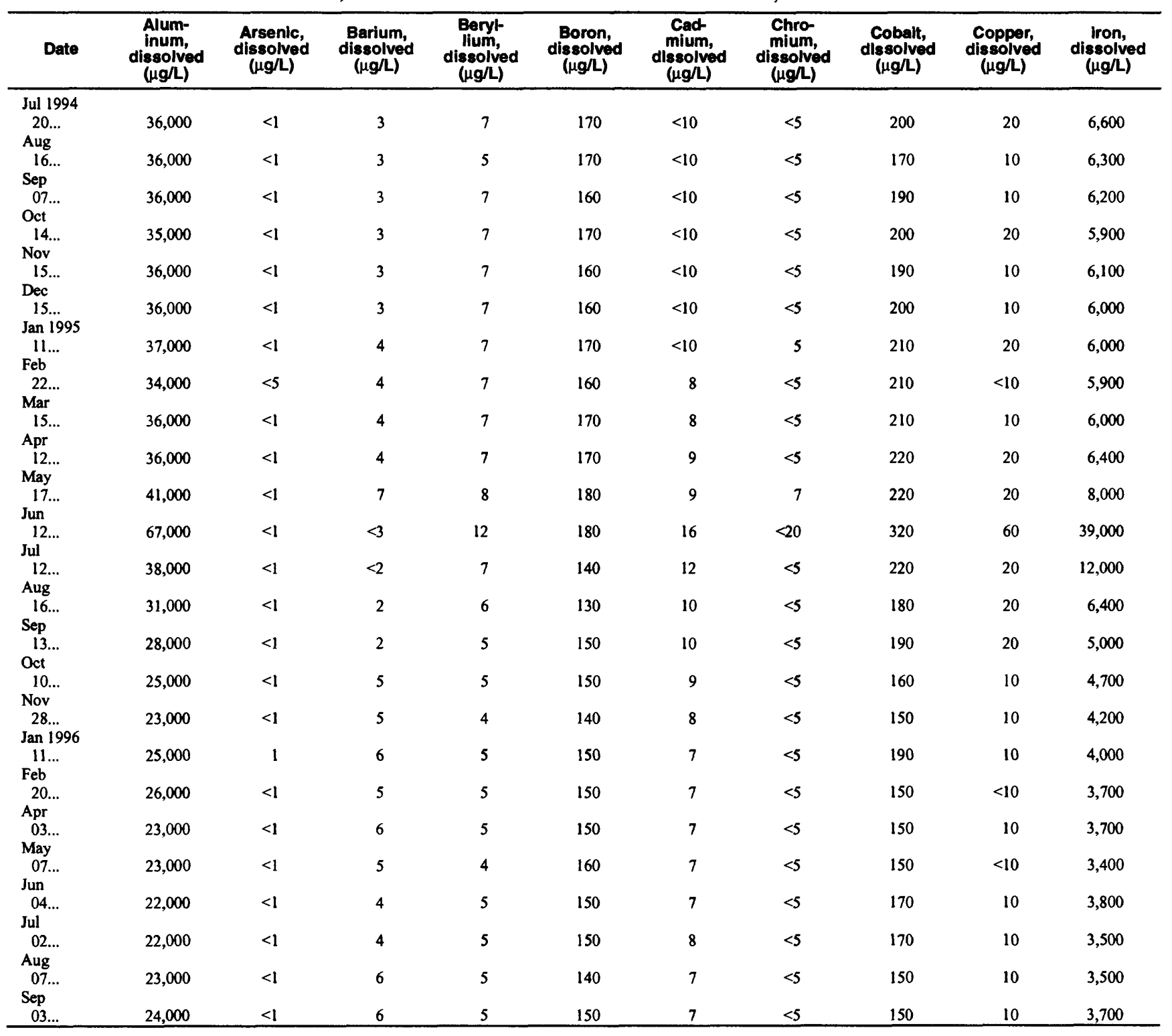


Table 3. Water-quality data for sites in the Sand Coulee Coal Area, Montana, July 1994 through September 1996 (Continued) SITE 22, 472446111085101--PIPE SPRING AT TRACY, MT--Continued

\begin{tabular}{|c|c|c|c|c|c|c|c|c|c|c|}
\hline Date & $\begin{array}{l}\text { Lead, } \\
\text { dissolved } \\
(\mu g /)\end{array}$ & $\begin{array}{l}\text { Llthium, } \\
\text { dissolved } \\
\left.\text { ( } \mu g^{\prime} h\right)\end{array}$ & $\begin{array}{l}\text { Manga- } \\
\text { nese, } \\
\text { dissolved } \\
(\mu g /)\end{array}$ & $\begin{array}{c}\text { Molyb- } \\
\text { denum, } \\
\text { dissolved } \\
(\mu g / L)\end{array}$ & $\begin{array}{c}\text { Nickel, } \\
\text { dissolved } \\
(\mu g /)\end{array}$ & $\begin{array}{c}\text { Sele- } \\
\text { nium, } \\
\text { dissolved } \\
(\mu g /)\end{array}$ & $\begin{array}{l}\text { Silver, } \\
\text { dissolved } \\
(\mu g / L)\end{array}$ & $\begin{array}{c}\text { Stron- } \\
\text { tium, } \\
\text { dissolved } \\
(\mu(\mu))\end{array}$ & $\begin{array}{c}\text { Vana- } \\
\text { dlum, } \\
\text { diseolved } \\
(\mu g /)\end{array}$ & $\begin{array}{l}\text { Zinc, } \\
\text { dissolved } \\
(\mu \mathrm{g} / \mathrm{L})\end{array}$ \\
\hline Jul 1994 & & & & & & & & & & \\
\hline $\begin{array}{l}20 \ldots \\
\text { Aug }\end{array}$ & $<10$ & 140 & 600 & $<10$ & 360 & $<1$ & 2 & 610 & $<6$ & 1,200 \\
\hline $16 \ldots$ & $<10$ & 140 & 590 & $<10$ & 350 & $<5$ & $<1$ & 610 & $<6$ & 1,100 \\
\hline $\begin{array}{l}\text { Sep } \\
07 \ldots\end{array}$ & $<10$ & 140 & 600 & $<10$ & 360 & $<1$ & 2 & 610 & $<6$ & 100 \\
\hline Oct & -10 & 140 & 000 & & & & & & & 1,100 \\
\hline $\begin{array}{l}14 \ldots \\
\text { Nov }\end{array}$ & $<10$ & 140 & 580 & $<10$ & 360 & $<2$ & $<1$ & 600 & $<6$ & 1,100 \\
\hline $\begin{array}{l}15 . . \\
\text { Dec }\end{array}$ & $<10$ & 150 & 630 & $<10$ & 380 & 1 & $<1$ & 610 & $<6$ & 1,200 \\
\hline $\begin{array}{l}15 \ldots \\
\text { Jan } 1995\end{array}$ & $<10$ & 150 & 610 & $<10$ & 380 & 1 & $<1$ & 620 & $<6$ & 1,100 \\
\hline $\begin{array}{l}11 \ldots \\
\text { Feb }\end{array}$ & $<10$ & 150 & 630 & $<10$ & 400 & $<2$ & $<1$ & 670 & $<6$ & 1,200 \\
\hline $22 \ldots$ & $<1$ & 160 & 600 & $<1$ & 360 & 1 & $<1$ & 650 & $<6$ & 1,100 \\
\hline $\begin{array}{l}15 \ldots \\
\text { Apr }\end{array}$ & $<1$ & 160 & 620 & $<1$ & 370 & 1 & $<1$ & 660 & $<6$ & 1,100 \\
\hline $\begin{array}{l}12 \ldots \\
\text { May }\end{array}$ & 1 & 160 & 650 & $<1$ & 400 & 1 & $<1$ & 670 & $<6$ & 1,200 \\
\hline $\begin{array}{c}17 \ldots \\
\text { Jun }\end{array}$ & $<1$ & 160 & 660 & $<1$ & 450 & $<1$ & $<1$ & 680 & $<6$ & 1,300 \\
\hline $12 \ldots$ & $<1$ & 160 & 930 & $<1$ & 600 & $<1$ & $<3$ & 670 & $<18$ & 1,900 \\
\hline $\begin{array}{l}12 \ldots \\
\text { Aug }\end{array}$ & $<1$ & 130 & 650 & $<1$ & 380 & - & $<1$ & 540 & $<6$ & 1,200 \\
\hline $\begin{array}{l}16 \ldots \\
\text { Sep }\end{array}$ & $<1$ & 120 & 570 & $<1$ & 330 & - & $<1$ & 530 & $<6$ & 1,000 \\
\hline $\begin{array}{l}13 \ldots \\
\text { Oct }\end{array}$ & $<1$ & 110 & 530 & $<2$ & 290 & -- & $<1$ & 500 & $<6$ & 890 \\
\hline $\begin{array}{l}10 \ldots \\
\text { Nov }\end{array}$ & $<1$ & 120 & 520 & $<1$ & 310 & - & 2 & 510 & $<6$ & 930 \\
\hline $\begin{array}{c}28 \ldots \\
\operatorname{Jan} 1996\end{array}$ & $<1$ & 120 & 500 & $<1$ & 280 & - & $<1$ & 540 & $<6$ & 840 \\
\hline $\begin{array}{l}11 \ldots \\
\text { Feb }\end{array}$ & $<1$ & 110 & 510 & $<1$ & 290 & - & $<1$ & 530 & $<6$ & 910 \\
\hline $\begin{array}{l}20 \ldots \\
\text { Apr }\end{array}$ & $<1$ & 120 & 500 & $<1$ & 290 & - & $<1$ & 550 & $<6$ & 850 \\
\hline $\begin{array}{l}03 \ldots \\
\text { May }\end{array}$ & $<1$ & 130 & 500 & $<1$ & 280 & - & $<1$ & 550 & $<6$ & 820 \\
\hline $\begin{array}{c}07 . . . \\
\text { Jun }\end{array}$ & 1 & 110 & 480 & 2 & 280 & - & $<1$ & 490 & $<6$ & 860 \\
\hline $\begin{array}{c}04 \ldots \\
\text { Jul }\end{array}$ & 2 & 130 & 500 & $<1$ & 290 & - & $<1$ & 550 & $<6$ & 860 \\
\hline Aug & $<1$ & 120 & 500 & $<1$ & 290 & - & $<1$ & 580 & $<6$ & 880 \\
\hline $\begin{array}{l}07 . . . \\
\text { Sep }\end{array}$ & $<1$ & 130 & 480 & $<1$ & 280 & - & $<1$ & 550 & $<6$ & 810 \\
\hline $03 \ldots$ & $<1$ & 130 & 520 & $<1$ & 300 & - & 1 & 580 & $<6$ & 920 \\
\hline
\end{tabular}


Table 3. Water-quality data for sites in the Sand Coulee Coal Area, Montana, July 1994 through September 1996 (Continued) SITE 23, 472447111085301--STOCK TANK SPRING AT TRACY, MT

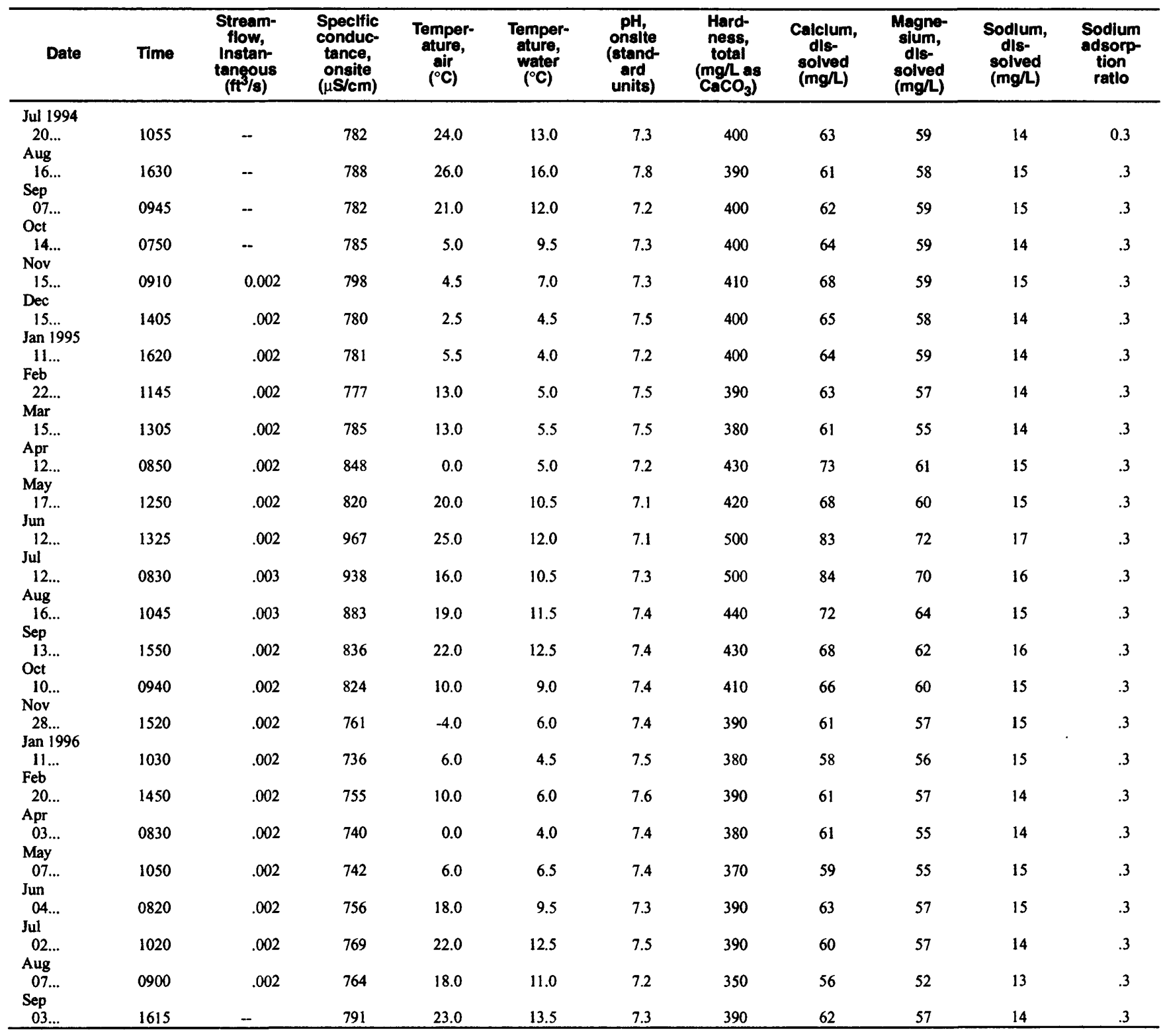


Table 3. Water-quality data for sites in the Sand Coulee Coal Area, Montana, July 1994 through September 1996 (Continued) SITE 23, 472447111085301--STOCK TANK SPRING AT TRACY, MT--Continued

\begin{tabular}{|c|c|c|c|c|c|c|c|c|c|c|}
\hline Date & $\begin{array}{l}\text { Potas- } \\
\text { sium, } \\
\text { dissolved } \\
\text { (mg/L) }\end{array}$ & $\begin{array}{c}\text { Acidity } \\
\left(\mathbf{m g}^{\prime} / \mathbf{L} \text { as }\right. \\
\left.\mathrm{H}^{+}\right)\end{array}$ & $\begin{array}{c}\text { Alkalinity, } \\
\text { lab } \\
\left(\mathrm{mgh}_{\mathrm{L}} \mathrm{as}\right. \\
\left.\mathrm{CaCO}_{3}\right)\end{array}$ & $\begin{array}{c}\text { Sulfate, } \\
\text { dissolved } \\
\text { (mg/L) }\end{array}$ & $\begin{array}{l}\text { Chloride, } \\
\text { dissolved } \\
\text { (mg/L) }\end{array}$ & $\begin{array}{l}\text { Fluoride, } \\
\text { dissolved } \\
\text { (mg/ll) }\end{array}$ & $\begin{array}{c}\text { Silica, } \\
\text { dissoived } \\
\text { (mg/ as as } \\
\left.\mathrm{SiO}_{2}\right)\end{array}$ & $\begin{array}{l}\text { Solids, } \\
\text { sum of } \\
\text { consti- } \\
\text { fuents, } \\
\text { dissolved } \\
\text { (mg/l) }\end{array}$ & $\begin{array}{l}\text { Solids, } \\
\text { dissolved } \\
\text { (ton/ } \\
\text { acre-ft) }\end{array}$ & $\begin{array}{l}\text { Solids, } \\
\text { dissolved } \\
\text { (ton/d) }\end{array}$ \\
\hline $\begin{array}{l}\text { Jul } 1994 \\
20 \ldots \\
\text { Aug }\end{array}$ & 2.1 & 0.3 & 230 & 170 & 5.7 & 0.8 & 10 & 463 & 0.63 & -- \\
\hline $\begin{array}{l}16 \ldots \\
\text { Sep }\end{array}$ & 2.1 & .2 & 229 & 170 & 5.9 & 1.0 & 9.6 & 461 & .63 & - \\
\hline $\begin{array}{l}07 \ldots \\
\text { Oct }\end{array}$ & 2.0 & .2 & 233 & 170 & 5.7 & .8 & 10 & 465 & .63 & -- \\
\hline $\begin{array}{l}14 \ldots \\
\text { Nov }\end{array}$ & 2.0 & .1 & 237 & 190 & 6.4 & .9 & 11 & 490 & .67 & -- \\
\hline $\begin{array}{l}15 \ldots \\
\text { Dec }\end{array}$ & 2.1 & .1 & 233 & 180 & 6.4 & 1.2 & 11 & 483 & .66 & $<.01$ \\
\hline $\begin{array}{c}15 \ldots \\
\text { Jan } 1995\end{array}$ & 2.0 & .2 & 227 & 170 & 6.1 & 1.0 & 10 & 463 & .63 & $<.01$ \\
\hline $\begin{array}{l}11 \ldots \\
\text { Feb }\end{array}$ & 1.9 & .3 & 225 & 170 & 6.5 & 1.1 & 10 & 462 & .63 & $<.01$ \\
\hline $\begin{array}{l}22 \ldots \\
\text { Mar }\end{array}$ & 1.8 & $<.1$ & 226 & 140 & 5.6 & .8 & 10 & 428 & .58 & $<.01$ \\
\hline $\begin{array}{l}15 \ldots \\
\text { Apr }\end{array}$ & 2.0 & $<.1$ & 227 & 140 & 5.5 & .9 & 9.6 & 425 & .58 & $<.01$ \\
\hline $\begin{array}{l}12 \ldots \\
\text { May }\end{array}$ & 2.4 & $<.1$ & 235 & 170 & 6.1 & .9 & 11 & 481 & .65 & $<.01$ \\
\hline $\begin{array}{l}17 \ldots \\
\text { Jun }\end{array}$ & 2.1 & $<.1$ & 231 & 190 & 6.4 & 1.0 & 10 & 492 & .67 & $<.01$ \\
\hline $\begin{array}{c}12 \ldots \\
\text { Jul }\end{array}$ & 2.3 & .1 & 217 & 290 & 5.7 & .4 & 11 & 612 & .83 & $<.01$ \\
\hline $\begin{array}{l}12 \ldots \\
\text { Aug }\end{array}$ & 2.7 & .1 & 216 & 300 & 5.4 & .8 & 13 & 622 & .85 & $<.01$ \\
\hline $\begin{array}{l}16 \ldots \\
\text { Sep }\end{array}$ & 2.3 & .2 & 222 & 240 & 5.8 & 1.0 & 12 & 546 & .74 & $<.01$ \\
\hline $\begin{array}{l}13 \ldots \\
\text { Oct }\end{array}$ & 2.4 & $<.1$ & 226 & 210 & 5.8 & 1.0 & 12 & 513 & .70 & $<.01$ \\
\hline $\begin{array}{l}10 . . \\
\text { Nov }\end{array}$ & 2.5 & $<.1$ & 227 & 200 & 6.0 & 1.0 & 12 & 499 & .68 & $<.01$ \\
\hline $\begin{array}{l}28 \ldots \\
\text { Jan } 1996\end{array}$ & - & - & -- & - & -- & -- & 11 & - & -- & - \\
\hline $\begin{array}{l}11 \ldots \\
\text { Feb }\end{array}$ & 1.9 & .4 & 222 & 160 & 5.3 & 1.1 & 11 & 442 & .60 & $<.01$ \\
\hline $\begin{array}{l}20 \ldots \\
\text { Apr }\end{array}$ & 1.9 & .3 & 225 & 160 & 5.9 & .5 & 11 & 447 & .61 & $<.01$ \\
\hline $\begin{array}{l}03 \ldots \\
\text { May }\end{array}$ & 2.0 & $<.1$ & 224 & 160 & 5.9 & 1.2 & 11 & 445 & .61 & $<.01$ \\
\hline $\begin{array}{c}07 . . . \\
\text { Jun }\end{array}$ & 2.0 & .2 & 228 & 160 & 5.9 & 1.1 & 11 & 446 & .61 & $<.01$ \\
\hline $\begin{array}{c}04 \ldots \\
\text { Jul }\end{array}$ & 2.0 & $<.1$ & 234 & 160 & 5.5 & 1.1 & 11 & 456 & .62 & $<.01$ \\
\hline $\begin{array}{l}02 \ldots \\
\text { Aug }\end{array}$ & 1.8 & .2 & 235 & 150 & 4.9 & 1.1 & 11 & 441 & .60 & $<.01$ \\
\hline $\begin{array}{c}07 \ldots \\
\text { Sep }\end{array}$ & 2.3 & $<.1$ & 239 & 150 & 5.7 & 1.1 & 9.7 & 434 & .59 & $<.01$ \\
\hline $03 \ldots$ & 2.7 & $<.1$ & 236 & 160 & 6.3 & 1.0 & 11 & 456 & .62 & $<.01$ \\
\hline
\end{tabular}


Table 3. Water-quality data for sites in the Sand Coulee Coal Area, Montana, July 1994 through September 1996 (Continued) SITE 23, 472447111085301--STOCK TANK SPRING AT TRACY, MT--Continued

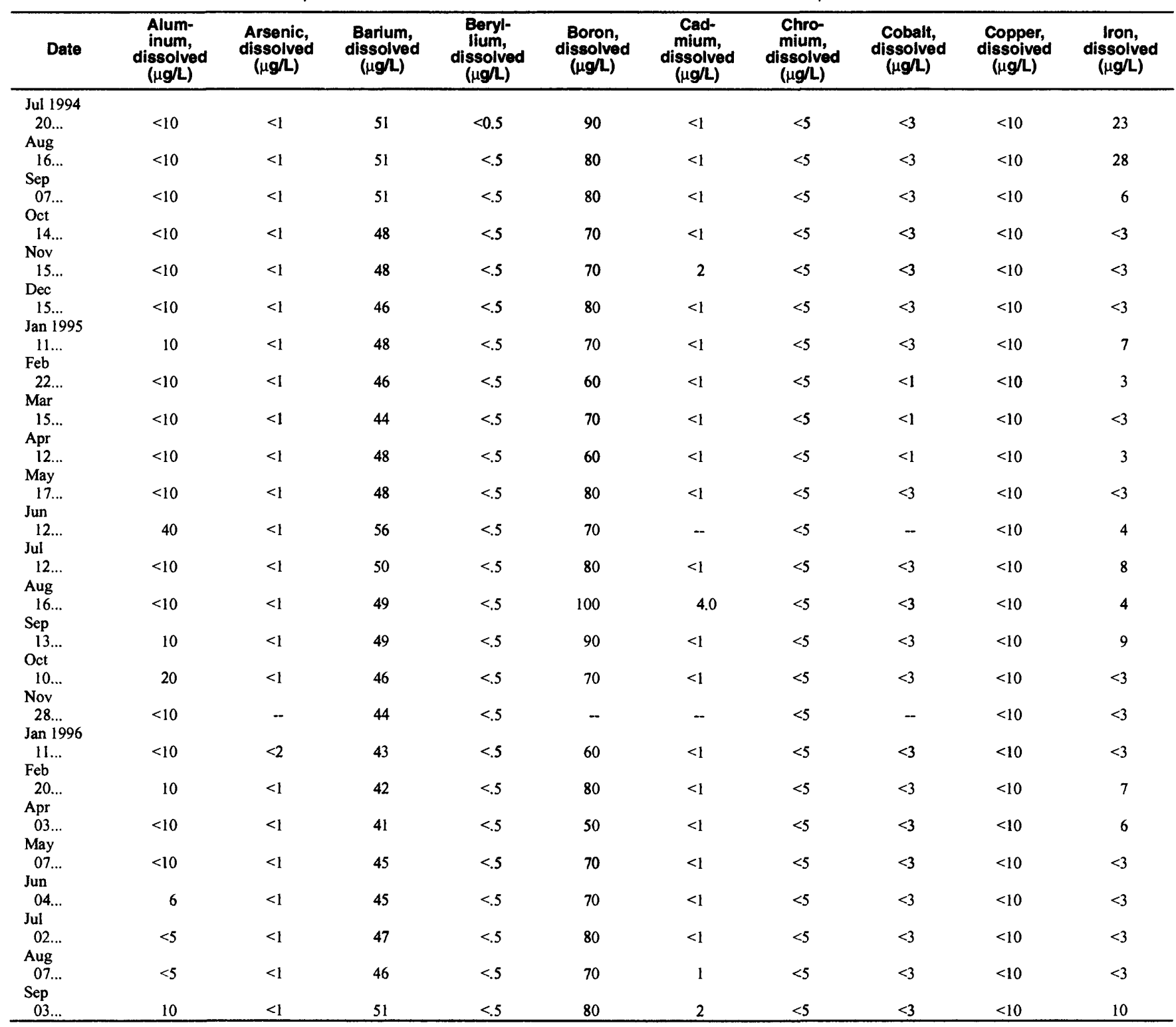


Table 3. Water-quality data for sites in the Sand Coulee Coal Area, Montana, July 1994 through September 1996 (Continued) SITE 23, 472447111085301--STOCK TANK SPRING AT TRACY, MT--Continued

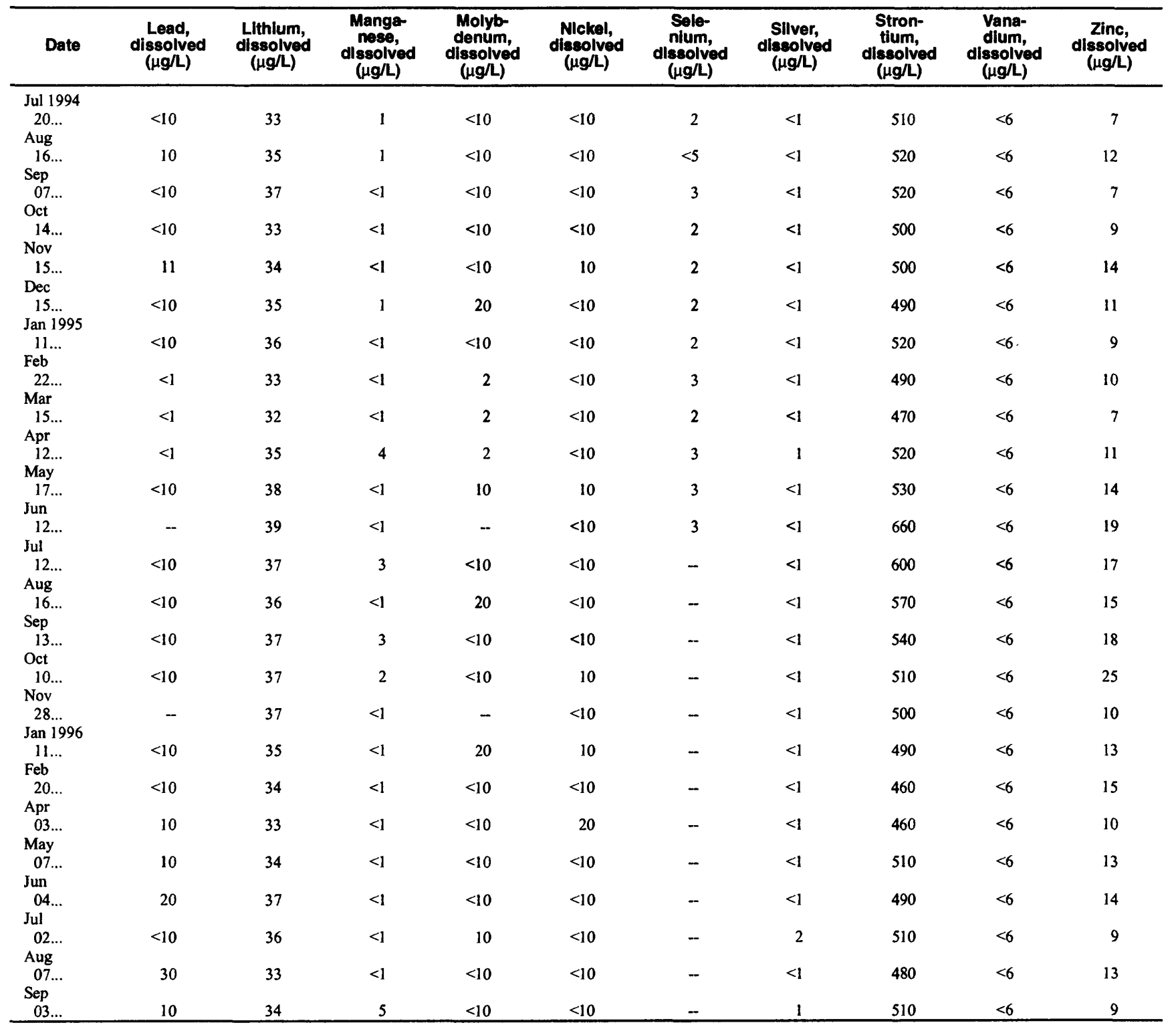


Table 3. Water-quality data for sites in the Sand Coulee Coal Area, Montana, July 1994 through September 1996 (Continued) SITE 24, 472513111082501--JOHNSON BADWATER MINE SMALL WETLANDS INFLOW NEAR TRACY, MT

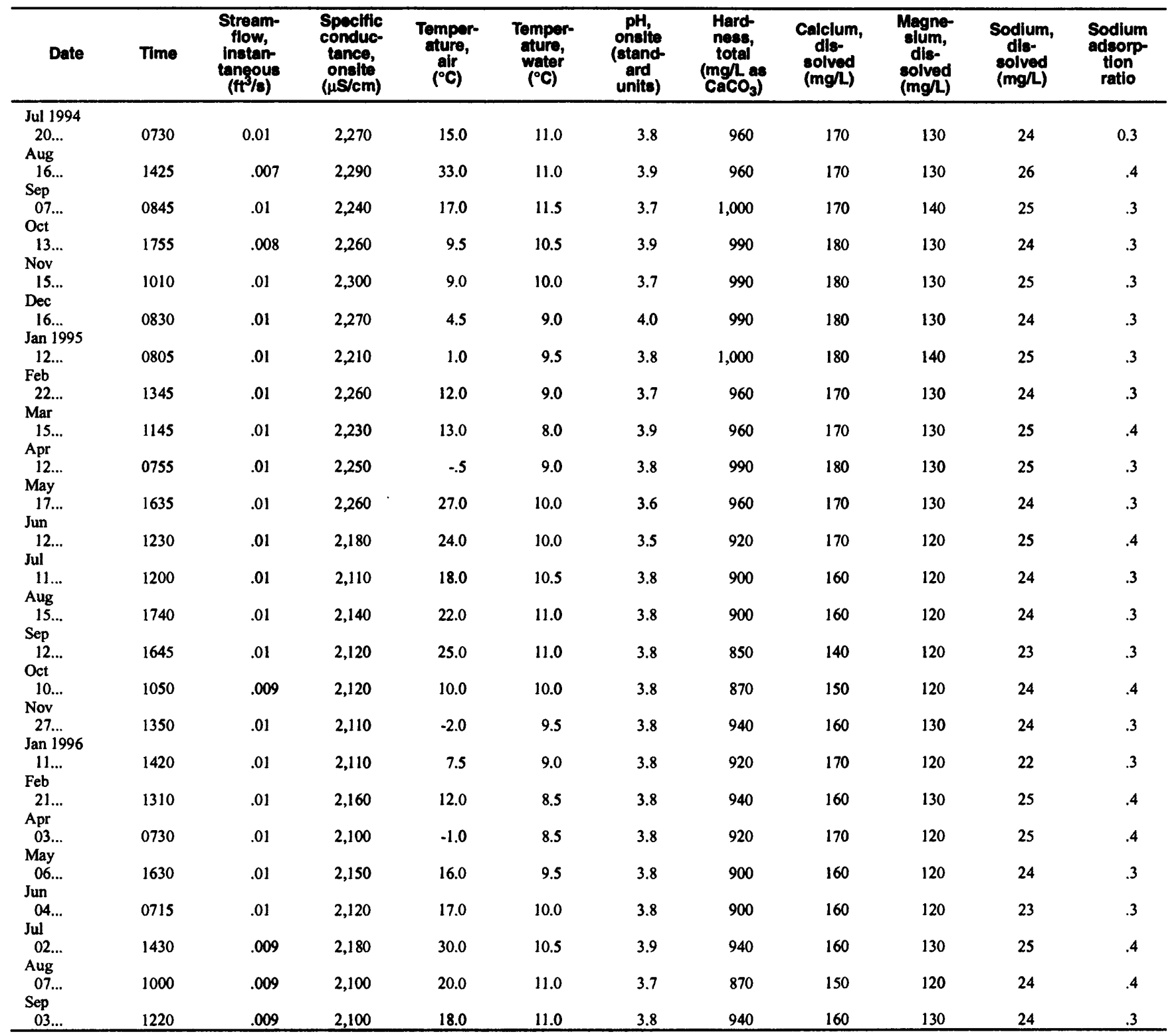


Table 3. Water-quality data for sites in the Sand Coulee Coal Area, Montana, July 1994 through September 1996 (Continued) SITE 24, 472513111082501--JOHNSON BADWATER MINE SMALL WETLANDS INFLOW NEAR TRACY, MT--Continued

\begin{tabular}{|c|c|c|c|c|c|c|c|c|c|c|}
\hline Date & $\begin{array}{l}\text { Potas- } \\
\text { sium, } \\
\text { dissolved } \\
\text { (mg/L) }\end{array}$ & $\begin{array}{l}\text { Acidity } \\
(\mathrm{mg} / \mathrm{L} \text { as } \\
\left.\mathrm{H}^{+}\right)\end{array}$ & $\begin{array}{c}\text { Alkalinity, } \\
\text { lab } \\
\left(\mathrm{mg}^{\prime} \mathrm{L} \text { as }\right. \\
\left.\mathrm{CaCO}_{3}\right)\end{array}$ & $\begin{array}{l}\text { Sulfate, } \\
\text { dissolved } \\
\text { (mg/ll) }\end{array}$ & $\begin{array}{l}\text { Chloride, } \\
\text { dissolved } \\
\text { (mg/l) }\end{array}$ & $\begin{array}{l}\text { Fluoride, } \\
\text { dissolved } \\
\text { (mg/L) }\end{array}$ & $\begin{array}{l}\text { Sillica, } \\
\text { dlssolved } \\
\text { (mg/l as } \\
\mathrm{SiO}_{2} \text { ) }\end{array}$ & $\begin{array}{l}\text { Solids, } \\
\text { sum of } \\
\text { constl- } \\
\text { tuents, } \\
\text { dissolved } \\
\text { (mg/L) }\end{array}$ & $\begin{array}{l}\text { Solids, } \\
\text { dissolved } \\
\text { (ton/ } \\
\text { acre-ft) }\end{array}$ & $\begin{array}{l}\text { Solids, } \\
\text { dissolved } \\
\text { (ton/d) }\end{array}$ \\
\hline $\begin{array}{c}\text { Jul } 1994 \\
20\end{array}$ & 75 & 12 & 1 & 1500 & 61 & 74 & 10 & $\mathrm{mmo}$ & 0.96 & 006 \\
\hline Aug & 7.5 & 12 & $e<1$ & 1,500 & 6.1 & 7.4 & 49 & $e 2,090$ & e2.85 & $\mathrm{e} 0.06$ \\
\hline $\begin{array}{l}16 \ldots \\
\text { Sep }\end{array}$ & 7.0 & 13 & $e<1$ & 1,600 & 5.9 & 5.1 & 48 & e2,190 & e2.98 & e. 04 \\
\hline $\begin{array}{l}07 . . . \\
\text { Oct }\end{array}$ & 7.5 & 13 & $e<1$ & 1,700 & 6.2 & 6.6 & 48 & e2,300 & e3.13 & e.07 \\
\hline $\begin{array}{l}13 \ldots \\
\text { Nov }\end{array}$ & 7.1 & 11 & $e<1$ & 2,000 & 6.6 & 7.0 & 51 & $e 2,590$ & e3.53 & e.06 \\
\hline $\begin{array}{l}15 . . \\
\text { Dec }\end{array}$ & 7.4 & 12 & $e<1$ & 1,800 & 6.8 & 6.3 & 48 & $e 2,400$ & e3.27 & e.06 \\
\hline $\begin{array}{l}16 \ldots \\
\operatorname{Jan} 1995\end{array}$ & 7.5 & 12 & $e<1$ & 1,600 & 6.3 & 4.6 & 49 & e2,190 & e2.99 & e.07 \\
\hline $\begin{array}{l}12 \ldots \\
\text { Feb }\end{array}$ & 6.8 & 14 & $e<1$ & 1,700 & 5.6 & 6.8 & 49 & e2,310 & e3.14 & e. 06 \\
\hline $\begin{array}{l}22 \ldots \\
\mathrm{Mar}\end{array}$ & 7.4 & 11 & $e<1$ & 1,600 & 6.2 & 6.1 & 49 & $e 2,180$ & e2.97 & e.06 \\
\hline $\begin{array}{l}15 \ldots \\
\text { Apr }\end{array}$ & 7.4 & 11 & $e<1$ & 2,000 & 8.1 & 5.6 & 47 & $e 2,580$ & e3.51 & $e .07$ \\
\hline $\begin{array}{l}12 \ldots \\
\text { May }\end{array}$ & 7.0 & 11 & $e<1$ & 1,800 & 8.9 & 6.2 & 49 & e2,390 & e3.26 & e.06 \\
\hline $\begin{array}{l}17 \ldots \\
\text { Jun }\end{array}$ & 7.0 & 12 & $e<1$ & 1,700 & 9.2 & 5.6 & 49 & e2,270 & e3.09 & e. 07 \\
\hline $\begin{array}{l}12 \ldots \\
\text { Jul }\end{array}$ & 6.9 & 11 & $e<1$ & 1,900 & 6.0 & 5.7 & 48 & $e 2,470$ & e3.36 & e. 07 \\
\hline $\begin{array}{l}11 \ldots \\
\text { Aug }\end{array}$ & 7.2 & 11 & $e<1$ & 1,600 & 6.4 & 3.2 & 50 & e2,150 & e2.92 & e.06 \\
\hline $\begin{array}{l}15 \ldots \\
\text { Sep }\end{array}$ & 7.5 & 11 & $e<1$ & 1,600 & 8.3 & 5.8 & 50 & $e 2,150$ & e2.93 & e.06 \\
\hline $\begin{array}{l}12 \ldots \\
\text { Oct }\end{array}$ & 6.0 & 10 & $e<1$ & 1,400 & 6.0 & 5.9 & 47 & el,920 & e2.61 & e.05 \\
\hline $\begin{array}{l}10 \ldots \\
\text { Nov }\end{array}$ & 6.9 & 12 & $\mathbf{e}<1$ & 1,500 & 5.4 & 5.4 & 48 & e2,040 & e2.77 & e. 05 \\
\hline $\begin{array}{l}27 \ldots \\
\text { Jan } 1996\end{array}$ & 6.4 & 12 & $e<1$ & 1,600 & 5.5 & 7.4 & 47 & e2,160 & e2.94 & e.06 \\
\hline $\begin{array}{l}11 \ldots \\
\text { Feb }\end{array}$ & 7.1 & 12 & $e<1$ & 1,700 & 9.8 & 5.4 & 50 & $\mathbf{e} 2,270$ & e3.09 & $e .06$ \\
\hline $\begin{array}{l}21 \ldots \\
\text { Apr }\end{array}$ & 6.6 & 11 & $e<1$ & 1,400 & 6.1 & 8.3 & 51 & el, 970 & e2.68 & $e .05$ \\
\hline $\begin{array}{l}03 \ldots \\
\text { May }\end{array}$ & 6.8 & 11 & $e<1$ & 1,500 & 7.6 & 6.7 & 50 & e2,060 & e2.81 & e.06 \\
\hline $\begin{array}{c}06 \ldots \\
\text { Jun }\end{array}$ & 6.8 & 11 & $e<1$ & 1,600 & 8.2 & 6.3 & 49 & e2,150 & e2.92 & e.06 \\
\hline $\begin{array}{c}04 \ldots \\
\text { Jul }\end{array}$ & 6.7 & 11 & $e<1$ & 1,400 & 6.3 & 7.0 & 49 & el,950 & e2.65 & e. 05 \\
\hline $\begin{array}{l}02 \ldots \\
\text { Aug }\end{array}$ & 7.4 & 11 & $e<1$ & 1,500 & 5.8 & 5.6 & 52 & e2,070 & e 2.82 & e. 05 \\
\hline $\begin{array}{l}07 . . \\
\text { Sep }\end{array}$ & 7.4 & 12 & $e<1$ & 1,500 & 7.2 & 6.4 & 47 & e2,040 & e2.77 & e.05 \\
\hline $03 \ldots$ & 7.1 & 9.7 & $e<1$ & 1,400 & 8.5 & 6.4 & 49 & el,960 & e2.67 & e. 05 \\
\hline
\end{tabular}


Table 3. Water-quality data for sites in the Sand Coulee Coal Area, Montana, July 1994 through September 1996 (Continued) SITE 24, 472513111082501--JOHNSON BADWATER MINE SMALL WETLANDS INFLOW NEAR TRACY, MT--Continued

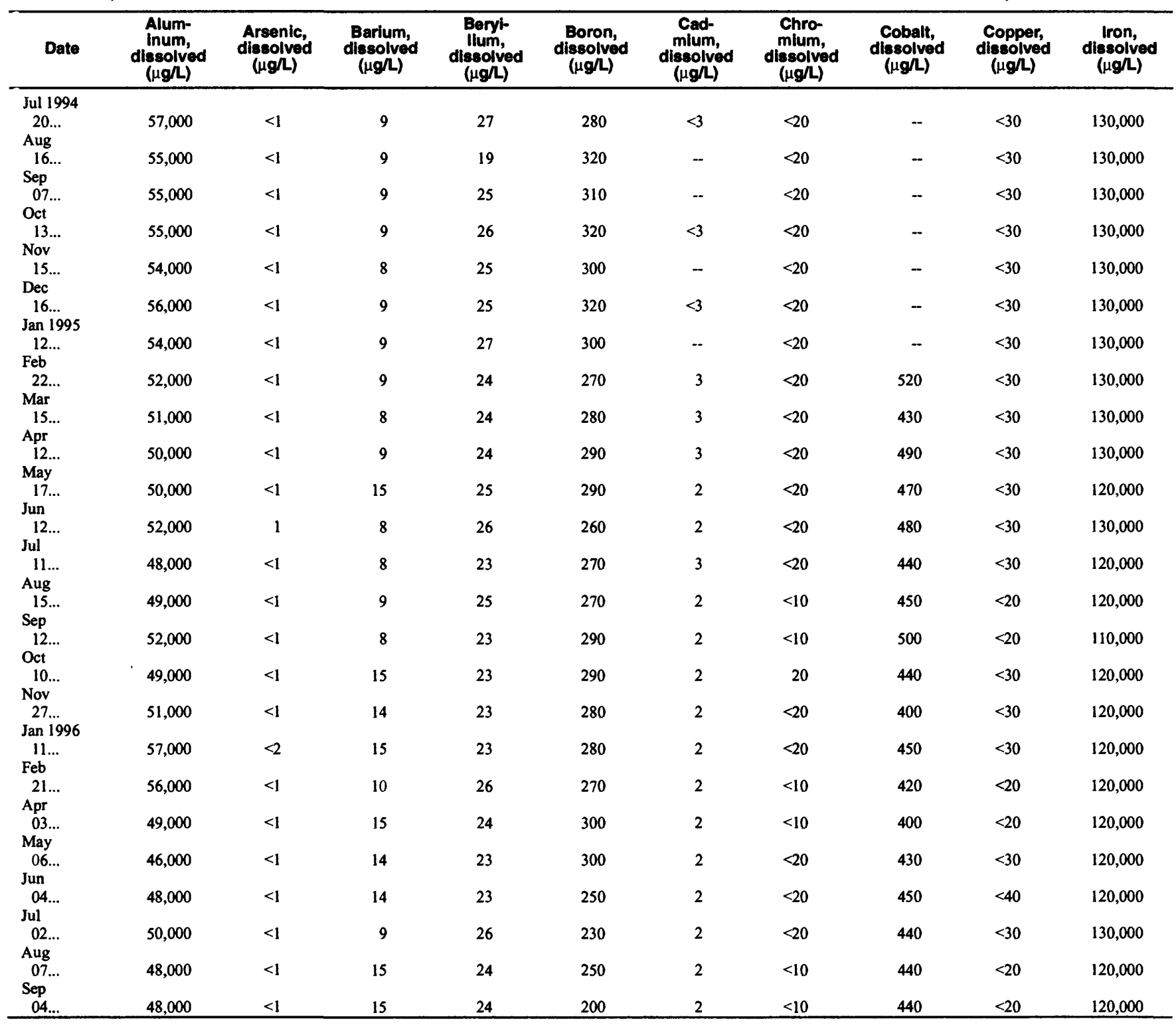


Table 3. Water-quality data for sites in the Sand Coulee Coal Area, Montana, July 1994 through September 1996 (Continued)

SITE 24, 472513111082501 --JOHNSON BADWATER MINE SMALL WETLANDS INFLOW NEAR TRACY, MT--Continued

\begin{tabular}{|c|c|c|c|c|c|c|c|c|c|c|}
\hline Date & $\begin{array}{l}\text { Load, } \\
\text { dissolved } \\
(\mu g / L)\end{array}$ & $\begin{array}{l}\text { Lithlum, } \\
\text { diseolved } \\
(\mu g /)\end{array}$ & $\begin{array}{c}\text { Manga- } \\
\text { nese, } \\
\text { dissolved } \\
(\mu g / L)\end{array}$ & $\begin{array}{l}\text { Molyb- } \\
\text { denum, } \\
\text { dissolved } \\
(\mu g /) \text { ( }\end{array}$ & $\begin{array}{l}\text { Nlckel, } \\
\text { dissolved } \\
(\mu g / L)\end{array}$ & $\begin{array}{c}\text { Sole- } \\
\text { nium, } \\
\text { dissolved } \\
(\mu g / 4)\end{array}$ & $\begin{array}{l}\text { Sllver, } \\
\text { dlasolved } \\
\left(\mu g^{\prime} L\right)\end{array}$ & $\begin{array}{c}\text { Stron- } \\
\text { tlum, } \\
\text { dlseolved } \\
(\mu g / L)\end{array}$ & $\begin{array}{c}\text { Vana- } \\
\text { dlum, } \\
\text { diseolved } \\
(\mu \mathrm{g} / \mathrm{L})\end{array}$ & $\begin{array}{c}\text { Zinc, } \\
\text { dissolved } \\
(\mu \mathrm{g} / \mathrm{L})\end{array}$ \\
\hline $\begin{array}{l}\text { Jul } 1994 \\
20 \ldots \\
\text { Aug }\end{array}$ & $<10$ & 360 & 980 & $<10$ & 1,100 & $<1$ & $<3$ & 1,200 & 19 & 3,100 \\
\hline $\begin{array}{l}16 \ldots \\
\text { Sep }\end{array}$ & $<10$ & 380 & 970 & $<10$ & 980 & $<5$ & $<3$ & 1,200 & 32 & 2,900 \\
\hline $\begin{array}{l}07 . . \\
\text { Oct }\end{array}$ & $<10$ & 380 & 990 & $<10$ & 1,100 & $<1$ & 4 & 1,200 & 33 & 3,200 \\
\hline $\begin{array}{l}13 \ldots \\
\text { Nov }\end{array}$ & $<10$ & 360 & 970 & $<10$ & 1,000 & $<1$ & $<$ & 1,200 & 26 & 3,200 \\
\hline $\begin{array}{l}15 \ldots \\
\text { Dec }\end{array}$ & $<10$ & 390 & 1,000 & $<10$ & 1,100 & $<1$ & $<3$ & 1,200 & 25 & 3,300 \\
\hline $\begin{array}{l}16 \ldots \\
\operatorname{Jan} 1995\end{array}$ & $<10$ & 370 & 970 & $<10$ & 980 & $<1$ & $<$ & 1,200 & 21 & 3,000 \\
\hline $\begin{array}{l}12 \ldots \\
\text { Feb }\end{array}$ & $<10$ & 380 & 1,000 & $<10$ & 1,100 & $<1$ & $<3$ & 1,300 & 34 & 3,200 \\
\hline $\begin{array}{c}22 \ldots \\
\text { Mar }\end{array}$ & $<1$ & 370 & 970 & 1 & 1,000 & $<1$ & - & 1,200 & 21 & 3,100 \\
\hline $\begin{array}{l}15 \ldots \\
\text { Apr }\end{array}$ & $<1$ & 380 & 950 & $<1$ & 960 & $<1$ & $<3$ & 1,200 & 6 & 2,800 \\
\hline $\begin{array}{l}12 \ldots \\
\text { May }\end{array}$ & $<1$ & 370 & 980 & $<1$ & 1,000 & $<1$ & $<3$ & 1,200 & $<6$ & 3,000 \\
\hline $\begin{array}{l}17 . . . \\
\text { Jun }\end{array}$ & $<1$ & 380 & 970 & $<1$ & 1,000 & $<1$ & $<3$ & 1,200 & 10 & 2,900 \\
\hline $\begin{array}{c}12 \ldots \\
\text { Jul }\end{array}$ & $<1$ & 380 & 960 & $<1$ & 980 & $<1$ & $<3$ & 1,200 & $<18$ & 3,000 \\
\hline $\begin{array}{l}11 \ldots \\
\text { Aug }\end{array}$ & $<1$ & 360 & 940 & $<1$ & 890 & - & $<3$ & 1,100 & $<18$ & 2,800 \\
\hline $\begin{array}{l}15 . . \\
\text { Sep }\end{array}$ & $<1$ & 360 & 890 & $<1$ & 920 & - & $<2$ & 1,100 & 13 & 2,700 \\
\hline $\begin{array}{l}12 \ldots \\
\text { Oct }\end{array}$ & $<1$ & 340 & 840 & $<1$ & 910 & - & $<2$ & 1,000 & $<12$ & 2,500 \\
\hline $\begin{array}{l}10 . . \\
\text { Nov }\end{array}$ & $<1$ & 360 & 890 & $<1$ & 880 & - & $<$ & 1,100 & $<18$ & 2,800 \\
\hline $\begin{array}{c}27 . . \\
\text { Jan } 1996\end{array}$ & $<1$ & 370 & 920 & $<1$ & 940 & - & $<3$ & 1,200 & $<18$ & 2,800 \\
\hline $\begin{array}{l}11 \ldots \\
\text { Feb }\end{array}$ & $<1$ & 320 & 970 & 1 & 980 & - & $<3$ & 1,100 & $<18$ & 3,200 \\
\hline $\begin{array}{l}21 \ldots \\
\text { Apr }\end{array}$ & $<1$ & 370 & 940 & $<1$ & 970 & -. & 3 & 1,100 & 15 & 2,800 \\
\hline $\begin{array}{l}03 \ldots \\
\text { May }\end{array}$ & $<1$ & 370 & 940 & $<1$ & 970 & - & $<2$ & 1,200 & $<12$ & 2,800 \\
\hline $\begin{array}{l}06 \ldots \\
\text { Jun }\end{array}$ & $<1$ & 380 & 910 & $<1$ & 970 & -- & $<3$ & 1,100 & $<18$ & 2,700 \\
\hline $\begin{array}{l}04 \ldots \\
\text { Jul }\end{array}$ & $<1$ & 360 & 910 & $<1$ & 960 & - & $<4$ & 1,100 & $<24$ & 2,800 \\
\hline $\begin{array}{l}02 \ldots \\
\text { Aug }\end{array}$ & $<1$ & 360 & 930 & $<1$ & 970 & - & $<3$ & 1,200 & $<18$ & 2,900 \\
\hline $\begin{array}{l}07 . . \\
\text { Sep }\end{array}$ & $<1$ & 370 & 860 & $<1$ & 900 & - & $<2$ & 1,100 & $<12$ & 2,600 \\
\hline $03 \ldots$ & $<1$ & 360 & 900 & $<1$ & 930 & -- & 3 & 1,100 & $<12$ & 2,800 \\
\hline
\end{tabular}


Table 3. Water-quality data for sites in the Sand Coulee Coal Area, Montana, July 1994 through September 1996 (Continued)

SITE 25, $472513111082901--J O H N S O N$ BADWATER MINE LARGE WETLANDS INFLOW NEAR TRACY, MT

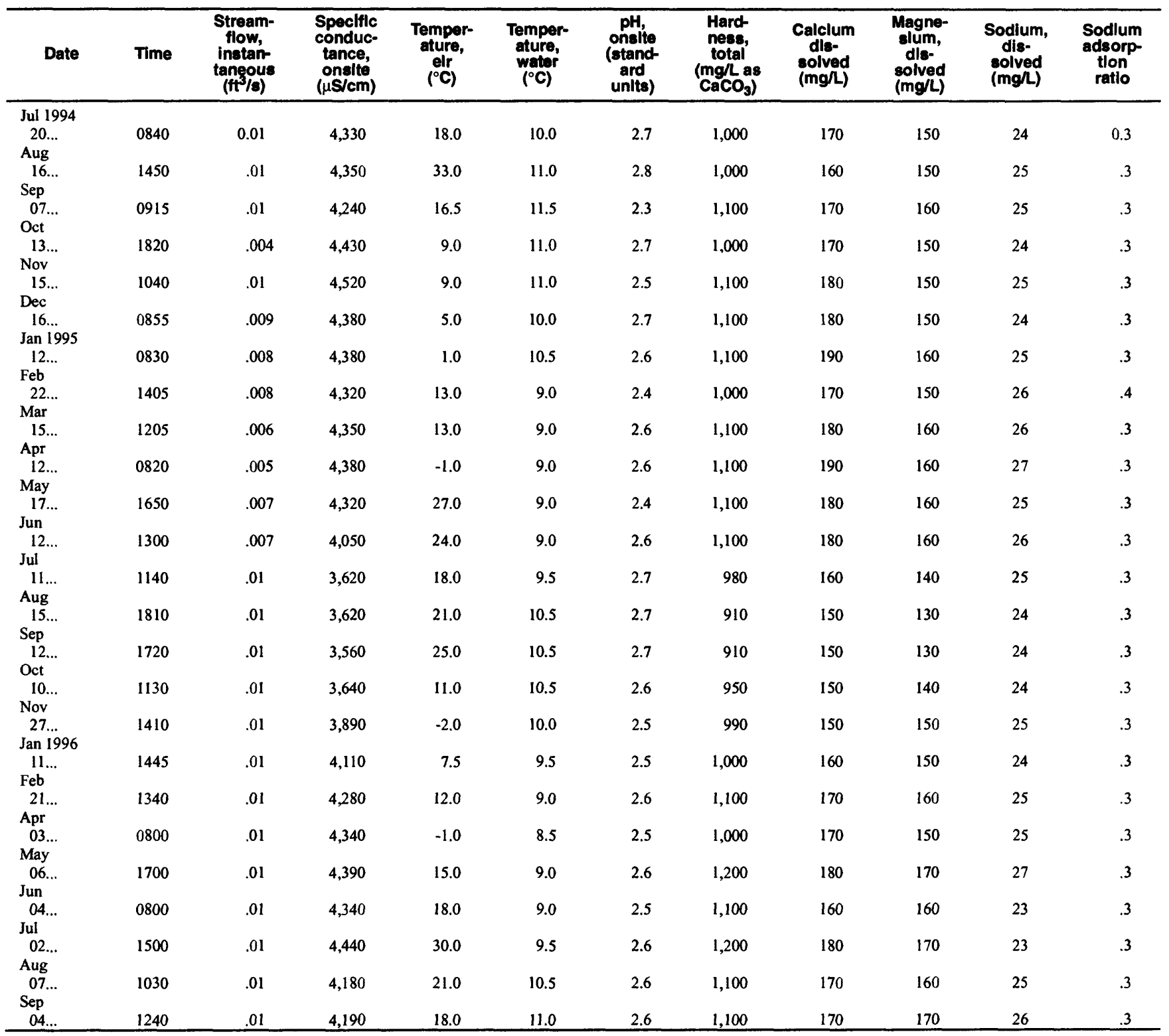


Table 3. Water-quality data for sites in the Sand Coulee Coal Area, Montana, July 1994 through September 1996 (Continued) SITE 25, 472513111082901--JOHNSON BADWATER MINE LARGE WETLANDS INFLOW NEAR TRACY, MT--Continued

\begin{tabular}{|c|c|c|c|c|c|c|c|c|c|c|}
\hline Date & $\begin{array}{l}\text { Potas- } \\
\text { slum, } \\
\text { dissolved } \\
\text { (mglt) }\end{array}$ & $\begin{array}{c}\text { Acidity } \\
\left(\mathbf{m g h}_{\mathbf{H}} \text { as }\right.\end{array}$ & $\begin{array}{c}\text { Alkalinity, } \\
\text { lab } \\
\left(\mathrm{mgl}_{\mathrm{L}} \text { as }\right. \\
\left.\mathrm{CaCO}_{3}\right)\end{array}$ & $\begin{array}{l}\text { Sulfate, } \\
\text { dissolved } \\
\text { (mg/ll) }\end{array}$ & $\begin{array}{l}\text { Chloride, } \\
\text { dissolved } \\
\text { (mg/L) }\end{array}$ & $\begin{array}{l}\text { Fluoride, } \\
\text { dissolved } \\
\text { (mg/L) }\end{array}$ & $\begin{array}{c}\text { Silica, } \\
\text { dissolved } \\
\text { (mg/h as } \\
\mathrm{SiO}_{2} \text { ) }\end{array}$ & $\begin{array}{l}\text { Solids, } \\
\text { sum of } \\
\text { consti- } \\
\text { tuents, } \\
\text { dissolved } \\
\text { (mg/L) }\end{array}$ & $\begin{array}{l}\text { Solids, } \\
\text { dissolved } \\
\text { (ton/ } \\
\text { acre-ft) }\end{array}$ & $\begin{array}{l}\text { Solids, } \\
\text { dissolved } \\
\text { (ton/d) }\end{array}$ \\
\hline Jul 1994 & & & & & & & & & & \\
\hline $\begin{array}{l}20 \ldots \\
\text { Aug }\end{array}$ & 1.4 & 48 & $e<1$ & 3,200 & 6.7 & $<1$ & 77 & $\mathrm{e} 4,220$ & es.74 & $\mathrm{e} 0.15$ \\
\hline $16 \ldots$ & 1.3 & 48 & $e<1$ & 3,300 & 6.7 & $<1$ & 76 & $\mathrm{e} 4,320$ & e5.88 & e. 15 \\
\hline Sep & & & & & & & & & & \\
\hline $\begin{array}{c}07 \ldots \\
\text { Oct }\end{array}$ & 1.3 & 52 & $e<1$ & 3,800 & 7.1 & 2.9 & 76 & e 4,860 & e6.61 & e. 16 \\
\hline $\begin{array}{l}13 . . \\
\text { Nov }\end{array}$ & 1.3 & 46 & $e<1$ & 4,000 & 7.2 & 3.9 & 81 & $e 5,060$ & e6.88 & e. 05 \\
\hline $\begin{array}{c}15 \ldots \\
\text { Dec }\end{array}$ & 1.2 & 47 & $e<1$ & 3,900 & 6.0 & 2.8 & 76 & $\mathrm{e} 4,980$ & e6. 77 & e. 13 \\
\hline $\begin{array}{l}16 \ldots \\
\operatorname{Jan} 1995\end{array}$ & 1.5 & 47 & $e<1$ & 3,400 & 6.1 & - & 77 & $\mathrm{e} 4,450$ & e6.05 & e.11 \\
\hline $\begin{array}{l}12 \ldots \\
\text { Feb }\end{array}$ & 1.1 & 46 & $e<1$ & 3,700 & 5.4 & 3.4 & 77 & $\mathrm{e} 4,780$ & e6.50 & e.11 \\
\hline $\begin{array}{l}22 \ldots \\
\text { Mar }\end{array}$ & 1.8 & 47 & $e<1$ & 4,700 & 21 & 5.0 & 74 & e5,730 & e 7.80 & e. 12 \\
\hline $\begin{array}{l}15 \ldots \\
\mathrm{Apr}\end{array}$ & 1.7 & 44 & $e<1$ & 4,600 & 7.6 & 5.1 & 74 & e 5,620 & e7.64 & e. 10 \\
\hline $\begin{array}{l}12 \ldots \\
\text { May }\end{array}$ & 1.9 & 44 & $e<1$ & 4,700 & 20 & 5.1 & 74 & $e 5,760$ & e 7.84 & e. 08 \\
\hline $\begin{array}{l}17 . . . \\
\text { Jun }\end{array}$ & 2.0 & 42 & $e<1$ & 4,300 & 14 & 5.1 & 74 & e5,330 & e 7.24 & e. 10 \\
\hline$\underset{\text { Jul }}{12 \ldots}$ & 2.3 & 42 & $e<1$ & 4,200 & 5.7 & 6.1 & 74 & $e 5,260$ & e7.15 & e. 10 \\
\hline $\begin{array}{l}11 \ldots \\
\text { Aug }\end{array}$ & 1.9 & 32 & $e<1$ & 2,800 & 5.6 & 2.2 & 72 & e3,630 & e4.94 & e. 12 \\
\hline $\begin{array}{l}15 . . \\
\text { Sep }\end{array}$ & 1.0 & 30 & $e<1$ & 2,800 & 12 & $<1$ & 73 & $\mathrm{e} 3,530$ & e 4.80 & e.11 \\
\hline $\begin{array}{l}12 \ldots \\
\text { Oct }\end{array}$ & 1.1 & 30 & $\mathrm{e}<1$ & 2,600 & 6.3 & 3.2 & 71 & $\mathrm{e} 3,340$ & e4.54 & e. 10 \\
\hline $\begin{array}{l}10 \ldots \\
\text { Nov }\end{array}$ & 1.5 & 32 & $e<1$ & 2,600 & 6.1 & 1.8 & 73 & e3,380 & e 4.60 & e. 10 \\
\hline $\begin{array}{c}27 \ldots \\
\text { Jan } 1996\end{array}$ & 1.2 & 38 & $e<1$ & 2,900 & 5.7 & 3.1 & 73 & e 3,750 & e5.10 & e.11 \\
\hline $\begin{array}{l}11 \ldots \\
\text { Feb }\end{array}$ & 1.2 & 44 & $e<1$ & 3,400 & 9.8 & 1.9 & 79 & $\mathrm{e} 4,370$ & e5.94 & e.13 \\
\hline $\begin{array}{l}21 \ldots \\
\mathrm{Apr}\end{array}$ & 1.3 & 46 & $e<1$ & 3,500 & 6.2 & 3.7 & 83 & $\mathrm{e} 4,550$ & e6.18 & e. 14 \\
\hline $\begin{array}{l}03 \ldots \\
\text { May }\end{array}$ & 1.4 & 45 & $\mathrm{e}<1$ & 3,200 & 8.2 & $<3$ & 79 & $\mathrm{e} 4,250$ & e5.78 & e. 13 \\
\hline $\begin{array}{l}06 . . . \\
\text { Jun }\end{array}$ & 1.6 & 46 & $e<1$ & 3,500 & 7.2 & 3.3 & 87 & $\mathrm{e} 4,570$ & e6.22 & e. 12 \\
\hline $\begin{array}{l}04 \ldots \\
\text { Jul }\end{array}$ & 1.5 & 47 & $e<1$ & 3,300 & 5.8 & 2.4 & 74 & $\mathrm{e} 4,350$ & e5.92 & e. 12 \\
\hline $\begin{array}{l}02 \ldots \\
\text { Aug }\end{array}$ & 1.7 & 48 & $e<1$ & 3,500 & 4.9 & 1.1 & 84 & e 4,650 & e6.32 & e. 13 \\
\hline $\begin{array}{l}07 . . \\
\text { Sep }\end{array}$ & 2.0 & 52 & $e<1$ & 3,200 & 5.9 & 4.3 & 75 & $\mathrm{e} 4,250$ & e5.78 & e.11 \\
\hline $04 \ldots$ & 1.8 & 43 & $\mathrm{e}<1$ & 3,300 & 8.5 & 4.6 & 77 & e 4,360 & e 5.92 & e. 12 \\
\hline
\end{tabular}


Table 3. Water-quality data for sites in the Sand Coulee Coal Area, Montana, July 1994 through September 1996 (Continued)

SITE 25, 472513111082901--JOHNSON BADWATER MINE LARGE WETLANDS INFLOW NEAR TRACY, MT--Continued

\begin{tabular}{|c|c|c|c|c|c|c|c|c|c|c|}
\hline Date & $\begin{array}{c}\text { Alum- } \\
\text { inum, } \\
\text { dissolved } \\
(\mu \mathrm{g} / \mathrm{L})\end{array}$ & $\begin{array}{c}\text { Arsenlc, } \\
\text { dissolved } \\
(\mu \mathrm{g} / \mathrm{L})\end{array}$ & $\begin{array}{l}\text { Barium, } \\
\text { dissolved } \\
(\mu \mathrm{g} / \mathrm{L})\end{array}$ & $\begin{array}{l}\text { Beryl- } \\
\text { lium, } \\
\text { dissolved } \\
(\mu g / L)\end{array}$ & $\begin{array}{c}\text { Boron, } \\
\text { dissolved } \\
(\mu g / L)\end{array}$ & $\begin{array}{c}\text { Cad- } \\
\text { mium, } \\
\text { disesolved } \\
\left(\mu g^{\prime} L\right)\end{array}$ & $\begin{array}{c}\text { Chro- } \\
\text { mium, } \\
\text { dissolved } \\
(\mu g / L)\end{array}$ & $\begin{array}{l}\text { Cobalt, } \\
\text { dissolved } \\
\left(\mu g^{\prime}(\mathrm{L})\right.\end{array}$ & $\begin{array}{l}\text { Copper, } \\
\text { dissolved } \\
(\mu \mathrm{g} / \mathrm{L})\end{array}$ & $\begin{array}{c}\text { Iron, } \\
\text { dissolved } \\
(\mu g / L)\end{array}$ \\
\hline $\begin{array}{l}\text { Jul } 1994 \\
20 \ldots \\
\text { Aug }\end{array}$ & 230,000 & $<1$ & $<3$ & 29 & 280 & 61 & 40 & 2,400 & 190 & 350,000 \\
\hline $\begin{array}{l}\text { Aug } \\
16 \ldots \\
\text { Sep }\end{array}$ & 230,000 & $<1$ & $<3$ & 21 & 350 & 100 & 40 & 1,100 & 200 & 360,000 \\
\hline $\begin{array}{c}07 \ldots \\
\text { Oct }\end{array}$ & 240,000 & $<1$ & $<3$ & 30 & 380 & 95 & 30 & 1,800 & 180 & 360,000 \\
\hline $\begin{array}{l}13 \ldots \\
\text { Nov }\end{array}$ & 240,000 & $<1$ & $<4$ & 32 & 380 & 58 & 30 & 3,800 & 180 & 360,000 \\
\hline $\begin{array}{c}15 \ldots \\
\text { Dec }\end{array}$ & 230,000 & $<1$ & $<10$ & 32 & 360 & 100 & $<50$ & 2,000 & 160 & 390,000 \\
\hline $\begin{array}{l}16 \ldots \\
\text { Jan } 1995\end{array}$ & 230,000 & $<1$ & $<3$ & 32 & 380 & 70 & 40 & 2,400 & 150 & 360,000 \\
\hline $\begin{array}{l}12 \ldots \\
\text { Feb }\end{array}$ & 230,000 & $<1$ & $<4$ & 36 & 330 & 95 & 30 & 2,000 & 140 & 370,000 \\
\hline $\begin{array}{c}22 \ldots \\
\mathrm{Mar}\end{array}$ & 220,000 & $<1$ & $<3$ & 34 & 300 & 81 & 20 & 1,100 & 130 & 350,000 \\
\hline $\begin{array}{l}15 \ldots \\
\text { Apr }\end{array}$ & 200,000 & $<1$ & $<3$ & 34 & 310 & 80 & 20 & 920 & -- & 350,000 \\
\hline $\begin{array}{l}12 \ldots \\
\text { May }\end{array}$ & 210,000 & $<1$ & $<3$ & 35 & 310 & 80 & 20 & 1,000 & 130 & 360,000 \\
\hline $\begin{array}{l}17 \ldots \\
\text { Jun }\end{array}$ & 210,000 & $<1$ & $<10$ & 40 & 320 & 69 & $<50$ & 860 & 120 & 340,000 \\
\hline $\begin{array}{l}12 \ldots \\
\text { Jul }\end{array}$ & 230,000 & $<1$ & $<4$ & 37 & 280 & 70 & 30 & 1,000 & 120 & 360,000 \\
\hline $\begin{array}{l}11 \ldots \\
\text { Aug }\end{array}$ & 170,000 & $<1$ & $<3$ & 25 & 250 & 63 & 20 & 810 & 100 & 240,000 \\
\hline $\begin{array}{l}15 \ldots \\
\text { Sep }\end{array}$ & 150,000 & $<1$ & $<2$ & 24 & 240 & 68 & 20 & 780 & 110 & 180,000 \\
\hline $\begin{array}{l}12 \ldots \\
\text { Oct }\end{array}$ & 170,000 & $<2$ & $<3$ & 22 & 240 & 74 & 40 & 750 & 120 & 170,000 \\
\hline $\begin{array}{l}10 \ldots \\
\text { Nov }\end{array}$ & 160,000 & $<1$ & 17 & 24 & 260 & 77 & $<20$ & 830 & 120 & 210,000 \\
\hline $\begin{array}{l}27 \ldots \\
\text { Jan } 1996\end{array}$ & 190,000 & $<1$ & 18 & 26 & 290 & 80 & 30 & 940 & 140 & 240,000 \\
\hline $\begin{array}{l}11 \ldots \\
\text { Feb }\end{array}$ & 230,000 & $<2$ & 9 & 28 & 280 & 67 & 40 & 940 & 140 & 300,000 \\
\hline $\begin{array}{l}21 \ldots \\
\text { Apr }\end{array}$ & 230,000 & $<1$ & $<5$ & 33 & 290 & 69 & 40 & 930 & 160 & 350,000 \\
\hline $\begin{array}{l}03 \ldots \\
\text { May }\end{array}$ & 250,000 & $<1$ & 21 & 31 & 300 & 86 & 40 & 1,200 & 140 & 350,000 \\
\hline $\begin{array}{l}06 \ldots \\
\text { Jun }\end{array}$ & 240,000 & $<1$ & 21 & 34 & 350 & 85 & 50 & 990 & 160 & 340,000 \\
\hline $\begin{array}{c}04 \ldots \\
\text { Jul }\end{array}$ & 230,000 & $<1$ & 23 & 32 & 270 & 87 & 60 & 1,100 & 120 & 380,000 \\
\hline $\begin{array}{l}02 \ldots \\
\text { Aug }\end{array}$ & 250,000 & $<1$ & $<10$ & 37 & - & 83 & $<50$ & 1,100 & 150 & 410,000 \\
\hline $\begin{array}{l}07 . . . \\
\text { Sep }\end{array}$ & 220,000 & $<5$ & 21 & 36 & - & 76 & 40 & 1,100 & 110 & 370,000 \\
\hline $04 \ldots$ & 210,000 & $<1$ & 20 & 32 & - & 71 & 30 & 960 & 130 & 370,000 \\
\hline
\end{tabular}


Table 3. Water-quality data for sites in the Sand Coulee Coal Area, Montana, July 1994 through September 1996 (Continued)

SITE 25, 472513111082901--JOHNSON BADWATER MINE LARGE WETLANDS INFLOW NEAR TRACY, MT--Continued

\begin{tabular}{|c|c|c|c|c|c|c|c|c|c|c|}
\hline Date & $\begin{array}{l}\text { Lead, } \\
\text { diseolved } \\
(\mu g / L)\end{array}$ & $\begin{array}{l}\text { Llthlum, } \\
\text { dlesolved } \\
(\mu g / L)\end{array}$ & $\begin{array}{l}\text { Manga- } \\
\text { nese, } \\
\text { dissolved } \\
(\mu g / L)\end{array}$ & $\begin{array}{c}\text { Molyb- } \\
\text { denum, } \\
\text { dissolved } \\
(\mu g / L)\end{array}$ & $\begin{array}{c}\text { Nlckel, } \\
\text { dissolved } \\
(\mu \mathrm{g} / \mathrm{L})\end{array}$ & $\begin{array}{c}\text { Sele- } \\
\text { nium, } \\
\text { dissolved } \\
(\mu g / L)\end{array}$ & $\begin{array}{c}\text { Silver, } \\
\text { dlissolved } \\
(\mu g / L)\end{array}$ & $\begin{array}{c}\text { Stron- } \\
\text { tlum, } \\
\text { dissolved } \\
(\mu g / L)\end{array}$ & $\begin{array}{c}\text { Vana- } \\
\text { dlum, } \\
\text { dissolved } \\
(\mu g / L)\end{array}$ & $\begin{array}{l}\text { Zlnc, } \\
\text { dlssolved } \\
(\mu g / L)\end{array}$ \\
\hline $\begin{array}{l}\text { Jul } 1994 \\
20 \ldots \\
\text { Aug }\end{array}$ & $<10$ & 390 & 1,400 & $<10$ & 2,200 & $<2$ & $<3$ & 980 & $<100$ & 9,000 \\
\hline $\begin{array}{l}16 \ldots \\
\text { Sep }\end{array}$ & $<10$ & 420 & 1,500 & $<10$ & 2,200 & $<5$ & $<3$ & 1,000 & $<100$ & 8,400 \\
\hline $\begin{array}{l}07 \ldots \\
\text { Oct }\end{array}$ & $<10$ & 420 & 1,500 & $<10$ & 2,200 & $<2$ & 4 & 1,000 & $<100$ & 8,900 \\
\hline $\begin{array}{l}13 \ldots \\
\text { Nov }\end{array}$ & $<10$ & 400 & 1,500 & $<10$ & 2,300 & $<2$ & $<4$ & 1,000 & $<100$ & 9,400 \\
\hline $\begin{array}{l}15 \ldots \\
\text { Dec }\end{array}$ & $<10$ & 470 & 1,600 & $<10$ & 2,300 & $<2$ & $<10$ & 1,000 & $<100$ & 9,900 \\
\hline $\begin{array}{l}16 \ldots \\
\operatorname{Jan} 1995\end{array}$ & $<10$ & 440 & 1,500 & $<10$ & 2,200 & $<1$ & $<3$ & 1,000 & $<100$ & 8,900 \\
\hline $\begin{array}{l}12 \ldots \\
\text { Feb }\end{array}$ & $<10$ & 450 & 1,600 & $<10$ & 2,400 & $<2$ & $<4$ & 1,100 & $<100$ & 9,400 \\
\hline $\begin{array}{l}22 \ldots \\
\text { Mar }\end{array}$ & $<1$ & 450 & 1,500 & 1 & 2,100 & 1 & $<3$ & 1,100 & 30 & 8,800 \\
\hline $\begin{array}{l}15 \ldots \\
\text { Apr }\end{array}$ & $<1$ & 450 & 1,500 & $<1$ & 2,100 & $<1$ & $<3$ & 1,100 & 43 & 8,800 \\
\hline $\begin{array}{l}12 \ldots \\
\text { May }\end{array}$ & $<1$ & 460 & 1,600 & $<1$ & 2,100 & $<2$ & $<3$ & 1,200 & 38 & 8,800 \\
\hline $\begin{array}{l}17 \ldots \\
\text { Jun }\end{array}$ & $<1$ & 460 & 1,500 & $<1$ & 2,100 & $<2$ & $<10$ & 1,100 & $<60$ & 7,900 \\
\hline $\begin{array}{c}12 \ldots \\
\text { Jul }\end{array}$ & 1 & 460 & 1,600 & $<1$ & 2,200 & $<5$ & $<4$ & 1,100 & 44 & 8,600 \\
\hline $\begin{array}{l}11 \ldots \\
\text { Aug }\end{array}$ & 1 & 390 & 1,300 & $<1$ & 1,700 & -- & $<3$ & 980 & 25 & 6,800 \\
\hline $\begin{array}{l}15 \ldots \\
\text { Sep }\end{array}$ & $<1$ & 350 & 1,100 & $<1$ & 1,600 & - & $<2$ & 960 & 18 & 6,200 \\
\hline $\begin{array}{l}12 \ldots \\
\text { Oct }\end{array}$ & $<1$ & 330 & 1,100 & $<1$ & 1,600 & - & $<3$ & 910 & 22 & 6,200 \\
\hline $\begin{array}{l}10 \ldots \\
\text { Nov }\end{array}$ & 2 & 360 & 1,200 & $<1$ & 1,700 & - & $<3$ & 980 & 24 & 6,800 \\
\hline $\begin{array}{l}27 \ldots \\
\text { Jan } 1996\end{array}$ & $<1$ & 390 & 1,300 & $<1$ & 1,900 & -- & $<3$ & 1,000 & 24 & 7,200 \\
\hline $\begin{array}{l}11 \ldots \\
\text { Feb }\end{array}$ & 1 & 390 & 1,400 & $<1$ & 2,100 & - & $<3$ & 1,000 & 27 & 8,300 \\
\hline $\begin{array}{l}21 \ldots \\
\text { Apr }\end{array}$ & $<1$ & 410 & 1,560 & $<1$ & 2,300 & - & $<5$ & 1,000 & 47 & 8,900 \\
\hline $\begin{array}{l}03 \ldots \\
\text { May }\end{array}$ & 1 & 420 & 1,500 & $<1$ & 2,200 & - & $<5$ & 1,000 & 34 & 8,400 \\
\hline $\begin{array}{l}06 . . \\
\text { Jun }\end{array}$ & 2 & 490 & 1,700 & 2 & 2,500 & - & $<5$ & 1,200 & 42 & 9,100 \\
\hline$\underset{\text { Jul }}{04 \ldots}$ & 1 & 420 & 1,500 & $<1$ & 2,200 & - & $<10$ & 1,000 & 64 & 8,900 \\
\hline $\begin{array}{l}02 \ldots . . \\
\text { Aug }\end{array}$ & 1 & 380 & 1,700 & $<1$ & 2,400 & - & $<10$ & 1,100 & $<60$ & 10,000 \\
\hline $\begin{array}{l}07 . . . \\
\text { Sep }\end{array}$ & $<1$ & 440 & 1,500 & $<1$ & 2,100 & -- & $<6$ & 1,100 & $<36$ & 8,600 \\
\hline $04 \ldots$ & $<2$ & 450 & 1,600 & $<1$ & 2,200 & - & 9 & 1,100 & $<36$ & 8,400 \\
\hline
\end{tabular}


Table 3. Water-quality data for sites in the Sand Coulee Coal Area, Montana, July 1994 through September 1996 (Continued)

SITE 26, 472514111082301--JOHNSON BADWATER MINE SMALL WETLANDS OUTFLOW NEAR TRACY, MT

\begin{tabular}{|c|c|c|c|c|c|c|c|c|c|c|c|}
\hline Date & Time & $\begin{array}{l}\text { Stream- } \\
\text { flow, } \\
\text { Instan- } \\
\text { taneous } \\
\left(f^{3} / s\right)\end{array}$ & $\begin{array}{c}\text { Specliflc } \\
\text { conduc- } \\
\text { tance, } \\
\text { onsite } \\
(\mu \mathrm{S} / \mathrm{cm})\end{array}$ & $\begin{array}{l}\text { Temper- } \\
\text { ature, } \\
\text { air } \\
\left({ }^{\circ} \mathrm{C}\right)\end{array}$ & $\begin{array}{c}\text { Temper- } \\
\text { ature, } \\
\text { water } \\
\left({ }^{\circ} \mathrm{C}\right)\end{array}$ & $\begin{array}{l}\text { pH, } \\
\text { onsite } \\
\text { (stand- } \\
\text { ard } \\
\text { unlts) }\end{array}$ & $\begin{array}{c}\text { Hard- } \\
\text { ness, } \\
\text { total } \\
(\mathrm{mgl} / \mathrm{as} \\
\mathrm{CaCO}_{3} \text { ) }\end{array}$ & $\begin{array}{l}\text { Calcium, } \\
\text { dls- } \\
\text { solved } \\
\text { (mgh) }\end{array}$ & $\begin{array}{l}\text { Magne- } \\
\text { slum, } \\
\text { dis- } \\
\text { solved } \\
\text { ( } m g / \text { L) }\end{array}$ & $\begin{array}{l}\text { Sodlum, } \\
\text { dls- } \\
\text { solved } \\
\text { (mgl) }\end{array}$ & $\begin{array}{c}\text { Sodlum } \\
\text { adsorp- } \\
\text { tlon } \\
\text { ratio }\end{array}$ \\
\hline $\begin{array}{l}\text { Sep } 1994 \\
07 \ldots \\
\text { Oct }\end{array}$ & 0855 & 0.002 & 2,580 & 17.0 & 12.0 & 2.8 & 1,200 & 220 & 150 & 28 & 0.4 \\
\hline $\begin{array}{l}13 \ldots \\
\text { Nov }\end{array}$ & 1805 & .003 & 2,610 & 9.0 & 7.5 & 3.0 & 1,100 & 200 & 140 & 26 & .3 \\
\hline $\begin{array}{c}15 \ldots \\
\text { Jan } 1995\end{array}$ & 1020 & .006 & 2,610 & 9.0 & 1.5 & 3.0 & 1,100 & 210 & 150 & 28 & .4 \\
\hline $\begin{array}{l}12 \ldots \\
\text { Feb }\end{array}$ & 0810 & .005 & 2,390 & 1.0 & 1.0 & 3.2 & 1,200 & 230 & 140 & 26 & .3 \\
\hline $\begin{array}{l}22 \ldots \\
\text { Mar }\end{array}$ & 1350 & .006 & 2,220 & 12.0 & 2.5 & 3.0 & 950 & 180 & 120 & 22 & .3 \\
\hline $\begin{array}{l}15 \ldots \\
\text { Apr }\end{array}$ & 1150 & .004 & 2,530 & 13.0 & 4.0 & 3.0 & 1,100 & 200 & 140 & 26 & .3 \\
\hline $\begin{array}{l}12 \ldots \\
\text { May }\end{array}$ & 0810 & .005 & 2,510 & -.5 & 1.5 & 3.1 & 1,200 & 230 & 150 & 28 & .4 \\
\hline $\begin{array}{c}17 \ldots \\
\text { Jun }\end{array}$ & 1645 & .003 & 2,780 & 27.0 & 19.0 & 2.6 & 1,200 & 250 & 150 & 28 & .3 \\
\hline $\begin{array}{c}12 \ldots \\
\text { Jul }\end{array}$ & 1240 & .004 & 2,550 & 24.0 & 19.5 & 2.7 & 1,200 & 230 & 140 & 27 & .3 \\
\hline $\begin{array}{l}11 \ldots \\
\text { Aug }\end{array}$ & 1210 & .003 & 2,530 & 19.0 & 19.5 & 3.0 & 1,000 & 200 & 130 & 26 & .4 \\
\hline $\begin{array}{l}15 . . \\
\text { Sep }\end{array}$ & 1750 & $<.001$ & 2,690 & 21.0 & 17.0 & 5.9 & 1,600 & 370 & 170 & 31 & .3 \\
\hline $\begin{array}{l}12 \ldots \\
\text { Oct }\end{array}$ & 1700 & $<.001$ & 2,490 & 25.0 & 16.5 & 3.1 & 1,200 & 220 & 150 & 29 & .4 \\
\hline $\begin{array}{l}10 \ldots \\
\text { Nov }\end{array}$ & 1100 & .004 & 2,450 & 10.0 & 8.0 & 2.9 & 960 & 170 & 130 & 25 & .4 \\
\hline $\begin{array}{c}27 \ldots \\
\text { Jan } 1996\end{array}$ & 1400 & .007 & 2,340 & -2.0 & 0.0 & 3.0 & 960 & 170 & 130 & 26 & .4 \\
\hline $\begin{array}{l}11 \ldots \\
\text { Feb }\end{array}$ & 1430 & .003 & 2,170 & 7.5 & .5 & 3.6 & 1,200 & 270 & 130 & 23 & .3 \\
\hline $\begin{array}{l}21 \ldots \\
\text { Apr }\end{array}$ & 1320 & .007 & 2,110 & 12.0 & 1.5 & 3.5 & 1,100 & 220 & 130 & 23 & .3 \\
\hline $\begin{array}{l}03 \ldots \\
\text { May }\end{array}$ & 0750 & .005 & 2,100 & -1.0 & .5 & 3.7 & 1,100 & 240 & 130 & 24 & .3 \\
\hline $\begin{array}{l}06 \ldots \\
\text { Jun }\end{array}$ & 1640 & .001 & 2,650 & 16.0 & 10.0 & 3.5 & 510 & 100 & 64 & 11 & .2 \\
\hline $04 \ldots$ & 0730 & .001 & 2,720 & 18.0 & 13.5 & 3.9 & 1,700 & 340 & 200 & 33 & .4 \\
\hline
\end{tabular}


Table 3. Water-quality data for sites in the Sand Coulee Area, Montana, July 1994 through September 1996 (Continued) SITE 26, 472514111082301--JOHNSON BADWATER MINE SMALL WETLANDS OUTFLOW NEAR TRACY, MT--Continued

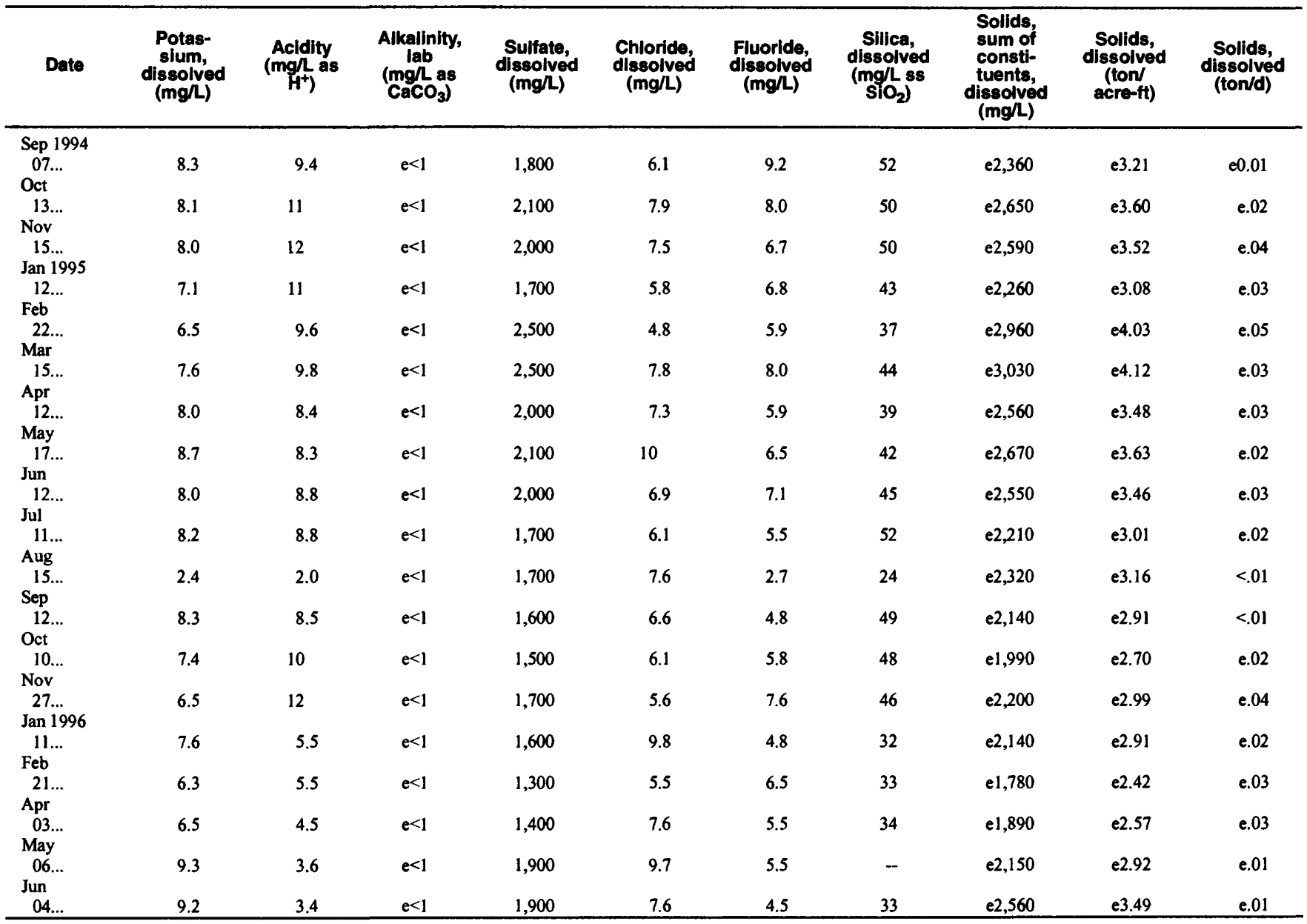


Table 3. Water-quality data for sites in the Sand Coulee Coal Area, Montana, July 1994 through September 1996 (Continued) SITE 26, 472514111082301--JOHNSON BADWATER MINE SMALL WETLANDS OUTFLOW NEAR TRACY, MT--Continued

\begin{tabular}{|c|c|c|c|c|c|c|c|c|c|c|}
\hline Date & $\begin{array}{c}\text { Alum- } \\
\text { inum, } \\
\text { dissolved } \\
(\mu g / L)\end{array}$ & $\begin{array}{l}\text { Arsenic, } \\
\text { dissolved } \\
(\mu g / L)\end{array}$ & $\begin{array}{c}\text { Bsrium, } \\
\text { dissolved } \\
(\mu \mathrm{g} / \mathrm{L})\end{array}$ & $\begin{array}{c}\text { Beryl- } \\
\text { lium, } \\
\text { dissolved } \\
(\mu g / L)\end{array}$ & $\begin{array}{l}\text { Boron, } \\
\text { disagolved } \\
(\mu \mathrm{g} / \mathrm{L})\end{array}$ & $\begin{array}{c}\text { Cad- } \\
\text { mium, } \\
\text { dissolved } \\
(\mu \mathrm{g} / \mathrm{L})\end{array}$ & $\begin{array}{c}\text { Chro- } \\
\text { milum, } \\
\text { dissolved } \\
(\mu g / L)\end{array}$ & $\begin{array}{l}\text { Cobait, } \\
\text { dissolved } \\
(\mu g / L)\end{array}$ & $\begin{array}{l}\text { Copper, } \\
\text { dissolved } \\
(\mu \mathrm{g} / \mathrm{L})\end{array}$ & $\begin{array}{l}\text { Iron, } \\
\text { dissolved } \\
(\mu g / L)\end{array}$ \\
\hline $\begin{array}{l}\text { Sep } 1994 \\
07 \ldots \\
\text { Oct }\end{array}$ & 61,000 & $<1$ & 14 & 27 & 330 & $<3$ & $<20$ & 520 & $<30$ & 17,000 \\
\hline $\begin{array}{l}13 \ldots \\
\text { Nov }\end{array}$ & 59,000 & $<1$ & 9 & 27 & 310 & $<3$ & $<20$ & 670 & $<30$ & 38,000 \\
\hline $\begin{array}{c}15 \ldots \\
\operatorname{Jan} 1995\end{array}$ & 57,000 & $<1$ & 7 & 27 & 290 & 5 & $<20$ & 660 & $<30$ & 60,000 \\
\hline $\begin{array}{l}12 \ldots \\
\text { Feb }\end{array}$ & 48,000 & $<1$ & 8 & 23 & 250 & $<3$ & $<20$ & 540 & $<30$ & 47,000 \\
\hline $\begin{array}{l}22 \ldots \\
\text { Mar }\end{array}$ & 42,000 & $<1$ & 6 & 18 & 220 & 2 & $<20$ & 390 & $<30$ & 37,000 \\
\hline $\begin{array}{c}15 \ldots \\
\text { Apr }\end{array}$ & 50,000 & $<1$ & 7 & 22 & 270 & 2 & $<20$ & 440 & $<30$ & 41,000 \\
\hline $\begin{array}{l}12 \ldots \\
\text { May }\end{array}$ & 43,000 & $<1$ & 7 & 20 & 250 & 2 & $<20$ & 400 & $<30$ & 40,000 \\
\hline $\begin{array}{l}17 \ldots \\
\text { Jun }\end{array}$ & 41,000 & $<1$ & 15 & 22 & 290 & 2 & $<20$ & 280 & $<30$ & 27,000 \\
\hline $\begin{array}{c}12 \ldots \\
\text { Jul }\end{array}$ & 44,000 & $<1$ & 10 & 22 & 260 & 2 & $<20$ & 350 & $<30$ & 32,000 \\
\hline $\begin{array}{l}11 \ldots \\
\text { Aug }\end{array}$ & 43,000 & $<1$ & 9 & 20 & 280 & 2 & $<20$ & 390 & $<30$ & 32,000 \\
\hline $\begin{array}{l}15 \ldots \\
\text { Sep }\end{array}$ & 3,300 & $<1$ & 14 & 3 & 300 & $<1$ & $<10$ & 200 & $<20$ & 4,800 \\
\hline $\begin{array}{l}12 \ldots \\
\text { Oct }\end{array}$ & 46,000 & $<1$ & 13 & 23 & 290 & 2 & $<10$ & 410 & $<20$ & 22,000 \\
\hline $\begin{array}{l}10 \ldots \\
\text { Nov }\end{array}$ & 47,000 & $<1$ & 13 & 24 & 280 & 2 & 20 & 430 & $<30$ & 39,000 \\
\hline $\begin{array}{c}27 \ldots \\
\text { Jan } 1996\end{array}$ & 48,000 & $<1$ & 12 & 23 & 270 & 2 & $<5$ & 400 & $<10$ & 50,000 \\
\hline $\begin{array}{l}11 \ldots \\
\text { Feb }\end{array}$ & 33,000 & $<1$ & 12 & 15 & 200 & 1 & $<20$ & 400 & $<30$ & 22,000 \\
\hline $\begin{array}{l}21 \ldots \\
\text { Apr }\end{array}$ & 34,000 & $<1$ & 8 & 16 & 190 & 2 & $<5$ & 330 & $<10$ & 12,000 \\
\hline $\begin{array}{c}03 \ldots \\
\text { May }\end{array}$ & 32,000 & $<1$ & 11 & 14 & 210 & 1 & $<5$ & 210 & $<10$ & 5,900 \\
\hline $\begin{array}{l}06 \ldots \\
\text { Jun }\end{array}$ & 33,000 & $<1$ & 4 & 5 & 270 & 1 & $<5$ & 340 & $<10$ & 4,200 \\
\hline $04 \ldots$ & 26,000 & $<1$ & 16 & 13 & 320 & $<1$ & $<15$ & 320 & $<30$ & 2,900 \\
\hline
\end{tabular}


Table 3. Water-quality data for sites in the Sand Coulee Coal Area, Montana, July 1994 through September 1996 (Continued) SITE 26, 472514111082301--JOHNSON BADWATER MINE SMALL WETLANDS OUTFLOW NEAR TRACY, MT--Continued

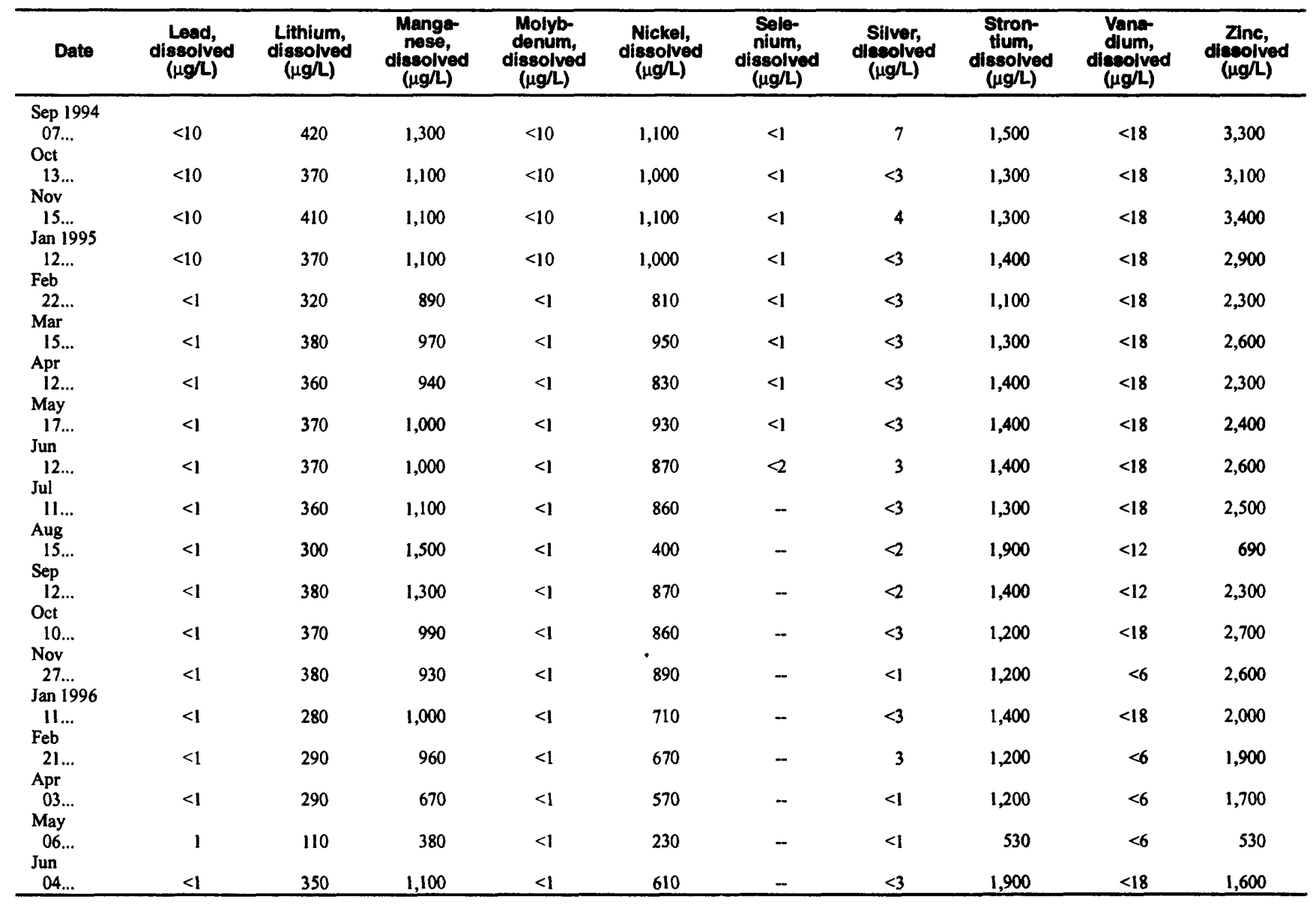


Table 3. Water-quality data for sites in the Sand Coulee Coal Area, Montana, July 1994 through September 1996 (Continued) SITE 27, 472517111081001--JOHNSON GOODWATER MINE SMALL WETLANDS INFLOW NEAR TRACY, MT

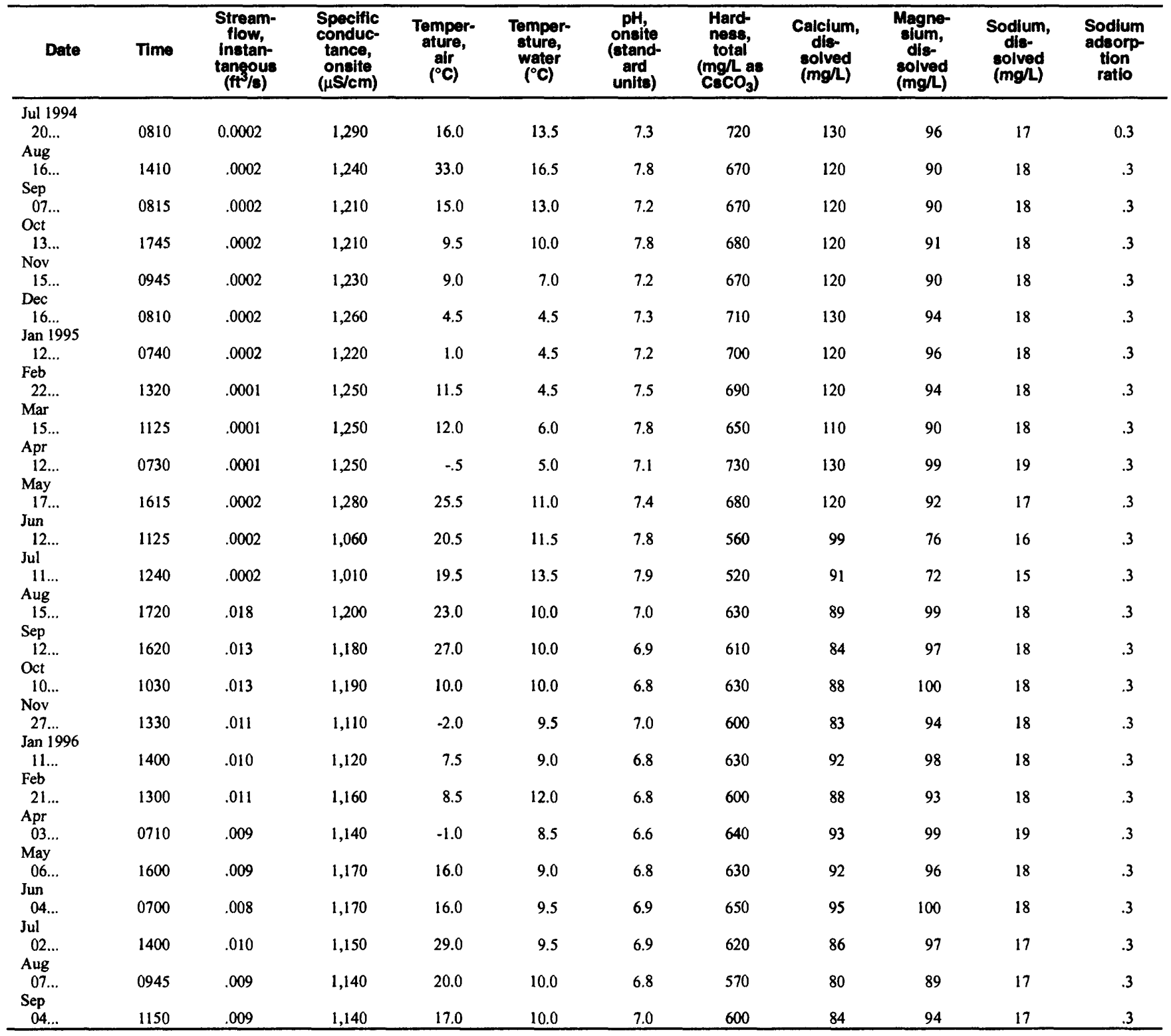


Table 3. Water-quality data for sites in the Sand Coulee Coal Area, Montana, July 1994 through September 1996 (Continued) SITE 27, 472517111081001-.JOHNSON GOODWATER MINE SMALL WETLANDS INFLOW NEAR TRACY, MT--Continued

\begin{tabular}{|c|c|c|c|c|c|c|c|c|c|c|}
\hline Date & $\begin{array}{l}\text { Potas- } \\
\text { sium, } \\
\text { dissolved } \\
\text { (mg/L) }\end{array}$ & $\begin{array}{c}\text { Acidity } \\
\left(\mathrm{mg}_{\mathrm{H}^{+}} \text {) as }\right.\end{array}$ & $\begin{array}{c}\text { Alkalinity, } \\
\text { lab } \\
\left(\mathrm{mg}^{\prime} \mathrm{L} \text { as }\right. \\
\left.\mathrm{CaCO}_{3}\right)\end{array}$ & $\begin{array}{l}\text { Sulfate, } \\
\text { dissolved } \\
\text { (mg/L) }\end{array}$ & $\begin{array}{l}\text { Chloride, } \\
\text { dissolved } \\
\text { (mg/h) }\end{array}$ & $\begin{array}{l}\text { Fluoride, } \\
\text { dissolved } \\
\text { (mg/l) }\end{array}$ & $\begin{array}{c}\text { Sillica, } \\
\text { dissolved } \\
\text { (mg/h as } \\
\left.\mathrm{SiO}_{2}\right)\end{array}$ & $\begin{array}{l}\text { Sollds, } \\
\text { sum of } \\
\text { constl- } \\
\text { tuents, } \\
\text { dissolved } \\
\text { (mg/L) }\end{array}$ & $\begin{array}{l}\text { Solids, } \\
\text { dissolved } \\
\text { (ton/ } \\
\text { acre-ft) }\end{array}$ & $\begin{array}{l}\text { Solids, } \\
\text { dissolved } \\
\text { (ton/d) }\end{array}$ \\
\hline $\begin{array}{c}\text { Jul } 1994 \\
20 \ldots\end{array}$ & 5.5 & 0.4 & 270 & 480 & 7.7 & 1.2 & 12 & 912 & 1.24 & $<.01$ \\
\hline Aug & & & & & & & & & & \\
\hline $\begin{array}{l}16 \ldots \\
\text { Sep }\end{array}$ & 5.3 & .2 & 264 & 450 & 8.1 & 1.1 & 11 & 863 & 1.17 & $<.01$ \\
\hline $\begin{array}{l}07 \ldots \\
\text { Oct }\end{array}$ & 5.3 & .2 & 270 & 430 & 7.5 & 1.2 & 11 & 846 & 1.15 & $<.01$ \\
\hline $\begin{array}{l}13 \ldots \\
\text { Nov }\end{array}$ & 5.1 & .1 & 275 & 450 & 8.1 & 1.2 & 12 & 871 & 1.18 & $<.01$ \\
\hline $\begin{array}{l}15 \ldots \\
\text { Dec }\end{array}$ & 5.1 & .4 & 274 & 430 & 7.6 & 1.4 & 11 & 848 & 1.15 & $<.01$ \\
\hline $\begin{array}{l}16 \ldots \\
\text { Jan } 1995\end{array}$ & 5.3 & .2 & 267 & 450 & 7.7 & 1.3 & 12 & 879 & 1.20 & $<.01$ \\
\hline $\begin{array}{l}12 \ldots \\
\text { Feb }\end{array}$ & 4.8 & 6 & 266 & 450 & 7.2 & 1.2 & 11 & 869 & 1.18 & $<.01$ \\
\hline $\begin{array}{l}22 \ldots \\
\text { Mar }\end{array}$ & 5.0 & $<.1$ & 265 & 460 & 7.6 & 1.2 & 11 & 877 & 1.19 & $<.01$ \\
\hline $\begin{array}{l}15 \ldots \\
\text { Apr }\end{array}$ & 5.0 & $<.1$ & 264 & 420 & 8.3 & 1.3 & 11 & 823 & 1.12 & $<.01$ \\
\hline $\begin{array}{l}12 \ldots . . \\
\text { May }\end{array}$ & 4.8 & .1 & 268 & 420 & 6.3 & 1.2 & 12 & 854 & 1.16 & $<.01$ \\
\hline $\begin{array}{l}17 \ldots \\
\text { Jun }\end{array}$ & 4.9 & $<.1$ & 255 & 420 & 8.8 & 1.2 & 11 & 829 & 1.13 & $<.01$ \\
\hline Jul & 5.0 & $<.1$ & 270 & 290 & 6.7 & 1.6 & 11 & 668 & .91 & $<.01$ \\
\hline $\begin{array}{l}11 \ldots \\
\text { Aug }\end{array}$ & 5.1 & $<.1$ & 281 & 270 & 6.4 & 1.5 & 10 & 640 & .87 & $<.01$ \\
\hline $\begin{array}{l}15 \ldots \\
\text { Sep }\end{array}$ & 4.5 & .4 & 174 & 450 & 6.9 & 1.4 & 9.1 & 787 & 1.07 & .04 \\
\hline $\begin{array}{l}12 \ldots \\
\text { Oct }\end{array}$ & 4.6 & .1 & 180 & 480 & 6.9 & 1.2 & 8.4 & 811 & 1.10 & .03 \\
\hline $\begin{array}{l}10 \ldots \\
\text { Nov }\end{array}$ & 4.3 & .1 & 184 & 470 & 7.4 & 1.3 & 8.4 & 810 & 1.10 & .03 \\
\hline $\begin{array}{l}27 \ldots \\
\text { Jan } 1996\end{array}$ & 4.2 & 1.7 & 208 & 440 & 7.1 & 1.5 & 8.3 & 782 & 1.06 & .02 \\
\hline $\begin{array}{l}11 \ldots \\
\text { Feb }\end{array}$ & 4.4 & .1 & 200 & 460 & 6.2 & 1.5 & 8.7 & 810 & 1.10 & .02 \\
\hline $\begin{array}{l}21 \ldots \\
\text { Apr }\end{array}$ & 4.1 & .4 & 202 & 440 & 7.2 & 1.4 & 8.4 & 783 & 1.06 & .02 \\
\hline $\begin{array}{l}03 \ldots \\
\text { May }\end{array}$ & 4.2 & .4 & 199 & 450 & 7.0 & 1.5 & 8.8 & 803 & 1.09 & .02 \\
\hline $\begin{array}{c}06 \ldots \\
\text { Jun }\end{array}$ & 4.4 & .3 & 201 & 450 & 6.9 & 1.5 & 8.6 & 799 & 1.09 & .02 \\
\hline $\begin{array}{c}04 \ldots \\
\text { Jul }\end{array}$ & 4.5 & $<.1$ & 203 & 460 & 6.8 & 1.3 & 8.8 & 818 & 1.11 & .02 \\
\hline $\begin{array}{l}02 \ldots \\
\text { Aug }\end{array}$ & 4.3 & .2 & 211 & 420 & 6.5 & 1.4 & 8.7 & 769 & 1.05 & .02 \\
\hline $\begin{array}{l}07 \ldots \\
\text { Sep }\end{array}$ & 4.8 & $<.1$ & 211 & 430 & 6.9 & 1.3 & 7.7 & 764 & 1.04 & .02 \\
\hline $04 \ldots$ & 4.2 & .3 & 213 & 430 & 7.1 & 1.3 & 7.9 & 774 & 1.05 & .02 \\
\hline
\end{tabular}


Table 3. Water-quality data for sites in the Sand Coulee Coal Area, Montana, July 1994 through September 1996 (Continued) SITE 27, 472517111081001--JOHNSON GOODWATER MINE SMALL WETLANDS INFLOW NEAR TRACY, MT--Continued

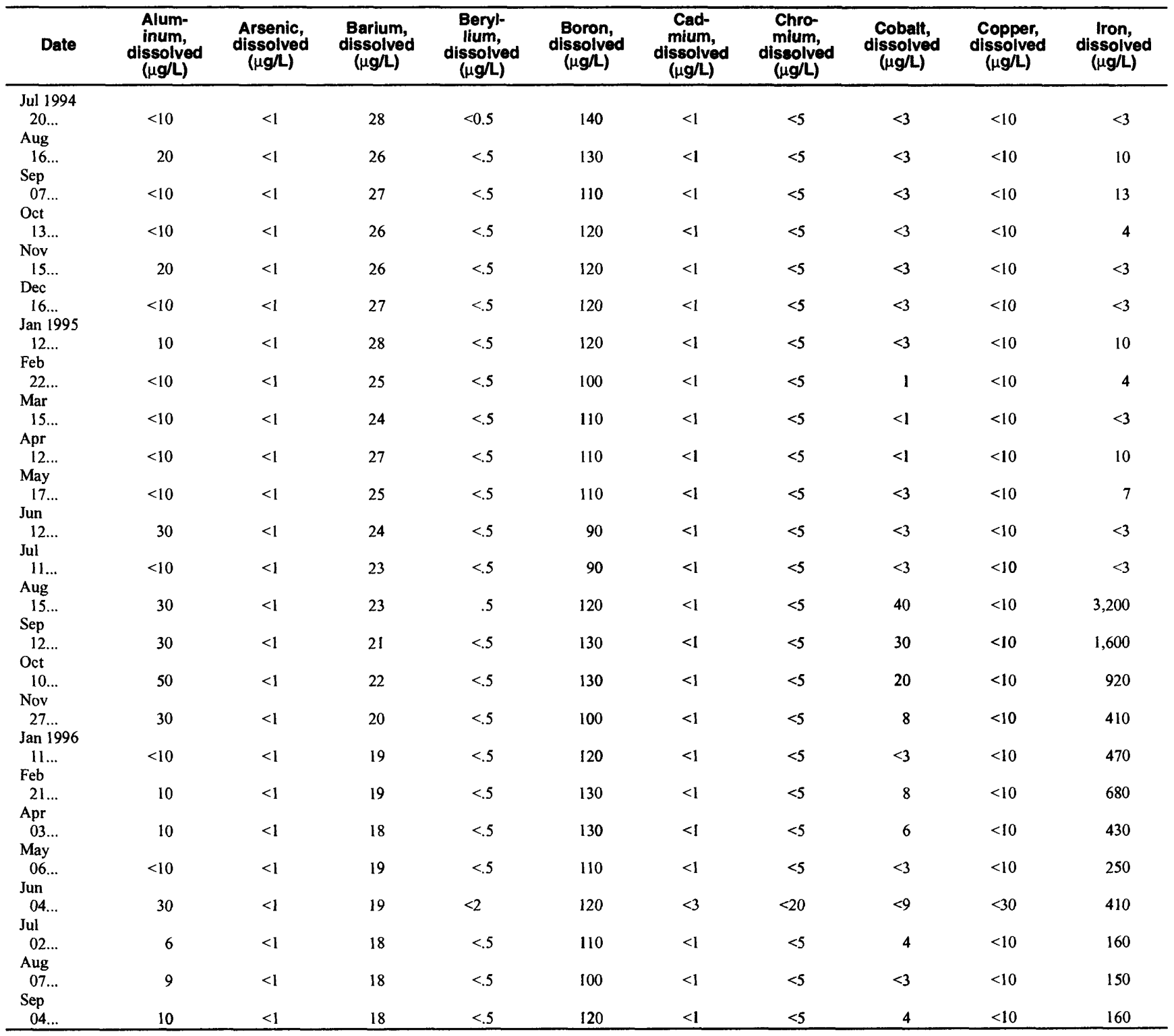


Table 3. Water-quality data for sites in the Sand Coulee Coal Area, Montana, July 1994 through September 1996 (Continued) SITE 27, 472517111081001--JOHNSON GOODWATER MINE SMALL WETLANDS INFLOW NEAR TRACY, MT--Continued

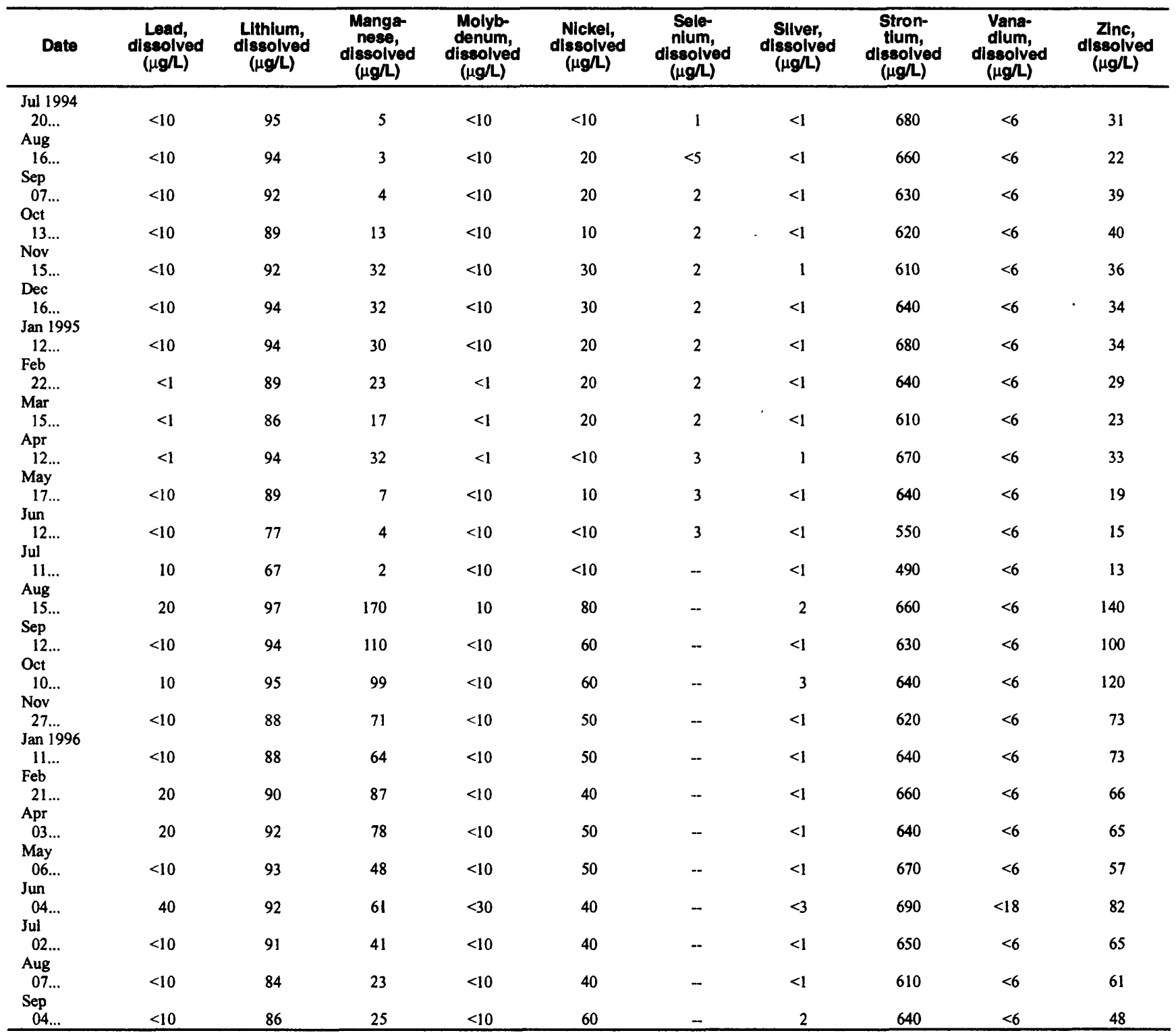


Table 4. Chemical analyses of field replicates for water samples from sites in the Sand Coulee Coal Area, Montana, July 1994 through September 1996

[Site number shown in figure 2 or 3 . Abbreviations: $\mathrm{ft}^{3} / \mathrm{s}$, cubic feet per second; ${ }^{\circ} \mathrm{C}$, degrees Celsius; e, estimated; lab, laboratory; $\mu \mathrm{g} / \mathrm{L}$, micrograms per liter; $\mu \mathrm{S} / \mathrm{cm}$, microsimens per centimeter at $25^{\circ} \mathrm{C} ; \mathrm{mg} / \mathrm{L}$, milligrams per liter. Symbols: <, less than; --, no data]

SITE 2, 06078250--COTTONWOOD CREEK NEAR STOCKETT, MT

\begin{tabular}{|c|c|c|c|c|c|c|c|c|c|c|}
\hline Date & Time & $\begin{array}{c}\text { Calcium, } \\
\text { dis- } \\
\text { solved } \\
\text { (mg/L) }\end{array}$ & $\begin{array}{l}\text { Magne- } \\
\text { sium, } \\
\text { dis- } \\
\text { solved } \\
\text { (mg/l) }\end{array}$ & $\begin{array}{l}\text { Sodium, } \\
\text { dis- } \\
\text { solved } \\
\text { (mg/l) }\end{array}$ & $\begin{array}{l}\text { Potas- } \\
\text { sium, } \\
\text { dissolved } \\
\text { (mg/L) }\end{array}$ & $\begin{array}{l}\text { Acidity } \\
\text { (mg/L) }\end{array}$ & $\begin{array}{c}\text { Alkalinity, } \\
\text { lab } \\
(\underset{\mathrm{mg} / \mathrm{L}}{\mathrm{as}} \\
\left.\mathrm{CsCO}_{3}\right)\end{array}$ & $\begin{array}{l}\text { Sulfate, } \\
\text { dissolved } \\
\text { (mg/L) }\end{array}$ & $\begin{array}{l}\text { Chloride, } \\
\text { dissolived } \\
\text { (mg/L) }\end{array}$ & $\begin{array}{l}\text { Fluoride, } \\
\text { dissolved } \\
\text { (mg/L) }\end{array}$ \\
\hline \multicolumn{11}{|l|}{ May 1996} \\
\hline $06 \ldots$ & 1300 & 160 & 73 & 11 & 4.4 & $<0.1$ & 153 & 550 & 5.6 & 0.8 \\
\hline $06 \ldots$ & 1305 & 160 & 74 & 11 & 4.5 & .1 & 152 & 540 & 5.5 & .8 \\
\hline
\end{tabular}

\begin{tabular}{|c|c|c|c|c|c|c|c|c|c|c|}
\hline Date & $\begin{array}{l}\text { Sillica, } \\
\text { dissolved } \\
\text { (mg/L) }\end{array}$ & $\begin{array}{l}\text { Alum- } \\
\text { Inum, } \\
\text { dissolved } \\
(\mu g / L)\end{array}$ & $\begin{array}{c}\text { Arsenic, } \\
\text { dissolved } \\
(\mu g / L)\end{array}$ & $\begin{array}{l}\text { Barium, } \\
\text { dissolved } \\
(\mu g / L)\end{array}$ & $\begin{array}{l}\text { Beryl- } \\
\text { lium, } \\
\text { dissolved } \\
(\mu g / L)\end{array}$ & $\begin{array}{l}\text { Boron, } \\
\text { dissolved } \\
(\mu \mathrm{g} / \mathrm{L})\end{array}$ & $\begin{array}{c}\text { Csd- } \\
\text { mium, } \\
\text { dissolved } \\
(\mu g / L)\end{array}$ & $\begin{array}{c}\text { Chro- } \\
\text { mium, } \\
\text { dissolved } \\
(\mu g / L)\end{array}$ & $\begin{array}{l}\text { Cobalt, } \\
\text { dissolved } \\
(\mu g / L)\end{array}$ & $\begin{array}{l}\text { Copper, } \\
\text { dissolved } \\
(\mu g / L)\end{array}$ \\
\hline $\begin{array}{c}\text { May } 1996 \\
06 . . \\
06 . .\end{array}$ & $\begin{array}{l}3.5 \\
3.5\end{array}$ & $\begin{array}{l}210 \\
240\end{array}$ & $\begin{array}{l}<1 \\
<1\end{array}$ & $\begin{array}{l}24 \\
25\end{array}$ & $\begin{array}{r}<0.5 \\
<.5\end{array}$ & $\begin{array}{l}80 \\
80\end{array}$ & $\begin{array}{l}<1 \\
<1\end{array}$ & $\begin{array}{l}<5 \\
<5\end{array}$ & $\begin{array}{l}60 \\
60\end{array}$ & $\begin{array}{l}<10 \\
<10\end{array}$ \\
\hline
\end{tabular}

\begin{tabular}{|c|c|c|c|c|c|c|c|c|c|c|}
\hline Dste & $\begin{array}{l}\text { Iron, } \\
\text { dissolved } \\
(\mu g / L)\end{array}$ & $\begin{array}{l}\text { Lead, } \\
\text { dissolved } \\
(\mu \mathrm{g} / \mathrm{L})\end{array}$ & $\begin{array}{c}\text { Lithium, } \\
\text { dissolved } \\
(\mu \mathrm{g} / \mathrm{L})\end{array}$ & $\begin{array}{l}\text { Manga- } \\
\text { nese, } \\
\text { dissolved } \\
(\mu g / L)\end{array}$ & $\begin{array}{l}\text { Molyb- } \\
\text { denum, } \\
\text { dissolved } \\
(\mu g / L)\end{array}$ & $\begin{array}{c}\text { Nickel, } \\
\text { dissolved } \\
(\mu \mathrm{g} / \mathrm{L})\end{array}$ & $\begin{array}{l}\text { Silver, } \\
\text { dissolved } \\
(\mu g / L)\end{array}$ & $\begin{array}{c}\text { Stron- } \\
\text { tium, } \\
\text { dissolved } \\
(\mu \mathrm{g} / \mathrm{L})\end{array}$ & $\begin{array}{l}\text { Vana- } \\
\text { dium, } \\
\text { dissolved } \\
(\mu g / L)\end{array}$ & $\begin{array}{l}\text { Zinc, } \\
\text { dissolved } \\
(\mu g / L)\end{array}$ \\
\hline May 1996 & & & & & & & & & & \\
\hline
\end{tabular}

SITE 4, 06078270--SAND COULEE AT SAND COULEE, MT

\begin{tabular}{|c|c|c|c|c|c|c|c|c|c|c|}
\hline Date & Time & $\begin{array}{l}\text { Csiclum, } \\
\text { dis- } \\
\text { solved } \\
\text { (mg/L) }\end{array}$ & $\begin{array}{l}\text { Magne- } \\
\text { slum, } \\
\text { dis- } \\
\text { solved } \\
\text { (mg/L) }\end{array}$ & $\begin{array}{l}\text { Sodium, } \\
\text { dis- } \\
\text { solved } \\
\text { (mgll) }\end{array}$ & $\begin{array}{l}\text { Potas- } \\
\text { sium, } \\
\text { dissolved } \\
\text { (mg/l) }\end{array}$ & $\begin{array}{l}\text { Acidity } \\
\text { (mg/L) }\end{array}$ & $\begin{array}{c}\text { Aikalinity, } \\
\text { leb } \\
\text { (mg/l as } \\
\left.\mathrm{CaCO}_{3}\right)\end{array}$ & $\begin{array}{l}\text { Sulfate, } \\
\text { dissolved } \\
\text { (mg/L) }\end{array}$ & $\begin{array}{l}\text { Chloride, } \\
\text { dissolved } \\
\text { (mg/L) }\end{array}$ & $\begin{array}{l}\text { Fluoride, } \\
\text { dissolved } \\
\text { (mg/L) }\end{array}$ \\
\hline \multicolumn{11}{|l|}{ Jun 1995} \\
\hline $13 \ldots$ & 1150 & 110 & 85 & 20 & 3.5 & 11 & $e<1$ & 1,700 & 8.5 & 1.7 \\
\hline $13 \ldots$ & 1155 & 120 & 88 & 21 & 3.5 & 12 & $e<1$ & 1,600 & 8.6 & 2.5 \\
\hline \multicolumn{11}{|l|}{ Jul 1996} \\
\hline $02 \ldots$ & 0820 & 180 & 120 & 22 & 2.6 & 33 & $e<1$ & 2,700 & 7.1 & .7 \\
\hline $02 \ldots$ & 0825 & 190 & 130 & 22 & 2.5 & 32 & $e<1$ & 2,600 & 7.7 & .7 \\
\hline
\end{tabular}

\begin{tabular}{|c|c|c|c|c|c|c|c|c|c|c|}
\hline Date & $\begin{array}{l}\text { Sillca, } \\
\text { dissolved } \\
\text { (mg/L) }\end{array}$ & $\begin{array}{c}\text { Alum- } \\
\text { inum, } \\
\text { dissolved } \\
(\mu g / L)\end{array}$ & $\begin{array}{l}\text { Arsenic, } \\
\text { dlesolved } \\
\text { ( } \mu \mathrm{g} / \mathrm{L})\end{array}$ & $\begin{array}{l}\text { Barium, } \\
\text { dissolved } \\
\text { ( } \mu \mathrm{g} / \mathrm{L})\end{array}$ & $\begin{array}{l}\text { Beryl- } \\
\text { fium, } \\
\text { dissolved } \\
\text { ( } \mu g / L)\end{array}$ & $\begin{array}{l}\text { Boron, } \\
\text { diseolved } \\
\text { ( } \mu \mathrm{g} / \mathrm{L})\end{array}$ & $\begin{array}{l}\text { Cad- } \\
\text { mium, } \\
\text { dissolved } \\
(\mu g / L)\end{array}$ & $\begin{array}{c}\text { Chro- } \\
\text { mium, } \\
\text { dissolved } \\
(\mu g / \mathrm{L})\end{array}$ & 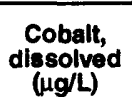 & $\begin{array}{l}\text { Copper, } \\
\text { dissolved } \\
(\mu \mathrm{g} / \mathrm{L})\end{array}$ \\
\hline \multicolumn{11}{|l|}{ Jun 1995} \\
\hline $13 \ldots$ & 26 & 73,000 & $<1$ & 120 & 11 & 140 & 9 & $<5$ & 270 & 20 \\
\hline $13 \ldots$ & 27 & 74,000 & $<1$ & 130 & 12 & 120 & 9 & $<5$ & 260 & 20 \\
\hline \multicolumn{11}{|l|}{ Jul 1996} \\
\hline $02 \ldots$ & 57 & 190,000 & 2 & 11 & 26 & 200 & 21 & 60 & 790 & $<80$ \\
\hline $02 \ldots$ & 58 & 190,000 & 2 & 9 & 26 & 160 & 22 & $<40$ & 800 & $<80$ \\
\hline
\end{tabular}

\begin{tabular}{|c|c|c|c|c|c|c|c|c|c|c|c|}
\hline Date & $\begin{array}{l}\text { Iron, } \\
\text { diessolved } \\
\text { ( } \mu \mathrm{g} / \mathrm{L})\end{array}$ & $\begin{array}{l}\text { Lead, } \\
\text { dleselved } \\
\text { ( } \mu \mathrm{g} / \mathrm{L})\end{array}$ & $\begin{array}{l}\text { Lithium, } \\
\text { diseolved } \\
(\mu \mathrm{g} / \mathrm{L})\end{array}$ & $\begin{array}{l}\text { Manga- } \\
\text { nese, } \\
\text { dissolved } \\
(\mu g / L)\end{array}$ & $\begin{array}{l}\text { Molyb- } \\
\text { denum, } \\
\text { dissolved } \\
(\mu \mathrm{g} / \mathrm{L})\end{array}$ & $\begin{array}{l}\text { Nickel, } \\
\text { diseolved } \\
\text { ( } \mu \mathrm{g} / \mathrm{L})\end{array}$ & $\begin{array}{l}\text { Selenium, } \\
\text { dissolved } \\
\text { ( } \mu \mathrm{g} / \mathrm{L})\end{array}$ & $\begin{array}{l}\text { Silver, } \\
\text { dissolved } \\
(\mu \mathrm{g} / \mathrm{l})\end{array}$ & $\begin{array}{c}\text { Stron- } \\
\text { tium, } \\
\text { dissolved } \\
(\mu \mathrm{g} / \mathrm{L})\end{array}$ & $\begin{array}{c}\text { Vana- } \\
\text { dium, } \\
\text { dissolved } \\
(\mu g / L)\end{array}$ & $\begin{array}{c}\text { Zinc, } \\
\text { dissolved } \\
(\mu g / L)\end{array}$ \\
\hline \multicolumn{12}{|l|}{ Jun 1995} \\
\hline $13 \ldots$ & 86,000 & $<1$ & 190 & 1,100 & $<1$ & 750 & $<1$ & $<1$ & 730 & $<6$ & 3,000 \\
\hline $13 \ldots$ & 88,000 & $<1$ & 200 & 1,100 & $<1$ & 760 & $<1$ & $<1$ & 760 & $<6$ & 3,000 \\
\hline \multicolumn{12}{|l|}{ Jul 1996} \\
\hline $02 \ldots$ & 150,000 & 1 & 380 & 2,600 & $<1$ & 1,600 & -- & 10 & 950 & $<48$ & 6,800 \\
\hline $02 \ldots$ & 150,000 & $<1$ & 380 & 2,700 & $<1$ & 1,700 & - & $<8$ & 970 & $<48$ & 6,900 \\
\hline
\end{tabular}


Table 4. Chemical analyses of field replicates for water samples from sites in the Sand Coulee Coal Area, July 1994 through September 1996 (Continued)

SITE 5, 06090590--ANACONDA DRAIN AT BELT, MT

\begin{tabular}{|c|c|c|c|c|c|c|c|c|c|c|}
\hline Date & Time & $\begin{array}{c}\text { Calclum, } \\
\text { dls- } \\
\text { solved } \\
\text { (mg/l) }\end{array}$ & $\begin{array}{l}\text { Magne- } \\
\text { sium, } \\
\text { dis- } \\
\text { solved } \\
\text { (mg/l) }\end{array}$ & $\begin{array}{c}\text { Sodlum, } \\
\text { dls- } \\
\text { solved } \\
\text { (mg/l) }\end{array}$ & $\begin{array}{l}\text { Potas- } \\
\text { sium, } \\
\text { dissolved } \\
\text { (mg/ll) }\end{array}$ & $\begin{array}{l}\text { Acldity } \\
\text { (mg/L) }\end{array}$ & $\begin{array}{c}\text { Alkalinity, } \\
\text { lab } \\
\left(\mathrm{mg}_{\mathrm{L}} \text { as }\right. \\
\left.\mathrm{CaCO}_{3}\right)\end{array}$ & $\begin{array}{l}\text { Sulfate, } \\
\text { dissolved } \\
\text { (mg/l) }\end{array}$ & $\begin{array}{l}\text { Chloride, } \\
\text { dlseolved } \\
\text { (mg/l) }\end{array}$ & $\begin{array}{c}\text { Fluoride, } \\
\text { dissolved } \\
(\mathrm{mg} / \mathrm{L})\end{array}$ \\
\hline Oct 1995 & & & & & & & & & & \\
\hline $12 \ldots$ & 1145 & 150 & 67 & 9.7 & 2.7 & 23 & $e<1$ & 1,900 & 2.5 & 1.5 \\
\hline $12 \ldots$ & 1150 & 150 & 69 & 10 & 2.7 & 22 & $\mathrm{e}<1$ & 1,800 & 2.6 & 1.2 \\
\hline
\end{tabular}

\begin{tabular}{|c|c|c|c|c|c|c|c|c|c|c|}
\hline Date & $\begin{array}{l}\text { Silica, } \\
\text { dissolved } \\
\text { (mg/l) }\end{array}$ & $\begin{array}{c}\text { Alum- } \\
\text { Inum, } \\
\text { dissolved } \\
(\mu g / L)\end{array}$ & $\begin{array}{l}\text { Arsenlc, } \\
\text { dissolved } \\
\text { ( } \mu \mathrm{g} / \mathrm{L})\end{array}$ & $\begin{array}{c}\text { Barium, } \\
\text { dissolved } \\
(\mu g / L)\end{array}$ & $\begin{array}{c}\text { Beryl- } \\
\text { llum, } \\
\text { diseolved } \\
(\mu \mathrm{g} / \mathrm{L})\end{array}$ & $\begin{array}{l}\text { Boron, } \\
\text { dissolved } \\
(\mu \mathrm{g} / \mathrm{L})\end{array}$ & $\begin{array}{l}\text { Cad- } \\
\text { mium, } \\
\text { dissolved } \\
(\mu \mathrm{g} / \mathrm{L})\end{array}$ & $\begin{array}{c}\text { Chro- } \\
\text { mium, } \\
\text { dissolved } \\
(\mu \mathrm{g} / \mathrm{L})\end{array}$ & $\begin{array}{l}\text { Cobalt, } \\
\text { dlssolved } \\
\text { ( } \mu \mathrm{g} / \mathrm{L})\end{array}$ & $\begin{array}{l}\text { Copper, } \\
\text { dlssolved } \\
(\mu \mathrm{g} / \mathrm{L})\end{array}$ \\
\hline \multicolumn{11}{|l|}{ Oct 1995} \\
\hline $12 \ldots$ & 58 & 110,000 & 1 & 11 & 16 & 180 & 10 & 50 & 360 & 40 \\
\hline $12 \ldots$ & 58 & 110,000 & 2 & 10 & 17 & 170 & 10 & 40 & 360 & $<30$ \\
\hline
\end{tabular}

\begin{tabular}{|c|c|c|c|c|c|c|c|c|c|c|c|}
\hline Date & $\begin{array}{l}\text { Iron, } \\
\text { dissolved } \\
(\mu \mathrm{g} / \mathrm{L})\end{array}$ & $\begin{array}{l}\text { Lead, } \\
\text { diseolved } \\
(\mu g / \mathrm{L})\end{array}$ & $\begin{array}{l}\text { Lithlum, } \\
\text { dissolved } \\
(\mu \mathrm{g} / \mathrm{L})\end{array}$ & $\begin{array}{l}\text { Manga- } \\
\text { nese, } \\
\text { dissolved } \\
(\mu \mathrm{g} / \mathrm{L})\end{array}$ & $\begin{array}{c}\text { Molyb- } \\
\text { denum, } \\
\text { dissolved } \\
(\mu g / L)\end{array}$ & $\begin{array}{l}\text { Nickel, } \\
\text { dlseolved } \\
\text { ( } \mu \mathrm{g} / \mathrm{L})\end{array}$ & $\begin{array}{l}\text { Selenium, } \\
\text { dlseolved } \\
\text { ( } \mu \mathrm{g} / \mathrm{L})\end{array}$ & $\begin{array}{l}\text { Sllver, } \\
\text { dlssolved } \\
(\mu \mathrm{g} / \mathrm{L})\end{array}$ & $\begin{array}{c}\text { Stron- } \\
\text { tlum, } \\
\text { dissolved } \\
(\mu \mathrm{g} / \mathrm{L})\end{array}$ & $\begin{array}{l}\text { Vana- } \\
\text { dlum, } \\
\text { dissolved } \\
(\mu \mathrm{g} / \mathrm{L})\end{array}$ & $\begin{array}{c}\text { Zinc, } \\
\text { dissolved } \\
(\mu \mathrm{g} / L)\end{array}$ \\
\hline \multicolumn{12}{|l|}{ Oct 1995} \\
\hline $12 \ldots$ & 180,000 & $<1$ & 190 & 410 & $<1$ & 820 & - & $<3$ & 1,500 & $<18$ & 3,600 \\
\hline $12 \ldots$ & 180,000 & 1.0 & 200 & 420 & $<1$ & 800 & - & $<3$ & 1,500 & 32 & 3,600 \\
\hline
\end{tabular}

SITE 6, 471851111111101--GIFFEN SPRING NEAR STOCKETT, MT

\begin{tabular}{|c|c|c|c|c|c|c|c|c|c|c|}
\hline Date & Time & $\begin{array}{l}\text { Calclum, } \\
\text { dls- } \\
\text { solved } \\
\text { (mg/l) }\end{array}$ & $\begin{array}{l}\text { Magne- } \\
\text { slum, } \\
\text { dls- } \\
\text { solved } \\
\text { (mg/h) }\end{array}$ & $\begin{array}{c}\text { Sodium, } \\
\text { dis- } \\
\text { solved } \\
\text { (mg/l) }\end{array}$ & $\begin{array}{l}\text { Potas- } \\
\text { sium, } \\
\text { dissolved } \\
\text { (mg/L) }\end{array}$ & $\begin{array}{l}\text { Acidlty } \\
\text { (mg/l) }\end{array}$ & $\begin{array}{c}\text { Alkalinity, } \\
\text { lab } \\
\left(\begin{array}{c}\text { mg/l as } \\
\text { Caco }\end{array}\right)\end{array}$ & $\begin{array}{l}\text { Sulfate, } \\
\text { dissolved } \\
\text { (mg/l) }\end{array}$ & $\begin{array}{l}\text { Chioride, } \\
\text { diseolved } \\
\text { (mg/L) }\end{array}$ & $\begin{array}{l}\text { Fluoride, } \\
\text { dissolved } \\
\text { (mg/L) }\end{array}$ \\
\hline \multicolumn{11}{|l|}{ Jul 1994} \\
\hline $21 \ldots$ & 0845 & 110 & 42 & 14 & 5.4 & 4.5 & $e<1$ & 740 & 3.1 & 0.4 \\
\hline $21 \ldots$ & 0850 & 120 & 42 & 14 & 5.3 & 4.5 & $<1$ & 740 & 3.2 & .5 \\
\hline \multicolumn{11}{|l|}{ Sep 1996} \\
\hline $03 \ldots$ & 1015 & 110 & 43 & 15 & 5.3 & 1.5 & $e<1$ & 550 & 4.0 & .8 \\
\hline $03 \ldots$ & 1015 & 110 & 41 & 14 & 5.5 & 1.5 & $e<1$ & 540 & 3.6 & .8 \\
\hline
\end{tabular}

\begin{tabular}{|c|c|c|c|c|c|c|c|c|c|c|}
\hline Date & $\begin{array}{l}\text { Silica, } \\
\text { dissolved } \\
\text { (mg/L) }\end{array}$ & $\begin{array}{l}\text { Alum- } \\
\text { Inum, } \\
\text { dissolved } \\
(\mu g / \mathrm{L})\end{array}$ & $\begin{array}{l}\text { Arsenic, } \\
\text { dlssolved } \\
(\mu \mathrm{g} / \mathrm{L})\end{array}$ & $\begin{array}{l}\text { Barlum, } \\
\text { dissolved } \\
(\mu \mathrm{g} / \mathrm{L})\end{array}$ & $\begin{array}{c}\text { Beryl- } \\
\text { llum, } \\
\text { dissolved } \\
(\mu \mathrm{g} / \mathrm{L})\end{array}$ & $\begin{array}{l}\text { Boron, } \\
\text { dissolved } \\
(\mu \mathrm{g} / \mathrm{L})\end{array}$ & $\begin{array}{c}\text { Cad- } \\
\text { mium, } \\
\text { dissolved } \\
(\mu \mathrm{g} / \mathrm{L})\end{array}$ & $\begin{array}{c}\text { Chro- } \\
\text { mium, } \\
\text { dlssolved } \\
(\mu \mathrm{g} / \mathrm{L})\end{array}$ & $\begin{array}{l}\text { Cobalt, } \\
\text { dlssolved } \\
(\mu \mathrm{g} / \mathrm{L})\end{array}$ & $\begin{array}{l}\text { Copper, } \\
\text { dissolved } \\
(\mu \mathrm{g} / \mathrm{L})\end{array}$ \\
\hline \multicolumn{11}{|l|}{ Jul 1994} \\
\hline $21 \ldots$ & 21 & 15,000 & $<1$ & 24 & 6 & 90 & 8 & $<5$ & 380 & 20 \\
\hline $2 \mathrm{I} \ldots$ & 21 & 15,000 & $<1$ & 24 & 6 & 110 & 6 & $<5$ & 500 & 20 \\
\hline \multicolumn{11}{|l|}{ Sep 1996} \\
\hline $03 \ldots$ & 18 & 2,000 & $<1$ & 25 & 3 & 50 & 3 & $<5$ & 130 & $<10$ \\
\hline $03 \ldots$ & 18 & 2,000 & 1 & 25 & 2 & 50 & 3 & $<5$ & 150 & $<10$ \\
\hline
\end{tabular}

\begin{tabular}{|c|c|c|c|c|c|c|c|c|c|c|c|}
\hline Date & $\begin{array}{l}\text { Iron, } \\
\text { dissolved } \\
\text { ( } \mu g / \mathrm{L})\end{array}$ & $\begin{array}{l}\text { Lead, } \\
\text { diseolved } \\
\text { ( } \mu g / \text { L) }\end{array}$ & $\begin{array}{l}\text { Llthium, } \\
\text { dlseolved } \\
\text { ( } \mu g / L)\end{array}$ & $\begin{array}{c}\text { Manga- } \\
\text { nese, } \\
\text { dissolved } \\
\text { ( } \mu \mathrm{g} / \mathrm{L})\end{array}$ & $\begin{array}{l}\text { Molyb- } \\
\text { denum, } \\
\text { dissolved } \\
(\mu \mathrm{g} / \mathrm{L})\end{array}$ & $\begin{array}{l}\text { Nickel, } \\
\text { dlssolved } \\
\text { ( } \mathrm{g} / \mathrm{h})\end{array}$ & $\begin{array}{l}\text { Selenium, } \\
\text { dissolved } \\
\text { ( } \mathrm{g} / \mathrm{L})\end{array}$ & $\begin{array}{l}\text { Silver, } \\
\text { dissolved } \\
\text { ( } \mu \mathrm{g} / \mathrm{L})\end{array}$ & $\begin{array}{c}\text { Stron- } \\
\text { tium, } \\
\text { dissolved } \\
(\mu \mathrm{g} / \mathrm{L})\end{array}$ & $\begin{array}{l}\text { Vana- } \\
\text { dlum, } \\
\text { dissolved } \\
(\mu g / L)\end{array}$ & $\begin{array}{l}\text { Zinc, } \\
\text { dissolved } \\
(\mu g / L)\end{array}$ \\
\hline \multicolumn{12}{|l|}{ Jul 1994} \\
\hline $21 \ldots$ & 81,000 & $<10$ & 68 & 370 & $<10$ & 390 & $<1$ & 2 & 350 & 17 & 1,600 \\
\hline $21 \ldots$ & 83,000 & $<10$ & 75 & 380 & $<10$ & 430 & $<1$ & $<\mathrm{I}$ & 360 & 13 & 1,600 \\
\hline \multicolumn{12}{|l|}{ Sep 1996} \\
\hline $03 \ldots$ & 54,000 & $<1$ & 67 & 340 & $<1$ & 270 & -- & $<1$ & 340 & $<6$ & 1,100 \\
\hline $03 \ldots$ & 52,000 & $<1$ & 66 & 330 & 1 & 270 & -- & $<1$ & 330 & $<6$ & 1,100 \\
\hline
\end{tabular}


Table 4. Chemical analyses of field replicates for water samples from sites in the Sand Coulee Coal Area, July 1994 through September 1996 (Continued)

\section{SITE 7, 472016111085701--COTTONWOOD MINE NO. 6 DRAIN TO COTTONWOOD CREEK NEAR STOCKETT, MT}

\begin{tabular}{|c|c|c|c|c|c|c|c|c|c|c|}
\hline Date & Time & $\begin{array}{c}\text { Calcium, } \\
\text { dis- } \\
\text { solved } \\
\text { (mg/l) }\end{array}$ & $\begin{array}{l}\text { Magne- } \\
\text { slum, } \\
\text { dis"- } \\
\text { solved } \\
\text { (mg/L) }\end{array}$ & $\begin{array}{c}\text { Sodium, } \\
\text { dis- } \\
\text { solved } \\
\text { (mg/l) }\end{array}$ & $\begin{array}{l}\text { Potas- } \\
\text { sium, } \\
\text { dissolved } \\
\text { (mg/l) }\end{array}$ & $\begin{array}{l}\text { Acidity } \\
\text { ( } \mathrm{mg} / \mathrm{l})\end{array}$ & $\begin{array}{c}\text { Alkalinity, } \\
\text { lab } \\
\left(\mathrm{mgll}_{\mathrm{L}} \text { as }\right. \\
\left.\mathrm{CaCO}_{3}\right)\end{array}$ & $\begin{array}{l}\text { Sulfate, } \\
\text { dissolved } \\
\text { (mg/L) }\end{array}$ & $\begin{array}{l}\text { Chloride, } \\
\text { dissolved } \\
\text { (mg/L) }\end{array}$ & $\begin{array}{l}\text { Fluoride, } \\
\text { dissolved } \\
\text { (mg/L) }\end{array}$ \\
\hline $10 \ldots$ & 1455 & 350 & 140 & 14 & 2.4 & 86 & $e<1$ & 6,000 & $<.10$ & - \\
\hline \multicolumn{11}{|l|}{ Jun } \\
\hline $03 \ldots$ & 1710 & 350 & 140 & 14 & 3.0 & 85 & $e<1$ & 7,200 & 3.8 & $<1$ \\
\hline
\end{tabular}

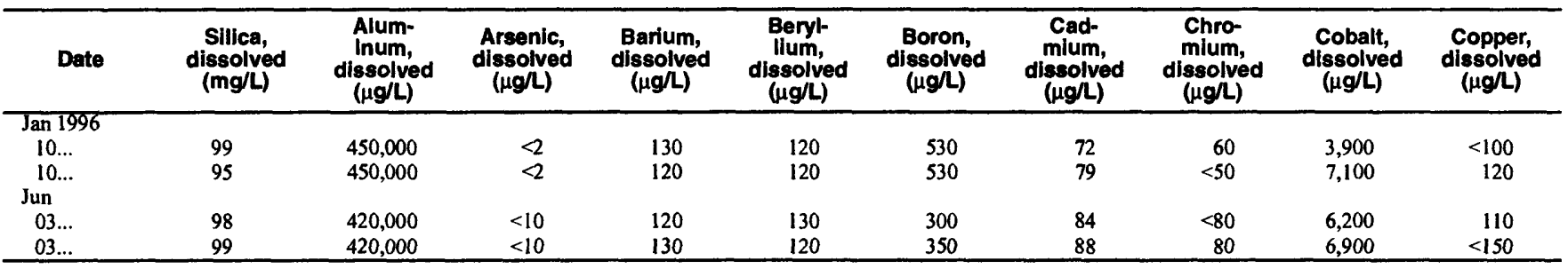

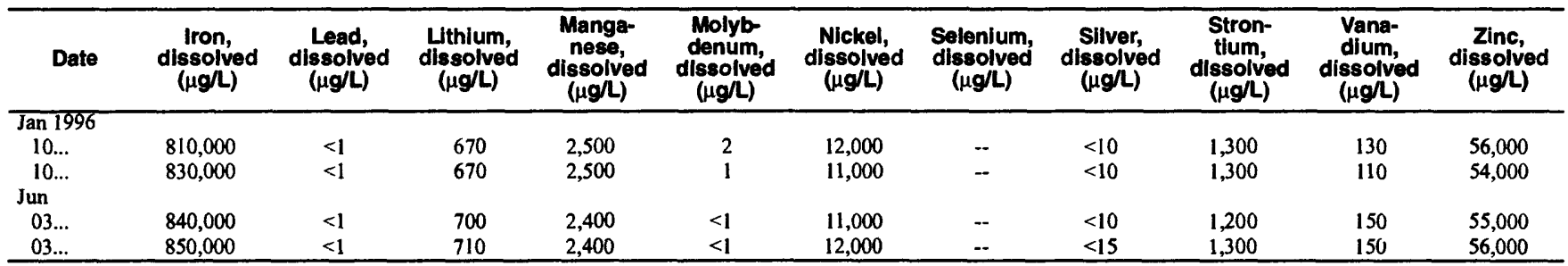

SITE 9, 472212111093301--NUMBER FIVE COULEE NEAR STOCKETT, MT

\begin{tabular}{|c|c|c|c|c|c|c|c|c|c|c|}
\hline Date & Time & $\begin{array}{c}\text { Calcium, } \\
\text { dis- } \\
\text { solved } \\
\text { (mg/L) }\end{array}$ & $\begin{array}{l}\text { Magne- } \\
\text { sjum, } \\
\text { dis- } \\
\text { solved } \\
\text { ( } \mathrm{mg} \text { /L) }\end{array}$ & $\begin{array}{l}\text { Sodium, } \\
\text { dis- } \\
\text { solved } \\
\text { (mg/L) }\end{array}$ & $\begin{array}{l}\text { Potas- } \\
\text { sium, } \\
\text { dissolved } \\
\text { (mg/L) }\end{array}$ & $\begin{array}{l}\text { Acidity } \\
\text { (mg/L) }\end{array}$ & $\begin{array}{c}\text { Alkalinity, } \\
\text { lab } \\
\left(\mathrm{mgll}_{\mathrm{L}} \mathrm{as}\right. \\
\left.\mathrm{CaCO}_{3}\right)\end{array}$ & $\begin{array}{c}\text { Sulfate, } \\
\text { dissolved } \\
\text { (mg/ll) }\end{array}$ & $\begin{array}{l}\text { Chioride, } \\
\text { dissolved } \\
\text { (mg/ll) }\end{array}$ & $\begin{array}{c}\text { Fiuoride, } \\
\text { dissolved } \\
\text { (mg/L) }\end{array}$ \\
\hline \multicolumn{11}{|l|}{ Apr 1995} \\
\hline $11 \ldots$ & 1800 & 98 & 35 & 24 & 5.4 & $<0.1$ & 33 & 380 & 4.4 & 0.4 \\
\hline $11 \ldots$ & 1805 & 95 & 34 & 24 & 5.2 & $<.1$ & 33 & 360 & 3.6 & .5 \\
\hline
\end{tabular}

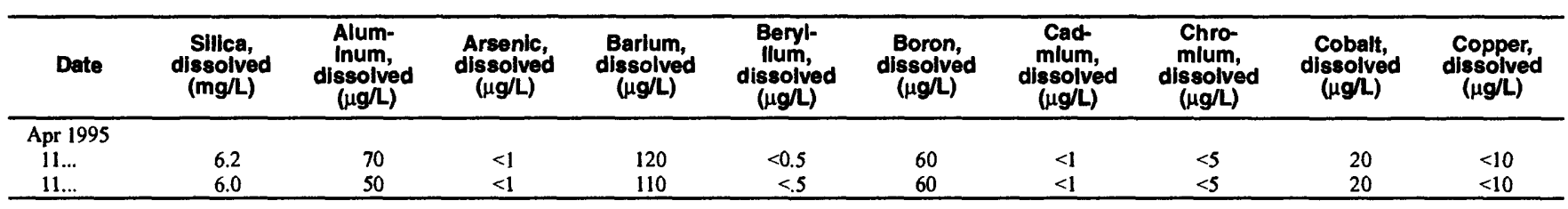

\begin{tabular}{|c|c|c|c|c|c|c|c|c|c|c|c|}
\hline Date & $\begin{array}{c}\text { Iron, } \\
\text { dissolved } \\
(\mu g / L)\end{array}$ & $\begin{array}{l}\text { Lead, } \\
\text { dissolved } \\
(\mu g / L)\end{array}$ & $\begin{array}{l}\text { Lithlum, } \\
\text { dissolved } \\
(\mu g / L)\end{array}$ & $\begin{array}{c}\text { Mange- } \\
\text { nese, } \\
\text { dissolved } \\
(\mu g / L)\end{array}$ & $\begin{array}{c}\text { Molyb- } \\
\text { denum, } \\
\text { dlssolved } \\
(\mu g / L)\end{array}$ & $\begin{array}{c}\text { Nickel, } \\
\text { dissolved } \\
(\mu \mathrm{g} / \mathrm{L})\end{array}$ & $\begin{array}{l}\text { Selenium, } \\
\text { dissolved } \\
(\mu g / L)\end{array}$ & $\begin{array}{c}\text { Silver, } \\
\text { dissolved } \\
(\mu g / L)\end{array}$ & $\begin{array}{c}\text { Stron- } \\
\text { tium, } \\
\text { dissolved } \\
(\mu \mathrm{g} / \mathrm{L})\end{array}$ & $\begin{array}{c}\text { Vana- } \\
\text { dium, } \\
\text { dlssolved } \\
(\mu g / L)\end{array}$ & $\begin{array}{l}\text { Zinc, } \\
\text { dissolved } \\
(\mu g / L)\end{array}$ \\
\hline \multicolumn{12}{|l|}{ Apr 1995} \\
\hline
\end{tabular}


Table 4. Chemical analyses of field replicates for water samples from sites in the Sand Coulee Coal Area, Montana, July 1994 through September 1996 (Continued)

SITE 10, $472233110552601--F R E N C H$ COULEE WETLANDS OUTFLOW AT BELT, MT

\begin{tabular}{|c|c|c|c|c|c|c|c|c|c|c|}
\hline Date & Time & $\begin{array}{c}\text { Calcium, } \\
\text { dls- } \\
\text { solved } \\
\text { (mg/l) }\end{array}$ & $\begin{array}{l}\text { Magne- } \\
\text { slum, } \\
\text { dls- } \\
\text { solved } \\
\text { (mg/L) }\end{array}$ & $\begin{array}{l}\text { Sodium, } \\
\text { dls- } \\
\text { solved } \\
\text { (mg/h) }\end{array}$ & $\begin{array}{l}\text { Potas- } \\
\text { slum, } \\
\text { dlssolved } \\
\text { (mg/l) }\end{array}$ & $\begin{array}{l}\text { Acldity } \\
\text { (mg/l) }\end{array}$ & $\begin{array}{c}\text { Alkalinity, } \\
\text { lab } \\
\left(\mathrm{mgh}_{\mathrm{as}}\right. \\
\left.\mathrm{CaCO}_{3}\right)\end{array}$ & $\begin{array}{l}\text { Sulfate, } \\
\text { dissolved } \\
\text { (mg/l) }\end{array}$ & $\begin{array}{l}\text { Chlorlde, } \\
\text { dissolved } \\
\text { (mg/ll) }\end{array}$ & $\begin{array}{l}\text { Fuoride, } \\
\text { dissolved } \\
\text { (mg/L) }\end{array}$ \\
\hline
\end{tabular}

\begin{tabular}{|c|c|c|c|c|c|c|c|c|c|c|}
\hline Date & $\begin{array}{l}\text { Silica, } \\
\text { dissolved } \\
\text { (mg/L) }\end{array}$ & $\begin{array}{c}\text { Alum- } \\
\text { inum, } \\
\text { dissolved } \\
(\mu g / L)\end{array}$ & $\begin{array}{c}\text { Arsenic, } \\
\text { dissolved } \\
(\mu g / L)\end{array}$ & $\begin{array}{l}\text { Barium, } \\
\text { dissolved } \\
(\mu \mathrm{g} / L)\end{array}$ & $\begin{array}{c}\text { Beryl- } \\
\text { lium, } \\
\text { dissolved } \\
(\mu g / L)\end{array}$ & $\begin{array}{c}\text { Boron, } \\
\text { dissolved } \\
(\mu g / L)\end{array}$ & $\begin{array}{c}\text { Cad- } \\
\text { mlum, } \\
\text { dissolved } \\
(\mu g h)\end{array}$ & $\begin{array}{c}\text { Chro- } \\
\text { mlum, } \\
\text { dissolved } \\
(\mu g /)\end{array}$ & $\begin{array}{l}\text { Cobalt, } \\
\text { dissolved } \\
\left(\mu g^{\prime} L\right)\end{array}$ & $\begin{array}{l}\text { Copper, } \\
\text { dlssolved } \\
(\mu g / L)\end{array}$ \\
\hline $\begin{array}{c}\text { Sep } 1995 \\
14 \ldots \\
14 \ldots\end{array}$ & $\begin{array}{l}86 \\
88\end{array}$ & $\begin{array}{l}240,000 \\
230,000\end{array}$ & $\begin{array}{l}<2 \\
<2\end{array}$ & $\begin{array}{l}4 \\
4\end{array}$ & $\begin{array}{l}26 \\
27\end{array}$ & $\begin{array}{l}170 \\
180\end{array}$ & $\begin{array}{l}3 \\
3\end{array}$ & $\begin{array}{l}50 \\
60\end{array}$ & $\begin{array}{l}180 \\
180\end{array}$ & $\begin{array}{r}30 \\
<30\end{array}$ \\
\hline
\end{tabular}

\begin{tabular}{|c|c|c|c|c|c|c|c|c|c|c|}
\hline Date & $\begin{array}{l}\text { Iron, } \\
\text { dissolved } \\
(\mu \mathrm{g} / L)\end{array}$ & $\begin{array}{l}\text { Lead, } \\
\text { dissolved } \\
(\mu g / L)\end{array}$ & $\begin{array}{l}\text { Lithlum, } \\
\text { dissolved } \\
(\mu \mathrm{g} / \mathrm{L})\end{array}$ & $\begin{array}{l}\text { Manga- } \\
\text { nese, } \\
\text { dissolved } \\
(\mu g / L)\end{array}$ & $\begin{array}{c}\text { Molyb- } \\
\text { denumm } \\
\text { dissolved } \\
(\mu \mathrm{g} / \mathrm{L})\end{array}$ & $\begin{array}{c}\text { Nickel, } \\
\text { dissolved } \\
(\mu \mathrm{g} / \mathrm{L})\end{array}$ & $\begin{array}{l}\text { Silver, } \\
\text { dissolved } \\
\left(\mu g^{\prime} / L\right)\end{array}$ & $\begin{array}{c}\text { Stron- } \\
\text { tium, } \\
\text { dlssolved } \\
(\mu g / L)\end{array}$ & $\begin{array}{c}\text { Vana- } \\
\text { dium, } \\
\text { dissolved } \\
(\mu g / L)\end{array}$ & $\begin{array}{l}\text { Zinc, } \\
\text { dissolved } \\
(\mu g / L)\end{array}$ \\
\hline $\begin{array}{c}\text { Sep } 1995 \\
14 \ldots \\
14 \ldots\end{array}$ & $\begin{array}{l}170,000 \\
170,000\end{array}$ & $\begin{array}{l}<1 \\
<1\end{array}$ & $\begin{array}{l}280 \\
310\end{array}$ & $\begin{array}{l}1,100 \\
1,100\end{array}$ & $\begin{array}{l}<1 \\
<1\end{array}$ & $\begin{array}{l}350 \\
380\end{array}$ & $\begin{array}{l}<2 \\
<3\end{array}$ & $\begin{array}{l}1,300 \\
1,400\end{array}$ & $\begin{array}{l}<12 \\
<18\end{array}$ & $\begin{array}{l}1,300 \\
1,300\end{array}$ \\
\hline
\end{tabular}

SITE 12, 472235110553202--FRENCH COULEE WETLANDS INFLOW NO. 2 AT BELT, MT

\begin{tabular}{|c|c|c|c|c|c|c|c|c|c|c|}
\hline Date & Time & $\begin{array}{l}\text { Calcium, } \\
\text { dis- } \\
\text { solved } \\
\text { (mg/l) }\end{array}$ & $\begin{array}{l}\text { Magne- } \\
\text { slum, } \\
\text { dis- } \\
\text { solved } \\
\text { (mg/L) }\end{array}$ & $\begin{array}{l}\text { Sodium, } \\
\text { dis- } \\
\text { solved } \\
\text { (mg/l) }\end{array}$ & $\begin{array}{l}\text { Potas- } \\
\text { sium, } \\
\text { dissolved } \\
\text { (mg/l) }\end{array}$ & $\begin{array}{l}\text { Acidity } \\
\text { (mg/l) }\end{array}$ & $\begin{array}{c}\text { Alkalinity, } \\
\text { lab } \\
(\underset{\mathrm{mgl}}{\mathrm{maCO}} \text { as } \\
\left.\mathrm{CoO}_{3}\right)\end{array}$ & $\begin{array}{l}\text { Sulfate, } \\
\text { dissolved } \\
\text { (mg/L) }\end{array}$ & $\begin{array}{l}\text { Chlorlde, } \\
\text { dlssolved } \\
\text { (mg/L) }\end{array}$ & $\begin{array}{l}\text { Fluoride, } \\
\text { dissolved } \\
\text { (mg/L) }\end{array}$ \\
\hline \multicolumn{11}{|l|}{ Apr 1996} \\
\hline $04 \ldots$ & 1130 & 190 & 94 & 13 & 5.3 & 79 & $e<1$ & 5,300 & 9.6 & 3.9 \\
\hline $04 \ldots$ & 1135 & 190 & 92 & 12 & 5.4 & 78 & $e<1$ & 5,000 & 9.3 & 4.0 \\
\hline
\end{tabular}

\begin{tabular}{|c|c|c|c|c|c|c|c|c|c|c|}
\hline Date & $\begin{array}{l}\text { Sillca, } \\
\text { diseolved } \\
\text { (mg/l) }\end{array}$ & $\begin{array}{c}\text { Alum- } \\
\text { inum, } \\
\text { dissolved } \\
(\mu g / L)\end{array}$ & $\begin{array}{l}\text { Arsenlc, } \\
\text { dlssolved } \\
\text { ( } \mu \mathrm{g} / \mathrm{L})\end{array}$ & $\begin{array}{l}\text { Barium, } \\
\text { dissolved } \\
\text { ( } \mu \mathrm{g} / \mathrm{L})\end{array}$ & $\begin{array}{c}\text { Beryl- } \\
\text { llum, } \\
\text { dissolved } \\
(\mu g / h)\end{array}$ & $\begin{array}{l}\text { Boron, } \\
\text { dissolved } \\
\text { ( } \mu \mathrm{g} / \mathrm{L})\end{array}$ & $\begin{array}{c}\text { Cad- } \\
\text { mium, } \\
\text { dlseolved } \\
(\mu \mathrm{g} / \mathrm{L})\end{array}$ & $\begin{array}{c}\text { Chro- } \\
\text { mium, } \\
\text { dissolved } \\
(\mu \mathrm{g} / \mathrm{L})\end{array}$ & $\begin{array}{l}\text { Cobalt, } \\
\text { dissolved } \\
(\mu \mathrm{g} / \mathrm{L})\end{array}$ & $\begin{array}{l}\text { Copper, } \\
\text { dissolved } \\
(\mu g / L)\end{array}$ \\
\hline \multicolumn{11}{|l|}{ Apr 1996} \\
\hline $04 \ldots$ & 100 & 430,000 & $<2$ & 13 & 48 & 330 & 7 & 140 & 350 & $<100$ \\
\hline $04 \ldots$ & 100 & 410,000 & $<2$ & 14 & 43 & 340 & 6 & 150 & 360 & $<100$ \\
\hline
\end{tabular}

\begin{tabular}{|c|c|c|c|c|c|c|c|c|c|c|}
\hline Dente & $\begin{array}{l}\text { Iron, } \\
\text { dissolved } \\
\text { ( } \mu \mathrm{g} / \mathrm{L})\end{array}$ & $\begin{array}{l}\text { Lead, } \\
\text { diseolved } \\
\text { ( } \mu \mathrm{g} / \mathrm{L} \text { ) }\end{array}$ & $\begin{array}{l}\text { Lithium, } \\
\text { dlseolved } \\
\text { ( } \mu \mathrm{g} / \mathrm{L})\end{array}$ & $\begin{array}{c}\text { Manga- } \\
\text { nese, } \\
\text { dissolved } \\
(\mu g / l)\end{array}$ & $\begin{array}{c}\text { Molyb- } \\
\text { denum, } \\
\text { dissolved } \\
(\mu \mathrm{g} / \mathrm{L})\end{array}$ & $\begin{array}{c}\text { Nickel, } \\
\text { dlseolved } \\
\text { ( } \mu g / \mathrm{L})\end{array}$ & $\begin{array}{l}\text { Silver, } \\
\text { dlssolved } \\
\text { ( } \mu \mathrm{g} / \mathrm{L})\end{array}$ & $\begin{array}{c}\text { Stron- } \\
\text { tium, } \\
\text { dissolved } \\
(\mu \mathrm{g} / \mathrm{L})\end{array}$ & $\begin{array}{c}\text { Vana- } \\
\text { dium, } \\
\text { diseolved } \\
(\mu g / L)\end{array}$ & $\begin{array}{c}\text { Znc, } \\
\text { dissolved } \\
(\mu g / L)\end{array}$ \\
\hline \multicolumn{11}{|l|}{ Apr 1996} \\
\hline $04 \ldots$ & $\begin{array}{l}870,000 \\
850,000\end{array}$ & $\begin{array}{l}2 \\
2\end{array}$ & $\begin{array}{l}520 \\
520\end{array}$ & $\begin{array}{l}750 \\
730\end{array}$ & $\begin{array}{l}<1 \\
<1\end{array}$ & $\begin{array}{l}870 \\
840\end{array}$ & $\begin{array}{l}<10 \\
<10\end{array}$ & $\begin{array}{l}1,800 \\
1,800\end{array}$ & $\begin{array}{l}72 \\
95\end{array}$ & $\begin{array}{l}4,000 \\
4,100\end{array}$ \\
\hline
\end{tabular}


Table 4. Chemical analyses of field replicates for water samples from sites in the Sand Coulee Coal Area, Montana, July 1994 through September 1996 (Continued)

SITE 13, 472305110551701 --LEWIS COULEE ABOVE CASTNER PARK, AT BELT, MT

\begin{tabular}{|c|c|c|c|c|c|c|c|c|c|c|}
\hline Date & Time & $\begin{array}{l}\text { Calcium, } \\
\text { dis- } \\
\text { solved } \\
\text { (mg/L) }\end{array}$ & $\begin{array}{l}\text { Magne- } \\
\text { sium, } \\
\text { dis- } \\
\text { solved } \\
\text { (mg/L) }\end{array}$ & $\begin{array}{l}\text { Sodium, } \\
\text { dis- } \\
\text { solved } \\
\text { (mg/L) }\end{array}$ & $\begin{array}{l}\text { Potas- } \\
\text { sium, } \\
\text { dissolved } \\
\text { (mg/l) }\end{array}$ & $\begin{array}{l}\text { Acidity } \\
\text { (mg/lL) }\end{array}$ & $\begin{array}{c}\text { Alkalinity, } \\
\text { lab } \\
\left(\mathrm{mg}^{\prime} \mathrm{L} \text { as }\right. \\
\left.\mathrm{CaCO}_{3}\right)\end{array}$ & $\begin{array}{l}\text { Sulfate, } \\
\text { dissolved } \\
\text { (mg/L) }\end{array}$ & $\begin{array}{l}\text { Chloride, } \\
\text { dlssolved } \\
\text { (mg/l) }\end{array}$ & $\begin{array}{l}\text { Fluoride, } \\
\text { dlssolved } \\
\text { (mg/L) }\end{array}$ \\
\hline \multicolumn{11}{|l|}{ Jul 1995} \\
\hline $13 \ldots$ & 0950 & 190 & 120 & 27 & 1.1 & 63 & $e<1$ & 4,800 & 5.2 & $<.01$ \\
\hline $13 \ldots$ & 0955 & 180 & 120 & 27 & 1.1 & 62 & $e<1$ & 4,600 & 5.0 & $<.1$ \\
\hline
\end{tabular}

\begin{tabular}{|c|c|c|c|c|c|c|c|c|c|c|}
\hline Date & $\begin{array}{l}\text { Sillca, } \\
\text { djssolved } \\
\text { (mg/l) }\end{array}$ & $\begin{array}{l}\text { Alum- } \\
\text { Inum, } \\
\text { dissolved } \\
(\mu \mathrm{g} / \mathrm{L})\end{array}$ & $\begin{array}{c}\text { Arsenlc, } \\
\text { dissolved } \\
(\mu \mathrm{g} / \mathrm{L})\end{array}$ & $\begin{array}{c}\text { Barlum, } \\
\text { dissoived } \\
(\mu g / L)\end{array}$ & $\begin{array}{c}\text { Beryl- } \\
\text { Ilum, } \\
\text { dlssolved } \\
\text { ( } \mu \mathrm{g} / \mathrm{L})\end{array}$ & $\begin{array}{l}\text { Boron, } \\
\text { dissolved } \\
(\mu \mathrm{g} / \mathrm{L})\end{array}$ & $\begin{array}{l}\text { Cad- } \\
\text { mlum, } \\
\text { dissolved } \\
(\mu \mathrm{g} / \mathrm{L})\end{array}$ & $\begin{array}{l}\text { Chro- } \\
\text { mlum, } \\
\text { dlssolved } \\
(\mu \mathrm{g} / \mathrm{L})\end{array}$ & $\begin{array}{l}\text { Cobalt, } \\
\text { dissolved } \\
\text { ( } \mu \mathrm{g} / \mathrm{L})\end{array}$ & $\begin{array}{l}\text { Copper, } \\
\text { dissolved } \\
(\mu g / L)\end{array}$ \\
\hline \multicolumn{11}{|l|}{ Jul 1995} \\
\hline $13 \ldots$ & 87 & 320,000 & $<5$ & $<10$ & 21 & 270 & 63 & 170 & 950 & 150 \\
\hline $13 \ldots$ & 85 & 320,000 & $<5$ & $<10$ & 20 & 250 & 67 & 160 & 1,000 & 150 \\
\hline
\end{tabular}

\begin{tabular}{|c|c|c|c|c|c|c|c|c|c|c|}
\hline Date & $\begin{array}{l}\text { Iron, } \\
\text { dissolved } \\
(\mu \mathrm{g} / \mathrm{L})\end{array}$ & $\begin{array}{l}\text { Lead, } \\
\text { dissolved } \\
(\mu \mathrm{g} / \mathrm{L})\end{array}$ & $\begin{array}{l}\text { Lithium, } \\
\text { dlssolved } \\
(\mu \mathrm{g} / \mathrm{L})\end{array}$ & $\begin{array}{l}\text { Manga- } \\
\text { nese, } \\
\text { dlssolved } \\
(\mu g / L)\end{array}$ & $\begin{array}{l}\text { Molyb- } \\
\text { denum, } \\
\text { dissolved } \\
(\mu \mathrm{g} / \mathrm{L})\end{array}$ & $\begin{array}{c}\text { Nickel, } \\
\text { dlssolved } \\
(\mu \mathrm{g} / \mathrm{L})\end{array}$ & $\begin{array}{l}\text { Silver, } \\
\text { dlssolved } \\
(\mu \mathrm{g} / \mathrm{L})\end{array}$ & $\begin{array}{l}\text { Stron- } \\
\text { tlum, } \\
\text { dlssolved } \\
(\mu \mathrm{g} / \mathrm{L})\end{array}$ & $\begin{array}{c}\text { Vana- } \\
\text { dium, } \\
\text { dlssolved } \\
(\mu \mathrm{g} / \mathrm{L})\end{array}$ & $\begin{array}{c}\text { Zlnc, } \\
\text { dlssolved } \\
(\mu g / L)\end{array}$ \\
\hline \multicolumn{11}{|l|}{ Jul 1995} \\
\hline $13 \ldots$ & 560,000 & $<1$ & 470 & 1,000 & $<1$ & 2,100 & 10 & 1,500 & $<60$ & 7,700 \\
\hline $13 \ldots$ & 550,000 & 1 & 470 & 1,000 & 1 & 2,200 & 10 & 1,500 & $<60$ & 7,700 \\
\hline
\end{tabular}

SITE 14,472306111103601 --MINE DRAIN TO MINING COULEE NEAR SAND COULEE, MT

\begin{tabular}{|c|c|c|c|c|c|c|c|c|c|c|}
\hline Date & Time & $\begin{array}{l}\text { Calcium, } \\
\text { dis- } \\
\text { solved } \\
\text { (mg/L) }\end{array}$ & $\begin{array}{l}\text { Magne- } \\
\text { slum, } \\
\text { dls- } \\
\text { solved } \\
\text { (mg/L) }\end{array}$ & $\begin{array}{l}\text { Sodium, } \\
\text { dls- } \\
\text { solved } \\
\text { (mg/L) }\end{array}$ & $\begin{array}{l}\text { Potas- } \\
\text { slum, } \\
\text { dlssolved } \\
\text { (mg/l) }\end{array}$ & $\begin{array}{l}\text { Acldity } \\
\text { (mg/L) }\end{array}$ & $\begin{array}{c}\text { Alkalinity, } \\
\text { lab } \\
\left(\mathrm{mg} / \mathrm{L}_{\mathrm{as}}\right. \\
\left.\mathrm{CaCO}_{3}\right)\end{array}$ & $\begin{array}{l}\text { Sulfate, } \\
\text { dlssolved } \\
\text { (mg/l) }\end{array}$ & $\begin{array}{l}\text { Chlorlde, } \\
\text { dissolved } \\
\text { (mg/L) }\end{array}$ & $\begin{array}{l}\text { Fluorlde, } \\
\text { dissolved } \\
\text { (mg/L) }\end{array}$ \\
\hline $\begin{array}{c}\text { Feb } 1995 \\
21 \ldots \\
21 \ldots\end{array}$ & $\begin{array}{l}1410 \\
1415\end{array}$ & $\begin{array}{l}270 \\
290\end{array}$ & $\begin{array}{l}190 \\
200\end{array}$ & $\begin{array}{l}19 \\
20\end{array}$ & $\begin{array}{r}0.2 \\
.2\end{array}$ & $\begin{array}{l}150 \\
150\end{array}$ & $\begin{array}{l}e<1 \\
e<1\end{array}$ & $\begin{array}{l}11,000 \\
12,000\end{array}$ & $\begin{array}{l}25 \\
<.1\end{array}$ & $\begin{array}{l}2.6 \\
2.6\end{array}$ \\
\hline
\end{tabular}

\begin{tabular}{|c|c|c|c|c|c|c|c|c|c|c|}
\hline Date & $\begin{array}{l}\text { Sllica, } \\
\text { dlssolved } \\
\text { (mg/L) }\end{array}$ & $\begin{array}{c}\text { Alum- } \\
\text { inum, } \\
\text { dissoived } \\
(\mu \mathrm{g} / \mathrm{L})\end{array}$ & $\begin{array}{l}\text { Arsenlc, } \\
\text { dlssolved } \\
(\mu g / L)\end{array}$ & $\begin{array}{l}\text { Barium, } \\
\text { dissolved } \\
\text { ( } \mu \mathrm{g} / \mathrm{L})\end{array}$ & $\begin{array}{c}\text { Beryl- } \\
\text { llum, } \\
\text { dissolved } \\
\text { ( } \mu \mathrm{g} / \mathrm{L})\end{array}$ & $\begin{array}{l}\text { Boron, } \\
\text { dlssolved } \\
(\mu \mathrm{g} / \mathrm{L})\end{array}$ & $\begin{array}{c}\text { Cad- } \\
\text { mium, } \\
\text { dissolved } \\
(\mu g / L)\end{array}$ & $\begin{array}{c}\text { Chro- } \\
\text { mium, } \\
\text { dissoived } \\
(\mu \mathrm{g} / \mathrm{L})\end{array}$ & $\begin{array}{l}\text { Cobalt, } \\
\text { dlssolved } \\
(\mu \mathrm{g} / \mathrm{L})\end{array}$ & $\begin{array}{c}\text { Copper, } \\
\text { dissolved } \\
(\mu g / L)\end{array}$ \\
\hline \multicolumn{11}{|l|}{ Feb 1995} \\
\hline $\begin{array}{l}21 \ldots \\
21 \ldots\end{array}$ & $\begin{array}{l}120 \\
130\end{array}$ & $\begin{array}{l}860,000 \\
870,000\end{array}$ & $\begin{array}{l}<10 \\
<10\end{array}$ & $\begin{array}{r}<100 \\
<10\end{array}$ & $\begin{array}{l}95 \\
92\end{array}$ & $\begin{array}{l}720 \\
720\end{array}$ & $\begin{array}{l}83 \\
88\end{array}$ & $\begin{array}{l}310 \\
320\end{array}$ & $\begin{array}{l}3,300 \\
3,400\end{array}$ & $\begin{array}{r}100 \\
<100\end{array}$ \\
\hline
\end{tabular}

\begin{tabular}{|c|c|c|c|c|c|c|c|c|c|c|c|}
\hline Date & $\begin{array}{l}\text { Iron, } \\
\text { dissolved } \\
(\mu g / L)\end{array}$ & $\begin{array}{l}\text { Lead, } \\
\text { dlseolved } \\
(\mu g / L)\end{array}$ & $\begin{array}{l}\text { Lithium, } \\
\text { dissolved } \\
\text { ( } \mu \mathrm{g} / \mathrm{L})\end{array}$ & $\begin{array}{l}\text { Manga- } \\
\text { nese, } \\
\text { dissolved } \\
(\mu \mathrm{g} / \mathrm{L})\end{array}$ & $\begin{array}{l}\text { Molyb- } \\
\text { denum, } \\
\text { dlssolved } \\
\text { ( } \mu \mathrm{g} / \mathrm{L})\end{array}$ & $\begin{array}{l}\text { Nlckel, } \\
\text { dissolved } \\
(\mu \mathrm{g} / \mathrm{L})\end{array}$ & $\begin{array}{c}\text { Selenium, } \\
\text { dlssolved } \\
\text { ( } \mu \mathrm{g} / \mathrm{L})\end{array}$ & $\begin{array}{l}\text { Sllver, } \\
\text { dlssolved } \\
\text { ( } \mu \mathrm{g} / \mathrm{L})\end{array}$ & $\begin{array}{l}\text { Stron- } \\
\text { tium, } \\
\text { dissolved } \\
\text { ( } \mu \mathrm{g} / \mathrm{L})\end{array}$ & $\begin{array}{l}\text { Vana- } \\
\text { dium, } \\
\text { dissolved } \\
(\mu \mathrm{g} / \mathrm{L})\end{array}$ & $\begin{array}{c}\text { Zinc, } \\
\text { dissolved } \\
(\mu g / L)\end{array}$ \\
\hline \multicolumn{12}{|l|}{ Feb 1995} \\
\hline $21 \ldots$ & 950,000 & $<1$ & 1,200 & 3,200 & $<2$ & 7,300 & $<2$ & $<4$ & 1,200 & 320 & 33,000 \\
\hline $21 \ldots$ & $1,000,000$ & $<1$ & 1,200 & 3,400 & 2 & 7,500 & $<1$ & $<10$ & 1,200 & 330 & 32,000 \\
\hline
\end{tabular}


Table 4. Chemical analyses of field replicates for water samples from sites in the Sand Coulee Coal Area, Montana, July 1994 through September 1996 (Continued)

SITE 21, 472309110551201--LEWIS COULEE BELOW MINE ADIT, AT BELT, MT

\begin{tabular}{|c|c|c|c|c|c|c|c|c|c|c|}
\hline Date & Time & $\begin{array}{c}\text { Calcium, } \\
\text { dls- } \\
\text { solved } \\
\text { (mg/l) }\end{array}$ & $\begin{array}{l}\text { Magne- } \\
\text { slum, } \\
\text { dls- } \\
\text { solved } \\
\text { (mg/L) }\end{array}$ & $\begin{array}{c}\text { Sodium, } \\
\text { dls- } \\
\text { solved } \\
\text { (mg/L) }\end{array}$ & $\begin{array}{l}\text { Potas- } \\
\text { sium, } \\
\text { dissolved } \\
\text { (mgll) }\end{array}$ & $\begin{array}{l}\text { Acidity } \\
\text { (mg/L) }\end{array}$ & $\begin{array}{c}\text { Alkalinity, } \\
\text { lab } \\
\left(\mathrm{mgg}^{\prime} \mathrm{Las}\right. \\
\left.\mathrm{CaCO}_{3}\right)\end{array}$ & $\begin{array}{l}\text { Sulfate, } \\
\text { dissolved } \\
\text { (mg/L) }\end{array}$ & $\begin{array}{l}\text { Chloride, } \\
\text { dissolved } \\
\text { (mg/L) }\end{array}$ & $\begin{array}{l}\text { Fluoride, } \\
\text { dissolved } \\
\text { (mg/L) }\end{array}$ \\
\hline \multicolumn{11}{|l|}{ May 1995} \\
\hline $17 \ldots$ & 0900 & 62 & 62 & 19 & 4.4 & $<0.1$ & 246 & 180 & 14 & 0.4 \\
\hline $17 \ldots$ & 0905 & 63 & 63 & 19 & 4.3 & $<.1$ & 246 & 180 & 14 & .5 \\
\hline \multicolumn{11}{|l|}{ Feb 1996} \\
\hline $21 \ldots$ & 0945 & 200 & 130 & 22 & 5.8 & 52 & $\mathrm{e}<1$ & 4,000 & 7.9 & $<1$ \\
\hline $21 \ldots$ & 0950 & 200 & 140 & 23 & 5.3 & 52 & $e<1$ & 3,900 & 8.3 & $<\mathrm{l}$ \\
\hline
\end{tabular}

\begin{tabular}{|c|c|c|c|c|c|c|c|c|c|c|}
\hline Date & $\begin{array}{l}\text { Silica, } \\
\text { dissolved } \\
\text { (mg/L) }\end{array}$ & $\begin{array}{c}\text { Alum- } \\
\text { inum, } \\
\text { dissolved } \\
(\mu g / L)\end{array}$ & $\begin{array}{c}\text { Arsenic, } \\
\text { dissolved } \\
(\mu \mathrm{g} / \mathrm{L})\end{array}$ & $\begin{array}{c}\text { Barium, } \\
\text { dissolved } \\
(\mu g / L)\end{array}$ & $\begin{array}{c}\text { Beryl- } \\
\text { lium, } \\
\text { dissolved } \\
(\mu \mathrm{g} / \mathrm{L})\end{array}$ & $\begin{array}{l}\text { Boron, } \\
\text { dissolved } \\
\left(\mu g^{\prime} L\right)\end{array}$ & $\begin{array}{c}\text { Cad- } \\
\text { mium, } \\
\text { dissolved } \\
(\mu \mathrm{g} / \mathrm{L})\end{array}$ & $\begin{array}{c}\text { Chro- } \\
\text { mium, } \\
\text { dissolved } \\
(\mu g / L)\end{array}$ & $\begin{array}{l}\text { Cobalt, } \\
\text { dissolved } \\
(\mu \mathrm{g} / \mathrm{L})\end{array}$ & $\begin{array}{l}\text { Copper, } \\
\text { dissolved } \\
(\mu g / L)\end{array}$ \\
\hline \multicolumn{11}{|l|}{ May 1995} \\
\hline $17 \ldots$ & 8.1 & 40 & $<1$ & 75 & $<.5$ & 50 & $<1$ & $<5$ & 10 & $<10$ \\
\hline \multicolumn{11}{|l|}{ Feb 1996} \\
\hline $21 \ldots$ & 75 & 280,000 & $<2$ & 21 & 25 & 250 & 15 & 50 & 660 & $<50$ \\
\hline $21 \ldots$ & 74 & 260,000 & $<1$ & 12 & 21 & 260 & 19 & 80 & 830 & $<100$ \\
\hline
\end{tabular}

\begin{tabular}{|c|c|c|c|c|c|c|c|c|c|c|c|}
\hline Date & $\begin{array}{l}\text { Iron, } \\
\text { dissolved } \\
\left(\mu \mathbf{g}^{\prime} / L\right)\end{array}$ & $\begin{array}{l}\text { Lead, } \\
\text { dissolved } \\
(\mu \mathrm{g} / L)\end{array}$ & $\begin{array}{l}\text { Lithium, } \\
\text { dissolved } \\
(\mu g / L)\end{array}$ & $\begin{array}{l}\text { Manga- } \\
\text { nese, } \\
\text { dlissolved } \\
(\mu \mathrm{g} / \mathrm{L})\end{array}$ & $\begin{array}{l}\text { Molyb- } \\
\text { denum, } \\
\text { dissolved } \\
(\mu g / L)\end{array}$ & $\begin{array}{c}\text { Nickel, } \\
\text { dissolved } \\
\left(\mu g^{\prime} / L\right)\end{array}$ & $\begin{array}{l}\text { Selenlum, } \\
\text { dissolved } \\
\left(\mu g^{\prime} / L\right)\end{array}$ & $\begin{array}{l}\text { Silver, } \\
\text { dissolved } \\
(\mu \mathrm{g} / \mathrm{L})\end{array}$ & $\begin{array}{c}\text { Stron- } \\
\text { tium, } \\
\text { dissolved } \\
(\mu \mathrm{g} / \mathrm{L})\end{array}$ & $\begin{array}{c}\text { Vana- } \\
\text { dium, } \\
\text { dissolved } \\
(\mu \mathrm{g} / \mathrm{L})\end{array}$ & $\begin{array}{c}\text { Zinc, } \\
\text { dissolved } \\
(\mu \mathrm{g} / \mathrm{L})\end{array}$ \\
\hline \multicolumn{12}{|l|}{ May 1995} \\
\hline $17 \ldots$ & 5,700 & $<1$ & 54 & 57 & 2 & 30 & 2 & $<1$ & 470 & $<6$ & 54 \\
\hline \multicolumn{12}{|l|}{ Feb 1996} \\
\hline $21 \ldots$ & 560,000 & 2 & 440 & 1,500 & $<1$ & 1,600 & -- & $<5$ & 1,400 & 59 & 5,600 \\
\hline $21 \ldots$ & 550,000 & 2 & 420 & 1,500 & $<1$ & 1,600 & -- & $<10$ & 1,300 & 76 & 5,300 \\
\hline
\end{tabular}


Table 4. Chemical analyses of field replicates for water samples from sites in the Sand Coulee Coal Area, Montana, July 1994 through September 1996 (Continued)

SITE 16,472313111104901 --MINE DRAIN TO SAND COULEE NEAR SAND COULEE, MT

\begin{tabular}{|c|c|c|c|c|c|c|c|c|c|c|}
\hline Date & Time & $\begin{array}{l}\text { Calcium, } \\
\text { dls- } \\
\text { solved } \\
\text { (mg/l) }\end{array}$ & $\begin{array}{l}\text { Magne- } \\
\text { sium, } \\
\text { dlis- } \\
\text { solved } \\
\text { (mg/L) }\end{array}$ & $\begin{array}{l}\text { Sodium, } \\
\text { dis- } \\
\text { solved } \\
\text { (mgh) }\end{array}$ & $\begin{array}{l}\text { Potas- } \\
\text { sium, } \\
\text { dissolved } \\
\text { (mg/l) }\end{array}$ & $\begin{array}{l}\text { Acldity } \\
\text { (mg/L) }\end{array}$ & $\begin{array}{c}\text { Alkelinity, } \\
\text { lab } \\
(\mathrm{mg} / \mathrm{L} \text { as } \\
\left.\mathrm{CsCO}_{3}\right)\end{array}$ & $\begin{array}{l}\text { Sulfate, } \\
\text { dissolved } \\
\text { (mg/L) }\end{array}$ & $\begin{array}{l}\text { Chloride, } \\
\text { dlssolved } \\
\text { (mg/L) }\end{array}$ & $\begin{array}{l}\text { Fluoride, } \\
\text { dissolved } \\
\text { (mg/L) }\end{array}$ \\
\hline \multicolumn{11}{|l|}{ Sep 1994} \\
\hline $06 \ldots$ & 1730 & 160 & 100 & 18 & 1.7 & 42 & $e<1$ & 3,200 & 15 & 0.5 \\
\hline $06 \ldots$ & 1735 & 160 & 100 & 18 & 1.8 & 43 & $e<1$ & 2,800 & 4.6 & .6 \\
\hline \multicolumn{11}{|l|}{ Aug 1995} \\
\hline $16 \ldots$ & 1520 & 180 & 110 & 20 & 2.0 & 43 & $e<1$ & 3,600 & 13 & $<.1$ \\
\hline $16 \ldots$ & 1525 & 170 & 110 & 20 & 2.2 & 42 & e $<1$ & 3,300 & 12 & - \\
\hline
\end{tabular}

\begin{tabular}{|c|c|c|c|c|c|c|c|c|c|c|}
\hline Date & $\begin{array}{l}\text { Silica, } \\
\text { dissolved } \\
\text { (mg/L) }\end{array}$ & $\begin{array}{l}\text { Alum- } \\
\text { inum, } \\
\text { dissolved } \\
(\mu \mathrm{g} / \mathrm{L})\end{array}$ & $\begin{array}{l}\text { Areenic, } \\
\text { diseolved } \\
\text { ( } \mu \mathrm{g} / \mathrm{L})\end{array}$ & $\begin{array}{l}\text { Barium, } \\
\text { diseolved } \\
\text { ( } \mu \mathrm{g} / \mathrm{L})\end{array}$ & $\begin{array}{c}\text { Beryl- } \\
\text { lium, } \\
\text { dissolved } \\
(\mu \mathrm{g} / \mathrm{L})\end{array}$ & $\begin{array}{l}\text { Boron, } \\
\text { diseolved } \\
(\mu \mathrm{g} / \mathrm{L})\end{array}$ & $\begin{array}{c}\text { Ced- } \\
\text { mium, } \\
\text { dissolved } \\
(\mu g / L)\end{array}$ & $\begin{array}{c}\text { Chro- } \\
\text { mlum, } \\
\text { dissolved } \\
(\mu g / L)\end{array}$ & $\begin{array}{l}\text { Cobalt, } \\
\text { dissolved } \\
(\mu \mathrm{g} / \mathrm{L})\end{array}$ & $\begin{array}{l}\text { Copper, } \\
\text { dissolved } \\
(\mu \mathrm{g} / \mathrm{L})\end{array}$ \\
\hline \multicolumn{11}{|l|}{ Sep 1994} \\
\hline $06 \ldots$ & 66 & 220,000 & 4 & 4 & 28 & 320 & 39 & 80 & 1,500 & $<30$ \\
\hline $06 \ldots$ & 66 & 220,000 & 5 & 4 & 28 & 310 & 36 & 70 & 1,500 & $<30$ \\
\hline \multicolumn{11}{|l|}{ Aug 1995} \\
\hline $16 \ldots$ & 69 & 250,000 & $<5$ & 8 & 34 & 360 & 31 & 100 & 1,200 & $<50$ \\
\hline $16 \ldots$ & 69 & 240,000 & $<5$ & 10 & 36 & 310 & 32 & 90 & 1,200 & $<50$ \\
\hline
\end{tabular}

\begin{tabular}{|c|c|c|c|c|c|c|c|c|c|c|c|}
\hline Date & 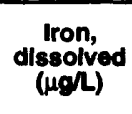 & 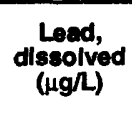 & $\begin{array}{l}\text { Lithium, } \\
\text { dlseolved } \\
\text { ( } \mu \mathrm{g} / \mathrm{L})\end{array}$ & $\begin{array}{l}\text { Mange- } \\
\text { nese, } \\
\text { dissolved } \\
(\mu g / L)\end{array}$ & $\begin{array}{c}\text { Molyb- } \\
\text { denum, } \\
\text { dissolved } \\
(\mu g / L)\end{array}$ & $\begin{array}{l}\text { Nickel, } \\
\text { dlssolved } \\
\text { ( } \mu \mathrm{g} / \mathrm{L})\end{array}$ & $\begin{array}{l}\text { Selenium, } \\
\text { dlssolved } \\
\text { ( } \mu \mathrm{g} / \mathrm{L})\end{array}$ & $\begin{array}{l}\text { Silver, } \\
\text { dissolved } \\
(\mu \mathrm{g} / \mathrm{L})\end{array}$ & $\begin{array}{c}\text { Stron- } \\
\text { tium, } \\
\text { dissolved } \\
(\mu \mathrm{g} / \mathrm{L})\end{array}$ & $\begin{array}{l}\text { Vana- } \\
\text { dium, } \\
\text { dissolved } \\
(\mu g / L)\end{array}$ & $\begin{array}{l}\text { Zinc, } \\
\text { dissolved } \\
(\mu g / L)\end{array}$ \\
\hline \multicolumn{12}{|l|}{ Sep 1994} \\
\hline $06 \ldots$ & 320,000 & $<10$ & 360 & 1,200 & $<10$ & 2,300 & $<5$ & $<3$ & 990 & 150 & 10,000 \\
\hline $06 \ldots$ & 310,000 & $<10$ & 360 & 1,200 & $<10$ & 2,300 & $<5$ & $<3$ & 990 & 150 & 10,000 \\
\hline \multicolumn{12}{|l|}{ Aug 1995} \\
\hline $16 \ldots$ & 390,000 & $<1$ & 400 & 1,400 & $<1$ & 2,600 & - & $<5$ & 1,100 & 110 & 11,000 \\
\hline $16 \ldots$ & 390,000 & $<1$ & 400 & 1,400 & $<1$ & 2,600 & - & $<5$ & 1,100 & 110 & 11,000 \\
\hline
\end{tabular}


Table 4. Chemical analyses of field replicates for water samples from sites in the Sand Coulee Coal Area, Montana, July 1994 through September 1996 (Continued)

SITE 17, 472330111082801--CENTERVILLE WETLANDS INFLOW AT CENTERVILLE, MT

\begin{tabular}{|c|c|c|c|c|c|c|c|c|c|c|}
\hline Date & Time & $\begin{array}{l}\text { Calcium, } \\
\text { dls- } \\
\text { solved } \\
\text { (mg/l) }\end{array}$ & $\begin{array}{l}\text { Magne- } \\
\text { slum, } \\
\text { dis- } \\
\text { solved } \\
\text { (mg/h) }\end{array}$ & $\begin{array}{l}\text { Sodium, } \\
\text { dis- } \\
\text { solved } \\
\text { (mgh) }\end{array}$ & $\begin{array}{l}\text { Potas- } \\
\text { sium, } \\
\text { dissolved } \\
\text { (mg/l) }\end{array}$ & $\begin{array}{l}\text { Acidlty } \\
\text { (mg/L) }\end{array}$ & $\begin{array}{c}\text { Alkalinity, } \\
\text { lab } \\
\left.(\underset{\mathrm{mg} / \mathrm{h}}{\mathrm{macO}})_{3}\right)\end{array}$ & $\begin{array}{l}\text { Sulfate, } \\
\text { dissolved } \\
\text { (mg/h) }\end{array}$ & $\begin{array}{l}\text { Chloride, } \\
\text { dlssolved } \\
\text { (mg/L) }\end{array}$ & $\begin{array}{l}\text { Fluoride, } \\
\text { dissolved } \\
\text { (mg/L) }\end{array}$ \\
\hline \multicolumn{11}{|l|}{ Mar 1995} \\
\hline $15 \ldots$ & 1700 & 180 & 81 & 14 & 1.0 & 42 & $e<1$ & 3,700 & 3.2 & 0.8 \\
\hline $15 \ldots$ & 1705 & 190 & 84 & 15 & 1.1 & 42 & $e<1$ & 4,300 & 17 & 2.8 \\
\hline
\end{tabular}

\begin{tabular}{|c|c|c|c|c|c|c|c|c|c|c|}
\hline Date & $\begin{array}{l}\text { Sllica, } \\
\text { dissolved } \\
\text { (mg/L) }\end{array}$ & $\begin{array}{l}\text { Alum- } \\
\text { inum, } \\
\text { dissolved } \\
(\mu \mathrm{g} / \mathrm{L})\end{array}$ & $\begin{array}{l}\text { Arsenic, } \\
\text { dissolved } \\
\left.\text { ( } \mu g^{\prime} / 4\right)\end{array}$ & $\begin{array}{l}\text { Barium, } \\
\text { dissolved } \\
\text { ( } \mu \mathrm{g} / \mathrm{L})\end{array}$ & $\begin{array}{l}\text { Beryl- } \\
\text { lium, } \\
\text { dissolved } \\
(\mu \mathrm{g} / \mathrm{L})\end{array}$ & $\begin{array}{l}\text { Boron, } \\
\text { dissolved } \\
\text { ( } \mu \mathrm{g} / \mathrm{L})\end{array}$ & $\begin{array}{c}\text { Cad- } \\
\text { mium, } \\
\text { dissojved } \\
(\mu g / L)\end{array}$ & $\begin{array}{c}\text { Chro- } \\
\text { mium, } \\
\text { dissolved } \\
(\mu \mathrm{g} / \mathrm{L})\end{array}$ & $\begin{array}{l}\text { Cobalt, } \\
\text { dissolved } \\
\text { ( } \mu \mathrm{g} / \mathrm{L})\end{array}$ & $\begin{array}{l}\text { Copper, } \\
\text { dissolved } \\
(\mu g / L)\end{array}$ \\
\hline \multicolumn{11}{|l|}{ Mar 1995} \\
\hline $15 \ldots$ & 87 & 220,000 & $<1$ & 5 & 31 & 180 & 32 & $<20$ & 470 & 110 \\
\hline $15 \ldots$ & 90 & 220,000 & $<1$ & 6 & 32 & 180 & 30 & 20 & 410 & 120 \\
\hline
\end{tabular}

\begin{tabular}{|c|c|c|c|c|c|c|c|c|c|c|c|}
\hline Date & $\begin{array}{l}\text { Iron, } \\
\text { dissolved } \\
(\mu \mathrm{g} / \mathrm{L})\end{array}$ & $\begin{array}{l}\text { Lead, } \\
\text { dissolved } \\
(\mu g / L)\end{array}$ & $\begin{array}{l}\text { Lithium, } \\
\text { dissolved } \\
(\mu g / L)\end{array}$ & $\begin{array}{l}\text { Manga- } \\
\text { nese, } \\
\text { dissolved } \\
(\mu \mathrm{g} / \mathrm{L})\end{array}$ & $\begin{array}{c}\text { Molyb- } \\
\text { denum, } \\
\text { dissolved } \\
(\mu \mathrm{g} / \mathrm{L})\end{array}$ & $\begin{array}{l}\text { Nickel, } \\
\text { dissolved } \\
\text { ( } \mu \mathrm{g} / \mathrm{L})\end{array}$ & $\begin{array}{l}\text { Selenium, } \\
\text { dissolved } \\
(\mu \mathrm{g} / \mathrm{h})\end{array}$ & $\begin{array}{l}\text { Silver, } \\
\text { dissolved } \\
(\mu g / h)\end{array}$ & $\begin{array}{l}\text { Stron- } \\
\text { tium, } \\
\text { dissolved } \\
(\mu g / L)\end{array}$ & $\begin{array}{c}\text { Vana- } \\
\text { dium, } \\
\text { dissolved } \\
(\mu \mathrm{g} / \mathrm{L})\end{array}$ & $\begin{array}{c}\text { Zinc, } \\
\text { dissolved } \\
(\mu \mathrm{g} / \mathrm{L})\end{array}$ \\
\hline \multicolumn{12}{|l|}{ Mar 1995} \\
\hline $\begin{array}{l}15 \ldots \\
15 \ldots\end{array}$ & $\begin{array}{l}250,000 \\
260,000\end{array}$ & $\begin{array}{l}3 \\
2\end{array}$ & $\begin{array}{l}340 \\
360\end{array}$ & $\begin{array}{l}1,500 \\
1,500\end{array}$ & $\begin{array}{l}<1 \\
<1\end{array}$ & $\begin{array}{l}800 \\
850\end{array}$ & $\begin{array}{l}2 \\
1\end{array}$ & $\begin{array}{l}<3 \\
<3\end{array}$ & $\begin{array}{l}910 \\
940\end{array}$ & $\begin{array}{l}17 \\
23\end{array}$ & $\begin{array}{l}2,400 \\
2,500\end{array}$ \\
\hline
\end{tabular}


Table 4. Chemical analyses of field replicates for water samples from sites in the Sand Coulee Coai Area, Montana, July 1994 through September 1996 (Continued)

SITE 19, $472334111104401-$ MOUNT OREGON MINE DRAIN TO KATE'S COULEE AT SAND COULEE, MT

\begin{tabular}{|c|c|c|c|c|c|c|c|c|c|c|}
\hline Date & Time & $\begin{array}{l}\text { Calcium, } \\
\text { dis- } \\
\text { solved } \\
\text { (mg/L) }\end{array}$ & $\begin{array}{l}\text { Magne- } \\
\text { slum, } \\
\text { dis- } \\
\text { solved } \\
\text { (mghl) }\end{array}$ & $\begin{array}{l}\text { Sodlum, } \\
\text { dls- } \\
\text { solved } \\
\text { (mg/L) }\end{array}$ & $\begin{array}{l}\text { Potas- } \\
\text { slum, } \\
\text { dissolved } \\
\text { (mg/L) }\end{array}$ & $\begin{array}{l}\text { Acldity } \\
\text { (mg/L) }\end{array}$ & $\begin{array}{c}\text { Alkallnity, } \\
\text { lab } \\
(\underset{\mathrm{mg} / \mathrm{L}}{\mathrm{Cs}}) \\
\left.\mathrm{CoO}_{3}\right)\end{array}$ & $\begin{array}{l}\text { Sulfate, } \\
\text { dissolved } \\
\text { (mg/l) }\end{array}$ & $\begin{array}{l}\text { Chloride, } \\
\text { dissolved } \\
\text { (mg/L) }\end{array}$ & $\begin{array}{l}\text { Fuoride, } \\
\text { dissolved } \\
\text { (mg/L) }\end{array}$ \\
\hline \multicolumn{11}{|l|}{ Aug 1994} \\
\hline $17 \ldots$ & 1115 & 170 & 130 & 24 & 5.2 & 33 & $\mathbf{e}<1$ & 2,700 & 4.3 & 0.1 \\
\hline $17 \ldots$ & 1120 & 170 & 130 & 24 & 5.3 & 34 & $e<1$ & 2,500 & 4.1 & $<1$ \\
\hline \multicolumn{11}{|l|}{ Nov 1995} \\
\hline $28 .$. & 1100 & 160 & 130 & 24 & 4.2 & 30 & $e<1$ & 2,400 & 3.7 & 1.4 \\
\hline $28 \ldots$ & 1105 & 160 & 130 & 24 & 4.6 & 31 & $e<1$ & 2,500 & 3.3 & 1.5 \\
\hline \multicolumn{11}{|l|}{ Aug 1996} \\
\hline $07 \ldots$ & 0810 & 160 & 120 & 22 & 4.8 & 29 & $e<1$ & 2,200 & 5.0 & 2.5 \\
\hline $07 \ldots$ & 0815 & 150 & 120 & 24 & 4.7 & 30 & $e<1$ & 2,300 & 5.0 & 2.7 \\
\hline
\end{tabular}

\begin{tabular}{|c|c|c|c|c|c|c|c|c|c|c|}
\hline Date & $\begin{array}{l}\text { Sllloe, } \\
\text { diseolved } \\
\text { (mg/h) }\end{array}$ & $\begin{array}{l}\text { Alum- } \\
\text { inum, } \\
\text { dissolved } \\
\text { ( } \mu \mathrm{g} / \mathrm{L})\end{array}$ & $\begin{array}{l}\text { Aranic, } \\
\text { dissolved } \\
(\mu \mathrm{g} / \mathrm{L})\end{array}$ & 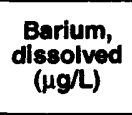 & $\begin{array}{l}\text { Beryj- } \\
\text { lium, } \\
\text { diesolved } \\
(\mu g / h)\end{array}$ & $\begin{array}{l}\text { Boron, } \\
\text { dlesolved } \\
(\mu \mathrm{g} / \mathrm{L})\end{array}$ & $\begin{array}{l}\text { Cad- } \\
\text { mium, } \\
\text { dissolved } \\
(\mu g / L)\end{array}$ & $\begin{array}{c}\text { Chro- } \\
\text { mium, } \\
\text { dissolved } \\
\text { ( } \mu \mathrm{g} / \mathrm{L} \text { ) }\end{array}$ & $\begin{array}{l}\text { Cobalt, } \\
\text { dlesolved } \\
(\mu g / \mathrm{L})\end{array}$ & $\begin{array}{l}\text { Copper, } \\
\text { dissolved } \\
(\mu \mathrm{g} / \mathrm{L})\end{array}$ \\
\hline \multicolumn{11}{|l|}{ Aug 1994} \\
\hline $17 \ldots$ & 39 & 170,000 & 14 & 14 & 26 & 270 & 29 & 30 & 2,000 & $<30$ \\
\hline \multicolumn{11}{|l|}{ Nov 1995} \\
\hline 28 ... & 37 & 180,000 & 11 & 27 & 23 & 260 & 8 & 20 & 670 & $<30$ \\
\hline $28 \ldots$ & 38 & 160,000 & 10 & 27 & 23 & 290 & 8 & 30 & 710 & $<30$ \\
\hline \multicolumn{11}{|l|}{ Aug 1996} \\
\hline $07 \ldots$ & 36 & 150,000 & 9 & 26 & 23 & 170 & 8 & 30 & 660 & $<40$ \\
\hline $07 \ldots$ & 36 & 150,000 & 14 & 27 & 25 & 180 & 8 & 40 & 670 & $<40$ \\
\hline
\end{tabular}

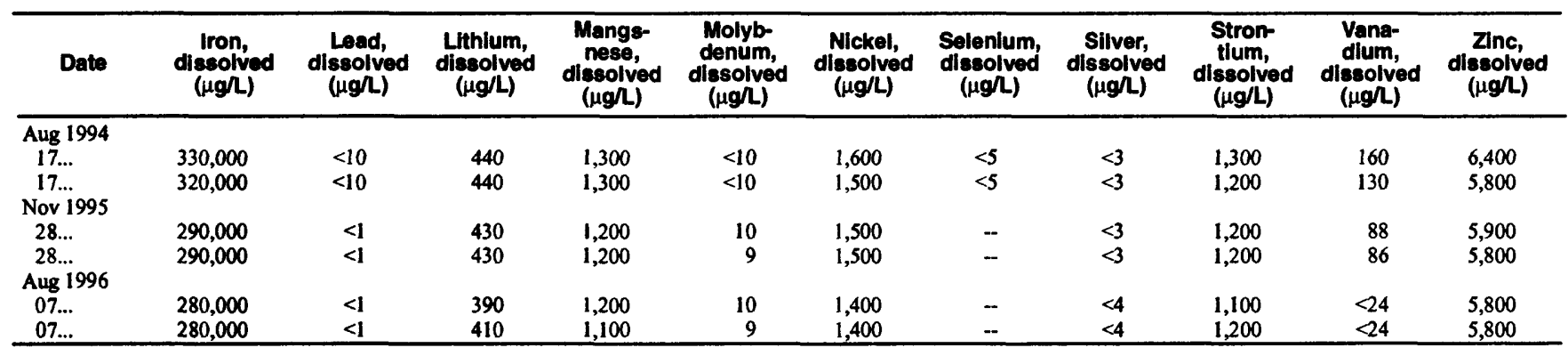


Table 4. Chemical analyses of field replicates for water samples from sites in the Sand Coulee Coal Area, Montana, July 1994 through September 1996 (Continued)

\section{SITE 20, 472346111102401--NELSON MINE DRAIN TO SAND COULEE AT SAND COULEE, MT}

\begin{tabular}{|c|c|c|c|c|c|c|c|c|c|c|}
\hline Date & Time & $\begin{array}{c}\text { Caicium, } \\
\text { dis- } \\
\text { solved } \\
\text { (mg/l) }\end{array}$ & $\begin{array}{l}\text { Magno- } \\
\text { sium, } \\
\text { dis- } \\
\text { solved } \\
\text { (mg/h) }\end{array}$ & $\begin{array}{l}\text { Sodium, } \\
\text { dis- } \\
\text { solved } \\
\text { (mg/l) }\end{array}$ & $\begin{array}{l}\text { Potas- } \\
\text { sium, } \\
\text { dlesolved } \\
\text { (mg/l) }\end{array}$ & $\begin{array}{l}\text { Acidity } \\
\text { (mg/l) }\end{array}$ & $\begin{array}{c}\text { Alkallnity, } \\
\text { lab } \\
\text { (mg/las as } \\
\text { Caco })\end{array}$ & $\begin{array}{l}\text { Sulfate, } \\
\text { diseolved } \\
\text { (mg/l) }\end{array}$ & $\begin{array}{l}\text { Chloride, } \\
\text { dissolved } \\
\text { (mg/h) }\end{array}$ & $\begin{array}{l}\text { Fluoride, } \\
\text { diseolved } \\
\text { (mg/l) }\end{array}$ \\
\hline \multicolumn{11}{|l|}{ Jan 1995} \\
\hline $\begin{array}{l}11 \ldots \\
11 \ldots\end{array}$ & $\begin{array}{l}1500 \\
1505\end{array}$ & $\begin{array}{l}250 \\
250\end{array}$ & $\begin{array}{l}230 \\
230\end{array}$ & $\begin{array}{l}16 \\
17\end{array}$ & $\begin{array}{l}0.9 \\
1.0\end{array}$ & $\begin{array}{l}170 \\
170\end{array}$ & $\begin{array}{l}e<1 \\
e<1\end{array}$ & $\begin{array}{l}11,000 \\
11,000\end{array}$ & $\begin{array}{l}38 \\
40\end{array}$ & $\begin{array}{l}7.3 \\
5.9\end{array}$ \\
\hline
\end{tabular}

\begin{tabular}{|c|c|c|c|c|c|c|c|c|c|c|}
\hline Date & $\begin{array}{l}\text { Sllica, } \\
\text { dissolved } \\
\text { (mg/L) }\end{array}$ & $\begin{array}{l}\text { Alum- } \\
\text { Inum, } \\
\text { dissolved } \\
\left(\mu g^{\prime} L\right)\end{array}$ & 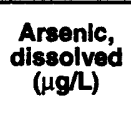 & $\begin{array}{l}\text { Berium, } \\
\text { dlesolved } \\
\text { ( } \mu \mathrm{g} / \mathrm{L})\end{array}$ & $\begin{array}{c}\text { Beryl- } \\
\text { llum, } \\
\text { dissolved } \\
(\mu \mathrm{g} / \mathrm{L})\end{array}$ & $\begin{array}{l}\text { Boron, } \\
\text { dissolved } \\
\text { ( } \mu \mathrm{g} / \mathrm{L})\end{array}$ & $\begin{array}{c}\text { Cad- } \\
\text { mium, } \\
\text { dissolved } \\
(\mu g / L)\end{array}$ & 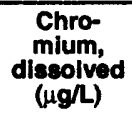 & $\begin{array}{l}\text { Cobalt, } \\
\text { dissolved } \\
(\mu g / L)\end{array}$ & $\begin{array}{l}\text { Copper, } \\
\text { diseolved } \\
(\mu g / L)\end{array}$ \\
\hline $\begin{array}{c}\text { Jan } 1995 \\
11 \ldots \\
11 \ldots \\
\end{array}$ & $\begin{array}{l}59 \\
70 \\
\end{array}$ & $\begin{array}{l}920,000 \\
870,000 \\
\end{array}$ & $\begin{array}{l}63 \\
58 \\
\end{array}$ & $\begin{array}{l}<100 \\
<100 \\
\end{array}$ & $\begin{array}{l}80 \\
80 \\
\end{array}$ & $\begin{array}{l}830 \\
880 \\
\end{array}$ & $\begin{array}{l}110 \\
110 \\
\end{array}$ & $\begin{array}{l}320 \\
310 \\
\end{array}$ & $\begin{array}{l}2,200 \\
2,200 \\
\end{array}$ & $\begin{array}{l}300 \\
310 \\
\end{array}$ \\
\hline
\end{tabular}

\begin{tabular}{|c|c|c|c|c|c|c|c|c|c|c|c|}
\hline Date & $\begin{array}{l}\text { Iron, } \\
\text { dissolved } \\
(\mu g / L)\end{array}$ & $\begin{array}{l}\text { Lead, } \\
\text { dissolved } \\
(\mu \mathrm{g} / \mathrm{L})\end{array}$ & $\begin{array}{l}\text { Lithium, } \\
\text { dissolved } \\
\text { ( } \mu g / L)\end{array}$ & $\begin{array}{l}\text { Mange- } \\
\text { nese, } \\
\text { dissolved } \\
(\mu g / L)\end{array}$ & $\begin{array}{c}\text { Molyb- } \\
\text { denum, } \\
\text { dissolved } \\
(\mu g \Omega)\end{array}$ & $\begin{array}{l}\text { Nickel, } \\
\text { dissolved } \\
\text { ( } \mu g / \text { L) }\end{array}$ & $\begin{array}{l}\text { Selenlum, } \\
\text { dissolved } \\
(\mu g / L)\end{array}$ & $\begin{array}{c}\text { Silver, } \\
\text { dissolved } \\
(\mu \mathrm{g} / \mathrm{L})\end{array}$ & $\begin{array}{l}\text { Stron- } \\
\text { tlum, } \\
\text { diseolved } \\
\left(\mu g^{\prime} L\right)\end{array}$ & $\begin{array}{c}\text { Vana- } \\
\text { dium, } \\
\text { dissolved } \\
(\mu g / L)\end{array}$ & $\begin{array}{c}\text { Zinc, } \\
\text { dissolved } \\
(\mu g / L)\end{array}$ \\
\hline Jan 1995 & & & & & & & & & & & \\
\hline
\end{tabular}

SITE 22, 472446111085101--PIPE SPRING AT TRACY, MT

\begin{tabular}{|c|c|c|c|c|c|c|c|c|c|c|}
\hline Date & Time & $\begin{array}{c}\text { Caicium, } \\
\text { dis- } \\
\text { solved } \\
\text { (mg/L) }\end{array}$ & $\begin{array}{l}\text { Magne- } \\
\text { sium, } \\
\text { dls- } \\
\text { solved } \\
\text { (mg/h) }\end{array}$ & $\begin{array}{l}\text { Sodium, } \\
\text { dis- } \\
\text { solved } \\
\text { (mg/l) }\end{array}$ & $\begin{array}{l}\text { Potas- } \\
\text { sium, } \\
\text { dissolved } \\
\text { (mg/L) }\end{array}$ & $\begin{array}{l}\text { Acldity } \\
\text { (mg/l) }\end{array}$ & $\begin{array}{c}\text { Alkalinity, } \\
\text { lab } \\
\left(\mathrm{mgh}_{\mathrm{as}}\right. \\
\left.\mathrm{CaCO}_{3}\right)\end{array}$ & $\begin{array}{l}\text { Sulfate, } \\
\text { dissolved } \\
\text { (mg/L) }\end{array}$ & $\begin{array}{l}\text { Chioride, } \\
\text { dissolved } \\
\text { (mg/L) }\end{array}$ & $\begin{array}{l}\text { Fluoride, } \\
\text { dissolved } \\
\text { (mg/L) }\end{array}$ \\
\hline $\begin{array}{c}\text { Oct } 1994 \\
14 \ldots \\
14 \ldots\end{array}$ & $\begin{array}{l}0800 \\
0805\end{array}$ & $\begin{array}{l}79 \\
80 \\
\end{array}$ & $\begin{array}{l}68 \\
67\end{array}$ & $\begin{array}{l}21 \\
21 \\
\end{array}$ & $\begin{array}{l}2.5 \\
2.4 \\
\end{array}$ & $\begin{array}{l}6.1 \\
6.6 \\
\end{array}$ & $\begin{array}{l}e<1 \\
e<1\end{array}$ & $\begin{array}{l}910 \\
930 \\
\end{array}$ & $\begin{array}{l}5.6 \\
5.8 \\
\end{array}$ & $\begin{array}{l}2.0 \\
2.0 \\
\end{array}$ \\
\hline
\end{tabular}

\begin{tabular}{|c|c|c|c|c|c|c|c|c|c|c|}
\hline Date & $\begin{array}{l}\text { Silica, } \\
\text { dissolved } \\
\text { (mg/L) }\end{array}$ & $\begin{array}{c}\text { Alum- } \\
\text { Inum, } \\
\text { dissolved } \\
(\mu g / L)\end{array}$ & $\begin{array}{l}\text { Arsenic, } \\
\text { dissolved } \\
(\mu g / L)\end{array}$ & $\begin{array}{c}\text { Barium, } \\
\text { dissolved } \\
(\mu g / L)\end{array}$ & $\begin{array}{c}\text { Beryl- } \\
\text { llum, } \\
\text { dissolved } \\
(\mu g / L)\end{array}$ & $\begin{array}{c}\text { Boron, } \\
\text { dissolved } \\
(\mu \mathrm{g} / \mathrm{L})\end{array}$ & $\begin{array}{c}\text { Cad- } \\
\text { mium, } \\
\text { dissolved } \\
(\mu g / L)\end{array}$ & $\begin{array}{c}\text { Chro- } \\
\text { mium, } \\
\text { dissolved } \\
(\mu g / L)\end{array}$ & $\begin{array}{c}\text { Cobalt, } \\
\text { dissolved } \\
(\mu g / L)\end{array}$ & $\begin{array}{c}\text { Copper, } \\
\text { dissolved } \\
\left(\mu g^{\prime} L\right)\end{array}$ \\
\hline $\begin{array}{c}\text { Oet } 1994 \\
14 \ldots \\
14 \ldots\end{array}$ & $\begin{array}{l}50 \\
50\end{array}$ & $\begin{array}{l}35,000 \\
36,000\end{array}$ & $\begin{array}{l}<1 \\
<1\end{array}$ & $\begin{array}{l}3 \\
3\end{array}$ & $\begin{array}{l}7 \\
7\end{array}$ & $\begin{array}{l}170 \\
180\end{array}$ & $\begin{array}{l}<1 \\
<1\end{array}$ & $\begin{array}{l}<5 \\
<5\end{array}$ & $\begin{array}{l}200 \\
220\end{array}$ & $\begin{array}{l}20 \\
10\end{array}$ \\
\hline
\end{tabular}

\begin{tabular}{|c|c|c|c|c|c|c|c|c|c|c|c|}
\hline Date & $\begin{array}{l}\text { Iron, } \\
\text { dissolved } \\
(\mu g / L)\end{array}$ & $\begin{array}{l}\text { Lesd, } \\
\text { diseolved } \\
(\mu \mathrm{g} / \mathrm{L})\end{array}$ & $\begin{array}{l}\text { Lithlum, } \\
\text { dissolved } \\
(\mu \mathrm{g} / \mathrm{L})\end{array}$ & $\begin{array}{l}\text { Manga- } \\
\text { nese, } \\
\text { dliseolved } \\
(\mu g / L)\end{array}$ & $\begin{array}{l}\text { Molyb- } \\
\text { denum, } \\
\text { dissolved } \\
(\mu g / L)\end{array}$ & $\begin{array}{c}\text { Nickel, } \\
\text { dissolved } \\
(\mu \mathrm{g} L)\end{array}$ & $\begin{array}{l}\text { Selenium, } \\
\text { dissolved } \\
(\mu g / L)\end{array}$ & $\begin{array}{l}\text { Silver, } \\
\text { diseolved } \\
(\mu g / \text { L })\end{array}$ & $\begin{array}{c}\text { Stron- } \\
\text { tium, } \\
\text { dissolved } \\
(\mu g / L)\end{array}$ & $\begin{array}{c}\text { Vena- } \\
\text { dlum, } \\
\text { dissolved } \\
(\mu g / L)\end{array}$ & $\begin{array}{l}\text { Zinc, } \\
\text { dissolved } \\
(\mu g /))\end{array}$ \\
\hline \multicolumn{12}{|l|}{ Oct 1994} \\
\hline $14 \ldots$ & 5,900 & $<10$ & 140 & 580 & $<10$ & 360 & $<2$ & $<1$ & 600 & $<6$ & 1,100 \\
\hline $14 \ldots$ & 5,800 & $<10$ & 130 & 580 & $<10$ & 350 & $<2$ & $<1$ & 590 & $<6$ & 1,200 \\
\hline
\end{tabular}


Table 4. Chemical analyses of field replicates for water samples from sites in the Sand Coulee Coal Area, Montana, July 1994 through September 1996 (Continued)

SITE 23, 472447111085301--STOCK TANK SPRING AT TRACY, MT

\begin{tabular}{|c|c|c|c|c|c|c|c|c|c|c|}
\hline Date & Time & $\begin{array}{l}\text { Calcium, } \\
\text { dls- } \\
\text { solved } \\
\text { (mg/L) }\end{array}$ & $\begin{array}{l}\text { Magne- } \\
\text { sium, } \\
\text { dis- } \\
\text { solved } \\
\text { (mg/l) }\end{array}$ & $\begin{array}{l}\text { Sodium, } \\
\text { dis- } \\
\text { solved } \\
\text { (mg/l) }\end{array}$ & $\begin{array}{l}\text { Potas- } \\
\text { slum, } \\
\text { dissolved } \\
\text { (mg/L) }\end{array}$ & $\begin{array}{l}\text { Acldity } \\
\text { (mg/L) }\end{array}$ & $\begin{array}{c}\text { Alkalinity, } \\
\text { lab } \\
\left(\begin{array}{c}\mathrm{mg}^{\prime} / \mathrm{L} \text { as } \\
\left.\mathrm{CaCO}_{3}\right)\end{array}\right.\end{array}$ & $\begin{array}{l}\text { Sulfate, } \\
\text { dissolved } \\
\text { (mg/L) }\end{array}$ & $\begin{array}{l}\text { Chioride, } \\
\text { dissolved } \\
\text { (mg/L) }\end{array}$ & $\begin{array}{c}\text { Fuoride, } \\
\text { dissolved } \\
\text { (mg/l) }\end{array}$ \\
\hline \multicolumn{11}{|l|}{$\operatorname{Dec} 1994$} \\
\hline $15 \ldots$ & 1405 & 65 & 58 & 14 & 2.0 & 0.2 & 227 & 170 & 6.1 & 1.0 \\
\hline $15 \ldots$ & 1410 & 64 & 56 & 14 & 2.0 & .1 & 226 & 170 & 6.2 & 1.0 \\
\hline
\end{tabular}

\begin{tabular}{|c|c|c|c|c|c|c|c|c|c|c|}
\hline Date & $\begin{array}{l}\text { Sllica, } \\
\text { dissolved } \\
\text { (mg/L) }\end{array}$ & $\begin{array}{l}\text { Alum- } \\
\text { Inum, } \\
\text { dissolved } \\
\text { ( } \mu \mathrm{g} / \mathrm{L})\end{array}$ & $\begin{array}{l}\text { Arsenic, } \\
\text { dlssolved } \\
\text { ( } \mu \mathrm{g} / \mathrm{L})\end{array}$ & $\begin{array}{l}\text { Barium, } \\
\text { dissolved } \\
\text { ( } \mu \mathrm{g} / \mathrm{L})\end{array}$ & $\begin{array}{l}\text { Beryl- } \\
\text { Ilum, } \\
\text { dissolved } \\
\text { ( } \mu \mathrm{g} / \mathrm{L})\end{array}$ & $\begin{array}{l}\text { Boron, } \\
\text { dissolved } \\
\text { ( } \mu \mathrm{g} / \mathrm{L})\end{array}$ & $\begin{array}{c}\text { Cad- } \\
\text { mlum, } \\
\text { dissolved } \\
(\mu \mathrm{g} / \mathrm{L})\end{array}$ & $\begin{array}{c}\text { Chro- } \\
\text { mlum, } \\
\text { dlssolved } \\
(\mu \mathrm{g} / \mathrm{L} \text { ) }\end{array}$ & $\begin{array}{l}\text { Cobalt, } \\
\text { dlssolved } \\
(\mu g / L)\end{array}$ & $\begin{array}{l}\text { Copper, } \\
\text { dissolved } \\
(\mu \mathrm{g} / \mathrm{L})\end{array}$ \\
\hline $\begin{array}{c}\text { Dec } 1994 \\
15 \ldots \\
15 \ldots\end{array}$ & $\begin{array}{l}10 \\
10\end{array}$ & $\begin{array}{l}<10 \\
<10\end{array}$ & $\begin{array}{l}<1 \\
<1\end{array}$ & $\begin{array}{l}46 \\
45\end{array}$ & $\begin{array}{r}<0.5 \\
<.5\end{array}$ & $\begin{array}{l}80 \\
70\end{array}$ & $\begin{array}{l}<1 \\
<1\end{array}$ & $\begin{array}{l}<5 \\
<5\end{array}$ & $\begin{array}{r}<3 \\
3\end{array}$ & $\begin{array}{l}<10 \\
<10\end{array}$ \\
\hline
\end{tabular}

\begin{tabular}{|c|c|c|c|c|c|c|c|c|c|c|c|}
\hline Date & $\begin{array}{l}\text { Iron, } \\
\text { dissolved } \\
(\mu g / L)\end{array}$ & $\begin{array}{l}\text { Lead, } \\
\text { dissolved } \\
\text { ( } \mu \mathrm{g} / \mathrm{L})\end{array}$ & $\begin{array}{l}\text { Lithium, } \\
\text { dissolved } \\
\text { ( } \mu \mathrm{g} / \mathrm{L})\end{array}$ & $\begin{array}{l}\text { Manga- } \\
\text { nese, } \\
\text { dlssolved } \\
(\mu g / L)\end{array}$ & $\begin{array}{l}\text { Molyb- } \\
\text { denum, } \\
\text { dissolved } \\
\text { ( } \mu \mathrm{g} / \mathrm{L})\end{array}$ & $\begin{array}{l}\text { Nickel, } \\
\text { dissolved } \\
(\mu \mathrm{g} / \mathrm{L})\end{array}$ & $\begin{array}{l}\text { Selenium, } \\
\text { dissolved } \\
\text { ( } \mu \mathrm{g} / \mathrm{L})\end{array}$ & $\begin{array}{l}\text { Sllver, } \\
\text { dissolved } \\
\text { ( } \mu \mathrm{g} / \mathrm{L})\end{array}$ & $\begin{array}{c}\text { Stron- } \\
\text { tlum, } \\
\text { dissolved } \\
\text { ( } \mu \mathrm{g} / \mathrm{L})\end{array}$ & $\begin{array}{l}\text { Vana- } \\
\text { dium, } \\
\text { dissolved } \\
\text { ( } \mu \mathrm{g} / \mathrm{L})\end{array}$ & $\begin{array}{l}\text { Zinc, } \\
\text { dissolved } \\
(\mu \mathrm{g} / \mathrm{L})\end{array}$ \\
\hline \multicolumn{12}{|l|}{ Dec 1994} \\
\hline $15 \ldots$ & $<3$ & $<10$ & 35 & 1 & 20 & $<10$ & 2 & $<1$ & 490 & $<6$ & 11 \\
\hline $15 \ldots$ & 5 & $<10$ & 35 & 1 & $<10$ & $<10$ & 2 & $<1$ & 480 & $<6$ & 5 \\
\hline
\end{tabular}

SITE 24, 472513111082501--JOHNSON BADWATER MINE SMALL WETLANDS INFLOW NEAR TRACY, MT

\begin{tabular}{|c|c|c|c|c|c|c|c|c|c|c|}
\hline Date & Time & $\begin{array}{l}\text { Calclum, } \\
\text { dis- } \\
\text { solved } \\
\text { (mg/L) }\end{array}$ & $\begin{array}{l}\text { Magne- } \\
\text { slum, } \\
\text { dls- } \\
\text { solved } \\
\text { (mg/L) }\end{array}$ & $\begin{array}{l}\text { Sodium, } \\
\text { dlis- } \\
\text { solved } \\
\text { (mg/L) }\end{array}$ & $\begin{array}{l}\text { Potas- } \\
\text { slum, } \\
\text { dissolved } \\
\text { (mgl) }\end{array}$ & $\begin{array}{l}\text { Acidity } \\
\text { (mg/l) }\end{array}$ & 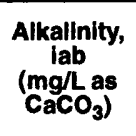 & $\begin{array}{l}\text { Sulfate, } \\
\text { dlissolved } \\
\text { (mg/L) }\end{array}$ & $\begin{array}{l}\text { Chloride, } \\
\text { dissolved } \\
\text { (mg/L) }\end{array}$ & $\begin{array}{c}\text { Fluoride, } \\
\text { dissolved } \\
\text { (mg/L) }\end{array}$ \\
\hline \multicolumn{11}{|l|}{ Nov 1994} \\
\hline $15 \ldots$ & 1010 & 180 & 130 & 25 & 7.4 & 12 & $e<1$ & 1,800 & 6.8 & 6.3 \\
\hline $15 \ldots$ & 1015 & 180 & 140 & 24 & 7.5 & 12 & $\cdots$ & 2,100 & 7.5 & 5.9 \\
\hline
\end{tabular}

\begin{tabular}{|c|c|c|c|c|c|c|c|c|c|c|}
\hline Date & $\begin{array}{l}\text { Slllca, } \\
\text { dissolved } \\
\text { (mg/L) }\end{array}$ & $\begin{array}{c}\text { Alum- } \\
\text { inum, } \\
\text { dissolved } \\
\text { ( } \mu \mathrm{g} / \mathrm{L})\end{array}$ & $\begin{array}{l}\text { Arsenic, } \\
\text { dissolved } \\
(\mu g / L)\end{array}$ & $\begin{array}{l}\text { Barium, } \\
\text { dlssolved } \\
\text { ( } \mu \mathrm{g} / \mathrm{L} \text { ) }\end{array}$ & $\begin{array}{c}\text { Beryi- } \\
\text { lium, } \\
\text { dissolved } \\
\text { ( } \mu \mathrm{g} / \mathrm{L} \text { ) }\end{array}$ & $\begin{array}{l}\text { Boron, } \\
\text { dissolved } \\
\text { ( } \mu g / \mathrm{L})\end{array}$ & $\begin{array}{c}\text { Cad- } \\
\text { mlum, } \\
\text { dissolved } \\
(\mu \mathrm{g} / \mathrm{L})\end{array}$ & $\begin{array}{c}\text { Chro- } \\
\text { mlum, } \\
\text { dlissolved } \\
\text { ( } \mu \mathrm{g} / \mathrm{L} \text { ) }\end{array}$ & $\begin{array}{l}\text { Cobalt, } \\
\text { dissolved } \\
(\mu \mathrm{g} / \mathrm{L})\end{array}$ & $\begin{array}{l}\text { Copper, } \\
\text { dissolved } \\
(\mu \mathrm{g} / \mathrm{L})\end{array}$ \\
\hline \multicolumn{11}{|l|}{ Nov 1994} \\
\hline $15 \ldots$ & 48 & 54,000 & $<1$ & 8 & 25 & 300 & 9 & $<20$ & 790 & $<30$ \\
\hline $15 \ldots$ & 47 & 55,000 & $<1$ & 8 & 27 & 300 & 7 & $<20$ & 730 & $<30$ \\
\hline
\end{tabular}

\begin{tabular}{|c|c|c|c|c|c|c|c|c|c|c|c|}
\hline Date & $\begin{array}{l}\text { Iron, } \\
\text { dissolved } \\
\text { ( } \mu g / \mathrm{L})\end{array}$ & $\begin{array}{l}\text { Lead, } \\
\text { dissolved } \\
(\mu g / L)\end{array}$ & $\begin{array}{l}\text { Lithlum, } \\
\text { dissolved } \\
(\mu \mathrm{g} / \mathrm{L})\end{array}$ & $\begin{array}{l}\text { Manga- } \\
\text { nese, } \\
\text { dissolved } \\
\text { ( } \mu \mathrm{g} / \mathrm{L})\end{array}$ & $\begin{array}{l}\text { Molyb- } \\
\text { denum, } \\
\text { dissolved } \\
(\mu g / L)\end{array}$ & $\begin{array}{c}\text { Nickel, } \\
\text { dissolved } \\
\text { ( } \mu \mathrm{g} / \mathrm{L})\end{array}$ & $\begin{array}{c}\text { Selenium, } \\
\text { dissolved } \\
\text { ( } \mu \mathrm{g} / \mathrm{L})\end{array}$ & $\begin{array}{l}\text { Sllver, } \\
\text { dissolved } \\
(\mu \mathrm{g} / \mathrm{L})\end{array}$ & $\begin{array}{c}\text { Stron- } \\
\text { tium, } \\
\text { dissolved } \\
(\mu g / L)\end{array}$ & $\begin{array}{l}\text { Vana- } \\
\text { dlum, } \\
\text { dissolved } \\
(\mu g / L)\end{array}$ & $\begin{array}{c}\text { Zinc, } \\
\text { dissolved } \\
(\mu g / L)\end{array}$ \\
\hline \multicolumn{12}{|l|}{ Nov 1994} \\
\hline $15 \ldots$ & 130,000 & $<10$ & 390 & 1,000 & $<10$ & 1,100 & $<1$ & $<3$ & 1,200 & 25 & 3,300 \\
\hline $15 \ldots$ & 140,000 & $<10$ & 390 & 990 & $<10$ & 1,100 & $<1$ & $<3$ & 1,300 & 28 & 3,200 \\
\hline
\end{tabular}


Table 5. Chemical analyses of field blanks for water samples

[Abbreviations: lab, laboratory; $\mu \mathrm{g} / \mathrm{L}$, micrograms per liter; $\mu \mathrm{S} / \mathrm{cm}$, microsiemens per centimeter at $25^{\circ} \mathrm{C} ; \mathrm{mg} / \mathrm{L}$, milligrams per liter. Symbols: $<$, less than minimum reporting level; --, no data]

\begin{tabular}{|c|c|c|c|c|c|c|c|c|c|c|c|}
\hline Date & Time & $\begin{array}{c}\text { Specific } \\
\text { conduc- } \\
\text { tance, } \\
\text { onsite } \\
(\mu S / \mathrm{cm})\end{array}$ & $\begin{array}{c}\text { pH, } \\
\text { onsite } \\
\text { (stand- } \\
\text { ard } \\
\text { units) }\end{array}$ & $\begin{array}{l}\text { Calcium, } \\
\text { dis- } \\
\text { solved } \\
\text { (mg/L) }\end{array}$ & $\begin{array}{l}\text { Magne- } \\
\text { sium, } \\
\text { dis- } \\
\text { solved } \\
\text { (mg/L) }\end{array}$ & $\begin{array}{l}\text { Sodium, } \\
\text { dis- } \\
\text { solved } \\
\text { (mgl) }\end{array}$ & $\begin{array}{l}\text { Potas- } \\
\text { slum, } \\
\text { dissolved } \\
\text { (mg/L) }\end{array}$ & $\begin{array}{l}\text { Acidity } \\
\text { (mg/h) }\end{array}$ & $\begin{array}{c}\text { Alkalinity, } \\
\text { lab } \\
\left(\mathrm{mgl}_{\text {as }}\right. \\
\left.\mathrm{CaCO}_{3}\right)\end{array}$ & $\begin{array}{l}\text { Sulfate, } \\
\text { dissolved } \\
\text { (mg/L) }\end{array}$ & $\begin{array}{l}\text { Chloride, } \\
\text { dissolved } \\
\text { (mg/L) }\end{array}$ \\
\hline $\begin{array}{l}\text { Jul } 1994 \\
20 \ldots \\
\text { Aug }\end{array}$ & 0945 & 2 & 6.8 & $<0.02$ & $<0.01$ & $<0.20$ & $<0.10$ & 0.2 & 1.7 & $<0.10$ & $<0.10$ \\
\hline $\begin{array}{l}\text { Aug } \\
17 \ldots \\
\text { Sep }\end{array}$ & 1440 & 1 & 6.8 & .02 & $<.01$ & $<.20$ & $<.10$ & $<.1$ & 1.3 & $<.10$ & $<.10$ \\
\hline $\begin{array}{c}07 . . . \\
\text { Oct }\end{array}$ & 1040 & 1 & 6.0 & $<.02$ & $<.01$ & $<.20$ & $<.10$ & $<.1$ & 1.5 & 2.4 & $<.10$ \\
\hline $\begin{array}{l}14 \ldots \\
\text { Nov }\end{array}$ & 1100 & 2 & 6.0 & $<.02$ & $<.01$ & $<.20$ & $<.10$ & .1 & 1.6 & $<.10$ & $<.10$ \\
\hline $\begin{array}{l}15 \ldots \\
\text { Dec }\end{array}$ & 1220 & 3 & 7.1 & $<.02$ & $<.01$ & $<.20$ & $<.10$ & $<.1$ & 2.0 & .80 & $<10$ \\
\hline $\begin{array}{l}16 \ldots \\
\operatorname{Jan} 1995\end{array}$ & 1215 & 2 & 5.6 & $<.02$ & $<.01$ & $<.20$ & $<.10$ & .1 & 1.6 & $<.10$ & $<.10$ \\
\hline $\begin{array}{l}11 \ldots \\
\text { Feb }\end{array}$ & 1410 & 2 & 5.8 & $<.02$ & $<.01$ & $<.20$ & $<.10$ & .1 & - & $<.10$ & $<.10$ \\
\hline $\begin{array}{c}21 \ldots \\
\text { Mar }\end{array}$ & 1810 & 2 & 5.8 & $<.02$ & $<.01$ & $<.20$ & $<.10$ & $<.1$ & 1.3 & $<.10$ & $<.10$ \\
\hline $\begin{array}{l}15 \ldots \\
\text { Apr }\end{array}$ & 1325 & 1 & 5.5 & $<.02$ & $<.01$ & $<.20$ & $<.10$ & $<.1$ & 1.4 & $<.10$ & $<.10$ \\
\hline $\begin{array}{l}12 \ldots \\
\text { May }\end{array}$ & 1030 & 2 & 5.3 & $<.02$ & $<.01$ & $<.20$ & $<.10$ & $<.1$ & 1.4 & $<.10$ & $<10$ \\
\hline $\begin{array}{l}17 \ldots \\
\text { Jun }\end{array}$ & 1415 & 2 & 6.1 & .02 & $<.01$ & $<.20$ & $<.10$ & $<.1$ & 1.3 & .10 & $<.10$ \\
\hline $\begin{array}{l}13 \ldots \\
\text { Jul }\end{array}$ & 1605 & 2 & 7.3 & $<.02$ & $<.01$ & $<.20$ & $<.10$ & $<.1$ & 1.8 & $<.10$ & $<.10$ \\
\hline $\begin{array}{l}13 . . \\
\text { Aug }\end{array}$ & 1100 & 2 & 5.5 & $<.02$ & $<.01$ & $<.20$ & $<.10$ & $<.1$ & - & 7.2 & $<.10$ \\
\hline $\begin{array}{l}16 \ldots \\
\text { Sep }\end{array}$ & 1200 & 2 & 6.0 & $<.02$ & $<.01$ & $<.20$ & $<.10$ & $<.1$ & 1.6 & $<.10$ & $<.10$ \\
\hline $\begin{array}{l}13 \ldots \\
\text { Oct }\end{array}$ & 1110 & 2 & 5.9 & .04 & $<.01$ & $<.20$ & $<.10$ & $<.1$ & - & 1.0 & $<.10$ \\
\hline $\begin{array}{l}10 \ldots \\
\text { Nov }\end{array}$ & 1230 & 1 & 5.9 & $<.02$ & $<.01$ & $<.20$ & $<.10$ & $<.1$ & 1.3 & 7.5 & $<.10$ \\
\hline $\begin{array}{c}29 \ldots \\
\operatorname{Jan} 1996\end{array}$ & 1500 & 2 & 5.8 & $<.02$ & $<.01$ & $<.20$ & $<.10$ & .3 & $<1.0$ & $<.10$ & $<.10$ \\
\hline $\begin{array}{l}11 \ldots \\
\text { Feb }\end{array}$ & 1100 & 2 & 5.7 & $<.02$ & $<.01$ & $<.20$ & $<.10$ & .1 & 1.6 & $<.10$ & .04 \\
\hline $\begin{array}{l}21 . . . \\
\mathrm{Apr}\end{array}$ & 1430 & 2 & 6.6 & $<.02$ & $<.01$ & $<.20$ & $<.10$ & .1 & 1.8 & $<.10$ & $<.10$ \\
\hline $\begin{array}{l}03 . . . \\
\text { Jun }\end{array}$ & 0930 & 2 & 5.2 & $<.02$ & $<.01$ & $<.20$ & $<.10$ & $<.1$ & 1.8 & $<10$ & $<.10$ \\
\hline $\begin{array}{c}04 \ldots \\
\text { Jul }\end{array}$ & 0900 & 1 & 6.3 & $<.02$ & $<.01$ & $<.20$ & $<.10$ & $<.1$ & $<1.0$ & $<.10$ & $<.10$ \\
\hline $\begin{array}{l}03 \ldots \\
\text { Aug }\end{array}$ & 1100 & 1 & 6.5 & $<.02$ & $<.01$ & $<.20$ & $<.10$ & $<.1$ & 1.5 & $<.10$ & $<.10$ \\
\hline $\begin{array}{l}06 . . . \\
\text { Sep }\end{array}$ & 1800 & 2 & 5.8 & $<.02$ & $<.01$ & $<.20$ & $<.10$ & .2 & 1.6 & $<.10$ & $<.10$ \\
\hline $04 \ldots$ & 1300 & 2 & 5.5 & $<.02$ & $<.01$ & $<.20$ & $<.10$ & $<.1$ & 1.3 & $<10$ & $<.10$ \\
\hline
\end{tabular}


Table 5. Chemical analyses of field blanks for water samples--Continued

\begin{tabular}{|c|c|c|c|c|c|c|c|c|c|c|c|}
\hline Date & $\begin{array}{l}\text { Fluoride, } \\
\text { dis- } \\
\text { solved } \\
\text { (mg/L) }\end{array}$ & $\begin{array}{l}\text { Sllica, } \\
\text { dis- } \\
\text { solved } \\
\text { (mg/L) }\end{array}$ & $\begin{array}{l}\text { Alum- } \\
\text { inum, } \\
\text { dis- } \\
\text { solved } \\
\text { ( } \mu g / L)\end{array}$ & $\begin{array}{c}\text { Arsenic, } \\
\text { dis- } \\
\text { solved } \\
(\mu g / L)\end{array}$ & $\begin{array}{c}\text { Barium, } \\
\text { dls- } \\
\text { solved } \\
(\mu g / L)\end{array}$ & $\begin{array}{l}\text { Beryl- } \\
\text { lium, } \\
\text { dls- } \\
\text { solved } \\
(\mu g / L)\end{array}$ & $\begin{array}{l}\text { Boron, } \\
\text { dls- } \\
\text { solved } \\
(\mu g / L)\end{array}$ & $\begin{array}{c}\text { Cad- } \\
\text { mium, } \\
\text { dis- } \\
\text { solved } \\
\left(\mu g^{\prime} L\right)\end{array}$ & $\begin{array}{l}\text { Chro- } \\
\text { mium, } \\
\text { dis- } \\
\text { solved } \\
(\mu g / L)\end{array}$ & $\begin{array}{c}\text { Cobalt, } \\
\text { dis- } \\
\text { solved } \\
(\mu g /)\end{array}$ & $\begin{array}{c}\text { Copper, } \\
\text { dls- } \\
\text { solved } \\
(\mu g / L)\end{array}$ \\
\hline $\begin{array}{c}\text { Jul } 1994 \\
20 \ldots \\
\text { Aug }\end{array}$ & $<0.10$ & $<0.01$ & $<10$ & $<1$ & $<2$ & $<0.5$ & $<10$ & $<1$ & $<5$ & $<3$ & $<10$ \\
\hline $\begin{array}{l}17 \ldots \\
\text { Sep }\end{array}$ & $<.10$ & $<.01$ & $<10$ & $<1$ & $<2$ & $<.5$ & $<10$ & $<1$ & $<5$ & $<3$ & $<10$ \\
\hline $\begin{array}{l}07 \ldots \\
\text { Oct }\end{array}$ & $<.10$ & .10 & $<10$ & $<1$ & $<2$ & $<.5$ & $<10$ & $<1$ & $<5$ & $<3$ & $<10$ \\
\hline $\begin{array}{l}14 \ldots \\
\text { Nov }\end{array}$ & $<.10$ & .05 & $<10$ & $<1$ & $<2$ & $<.5$ & $<10$ & $<1$ & $<5$ & $<3$ & $<10$ \\
\hline $\begin{array}{c}15 \ldots \\
\mathrm{Dec}\end{array}$ & $<.10$ & .02 & $<10$ & $<1$ & $<2$ & $<.5$ & $<10$ & $<1$ & $<5$ & $<3$ & $<10$ \\
\hline $\begin{array}{c}16 \ldots \\
\operatorname{Jan} 1995\end{array}$ & $<.10$ & $<.01$ & $<10$ & $<1$ & $<2$ & $<.5$ & $<10$ & $<1$ & $<5$ & $<3$ & $<10$ \\
\hline $\begin{array}{l}11 \ldots \\
\text { Feb }\end{array}$ & $<.10$ & .05 & $<10$ & $<1$ & $<2$ & $<.5$ & $<10$ & $<1$ & $<5$ & $<3$ & $<10$ \\
\hline $\begin{array}{l}21 \ldots \\
\text { Mar }\end{array}$ & $<.10$ & .03 & $<10$ & $<1$ & $<2$ & $<.5$ & $<10$ & $<1$ & $<5$ & $<1$ & $<10$ \\
\hline $\begin{array}{l}15 \ldots \\
\text { Apr }\end{array}$ & $<.10$ & $<.01$ & $<10$ & $<1$ & $<2$ & $<.5$ & $<10$ & $<1$ & $<5$ & 4 & $<10$ \\
\hline $\begin{array}{l}12 \ldots \\
\text { May }\end{array}$ & $<.10$ & .02 & 20 & $<1$ & $<2$ & $<.5$ & $<10$ & $<1$ & $<5$ & $<1$ & $<10$ \\
\hline $\begin{array}{l}17 \ldots \\
\text { Jun }\end{array}$ & .10 & .04 & $<10$ & $<1$ & $<2$ & $<.5$ & $<10$ & $<1$ & $<5$ & $<3$ & $<10$ \\
\hline$\underset{\mathrm{Jul}}{13 \ldots}$ & $<.10$ & 14 & $<10$ & $<1$ & $<2$ & $<.5$ & $<10$ & $<1$ & $<5$ & $<3$ & $<10$ \\
\hline $\begin{array}{l}13 \ldots \\
\text { Aug }\end{array}$ & $<.10$ & 21 & $<10$ & $<1$ & $<2$ & $<.5$ & $<10$ & $<1$ & $<5$ & $<3$ & $<10$ \\
\hline $\begin{array}{l}16 \ldots \\
\text { Sep }\end{array}$ & $<.10$ & 3.2 & $<10$ & $<1$ & $<2$ & $<.5$ & $<10$ & $<1$ & $<5$ & $<3$ & $<10$ \\
\hline $\begin{array}{l}13 \ldots \\
\text { Oct }\end{array}$ & $<.10$ & 8.0 & -. & $<1$ & $<2$ & $<.5$ & $<10$ & 3 & $<5$ & $<3$ & $<10$ \\
\hline $\begin{array}{l}10 \ldots \\
\text { Nov }\end{array}$ & $<.10$ & $<.01$ & $<10$ & $<1$ & $<2$ & $<.5$ & $<10$ & $<1$ & $<5$ & $<3$ & $<10$ \\
\hline $\begin{array}{c}29 \ldots \\
\text { Jan } 1996\end{array}$ & $<.10$ & .11 & $<10$ & $<1$ & $<2$ & $<.5$ & $<10$ & 1 & $<5$ & $<3$ & $<10$ \\
\hline $\begin{array}{l}11 \ldots \\
\text { Feb }\end{array}$ & $<.10$ & .02 & $<10$ & $<2$ & $<2$ & $<.5$ & $<10$ & $<1$ & $<5$ & $<3$ & $<10$ \\
\hline $\begin{array}{l}21 \ldots \\
\text { Apr }\end{array}$ & $<.10$ & .42 & $<10$ & $<1$ & $<2$ & $<.5$ & $<10$ & $<1$ & $<5$ & $<3$ & $<10$ \\
\hline $\begin{array}{c}03 \ldots \\
\text { Jun }\end{array}$ & $<.10$ & $<.01$ & $<10$ & $<1$ & $<2$ & $<.5$ & $<10$ & $<1$ & $<5$ & $<3$ & $<10$ \\
\hline $\begin{array}{c}04 \ldots \\
\text { Jul }\end{array}$ & $<.10$ & .06 & $<5$ & $<1$ & $<2$ & $<.5$ & $<4$ & 1 & $<5$ & $<3$ & $<10$ \\
\hline $\begin{array}{l}03 \ldots \\
\text { Aug }\end{array}$ & $<.10$ & .03 & $<5$ & $<1$ & $<2$ & $<.5$ & 5 & $<1$ & $<5$ & $<3$ & $<10$ \\
\hline $\begin{array}{l}06 . . \\
\text { Sep }\end{array}$ & $<.10$ & .01 & $<5$ & $<1$ & $<2$ & $<.5$ & $<4$ & 1 & $<5$ & $<3$ & $<10$ \\
\hline $04 \ldots$ & $<.10$ & .02 & $<5$ & $<1$ & $<2$ & $<.5$ & $<4$ & $<1$ & $<5$ & $<3$ & $<10$ \\
\hline
\end{tabular}


Table 5. Chemical analyses of field blanks for water samples--Continued

\begin{tabular}{|c|c|c|c|c|c|c|c|c|c|c|c|}
\hline Date & $\begin{array}{l}\text { Iron, } \\
\text { dis- } \\
\text { aoived } \\
(\mu g / L)\end{array}$ & $\begin{array}{l}\text { Lead, } \\
\text { dis- } \\
\text { solved } \\
\text { ( } \mu g / L)\end{array}$ & $\begin{array}{l}\text { Lithium, } \\
\text { dis- } \\
\text { aolved } \\
\left(\mu g^{\prime} L\right)\end{array}$ & $\begin{array}{l}\text { Manga- } \\
\text { nese, } \\
\text { dia- } \\
\text { solved } \\
(\mu g / L)\end{array}$ & $\begin{array}{c}\text { Molyb- } \\
\text { denum, } \\
\text { dis- } \\
\text { solved } \\
(\mu g / L)\end{array}$ & $\begin{array}{c}\text { Nickel, } \\
\text { dis-- } \\
\text { solved } \\
(\mu g / L)\end{array}$ & $\begin{array}{l}\text { Selenium, } \\
\text { dissolved } \\
(\mu \mathrm{g} / \mathrm{L})\end{array}$ & $\begin{array}{c}\text { Silver, } \\
\text { dis- } \\
\text { solved } \\
(\mu \mathrm{g} / L)\end{array}$ & $\begin{array}{l}\text { Stron- } \\
\text { tium, } \\
\text { dis- } \\
\text { solved } \\
(\mu g / L)\end{array}$ & $\begin{array}{l}\text { Vana- } \\
\text { dium, } \\
\text { dis- } \\
\text { solved } \\
(\mu g / L)\end{array}$ & $\begin{array}{c}\text { Zinc, } \\
\text { dis- } \\
\text { solved } \\
(\mu g / L)\end{array}$ \\
\hline Jul 1994 & & & & & & & & & & & \\
\hline $20 \ldots$ & $<3$ & $<10$ & $<4$ & $<1$ & $<10$ & $<10$ & $<1$ & 1 & $<1$ & $<6$ & $<3$ \\
\hline Aug & & & & & & & & & & & \\
\hline $\begin{array}{l}17 \ldots \\
\text { Sep }\end{array}$ & 5 & $<10$ & $<4$ & $<1$ & $<10$ & $<10$ & $<5$ & $<1$ & $<1$ & $<6$ & $<3$ \\
\hline $\begin{array}{c}07 \ldots \\
\text { Oct }\end{array}$ & 20 & $<10$ & $<4$ & $<1$ & $<10$ & $<10$ & $<1$ & $<1$ & $<1$ & $<6$ & $<3$ \\
\hline $\begin{array}{l}14 \ldots \\
\text { Nov }\end{array}$ & 6 & $<10$ & $<4$ & $<1$ & $<10$ & $<10$ & $<1$ & $<1$ & $<1$ & $<6$ & 5 \\
\hline $\begin{array}{l}15 \ldots \\
\text { Dec }\end{array}$ & $<3$ & $<10$ & $<4$ & $<1$ & $<10$ & $<10$ & $<1$ & $<1$ & $<1$ & $<6$ & $<3$ \\
\hline $\begin{array}{l}16 \ldots \\
\operatorname{Jan} 1995\end{array}$ & $<3$ & $<10$ & $<4$ & $<1$ & $<10$ & $<10$ & $<1$ & $<1$ & $<1$ & $<6$ & $<3$ \\
\hline $\begin{array}{l}11 \ldots \\
\text { Feb }\end{array}$ & $<3$ & $<10$ & $<4$ & $<1$ & $<10$ & $<10$ & $<1$ & $<1$ & $<1$ & $<6$ & $<3$ \\
\hline $21 \ldots$ & 7 & $<10$ & $<4$ & $<1$ & $<10$ & $<10$ & $<1$ & $<1$ & 1 & $<6$ & 4 \\
\hline $\begin{array}{l}15 \ldots \\
\text { Apr }\end{array}$ & $<3$ & $<10$ & $<4$ & $<1$ & $<10$ & $<10$ & $<1$ & $<1$ & $<1$ & $<6$ & $<3$ \\
\hline $\begin{array}{l}12 \ldots \\
\text { May }\end{array}$ & $<3$ & $<10$ & $<4$ & $<1$ & $<10$ & $<10$ & $<1$ & $<1$ & $<1$ & $<6$ & $<3$ \\
\hline $\begin{array}{l}17 \ldots \\
\text { Jun }\end{array}$ & 4 & 20 & $<4$ & $<1$ & $<10$ & $<10$ & $<1$ & $<1$ & $<1$ & $<6$ & 4 \\
\hline$\underset{\text { Jul }}{13 \ldots}$ & 5 & $<10$ & $<4$ & $<1$ & $<10$ & $<10$ & $<1$ & $<1$ & $<1$ & $<6$ & $<3$ \\
\hline $\begin{array}{l}13 \ldots \\
\text { Aug }\end{array}$ & 8 & $<10$ & $<4$ & $<1$ & $<10$ & $<10$ & $<1$ & $<1$ & $<1$ & $<6$ & $<3$ \\
\hline $\begin{array}{l}16 . . \\
\text { Sep }\end{array}$ & $<3$ & $<10$ & $<4$ & $<1$ & $<10$ & $<10$ & - & 1 & $<1$ & $<6$ & $<3$ \\
\hline $\begin{array}{l}13 \ldots \\
\text { Oet }\end{array}$ & 4 & 10 & $<4$ & 2 & $<10$ & $<10$ & - & 1 & $<1$ & $<6$ & $<3$ \\
\hline $\begin{array}{l}10 \ldots \\
\text { Nov }\end{array}$ & $<3$ & 30 & $<4$ & $<1$ & $<10$ & $<10$ & - & $<1$ & $<1$ & $<6$ & 7 \\
\hline $\begin{array}{c}29 \ldots \\
\operatorname{Jan} 1996\end{array}$ & $<3$ & $<10$ & $<4$ & $<1$ & $<10$ & $<10$ & - & $<1$ & $<1$ & $<6$ & 4 \\
\hline $\begin{array}{l}11 \ldots \\
\text { Feb }\end{array}$ & $<3$ & $<10$ & $<4$ & $<1$ & $<10$ & $<10$ & - & $<1$ & $<1$ & $<6$ & $<3$ \\
\hline $\begin{array}{l}21 \ldots \\
\text { Apr }\end{array}$ & $<3$ & $<10$ & $<4$ & $<1$ & $<10$ & $<10$ & - & $<1$ & $<1$ & $<6$ & $<3$ \\
\hline $\begin{array}{c}03 \ldots \\
\text { Jun }\end{array}$ & 3 & $<10$ & $<4$ & $<1$ & $<10$ & $<10$ & - & $<1$ & $<1$ & $<6$ & $<3$ \\
\hline $\begin{array}{c}04 \ldots \\
\text { Jul }\end{array}$ & $<3$ & $<10$ & $<4$ & $<1$ & $<10$ & $<10$ & - & $<1$ & $<1$ & $<6$ & $<3$ \\
\hline $\begin{array}{l}03 \ldots \\
\text { Aug }\end{array}$ & $<3$ & $<10$ & $<4$ & $<1$ & $<10$ & $<10$ & - & $<1$ & $<1$ & $<6$ & $<3$ \\
\hline $\begin{array}{l}06 . . . \\
\text { Sep }\end{array}$ & $<3$ & 10 & $<4$ & $<1$ & $<10$ & $<10$ & - & 1 & $<1$ & $<6$ & 7 \\
\hline $04 \ldots$ & $<3$ & $<10$ & $<4$ & $<1$ & $<10$ & $<10$ & - & $<1$ & $<1$ & $<6$ & $<3$ \\
\hline
\end{tabular}


Table 6. Statistical summary of water-quality data for sites in the Sand Coulee Coal Area, Montana, July 1994 through September 1996

[Site number shown in figure 2 or 3. Abbreviations: $\mathrm{ft}^{3} / \mathrm{s}$, cubic feet per second; ${ }^{\circ} \mathrm{C}$, degrees Celsius; $\mu \mathrm{g} / \mathrm{L}$, micrograms per liter; $\mu \mathrm{S} / \mathrm{cm}$, microsiemens per centimeter at $25^{\circ} \mathrm{C} ; \mathrm{mg} / \mathrm{L}$, milligrams per liter. Symbols: <, less than minimum reporting level; --, indicates insufficient data greater than minimum reporting level to compute statistic; e, estimated. Note: Multiple minimum reporting levels during the period of record may result in varying values identified with a less-than $(<)$ symbol]

\begin{tabular}{|c|c|c|c|c|c|}
\hline $\begin{array}{l}\text { Property or constituent } \\
\text { (reporting unit) }\end{array}$ & $\begin{array}{c}\text { Number } \\
\text { of } \\
\text { samples }\end{array}$ & Maximum & Minimum & Mean & Median \\
\hline \multicolumn{6}{|c|}{ Site 1, 06078230-Sand Coulee Creek above Cottonwood Creek, at Centerville, Mont. } \\
\hline Streamflow, instantaneous $\left(\mathrm{ft}^{3} / \mathrm{s}\right)$ & 25 & 30 & 0.00 & 4.7 & 0.00 \\
\hline Specific conductance, onsite $(\mu \mathrm{S} / \mathrm{cm})$ & 12 & 485 & 310 & 396 & 388 \\
\hline Temperature, water $\left({ }^{\circ} \mathrm{C}\right)$ & 12 & 30.5 & 0.0 & 15.8 & 16.5 \\
\hline $\mathrm{pH}$, onsite (standard units) & 10 & 8.8 & 8.0 & 8.4 & 8.4 \\
\hline Hardness, total $\left(\mathrm{mg} / \mathrm{L}\right.$ as $\left.\mathrm{CaCO}_{3}\right)$ & 10 & 250 & 160 & 208 & 200 \\
\hline Calcium, dissolved (mg/L as $\mathrm{Ca}$ ) & 10 & 63 & 39 & 50 & 51 \\
\hline Magnesium, dissolved ( $\mathrm{mg} / \mathrm{L}$ as $\mathrm{Mg}$ ) & 10 & 25 & 12 & 20 & 22 \\
\hline Sodium, dissolved (mg/L as $\mathrm{Na}$ ) & 10 & 5.9 & 3.3 & 4.8 & 5.3 \\
\hline Potassium, dissolved ( $\mathrm{mg} / \mathrm{L}$ as $\mathrm{K}$ ) & 10 & 4.2 & 2.0 & 2.7 & 2.4 \\
\hline Acidity $\left(\mathrm{mg} / \mathrm{L}\right.$ as $\left.\mathrm{H}^{+}\right)$ & 10 & $<.1$ & $<.1$ & -- & $<.1$ \\
\hline Alkalinity $\left(\mathrm{mg} / \mathrm{L}\right.$ as $\left.\mathrm{CaCO}_{3}\right)$ & 10 & 208 & 120 & 162 & 160 \\
\hline Sulfate, dissolved (mg/L as $\mathrm{SO}_{4}$ ) & 10 & 88 & 24 & 54 & 56 \\
\hline Chloride, dissolved ( $\mathrm{mg} / \mathrm{L}$ as $\mathrm{Cl}$ ) & 10 & 2.4 & 1.5 & 2.0 & 2.1 \\
\hline Fluoride, dissolved (mg/L as Fl) & 10 & 6 & .2 & .4 & .3 \\
\hline Silica, dissolved $\left(\mathrm{mg} / \mathrm{L}\right.$ as $\left.\mathrm{SiO}_{2}\right)$ & 10 & 11 & 3.2 & 7.2 & 7.7 \\
\hline Dissolved solids, calculated (mg/L) & 10 & 284 & 184 & 240 & 240 \\
\hline Aluminum, dissolved ( $\mu \mathrm{g} / \mathrm{L}$ as $\mathrm{Al})$ & 10 & 430 & 20 & 147 & 95 \\
\hline Arsenic, dissolved ( $\mu \mathrm{g} / \mathrm{L}$ as As) & 10 & 2 & $<1$ & ${ }^{1} 1$ & 1 \\
\hline Barium, dissolved $(\mu \mathrm{g} / \mathrm{L}$ as $\mathrm{Ba})$ & 10 & 210 & 150 & 167 & 160 \\
\hline Beryllium, dissolved $(\mu \mathrm{g} / \mathrm{L}$ as $\mathrm{Be})$ & 10 & .5 & $<.5$ & -- & $<.5$ \\
\hline Boron, dissolved $(\mu \mathrm{g} / \mathrm{L}$ as $\mathrm{B})$ & 10 & 50 & 20 & 32 & 30 \\
\hline Cadmium, dissolved $(\mu \mathrm{g} / \mathrm{L}$ as $\mathrm{Cd})$ & 10 & 2 & $<1$ & -- & $<1$ \\
\hline Chromium, dissolved ( $\mu \mathrm{g} / \mathrm{L}$ as $\mathrm{Cr}$ ) & 10 & $<5$ & $<5$ & -- & $<5$ \\
\hline Cobalt, dissolved ( $\mu \mathrm{g} / \mathrm{L}$ as $\mathrm{Co})$ & 10 & $<3$ & $<3$ & -- & $<3$ \\
\hline Copper, dissolved $(\mu \mathrm{g} / \mathrm{L}$ as $\mathrm{Cu})$ & 10 & $<10$ & $<10$ & -- & $<10$ \\
\hline Iron, dissolved $(\mu \mathrm{g} / \mathrm{L}$ as $\mathrm{Fe})$ & 10 & 240 & 6 & 69 & 58 \\
\hline Lead, dissolved $(\mu \mathrm{g} / \mathrm{L}$ as $\mathrm{Pb})$ & 10 & 10 & $<10$ & -- & $<10$ \\
\hline Lithium, dissolved $(\mu \mathrm{g} / \mathrm{L}$ as $\mathrm{Li})$ & 10 & 12 & 4 & 8 & 8 \\
\hline Manganese, dissolved ( $\mu \mathrm{g} / \mathrm{L}$ as $\mathrm{Mn})$ & 10 & 23 & 3 & 9 & 9 \\
\hline Molybdenum, dissolved ( $\mu \mathrm{g} / \mathrm{L}$ as $\mathrm{Mo}$ ) & 10 & 10 & $<10$ & - & $<10$ \\
\hline Nickel, dissolved $(\mu \mathrm{g} / \mathrm{L}$ as $\mathrm{Ni})$ & 10 & 20 & $<10$ & -- & $<10$ \\
\hline Selenium, dissolved $(\mu \mathrm{g} / \mathrm{L}$ as $\mathrm{Se})$ & 3 & $<1$ & $<1$ & -- & -- \\
\hline Silver, dissolved ( $\mu \mathrm{g} / \mathrm{L}$ as $\mathrm{Ag})$ & 10 & 1 & $<1$ & -- & $<1$ \\
\hline Strontium, dissolved ( $\mu \mathrm{g} / \mathrm{L}$ as $\mathrm{Sr})$ & 10 & 720 & 300 & 557 & 635 \\
\hline Vanadium, dissolved $(\mu g / L$ as $V)$ & 10 & 6 & $<6$ & -- & $<6$ \\
\hline Zinc, dissolved ( $\mu \mathrm{g} / \mathrm{L}$ as $\mathrm{Zn})$ & 10 & 8 & $<3$ & $1_{4}$ & 3 \\
\hline
\end{tabular}


Table 6. Statistical summary of water-quality data for sites in the Sand Coulee Coal Area, Montana, July 1994 through September 1996 (Continued)

\begin{tabular}{|c|c|c|c|c|c|}
\hline $\begin{array}{l}\text { Property or constituent } \\
\text { (reporting unit) }\end{array}$ & $\begin{array}{l}\text { Number } \\
\text { of } \\
\text { samples }\end{array}$ & Maximum & Minimum & Mean & Median \\
\hline \multicolumn{6}{|c|}{ Site 2, 06078250-Cottonwood Creek near Stockett, Mont. } \\
\hline Streamflow, instantaneous $\left(\mathrm{ft}^{3} / \mathrm{s}\right)$ & 26 & 17 & 0.00 & 1.6 & 0.09 \\
\hline Specific conductance, onsite $(\mu \mathrm{S} / \mathrm{cm})$ & 13 & 1,360 & 656 & 1,020 & 1,000 \\
\hline Temperature, water $\left({ }^{\circ} \mathrm{C}\right)$ & 14 & 28.0 & 0.0 & 15.0 & 15.5 \\
\hline pH, onsite (standard units) & 11 & 8.3 & 4.0 & 6.7 & 7.5 \\
\hline Hardness, total (mg/L as $\left.\mathrm{CaCO}_{3}\right)$ & 11 & 700 & 320 & 504 & 510 \\
\hline Calcium, dissolved (mg/L as $\mathrm{Ca}$ ) & 11 & 160 & 71 & 108 & 110 \\
\hline Magnesium, dissolved ( $\mathrm{mg} / \mathrm{L}$ as $\mathrm{Mg}$ ) & 11 & 73 & 34 & 57 & 57 \\
\hline Sodium, dissolved ( $\mathrm{mg} / \mathrm{L}$ as $\mathrm{Na}$ ) & 11 & 13 & 10 & 12 & 12 \\
\hline Potassium, dissolved ( $\mathrm{mg} / \mathrm{L}$ as $\mathrm{K}$ ) & 11 & 5 & 3.4 & 4.1 & 4 \\
\hline Acidity $\left(\mathrm{mg} / \mathrm{L}\right.$ as $\left.\mathrm{H}^{+}\right)$ & 11 & 7.7 & $<.1$ & $1_{2.0}$ & .1 \\
\hline Alkalinity $\left(\mathrm{mg} / \mathrm{L}\right.$ and $\left.\mathrm{CaCO}_{3}\right)$ & 11 & 229 & $e<1$ & 114 & 153 \\
\hline Sulfate, dissolved ( $\mathrm{mg} / \mathrm{L}$ as $\mathrm{SO}_{4}$ ) & 11 & 950 & 97 & 485 & 370 \\
\hline Chloride, dissolved ( $\mathrm{mg} / \mathrm{L}$ as $\mathrm{Cl}$ ) & 11 & 5.6 & 4.0 & 4.6 & 4.6 \\
\hline Fluoride, dissolved (mg/L as F) & 11 & 1.7 & .3 & .8 & .7 \\
\hline Silica, dissolved (mg/L as $\left.\mathrm{SiO}_{2}\right)$ & 11 & 17 & 2.6 & 8.1 & 6.4 \\
\hline Dissolved solids, calculated (mg/L) & 11 & el, 180 & 366 & 762 & 683 \\
\hline Aluminum, dissolved ( $\mu \mathrm{g} / \mathrm{L}$ as $\mathrm{Al}$ ) & 11 & 54,000 & $<10$ & ${ }^{1} 9,380$ & 210 \\
\hline Arsenic, dissolved $(\mu \mathrm{g} / \mathrm{L}$ as As) & 11 & 3 & $<1$ & -- & $<1$ \\
\hline Barium, dissolved $(\mu \mathrm{g} / \mathrm{L}$ as $\mathrm{Ba})$ & 11 & 220 & 24 & 127 & 130 \\
\hline Beryllium, dissolved ( $\mu \mathrm{g} / \mathrm{L}$ as $\mathrm{Be})$ & 11 & 12 & $<.5$ & -- & $<.5$ \\
\hline Boron, dissolved ( $\mu \mathrm{g} / \mathrm{L}$ as $\mathrm{B})$ & 11 & 120 & 50 & 83 & 80 \\
\hline Cadmium, dissolved $(\mu \mathrm{g} / \mathrm{L}$ as $\mathrm{Cd})$ & 11 & 11 & $<1$ & $1_{3}$ & 2 \\
\hline Chromium, dissolved ( $\mu \mathrm{g} / \mathrm{L}$ as $\mathrm{Cr}$ ) & 11 & $<5$ & $<5$ & -- & $<5$ \\
\hline Cobalt, dissolved ( $\mu \mathrm{g} / \mathrm{L}$ as Co $)$ & 9 & 560 & 6 & 122 & 50 \\
\hline Copper, dissolved $(\mu \mathrm{g} / \mathrm{L}$ as $\mathrm{Cu})$ & 11 & 50 & $<10$ & -- & $<10$ \\
\hline Iron, dissolved ( $\mu \mathrm{g} / \mathrm{L}$ as $\mathrm{Fe})$ & 11 & 7,400 & $<3$ & ${ }^{1} 1,510$ & 6 \\
\hline Lead, dissolved $(\mu \mathrm{g} / \mathrm{L}$ as $\mathrm{Pb})$ & 11 & $<10$ & $<10$ & -- & $<10$ \\
\hline Lithium, dissolved $(\mu \mathrm{g} / \mathrm{L}$ as $\mathrm{Li})$ & 11 & 110 & 16 & 57 & 46 \\
\hline Manganese, dissolved ( $\mu \mathrm{g} / \mathrm{L}$ as $\mathrm{Mn})$ & 11 & 430 & 25 & 208 & 150 \\
\hline Molybdenum, dissolved ( $\mu \mathrm{g} / \mathrm{L}$ as Mo) & 11 & $<10$ & $<10$ & - & $<10$ \\
\hline Nickel, dissolved $(\mu \mathrm{g} / \mathrm{L}$ as $\mathrm{Ni})$ & 11 & 1,000 & 20 & 338 & 120 \\
\hline Selenium, dissolved $(\mu \mathrm{g} / \mathrm{L}$ as Se) & 4 & $<1$ & $<1$ & -- & - \\
\hline Silver, dissolved ( $\mu \mathrm{g} / \mathrm{L}$ as $\mathrm{Ag})$ & 11 & 1 & $<1$ & - & $<1$ \\
\hline Strontium, dissolved ( $\mu \mathrm{g} / \mathrm{L}$ as $\mathrm{Sr}$ ) & 11 & 480 & 300 & 408 & 440 \\
\hline Vanadium, dissolved $(\mu \mathrm{g} / \mathrm{L}$ as $\mathrm{V})$ & 11 & $<6$ & $<6$ & -- & $<6$ \\
\hline Zinc, dissolved $(\mu \mathrm{g} / \mathrm{L}$ as $\mathrm{Zn})$ & 11 & 4,800 & 7 & 1,250 & 68 \\
\hline
\end{tabular}


Table 6. Statistical summary of water-quality data for sites in the Sand Coulee Coal Area, Montana, July 1994 through September 1996 (Continued)

\begin{tabular}{|c|c|c|c|c|c|}
\hline $\begin{array}{l}\text { Property or constituent } \\
\text { (reporting unit) }\end{array}$ & $\begin{array}{c}\text { Number } \\
\text { of } \\
\text { samples }\end{array}$ & Maximum & MInimum & Mean & Medlan \\
\hline \multicolumn{6}{|c|}{ Slte 3, 06078260-Number Flve Couice below Glffen Spring, near Stockett, Mont. } \\
\hline Streamflow, instantaneous $\left(\mathrm{ft}^{3} / \mathrm{s}\right)$ & 29 & 8.3 & 0.60 & 2.0 & 0.87 \\
\hline Specific conductance, onsite $(\mu \mathrm{S} / \mathrm{cm})$ & 29 & 1,250 & 714 & 1,060 & 1,150 \\
\hline Temperature, water $\left({ }^{\circ} \mathrm{C}\right)$ & 29 & 18.0 & 4.5 & 10.0 & 9.5 \\
\hline $\mathrm{pH}$, onsite (standard units) & 25 & 7.9 & 6.0 & 6.5 & 6.5 \\
\hline Hardness, total $\left(\mathrm{mg} / \mathrm{L}\right.$ as $\left.\mathrm{CaCO}_{3}\right)$ & 25 & 600 & 330 & 516 & 530 \\
\hline Calcium, dissolved (mg/L as $\mathrm{Ca}$ ) & 25 & 160 & 74 & 134 & 140 \\
\hline Magnesium, dissolved ( $\mathrm{mg} / \mathrm{L}$ as $\mathrm{Mg}$ ) & 25 & 49 & 36 & 44 & 45 \\
\hline Sodium, dissolved (mg/L as Na) & 25 & 20 & 14 & 16 & 16 \\
\hline Potassium, dissolved (mg/L as $\mathrm{K}$ ) & 25 & 7.0 & 4.1 & 5.6 & 5.7 \\
\hline Acidity $\left(\mathrm{mg} / \mathrm{L}\right.$ as $\left.\mathrm{H}^{+}\right)$ & 25 & 3.2 & $<.1$ & 1.9 & .6 \\
\hline Alkalinity $\left(\mathrm{mg} / \mathrm{L}\right.$ and $\mathrm{CaCO}_{3}$ ) & 25 & 257 & $e<1$ & 44.1 & $e<1$ \\
\hline Sulfate, dissolved ( $\mathrm{mg} / \mathrm{L}$ as $\mathrm{SO}_{4}$ ) & 25 & 790 & 110 & 572 & 630 \\
\hline Chloride, dissolved (mg/L as $\mathrm{Cl}$ ) & 25 & 7.3 & 3.3 & 4.4 & 4.3 \\
\hline Fluoride, dissolved (mg/L as F) & 24 & 1.3 & .2 & 6 & .6 \\
\hline Silica, dissolved (mg/L as $\mathrm{SiO}_{2}$ ) & 25 & 17 & 8.3 & 13 & 14 \\
\hline Dissolved solids, calculated (mg/L) & 25 & el, 110 & 415 & 854 & e919 \\
\hline Aluminum, dissolved ( $\mu \mathrm{g} / \mathrm{L}$ as $\mathrm{Al})$ & 25 & 400 & 20 & 123 & 70 \\
\hline Arsenic, dissolved ( $\mu \mathrm{g} / \mathrm{L}$ as As) & 25 & $<2$ & $<1$ & -- & $<1$ \\
\hline Barium, dissolved $(\mu \mathrm{g} / \mathrm{L}$ as $\mathrm{Ba})$ & 25 & 210 & 22 & 56 & 35 \\
\hline Beryllium, dissolved ( $\mu \mathrm{g} / \mathrm{L}$ as $\mathrm{Be})$ & 25 & 1 & $<.5$ & ${ }^{1} .8$ & .6 \\
\hline Boron, dissolved ( $\mu \mathrm{g} / \mathrm{L}$ as $\mathrm{B})$ & 25 & 100 & 50 & 77 & 80 \\
\hline Cadmium, dissolved $(\mu \mathrm{g} / \mathrm{L}$ as $\mathrm{Cd})$ & 25 & 14 & $<1$ & ${ }^{1} 4$ & 3 \\
\hline Chromium, dissolved ( $\mu \mathrm{g} / \mathrm{L}$ as $\mathrm{Cr})$ & 25 & $<5$ & $<5$ & -- & $<5$ \\
\hline Cobalt, dissolved ( $\mu \mathrm{g} / \mathrm{L}$ as $\mathrm{Co})$ & 25 & 410 & 10 & 194 & 190 \\
\hline Copper, dissolved ( $\mu \mathrm{g} / \mathrm{L}$ as $\mathrm{Cu})$ & 25 & $<10$ & $<10$ & -- & $<10$ \\
\hline Iron, dissolved $(\mu \mathrm{g} / \mathrm{L}$ as $\mathrm{Fe})$ & 25 & 63,000 & 340 & 37,200 & 44,000 \\
\hline Lead, dissolved ( $\mu \mathrm{g} / \mathrm{L}$ as $\mathrm{Pb})$ & 25 & $<10$ & $<1$ & -- & $<1$ \\
\hline Lithium, dissolved $(\mu \mathrm{g} / \mathrm{L}$ as $\mathrm{Li})$ & 25 & 69 & 11 & 57 & 62 \\
\hline Manganese, dissolved ( $\mu \mathrm{g} / \mathrm{L}$ as $\mathrm{Mn}$ ) & 25 & 1,300 & 130 & 795 & 830 \\
\hline Molybdenum, dissolved ( $\mu \mathrm{g} / \mathrm{L}$ as $\mathrm{Mo}$ ) & 25 & 1 & $<1$ & - & $<1$ \\
\hline Nickel, dissolved $(\mu \mathrm{g} / \mathrm{L}$ as $\mathrm{Ni})$ & 25 & 470 & 30 & 285 & 310 \\
\hline Selenium, dissolved $(\mu \mathrm{g} / \mathrm{L}$ as Se) & 11 & 1 & $<1$ & - & $<1$ \\
\hline Silver, dissolved $(\mu \mathrm{g} / \mathrm{L}$ as $\mathbf{A g})$ & 25 & 2 & $<1$ & - & $<1$ \\
\hline Strontium, dissolved ( $\mu \mathrm{g} / \mathrm{L}$ as $\mathrm{Sr})$ & 25 & 430 & 290 & 368 & 380 \\
\hline Vanadium, dissolved $(\mu \mathrm{g} / \mathrm{L}$ as $\mathrm{V})$ & 25 & 12 & $<6$ & ${ }^{16}$ & $<6$ \\
\hline Zinc, dissolved $(\mu \mathrm{g} / \mathrm{L}$ as $\mathrm{Zn})$ & 25 & 1,900 & 30 & 1,040 & 1,100 \\
\hline
\end{tabular}


Table 6. Statistical summary of water-quality data for sites in the Sand Coulee Coal Area, Montana, July 1994 through September 1996 (Continued)

\begin{tabular}{|c|c|c|c|c|c|}
\hline $\begin{array}{l}\text { Property or constituent } \\
\text { (reporting unit) }\end{array}$ & $\begin{array}{c}\text { Number } \\
\text { of } \\
\text { sampies }\end{array}$ & Maximum & MInImum & Mean & Median \\
\hline \multicolumn{6}{|c|}{ Site 4, 06078270-Sand Coulee at Sand Coulee, Mont. } \\
\hline Streamflow, instantaneous $\left(\mathrm{ft}^{3} / \mathrm{s}\right)$ & 26 & 2.5 & 0.00 & 0.25 & 0.02 \\
\hline Specific conductance, onsite $(\mu \mathrm{S} / \mathrm{cm})$ & 15 & 5,460 & 1,020 & 3,630 & 3,930 \\
\hline Temperature, water $\left({ }^{\circ} \mathrm{C}\right)$ & 15 & 30.0 & 3.0 & 16.2 & 17.0 \\
\hline $\mathrm{pH}$, onsite (standard units) & 13 & 6.1 & 2.2 & 3.1 & 2.7 \\
\hline Hardness, total $\left(\mathrm{mg} / \mathrm{L}\right.$ as $\left.\mathrm{CaCO}_{3}\right)$ & 13 & 1,400 & 480 & 1,040 & 1,200 \\
\hline Calcium, dissolved $(\mathrm{mg} / \mathrm{L}$ as $\mathrm{Ca})$ & 13 & 250 & 86 & 193 & 220 \\
\hline Magnesium, dissolved (mg/L as $\mathrm{Mg}$ ) & 13 & 180 & 65 & 136 & 160 \\
\hline Sodium, dissolved (mg/L as $\mathrm{Na}$ ) & 13 & 26 & 16 & 22 & 23 \\
\hline Potassium, dissolved (mg/L as $\mathrm{K})$ & 13 & 7.1 & 2.2 & 3.2 & 2.9 \\
\hline Acidity $\left(\mathrm{mg} / \mathrm{L}\right.$ as $\mathrm{H}^{+}$) & 13 & 84 & $<.1$ & ${ }^{1} 50$ & 38 \\
\hline Alkalinity $\left(\mathrm{mg} / \mathrm{L}\right.$ as $\left.\mathrm{CaCO}_{3}\right)$ & 13 & $e<1$ & $e<1$ & -- & $e<1$ \\
\hline Sulfate, dissolved (mg/L as $\mathrm{SO}_{4}$ ) & 13 & 6,800 & 470 & 4,020 & 4,500 \\
\hline Chloride, dissolved (mg/L as $\mathrm{Cl}$ ) & 13 & 21 & 3.1 & 8.2 & 7.7 \\
\hline Fluoride, dissolved (mg/L as F) & 12 & 2.4 & $<.1$ & ${ }^{1} 1.0$ & .7 \\
\hline Silica, dissolved (mg/L as $\left.\mathrm{SiO}_{2}\right)$ & 13 & 86 & 9.9 & 61 & 77 \\
\hline Dissolved solids, calculated (mg/L) & 13 & $\mathrm{e} 8,290$ & e683 & $\mathrm{e} 5,080$ & e5,810 \\
\hline Aluminum, dissolved $(\mu \mathrm{g} / \mathrm{L}$ as $\mathrm{Al})$ & 13 & 490,000 & 30 & 301,000 & 410,000 \\
\hline Arsenic, dissolved ( $\mu \mathrm{g} / \mathrm{L}$ as As) & 13 & 11 & $<1$ & 14.3 & 4 \\
\hline Barium, dissolved $(\mu \mathrm{g} / \mathrm{L}$ as $\mathrm{Ba})$ & 13 & 120 & $<10$ & 139 & 11 \\
\hline Beryllium, dissolved ( $\mu \mathrm{g} / \mathrm{L}$ as $\mathrm{Be}$ ) & 13 & 60 & $<.5$ & $1_{38}$ & 49 \\
\hline Boron, dissolved ( $\mu \mathrm{g} / \mathrm{L}$ as $\mathrm{B})$ & 13 & 490 & 80 & 322 & 420 \\
\hline Cadmium, dissolved ( $\mu \mathrm{g} / \mathrm{L}$ as $\mathrm{Cd})$ & 11 & 49 & 2 & 26 & 21 \\
\hline Chromium, dissolved ( $\mu \mathrm{g} / \mathrm{L}$ as $\mathrm{Cr})$ & 13 & 160 & $<5$ & ${ }^{1} 87$ & 110 \\
\hline Cobalt, dissolved ( $\mu \mathrm{g} / \mathrm{L}$ as Co) & 13 & 3,000 & 110 & 1,410 & 1,400 \\
\hline Copper, dissolved ( $\mu \mathrm{g} / \mathrm{L}$ as $\mathrm{Cu})$ & 12 & 160 & $<10$ & ${ }^{1} 91$ & 110 \\
\hline Iron, dissolved $(\mu \mathrm{g} / \mathrm{L}$ as $\mathrm{Fe})$ & 13 & 600,000 & 7,700 & 312,000 & 360,000 \\
\hline Lead, dissolved $(\mu \mathrm{g} / \mathrm{L}$ as $\mathrm{Pb})$ & 13 & 2 & $<1$ & - & $<1$ \\
\hline Lithium, dissolved $(\mu \mathrm{g} / \mathrm{L}$ as $\mathrm{Li})$ & 13 & 700 & 85 & 470 & 610 \\
\hline Manganese, dissolved $(\mu \mathrm{g} / \mathrm{L}$ as $\mathrm{Mn})$ & 13 & 4,900 & 450 & 3,240 & 4,000 \\
\hline Molybdenum, dissolved ( $\mu \mathrm{g} / \mathrm{L}$ as $\mathrm{Mo}$ ) & 13 & 2 & $<1$ & -- & $<1$ \\
\hline Nickel, dissolved ( $\mu \mathrm{g} / \mathrm{L}$ as $\mathrm{Ni})$ & 13 & 3,400 & 240 & 2,180 & 2,800 \\
\hline Selenium, dissolved $(\mu \mathrm{g} / \mathrm{L}$ as $\mathrm{Se})$ & 7 & 1 & $<1$ & - & $<2$ \\
\hline Silver, dissolved ( $\mu \mathrm{g} / \mathrm{L}$ as $\mathrm{Ag})$ & 13 & $<10$ & $<1$ & - & $<3$ \\
\hline Strontium, dissolved ( $\mu \mathrm{g} / \mathrm{L}$ as $\mathrm{Sr})$ & 13 & 1,400 & 530 & 1,090 & 1,200 \\
\hline Vanadium, dissolved ( $\mu \mathrm{g} / \mathrm{L}$ as $\mathrm{V})$ & 13 & 130 & $<6$ & 165 & 57 \\
\hline Zinc, dissolved $(\mu \mathrm{g} / \mathrm{L}$ as $\mathrm{Zn})$ & 13 & 14,000 & 420 & 8,720 & 11,000 \\
\hline
\end{tabular}


Table 6. Statistical summary of water-quality data for sites in the Sand Coulee Coal Area, Montana, July 1994 through September 1996 (Continued)

\begin{tabular}{|c|c|c|c|c|c|}
\hline $\begin{array}{l}\text { Property or constituent } \\
\text { (reporting unit) }\end{array}$ & $\begin{array}{l}\text { Number } \\
\text { of } \\
\text { samples }\end{array}$ & Maximum & Minimum & Mean & Median \\
\hline \multicolumn{6}{|c|}{ Site 5, $06090590-$ Anaconda drain at Belt, Mont. } \\
\hline Streamflow, instantaneous $\left(\mathrm{ft}^{3} / \mathrm{s}\right)$ & 25 & 0.29 & 0.15 & 0.22 & 0.22 \\
\hline Specific conductance, onsite $(\mu \mathrm{S} / \mathrm{cm})$ & 26 & 2,460 & 2,280 & 2,380 & 2,380 \\
\hline Temperature, water $\left({ }^{\circ} \mathrm{C}\right)$ & 26 & 18.5 & 6.5 & 12.3 & 12.5 \\
\hline $\mathrm{pH}$, onsite (standard units) & 25 & 3.0 & 2.8 & 2.9 & 2.9 \\
\hline Hardness, total (mg/L as $\left.\mathrm{CaCO}_{3}\right)$ & 25 & 720 & 610 & 669 & 670 \\
\hline Calcium, dissolved (mg/L as $\mathrm{Ca}$ ) & 25 & 170 & 140 & 157 & 160 \\
\hline Magnesium, dissolved ( $\mathrm{mg} / \mathrm{L}$ as $\mathrm{Mg}$ ) & 25 & 72 & 63 & 67 & 67 \\
\hline Sodium, dissolved ( $\mathrm{mg} / \mathrm{L}$ as $\mathrm{Na})$ & 25 & 11 & 9.4 & 9.9 & 10 \\
\hline Potassium, dissolved (mg/L as $\mathrm{K}$ ) & 25 & 3.7 & 2.5 & 3.0 & 3.0 \\
\hline Acidity $\left(\mathrm{mg} / \mathrm{L}\right.$ as $\left.\mathrm{H}^{+}\right)$ & 25 & 25 & 18 & 21 & 21 \\
\hline Alkalinity $\left(\mathrm{mg} / \mathrm{L}\right.$ as $\left.\mathrm{CaCO}_{3}\right)$ & 25 & $e<1$ & $e<1$ & - & $e<1$ \\
\hline Sulfate, dissolved ( $\mathrm{mg} / \mathrm{L}$ as $\mathrm{SO}_{4}$ ) & 25 & 2,700 & 1,700 & 1,960 & 1,900 \\
\hline Chloride, dissolved ( $\mathrm{mg} / \mathrm{L}$ as $\mathrm{Cl})$ & 25 & 7.9 & 2.2 & 4.5 & 4.5 \\
\hline Fluoride, dissolved ( $\mathrm{mg} / \mathrm{L}$ as $\mathrm{F}$ ) & 23 & 2.7 & .6 & 1.6 & 1.7 \\
\hline Silica, dissolved (mg/L as $\left.\mathrm{SiO}_{2}\right)$ & 25 & 60 & 51 & 57 & 57 \\
\hline Dissolved solids, calculated $(\mathrm{mg} / \mathrm{L})$ & 25 & $\mathrm{e} 3,290$ & $\mathrm{e} 2,240$ & $\mathrm{e} 2,540$ & $\mathrm{e} 2,490$ \\
\hline Aluminum, dissolved $(\mu \mathrm{g} / \mathrm{L}$ as $\mathrm{Al})$ & 25 & 120,000 & 92,000 & 106,000 & 110,000 \\
\hline Arsenic, dissolved $(\mu \mathrm{g} / \mathrm{L}$ as As) & 25 & 3 & $<1$ & $1_{1}$ & 1 \\
\hline Barium, dissolved $(\mu \mathrm{g} / \mathrm{L}$ as $\mathrm{Ba})$ & 25 & 12 & $<2$ & ${ }^{16}$ & 3 \\
\hline Beryllium, dissolved $(\mu \mathrm{g} / \mathrm{L}$ as $\mathrm{Be})$ & 25 & 23 & 15 & 18 & 17 \\
\hline Boron, dissolved $(\mu \mathrm{g} / \mathrm{L}$ as $\mathrm{B})$ & 25 & 320 & 60 & 154 & 150 \\
\hline Cadmium, dissolved $(\mu \mathrm{g} / \mathrm{L}$ as $\mathrm{Cd})$ & 20 & 13 & 7 & 9 & 8 \\
\hline Chromium, dissolved ( $\mu \mathrm{g} / \mathrm{L}$ as $\mathrm{Cr})$ & 25 & 50 & 30 & 43 & 40 \\
\hline Cobalt, dissolved $(\mu \mathrm{g} / \mathrm{L}$ as $\mathrm{Co})$ & 21 & 500 & 280 & 347 & 330 \\
\hline Copper, dissolved $(\mu \mathrm{g} / \mathrm{L}$ as $\mathrm{Cu})$ & 25 & 40 & $<30$ & ${ }^{1} 27$ & $<30$ \\
\hline Iron, dissolved $(\mu \mathrm{g} / \mathrm{L}$ as $\mathrm{Fe})$ & 25 & 190,000 & 150,000 & 169,000 & 170,000 \\
\hline Lead, dissolved $(\mu \mathrm{g} / \mathrm{L}$ as $\mathrm{Pb})$ & 25 & 2 & $<1$ & ${ }^{1} 1$ & $<30$ \\
\hline Lithium, dissolved $(\mu \mathrm{g} / \mathrm{L}$ as $\mathrm{Li})$ & 25 & 210 & 170 & 194 & 200 \\
\hline Manganese, dissolved $(\mu \mathrm{g} / \mathrm{L}$ as $\mathrm{Mn})$ & 25 & 450 & 380 & 418 & 420 \\
\hline Molybdenum, dissolved ( $\mu \mathrm{g} / \mathrm{L}$ as $\mathrm{Mo})$ & 25 & 1 & $<1$ & -- & $<1$ \\
\hline Nickel, dissolved ( $\mu \mathrm{g} / \mathrm{L}$ as $\mathrm{Ni})$ & 25 & 850 & 700 & 785 & 790 \\
\hline Selenium, dissolved $(\mu \mathrm{g} / \mathrm{L}$ as $\mathrm{Se})$ & 12 & $<5$ & $<1$ & - & $<1$ \\
\hline Silver, dissolved ( $\mu \mathrm{g} / \mathrm{L}$ as $\mathrm{Ag}$ ) & 25 & 5 & $<2$ & - & $<3$ \\
\hline Strontium, dissolved $(\mu \mathrm{g} / \mathrm{L}$ as $\mathrm{Sr})$ & 25 & 1,700 & 1,400 & 1,540 & 1,600 \\
\hline Vanadium, dissolved ( $\mu \mathrm{g} / \mathrm{L}$ as $\mathrm{V})$ & 25 & 25 & $<18$ & ${ }^{1} 21$ & $<60$ \\
\hline Zinc, dissolved ( $\mu \mathrm{g} / \mathrm{L}$ as $\mathrm{Zn})$ & 25 & 3,800 & 3,100 & 3,450 & 3,500 \\
\hline
\end{tabular}


Table 6. Statistical summary of water-quality data for sites in the Sand Coulee Coal Area, Montana, July 1994 through September 1996 (Continued)

\begin{tabular}{|c|c|c|c|c|c|}
\hline $\begin{array}{l}\text { Property or constituent } \\
\text { (reporting unit) }\end{array}$ & $\begin{array}{c}\text { Number } \\
\text { of } \\
\text { samples }\end{array}$ & Maximum & Minimum & Mean & Median \\
\hline \multicolumn{6}{|c|}{ Site 6, 471851111111101-Giffen Spring near Stockett, Mont. } \\
\hline Streamflow, instantaneous $\left(\mathrm{ft}^{3} / \mathrm{s}\right)$ & 25 & 0.55 & 0.38 & 0.49 & 0.50 \\
\hline Specific conductance, onsite $(\mu \mathrm{S} / \mathrm{cm})$ & 25 & 1,400 & 982 & 1,160 & 1,130 \\
\hline Temperature, water $\left({ }^{\circ} \mathrm{C}\right)$ & 25 & 11.0 & 8.5 & 9.2 & 9.0 \\
\hline pH, onsite (standard units) & 25 & 5.8 & 3.7 & 4.9 & 5.0 \\
\hline Hardness, total $\left(\mathrm{mg} / \mathrm{L}\right.$ as $\left.\mathrm{CaCO}_{3}\right)$ & 25 & 480 & 440 & 459 & 460 \\
\hline Calcium, dissolved (mg/L as Ca) & 25 & 120 & 110 & 115 & 110 \\
\hline Magnesium, dissolved (mg/L as $\mathrm{Mg}$ ) & 25 & 44 & 39 & 42 & 42 \\
\hline Sodium, dissolved (mg/L as $\mathrm{Na}$ ) & 25 & 17 & 11 & 14 & 14 \\
\hline Potassium, dissolved (mg/L as $\mathrm{K}$ ) & 25 & 6.3 & 4.4 & 5.3 & 5.4 \\
\hline Acidity $\left(\mathrm{mg} / \mathrm{L}\right.$ as $\left.\mathbf{H}^{+}\right)$ & 25 & 9.5 & .6 & 4.3 & 4.5 \\
\hline Alkalinity ( $\mathrm{mg} / \mathrm{L}$ as $\mathrm{CaCO}_{3}$ ) & 25 & $e<1$ & $e<1$ & -- & $\mathrm{e}<1$ \\
\hline Sulfate, dissolved (mg/L as $\mathrm{SO}_{4}$ ) & 25 & 1,000 & 480 & 729 & 740 \\
\hline Chloride, dissolved (mg/L as $\mathrm{Cl}$ ) & 25 & 6.1 & 2.7 & 3.9 & 3.7 \\
\hline Fluoride, dissolved (mg/L as F) & 24 & 1.5 & .2 & .9 & .9 \\
\hline Silica, dissolved (mg/L as $\mathrm{SiO}_{2}$ ) & 25 & 25 & 16 & 19.8 & 19 \\
\hline Dissolved solids, calculated (mg/L) & 25 & el, 300 & e715 & el,, 020 & $\mathrm{e} 1,020$ \\
\hline Aluminum, dissolved $(\mu \mathrm{g} / \mathrm{L}$ as $\mathrm{Al})$ & 25 & 35,000 & 1,100 & 11,800 & 6,900 \\
\hline Arsenic, dissolved ( $\mu \mathrm{g} / \mathrm{L}$ as As) & 25 & 1 & $<1$ & -- & $<1$ \\
\hline Barium, dissolved ( $\mu \mathrm{g} / \mathrm{L}$ as $\mathrm{Ba})$ & 25 & 40 & 23 & 28 & 26 \\
\hline Beryllium, dissolved ( $\mu \mathrm{g} / \mathrm{L}$ as $\mathrm{Be})$ & 25 & 11 & 2 & 5 & 5 \\
\hline Boron, dissolved ( $\mu \mathrm{g} / \mathrm{L}$ as $\mathrm{B})$ & 24 & 120 & 50 & 86 & 85 \\
\hline Cadmium, dissolved $(\mu \mathrm{g} / \mathrm{L}$ as $\mathrm{Cd})$ & 25 & 18 & 1 & 7 & 8 \\
\hline Chromium, dissolved ( $\mu \mathrm{g} / \mathrm{L}$ as $\mathrm{Cr}$ ) & 25 & 7 & $<5$ & $1_{4}$ & $<5$ \\
\hline Cobalt, dissolved ( $\mu \mathrm{g} / \mathrm{L}$ as $\mathrm{Co})$ & 25 & 510 & 110 & 250 & 250 \\
\hline Copper, dissolved $(\mu \mathrm{g} / \mathrm{L}$ as $\mathrm{Cu})$ & 25 & 80 & $<10$ & ${ }^{1} 17$ & $<10$ \\
\hline Iron, dissolved $(\mu \mathrm{g} / \mathrm{L}$ as $\mathrm{Fe})$ & 25 & 110,000 & 40,000 & 72,200 & 71,000 \\
\hline Lead, dissolved $(\mu \mathrm{g} / \mathrm{L}$ as $\mathrm{Pb})$ & 25 & $<10$ & $<1$ & - & $<1$ \\
\hline Lithium, dissolved $(\mu \mathrm{g} / \mathrm{L}$ as $\mathrm{Li})$ & 25 & 86 & 60 & 73 & 73 \\
\hline Manganese, dissolved $(\mu \mathrm{g} / \mathrm{L}$ as $\mathrm{Mn})$ & 25 & 510 & 320 & 384 & 370 \\
\hline Molybdenum, dissolved ( $\mu \mathrm{g} / \mathrm{L}$ as $\mathrm{Mo})$ & 25 & 2 & $<1$ & - & $<1$ \\
\hline Nickel, dissolved ( $\mu \mathrm{g} / \mathrm{L}$ as $\mathrm{Ni})$ & 25 & 570 & 230 & 369 & 340 \\
\hline Selenium, dissolved $(\mu \mathrm{g} / \mathrm{L}$ as $\mathrm{Se})$ & 12 & $<5$ & $<1$ & - & $<1$ \\
\hline Silver, dissolved ( $\mu \mathrm{g} / \mathrm{L}$ as $\mathrm{Ag})$ & 25 & 2 & $<1$ & - & $<1$ \\
\hline Strontium, dissolved ( $\mu \mathrm{g} / \mathrm{L}$ as $\mathrm{Sr})$ & 25 & 380 & 310 & 352 & 350 \\
\hline Vanadium, dissolved ( $\mu \mathrm{g} / \mathrm{L}$ as $\mathrm{V})$ & 25 & 8 & $<6$ & - & $<6$ \\
\hline $\mathrm{Zinc}$, dissolved ( $\mu \mathrm{g} / \mathrm{L}$ as $\mathrm{Zn})$ & 25 & 2,500 & 830 & 1,480 & 1,400 \\
\hline
\end{tabular}


Table 6. Statistical summary of water-quality data for sites in the Sand Coulee Coal Area, Montana, July 1994 through September 1996 (Continued)

\begin{tabular}{|c|c|c|c|c|c|}
\hline $\begin{array}{l}\text { Property or constituent } \\
\text { (reporting unit) }\end{array}$ & $\begin{array}{c}\text { Number } \\
\text { of } \\
\text { samples }\end{array}$ & Maximum & Minimum & Mean & Median \\
\hline \multicolumn{6}{|c|}{ Site 7, 472016111085701-Cottonwood Mine No. 6 drain to Cottonwood Creek near Stockett, Mont. } \\
\hline Streamflow, instantaneous $\left(\mathrm{ft}^{3} / \mathrm{s}\right)$ & 18 & 0.15 & 0.02 & 0.05 & 0.03 \\
\hline Specific conductance, onsite $(\mu \mathrm{S} / \mathrm{cm})$ & 18 & 6,020 & 5,570 & 5,788 & 5,790 \\
\hline Temperature, water $\left({ }^{\circ} \mathrm{C}\right)$ & 18 & 11.5 & 6.0 & 9.6 & 10.0 \\
\hline pH, onsite (standard units) & 18 & 2.8 & 2.5 & 2.6 & 2.6 \\
\hline Hardness, total $\left(\mathrm{mg} / \mathrm{L}\right.$ as $\left.\mathrm{CaCO}_{3}\right)$ & 18 & 1,500 & 1,300 & 1,420 & 1,400 \\
\hline Calcium, dissolved (mg/L as Ca) & 18 & 380 & 330 & 348 & 350 \\
\hline Magnesium, dissolved (mg/L as $\mathrm{Mg}$ ) & 18 & 140 & 120 & 133 & 130 \\
\hline Sodium, dissolved (mg/L as $\mathrm{Na}$ ) & 18 & 15 & 13 & 14 & 14 \\
\hline Potassium, dissolved ( $\mathrm{mg} / \mathrm{L}$ as $\mathrm{K}$ ) & 18 & 5.7 & .5 & 2.7 & 2.5 \\
\hline Acidity $\left(\mathrm{mg} / \mathrm{L}\right.$ as $\left.\mathrm{H}^{+}\right)$ & 18 & 87 & 74 & 82 & 83 \\
\hline Alkalinity $\left(\mathrm{mg} / \mathrm{L}\right.$ as $\left.\mathrm{CaCO}_{3}\right)$ & 18 & $e<1$ & $e<1$ & -- & $\mathrm{e}<1$ \\
\hline Sulfate, dissolved ( $\mathrm{mg} / \mathrm{L}$ as $\left.\mathrm{SO}_{4}\right)$ & 18 & 7,200 & 5,400 & 6,040 & 5,850 \\
\hline Chloride, dissolved (mg/L as $\mathrm{Cl}$ ) & 18 & 20 & $<1$ & ${ }^{1} 6.7$ & 3.6 \\
\hline Fluoride, dissolved (mg/L as $\mathrm{F}$ ) & 15 & 5.6 & $<1$ & ${ }^{1} 2.5$ & 2.8 \\
\hline Silica, dissolved $\left(\mathrm{mg} / \mathrm{L}\right.$ as $\left.\mathrm{SiO}_{2}\right)$ & 18 & 100 & 83 & 93 & 95 \\
\hline Dissolved solids, calculated (mg/L) & 18 & e9,140 & e6,530 & $\mathrm{e} 7,810$ & e7,700 \\
\hline Aluminum, dissolved $(\mu \mathrm{g} / \mathrm{L}$ as $\mathrm{Al})$ & 18 & 450,000 & 350,000 & 401,000 & 405,000 \\
\hline Arsenic, dissolved ( $\mu \mathrm{g} / \mathrm{L}$ as As) & 18 & $<25$ & $<1$ & - & $<5$ \\
\hline Barium, dissolved ( $\mu \mathrm{g} / \mathrm{L}$ as $\mathrm{Ba})$ & 11 & 130 & 100 & 116 & 120 \\
\hline Beryllium, dissolved ( $\mu \mathrm{g} / \mathrm{L}$ as $\mathrm{Be})$ & 18 & 130 & 94 & 116 & 120 \\
\hline Boron, dissolved ( $\mu \mathrm{g} / \mathrm{L}$ as $\mathrm{B})$ & 15 & 570 & 470 & 509 & 510 \\
\hline Cadmium, dissolved $(\mu \mathrm{g} / \mathrm{L}$ as $\mathrm{Cd})$ & 18 & 110 & 72 & 84 & 80 \\
\hline Chromium, dissolved ( $\mu \mathrm{g} / \mathrm{L}$ as $\mathrm{Cr})$ & 18 & 80 & $<50$ & $1_{49}$ & $<80$ \\
\hline Cobalt, dissolved $(\mu \mathrm{g} / \mathrm{L}$ as $\mathrm{Co})$ & 17 & 9,700 & 3,900 & 6,220 & 6,200 \\
\hline Copper, dissolved $(\mu \mathrm{g} / \mathrm{L}$ as $\mathrm{Cu})$ & 18 & 210 & $<100$ & ${ }^{1} 113$ & 100 \\
\hline Iron, dissolved $(\mu \mathrm{g} / \mathrm{L}$ as $\mathrm{Fe})$ & 18 & 840,000 & 660,000 & 774,000 & 785,000 \\
\hline Lead, dissolved $(\mu \mathrm{g} / \mathrm{L}$ as $\mathrm{Pb})$ & 18 & $<4$ & $<1$ & - & $<1$ \\
\hline Lithium, dissolved $(\mu \mathrm{g} / \mathrm{L}$ as $\mathrm{Li})$ & 18 & 730 & 550 & 652 & 665 \\
\hline Manganese, dissolved ( $\mu \mathrm{g} / \mathrm{L}$ as $\mathrm{Mn})$ & 18 & 2,500 & 2,100 & 2,350 & 2,350 \\
\hline Molybdenum, dissolved ( $\mu \mathrm{g} / \mathrm{L}$ as $\mathrm{Mo}$ ) & 18 & 5 & $<1$ & 2 & 1 \\
\hline Nickel, dissolved $(\mu \mathrm{g} / \mathrm{L}$ as $\mathrm{Ni})$ & 18 & 12,000 & 9,700 & 10,800 & 11,000 \\
\hline Selenium, dissolved ( $\mu \mathrm{g} / \mathrm{L}$ as $\mathrm{Se})$ & 5 & 4 & $<1$ & - & -- \\
\hline Silver, dissolved $(\mu \mathrm{g} / \mathrm{L}$ as $\mathrm{Ag})$ & 18 & 11 & $<3$ & -- & $<10$ \\
\hline Strontium, dissolved ( $\mu \mathrm{g} / \mathrm{L}$ as $\mathrm{Sr}$ ) & 18 & 1,500 & 1,100 & 1,240 & 1,200 \\
\hline Vanadium, dissolved $(\mu \mathrm{g} / \mathrm{L}$ as $\mathrm{V})$ & 18 & 240 & $<60$ & ${ }^{1} 138$ & 140 \\
\hline Zinc, dissolved $(\mu \mathrm{g} / \mathrm{L}$ as $\mathrm{Zn})$ & 18 & 56,000 & 44,000 & 50,500 & 51,000 \\
\hline
\end{tabular}


Table 6. Statistical summary of water-quality data for sites in the Sand Coulee Coal Area, Montana, July 1994 through September 1996 (Continued)

\begin{tabular}{|c|c|c|c|c|c|}
\hline $\begin{array}{l}\text { Property or constituent } \\
\text { (reporting unit) }\end{array}$ & $\begin{array}{c}\text { Number } \\
\text { of } \\
\text { samples }\end{array}$ & Maximum & Minimum & Mean & Median \\
\hline \multicolumn{6}{|c|}{ Site 8, 472114111095001-Cottonwood Mine No. 2 drain to Ladd Coulee at Stockett, Mont. } \\
\hline Streamflow, instantaneous $\left(\mathrm{ft}^{3} / \mathrm{s}\right)$ & 24 & 0.10 & 0.001 & 0.02 & 0.01 \\
\hline Specific conductance, onsite $(\mu \mathrm{S} / \mathrm{cm})$ & 25 & 10,800 & 6,470 & 8,920 & 8,750 \\
\hline Temperature, water $\left({ }^{\circ} \mathrm{C}\right)$ & 25 & 26.0 & 0.0 & 11.7 & 11.0 \\
\hline pH, onsite (standard units) & 25 & 2.8 & 2.2 & 2.5 & 2.5 \\
\hline Hardness, total $\left(\mathrm{mg} / \mathrm{L}\right.$ as $\left.\mathrm{CaCO}_{3}\right)$ & 25 & 3,100 & 1,600 & 2,300 & 2,300 \\
\hline Calcium, dissolved (mg/L as Ca) & 25 & 520 & 320 & 425 & 430 \\
\hline Magnesium, dissolved ( $\mathrm{mg} / \mathrm{L}$ as $\mathrm{Mg}$ ) & 25 & 430 & 200 & 300 & 310 \\
\hline Sodium, dissolved ( $\mathrm{mg} / \mathrm{L}$ as $\mathrm{Na})$ & 25 & 12 & 4.7 & 8.8 & 8.9 \\
\hline Potassium, dissolved (mg/L as $\mathrm{K}$ ) & 24 & 5.7 & .3 & 2.2 & 1.8 \\
\hline Acidity (mg/L as $\mathrm{H}^{+}$) & 25 & 270 & 100 & 200 & 210 \\
\hline Alkalinity $\left(\mathrm{mg} / \mathrm{L}\right.$ as $\mathrm{CaCO}_{3}$ ) & 25 & $e<1$ & $e<1$ & -- & $e<1$ \\
\hline Sulfate, dissolved $\left(\mathrm{mg} / \mathrm{L}\right.$ as $\left.\mathrm{SO}_{4}\right)$ & 25 & 16,000 & 8,600 & 13,100 & 14,000 \\
\hline Fluoride, dissolved (mg/L as F) & 20 & 8.5 & $<1$ & 13.0 & 2.0 \\
\hline Silica, dissolved (mg/L as $\left.\mathrm{SiO}_{2}\right)$ & 25 & 140 & 38 & 97 & 100 \\
\hline Dissolved solids, calculated (mg/L) & 25 & $\mathrm{e} 20,700$ & e10,800 & el6,600 & el 7,400 \\
\hline Aluminum, dissolved $(\mu \mathrm{g} / \mathrm{L}$ as $\mathrm{Al})$ & 25 & $1,600,000$ & 740,000 & $1,180,000$ & $1,200,000$ \\
\hline Arsenic, dissolved ( $\mu \mathrm{g} / \mathrm{L}$ as $\mathrm{As})$ & 25 & 11 & $<1$ & ${ }^{1} 2$ & $<5$ \\
\hline Barium, dissolved $(\mu \mathrm{g} / \mathrm{L}$ as $\mathrm{Ba})$ & 25 & 200 & $<200$ & ${ }^{1} 150$ & $<200$ \\
\hline Beryllium, dissolved $(\mu \mathrm{g} / \mathrm{L}$ as $\mathrm{Be})$ & 25 & 300 & 110 & 176 & 170 \\
\hline Boron, dissolved $(\mu \mathrm{g} / \mathrm{L}$ as $\mathrm{B})$ & 23 & 1,000 & 390 & 727 & 760 \\
\hline Cadmium, dissolved $(\mu \mathrm{g} / \mathrm{L}$ as $\mathrm{Cd})$ & 24 & 510 & 160 & 306 & 310 \\
\hline Chromium, dissolved ( $\mu \mathrm{g} / \mathrm{L}$ as $\mathrm{Cr})$ & 25 & 270 & $<100$ & ${ }^{1} 129$ & 110 \\
\hline Cobalt, dissolved ( $\mu \mathrm{g} / \mathrm{L}$ as Co $)$ & 24 & 11,000 & 4,900 & 8,050 & 8,000 \\
\hline Copper, dissolved $(\mu \mathrm{g} / \mathrm{L}$ as $\mathrm{Cu})$ & 25 & 820 & 200 & 450 & 420 \\
\hline Iron, dissolved $(\mu \mathrm{g} / \mathrm{L}$ as $\mathrm{Fe})$ & 25 & $2,000,000$ & 720,000 & $1,380,000$ & $1,400,000$ \\
\hline Lead, dissolved $(\mu \mathrm{g} / \mathrm{L}$ as $\mathrm{Pb})$ & 24 & 1 & $<1$ & - & $<1$ \\
\hline Lithium, dissolved $(\mu \mathrm{g} / \mathrm{L}$ as $\mathrm{Li})$ & 25 & 2,000 & 1,100 & 1,540 & 1,500 \\
\hline Manganese, dissolved $(\mu \mathrm{g} / \mathrm{L}$ as $\mathrm{Mn})$ & 24 & 17,000 & 5,400 & 8,480 & 7,900 \\
\hline Molybdenum, dissolved ( $\mu \mathrm{g} / \mathrm{L}$ as $\mathrm{Mo})$ & 24 & 6 & $<1$ & ${ }^{1} 1$ & $<2$ \\
\hline Nickel, dissolved $(\mu \mathrm{g} / \mathrm{L}$ as $\mathrm{Ni})$ & 23 & 17,000 & 8,500 & 12,600 & 13,000 \\
\hline Selenium, dissolved ( $\mu \mathrm{g} / \mathrm{L}$ as $\mathrm{Se})$ & 12 & 4 & $<1$ & -- & $<5$ \\
\hline Silver, dissolved $(\mu \mathrm{g} / \mathrm{L}$ as $\mathrm{Ag})$ & 25 & $<40$ & $<1$ & - & $<10$ \\
\hline Strontium, dissolved ( $\mu \mathrm{g} / \mathrm{L}$ as $\mathrm{Sr}$ ) & 25 & 2,500 & 1,200 & 1,800 & 1,900 \\
\hline Vanadium, dissolved $(\mu \mathrm{g} / \mathrm{L}$ as $\mathrm{V})$ & 19 & 230 & $<6$ & -- & $<120$ \\
\hline Zinc, dissolved ( $\mu \mathrm{g} / \mathrm{L}$ as $\mathrm{Zn})$ & 25 & 87,000 & 42,000 & 63,200 & 62,000 \\
\hline
\end{tabular}


Table 6. Statistical summary of water-quality data for sites in the Sand Coulee Coal Area, Montana, July 1994 through September 1996 (Continued)

\begin{tabular}{|c|c|c|c|c|c|}
\hline $\begin{array}{l}\text { Property or constituent } \\
\text { (reporting unit) }\end{array}$ & $\begin{array}{c}\text { Number } \\
\text { of } \\
\text { sampies }\end{array}$ & Maximum & Minimum & Mean & Median \\
\hline \multicolumn{6}{|c|}{ Site 9, 472212111093301-Number Five Coulee near Stockett, Mont. } \\
\hline Streamflow, instantaneous $\left(\mathrm{ft}^{3} / \mathrm{s}\right)$ & 23 & 9.5 & 0.00 & 1.2 & 0.02 \\
\hline Specific conductance, onsite $(\mu \mathrm{S} / \mathrm{cm})$ & 14 & 1,160 & 645 & 932 & 981 \\
\hline Temperature, water $\left({ }^{\circ} \mathrm{C}\right)$ & 14 & 29.0 & 0.0 & 12.2 & 14.8 \\
\hline pH, onsite (standard units) & 14 & 8.4 & 5.1 & 7.1 & 7.2 \\
\hline Hardness, total $\left(\mathrm{mg} / \mathrm{L}\right.$ as $\left.\mathrm{CaCO}_{3}\right)$ & 14 & 620 & 300 & 468 & 480 \\
\hline Calcium, dissolved (mg/L as $\mathrm{Ca}$ ) & 14 & 170 & 61 & 117 & 125 \\
\hline Magnesium, dissolved (mg/L as $\mathrm{Mg}$ ) & 14 & 50 & 34 & 42 & 44 \\
\hline Sodium, dissolved (mg/L as $\mathrm{Na}$ ) & 14 & 25 & 11 & 19 & 20 \\
\hline Potassium, dissolved (mg/L as $\mathrm{K}$ ) & 14 & 6.9 & 4.1 & 5.4 & 5.4 \\
\hline Acidity $\left(\mathrm{mg} / \mathrm{L}\right.$ as $\left.\mathrm{H}^{+}\right)$ & 14 & 1.5 & $<.1$ & ${ }^{1} .2$ & $<.1$ \\
\hline Alkalinity $\left(\mathrm{mg} / \mathrm{L}\right.$ as $\mathrm{CaCO}_{3}$ ) & 14 & 259 & 1 & 74 & 30 \\
\hline Sulfate, dissolved ( $\mathrm{mg} / \mathrm{L}$ as $\left.\mathrm{SO}_{4}\right)$ & 14 & 750 & 77 & 432 & 495 \\
\hline Chloride, dissolved ( $\mathrm{mg} / \mathrm{L}$ as $\mathrm{Cl})$ & 14 & 5.7 & 3.1 & 4.4 & 4.4 \\
\hline Fluoride, dissolved (mg/L as $\mathrm{F}$ ) & 14 & 1 & .4 & .6 & .6 \\
\hline Silica, dissolved (mg/L as $\mathrm{SiO}_{2}$ ) & 14 & 12 & 1.4 & 6.7 & 6.7 \\
\hline Dissolved solids, calculated (mg/L) & 14 & 1,030 & 371 & 678 & 715 \\
\hline Aluminum, dissolved ( $\mu \mathrm{g} / \mathrm{L}$ as $\mathrm{Al})$ & 14 & 1,700 & 30 & 264 & 105 \\
\hline Arsenic, dissolved $(\mu \mathrm{g} / \mathrm{L}$ as As) & 14 & $<2$ & $<1$ & - & $<1$ \\
\hline Barium, dissolved $(\mu \mathrm{g} / \mathrm{L}$ as $\mathrm{Ba})$ & 14 & 160 & 32 & 84 & 75 \\
\hline Beryllium, dissolved $(\mu \mathrm{g} / \mathrm{L}$ as $\mathrm{Be})$ & 14 & .9 & $<.5$ & -- & $<.5$ \\
\hline Boron, dissolved ( $\mu \mathrm{g} / \mathrm{L}$ as $\mathrm{B})$ & 14 & 100 & 40 & 69 & 75 \\
\hline Cadmium, dissolved $(\mu \mathrm{g} / \mathrm{L}$ as $\mathrm{Cd})$ & 14 & 9 & $<1$ & $\mathrm{I}_{2}$ & $<1$ \\
\hline Chromium, dissolved ( $\mu \mathrm{g} / \mathrm{L}$ as $\mathrm{Cr}$ ) & 14 & 7 & $<5$ & -- & $<5$ \\
\hline Cobalt, dissolved $(\mu \mathrm{g} / \mathrm{L}$ as $\mathrm{Co})$ & 12 & 210 & $<3$ & ${ }^{1} 74$ & 30 \\
\hline Copper, dissolved ( $\mu \mathrm{g} / \mathrm{L}$ as $\mathrm{Cu})$ & 14 & $<10$ & $<10$ & -- & $<10$ \\
\hline Iron, dissolved $(\mu \mathrm{g} / \mathrm{L}$ as $\mathrm{Fe})$ & 14 & 27,000 & $<3$ & $1_{3,250}$ & 43 \\
\hline Lead, dissolved $(\mu \mathrm{g} / \mathrm{L}$ as $\mathrm{Pb})$ & 14 & 10 & $<1$ & -- & $<10$ \\
\hline Lithium, dissolved ( $\mu \mathrm{g} / \mathrm{L}$ as $\mathrm{Li})$ & 14 & 66 & 16 & 47.8 & 51.5 \\
\hline Manganese, dissolved ( $\mu \mathrm{g} / \mathrm{L}$ as $\mathrm{Mn})$ & 14 & 1,500 & 9 & 536 & 420 \\
\hline Molybdenum, dissolved ( $\mu \mathrm{g} / \mathrm{L}$ as $\mathrm{Mo})$ & 14 & $<10$ & $<1$ & -- & $<10$ \\
\hline Nickel, dissolved ( $\mu \mathrm{g} / \mathrm{L}$ as $\mathrm{Ni})$ & 14 & 450 & $<10$ & ${ }^{1} 162$ & 130 \\
\hline Selenium, dissolved $(\mu \mathrm{g} / \mathrm{L}$ as $\mathrm{Se})$ & 5 & 1 & $<1$ & - & -- \\
\hline Silver, dissolved $(\mu \mathrm{g} / \mathrm{L}$ as $\mathrm{Ag})$ & 14 & 2 & $<1$ & - & $<1$ \\
\hline Strontium, dissolved $(\mu \mathrm{g} / \mathrm{L}$ as $\mathrm{Sr})$ & 14 & 410 & 250 & 334 & 345 \\
\hline Vanadium, dissolved ( $\mu \mathrm{g} / \mathrm{L}$ as $\mathrm{V})$ & 14 & $<6$ & $<6$ & - & $<6$ \\
\hline Zinc, dissolved $(\mu \mathrm{g} / \mathrm{L}$ as $\mathrm{Zn})$ & 14 & 1,900 & $<3$ & ${ }^{1} 442$ & 66 \\
\hline
\end{tabular}


Table 6. Statistical summary of water-quality data for sites in the Sand Coulee Coal Area, Montana, July 1994 through September 1996 (Continued)

\begin{tabular}{|c|c|c|c|c|c|}
\hline $\begin{array}{l}\text { Property or constltuent } \\
\text { (reporting unit) }\end{array}$ & $\begin{array}{c}\text { Number } \\
\text { of } \\
\text { samples }\end{array}$ & Maximum & Minimum & Mean & Median \\
\hline \multicolumn{6}{|c|}{ Site 10, $472233110552601-F r e n c h$ Coulee wetlands outflow at Belt, Mont. } \\
\hline Streamflow, instantaneous $\left(\mathrm{ft}^{3} / \mathrm{s}\right)$ & 24 & 0.09 & $<0.001$ & 0.03 & 0.02 \\
\hline Specific conductance, onsite $(\mu \mathrm{S} / \mathrm{cm})$ & 20 & 6,180 & 2,880 & 4,601 & 4,630 \\
\hline Temperature, water $\left({ }^{\circ} \mathrm{C}\right)$ & 20 & 20.0 & 0.0 & 8.0 & 7.2 \\
\hline pH, onsite (standard units) & 20 & 3.9 & 2.5 & 3.0 & 2.8 \\
\hline Hardness, total $\left(\mathrm{mg} / \mathrm{L}\right.$ as $\left.\mathrm{CaCO}_{3}\right)$ & 20 & 2,000 & 810 & 1,342 & 1,300 \\
\hline Calcium, dissolved (mg/L as Ca) & 20 & 490 & 200 & 324 & 315 \\
\hline Magnesium, dissolved (mg/L as $\mathrm{Mg}$ ) & 20 & 210 & 75 & 128 & 110 \\
\hline Sodium, dissolved (mg/L as $\mathrm{Na}$ ) & 20 & 44 & 12 & 23 & 19 \\
\hline Potassium, dissolved (mg/L as $\mathrm{K}$ ) & 20 & 33 & 3 & 12 & 8.4 \\
\hline Acidity $\left(\mathrm{mg} / \mathrm{L}\right.$ as $\left.\mathrm{H}^{+}\right)$ & 20 & 90 & 23 & 64 & 66 \\
\hline Alkalinity (mg/L as $\left.\mathrm{CaCO}_{3}\right)$ & 20 & $e<1$ & $e<1$ & -- & $e<1$ \\
\hline Sulfate, dissolved $\left(\mathrm{mg} / \mathrm{L}\right.$ as $\left.\mathrm{SO}_{4}\right)$ & 20 & 6,800 & 2,200 & 5,005 & 5,450 \\
\hline Chloride, dissolved ( $\mathrm{mg} / \mathrm{L}$ as $\mathrm{Cl}$ ) & 20 & 29 & 7.8 & 18 & 17 \\
\hline Fluoride, dissolved ( $\mathrm{mg} / \mathrm{L}$ as $\mathrm{F}$ ) & 18 & 4.5 & $<1$ & 12.0 & 1.6 \\
\hline Silica, dissolved ( $\mathrm{mg} / \mathrm{L}$ as $\mathrm{SiO}_{2}$ ) & 20 & 130 & 55 & 92.4 & 90 \\
\hline Dissolved solids, calculated (mg/L) & 20 & e8,950 & $\mathrm{e} 2,870$ & $e 6,360$ & e6,800 \\
\hline Aluminum, dissolved ( $\mu \mathrm{g} / \mathrm{L}$ as $\mathrm{Al})$ & 20 & 570,000 & 100,000 & 371,000 & 375,000 \\
\hline Arsenic, dissolved ( $\mu \mathrm{g} / \mathrm{L}$ as $\mathrm{As})$ & 20 & $<2$ & $<1$ & -- & $<1$ \\
\hline Barium, dissolved ( $\mu \mathrm{g} / \mathrm{L}$ as $\mathrm{Ba})$ & 20 & 21 & $<10$ & ${ }^{1} 12.4$ & 12 \\
\hline Beryllium, dissolved $(\mu \mathrm{g} / \mathrm{L}$ as $\mathrm{Be})$ & 20 & 62 & 15 & 40 & 40 \\
\hline Boron, dissolved $(\mu \mathrm{g} / \mathrm{L}$ as $\mathrm{B})$ & 20 & 510 & 150 & 283 & 255 \\
\hline Cadmium, dissolved $(\mu \mathrm{g} / \mathrm{L}$ as $\mathrm{Cd})$ & 20 & 9 & $<5$ & $1_{4}$ & 3 \\
\hline Chromium, dissolved $(\mu \mathrm{g} / \mathrm{L}$ as $\mathrm{Cr})$ & 20 & 110 & $<50$ & ${ }^{1} 58$ & 45 \\
\hline Cobalt, dissolved ( $\mu \mathrm{g} / \mathrm{L}$ as $\mathrm{Co})$ & 15 & 420 & 90 & 261 & 260 \\
\hline Copper, dissolved ( $\mu \mathrm{g} / \mathrm{L}$ as $\mathrm{Cu})$ & 20 & 140 & $<30$ & ${ }^{1} 41$ & $<100$ \\
\hline Iron, dissolved $(\mu \mathrm{g} / \mathrm{L}$ as $\mathrm{Fe})$ & 19 & 770,000 & 170,000 & 471,000 & 500,000 \\
\hline Lead, dissolved ( $\mu \mathrm{g} / \mathrm{L}$ as $\mathrm{Pb})$ & 20 & 6 & $<1$ & $1_{2}$ & $<10$ \\
\hline Lithium, dissolved $(\mu \mathrm{g} / \mathrm{L}$ as $\mathrm{Li})$ & 20 & 750 & 200 & 476 & 455 \\
\hline Manganese, dissolved ( $\mu \mathrm{g} / \mathrm{L}$ as $\mathrm{Mn})$ & 20 & 6,400 & 790 & 2,770 & 2,200 \\
\hline Molybdenum, dissolved ( $\mu \mathrm{g} / \mathrm{L}$ as $\mathrm{Mo}$ ) & 20 & $<10$ & $<1$ & - & $<1$ \\
\hline Nickel, dissolved ( $\mu \mathrm{g} / \mathrm{L}$ as $\mathrm{Ni})$ & 20 & 1,100 & 230 & 671 & 695 \\
\hline Selenium, dissolved $(\mu \mathrm{g} / \mathrm{L}$ as $\mathrm{Se})$ & 12 & $<10$ & $<1$ & -- & $<1$ \\
\hline Silver, dissolved ( $\mu \mathrm{g} / \mathrm{L}$ as $\mathrm{Ag})$ & 20 & 18 & $<1$ & -- & $<5$ \\
\hline Strontium, dissolved ( $\mu \mathrm{g} / \mathrm{L}$ as $\mathrm{Sr})$ & 20 & 2,900 & 1,100 & 2,010 & 1,950 \\
\hline Vanadium, dissolved ( $\mu \mathrm{g} / \mathrm{L}$ as $\mathrm{V})$ & 18 & 40 & $<12$ & - & $<60$ \\
\hline Zinc, dissolved ( $\mu \mathrm{g} / \mathrm{L}$ as $\mathrm{Zn})$ & 17 & 7,400 & 810 & 3,020 & 2,400 \\
\hline
\end{tabular}


Table 6. Statistical summary of water-quality data for sites in the Sand Coulee Coal Area, Montana, July 1994 through September 1996 (Continued)

\begin{tabular}{|c|c|c|c|c|c|}
\hline $\begin{array}{l}\text { Property or constituent } \\
\text { (reporting unit) }\end{array}$ & $\begin{array}{l}\text { Number } \\
\text { of } \\
\text { samples }\end{array}$ & Maximum & Minimum & Mean & Median \\
\hline \multicolumn{6}{|c|}{ Site 11, $472235110553201-F r e n c h$ Coulee wetlands inflow at Belt, Mont. } \\
\hline Streamflow, instantaneous $\left(\mathrm{f}^{3} / \mathrm{s}\right)$ & 19 & 0.12 & 0.02 & 0.05 & 0.04 \\
\hline Specific conductance, onsite $(\mu \mathrm{S} / \mathrm{cm})$ & 19 & 6,550 & 2,300 & 4,730 & 5,620 \\
\hline Temperature, water $\left({ }^{\circ} \mathrm{C}\right)$ & 18 & 12.5 & 7.5 & 10.1 & 10.0 \\
\hline pH, onsite (standard units) & 19 & 2.9 & 2.5 & 2.7 & 2.7 \\
\hline Hardness, total ( $\mathrm{mg} / \mathrm{L}$ as $\left.\mathrm{CaCO}_{3}\right)$ & 19 & 1,100 & 550 & 901 & 1,000 \\
\hline Calcium, dissolved ( $\mathrm{mg} / \mathrm{L}$ as $\mathrm{Ca})$ & 19 & 250 & 110 & 199 & 230 \\
\hline Magnesium, dissolved (mg/L as $\mathrm{Mg}$ ) & 19 & 120 & 66 & 96 & 100 \\
\hline Sodium, dissolved (mg/L as Na) & 19 & 17 & 11 & 14 & 13 \\
\hline Potassium, dissolved (mg/L as $\mathrm{K}$ ) & 19 & 5.9 & 2.8 & 4.6 & 5.2 \\
\hline Acidity $\left(\mathrm{mg} / \mathrm{L}\right.$ as $\left.\mathrm{H}^{+}\right)$ & 19 & 120 & 22 & 73 & 91 \\
\hline Alkalinity $\left(\mathrm{mg} / \mathrm{L}\right.$ as $\left.\mathrm{CaCO}_{3}\right)$ & 19 & $e<1$ & $e<1$ & - & $e<1$ \\
\hline Sulfate, dissolved ( $\mathrm{mg} / \mathrm{L}$ as $\left.\mathrm{SO}_{4}\right)$ & 19 & 7,400 & 2,000 & 4,950 & 6,000 \\
\hline Chloride, dissolved ( $\mathrm{mg} / \mathrm{L}$ as $\mathrm{Cl}$ ) & 19 & 38 & 4.5 & 15 & 12 \\
\hline Fluoride, dissolved (mg/L as F) & 17 & 3.6 & $<1$ & ${ }^{1} 1.3$ & 1.1 \\
\hline Silica, dissolved ( $\mathrm{mg} / \mathrm{L}$ as $\mathrm{SiO}_{2}$ ) & 19 & 120 & 41 & 87 & 100 \\
\hline Dissolved solids, calculated (mg/L) & 19 & $e 9,860$ & $\mathrm{e} 2,530$ & $e 6,360$ & e7,260 \\
\hline Aluminum, dissolved ( $\mu \mathrm{g} / \mathrm{L}$ as $\mathrm{Al})$ & 19 & 640,000 & 100,000 & 366,000 & 450,000 \\
\hline Arsenic, dissolved ( $\mu \mathrm{g} / \mathrm{L}$ as As) & 19 & 39 & 2 & 19 & 17 \\
\hline Barium, dissolved ( $\mu \mathrm{g} / \mathrm{L}$ as $\mathrm{Ba})$ & 19 & 17 & $<3$ & ${ }^{1} 6$ & $<10$ \\
\hline Beryllium, dissolved ( $\mu \mathrm{g} / \mathrm{L}$ as $\mathrm{Be})$ & 19 & 64 & 12 & 40 & 49 \\
\hline Boron, dissolved ( $\mu \mathrm{g} / \mathrm{L}$ as $\mathrm{B})$ & 19 & 470 & 90 & 318 & 360 \\
\hline Cadmium, dissolved ( $\mu \mathrm{g} / \mathrm{L}$ as Cd) & 15 & 11 & $<1$ & $1_{7}$ & 6 \\
\hline Chromium, dissolved ( $\mu \mathrm{g} / \mathrm{L}$ as $\mathrm{Cr})$ & 19 & 210 & 30 & 136 & 160 \\
\hline Cobalt, dissolved ( $\mu \mathrm{g} / \mathrm{L}$ as $\mathrm{Co})$ & 12 & 440 & 100 & 253 & 225 \\
\hline Copper, dissolved $(\mu \mathrm{g} / \mathrm{L}$ as $\mathrm{Cu})$ & 19 & 60 & $<30$ & ${ }^{1} 42$ & $<100$ \\
\hline Iron, dissolved ( $\mu \mathrm{g} / \mathrm{L}$ as $\mathrm{Fe}$ ) & 18 & $1,300,000$ & 170,000 & 709,000 & 900,000 \\
\hline Lead, dissolved $(\mu \mathrm{g} / \mathrm{L}$ as $\mathrm{Pb})$ & 19 & 3 & $<1$ & $1_{2}$ & $<10$ \\
\hline Lithium, dissolved $(\mu \mathrm{g} / \mathrm{L}$ as $\mathrm{Li})$ & 19 & 670 & 170 & 454 & 560 \\
\hline Manganese, dissolved ( $\mu \mathrm{g} / \mathrm{L}$ as Mn) & 19 & 1,100 & 200 & 673 & 830 \\
\hline Molybdenum, dissolved ( $\mu \mathrm{g} / \mathrm{L}$ as $\mathrm{Mo})$ & 19 & $<10$ & $<1$ & - & $<1$ \\
\hline Nickel, dissolved ( $\mu \mathrm{g} / \mathrm{L}$ as Ni) & 19 & 1,300 & 230 & 789 & 980 \\
\hline Selenium, dissolved ( $\mu \mathrm{g} / \mathrm{L}$ as $\mathrm{Se})$ & 12 & 2 & $<1$ & - & $<2$ \\
\hline Silver, dissolved $(\mu \mathrm{g} / \mathrm{L}$ as $\mathrm{Ag})$ & 19 & 7 & $<3$ & - & $<5$ \\
\hline Strontium, dissolved ( $\mu \mathrm{g} / \mathrm{L}$ as $\mathrm{Sr}$ ) & 19 & 2,400 & 890 & 1,820 & 2,200 \\
\hline Vanadium, dissolved ( $\mu \mathrm{g} / \mathrm{L}$ as $\mathrm{V}$ ) & 19 & 290 & $<18$ & ${ }^{1} 127$ & 93 \\
\hline Zinc, dissolved $(\mu \mathrm{g} / \mathrm{L}$ as $\mathrm{Zn})$ & 19 & 5,800 & 1,200 & 3,730 & 4,600 \\
\hline
\end{tabular}


Table 6. Statistical summary of water-quality data for sites in the Sand Coulee Coal Area, Montana, July 1994 through September 1996 (Continued)

\begin{tabular}{|c|c|c|c|c|c|}
\hline $\begin{array}{l}\text { Property or constltuent } \\
\text { (reporting unit) }\end{array}$ & $\begin{array}{c}\text { Number } \\
\text { of } \\
\text { samples }\end{array}$ & Maximum & Minimum & Mean & Median \\
\hline \multicolumn{6}{|c|}{ Site 12, $472235110553202-F r e n c h$ Coulee wetlands Inflow No. 2 at Belt, Mont. } \\
\hline Streamflow, instantaneous $\left(\mathrm{ft}^{3} / \mathrm{s}\right)$ & 6 & 0.04 & 0.03 & 0.03 & 0.03 \\
\hline Specific conductance, onsite $(\mu \mathrm{S} / \mathrm{cm})$ & 6 & 5,550 & 3,640 & 4,430 & 4,350 \\
\hline Temperature, water $\left({ }^{\circ} \mathrm{C}\right)$ & 6 & 13.0 & 8.5 & 11.0 & 11.5 \\
\hline $\mathrm{pH}$, onsite (standard units) & 6 & 2.8 & 2.6 & 2.8 & 2.8 \\
\hline Hardness, total ( $\mathrm{mg} / \mathrm{L}$ as $\left.\mathrm{CaCO}_{3}\right)$ & 6 & 1,000 & 660 & 827 & 860 \\
\hline Calcium, dissolved (mg/L as $\mathrm{Ca}$ ) & 6 & 230 & 140 & 183 & 190 \\
\hline Magnesium, dissolved (mg/L as $\mathrm{Mg}$ ) & 6 & 110 & 75 & 90 & 93 \\
\hline Sodium, dissolved (mg/L as $\mathrm{Na}$ ) & 6 & 13 & 12 & 12 & 12 \\
\hline Potassium, dissolved (mg/L as $\mathrm{K}$ ) & 6 & 5.8 & .8 & 4.3 & 4.8 \\
\hline Acidity $\left(\mathrm{mg} / \mathrm{L}\right.$ as $\left.\mathrm{H}^{+}\right)$ & 6 & 98 & 45 & 69 & 72 \\
\hline Sulfate, dissolved (mg/L as $\mathrm{SO}_{4}$ ) & 6 & 6,000 & 3,300 & 4,550 & 4,450 \\
\hline Chloride, dissolved (mg/L as $\mathrm{Cl}$ ) & 6 & 13 & 9.1 & 11.0 & 10.5 \\
\hline Fluoride, dissolved (mg/L as F) & 6 & 3.9 & $<1$ & ${ }^{1} 2.7$ & 2.2 \\
\hline Silica, dissolved $\left(\mathrm{mg} / \mathrm{L}\right.$ as $\left.\mathrm{SiO}_{2}\right)$ & 6 & 100 & 70 & 88 & 90 \\
\hline Aluminum, dissolved ( $\mu \mathrm{g} / \mathrm{L}$ as $\mathrm{Al})$ & 6 & 480,000 & 214,000 & 337,000 & 335,000 \\
\hline Arsenic, dissolved ( $\mu \mathrm{g} / \mathrm{L}$ as $\mathrm{As})$ & 6 & 22 & $<2$ & ${ }^{1} 9.3$ & 5 \\
\hline Barium, dissolved $(\mu \mathrm{g} / \mathrm{L}$ as $\mathrm{Ba})$ & 6 & 19 & 8 & 12.5 & 12 \\
\hline Beryllium, dissolved ( $\mu \mathrm{g} / \mathrm{L}$ as $\mathrm{Be})$ & 6 & 56 & 26 & 41 & 42 \\
\hline Boron, dissolved $(\mu \mathrm{g} / \mathrm{L}$ as B) & 4 & 390 & 230 & - & -- \\
\hline Cadmium, dissolved $(\mu \mathrm{g} / \mathrm{L}$ as $\mathrm{Cd})$ & 6 & 10 & 5 & 7.5 & 7.5 \\
\hline Chromium, dissolved $(\mu \mathrm{g} / \mathrm{L}$ as $\mathrm{Cr})$ & 6 & 190 & 100 & 130 & 125 \\
\hline Cobalt, dissolved ( $\mu \mathrm{g} / \mathrm{L}$ as $\mathrm{Co})$ & 6 & 480 & 220 & 323 & 335 \\
\hline Copper, dissolved ( $\mu \mathrm{g} / \mathrm{L}$ as $\mathrm{Cu})$ & 6 & $<120$ & $<50$ & - & $<100$ \\
\hline Iron, dissolved $(\mu \mathrm{g} / \mathrm{L}$ as $\mathrm{Fe})$ & 6 & $1,000,000$ & 440,000 & 693,000 & 700,000 \\
\hline Lead, dissolved ( $\mu \mathrm{g} / \mathrm{L}$ as $\mathrm{Pb})$ & 6 & 2 & $<4$ & ${ }_{1}^{1} .8$ & 2 \\
\hline Lithium, dissolved $(\mu \mathrm{g} / \mathrm{L}$ as $\mathrm{Li})$ & 6 & 650 & 320 & 460 & 460 \\
\hline Manganese, dissolved ( $\mu \mathrm{g} / \mathrm{L}$ as $\mathrm{Mn})$ & 6 & 930 & 430 & 650 & 670 \\
\hline Molybdenum, dissolved ( $\mu \mathrm{g} / \mathrm{L}$ as Mo) & 6 & 1.6 & $<1$ & - & $<1$ \\
\hline Nickel, dissolved $(\mu \mathrm{g} / \mathrm{L}$ as $\mathrm{Ni})$ & 6 & 1,100 & 490 & 780 & 840 \\
\hline Silver, dissolved $(\mu \mathrm{g} / \mathrm{L}$ as $\mathrm{Ag})$ & 6 & 29 & $<5$ & - & $<10$ \\
\hline Strontium, dissolved ( $\mu \mathrm{g} / \mathrm{L}$ as $\mathrm{Sr})$ & 6 & 2,300 & 1,200 & 1,720 & 1,750 \\
\hline Vanadium, dissolved ( $\mu \mathrm{g} / \mathrm{L}$ as $\mathrm{V})$ & 6 & 87 & $<60$ & -- & 62 \\
\hline Zinc, dissolved $(\mu \mathrm{g} / \mathrm{L}$ as $\mathrm{Zn})$ & 6 & 5,100 & 2,300 & 3,550 & 3,700 \\
\hline
\end{tabular}


Table 6. Statistical summary of water-quality data for sites in the Sand Coulee Coal Area, Montana, July 1994 through September 1996 (Continued)

\begin{tabular}{|c|c|c|c|c|c|}
\hline $\begin{array}{l}\text { Property or consttuent } \\
\text { (reporting unit) }\end{array}$ & $\begin{array}{c}\text { Number } \\
\text { of } \\
\text { samples }\end{array}$ & Maximum & Minimum & Mean & Median \\
\hline \multicolumn{6}{|c|}{ Site 13, 472305110551701-Lewls Coulee above Castner Park, at Belt, Mont. } \\
\hline Streamflow, instantaneous $\left(\mathrm{ft}^{3} / \mathrm{s}\right)$ & 24 & 0.02 & 0.002 & 0.01 & 0.01 \\
\hline Specific conductance, onsite $(\mu \mathrm{S} / \mathrm{cm})$ & 24 & 4,850 & 3,890 & 4,310 & 4,220 \\
\hline Temperature, water $\left({ }^{\circ} \mathrm{C}\right)$ & 24 & 14.0 & 6.5 & 9.7 & 9.5 \\
\hline pH, onsite (standard units) & 24 & 2.8 & 2.4 & 2.7 & 2.7 \\
\hline Hardness, total $\left(\mathrm{mg} / \mathrm{L}\right.$ as $\left.\mathrm{CaCO}_{3}\right)$ & 24 & 1,000 & 850 & 934 & 920 \\
\hline Calcium, dissolved (mg/L as $\mathrm{Ca}$ ) & 24 & 200 & 160 & 177 & 175 \\
\hline Magnesium, dissolved ( $\mathrm{mg} / \mathrm{L}$ as $\mathbf{M g}$ ) & 24 & 130 & 110 & 120 & 120 \\
\hline Sodium, dissolved ( $\mathrm{mg} / \mathrm{L}$ as $\mathrm{Na}$ ) & 24 & 27 & 24 & 26 & 26 \\
\hline Potassium, dissolved ( $\mathrm{mg} / \mathrm{L}$ as $\mathrm{K}$ ) & 23 & 2.8 & .8 & 1.9 & 2.2 \\
\hline Acidity $\left(\mathrm{mg} / \mathrm{L}\right.$ as $\left.\mathrm{H}^{+}\right)$ & 24 & 63 & 35 & 56 & 57 \\
\hline Alkalinity $\left(\mathrm{mg} / \mathrm{L}\right.$ as $\left.\mathrm{CaCO}_{3}\right)$ & 24 & $e<1$ & $e<1$ & - & $e<1$ \\
\hline Sulfate, dissolved $\left(\mathrm{mg} / \mathrm{L}\right.$ as $\left.\mathrm{SO}_{4}\right)$ & 24 & 5,700 & 3,500 & 4,290 & 4,200 \\
\hline Chloride, dissolved (mg/L as Cl) & 24 & 26 & 4.3 & 8.7 & 6.8 \\
\hline Fluoride, dissolved (mg/L as F) & 21 & 2.1 & $<1$ & ${ }^{1} 1.0$ & $<1$ \\
\hline Silica, dissolved (mg/L as $\mathrm{SiO}_{2}$ ) & 24 & 88 & 74 & 82 & 83 \\
\hline Dissolved solids, calculated (mg/L) & 24 & $e 6,960$ & $e 4,630$ & $e 5,460$ & $e 5,420$ \\
\hline Aluminum, dissolved ( $\mu \mathrm{g} / \mathrm{L}$ as $\mathrm{Al})$ & 24 & 530,000 & 250,000 & 300,000 & 290,000 \\
\hline Arsenic, dissolved ( $\mu \mathrm{g} / \mathrm{L}$ as As) & 24 & 2 & $<1$ & ${ }^{1} .9$ & $<2$ \\
\hline Barium, dissolved $(\mu \mathrm{g} / \mathrm{L}$ as $\mathrm{Ba})$ & 24 & 20 & $<3$ & ${ }^{1} 14$ & $<10$ \\
\hline Beryllium, dissolved ( $\mu \mathrm{g} / \mathrm{L}$ as $\mathrm{Be})$ & 24 & 31 & 20 & 23 & 22 \\
\hline Boron, dissolved ( $\mu \mathrm{g} / \mathrm{L}$ as B) & 21 & 320 & 220 & 267 & 270 \\
\hline Cadmium, dissolved ( $\mu \mathrm{g} / \mathrm{L}$ as $\mathrm{Cd})$ & 24 & 65 & 9 & 43 & 44 \\
\hline Chromium, dissolved ( $\mu \mathrm{g} / \mathrm{L}$ as $\mathrm{Cr}$ ) & 24 & 190 & 100 & 129 & 125 \\
\hline Cobalt, dissolved ( $\mu \mathrm{g} / \mathrm{L}$ as $\mathrm{Co})$ & 24 & 5,900 & 750 & 1,450 & 1,000 \\
\hline Copper, dissolved $(\mu \mathrm{g} / \mathrm{L}$ as $\mathrm{Cu})$ & 24 & 200 & $<80$ & ${ }^{1} 89$ & 70 \\
\hline Iron, dissolved ( $\mu \mathrm{g} / \mathrm{L}$ as $\mathrm{Fe})$ & 24 & 560,000 & 450,000 & 504,000 & 505,000 \\
\hline Lead, dissolved $(\mu \mathrm{g} / \mathrm{L}$ as $\mathrm{Pb})$ & 24 & 3 & $<1$ & ${ }^{1.7}$ & $<1$ \\
\hline Lithium, dissolved ( $\mu \mathrm{g} / \mathrm{L}$ as $\mathrm{Li})$ & 24 & 520 & 390 & 452 & 450 \\
\hline Manganese, dissolved ( $\mu \mathrm{g} / \mathrm{L}$ as $\mathrm{Mn})$ & 24 & 1,000 & 840 & 923 & 920 \\
\hline Molybdenum, dissolved ( $\mu \mathrm{g} / \mathrm{L}$ as $\mathrm{Mo}$ ) & 24 & 1 & $<1$ & - & $<1$ \\
\hline Nickel, dissolved ( $\mu \mathrm{g} / \mathrm{L}$ as $\mathrm{Ni})$ & 24 & 2,200 & 1,800 & 2,080 & 2,100 \\
\hline Selenium, dissolved ( $\mu \mathrm{g} / \mathrm{L}$ as Se) & 11 & $<10$ & $<1$ & - & $<1$ \\
\hline Silver, dissolved ( $\mu \mathrm{g} / \mathrm{L}$ as $\mathrm{Ag})$ & 24 & 11 & $<3$ & - & $<5$ \\
\hline Strontium, dissolved ( $\mu \mathrm{g} / \mathrm{L}$ as $\mathrm{Sr}$ ) & 24 & 1,600 & 1,300 & 1,490 & 1,500 \\
\hline Vanadium, dissolved ( $\mu \mathrm{g} / \mathrm{L}$ as $\mathrm{V})$ & 24 & 150 & $<48$ & ${ }^{1} 70$ & 41 \\
\hline Zinc, dissolved $(\mu \mathrm{g} / \mathrm{L}$ as $\mathrm{Zn})$ & 24 & 8,300 & 6,500 & 7,450 & 7,450 \\
\hline
\end{tabular}


Table 6. Statistical summary of water-quality data for sites in the Sand Coulee Coal Area, Montana, July 1994 through September 1996 (Continued)

\begin{tabular}{|c|c|c|c|c|c|}
\hline $\begin{array}{l}\text { Property or constituent } \\
\text { (reporting unit) }\end{array}$ & $\begin{array}{c}\text { Number } \\
\text { of } \\
\text { samples }\end{array}$ & Maximum & MinImum & Mean & Median \\
\hline \multicolumn{6}{|c|}{ Site 14, 472306111103601-Mine draln to Mining Coulee near Sand Coulee, Mont. } \\
\hline Streamflow, instantaneous $\left(\mathrm{ft}^{3} / \mathrm{s}\right)$ & 25 & 0.04 & 0.01 & 0.02 & 0.01 \\
\hline Specific conductance, onsite $(\mu \mathrm{S} / \mathrm{cm})$ & 25 & 7,620 & 6,680 & 7,310 & 7,300 \\
\hline Temperature, water $\left({ }^{\circ} \mathrm{C}\right)$ & 25 & 14.5 & 7.5 & 9.6 & 9.5 \\
\hline pH, onsite (standard units) & 25 & 3.0 & 2.4 & 2.6 & 2.6 \\
\hline Hardness, total (mg/L as $\mathrm{CaCO}_{3}$ ) & 25 & 1,600 & 1,300 & 1,530 & 1,500 \\
\hline Calcium, dissolved ( $\mathrm{mg} / \mathrm{L}$ as $\mathrm{Ca}$ ) & 25 & 310 & 210 & 281 & 280 \\
\hline Magnesium, dissolved ( $\mathrm{mg} / \mathrm{L}$ as $\mathrm{Mg}$ ) & 25 & 220 & 190 & 202 & 200 \\
\hline Sodium, dissolved (mg/L as $\mathrm{Na}$ ) & 25 & 23 & 17 & 20 & 20 \\
\hline Potassium, dissolved (mg/L as $\mathrm{K}$ ) & 25 & .4 & $<.1$ & ${ }^{1} .2$ & .2 \\
\hline Acidity (mg/L as $\mathbf{H}^{+}$) & 25 & 160 & 140 & 150 & 150 \\
\hline Alkalinity ( $\mathrm{mg} / \mathrm{L}$ as $\mathrm{CaCO}_{3}$ ) & 25 & $e<1$ & $e<1$ & -- & $e<1$ \\
\hline Sulfate, dissolved (mg/L as $\left.\mathrm{SO}_{4}\right)$ & 25 & 12,000 & 8,700 & 9,860 & 9,700 \\
\hline Fluoride, dissolved (mg/L as F) & 20 & 8.4 & $<1$ & $1_{2.3}$ & 1.4 \\
\hline Silica, dissolved (mg/L as $\left.\mathrm{SiO}_{2}\right)$ & 24 & 140 & 55 & 118 & 130 \\
\hline Dissolved solids, calculated (mg/L) & 25 & el4,600 & ell,200 & e12,500 & e12,300 \\
\hline Aluminum, dissolved ( $\mu \mathrm{g} / \mathrm{L}$ as $\mathrm{Al})$ & 25 & 990,000 & 860,000 & 893,000 & 890,000 \\
\hline Arsenic, dissolved $(\mu \mathrm{g} / \mathrm{L}$ as As) & 25 & 12 & $<1$ & $1_{2.8}$ & 1 \\
\hline Barium, dissolved $(\mu \mathrm{g} / \mathrm{L}$ as $\mathrm{Ba})$ & 25 & 85 & $<100$ & 176 & $<100$ \\
\hline Beryllium, dissolved $(\mu \mathrm{g} / \mathrm{L}$ as $\mathrm{Be})$ & 25 & 100 & 40 & 86 & 94 \\
\hline Boron, dissolved $(\mu \mathrm{g} / \mathrm{L}$ as $\mathrm{B})$ & 25 & 890 & 220 & 693 & 730 \\
\hline Cadmium, dissolved $(\mu \mathrm{g} / \mathrm{L}$ as $\mathrm{Cd})$ & 24 & 98 & 71 & 84 & 85 \\
\hline Chromium, dissolved ( $\mu \mathrm{g} / \mathrm{L}$ as $\mathrm{Cr})$ & 25 & 400 & 130 & 315 & 320 \\
\hline Cobalt, dissolved ( $\mu \mathrm{g} / \mathrm{L}$ as $\mathrm{Co})$ & 25 & 9,500 & 2,900 & 4,060 & 3,600 \\
\hline Copper, dissolved $(\mu \mathrm{g} / \mathrm{L}$ as $\mathrm{Cu})$ & 25 & 120 & $<100$ & ${ }^{1} 90$ & $<200$ \\
\hline Iron, dissolved ( $\mu \mathrm{g} / \mathrm{L}$ as $\mathrm{Fe})$ & 25 & $1,200,000$ & 940,000 & $1,060,000$ & $1,100,000$ \\
\hline Lead, dissolved $(\mu \mathrm{g} / \mathrm{L}$ as $\mathrm{Pb})$ & 25 & $<10$ & $<1$ & - & $<1$ \\
\hline Lithium, dissolved $(\mu \mathrm{g} / \mathrm{L}$ as $\mathrm{Li})$ & 25 & 1,500 & 1,100 & 1,210 & 1,200 \\
\hline Manganese, dissolved $(\mu \mathrm{g} / \mathrm{L}$ as $\mathrm{Mn})$ & 24 & 3,900 & 2,900 & 3,390 & 3,400 \\
\hline Molybdenum, dissolved ( $\mu \mathrm{g} / \mathrm{L}$ as $\mathrm{Mo})$ & 25 & 3 & $<1$ & ${ }^{1} 1.5$ & 1 \\
\hline Nickel, dissolved $(\mu \mathrm{g} / \mathrm{L}$ as $\mathrm{Ni})$ & 25 & 8,700 & 7,200 & 7,830 & 7,800 \\
\hline Selenium, dissolved $(\mu \mathrm{g} / \mathrm{L}$ as $\mathrm{Se})$ & 12 & $<10$ & $<1$ & - & $<2$ \\
\hline Silver, dissolved $(\mu \mathrm{g} / \mathrm{L}$ as $\mathrm{Ag})$ & 25 & 54 & $<1$ & - & $<10$ \\
\hline Strontium, dissolved $(\mu \mathrm{g} / \mathrm{L}$ as $\mathrm{Sr})$ & 25 & 1,300 & 1,100 & 1,220 & 1,200 \\
\hline Vanadium, dissolved $(\mu \mathrm{g} / \mathrm{L}$ as $\mathrm{V})$ & 17 & 490 & $<120$ & 1345 & 340 \\
\hline Zinc, dissolved ( $\mu \mathrm{g} / \mathrm{L}$ as $\mathrm{Zn})$ & 25 & 38,000 & 32,000 & 33,700 & 33,000 \\
\hline
\end{tabular}


Table 6. Statistical summary of water-quality data for sites in the Sand Coulee Coal Area, Montana, July 1994 through September 1996 (Continued)

\begin{tabular}{|c|c|c|c|c|c|}
\hline $\begin{array}{l}\text { Property or constituent } \\
\text { (reporting unit) }\end{array}$ & $\begin{array}{c}\text { Number } \\
\text { of } \\
\text { samples }\end{array}$ & Maximum & Minimum & Mean & Median \\
\hline \multicolumn{6}{|c|}{ Site 16, 472313111104901 Mine Draln to Sand Coulee near Sand Coulee, Mont. } \\
\hline Streamflow, instantaneous $\left(\mathrm{ft}^{3} / \mathrm{s}\right)$ & 25 & 0.11 & 0.00 & 0.03 & 0.02 \\
\hline Specific conductance, onsite $(\mu \mathrm{S} / \mathrm{cm})$ & 19 & 3,480 & 3,100 & 3,290 & 3,290 \\
\hline Temperature, water $\left({ }^{\circ} \mathrm{C}\right)$ & 19 & 23.0 & 7.5 & 11.1 & 10.5 \\
\hline $\mathrm{pH}$, onsite (standard units) & 19 & 3.4 & 2.9 & 3.1 & 3.2 \\
\hline Hardness, total $\left(\mathrm{mg} / \mathrm{L}\right.$ as $\left.\mathrm{CaCO}_{3}\right)$ & 19 & 950 & 790 & 849 & 850 \\
\hline Calcium, dissolved (mg/L as $\mathrm{Ca}$ ) & 19 & 180 & 160 & 167 & 160 \\
\hline Magnesium, dissolved (mg/L as $\mathrm{Mg}$ ) & 19 & 120 & 95 & 105 & 100 \\
\hline Sodium, dissolved (mg/L as $\mathrm{Na}$ ) & 19 & 21 & 18 & 19 & 19 \\
\hline Potassium, dissolved (mg/L as $\mathrm{K}$ ) & 19 & 2 & 1.6 & 1.8 & 1.8 \\
\hline Acidity $\left(\mathrm{mg} / \mathrm{L}\right.$ as $\left.\mathrm{H}^{+}\right)$ & 19 & 44 & 35 & 41 & 42 \\
\hline Alkalinity $\left(\mathrm{mg} / \mathrm{L}\right.$ as $\mathrm{CaCO}_{3}$ ) & 19 & $e<1$ & $e<1$ & -- & $e<1$ \\
\hline Sulfate, dissolved (mg/L as $\left.\mathrm{SO}_{4}\right)$ & 19 & 3,600 & 2,700 & 3,020 & 3,000 \\
\hline Chloride, dissolved (mg/L as $\mathrm{Cl}$ ) & 19 & 15 & 3.9 & 7.4 & 5.2 \\
\hline Fluoride, dissolved ( $\mathrm{mg} / \mathrm{L}$ as $\mathrm{F}$ ) & 19 & 3.1 & $<1$ & ${ }^{1} 1.5$ & 1.3 \\
\hline Silica, dissolved (mg/L as $\mathrm{SiO}_{2}$ ) & 19 & 71 & 53 & 64 & 64 \\
\hline Dissolved solids, calculated $(\mathrm{mg} / \mathrm{L})$ & 19 & $e 4,650$ & $e 3,570$ & e3,970 & $\mathrm{e} 3,880$ \\
\hline Aluminum, dissolved ( $\mu \mathrm{g} / \mathrm{L}$ as $\mathrm{Al})$ & 19 & 270,000 & 180,000 & 229,000 & 230,000 \\
\hline Arsenic, dissolved ( $\mu \mathrm{g} / \mathrm{L}$ as As) & 19 & 11 & $<1$ & $1_{4.6}$ & 4 \\
\hline Barium, dissolved $(\mu \mathrm{g} / \mathrm{L}$ as $\mathrm{Ba})$ & 19 & 30 & $<4$ & ${ }^{1} 18$ & 25 \\
\hline Beryllium, dissolved $(\mu \mathrm{g} / \mathrm{L}$ as $\mathrm{Be})$ & 19 & 34 & 26 & 31 & 30 \\
\hline Boron, dissolved ( $\mu \mathrm{g} / \mathrm{L}$ as $\mathrm{B})$ & 18 & 360 & 130 & 273 & 280 \\
\hline Cadmium, dissolved $(\mu \mathrm{g} / \mathrm{L}$ as $\mathrm{Cd})$ & 18 & 50 & 18 & 30 & 30 \\
\hline Chromium, dissolved ( $\mu \mathrm{g} / \mathrm{L}$ as $\mathrm{Cr}$ ) & 19 & 100 & 60 & 80 & 80 \\
\hline Cobalt, dissolved ( $\mu \mathrm{g} / \mathrm{L}$ as $\mathrm{Co})$ & 19 & 2,300 & 800 & 1,320 & 1,100 \\
\hline Copper, dissolved $(\mu \mathrm{g} / \mathrm{L}$ as $\mathrm{Cu})$ & 19 & $<60$ & $<30$ & -- & $<50$ \\
\hline Iron, dissolved ( $\mu \mathrm{g} / \mathrm{L}$ as $\mathrm{Fe})$ & 19 & 410,000 & 290,000 & 349,000 & 350,000 \\
\hline Lead, dissolved ( $\mu \mathrm{g} / \mathrm{L}$ as $\mathrm{Pb})$ & 19 & $<10$ & $<1$ & $\cdots$ & $<1$ \\
\hline Lithium, dissolved $(\mu \mathrm{g} / \mathrm{L}$ as $\mathrm{Li})$ & 19 & 400 & 310 & 364 & 370 \\
\hline Manganese, dissolved $(\mu \mathrm{g} / \mathrm{L}$ as $\mathrm{Mn})$ & 19 & 1,400 & 1,100 & 1,280 & 1,300 \\
\hline Molybdenum, dissolved ( $\mu \mathrm{g} / \mathrm{L}$ as Mo) & 19 & 2 & $<1$ & -- & $<1$ \\
\hline Nickel, dissolved ( $\mu \mathrm{g} / \mathrm{L}$ as $\mathrm{Ni})$ & 19 & 2,600 & 2,100 & 2,370 & 2,300 \\
\hline Selenium, dissolved $(\mu \mathrm{g} / \mathrm{L}$ as $\mathrm{Se})$ & 6 & $<5$ & $<1$ & -- & $<2$ \\
\hline Silver, dissolved $(\mu \mathrm{g} / \mathrm{L}$ as $\mathrm{Ag})$ & 18 & 3 & $<3$ & -- & $<5$ \\
\hline Strontium, dissolved ( $\mu \mathrm{g} / \mathrm{L}$ as $\mathrm{Sr})$ & 19 & 1,100 & 940 & 1,030 & 1,000 \\
\hline Vanadium, dissolved ( $\mu \mathrm{g} / \mathrm{L}$ as $\mathrm{V})$ & 19 & 160 & $<36$ & ${ }^{1} 104$ & 98 \\
\hline Zinc, dissolved ( $\mu \mathrm{g} / \mathrm{L}$ as $\mathrm{Zn})$ & 19 & 12,000 & 9,200 & 10,300 & 10,000 \\
\hline
\end{tabular}


Table 6. Statistical summary of water-quality data for sites in the Sand Coulee Coal Area, Montana, July 1994 through September 1996 (Continued)

\begin{tabular}{|c|c|c|c|c|c|}
\hline $\begin{array}{l}\text { Property or constituent } \\
\text { (reporting unit) }\end{array}$ & $\begin{array}{c}\text { Number } \\
\text { of } \\
\text { sampies }\end{array}$ & Maximum & Minimum & Mean & Median \\
\hline \multicolumn{6}{|c|}{ Site 17, 472330111082801-Centerville wetlands Inflow at Centervilie, Mont. } \\
\hline Streamflow, instantaneous $\left(\mathrm{ft}^{3} / \mathrm{s}\right)$ & 25 & 0.02 & 0.01 & 0.02 & 0.02 \\
\hline Specific conductance, onsite $(\mu \mathrm{S} / \mathrm{cm})$ & 25 & 3,690 & 2,970 & 3,310 & 3,300 \\
\hline Temperature, water $\left({ }^{\circ} \mathrm{C}\right)$ & 25 & 12.5 & 7.5 & 9.7 & 10.0 \\
\hline pH, onsite (standard units) & 25 & 2.8 & 2.5 & 2.6 & 2.6 \\
\hline Hardness, total $\left(\mathrm{mg} / \mathrm{L}\right.$ as $\left.\mathrm{CaCO}_{3}\right)$ & 25 & 1,100 & 610 & 764 & 760 \\
\hline Calcium, dissolved $(\mathrm{mg} / \mathrm{L}$ as $\mathrm{Ca})$ & 25 & 260 & 130 & 173 & 170 \\
\hline Magnesium, dissolved (mg/L as $\mathrm{Mg}$ ) & 25 & 100 & 70 & 80 & 78 \\
\hline Sodium, dissolved (mg/L as $\mathrm{Na}$ ) & 25 & 16 & 13 & 15 & 15 \\
\hline Potassium, dissolved (mg/L as $\mathrm{K}$ ) & 25 & 1.9 & .2 & 1.1 & 1 \\
\hline Acidity $\left(\mathrm{mg} / \mathrm{L}\right.$ as $\mathrm{H}^{+}$) & 25 & 77 & 33 & 42 & 42 \\
\hline Alkalinity $\left(\mathrm{mg} / \mathrm{L}\right.$ as $\left.\mathrm{CaCO}_{3}\right)$ & 25 & $e<1$ & $e<1$ & - & $e<1$ \\
\hline Sulfate, dissolved $\left(\mathrm{mg} / \mathrm{L}\right.$ as $\left.\mathrm{SO}_{4}\right)$ & 25 & 4,400 & 2,400 & 2,996 & 2,900 \\
\hline Chloride, dissolved ( $\mathrm{mg} / \mathrm{L}$ as $\mathrm{Cl})$ & 25 & 9.7 & 1.4 & 4.2 & 2.9 \\
\hline Fluoride, dissolved (mg/L as F) & 24 & 2.4 & $<1$ & ${ }^{1} 1.0$ & 1.0 \\
\hline Silica, dissolved $\left(\mathrm{mg} / \mathrm{L}\right.$ as $\left.\mathrm{SiO}_{2}\right)$ & 25 & 94 & 67 & 82 & 84 \\
\hline Dissolved solids, calculated (mg/L) & 25 & e5,330 & $e 3,070$ & e3,820 & $\mathrm{e} 3,740$ \\
\hline Aluminum, dissolved $(\mu \mathrm{g} / \mathrm{L}$ as $\mathrm{Al})$ & 25 & 260,000 & 190,000 & 223,000 & 230,000 \\
\hline Arsenic, dissolved $(\mu \mathrm{g} / \mathrm{L}$ as As) & 25 & 3 & $<1$ & - & $<1$ \\
\hline Barium, dissolved ( $\mu \mathrm{g} / \mathrm{L}$ as $\mathrm{Ba})$ & 25 & 12 & 5 & 8 & 7 \\
\hline Beryllium, dissolved $(\mu \mathrm{g} / \mathrm{L}$ as $\mathrm{Be})$ & 25 & 37 & 24 & 31 & 31 \\
\hline Boron, dissolved $(\mu \mathrm{g} / \mathrm{L}$ as $\mathrm{B})$ & 22 & 220 & 130 & 189 & 190 \\
\hline Cadmium, dissolved $(\mu \mathrm{g} / \mathrm{L}$ as $\mathrm{Cd})$ & 22 & 32 & 11 & 25 & 27 \\
\hline Chromium, dissolved ( $\mu \mathrm{g} / \mathrm{L}$ as $\mathrm{Cr}$ ) & 25 & 40 & $<20$ & ${ }^{1} 19$ & 20 \\
\hline Cobalt, dissolved $(\mu \mathrm{g} / \mathrm{L}$ as $\mathrm{Co})$ & 19 & 520 & 310 & 428 & 440 \\
\hline Copper, dissolved $(\mu \mathrm{g} / \mathrm{L}$ as $\mathrm{Cu})$ & 25 & 130 & 80 & 106 & 110 \\
\hline Iron, dissolved $(\mu \mathrm{g} / \mathrm{L}$ as $\mathrm{Fe})$ & 25 & 290,000 & 170,000 & 233,000 & 240,000 \\
\hline Lead, dissolved $(\mu \mathrm{g} / \mathrm{L}$ as $\mathrm{Pb})$ & 25 & 4 & $<1$ & ${ }^{1} 2.2$ & 2 \\
\hline Lithium, dissolved ( $\mu \mathrm{g} / \mathrm{L}$ as $\mathrm{Li})$ & 25 & 590 & 270 & 349 & 340 \\
\hline Manganese, dissolved $(\mu \mathrm{g} / \mathrm{L}$ as $\mathrm{Mn})$ & 25 & 2,200 & 1,100 & 1,430 & 1,400 \\
\hline Molybdenum, dissolved $(\mu \mathrm{g} / \mathrm{L}$ as $\mathrm{Mo})$ & 25 & 1 & $<1$ & - & $<1$ \\
\hline Nickel, dissolved ( $\mu \mathrm{g} / \mathrm{L}$ as $\mathrm{Ni})$ & 25 & 950 & 630 & 779 & 790 \\
\hline Selenium, dissolved $(\mu \mathrm{g} / \mathrm{L}$ as Se $)$ & 12 & 2 & $<2$ & ${ }^{1} 1.8$ & 2 \\
\hline Silver, dissolved $(\mu \mathrm{g} / \mathrm{L}$ as $\mathrm{Ag})$ & 25 & 3 & $<2$ & -- & $<3$ \\
\hline Strontium, dissolved $(\mu \mathrm{g} / \mathrm{L}$ as $\mathrm{Sr})$ & 25 & 1,100 & 760 & 890 & 880 \\
\hline Vanadium, dissolved $(\mu \mathrm{g} / \mathrm{L}$ as $\mathrm{V})$ & 25 & 71 & $<12$ & ${ }^{1} 27$ & 11 \\
\hline Zinc, dissolved $(\mu \mathrm{g} / \mathrm{L}$ as $\mathrm{Zn})$ & 25 & 2,500 & 1,700 & 2,240 & 2,300 \\
\hline
\end{tabular}


Table 6. Statistical summary of water-quality data for sites in the Sand Coulee Coal Area, Montana, July 1994 through September 1996 (Continued)

\begin{tabular}{|c|c|c|c|c|c|}
\hline $\begin{array}{l}\text { Property or constituent } \\
\text { (reporting unit) }\end{array}$ & $\begin{array}{c}\text { Number } \\
\text { of } \\
\text { samples }\end{array}$ & Maximum & Minimum & Mean & Median \\
\hline \multicolumn{6}{|c|}{ Site 18,472331111083001 -Centerville wetlands outflow at Centerville, Mont. } \\
\hline Streamflow, instantaneous $\left(\mathrm{ft}^{3} / \mathrm{s}\right)$ & 16 & 0.02 & 0.002 & 0.01 & 0.01 \\
\hline Specific conductance, onsite $(\mu \mathrm{S} / \mathrm{cm})$ & 24 & 5,370 & 1,760 & 3,596 & 3,425 \\
\hline Temperature, water $\left({ }^{\circ} \mathrm{C}\right)$ & 24 & 27.5 & 0.0 & 11.8 & 12.5 \\
\hline pH, onsite (standard units) & 24 & 4.3 & 2.4 & 2.8 & 2.7 \\
\hline Hardness, total (mg/L as $\left.\mathrm{CaCO}_{3}\right)$ & 24 & 2,200 & 370 & 1,210 & 1,050 \\
\hline Calcium, dissolved (mg/L as $\mathrm{Ca}$ ) & 24 & 550 & 90 & 304 & 280 \\
\hline Magnesium, dissolved (mg/L as $\mathrm{Mg}$ ) & 24 & 190 & 34 & 109 & 96 \\
\hline Sodium, dissolved (mg/L as $\mathrm{Na}$ ) & 24 & 45 & 7.0 & 23 & 19 \\
\hline Potassium, dissolved ( $\mathrm{mg} / \mathrm{L}$ as $\mathrm{K}$ ) & 24 & 140 & 4.2 & 41 & 28 \\
\hline Acidity $\left(\mathrm{mg} / \mathrm{L}\right.$ as $\left.\mathrm{H}^{+}\right)$ & 24 & 55 & 14 & 33 & 32 \\
\hline Alkalinity $\left(\mathrm{mg} / \mathrm{L}\right.$ as $\left.\mathrm{CaCO}_{3}\right)$ & 24 & $e<1$ & $e<1$ & - & $e<1$ \\
\hline Sulfate, dissolved ( $\mathrm{mg} / \mathrm{L}$ as $\mathrm{SO}_{4}$ ) & 24 & 4,600 & 1,100 & 3,130 & 3,050 \\
\hline Chloride, dissolved (mg/L as $\mathrm{Cl}$ ) & 24 & 44 & 2.4 & 18 & 14 \\
\hline Fluoride, dissolved (mg/L as F) & 23 & 1.5 & $<1$ & ${ }^{1} 1.0$ & $<1$ \\
\hline Silica, dissolved (mg/L as $\mathrm{SiO}_{2}$ ) & 23 & 75 & 26 & 56 & 61 \\
\hline Dissolved solids, calculated (mg/L) & 24 & e5,870 & $\mathrm{el}, 440$ & $e 4,010$ & e3,920 \\
\hline Aluminum, dissolved $(\mu \mathrm{g} / \mathrm{L}$ as $\mathrm{Al})$ & 24 & 280,000 & 84,000 & 195,000 & 200,000 \\
\hline Arsenic, dissolved ( $\mu \mathrm{g} / \mathrm{L}$ as As) & 24 & 3 & $<1$ & -- & $<1$ \\
\hline Barium, dissolved $(\mu \mathrm{g} / \mathrm{L}$ as $\mathrm{Ba})$ & 24 & 39 & $<3$ & ${ }^{1} 12$ & 8 \\
\hline Beryllium, dissolved $(\mu \mathrm{g} / \mathrm{L}$ as $\mathrm{Be})$ & 24 & 44 & 11 & 28 & 28 \\
\hline Boron, dissolved $(\mu \mathrm{g} / \mathrm{L}$ as $\mathrm{B})$ & 24 & 410 & 60 & 203 & 190 \\
\hline Cadmium, dissolved $(\mu \mathrm{g} / \mathrm{L}$ as $\mathrm{Cd})$ & 19 & 30 & 12 & 22 & 21 \\
\hline Chromium, dissolved ( $\mu \mathrm{g} / \mathrm{L}$ as $\mathrm{Cr})$ & 24 & 40 & $<20$ & ${ }^{1} 15$ & $<20$ \\
\hline Cobalt, dissolved ( $\mu \mathrm{g} / \mathrm{L}$ as $\mathrm{Co})$ & 24 & 640 & 190 & 422 & 400 \\
\hline Copper, dissolved $(\mu \mathrm{g} / \mathrm{L}$ as $\mathrm{Cu})$ & 24 & 130 & $<30$ & 174 & 70 \\
\hline Iron, dissolved ( $\mu \mathrm{g} / \mathrm{L}$ as $\mathrm{Fe})$ & 24 & 240,000 & 50,000 & 129,000 & 105,000 \\
\hline Lead, dissolved ( $\mu \mathrm{g} / \mathrm{L}$ as $\mathrm{Pb})$ & 24 & 1 & $<1$ & -- & $<1$ \\
\hline Lithium, dissolved $(\mu \mathrm{g} / \mathrm{L}$ as $\mathrm{Li})$ & 24 & 650 & 120 & 395 & 380 \\
\hline Manganese, dissolved ( $\mu \mathrm{g} / \mathrm{L}$ as $\mathrm{Mn})$ & 24 & 6,100 & 740 & 3,020 & 2,500 \\
\hline Molybdenum, dissolved ( $\mu \mathrm{g} / \mathrm{L}$ as $\mathrm{Mo})$ & 24 & 1 & $<1$ & - & $<1$ \\
\hline Nickel, dissolved ( $\mu \mathrm{g} / \mathrm{L}$ as $\mathrm{Ni})$ & 24 & 1,000 & 300 & 695 & 695 \\
\hline Selenium, dissolved $(\mu \mathrm{g} / \mathrm{L}$ as $\mathrm{Se})$ & 11 & 1 & $<1$ & - & $<2$ \\
\hline Silver, dissolved $(\mu \mathrm{g} / \mathrm{L}$ as $\mathrm{Ag})$ & 24 & 2 & $<2$ & - & $<3$ \\
\hline Strontium, dissolved ( $\mu \mathrm{g} / \mathrm{L}$ as $\mathrm{Sr})$ & 24 & 2,200 & 390 & 1,260 & 1,100 \\
\hline Vanadium, dissolved ( $\mu \mathrm{g} / \mathrm{L}$ as $\mathrm{V})$ & 24 & 30 & $<6$ & - & $<18$ \\
\hline Zinc, dissolved ( $\mu \mathrm{g} / \mathrm{L}$ as $\mathrm{Zn})$ & 24 & 2,500 & 820 & 1,750 & 1,700 \\
\hline
\end{tabular}


Table 6. Statistical summary of water-quality data for sites in the Sand Coulee Coal Area, Montana, July 1994 through September 1996 (Continued)

\begin{tabular}{|c|c|c|c|c|c|}
\hline $\begin{array}{l}\text { Property or constituent } \\
\text { (reporting unit) }\end{array}$ & $\begin{array}{c}\text { Number } \\
\text { of } \\
\text { samples }\end{array}$ & Maximum & Minimum & Mean & Medlan \\
\hline \multicolumn{6}{|c|}{ Site 19, 472334111104401-Mount Oregon Mine draln to Kate's Coulee at Sand Coulee, Mont. } \\
\hline Streamflow, instantaneous $\left(\mathrm{ft}^{3} / \mathrm{s}\right)$ & 25 & 0.18 & 0.02 & 0.07 & 0.07 \\
\hline Specific conductance, onsite $(\mu \mathrm{S} / \mathrm{cm})$ & 25 & 3,010 & 2,690 & 2,830 & 2,860 \\
\hline Temperature, water $\left({ }^{\circ} \mathrm{C}\right)$ & 25 & 15.0 & 10.0 & 11.4 & 11.0 \\
\hline $\mathrm{pH}$, onsite (standard units) & 25 & 4.7 & 4.0 & 4.2 & 4.1 \\
\hline Hardness, total $\left(\mathrm{mg} / \mathrm{L}\right.$ as $\left.\mathrm{CaCO}_{3}\right)$ & 25 & 960 & 900 & 939 & 940 \\
\hline Calcium, dissolved (mg/L as Ca) & 25 & 170 & 160 & 166 & 170 \\
\hline Magnesium, dissolved (mg/L as $\mathrm{Mg}$ ) & 25 & 130 & 120 & 127 & 130 \\
\hline Sodium, dissolved (mg/L as $\mathrm{Na})$ & 25 & 25 & 22 & 24 & 24 \\
\hline Potassium, dissolved (mg/L as $\mathrm{K}$ ) & 24 & 5.2 & 4.1 & 4.6 & 4.6 \\
\hline Acidity $\left(\mathrm{mg} / \mathrm{L}\right.$ as $\left.\mathrm{H}^{+}\right)$ & 25 & 33 & 27 & 30 & 30 \\
\hline Alkalinity $\left(\mathrm{mg} / \mathrm{L}\right.$ as $\left.\mathrm{CaCO}_{3}\right)$ & 25 & $e<1$ & $e<1$ & -- & $e<1$ \\
\hline Sulfate, dissolved (mg/L as $\left.\mathrm{SO}_{4}\right)$ & 25 & 3,700 & 2,200 & 2,700 & 2,500 \\
\hline Chloride, dissolved (mg/L as $\mathrm{Cl}$ ) & 25 & 17 & 3 & 6.2 & 4.5 \\
\hline Fluoride, dissolved (mg/L as F) & 24 & 3.4 & $<1$ & $1^{\prime} 2.0$ & 2.1 \\
\hline Silica, dissolved ( $\mathrm{mg} / \mathrm{L}$ as $\mathrm{SiO}_{2}$ ) & 25 & 41 & 34 & 38 & 37 \\
\hline Dissolved solids, calculated (mg/L) & 25 & $\mathrm{e} 4,500$ & e2,990 & $\mathrm{e} 3,540$ & e3,390 \\
\hline Aluminum, dissolved ( $\mu \mathrm{g} / \mathrm{L}$ as $\mathrm{Al})$ & 25 & 180,000 & 140,000 & 164,000 & 170,000 \\
\hline Arsenic, dissolved ( $\mu \mathrm{g} / \mathrm{L}$ as As) & 25 & 24 & 6 & 14 & 14 \\
\hline Barium, dissolved $(\mu \mathrm{g} / \mathrm{L}$ as $\mathrm{Ba})$ & 25 & 28 & 12 & 18 & 14 \\
\hline Beryllium, dissolved ( $\mu \mathrm{g} / \mathrm{L}$ as $\mathrm{Be})$ & 25 & 35 & 23 & 25 & 25 \\
\hline Boron, dissolved ( $\mu \mathrm{g} / \mathrm{L}$ as B) & 21 & 310 & 240 & 265 & 260 \\
\hline Cadmium, dissolved ( $\mu \mathrm{g} / \mathrm{L}$ as $\mathrm{Cd})$ & 18 & 9 & 6 & 8 & 8 \\
\hline Chromium, dissolved $(\mu \mathrm{g} / \mathrm{L}$ as $\mathrm{Cr})$ & 25 & 50 & 20 & 33 & 30 \\
\hline Cobalt, dissolved ( $\mu \mathrm{g} / \mathrm{L}$ as $\mathrm{Co})$ & 18 & 740 & 510 & 651 & 665 \\
\hline Copper, dissolved $(\mu \mathrm{g} / \mathrm{L}$ as $\mathrm{Cu})$ & 25 & $<60$ & $<30$ & -- & $<30$ \\
\hline Iron, dissolved ( $\mu \mathrm{g} / \mathrm{L}$ as $\mathrm{Fe})$ & 25 & 340,000 & 230,000 & 296,000 & 290,000 \\
\hline Lead, dissolved $(\mu \mathrm{g} / \mathrm{L}$ as $\mathrm{Pb})$ & 25 & 2 & $<1$ & 1.9 & $<10$ \\
\hline Lithium, dissolved ( $\mu \mathrm{g} / \mathrm{L}$ as $\mathrm{Li})$ & 25 & 470 & 380 & 418 & 420 \\
\hline Manganese, dissolved ( $\mu \mathrm{g} / \mathrm{L}$ as $\mathrm{Mn})$ & 25 & 1,300 & 1,200 & 1,240 & 1,200 \\
\hline Molybdenum, dissolved ( $\mu \mathrm{g} / \mathrm{L}$ as $\mathrm{Mo}$ ) & 25 & 12 & $<30$ & ${ }^{1} 11$ & 10 \\
\hline Nickel, dissolved $(\mu \mathrm{g} / \mathrm{L}$ as $\mathrm{Ni})$ & 25 & 1,600 & 1,400 & 1,530 & 1,500 \\
\hline Selenium, dissolved ( $\mu \mathrm{g} / \mathrm{L}$ as Se) & 12 & $<5$ & $<1$ & - & $<1$ \\
\hline Silver, dissolved $(\mu \mathrm{g} / \mathrm{L}$ as $\mathrm{Ag})$ & 25 & 10 & $<3$ & - & $<3$ \\
\hline Strontium, dissolved ( $\mu \mathrm{g} / \mathrm{L}$ as $\mathrm{Sr})$ & 25 & 1,300 & 1,100 & 1,180 & 1,200 \\
\hline Vanadium, dissolved $(\mu \mathrm{g} / \mathrm{L}$ as $\mathrm{V})$ & 25 & 160 & 24 & 102 & 94 \\
\hline Zinc, dissolved ( $\mu \mathrm{g} / \mathrm{L}$ as $\mathrm{Zn})$ & 25 & 6,600 & 5,500 & 6,080 & 6,100 \\
\hline
\end{tabular}


Table 6. Statistical summary of water-quality data for sites in the Sand Coulee Coal Area, Montana, July 1994 through September 1996 (Continued)

\begin{tabular}{|c|c|c|c|c|c|}
\hline $\begin{array}{l}\text { Property or constituent } \\
\text { (reporting unit) }\end{array}$ & $\begin{array}{c}\text { Number } \\
\text { of } \\
\text { samples }\end{array}$ & Maximum & Minimum & Mean & Median \\
\hline \multicolumn{6}{|c|}{ Site 20, 472346111102401-Nelson Mine draln to Sand Coulee at Sand Coulee, Mont. } \\
\hline Streamflow, instantaneous $\left(\mathrm{ft}^{3} / \mathrm{s}\right)$ & 25 & 0.08 & 0.009 & 0.03 & 0.02 \\
\hline Specific conductance, onsite $(\mu \mathrm{S} / \mathrm{cm})$ & 25 & $\mathbf{8 , 9 8 0}$ & 6,810 & 7,800 & 7,970 \\
\hline Temperature, water $\left({ }^{\circ} \mathrm{C}\right)$ & 25 & 17.5 & 5.5 & 11.6 & 11.5 \\
\hline pH, onsite (standard units) & 25 & 3.0 & 2.3 & 2.6 & 2.6 \\
\hline Hardness, total $\left(\mathrm{mg} / \mathrm{L}\right.$ as $\left.\mathrm{CaCO}_{3}\right)$ & 25 & 1,800 & 1,400 & 1,580 & 1,600 \\
\hline Calcium, dissolved (mg/L as $\mathrm{Ca}$ ) & 25 & 280 & 190 & 249 & 250 \\
\hline Magnesium, dissolved (mg/L as $\mathrm{Mg}$ ) & 25 & 270 & 210 & 232 & 230 \\
\hline Sodium, dissolved (mg/L as Na) & 25 & 24 & 16 & 20 & 20 \\
\hline Potassium, dissolved ( $\mathrm{mg} / \mathrm{L}$ as $\mathrm{K})$ & 25 & 2 & .3 & .8 & .7 \\
\hline Acidity (mg/L as $\mathrm{H}^{+}$) & 25 & 200 & 140 & 168 & 170 \\
\hline Alkalinity (mg/L as $\mathrm{CaCO}_{3}$ ) & 25 & $e<1$ & $e<1$ & -- & $e<1$ \\
\hline Sulfate, dissolved ( $\mathrm{mg} / \mathrm{L}$ as $\mathrm{SO}_{4}$ ) & 24 & 14,000 & 8,900 & 10,900 & 11,000 \\
\hline Fluoride, dissolved (mg/L as F) & 18 & 7.3 & $<1$ & ${ }^{1} 2.9$ & 1.6 \\
\hline Silica, dissolved (mg/L as $\mathrm{SiO}_{2}$ ) & 24 & 150 & 59 & 126 & 140 \\
\hline Dissolved solids, calculated $(\mathrm{mg} / \mathrm{L})$ & 24 & el7,200 & el1,600 & $\mathrm{e} 14,000$ & el4,000 \\
\hline Aluminum, dissolved $(\mu \mathrm{g} / \mathrm{L}$ as $\mathrm{Al})$ & 25 & $1,000,000$ & 740,000 & 889,000 & 890,000 \\
\hline Arsenic, dissolved ( $\mu \mathrm{g} / \mathrm{L}$ as As) & 24 & 76 & 16 & 53 & 51 \\
\hline Barium, dissolved $(\mu \mathrm{g} / \mathrm{L}$ as $\mathrm{Ba})$ & 25 & 44 & $<10$ & ${ }^{1} 36$ & $<100$ \\
\hline Beryllium, dissolved ( $\mu \mathrm{g} / \mathrm{L}$ as $\mathrm{Be})$ & 25 & 140 & 40 & 96 & 99 \\
\hline Boron, dissolved $(\mu \mathrm{g} / \mathrm{L}$ as B) & 21 & 1,000 & 620 & 781 & 790 \\
\hline Cadmium, dissolved ( $\mu \mathrm{g} / \mathrm{L}$ as $\mathrm{Cd})$ & 24 & 120 & 84 & 106 & 110 \\
\hline Chromium, dissolved ( $\mu \mathrm{g} / \mathrm{L}$ as $\mathrm{Cr})$ & 25 & 400 & 270 & 325 & 320 \\
\hline Cobalt, dissolved $(\mu \mathrm{g} / \mathrm{L}$ as Co) & 25 & 5,600 & 1,700 & 2,540 & 2,200 \\
\hline Copper, dissolved ( $\mu \mathrm{g} / \mathrm{L}$ as $\mathrm{Cu})$ & 25 & 650 & 230 & 369 & 370 \\
\hline Iron, dissolved $(\mu \mathrm{g} / \mathrm{L}$ as $\mathrm{Fe})$ & 25 & $2,000,000$ & $1,200,000$ & $1,390,000$ & $1,500,000$ \\
\hline Lead, dissolved $(\mu \mathrm{g} / \mathrm{L}$ as $\mathrm{Pb})$ & 25 & 1 & $<1$ & -. & $<1$ \\
\hline Lithium, dissolved ( $\mu \mathrm{g} / \mathrm{L}$ as $\mathrm{Li})$ & 25 & 1,400 & 880 & 1,010 & 970 \\
\hline Manganese, dissolved ( $\mu \mathrm{g} / \mathrm{L}$ as $\mathrm{Mn}$ ) & 25 & 12,000 & 7,100 & 8,650 & 8,200 \\
\hline Molybdenum, dissolved ( $\mu \mathrm{g} / \mathrm{L}$ as $\mathrm{Mo})$ & 25 & 6 & $<1$ & $1_{3}$ & 3 \\
\hline Nickel, dissolved ( $\mu \mathrm{g} / \mathrm{L}$ as $\mathrm{Ni})$ & 25 & 5,500 & 4,300 & 4,760 & 4,800 \\
\hline Selenium, dissolved ( $\mu \mathrm{g} / \mathrm{L}$ as $\mathrm{Se})$ & 12 & $<25$ & $<2$ & - & $<5$ \\
\hline Silver, dissolved $(\mu \mathrm{g} / \mathrm{L}$ as $\mathrm{Ag})$ & 24 & $<25$ & $<1$ & - & $<12$ \\
\hline Strontium, dissolved ( $\mu \mathrm{g} / \mathrm{L}$ as $\mathrm{Sr})$ & 25 & 1,800 & 1,400 & 1,560 & 1,600 \\
\hline Vanadium, dissolved ( $\mu \mathrm{g} / \mathrm{L}$ as V) & 18 & 660 & $<120$ & ${ }^{1} 385$ & 360 \\
\hline Zinc, dissolved $(\mu \mathrm{g} / \mathrm{L}$ as $\mathrm{Zn})$ & 25 & 19,000 & 15,000 & 17,600 & 18,000 \\
\hline
\end{tabular}


Table 6. Statistical summary of water-quality data for sites in the Sand Coulee Coal Area, Montana, July 1994 through September 1996 (Continued)

\begin{tabular}{|c|c|c|c|c|c|}
\hline $\begin{array}{l}\text { Property or constltuent } \\
\text { (reporting unit) }\end{array}$ & $\begin{array}{c}\text { Number } \\
\text { of } \\
\text { samples }\end{array}$ & Maximum & Minimum & Mean & Median \\
\hline \multicolumn{6}{|c|}{ Site 21, 472309110551201-Lewis Coulee below mine adit, at Belt, Mont. } \\
\hline Streamflow, instantaneous $\left(\mathrm{ft}^{3} / \mathrm{s}\right)$ & 25 & 0.30 & 0.004 & 0.04 & 0.01 \\
\hline Specific conductance, onsite $(\mu \mathrm{S} / \mathrm{cm})$ & 25 & 3,810 & 818 & 3,110 & 3,510 \\
\hline Temperature, water $\left({ }^{\circ} \mathrm{C}\right)$ & 25 & 24.0 & .5 & 11.4 & 11.5 \\
\hline pH, onsite (standard units) & 25 & 7.4 & 3 & 3.9 & 3.2 \\
\hline Hardness, total $\left(\mathrm{mg} / \mathrm{L}\right.$ as $\left.\mathrm{CaCO}_{3}\right)$ & 25 & 1,000 & 370 & 876 & 950 \\
\hline Calcium, dissolved ( $\mathrm{mg} / \mathrm{L}$ as $\mathrm{Ca}$ ) & 25 & 200 & 58 & 167 & 180 \\
\hline Magnesium, dissolved (mg/L as $\mathrm{Mg}$ ) & 25 & 130 & 54 & 111 & 120 \\
\hline Sodium, dissolved (mg/L as $\mathrm{Na}$ ) & 25 & 60 & 19 & 25 & 24 \\
\hline Potassium, dissolved ( $\mathrm{mg} / \mathrm{L}$ as $\mathrm{K}$ ) & 25 & 7.7 & 4.4 & 5.8 & 5.8 \\
\hline Acidity (mg/L as $\mathrm{H}^{+}$) & 25 & 55 & $<.1$ & 137 & 45 \\
\hline Alkalinity $\left(\mathrm{mg} / \mathrm{L}\right.$ as $\mathrm{CaCO}_{3}$ ) & 25 & $e<1$ & $e<1$ & -- & $e<1$ \\
\hline Sulfate, dissolved $\left(\mathrm{mg} / \mathrm{L}\right.$ as $\left.\mathrm{SO}_{4}\right)$ & 25 & 4,000 & 180 & 2,880 & 3,200 \\
\hline Chloride, dissolved (mg/L as $\mathrm{Cl}$ ) & 25 & 17 & 7.2 & 11 & 10 \\
\hline Fluoride, dissolved (mg/L as F) & 21 & 2.2 & $<1$ & ${ }^{1} 1.2$ & $<1$ \\
\hline Silica, dissolved (mg/L as $\mathrm{SiO}_{2}$ ) & 25 & 76 & 7.9 & 59 & 69 \\
\hline Dissolved solids, calculated (mg/L) & 25 & $\mathrm{e} 5,290$ & e485 & $\mathrm{e} 3,860$ & $\mathrm{e} 4,310$ \\
\hline Aluminum, dissolved $(\mu \mathrm{g} / \mathrm{L}$ as $\mathrm{Al})$ & 25 & 280,000 & 20 & 193,000 & 230,000 \\
\hline Arsenic, dissolved ( $\mu \mathrm{g} / \mathrm{L}$ as As) & 25 & 2 & $<1$ & -- & $<1$ \\
\hline Barium, dissolved $(\mu \mathrm{g} / \mathrm{L}$ as $\mathrm{Ba})$ & 25 & 130 & 9 & 29 & 20 \\
\hline Beryllium, dissolved $(\mu \mathrm{g} / \mathrm{L}$ as $\mathrm{Be})$ & 25 & 32 & $<.5$ & ${ }^{1} 19$ & 19 \\
\hline Boron, dissolved ( $\mu \mathrm{g} / \mathrm{L}$ as $\mathrm{B})$ & 22 & 290 & 40 & 220 & 250 \\
\hline Cadmium, dissolved $(\mu \mathrm{g} / \mathrm{L}$ as $\mathrm{Cd})$ & 23 & 21 & $<1$ & ${ }^{1} 11$ & 10 \\
\hline Chromium, dissolved ( $\mu \mathrm{g} / \mathrm{L}$ as $\mathrm{Cr}$ ) & 25 & 60 & $<5$ & ${ }^{1} 36$ & 30 \\
\hline Cobalt, dissolved ( $\mu \mathrm{g} / \mathrm{L}$ as $\mathrm{Co})$ & 25 & 3,300 & 20 & 871 & 750 \\
\hline Copper, dissolved $(\mu \mathrm{g} / \mathrm{L}$ as $\mathrm{Cu})$ & 25 & 80 & $<10$ & ${ }^{1} 29$ & $<50$ \\
\hline Iron, dissolved $(\mu \mathrm{g} / \mathrm{L}$ as $\mathrm{Fe})$ & 25 & 560,000 & 2,200 & 373,000 & 440,000 \\
\hline Lead, dissolved ( $\mu \mathrm{g} / \mathrm{L}$ as $\mathrm{Pb})$ & 25 & 2 & $<1$ & ${ }^{1} 1.6$ & $<10$ \\
\hline Lithium, dissolved ( $\mu \mathrm{g} / \mathrm{L}$ as $\mathrm{Li})$ & 25 & 480 & 47 & 360 & 410 \\
\hline Manganese, dissolved ( $\mu \mathrm{g} / \mathrm{L}$ as $\mathrm{Mn})$ & 25 & 2,100 & 56 & 1,320 & 1,500 \\
\hline Molybdenum, dissolved ( $\mu \mathrm{g} / \mathrm{L}$ as Mo) & 25 & 10 & $<1$ & ${ }^{1} .8$ & $<1$ \\
\hline Nickel, dissolved ( $\mu \mathrm{g} / \mathrm{L}$ as $\mathrm{Ni})$ & 25 & 1,600 & 20 & 1,160 & 1,300 \\
\hline Selenium, dissolved ( $\mu \mathrm{g} / \mathrm{L}$ as $\mathrm{Se})$ & 12 & 2 & $<1$ & - & $<1$ \\
\hline Silver, dissolved $(\mu \mathrm{g} / \mathrm{L}$ as $\mathrm{Ag})$ & 25 & 18 & $<1$ & ${ }^{1} 1.7$ & $<5$ \\
\hline Strontium, dissolved ( $\mu \mathrm{g} / \mathrm{L}$ as $\mathrm{Sr}$ ) & 25 & 1,400 & 400 & 1,150 & 1,200 \\
\hline Vanadium, dissolved ( $\mu \mathrm{g} / \mathrm{L}$ as $\mathrm{V})$ & 25 & 110 & $<6$ & ${ }^{1} 56$ & 59 \\
\hline Zinc, dissolved $(\mu \mathrm{g} / \mathrm{L}$ as $\mathrm{Zn})$ & 25 & 5,600 & 32 & 3,880 & 4,500 \\
\hline
\end{tabular}


Table 6. Statistical summary of water-quality data for sites in the Sand Coulee Coal Area, Montana, July 1994 through September 1996 (Continued)

\begin{tabular}{|c|c|c|c|c|c|}
\hline $\begin{array}{l}\text { Property or constituent } \\
\text { (reporting unit) }\end{array}$ & $\begin{array}{c}\text { Number } \\
\text { of } \\
\text { samples }\end{array}$ & Maximum & Minimum & Mean & Median \\
\hline \multicolumn{6}{|c|}{ Site 22, 472446111085101-Plpe spring at Tracy, Mont. } \\
\hline Streamflow, instantaneous $\left(\mathrm{ft}^{3} / \mathrm{s}\right)$ & 25 & 0.61 & 0.03 & 0.09 & 0.05 \\
\hline Specific conductance, onsite $(\mu \mathrm{S} / \mathrm{cm})$ & 25 & 2,360 & 1,410 & 1,620 & 1,620 \\
\hline Temperature, water $\left({ }^{\circ} \mathrm{C}\right)$ & 25 & 11.5 & 8.5 & 10.7 & 10.5 \\
\hline $\mathrm{pH}$, onsite (standard units) & 25 & 3.1 & 2.4 & 2.9 & 2.9 \\
\hline Hardness, total $\left(\mathrm{mg} / \mathrm{L}\right.$ as $\left.\mathrm{CaCO}_{3}\right)$ & 25 & 540 & 410 & 461 & 460 \\
\hline Calcium, dissolved (mg/L as $\mathrm{Ca}$ ) & 25 & 91 & 63 & 75 & 73 \\
\hline Magnesium, dissolved ( $\mathrm{mg} / \mathrm{L}$ as $\mathrm{Mg}$ ) & 25 & 76 & 57 & 66 & 67 \\
\hline Sodium, dissolved (mg/L as $\mathrm{Na}$ ) & 25 & 25 & 19 & 22 & 22 \\
\hline Potassium, dissolved (mg/L as $\mathrm{K})$ & 25 & 4.9 & 1.7 & 2.5 & 2.5 \\
\hline Acidity $\left(\mathrm{mg} / \mathrm{L}\right.$ as $\left.\mathrm{H}^{+}\right)$ & 24 & 13 & 4.2 & 5.9 & 6.0 \\
\hline Alkalinity $\left(\mathrm{mg} / \mathrm{L}\right.$ as $\left.\mathrm{CaCO}_{3}\right)$ & 25 & $e<1$ & $e<1$ & -- & $e<1$ \\
\hline Sulfate, dissolved $\left(\mathrm{mg} / \mathrm{L}\right.$ as $\left.\mathrm{SO}_{4}\right)$ & 25 & 1,300 & 640 & 831 & 810 \\
\hline Chloride, dissolved ( $\mathrm{mg} / \mathrm{L}$ as $\mathrm{Cl}$ ) & 25 & 8.1 & 5.4 & 6.2 & 5.9 \\
\hline Fluoride, dissolved (mg/L as F) & 25 & 2.5 & .5 & 1.2 & 1 \\
\hline Silica, dissolved (mg/L as $\mathrm{SiO}_{2}$ ) & 25 & 54 & 38 & 46 & 45 \\
\hline Dissolved solids, calculated (mg/L) & 25 & el,670 & e875 & el,090 & el, 080 \\
\hline Aluminum, dissolved ( $\mu \mathrm{g} / \mathrm{L}$ as $\mathrm{Al})$ & 25 & 67,000 & 22,000 & 32,000 & 34,000 \\
\hline Arsenic, dissolved ( $\mu \mathrm{g} / \mathrm{L}$ as As) & 25 & 1 & $<1$ & -- & $<1$ \\
\hline Barium, dissolved ( $\mu \mathrm{g} / \mathrm{L}$ as $\mathrm{Ba})$ & 25 & 7 & $<2$ & $t_{4}$ & 4 \\
\hline Beryllium, dissolved ( $\mu \mathrm{g} / \mathrm{L}$ as $\mathrm{Be})$ & 25 & 12 & 4 & 6 & 6 \\
\hline Boron, dissolved $(\mu \mathrm{g} / \mathrm{L}$ as $\mathrm{B})$ & 25 & 180 & 130 & 157 & 160 \\
\hline Cadmium, dissolved ( $\mu \mathrm{g} / \mathrm{L}$ as $\mathrm{Cd})$ & 25 & 16 & $<10$ & ${ }^{1} 8$ & 7 \\
\hline Chromium, dissolved $(\mu \mathrm{g} / \mathrm{L}$ as $\mathrm{Cr})$ & 25 & 7 & $<5$ & -- & $<5$ \\
\hline Cobalt, dissolved $(\mu \mathrm{g} / \mathrm{L}$ as $\mathrm{Co})$ & 25 & 320 & 150 & 189 & 190 \\
\hline Copper, dissolved $(\mu \mathrm{g} / \mathrm{L}$ as $\mathrm{Cu})$ & 25 & 60 & $<10$ & ${ }^{1} 15$ & 10 \\
\hline Iron, dissolved ( $\mu \mathrm{g} / \mathrm{L}$ as $\mathrm{Fe})$ & 25 & 39,000 & 3,400 & 6,800 & 5,900 \\
\hline Lead, dissolved ( $\mu \mathrm{g} / \mathrm{L}$ as $\mathrm{Pb})$ & 25 & 2 & $<1$ & -- & $<1$ \\
\hline Lithium, dissolved ( $\mu \mathrm{g} / \mathrm{L}$ as $\mathrm{Li})$ & 25 & 160 & 110 & 136 & 130 \\
\hline Manganese, dissolved ( $\mu \mathrm{g} / \mathrm{L}$ as $\mathrm{Mn})$ & 25 & 930 & 480 & 578 & 580 \\
\hline Molybdenum, dissolved ( $\mu \mathrm{g} / \mathrm{L}$ as $\mathrm{Mo})$ & 25 & 2 & $<1$ & - & $<1$ \\
\hline Nickel, dissolved $(\mu \mathrm{g} / \mathrm{L}$ as $\mathrm{Ni})$ & 25 & 600 & 280 & 346 & 350 \\
\hline Selenium, dissolved $(\mu \mathrm{g} / \mathrm{L}$ as Se $)$ & 12 & 1 & $<1$ & $\mathrm{I}_{1}$ & $<2$ \\
\hline Silver, dissolved $(\mu \mathrm{g} / \mathrm{L}$ as $\mathrm{Ag})$ & 25 & 2 & $<1$ & - & $<1$ \\
\hline Strontium, dissolved ( $\mu \mathrm{g} / \mathrm{L}$ as $\mathrm{Sr}$ ) & 25 & 680 & 490 & 586 & 580 \\
\hline Vanadium, dissolved ( $\mu \mathrm{g} / \mathrm{L}$ as $\mathrm{V})$ & 25 & $<18$ & $<6$ & -. & $<6$ \\
\hline Zinc, dissolved ( $\mu \mathrm{g} / \mathrm{L}$ as $\mathrm{Zn})$ & 25 & 1,900 & 810 & 1,050 & 1,100 \\
\hline
\end{tabular}


Table 6. Statistical summary of water-quality data for sites in the Sand Coulee Coal Area, Montana, July 1994 through September 1996 (Continued)

\begin{tabular}{|c|c|c|c|c|c|}
\hline $\begin{array}{l}\text { Property or constltuent } \\
\text { (reporting unit) }\end{array}$ & $\begin{array}{c}\text { Number } \\
\text { of } \\
\text { samples }\end{array}$ & Maximum & Minimum & Mean & Medlan \\
\hline \multicolumn{6}{|c|}{ Site $23,472447111085301-S t o c k$ tank spring at Tracy, Mont. } \\
\hline Streamflow, instantaneous $\left(\mathrm{ft}^{3} / \mathrm{s}\right)$ & 20 & 0.003 & 0.002 & 0.002 & 0.002 \\
\hline Specific conductance, onsite $(\mu \mathrm{S} / \mathrm{cm})$ & 25 & 967 & 736 & 800 & 782 \\
\hline Temperature, water $\left({ }^{\circ} \mathrm{C}\right)$ & 25 & 16.0 & 4.0 & 8.8 & 9.5 \\
\hline pH, onsite (standard units) & 25 & 7.8 & 7.1 & 7.4 & 7.4 \\
\hline Hardness, total $\left(\mathrm{mg} / \mathrm{L}\right.$ as $\left.\mathrm{CaCO}_{3}\right)$ & 25 & 500 & 350 & 405 & 400 \\
\hline Calcium, dissolved (mg/L as $\mathrm{Ca}$ ) & 25 & 84 & 56 & 65 & 63 \\
\hline Magnesium, dissolved (mg/L as $\mathrm{Mg}$ ) & 25 & 72 & 52 & 59 & 58 \\
\hline Sodium, dissolved (mg/L as $\mathrm{Na}$ ) & 25 & 17 & 13 & 15 & 15 \\
\hline Potassium, dissolved (mg/L as $\mathrm{K}$ ) & 24 & 2.7 & 1.8 & 2.1 & 2.0 \\
\hline Acidity $\left(\mathrm{mg} / \mathrm{L}\right.$ as $\left.\mathrm{H}^{+}\right)$ & 24 & .4 & $<.1$ & ${ }^{1} .1$ & .1 \\
\hline Alkalinity $\left(\mathrm{mg} / \mathrm{L}\right.$ as $\left.\mathrm{CaCO}_{3}\right)$ & 24 & 239 & 216 & 228 & 228 \\
\hline Sulfate, dissolved (mg/L as $\mathrm{SO}_{4}$ ) & 24 & 300 & 140 & 182 & 170 \\
\hline Chloride, dissolved (mg/L as $\mathrm{Cl}$ ) & 24 & 6.5 & 4.9 & 5.8 & 5.8 \\
\hline Fluoride, dissolved (mg/L as F) & 24 & 1.2 & .4 & 1.0 & 1.0 \\
\hline Silica, dissolved $\left(\mathrm{mg} / \mathrm{L}\right.$ as $\left.\mathrm{SiO}_{2}\right)$ & 25 & 13 & 9.6 & 11 & 11 \\
\hline Dissolved solids, calculated (mg/L) & 24 & 622 & 425 & 478 & 462 \\
\hline Aluminum, dissolved ( $\mu \mathrm{g} / \mathrm{L}$ as $\mathrm{Al})$ & 25 & 40 & $<5$ & ${ }^{16}$ & $<10$ \\
\hline Arsenic, dissolved ( $\mu \mathrm{g} / \mathrm{L}$ as As) & 24 & $<2$ & $<1$ & -- & $<1$ \\
\hline Barium, dissolved $(\mu \mathrm{g} / \mathrm{L}$ as $\mathrm{Ba})$ & 25 & 56 & 41 & 47 & 48 \\
\hline Beryllium, dissolved ( $\mu \mathrm{g} / \mathrm{L}$ as $\mathrm{Be})$ & 25 & $<.5$ & $<.5$ & -- & $<.5$ \\
\hline Boron, dissolved ( $\mu \mathrm{g} / \mathrm{L}$ as B) & 24 & 100 & 50 & 74 & 70 \\
\hline Cadmium, dissolved $(\mu \mathrm{g} / \mathrm{L}$ as $\mathrm{Cd})$ & 23 & 4 & $<1$ & -- & $<1$ \\
\hline Chromium, dissolved ( $\mu \mathrm{g} / \mathrm{L}$ as $\mathrm{Cr}$ ) & 25 & $<5$ & $<5$ & - & $<5$ \\
\hline Cobalt, dissolved ( $\mu \mathrm{g} / \mathrm{L}$ as $\mathrm{Co})$ & 23 & $<3$ & $<1$ & - & $<3$ \\
\hline Copper, dissolved $(\mu \mathrm{g} / \mathrm{L}$ as $\mathrm{Cu})$ & 25 & $<10$ & $<10$ & -- & $<10$ \\
\hline Iron, dissolved ( $\mu \mathrm{g} / \mathrm{L}$ as $\mathrm{Fe})$ & 25 & 28 & $<3$ & $1_{5}$ & 3 \\
\hline Lead, dissolved ( $\mu \mathrm{g} / \mathrm{L}$ as $\mathrm{Pb})$ & 23 & 30 & $<1$ & $1_{7}$ & $<10$ \\
\hline Lithium, dissolved ( $\mu \mathrm{g} / \mathrm{L}$ as $\mathrm{Li})$ & 25 & 39 & 32 & 35 & 35 \\
\hline Manganese, dissolved ( $\mu \mathrm{g} / \mathrm{L}$ as $\mathrm{Mn}$ ) & 25 & 5 & $<1$ & $1_{1}$ & $<1$ \\
\hline Molybdenum, dissolved ( $\mu \mathrm{g} / \mathrm{L}$ as $\mathrm{Mo})$ & 23 & 20 & $<10$ & ${ }^{1} 6$ & $<10$ \\
\hline Nickel, dissolved ( $\mu \mathrm{g} / \mathrm{L}$ as $\mathrm{Ni})$ & 25 & 20 & $<10$ & ${ }^{15}$ & $<10$ \\
\hline Selenium, dissolved $(\mu \mathrm{g} / \mathrm{L}$ as $\mathrm{Se})$ & 12 & 3 & $<5$ & $1_{2}$ & 2 \\
\hline Silver, dissolved $(\mu \mathrm{g} / \mathrm{L}$ as $\mathrm{Ag})$ & 25 & 2 & $<1$ & -- & $<1$ \\
\hline Strontium, dissolved ( $\mu \mathrm{g} / \mathrm{L}$ as $\mathrm{Sr}$ ) & 25 & 660 & 460 & 514 & 510 \\
\hline Vanadium, dissolved ( $\mu \mathrm{g} / \mathrm{L}$ as $\mathrm{V})$ & 25 & $<6$ & $<6$ & -- & $<6$ \\
\hline Zinc, dissolved $(\mu \mathrm{g} / \mathrm{L}$ as $\mathrm{Zn})$ & 25 & 25 & 7 & 12 & 12 \\
\hline
\end{tabular}


Table 6. Statistical summary of water-quality data for sites in the Sand Coulee Coal Area, Montana, July 1994 through September 1996 (Continued)

\begin{tabular}{|c|c|c|c|c|c|}
\hline $\begin{array}{l}\text { Property or constituent } \\
\text { (reporting unit) }\end{array}$ & $\begin{array}{c}\text { Number } \\
\text { of } \\
\text { sampies }\end{array}$ & Maximum & Minimum & Mean & Median \\
\hline \multicolumn{6}{|c|}{ Site 24, 472513111082501-Johnson Badwater Mine small wetlands Inflow near Tracy, Mont. } \\
\hline Streamflow, instantaneous $\left(\mathrm{ft}^{3} / \mathrm{s}\right)$ & 25 & 0.01 & 0.007 & 0.01 & 0.01 \\
\hline Specific conductance, onsite $(\mu \mathrm{S} / \mathrm{cm})$ & 25 & 2,300 & 2,100 & 2,190 & 2,180 \\
\hline Temperature, water $\left({ }^{\circ} \mathrm{C}\right)$ & 25 & 11.5 & 8.0 & 9.9 & 10.0 \\
\hline pH, onsite (standard units) & 25 & 4.0 & 3.5 & 3.8 & 3.8 \\
\hline Hardness, total $\left(\mathrm{mg} / \mathrm{L}\right.$ as $\left.\mathrm{CaCO}_{3}\right)$ & 25 & 1,000 & 850 & 939 & 940 \\
\hline Calcium, dissolved (mg/L as $\mathrm{Ca}$ ) & 25 & 180 & 140 & 166 & 170 \\
\hline Magnesium, dissolved (mg/L as $\mathrm{Mg}$ ) & 25 & 140 & 120 & 127 & 130 \\
\hline Sodium, dissolved ( $\mathrm{mg} / \mathrm{L}$ as $\mathrm{Na}$ ) & 25 & 26 & 22 & 24 & 24 \\
\hline Potassium, dissolved ( $\mathrm{mg} / \mathrm{L}$ as $\mathrm{K}$ ) & 25 & 7.5 & 6 & 7.1 & 7.1 \\
\hline Acidity $\left(\mathrm{mg} / \mathrm{L}\right.$ as $\left.\mathrm{H}^{+}\right)$ & 25 & 14 & 9.7 & 12 & 11 \\
\hline Alkalinity (mg/L as $\mathrm{CaCO}_{3}$ ) & 25 & $\mathrm{e}<1$ & $\mathrm{e}<1$ & -- & $\mathrm{e}<1$ \\
\hline Sulfate, dissolved $\left(\mathrm{mg} / \mathrm{L}\right.$ as $\left.\mathrm{SO}_{4}\right)$ & 25 & 2,000 & 1,400 & 1,620 & 1,600 \\
\hline Chloride, dissolved ( $\mathrm{mg} / \mathrm{L}$ as $\mathrm{Cl}$ ) & 25 & 9.8 & 5.4 & 6.9 & 6.3 \\
\hline Fluoride, dissolved ( $\mathrm{mg} / \mathrm{L}$ as $\mathrm{Fl}$ ) & 25 & 8.3 & 3.2 & 6.1 & 6.2 \\
\hline Silica, dissolved (mg/L as $\left.\mathrm{SiO}_{2}\right)$ & 25 & 52 & 47 & 49 & 49 \\
\hline Dissolved solids, calculated (mg/L) & 25 & $\mathrm{e} 2,590$ & $\mathrm{el}, 920$ & e2,190 & $\mathrm{e} 2,160$ \\
\hline Aluminum, dissolved $(\mu \mathrm{g} / \mathrm{L}$ as $\mathrm{Al})$ & 25 & 57,000 & 46,000 & 51,700 & 51,000 \\
\hline Arsenic, dissolved ( $\mu \mathrm{g} / \mathrm{L}$ as $A s)$ & 25 & 1 & $<1$ & -- & $<1$ \\
\hline Barium, dissolved $(\mu \mathrm{g} / \mathrm{L}$ as $\mathrm{Ba})$ & 25 & 15 & 8 & 11 & 9 \\
\hline Beryllium, dissolved $(\mu \mathrm{g} / \mathrm{L}$ as $\mathrm{Be})$ & 25 & 27 & 19 & 24 & 24 \\
\hline Boron, dissolved ( $\mu \mathrm{g} / \mathrm{L}$ as $\mathrm{B})$ & 25 & 320 & 200 & 281 & 280 \\
\hline Cadmium, dissolved $(\mu \mathrm{g} / \mathrm{L}$ as $\mathrm{Cd})$ & 21 & 3 & $<3$ & $1_{2}$ & 2 \\
\hline Chromium, dissolved ( $\mu \mathrm{g} / \mathrm{L}$ as $\mathrm{Cr}$ ) & 25 & 20 & $<10$ & -- & $<20$ \\
\hline Cobalt, dissolved $(\mu \mathrm{g} / \mathrm{L}$ as $\mathrm{Co})$ & 18 & 520 & 400 & 449 & 440 \\
\hline Copper, dissolved ( $\mu \mathrm{g} / \mathrm{L}$ as $\mathrm{Cu})$ & 25 & $<40$ & $<20$ & -- & $<30$ \\
\hline Iron, dissolved $(\mu \mathrm{g} / \mathrm{L}$ as $\mathbf{F e})$ & 25 & 130,000 & 110,000 & 124,000 & 120,000 \\
\hline Lead, dissolved ( $\mu \mathrm{g} / \mathrm{L}$ as $\mathrm{Pb})$ & 25 & $<10$ & $<1$ & -- & $<1$ \\
\hline Lithium, dissolved $(\mu \mathrm{g} / \mathrm{L}$ as $\mathrm{Li})$ & 25 & 390 & 320 & 367 & 370 \\
\hline Manganese, dissolved ( $\mu \mathrm{g} / \mathrm{L}$ as $\mathrm{Mn})$ & 25 & 1,000 & 840 & 942 & 950 \\
\hline Molybdenum, dissolved ( $\mu \mathrm{g} / \mathrm{L}$ as $\mathrm{Mo}$ ) & 25 & 1 & $<1$ & -- & $<1$ \\
\hline Nickel, dissolved ( $\mu \mathrm{g} / \mathrm{L}$ as $\mathrm{Ni})$ & 25 & 1,100 & 880 & 980 & 970 \\
\hline Selenium, dissolved ( $\mu \mathrm{g} / \mathrm{L}$ as $\mathrm{Se})$ & 12 & $<5$ & $<1$ & -- & $<1$ \\
\hline Silver, dissolved ( $\mu \mathrm{g} / \mathrm{L}$ as $\mathrm{Ag})$ & 24 & 4 & $<2$ & -- & $<3$ \\
\hline Strontium, dissolved ( $\mu \mathrm{g} / \mathrm{L}$ as $\mathrm{Sr}$ ) & 25 & 1,300 & 1,000 & 1,160 & 1,200 \\
\hline Vanadium, dissolved $(\mu \mathrm{g} / \mathrm{L}$ as $\mathrm{V})$ & 25 & 34 & $<6$ & ${ }^{1} 14$ & $<24$ \\
\hline Zinc, dissolved ( $\mu \mathrm{g} / \mathrm{L}$ as $\mathrm{Zn})$ & 25 & 3,300 & 2,500 & 2,920 & 2,900 \\
\hline
\end{tabular}


Table 6. Statistical summary of water-quality data for sites in the Sand Coulee Coal Area, Montana, July 1994 through September 1996 (Continued)

\begin{tabular}{|c|c|c|c|c|c|}
\hline $\begin{array}{l}\text { Property or constltuent } \\
\text { (reporting unit) }\end{array}$ & $\begin{array}{c}\text { Number } \\
\text { of } \\
\text { samples }\end{array}$ & Maximum & Minimum & Mean & Median \\
\hline \multicolumn{6}{|c|}{ Site 25, 472513111082901-Johnson Badwater Mine large wetlands inflow near Tracy, Mont. } \\
\hline Streamflow, instantaneous $\left(\mathrm{ft}^{3} / \mathrm{s}\right)$ & 25 & 0.01 & 0.004 & 0.01 & 0.01 \\
\hline Specific conductance, onsite $(\mu \mathrm{S} / \mathrm{cm})$ & 25 & 4,520 & 3,560 & 4,190 & 4,320 \\
\hline Temperature, water $\left({ }^{\circ} \mathrm{C}\right)$ & 25 & 11.5 & 8.5 & 9.9 & 10.0 \\
\hline $\mathrm{pH}$, onsite (standard units) & 25 & 2.8 & 2.3 & 2.6 & 2.6 \\
\hline Hardness, total (mg/L as $\left.\mathrm{CaCO}_{3}\right)$ & 25 & 1,200 & 910 & 1,050 & 1,100 \\
\hline Calcium, dissolved (mg/L as Ca) & 25 & 190 & 150 & 170 & 170 \\
\hline Magnesium, dissolved (mg/L as $\mathrm{Mg}$ ) & 25 & 170 & 130 & 154 & 150 \\
\hline Sodium, dissolved (mg/L as $\mathrm{Na}$ ) & 25 & 27 & 23 & 25 & 25 \\
\hline Potassium, dissolved (mg/L as $\mathrm{K}$ ) & 25 & 2.3 & 1.0 & 1.5 & 1.5 \\
\hline Acidity $\left(\mathrm{mg} / \mathrm{L}\right.$ as $\left.\mathrm{H}^{+}\right)$ & 25 & 52 & 30 & 43 & 46 \\
\hline Alkalinity $\left(\mathrm{mg} / \mathrm{L}\right.$ as $\left.\mathrm{CaCO}_{3}\right)$ & 25 & $e<1$ & $e<1$ & -- & $e<1$ \\
\hline Sulfate, dissolved $\left(\mathrm{mg} / \mathrm{L}\right.$ as $\left.\mathrm{SO}_{4}\right)$ & 25 & 4,700 & 2,600 & 3,540 & 3,400 \\
\hline Chloride, dissolved ( $\mathrm{mg} / \mathrm{L}$ as $\mathrm{Cl})$ & 25 & 21 & 4.9 & 8.2 & 6.7 \\
\hline Fluoride, dissolved ( $\mathrm{mg} / \mathrm{L}$ as $\mathrm{F})$ & 24 & 6.1 & $<1$ & $1_{3.1}$ & 3.1 \\
\hline Silica, dissolved (mg/L as $\mathrm{SiO}_{2}$ ) & 25 & 87 & 71 & 76 & 76 \\
\hline Dissolved solids, calculated (mg/L) & 25 & e5,760 & e3,340 & $\mathrm{e} 4,530$ & $\mathrm{e} 4,450$ \\
\hline Aluminum, dissolved ( $\mu \mathrm{g} / \mathrm{L}$ as $\mathrm{Al})$ & 25 & 250,000 & 150,000 & 216,000 & 230,000 \\
\hline Arsenic, dissolved ( $\mu \mathrm{g} / \mathrm{L}$ as As) & 25 & $<5$ & $<1$ & - & $<1$ \\
\hline Barium, dissolved $(\mu \mathrm{g} / \mathrm{L}$ as $\mathrm{Ba})$ & 25 & 23 & $<2$ & ${ }^{1} 12$ & $<4$ \\
\hline Beryllium, dissolved $(\mu \mathrm{g} / \mathrm{L}$ as $\mathrm{Be})$ & 25 & 40 & 21 & 31 & 32 \\
\hline Boron, dissolved ( $\mu \mathrm{g} / \mathrm{L}$ as $\mathrm{B})$ & 22 & 380 & 240 & 307 & 300 \\
\hline Cadmium, dissolved $(\mu \mathrm{g} / \mathrm{L}$ as $\mathrm{Cd})$ & 25 & 100 & 58 & 78 & 77 \\
\hline Chromium, dissolved ( $\mu \mathrm{g} / \mathrm{L}$ as $\mathrm{Cr})$ & 25 & 60 & $<20$ & ${ }^{1} 33$ & 30 \\
\hline Cobalt, dissolved $(\mu \mathrm{g} / \mathrm{L}$ as Co $)$ & 25 & 3,800 & 750 & 1,310 & 1,000 \\
\hline Copper, dissolved $(\mu \mathrm{g} / \mathrm{L}$ as $\mathrm{Cu})$ & 24 & 200 & 100 & 142 & 140 \\
\hline Iron, dissolved ( $\mu \mathrm{g} / \mathrm{L}$ as $\mathrm{Fe})$ & 25 & 410,000 & 170,000 & 329,000 & 350,000 \\
\hline Lead, dissolved $(\mu \mathrm{g} / \mathrm{L}$ as $\mathrm{Pb})$ & 25 & 2 & $<1$ & 1.9 & $<10$ \\
\hline Lithium, dissolved ( $\mu \mathrm{g} / \mathrm{L}$ as $\mathrm{Li})$ & 25 & 490 & 330 & 420 & 420 \\
\hline Manganese, dissolved ( $\mu \mathrm{g} / \mathrm{L}$ as $\mathrm{Mn})$ & 25 & 1,700 & 1,100 & 1,470 & 1,500 \\
\hline Molybdenum, dissolved ( $\mu \mathrm{g} / \mathrm{L}$ as $\mathrm{Mo}$ ) & 25 & 2 & $<1$ & -- & $<1$ \\
\hline Nickel, dissolved $(\mu \mathrm{g} / \mathrm{L}$ as $\mathrm{Ni})$ & 25 & 2,500 & 1,600 & 2,120 & 2,200 \\
\hline Selenium, dissolved ( $\mu \mathrm{g} / \mathrm{L}$ as $\mathrm{Se})$ & 12 & 1 & $<1$ & -- & $<2$ \\
\hline Silver, dissolved ( $\mu \mathrm{g} / \mathrm{L}$ as $\mathrm{Ag})$ & 25 & 9 & $<2$ & -- & $<4$ \\
\hline Strontium, dissolved ( $\mu \mathrm{g} / \mathrm{L}$ as $\mathrm{Sr}$ ) & 25 & 1,200 & 910 & 1,040 & 1,000 \\
\hline Vanadium, dissolved ( $\mu \mathrm{g} / \mathrm{L}$ as $\mathrm{V})$ & 25 & 64 & $<36$ & ${ }^{1} 33$ & 22 \\
\hline Zinc, dissolved ( $\mu \mathrm{g} / \mathrm{L}$ as $\mathrm{Zn})$ & 25 & 10,000 & 6,200 & 8,420 & 8,800 \\
\hline
\end{tabular}


Table 6. Statistical summary of water-quality data for sites in the Sand Coulee Coal Area, Montana, July 1994 through September 1996 (Continued)

\begin{tabular}{|c|c|c|c|c|c|}
\hline $\begin{array}{l}\text { Property or constituent } \\
\text { (reporting unit) }\end{array}$ & $\begin{array}{c}\text { Number } \\
\text { of } \\
\text { samples }\end{array}$ & Maximum & Minimum & Mean & Median \\
\hline \multicolumn{6}{|c|}{ Site 26, 472514111082301-Johnson Badwater Mine small wetlands outflow near Tracy, Mont. } \\
\hline Streamflow, instantaneous $\left(\mathrm{ft}^{3} / \mathrm{s}\right)$ & 23 & 0.007 & $<0.001$ & 0.003 & 0.003 \\
\hline Specific conductance, onsite $(\mu \mathrm{S} / \mathrm{cm})$ & 19 & 2,780 & 2,100 & 2,480 & 2,530 \\
\hline Temperature, water $\left({ }^{\circ} \mathrm{C}\right)$ & 19 & 19.5 & 0.0 & 8.1 & 7.5 \\
\hline pH, onsite (standard units) & 19 & 5.9 & 2.6 & 3.2 & 3.0 \\
\hline Hardness, total $\left(\mathrm{mg} / \mathrm{L}\right.$ as $\left.\mathrm{CaCO}_{3}\right)$ & 19 & 1,700 & 510 & 1,140 & 1,100 \\
\hline Calcium, dissolved (mg/L as $\mathrm{Ca}$ ) & 19 & 370 & 100 & 224 & 220 \\
\hline Magnesium, dissolved (mg/L as $\mathrm{Mg}$ ) & 19 & 200 & 64 & 139 & 140 \\
\hline Sodium, dissolved (mg/L as $\mathrm{Na}$ ) & 19 & 33 & 11 & 26 & 26 \\
\hline Potassium, dissolved (mg/L as $\mathrm{K}$ ) & 19 & 9.3 & 2.4 & 7.5 & 8.0 \\
\hline Acidity $\left(\mathrm{mg} / \mathrm{L}\right.$ as $\left.\mathrm{H}^{+}\right)$ & 19 & 12 & 2 & 8.0 & 8.8 \\
\hline Alkalinity (mg/L as $\left.\mathrm{CaCO}_{3}\right)$ & 19 & $e<1$ & $e<1$ & -- & $\mathrm{e}<1$ \\
\hline Sulfate, dissolved $\left(\mathrm{mg} / \mathrm{L}\right.$ as $\left.\mathrm{SO}_{4}\right)$ & 19 & 2,500 & 1,300 & 1,840 & 1,800 \\
\hline Chloride, dissolved (mg/L as $\mathrm{Cl}$ ) & 19 & 10 & 4.8 & 7.1 & 7.3 \\
\hline Fluoride, dissolved (mg/L as F) & 19 & 9.2 & 2.7 & 6.2 & 5.9 \\
\hline Silica, dissolved (mg/L as $\mathrm{SiO}_{2}$ ) & 18 & 52 & 24 & 42 & 44 \\
\hline Dissolved solids, calculated (mg/L) & 19 & $\mathrm{e} 3,030$ & el, 780 & $\mathrm{e} 2,370$ & $\mathrm{e} 2,320$ \\
\hline Aluminum, dissolved $(\mu \mathrm{g} / \mathrm{L}$ as $\mathrm{Al})$ & 19 & 61,000 & 3,300 & 41,600 & 43,000 \\
\hline Arsenic, dissolved ( $\mu \mathrm{g} / \mathrm{L}$ as As) & 19 & $<1$ & $<1$ & -- & $<1$ \\
\hline Barium, dissolved $(\mu \mathrm{g} / \mathrm{L}$ as $\mathrm{Ba})$ & 19 & 16 & 4 & 10 & 10 \\
\hline Beryllium, dissolved ( $\mu \mathrm{g} / \mathrm{L}$ as $\mathrm{Be}$ ) & 19 & 27 & 3 & 19 & 22 \\
\hline Boron, dissolved ( $\mu \mathrm{g} / \mathrm{L}$ as B) & 19 & 330 & 190 & 265 & 270 \\
\hline Cadmium, dissolved $(\mu \mathrm{g} / \mathrm{L}$ as $\mathrm{Cd})$ & 19 & 5 & $<1$ & ${ }^{1} 2$ & 2 \\
\hline Chromium, dissolved ( $\mu \mathrm{g} / \mathrm{L}$ as $\mathrm{Cr})$ & 19 & 20 & $<5$ & -- & $<20$ \\
\hline Cobalt, dissolved $(\mu \mathrm{g} / \mathrm{L}$ as $\mathrm{Co})$ & 19 & 670 & 200 & 404 & 400 \\
\hline Copper, dissolved $(\mu \mathrm{g} / \mathrm{L}$ as $\mathrm{Cu})$ & 19 & $<30$ & $<10$ & -- & $<30$ \\
\hline Iron, dissolved ( $\mu \mathrm{g} / \mathrm{L}$ as $\mathrm{Fe})$ & 19 & 60,000 & 2,900 & 28,100 & 32,000 \\
\hline Lead, dissolved $(\mu \mathrm{g} / \mathrm{L}$ as $\mathrm{Pb})$ & 19 & 1 & $<1$ & -- & $<1$ \\
\hline Lithium, dissolved ( $\mu \mathrm{g} / \mathrm{L}$ as $\mathrm{Li})$ & 19 & 420 & 110 & 341 & 370 \\
\hline Manganese, dissolved ( $\mu \mathrm{g} / \mathrm{L}$ as $\mathrm{Mn})$ & 19 & 1,500 & 380 & 1,020 & 1,000 \\
\hline Molybdenum, dissolved ( $\mu \mathrm{g} / \mathrm{L}$ as $\mathrm{Mo}$ ) & 19 & $<10$ & $<1$ & -- & $<1$ \\
\hline Nickel, dissolved ( $\mu \mathrm{g} / \mathrm{L}$ as $\mathrm{Ni})$ & 19 & 1,100 & 230 & 803 & 860 \\
\hline Selenium, dissolved $(\mu \mathrm{g} / \mathrm{L}$ as Se $)$ & 9 & $<2$ & $<1$ & - & $<1$ \\
\hline Silver, dissolved ( $\mu \mathrm{g} / \mathrm{L}$ as $\mathrm{Ag})$ & 19 & 7 & $<1$ & - & $<3$ \\
\hline Strontium, dissolved ( $\mu \mathrm{g} / \mathrm{L}$ as $\mathrm{Sr}$ ) & 19 & 1,900 & 530 & 1,330 & 1,300 \\
\hline Vanadium, dissolved ( $\mu \mathrm{g} / \mathrm{L}$ as $\mathrm{V})$ & 19 & $<18$ & $<6$ & - & $<18$ \\
\hline Zinc, dissolved ( $\mu \mathrm{g} / \mathrm{L}$ as $\mathrm{Zn})$ & 19 & 3,400 & 530 & 2,280 & 2,400 \\
\hline
\end{tabular}


Table 6. Statistical summary of water-quality data for sites in the Sand Coulee Coal Area, Montana, July 1994 through September 1996 (Continued)

\begin{tabular}{|c|c|c|c|c|c|}
\hline $\begin{array}{l}\text { Property or constituent } \\
\text { (reporting unit) }\end{array}$ & $\begin{array}{c}\text { Number } \\
\text { of } \\
\text { samples }\end{array}$ & Maximum & Minimum & Mean & Median \\
\hline \multicolumn{6}{|c|}{ Slte 27, 472517111081001-Johnson Goodwater Mine small wetlands Inflow near Tracy, Mont. } \\
\hline Streamflow, instantaneous $\left(\mathrm{ft}^{3} / \mathrm{s}\right)$ & 25 & 0.018 & 0.0001 & 0.005 & 0.0002 \\
\hline Specific conductance, onsite $(\mu \mathrm{S} / \mathrm{cm})$ & 25 & 1,290 & 1,010 & 1,190 & 1,190 \\
\hline Temperature, water $\left({ }^{\circ} \mathrm{C}\right)$ & 25 & 16.5 & 4.5 & 9.5 & 10.0 \\
\hline pH, onsite (standard units) & 25 & 7.9 & 6.6 & 7.2 & 7.1 \\
\hline Hardness, total $\left(\mathrm{mg} / \mathrm{L}\right.$ as $\left.\mathrm{CaCO}_{3}\right)$ & 25 & 730 & 520 & 642 & 640 \\
\hline Calcium, dissolved (mg/L as $\mathrm{Ca}$ ) & 25 & 130 & 80 & 103 & 95 \\
\hline Magnesium, dissolved (mg/L as $\mathrm{Mg}$ ) & 25 & 100 & 72 & 93 & 94 \\
\hline Sodium, dissolved (mg/L as $\mathrm{Na})$ & 25 & 19 & 15 & 18 & 18 \\
\hline Potassium, dissolved (mg/L as $\mathrm{K})$ & 25 & 5.5 & 4.1 & 4.7 & 4.8 \\
\hline Acidity (mg/L as $\left.\mathrm{H}^{+}\right)$ & 25 & 1.7 & $<.1$ & ${ }^{1} .3$ & .2 \\
\hline Alkalinity $\left(\mathrm{mg} / \mathrm{L}\right.$ as $\left.\mathrm{CaCO}_{3}\right)$ & 25 & 281 & 174 & 235 & 255 \\
\hline Sulfate, dissolved ( $\mathrm{mg} / \mathrm{L}$ as $\left.\mathrm{SO}_{4}\right)$ & 25 & 480 & 270 & 432 & 450 \\
\hline Chloride, dissolved ( $\mathrm{mg} / \mathrm{L}$ as $\mathrm{Cl}$ ) & 25 & 8.8 & 6.2 & 7.2 & 7.1 \\
\hline Fluoride, dissolved (mg/L as F) & 25 & 1.6 & 1.1 & 1.3 & 1.3 \\
\hline Silica, dissolved (mg/L as $\mathrm{SiO}_{2}$ ) & 25 & 12 & 7.7 & 9.9 & 10 \\
\hline Dissolved solids, calculated $(\mathrm{mg} / \mathrm{L})$ & 25 & 912 & 640 & 812 & 811 \\
\hline Aluminum, dissolved $(\mu \mathrm{g} / \mathrm{L}$ as $\mathrm{Al})$ & 25 & 50 & $<10$ & ${ }^{1} 15$ & 9 \\
\hline Arsenic, dissolved ( $\mu \mathrm{g} / \mathrm{L}$ as As) & 25 & $<1$ & $<1$ & -- & $<1$ \\
\hline Barium, dissolved ( $\mu \mathrm{g} / \mathrm{L}$ as Ba) & 25 & 28 & 18 & 23 & 23 \\
\hline Beryllium, dissolved ( $\mu \mathrm{g} / \mathrm{L}$ as $\mathrm{Be}$ ) & 25 & .5 & $<.5$ & - & $<.5$ \\
\hline Boron, dissolved ( $\mu \mathrm{g} / \mathrm{L}$ as $\mathrm{B})$ & 25 & 140 & 90 & 116 & 120 \\
\hline Cadmium, dissolved ( $\mu \mathrm{g} / \mathrm{L}$ as $\mathrm{Cd}$ ) & 25 & $<3$ & $<1$ & - & $<1$ \\
\hline Chromium, dissolved ( $\mu \mathrm{g} / \mathrm{L}$ as $\mathrm{Cr}$ ) & 25 & $<20$ & $<5$ & -- & $<5$ \\
\hline Cobalt, dissolved ( $\mu \mathrm{g} / \mathrm{L}$ as Co) & 25 & 40 & $<1$ & ${ }^{1} 5$ & $<3$ \\
\hline Copper, dissolved $(\mu \mathrm{g} / \mathrm{L}$ as $\mathrm{Cu})$ & 25 & $<30$ & $<10$ & -- & $<10$ \\
\hline Iron, dissolved $(\mu \mathrm{g} / \mathrm{L}$ as $\mathrm{Fe})$ & 25 & 3,200 & $<3$ & $1_{356}$ & 13 \\
\hline Lead, dissolved $(\mu \mathrm{g} / \mathrm{L}$ as $\mathrm{Pb})$ & 25 & 40 & $<1$ & ${ }^{1} 7$ & $<10$ \\
\hline Lithium, dissolved ( $\mu \mathrm{g} / \mathrm{L}$ as $\mathrm{Li})$ & 25 & 97 & 67 & 90 & 92 \\
\hline Manganese, dissolved ( $\mu \mathrm{g} / \mathrm{L}$ as $\mathrm{Mn}$ ) & 25 & 170 & 2 & 43 & 32 \\
\hline Molybdenum, dissolved ( $\mu \mathrm{g} / \mathrm{L}$ as Mo) & 25 & 10 & $<1$ & -- & $<10$ \\
\hline Nickel, dissolved ( $\mu \mathrm{g} / \mathrm{L}$ as Ni) & 25 & 80 & $<10$ & ${ }^{1} 33$ & 30 \\
\hline Selenium, dissolved ( $\mu \mathrm{g} / \mathrm{L}$ as Se) & 12 & 3 & $<5$ & ${ }^{1} 2$ & 2 \\
\hline Silver, dissolved $(\mu \mathrm{g} / \mathrm{L}$ as $\mathrm{Ag})$ & 25 & 3 & $<1$ & 1.7 & $<1$ \\
\hline Strontium, dissolved ( $\mu \mathrm{g} / \mathrm{L}$ as $\mathrm{Sr}$ ) & 25 & 690 & 490 & 635 & 640 \\
\hline Vanadium, dissolved $(\mu \mathrm{g} / \mathrm{L}$ as $\mathrm{V})$ & 25 & $<18$ & $<6$ & -- & $<6$ \\
\hline Zinc, dissolved $(\mu \mathrm{g} / \mathrm{L}$ as $\mathrm{Zn})$ & 25 & 140 & 13 & 53 & 40 \\
\hline
\end{tabular}

IValue is estimated by using a log-probability regression to predict the values of data less than the minimum reporting level (Helsel and Cohn, 1988). 